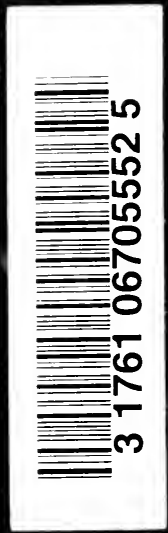




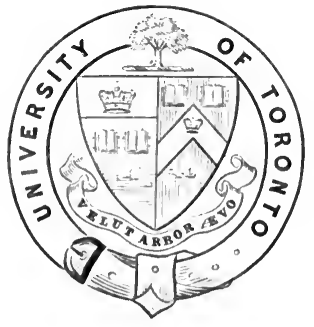

Dresented to

- The Tíbrary of the

\section{University of Toronto}

by

Mrs. Albert Gooderham 
$\$ 1.00$

Alver, pooderhauiff 
Digitized by the Internet Archive in 2007 with funding from Microsoft Corporation 


\section{ELEMENTARY LESSONS}

IN

\section{ELECTRICITY AND MAGNETISM}




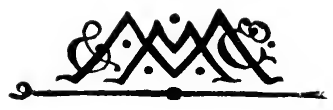




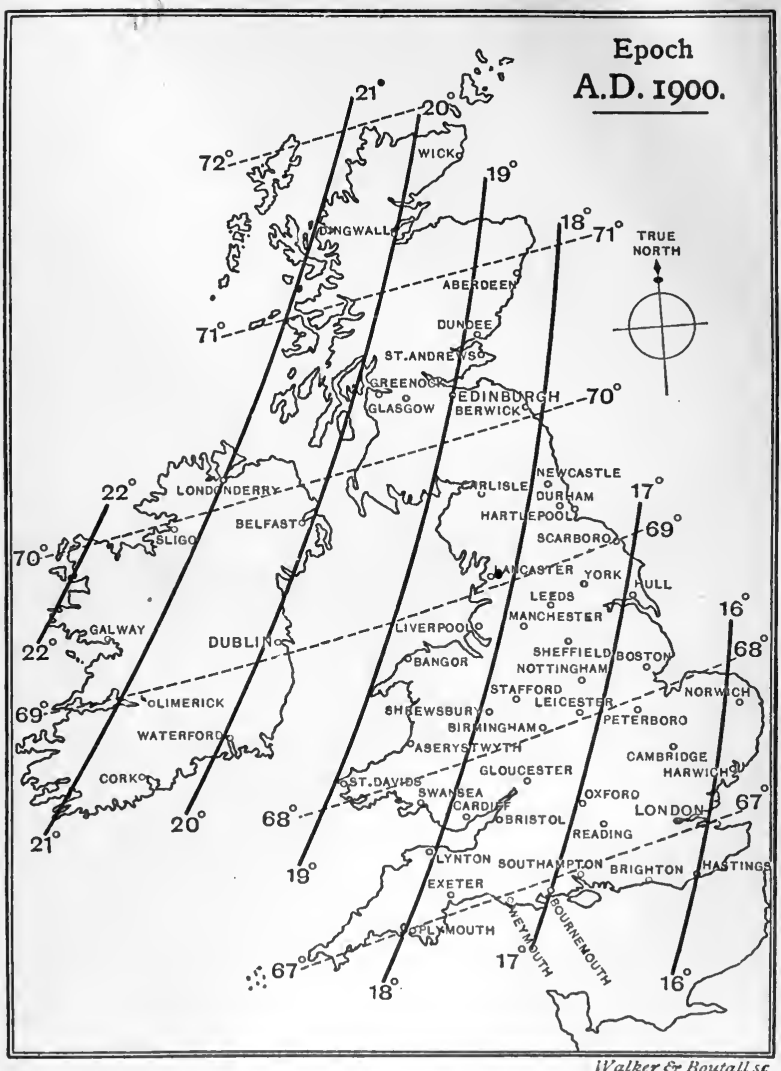

MAGNETIC CHART OF THE BRITISH ISLANDS,

SHOWING THE LINES OF EQUAL MAGNETIC DECLINATION AND THOSE OF EQUAI, MAGNETIC DIP. 


\section{PhyE}

\section{ELEMENTARY LESSONS}

IN

\section{ELECTRICITY \& MAGNETISM}

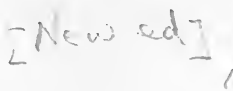

BY

\section{SILVANUS P. THOMPSON \\ D.Sc., B.A., F.R.S., F.R.A.S.}

PRINCIPAL OF AND PROFESSOR OF PHYSICS IN THE CITY AND GUILDS OF LONDON TECHNICAL COLLEGE, FINSBURY

LATE PROFESSOR OF EXPERIMENTAL PHYSICS IN UNIVERSITY COLLEGE, BRISTOL

\section{żandan}

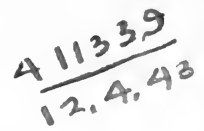

\section{MACMILLAN AND CO., Lmited}

NEW YORK: THE MACMILLAN COMPANY

1905 
First Edition printed 1881

Reprinted with corrctions and additions April 1882

With alterations and additions November 1882

Cancel inserted November 18s2, with alterations and additions 1883, 18st, 1855, 1886, 1887, 1889. Reprinted May and December 1890 ; April and August 1891 ; January, May, and

October 1S92, 1893, 1894

New Edition January 1895. Reprinted October 1895, 1897, 1899 $1900,1901,1902,1903,1905$ 


\section{PREFACE}

These Elementary Lessons have now been largely rewritten. The considerable changes made have been necessitated not only by the progress of the science but by the piracy, covert as well as open, to which since its appearance in 1881 the book has been subjected.

In the thirteen years which have elapsed much addition has been made to our knowledge, and many points then in controversy have been settled. The system of electric units, elaborated first by the British Association and subsequently in several International Congresses, is now legalized in the chief civilized countries. New magnetic surveys-in England by Thorpe and Rïcker, in the United States under Mendenhall-have enabled new magnetic charts to be prepared for the epoch 1900 A.D. The researches of Ewing, Hopkinson, and others on the magnetic properties of iron, and the general recognition of the principle of the magnetic circuit, have advanced the science of magnetism, to which also Ewing's molecular theory has given an added interest. The properties of alternate currents, of which in 1881 little was known, have been forced into study by the extension of their industrial uses in telephony and in electric lighting. 
Entirely new is the use of polyphase alternate currents and rotatory magnetic fields for the electric transmission of power. Transformers have come into extensive employment for the distribution at low pressure of electric energy which has been transmitted from a generating station at high pressure. Accumulators for the storage of electric energy have become of great commercial importance. Electric lamps, large and small, illuminate in millions our cities, towns, villages, and ships. Electric currents for lighting and power are now supplied publicly on a very large scale from central stations operated by steam or water power. Supply-meters are in regular use, and measuring instruments of many forms have come into the market.

Along with these advances in practice there has been a no less striking progress in theory. The ideas of Faraday, as enlarged and developed by Clerk Maxwell, were in 1881 only beginning to be understood and appreciated outside a narrow circle. In 1894, thanks largely to the labours of Heaviside, Hertz, Lodge, Poynting, Fitzgerald, Boltzmann, Poincaré, and others, they are everywhere accepted. In 1881 Maxwell's electromagnetic theory of light-a conception not less far-reaching than the theory of the conservation of energy-was deemed of doubtful probability: it was not yet accepted by such great masters as Lord Kelvin or Von Helmholtz. Though adopted by the younger generation of British physicists, it needed the experimental researches of Hertz and of Lodge upon the propagation of electric waves to demonstrate its truth to their brethren in Germany, France, and America. Even now, after the most convincing experimental verifications of Maxwell's splendid generalization that light-waves are 
really electric waves, many of the logical consequences of Maxwell's teaching are still ignored or misunderstood. It is still, to many, a hard saying that in an electric circuit the conducting wire though it guides does not carry the energy : that the energy-paths lie outside in the surrounding medium, not inside within the so-called conductor. That the guttapercha sheath, and not the copper wire within it, is the actual medium which conveys the impulse from one side of the Atlantic to the other in cable-telegraphy, is still incredible to those brought up in the older school of thought. But it is none the less a necessary consequence of the views which the inescapable logic of facts drove Maxwell and his followers to adopt.

This expansion of the science and of its practical applications has rendered more difficult than before the task of presenting with sufficient clearness, yet with necessary brevity, an elementary exposition of the leading phenomena, and of their relations to one another.

The author is under obligations to many scientific friends for data of which he has made use. He is under special obligations to his assistant, Mr. Miles Walker, for indefatigable proof-reading and revision of the Problems and Index.

London, September 1894. 


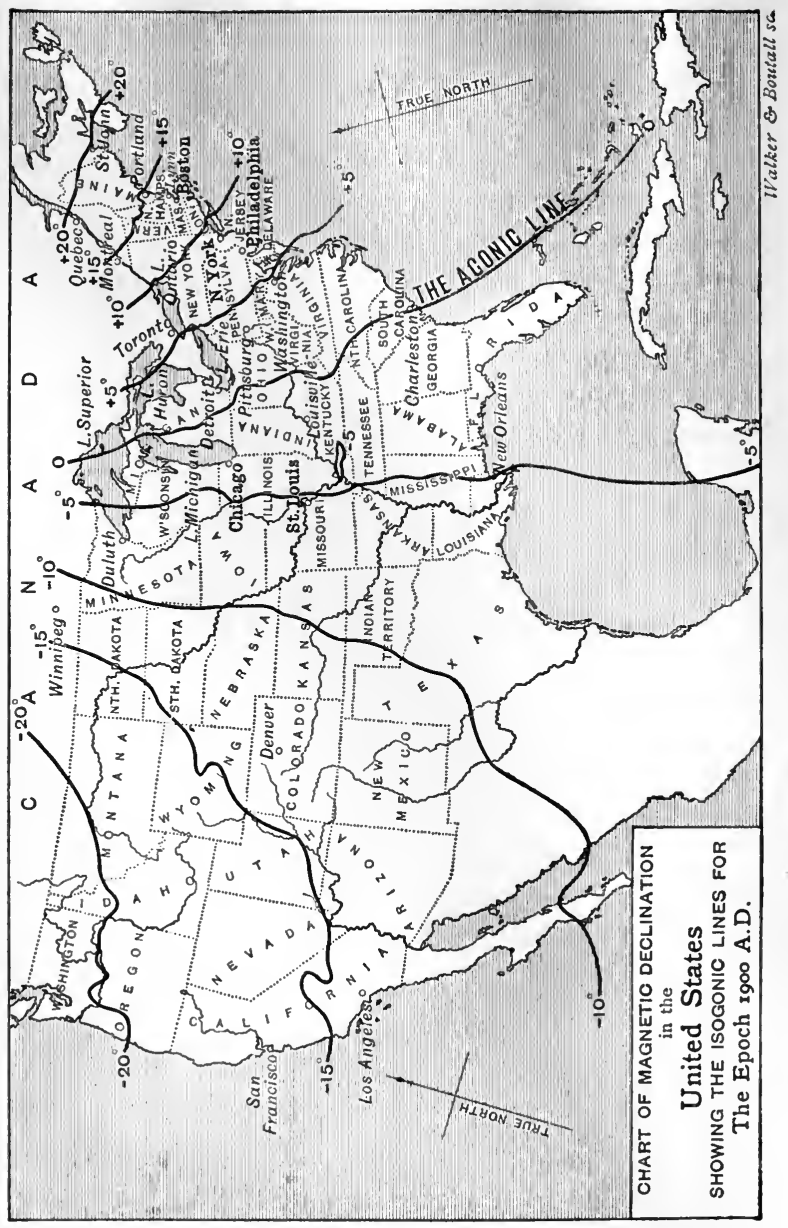




\title{
CONTENTS
}

\author{
抻art Jirst
}

CHAPTER I

Frictional Electricity

I. Electric Attraction and Repulsion . . 1

II. Electroscopes . . . . . . . 15

III. Electrification by Influence. • . . . 24

IV. Conduction and Distribution of Electricity . . 35

V. Electric Machines . . . . . . 47

VI. The Leyden Jar and other Condensers . ' 68

VII. Other Sources of Electrification . . . . 77

\section{CHAPTER II}

Magnetism

VIII. Magnetic Attraction and Repulsion . $\quad 89$

IX. Methods of making Magnets . . . . $\quad$. 99

X. Distribution of Magnetism . . . . . 106

XI. Laws of Magnetic Force . • • • . 117

Note on Ways of Reckoning Angles and Solid Angles 133

XII. Terrestrial Magnetism * . . . . . 136 


\section{CHAPTER III}

\section{Current Electricity}

LESSON

X1II. Simple Voltaic Cells

XIV. Chemical Actions in the Cell .

XV. Voltaic Cells .

XVI. Magnetic Actions of the Current . . . 181

XVII. Galvanometers . . . . • • 193

XVIII. Currents produced by Induction-. . . 210

XIX. Chemical Actions of Currents . . . 223

XX. Physical and Physiological Effects of the Current . . . . . . . 234

\section{护art Serond}

CHAPTER IV

Electrostatics

XXI. Theory of Potential . . . . . 244

Note on Fundamental and Derived Units . 263

XXII. Electrometers . . . . . . 267

XXIII. Dielectric Capacity, etc. . . . . . 277

XXIV. Phenomena of Discharge . . . , 293

XXV. Atmospheric Electricity. . . . • 316

\section{CHAPTER V}

\section{ELECTROMAGNETICS}

XXVI. Magnetic Potential . . . • . 327

XXVII. The Electromagnetic System of Units . . 344

XXVIII. Properties of Iron and Steel . . . . 354

XXIX. Diamagnetism . . . . . . 363

XXX. The Magnetic Circuit . . . . . 369

XXXI. Electromagnets . . . . . 374

XXXII. Electrodynamies . . . . . . 384 


\section{CHAPTER VI \\ Measurement of Currents, etc.}

LEsSON

XXXIII. Ohm's Law and its Consequences . $\quad 397$ XXXIV. Electrical Measurements . . . • 412

\section{CHA PTER VII}

\section{Thermo-ELECTRICITY}

\section{Thermo-Electric Currents .
CHAPTER VIII}

Heat, Power, and Light, from Electric Currents XXXVI. Heating Effects of Currents 435 XXXVII. Electric Energy : its Supply and Measurement 441 XXXVIII. Electric Motors (Electromagnetic Engines) • 448 XXXIX. Electric Light . . . . . . 455

\section{CHAPTER IX}

\section{INDUCTANCE}

XL. Mutual Induction . . $\quad{ }^{\circ}$. $\quad$. 464 XLI. Self-Induction . . . . . . $\quad$ • 468

\section{CHAPTER X}

\section{Dynamos and Transformers}

XLII. Magneto-electric and Dynamo-electric Generators

XLIII. Alternate Currents . . . . . 486

XLIV. Alternate-current Generators . . 495

XLV. Transformers . . . . . . 500

XLVI. Alternate-eurrent Motors . . . . 504 


\section{CHAPTER XI}

\section{Electro-Chemistry}

LESSON

PAGE

XLVII. Electrolysis .

508

XLVIII. Accumulators

522

XLIX. Electrodeposition . . . . . . 524

\section{CHAPTER XII}

\section{Telegraphy}

L. Electric Telegraphs — . . . $\quad 529$

LI. Cable Telegraphy . . . . . . 538

LII. Miscellaneous Telegraphs . . . . 540

\section{CHAPTER XIII}

TELEPHONY

LIII. Telephones . $\quad$ • $\quad$ - . $\quad$ - 544

\section{CHAPTER XIV}

Electric Waves

LIV. Oscillations and Waves . . . . . 551

LV. The Electromagnetic Theory of Light . . 555

LVI. Other Relations between Light and Electricity 563 


\section{APPENDIX}

Appendix A. Table of Angles and Solid Angles 570 Appendix B. Order in Council on Electrical Units and Standarid . $\quad . \quad$. $\quad$. 572

Appendix C. Official Specification for the Preparation of the Clark Standard Cell . . 575 Problems AND Exercises . . . . . . 578 $\operatorname{INDEX}$. . . . . . . 607

Magnetic Chart of the British Islands . Frontispiece Magnetic Map of the United States . . . p. x 



\title{
ELEMENTARY LESSONS
}

oN

\section{ELECTRICI'TY \& MAGNETISM}

\section{提art ffirst}

\author{
CHAPTER I
}

FRICTIONAL ELECTRICITY

Lesson I.-Electric Attraction and Repulsion

1. Electricity.-Electricity is the name given to an invisible agent known to us only by the effects which it produces and by various manifestations called electrical. These manifestations, at first obscure and even mysterious, are now. well understood, though little is yet known of the precise nature of electricity itself. It is neither matter nor energy; yet it apparently can be associated or combined with matter; and energy can be spent in moving it. Indeed its great importance to mankind arises from the circumstance that by its means energy spent in generating electric forces in one part of a system can be made to reappear as electric heat or light or work at some other part of the system ; such transfer of energy taking place even to very great distances at an enormous speed. Electricity is apparently as indestructible as 
matter or as energy. It call neither be created nor destroyed, but it can be transformed in its relations to natter and to energy, and it can be moved from one place to another. In many ways its behaviour resembles that of an incompressible liquid; in other ways that of a highly attenuated and weightless gas. It appears to exist distributed nearly uniformly thronghout all space. Many persons (including the author) are disposed to consider it as identical with the luminiferous ether. If it be not the same thing, there is an intimate relation between the two. That this must be so, is a mecessary result of the great discovery of Maxwell-the greatest scientific discovery of the nineteenth century - that light itself is an electric phenomenon, and that the light-waves are merely electric, or, as he put it, electromagnetic waves.

The name electricity is also given to that branch of science which deals with electric phenomena and theories. The phenomena, and the science which deals with them, fall under four heads. The manifestations of electricity when standing still are different from those of electricity moving or flowing along: hence we have to consider separately the properties of (i.) statical charges, and those of (ii.) currents. Further, electricity whirling round or in circulation possesses properties which were independently discovered under the name of (iii.) magnetism. Lastly, electricity when in a state of rapid vibration manifests new properties not possessed in any of the previous states, and causes the propagation of (iv.) waves. These four branches of the science of electricity are, however, closely connected. The object of the present work is to give the reader a general view of the main facts and their simple relations to one another.

In these first lessons we begin with charges of electricity, their production by friction, by influence, and by various other means, and shall study them mainly by the manifestations of attraction and repulsion to which they give rise. After that we go on to magnetism and 
currents, and the relations between them. The subject of electric waves is briefly discussed at the end of the book.

2. Electric Attraction.-If you take a piece of sealing-wax, or of resin, or a glass rod, and rub it upon a piece of flannel or silk, it will be found to have acquired a property which it did not previously possess : namely, the power of attracting to itself such light bodies as chaff, or dust, or bits of paper (Fig. 1). This curious power

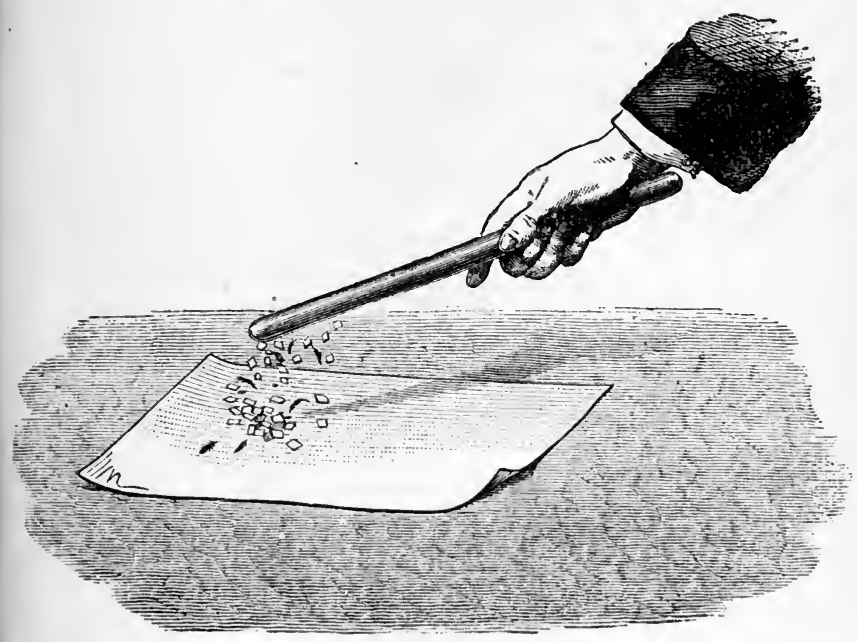

Fig. 1.

was originally discovered to be a property of amber, or, as the Greeks called it, $\eta \lambda_{\epsilon \kappa} \tau \rho o v$, which is mentioned by Thales of Miletus (B.c. 600), and by Theophrastus in his treatise on Gems, as attracting light bodies when rubbed. Although an enormous number of substances possess this property, amber and jet were the only two in which its existence had been recognised by the ancients, or even down to so late a date as the time of Queen Elizabeth. 
About the year 1600, Dr. Gilbert of Colchester discovered

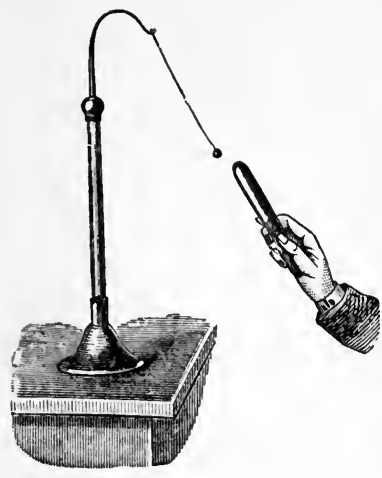

Fig. 2.

by experiment that not only amber and jet, but a very large number of substances, such as diamond, sapphire, rock-crystal, glass, sulphur, sealing-wax, resin, etc., which he styled electrics, ${ }^{*}$ possess the same property. Ever since his time the name electricity $\dagger$ has been employed to denote the agency at work in producing these phenomena. Gilbertalso remarked that these experiments are spoiled by the presence of moisture.

3. Further Experiments.-A better way of observing the attracting force is to enploy a small ball of elder pith, or of cork, hung by a fine thread from a support, as shown in Fig. 2. A dry warm glass tube, excited by rubbing it briskly with a silk handkerchief, will attract the pithball strongly, showing that it is highly electrified. The most suitable rubber, if a stick of sealing - wax is used, will be found to be

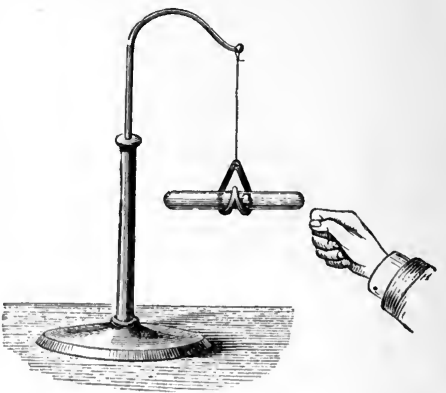

Fig. 3. flannel, woollen cloth, or, best of all, fur. Boyle discovered

* "Electrica; quæ attrahunt ealem ratione ut electrum" (Gilbert).

$\uparrow$ The first work in which this term was used is that of Robert Boyle, On the Mechanical Production of Electricity, published at Oxford in 1675. 
that an electrified body is itself attracted by one that has not been electrified. This may be verified (see Fig. 3) by rubbing a stick of sealing-wax, or a glass rod, and hanging it in a wire loop at the end of a silk thread. If, then, the hand be held out towards the suspended electrified body, the latter will turn round and approach the hand. So, again, a piece of silk ribbon, if rubbed with warm indiarubber, or even if drawn between two pieces of warm flannel, and then hung up by one end, will be found to be attracted by objects presented to it. If held near the wall of the room it will fly to it and stick to it. With proper precautions it can be shown that both the rubber and the thing rubbed are in an electrified state, for both will attract light bodies ; but to show this, eare must be taken not to handle the rubber too much. Thus, if it is desired to show that when a piece of fur is rubbed upon sealing-wax, the fur becomes also electrified, it is better not to take the fur in the hand, but to cement it to the end of a glass rod as a handle. The reason of this precaution will be explained toward the close of this lesson, and more fully in Lesson IV.

A large number of substances, including iron, gold, brass, and all the metals, when held in the hand and rubbed, exhibit no sign of electrification,- - that is to say, do not attract light bodies as rubbed amber and rubbed glass do. Gilbert mentions also pearls, marble, agate, and the lodestone, as substances not excited electrically by rubbing them. Such bodies were, on that account, formerly termed non-electrics; but the term is erroneous, for if they are mounted on glass handles and then rubbed with silk or fur, they behave as electrics.

4. Electric Repulsion.-When experimenting, as in Fig. 1, with a rubbed glass rod and bits of chopped paper, or straw, or bran, it will be noticed that these little bits are first attracted and fly up towards the excited rod, but that, having touched it, they are speedily repelled 
and fly back to the table. To show this repulsion better, let a small piece of feather or down be hung by a silk

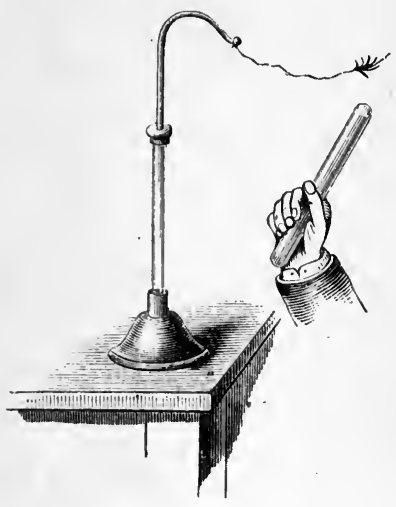

- Fig. 4. thread to a support, and let an electrified glass rod be held near it. It will dart towards the rod and stick to it, and a moment later will dart away from it, repelled by an invisible force (Fig. 4), nor will it again dart towards the rod. If the experiment be repeated with another feather, and a stick of sealing-wax rubbed on flannel, the same effects will occur. But, if now the hand be held towards the feather, it will rush toward the hand, as the rubbed body (in Fig. 3) did. This proves that the feather, though it has not itself been rubbed, possesses the property originally imparted to the rod by rubbing it. In fact, it has become electrified, by liaving touched an electrified body which has given part of its electricity to it. It would appear then that two hodies electrified with the same electrification repel one an-

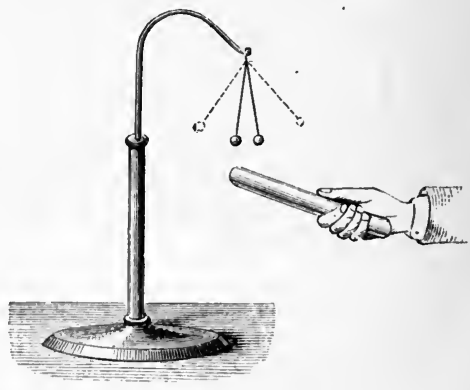

Fig. 5. other. This may be confirmed by a further experiment. A rubbed glass rod, hung up as in Fig. 3 , is repelled by a 
similar rubbed glass rod ; while a rubbed stick of sealingwax is repelled by a second rubbed stick of sealing-wax. Another way of showing the repulsion between two similarly electrified bodies is to hang a couple of small pith-balls, by thin linen threads, to a glass support, as in Fig. 5, and then touch them both with a rubbed glass rod. They repel one another and fly apart, instead of hanging down side by side, while the near presence of the glass rod will make them open ont still wider, for now it repels them both. The self-repulsion of the parts of an electrified body is beantifully illustrated by the experiment of electrifying a soap-bubble, which expands when electrifier.

5. Two Kinds of Electriflcation. - Electrified bodies do not, however, always repel one another. The feather which (see Fig. 4) has been touched by a rubbed glass rod, and which in consequence is repelled from the rubbed glass, will be attracted if a stick of rubbed sealingwax be presented to it ; and conversely, if the feather has been first electrified by touching it with the rubbed sealing-wax, it will be attracted to a rubbed glass rod, though repelled by the rubbed wax. So, again, a rubbed glass rod suspended as in Fig. 3 will be attracted by a rubbed piece of sealing-wax, or resin, or amber, though repelled by a rubbed piece of glass. The two pith-balls tonched (as in Fig. 5) with a rubbed glass rod fly from one another by repulsion, and, as we have seen, fly wider asunder when the excited glass rod is held near them; yet they fall nearer together when a rubbed piece of sealing-wax is held under them, being attracted by it. Symmer first observed such phenomena as these, and they were independently discovered by Du Fay, who suggested in explanation of them that there were two different kinds of electricity which attracted one another, while each repelled itself. The electricity produced on glass by rubbing it with silk he called vitreous electricity, supposing, though erroneously, that glass could yield no 
other kind; and the electricity excited in such substances as sealing-wax, resin, shellac, indiarubber, and amber, by rubbing them on wool or flannel, he termed resinous electricity. The kind of electricity produced is, however, found to depend not only on the thing rubbed but on the rubber also ; for glass yields "resinous" electricity when rubbed with a cat's skin, and resin yields "vitreous" electricity if rubbed with a soft amalgam of tin and mercury spread on leather. Hence these names have been abandoned in favour of the more appropriate terms introduced by Franklin, who called the electricity excited upon glass by rubbing it with silk, positive electricity, and that produced on resinous bodies by friction with wool or fur, negative electricity. The observations of Symmer and Du Fay may therefore be stated as follows:-Two positively electrified bodies apparently repel one another : two negatively electrified bodies apparently repel one another : but a positively electrified body and a negatively electrified body apparently attract one another. It is now known that these effects which appear like a repulsion and an attraction between bodies at a distance from one another, are really due to actions going on in the medium between them. The positive charge does not really attract the negative charge that is near it; but both are urged toward one another by stresses in the medium in the intervening space.

6. Simultaneous Production of both Electrical States.-Neither kind of electrification is produced alone; there is always an equal quantity of both kinds produced; one kind appearing on the thing rubbed and an equal amount of the other kind on the rubber. The clearest proof that these amounts are equal can be given in some cases. For it is found that if both the - electricity of the rubber and the + electricity of the thing rubbed be imparted to a third body, that third body will show no electrification at all, the two erpual and opposite electrifications having exactly neutralized each other. A simple 
experiment consists in rubbing together a disk of sealingwax and one covered with flannel, both being held by insulating handles. To test them is required an insulated pot and an electroscope, as in Fig. 29. If either disk be inserted in the pot the leaves of the electroscope will diverge; but if both are inserted at the same time the leaves do not diverge, showing that the two charges on the disks are equal and of opposite sign.

In the following list the bodies are arranged in such an order that if any two be rubbed together the one which stands earlier in the series becomes positively electrified, and the one that stands later negatively electrified :-Fur, wool, ivory, glass, silk, metals, sulphur, indiarubber, guttapercha, collodion, or celluloid.

7. Theories of Electricity.-Several theories have been advanced to account for these phenomena, but all are more or less unsatisfactory. Symmer proposed a "two-fluid" theory, according to which there are two imponderable electric fluids of opposite kinds, which neutralize one another when they combine, and which exist combined in equal quantities in all bodies until their condition is disturbed by friction. A modification of this theory was made by Franklin, who proposed instead a "one-fluid" theory, according to which there is a single electric fluid distributed usually uniformly in all bodies, but which, when they are subjected to friction, distributes itself unequally between the rubber and the thing rubbed, one having more of the fluid, the other less, than the average. Hence the terms positive and negative, which are still retained ; that body which is supposed to have an excess being said to be charged with positive electricity (usually denoted by the plus sign +), while that which is supposed to have less is said to be charged with negative electricity (and is denoted by the minus sign -). These terms are, however, purely arbitrary, for in the present state of science we do not know which of these two states really means more and 
which means less. In many ways electricity behaves as a weightless substance as incompressible as any material liquid. It is, however, quite certain that electricity is not a material fluid, whatever else it may be. For while it resembles a fluid in its property of apparently flowing from one point to another, it differs from every known fluid in almost every other respect. It possesses no weight ; it repels itself. It is, moreover, quite impossible to conceive of two fluids whose properties should in every respect be the precise opposites of one another. For these reasons it is clearly misleading to speak of an electric fluid or fluids, however convenient the term may seem to be. In metals and other gcod conductors electricity can apparently move and flow quite easily in currents. In transparent solids such as glass and resin; and in many transparent liquids such as oils, and in gases such as the air (if still, and not rarefied), electricity apparently cannot flow. Even a vacuum appears to be a non-conductor. In the case of all non-conductors electricity can only be moved by an action known as displacement (see Art. 57).

It appeàrs then that in metals electricity can easily pass from molecule to molecule; but in the case of nonconductors the electricity is in some way stuck to the molecules, or associated with them. Some electricians, notably Faraday, have propounded a molecular theory of electricity, according to which the electrical states are the result of certain peculiar conditions of the molecules of the surfaces that have been rubbed. Another view is to regard the state of electrification as related to the ether (the highly-attenuated medimm which fills all space, and is the vehicle by which light is transmitted), which is known to be associated with the molecules of matter. Some indeed hold that the ether itself is electricity ; and that the two states of positive and negrative electrification are simply due to displacement of the ether at the surfaces of bodies. In these lessons we shall avoid as 
far as possible all theories, and shall be content to use the term electricity.

8. Charge.-The quantity of electrification of either kind produced by friction or other means upon the surface of a body is spoken of as a charge, and a body when electrified is said to be charged. It is clear that there may be charges of different values as well as of either kind. When the charge of electricity is removed from a charged body it is said to be discharged. Good conductors of electricity are instantaneously discharged if touched by the hand or by any conductor in contact with the ground, the charge thus finding a means of escaping to earth or to surrounding walls. A body that is not a good conductor may be readily discharged by passing it rapidly through the flame of a spirit-lamp or a candle; for the hot gases instantly carry off the charge and dissipate it in the air.

Electricity may either reside upon the surface of bodies as a charge, or flow through their substance as a current. That branch of the science which treats of the laws of the charges, that is to say, of electricity at rest, upon the surface of bodies is termed electrostatics, and is dealt with in Chapter IV. The branch of the subject which treats of the flow of electricity in currents is dealt with in Chapter III., and other later portions of this book.

9. Modes of representing Electriflcation.Several modes are used to represent the electrification of surfaces. In Figs. 6, 7, and 8 are represented two disks, -A covered with woollen cloth, B of some resinous borty, - which have been rubbed together so that $\mathrm{A}$ has become positively, B negatively electrified. In

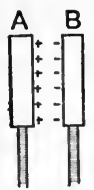

Fig. 6.

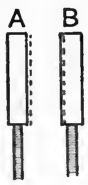

Fig. 7 .

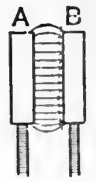

Fig. S. Fig. 6 the surfaces are marked with plus $(+)$ and minus ( - ) signs. In Fig. 7 dotted lines are drawn just outside 
the positively electrified surface and just within the negatively electrified surface, as though one had a surplus and the other a deficit of electricity. In Fig. 8 lines are drawn across the intervening space from the positively electrified surface to the opposite negative charge. The advantages of this last mode are explained in Art. 13.

10. Conductors and Insulators. - The term "conductors," used above, is applied to those bodies which readily allow electricity to flow through them. Roughly speaking, bodies may be divided into two classes-those which conduct and those which do not; though very many substances are partial conductors, and cannot well be classed in either category. All the metals conduct well ; the human body conducts, and so does water. On the other hand glass, sealing-wax, silk, shellac, guttapercha, indiarubber, resin, fatty substances generally, and the air, are non-conductors. On this account these substances are used to make supports and handles for electrical apparatus where it is important that the electricity should not leak away; hence they are sometimes callèd insulators or isolators. Faraday termed them dielectrics. We have remarked above that the name of non-electrics was given to those substances which, like the metals, yield no sign of electrification when held in the hand and rubbed. We now know the reason why they show no electrification; for, being good conductors, the electrification flows away as fast as it is generated. The observation of Gilbert that electrical experiments fail in damp weather is also explained by the knowledge that water is a conductor, the film of moisture on the surface of damp bodies causing the electricity produced by friction to leak away as fast as it is generated.

11. Other Electrical Effects.-The production of electricity by friction is attested by other effects than those of attraction and repulsion, which hitherto we have assumed to be the test of the presence of electricity. Otto von Guericke first observed that sparks and flashes 
of light could be obtained from highly electrified bodies at the moment when they were discharged. Such sparks are usually accompanied by a snapping sound, suggesting on a small scale the thunder accompanying the lightning spark, as was remarked by Newton and other early observers. Pale flashes of light are also produced by the discharge of electricity through tubes partially exhausted of air by the air-pump. Other effects will be noticed in due course.

12. Other Sources of Electrification. - The student must be reminded that friction is by no means the only source of electrification. The other sources, percussion, compression, heat, chemical action, physiological action, contact of metals, etc., will be treated of in Lesson VII. We will simply remark here that friction between two different substances always produces electrical separation, no matter what the substances may be. Symmer observed the production of electrification when a silk stocking was drawn over a woollen one, though woollen rubbed upon woollen, or silk rubbed upon silk, produces no electrical effect. If, however, a piece of rough glass be rubbed on a piece of smooth glass, electrification is observed ; and indeed the conditions of the surface play a very important part in the production of electrification by friction. In general, of two bodies thus rubbed together, that one becomes negatively electrical whose particles are the more easily removed by friction. Differences of temperature also affect the electrical conditions of borlies, a warm body being usually negative when rubbed on a cold piece of the same substance. The quantity of electrification produced is, however, not proportional to the amount of the actual mechanical friction; hence it appears doubtful whether friction is truly the cause of the electrification. Something certainly happens when the surfaces of two different substances are brought into intimate contact, which has the result that when they are drawn apart they are found (provided at least one of 
them is a non-conductor) to have acquired opposite charges of electrification; one surface having apparently taken some electricity from the other. But these opposite charges attract one another and cannot be drawn apart without there being mechanical work done upon the system. The work thus spent is stored up in the act of separating the charged surfaces; and as long as the charges remain separated they constitute a store of potential energy. The so-called frictional electric machines are therefore machines for bringing dissimilar substances into intimate contact, and then drawing apart the particles that have touched one another and become electrical.

If the two bodies that are rubbed together are both good conductors, they will not become strongly electrified, even if held on insulating handles. It is quite likely, however, that the heat produced by friction, as in the bearings of machinery, is due to electric currents generated where the surfaces meet and slip.

13. Electric Field. - Whenever two oppositely charged surfaces are placed near one another they tend to move together, and the space between them is found

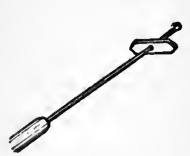

Fig. 9. to be thrown into a peeuliar state of stress, as though the medium in between had been stretched. To explore the space between two bodies one of which has been positively and the other negatively electrified, we may use a light pointer (Fig. 9) made of a small piece of very thin paper piereed with a hole through which passes a long thread of glass. It will be found that this pointer tends to point across from the positively electrified surface to the negatively electrified surface, along invisible lines of electric force. The space so filled with electric lines of foree is ealled an electric field. In Fig. $8 \mathrm{~A}$ and B represent two bodies the surfaces of which have been eleetrified, the one positively, the other negatively. In 
the field between them the electric lines pass across almost straight, except near the edges, where they are curved. Electric lines of force start from a positively charged surface at one end, and end on a negatively charged surface at the other end. They never meet or cross one another. Their direction indicates that of the resultant electric force at every point through which they pass. The stress in the medium thus mapped out by the lines of force acts as a tension along them, as though they tended to shorten themselves.

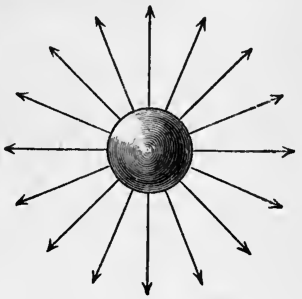

Fig. 10. In fact in Fig. 8 the tension in the medium draws the two surfaces together. There is also a pressure in the medium at right angles to the lines, tending to widen the distance between them. Fig. 10 represents a ball which has been positively electrified, and placed at a distance from other objects; the lines in the field being simply radial.

\section{Lesson II.-Electroscopes}

14. Simple Electroscopes.-An instrument for detecting whether a body is electrified or not, and whether the electrification is positive or negative, is termed an Electroscope. The feather which was attracted or repelled, and the two pith-balls which flew apart, as we found in Lesson I., are in reality simple electroscopes. There are, however, a number of pieces of apparatus better adapted for this particular purpose, some of which we will describe.

15. Needle Electroscope.-The earliest electroscope was that devised by Dr. Gilbert, and shown in Fig. 11, which consists of a stiff strip balanced lightly upon a sharp point. A thin strip of brass or wood, a straw, or 
even a goose quill, balanced upon a sewing needle, will serve equally well. When an electrified body is held near

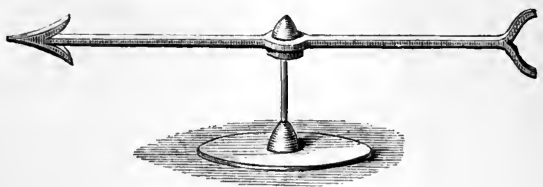

Fig. 11.

the electroscope it is attracted and turned round, and will thus indicate the presence of electric charges far too feeble to attract bits of paper from a table.

16. Gold-Leaf Electroscope.-A still more sensi-

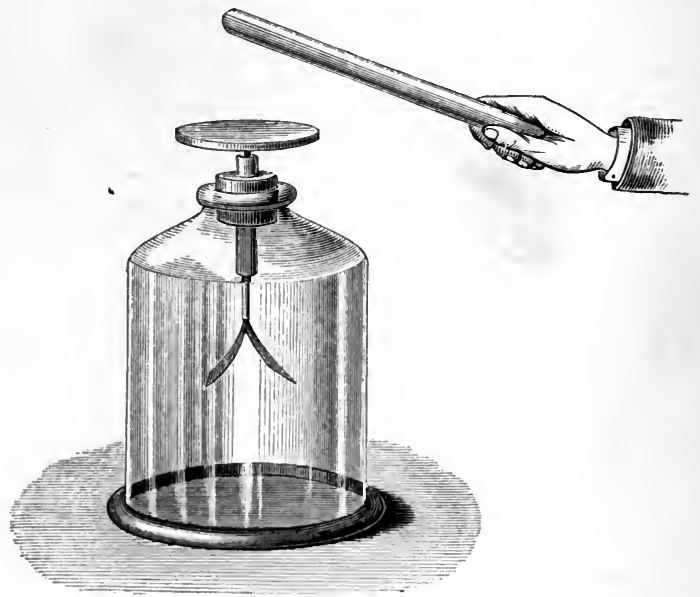

Fig. 12.

tive instrument is the Gold-Leaf Electroscope invented by Bennet, and shown in Fig. 12. We have seen how two pith-balls when similarly electrified repel 
one another and stand apart, gravity being partly overcome by the force of the electric repulsion. A couple of narrow strips of the thinnest tissue paper, hung upon a support, will behave similarly when electrified. But the best results are obtained with two strips of gold-leaf, which, being excessively thin, is much lighter than the thinnest paper. The Gold-Leaf Electroscope is conveniently made by suspending the two leaves within a wide-mouthed glass jar, which both serves to protect them from draughts of air and to support them from contact with the ground. The mouth of the jar should be closed by a plug of paraffin wax, through which is pushed a bit of varnished glass tube. Through this passes a stiff brass wire, the lower end of which is bent at a right angle to receive the two strips of gold-leaf, while the upper supports a flat plate of metal, or may be furnished with a brass knob. When kept dry and free from dust it will indicate excessively small quantities of electrification. A rubbed glass rod, even while two or three feet from the instrument, will cause the leaves to repel one another. The chips produced by sharpening a pencil, falling on the electroscope top, are seen to be electrified. If the knob be even brushed with a small camel's-hair brush, the slight friction produces a perceptible effect. With this instrument all kinds of friction can be shown to produce electrification. Let a person, standing upon an insulating support,- -such as a stool with glass legs, or a board supported on four glass tumblers,--be briskly struck with a silk handkerchief, or with a fox's tail, or even brushed with a clothes' brush, he will be electrified, as will be indicated by the electroscope if he place one hand on the knob at the top of it. The Gold-Leaf Electroscope can further be used to indicate the kind of electrification on an excited body. Thus, suppose we rubbed a piece of brown paper with a piece of indiarubber and desired to find out whether the electrification excited on the paper was + or - , we should 
proceed as follows:-First charge the gold leaves of the electroscope by touching the knols with a glass rod rubbed on silk. The leaves diverge, being electrified with + electrification. When they are thus charged the approach of a body which is positively electrified will cause them to diverge still more widely; while, on the approach of one negatively electrified, they will tend to close together. If now the brown paper be brought near the electroseope, the leaves will be seen to diverge more, proving the electrification of the paper to be of the same kind as that with which the electroscope is charged, or positive.

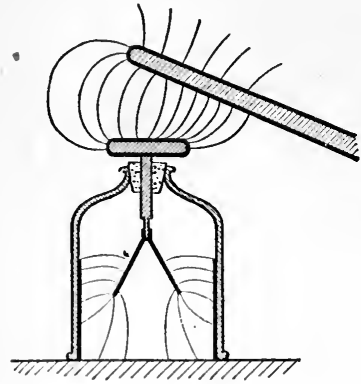

Fig. 13.

Sometimes the outer surface of the glass jar containing the gold leaves is covered with wire gauze or strips of foil to shield the leaves from the influence of external bodies. A preferable way is to use glass of a kind that conducts.

The part played by the surrounding medium in the operation of the electroscope is illustrated by Fig. 13. Of the electric lines in the field surrounding the rubbed rod a number will pass into the metal cap of the electroscope and emerge below, through the leaves. The nearer the rod is brought, the greater will be the number of electric lines thus affecting the instrument. There being a tension along the lines and a pressure across them, the effect is to draw the gold leaves apart as though they repelled each other.

The Gold-Leaf Electroscope will also indicate roughly the amount of electrification on a body placed in contact with it, for the gold leaves open out more widely when the charge thus imparted to them is greater. For exact measurement, however, of the degree of electrification, 
recourse must be had to the instruments known as Electrometers, deseribed in Lesson XXII.

In another form of electroscope (Bohnenberger's) a single gold leaf is used, and is suspended between two metallic plates, one of which can be positively, the other negatively electrified, by placing them in communication with the poles of a "dry pile" (Art. 193). If the gold leaf be charged positively or negatively it will be attracted to one side and repelled from the other, according to the law of attraction and repulsion mentioned in Art. 4.

17. Henley's Semaphore.-As an indicator for large charges of electricity there is sometimes used a semaphore like that shown in Fig. 14. It consists of a pith-ball at the end of a light arm fixed on a pivot to an upright. When the whole is electrified the pith-ball is repelled from the upright and flies out at an angle, indieated on a graduated scale or dial behind it. This little electroscope, which is seldom used except to show whether an electric machine or a Leyden battery is charged, must on no account be confused with the delicate "Quadrant Electrometer" deseribed in Lesson

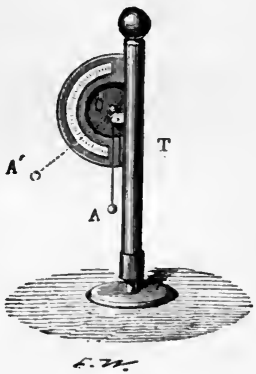

Fig. 14. XXII., whose object is to measure very small charges of electricity - not to indicate large ones.

18. The Torsion Balance.-Although more properly an Electrometer than a mere Electroscope, it will be most convenient to describe here the instrument known as the Torsion Balance (Fig. 15). This instrument, once famous, but now quite obsolete, served to measure the force of the repulsion between two similarly electrified bodies, by balaneing the repelling force against the force exerted by a fine wire in untwisting itself after it has been twisted. The torsion balance consists of a light arm 
or lever of shellac suspended within a cylindrical glass case by means of a fine silver wire. At one end this lever is furnished with a gilt pith-ball $n$. The upper end of the silver wire is fastened to a brass top, upon

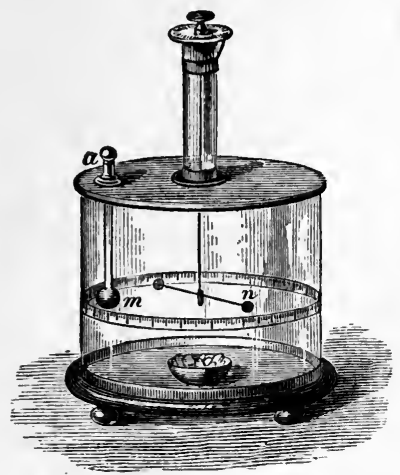

Fig. 15.

which a circle, divided into degrees, is cut. This top can be turned round in the tube which supports it, and iscalled the torsionhead. Through an aperture in the cover there can be introduced a second gilt pith-ball $m$, fixed to the end of a vertical glass rod a. Round the glass case, at the level of the pith - balls, a circle is drawn, and divided also into degrees.

In using the torsion balance to measure the amount of a charge of electricity, the following method is adopted :-First, the torsion-head is turned round until the two pith-balls $m$ and $n$ just touch one another. Then the glass rod $a$ is taken ont, and the charge of electricity to be measured is imparted to the ball $m$, which is then replaced in the balance. As soon as $m$ and $n$ touch one another, part of the charge passes from $m$ to $n$, and they repel one another because they are then similarly electrified. The ball $n$, therefore, is driven round and twists the wire up to a certain extent. The force of repulsion becomes less and less as $n$ gets farther and farther from $m$; but the force of the twist gets greater and greater the more the wire is twisted. Hence these two forces will balance one another when the balls are separated by a certain distance, and it is clear that a large charge of electricity will repel the ball $n$ with a greater force than a lesser charge would. The 
distance through which the ball is repelled is read off in angular degrees of the scale. When a wire is twisted, the force with which it tends to untwist is precisely proportional to the amount of the twist. The force required to twist the wire ten degrees is just ten times as great as the force required to twist it one degree. In other words, the force of torsion is proportional to the angle of torsion. The angular distance between the two balls is, when they are not very widely separated, very nearly proportional to the actual straight distance between them, and represents the force exerted between electrified balls at that distance apart. The student must, however, carefully distinguish between the measurement of the force and the measurement of the actual quantity of electricity with which the instrument is charged. For the force exerted betwen the electrified balls will vary at different distances according to a particular law known as the "law of inverse squares," which requires to be carefully explained.

19. The Law of Inverse Squares.-Coulomb proved, by means of the Torsion Balance, that the force exerted between two small electrified bodies varies inversely as the square of the distance between them when the distance is varied. Thus, suppose two small electrified bodies 1 inch apart repel one another with a certain force, at a distance of 2 inches the force will be found to be only one quarter as great as the force at 1 inch; and at 10 inches it will be only $\frac{1}{100}$ part as great as at 1 inch. This law is proved by the following experinent with the torsion balance. The two scales were adjusted to $0^{\circ}$, and a certain charge was then imparted to the balls. The ball $n$ was repelled round to a distance of $36^{\circ}$. The twist on the wire between its upper and lower ends was also $36^{\circ}$, or the force tending to repel was thirty-six times as great as the force required to twist the wire by $1^{\circ}$. The torsion-head was now turned round so as to twist the thread at the 
top and force the ball $n$ nearer to $m$, and was turned round until the distance between $n$ and $n$ was halved. To bring down this distance from $36^{\circ}$ to $18^{\circ}$, it was found needful to twist the torsion-head through $126^{\circ}$. The total twist between the upper and lower ends of the wire was now $126^{\circ}+18^{\circ}$, or $144^{\circ}$; and the force was 144 times as great as that force which would twist the wire $1^{\circ}$. But 144 is four times as great as 36 ; hence we see that while the distance had been reduced to one half, the force between the balls had become four times as great. Had we reduced the distance to one quarter, or $9^{\circ}$, the total torsion would have been found to be $576^{\circ}$, or sixteen times as great; proving the force to vary inversely as the square of the distance.

In practice it requires great experience and skill to obtain results as exact as this, for there are many sources of inaccuracy in the instrument. The balls must be very small, in proportion to the distances between them. The charges of electricity on the balls are found, moreover, to become gradually less and less, as if the electricity leaked away into the air. This loss is less if the apparatus be quite dry. It is therefore usual to dry the interior by placing inside the case a cup containing either chloride of calcium, or pumice stone soaked with strong sulphuric acid, to absorb the moisture.

Before leaving the subject of electric forces, it may be well to mention that the force of attraction between two oppositely electrified bodies varies also inversely as the square of the distance between them. And in every case, whether of attraction or repulsion, the force at any given distance is proportional to the product of the two quantities of electricity on the bodies. Thus, if we had separately given a charge of 2 to the ball $m$ and a charge of 3 to the ball $n$, the force between them will be $3 \times 2=6$ times as great as if each had had a charge of 1 given to it. It must be remembered, however, that the 
law of inverse squares is only true when applied to the case of bodies so small, as compared with the distance between them, that they are mere points. For flat, large, or elongated bodies the law of inverse squares does not hold good. The attraction between two large flat disks oppositely electrified with given charges, and placed near together, does not vary with the distance.

20. Field between two Balls.-The electric field (Art. 13) between two oppositely electrified balls is found to consist of curved lines. By the principle laid down in Art. 13, there is a tension along these lines so that they tend not only to draw the two balls together, but also to draw the electrifications on the surfaces of the balls toward one another.

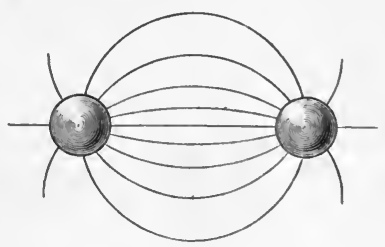

Fig. 16. There is also a lateral pressure in the medium tending to keep the electric lines apart from one another. One result of these actions is that the charges are no longer equally distributed over the surfaces, but are more dense on the parts that approach most nearly.

21. Unit Quantity of Electricity.-In consequence of these laws of attraction and repulsion, it is found most convenient to adopt the following definition for that quantity of electricity which we take for a unit or standard by which to measure other quantities of electricity. One (electrostatic) Unit of Electricity is that quantity which, when placed at a distance of one centimetre in air from a similar and equal quantity, repels it with a force of one dyne. If instead of air another medium occupies the space, the force will be different. For example, if petroleum is used the force exerted between given charges will be about half as great (see Art. 56). Further information about the measurement of electrical quantities is given in Lessons XXI. and XXII. 
2का

\section{Lesson III.-Electrification by Influence}

22. Influence. - We have now learned how two charged bodies may apparently attract or repel one another. It is sometimes said that it is the charges in the bodies which attract or repel one another; but as electrification is not known to exist except in or on material bodies, the proof that it is the charges themselves which are acted upon is only indirect. Nevertheless there are certain matters which support this view, one of these

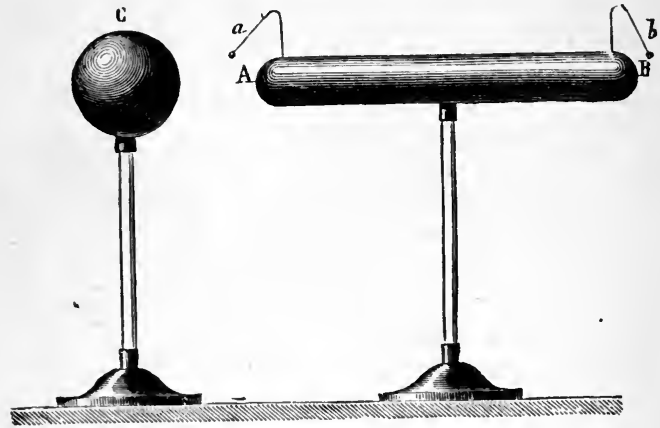

Fig. 17.

being the electric influence exerted by an electrified body upon one not electrified.

Suppose we electrify positively a ball C, shown in Fig. 17 , and hold it near to a body that has not been electrified, what will occur? We take for this experiment the apparatus shown on the right, consisting of a long sausageshaped piece of metal, either hollow or solid, held upon a glass support. This "conductor," so called because it is made of metal which permits electricity to pass freely through it or over its surface, is supported on glass to 
prevent the escape of electricity to the earth, glass being a non-conductor. The influence of the positive charge of the ball placed near this conductor is found to induce electrification on the conductor, which, although it has not been rubbed itself, will be found to behave at its two ends as an electrified body. The ends of the conductor will attract little bits of paper; and if pith-balls be hung to the ends they are found to be repelled. It will, however, be found that the middle region of the long-shaped conductor will give no sign of any electrification. Further examination will show that the two electrifications on the ends of the conductor are of opposite kinds, that nearest the excited glass ball being a negative charge, and that at the farthest end being an equal charge, but of positive sign. It appears then that a positive charge attracts negative and repels positive, and that this influence can be exerted at a distance from a body. If we had begun with a charge of negative electrification upon a stick of sealing-wax, the presence of the negative charge near the conductor would have induced a positive charge on the near end, and negative on the far end. This action, discovered in 1753 by John Canton, is spoken of as influence or electrostatic induction * It will take place across a considerable distance. Even if a large sheet of glass be placed between, the same effect will be produced. When the electrified body is removed both the charges disappear and leave no trace behind, and the glass ball is found to be just as much electrified as before; it has parted with none of its own charge. It

* The word induction originally used was intended to denote an action at a distance, as distinguished from conduction, which implied the conveyance of the action by a material conductor. But there were discovered other actions at a distance, namely, the indnction of currents by noving magnets, or by other currents, and the induction of magnetism in iron in the presence of a neighbouring magnet. As the term induction has now been officially adopted for the induction of currents, its use in other senses ought to be dropped. Hence the preference now given to the term influence for the induction of charges by charges. 
will be remembered that on one theory a body charged positively is regarded as having more electricity than the things round it, while one with a negative charge is regarded as having less. According to this view it would appear that when a body (such as the + electrified glass ball) having more electricity than things around it is placed near an insulated conductor, the uniform distribution of electricity in that conductor is disturbed, the electricity flowing away from that end which is near the + body, leaving less than usual at that end, and produeing

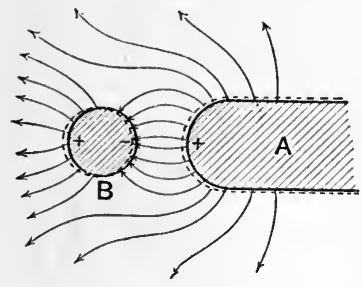

Fig. 18. more than usual at the other end. This view of things will account for the disappearance of all signs of electrification when the electrified body is removed, for then the conductor returns to its former condition; and being neither more nor less electrified than all the objects around on the surface of the earth, will show neither positive nor negative charge. The action is not, however, a mere action at a distance; it is one in which the intervening medium takes an essential part. Consider (Fig. 18) what takes place when an insulated, non-electrified metal ball $\mathrm{B}$ is brought under the influence of a positively electrified body A. At once some of the electric lines of the field that surrounds $A$ pass through $B$, entering it at the side nearer $A$, and leaving it at the farther side. As the ball $B$ has no charge of its own, as many electric lines will enter on one side as leave on the other; or, in other words, the induced negative charge on one side and the induced positive charge on the other will be exactly equal in amount. They will not, however, be quite equally distributed, the negative charge on the side nearer $A$ being more concentrated, and the lines in the field on that side denser. 
23. Effects of Influence. - If the conductor be made in two parts, which, while under the influence of the electrified body, are separated, then on the removal of the electrified body the two charges can no longer return to neutralize one another, but remain each on its own portion of the conductor.

If the conductor be not insulated on glass supports, but placed in contact with the ground, that end only which is nearest the electrified body will be found to be electrified. The repelled charge is indeed repelled as far as possible into the walls of the room ; or, if the experiment be performed in the open air, into the eartl. One kind of electrification only is under these circumstances to be found, namely, the opposite kind to that of the excited body, whichever this may be. The same effect occurs in this case as if an electrified body had the power of attracting up the opposite kind of charge out of the eartl.

The quantity of the two charges thus separated by influence on such a conductor in the presence of a charge of electricity, depends upon the amourt of the charge, and upon the distance of the charged body from the conductor. A highly electrified glass rod will exert a greater influence than a less highly electrified one; and it produces a greater effect as it is brought nearer and nearer. The utmost it can do will be to induce on the near end a negative charge equal in amount to its own positive charge, and a similar amount of positive electrification at the far end ; but usually, before the electrified body can be brought so near as to do this, something else occurs which entirely alters the condition of things. As the electrified body is brought nearer and nearer, the charges of opposite sign on the two opposed surfaces attract one another more and more strongly and accumulate more and more densely, until, as the electrified body approaches very near, a spark is seen to dart across, the two charges thus rushing together to neutralize one 
another, leaving the induced charge of positive electricity, which was formerly repelled to the other end of the conductor, as a permanent charge after the electrified body has been removed.

In Fig. 19 is illustrated the operation of gradually lowering down over a table a positively electrified metal ball. The nearer it approaches the table, the more does the electric field surrounding it concentrate itself in the

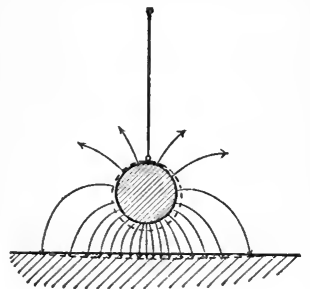

Fig. 19. gap between the ball and the table top; the latter becoming negatively electrified by influence. Where the electric lines are densest the tension in the medium is greatest, until when the ball is lowered still further the mechanical resistance of the air can no longer withstand the stress; it breaks down and the layer of air is pierced by a spark. If oil is used as a surrounding medium instead of air, it will be found to stand a much greater stress without being pierced.

24. Attraction due to Influence.-We are now able to apply the principle of influence to explain why an electrified body should attract things that have not been electrified at all. Fig. 18, on p. 26, may be taken to represent a light metal ball $B$ hung from a silk thread presented to the end of a rubbed glass rod A. The positive charge on A produces by influence a negative charge on the nearer side of $\mathrm{B}$ and an equal positive charge on the far side of $B$. The nearer half of the ball will therefore be attracted, and the farther half repelled; but the attraction will be stronger than the repulsion, because the attracted charge is nearer than the repelled. Hence on the whole the ball will be attracted. It can easily be observed that if a ball of non-conducting substance, such as wax, be employed, it is not 'attracted 
so much as a ball of conducting material. This in itself proves that influence really precedes attraction.

Another way of stating the facts is as follows:-The tension along the electric field on the right of $\mathrm{B}$ will be greater than that on the left, because of the greater concentration of the electric lines on the right.

25. Dielectric Power.-We have pointed out sereral times what part the intervening medium plays in these actions at a distance. The air, oil, glass, or other material between does not act simply as a non-conductor; it takes part in the propagation of the electric forces. Hence Faraday, who discovered this fact, termed such materials dielectrics. Had oil, or solid sulphur, or glass, been used insteal of air, the influence exerted by the presence of the electrified body at the same distance would have been greater. The power of a non-conducting substance to convey the influence of an electrified body across it is called its delectric power (or was formerly called its specific inductive capacity, see Art. 56 and Lesson XXIII.).

26. The Electrophorus. - We are now prepared to explain the operation of a simple and ingenious instrument, devised by Volta in 1775 , for the purpose of procuring, by the principle of influence, an unlimited number of charges of electricity from one single charge. This instrument * is the Electrophorus (Fig. 20). It consists of two parts, a round cake of resinous material cast in a metal dish or "sole," about 12 inches in diameter, and a round disk of slightly smaller diameter made of metal, or of wood covered with tinfoil, and provided with a glass handle. Shellac, or sealing-wax, or a mixture of resin, shellac, and Venice turpentine, may be used to make the cake. A slab of sulphur will also answer, but it is liable to crack. Sheets of hard ebonized indiarubber are excellent; but the surface of this substance

* Volta's electrophorus was announced in 1775. Its principle had alrealy been antieipated by Wilcke, who in 1762 described to the Swedish Academy of Sciences two "charging-machines" working by influence. 
requires occasional washing with ammonia and rubbing with paraffin oil, as the sulphur contained in it is liable to oxidize and to attract moisture. To use the electrophorus the resinous cake must be beaten or rubbed with a warm piece of woollen cloth, or, better still, with a cat's

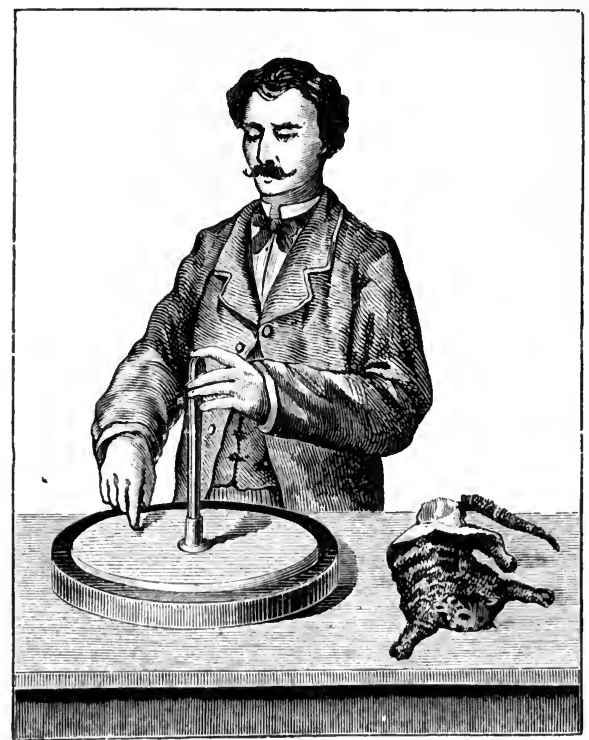

Fig. 20 .

skin. The disk or "cover" is then placed upon the cake, tonched momentarily with the finger, then removed by taking it up by the glass handle, when it is found to be powerfully electrified with a positive charge, so much so indeed as to yield a spark when the knuckle is presented to it. The "cover" may be replaced, touched, and once more removed, and will thus yield any number of sparks, 
the original charge on the resinous plate meanwhile remaining practically as strong as before.

The theory of the electrophorus is very simple, provided the student has clearly grasped the principle of influence explained above. When the resinous cake is first beaten with the cat's skin its surface is negatively electrified, as indicated in Fig. 21 . When the metal disk is placed down upon it, it rests really only on three or four points of the surface, and may be regarded as an insulated conductor in the presence of an electrified body. The negative electrification of the cake therefore acts by influence on the metallic disk or "cover," the natural electricity in it being displaced downwards, producing a positive charge on the under side, and leaving the upper

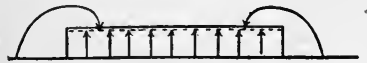

Fig. 21.

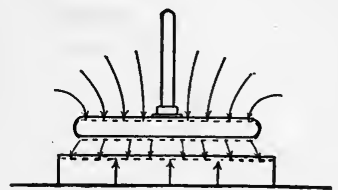

Fig. ‥

side negatively electrified. This state of things is shown in Fig. 22. If now the cover be touched for an instant with the finger, the negative charge of the upper surface will be neutralized by electricity flowing in from the earth through the hand and body of the experimenter. The attracted positive charge will, however, remain, being bound as it were by its attraction towards the negative charge on the cake. Fig. 23 shows the condition of things after the cover has been touched. If, finally, the cover be lifted by its handle, the remaining positive charge will be no longer "bound" on the lower surface by attraction, but will distribute itself on both sides of the cover, and may be used to give a spark, as already said. It is clear that no part of the original charge has been consumed in the process, which may be repeated as 
often as desired. As a matter of fact, the charge on the cake slowly dissipates-especially if the air be damp. Hence it is needful sometimes to renew the original charge by afresh beating the cake with the cat's skin. The labour of touching the cover with the finger at each operation may be saved by having a pin of brass or a strip of tinfoil projecting from the metallic "sole" on to the top of the cake, so that it touches the plate each time, and thus neutralizes the negative charge by allowing electricity to flow in from the earth.

The principle of the electrophorus may then be summed up in the following sentence. $A$ conductor if touched

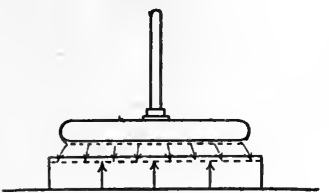

Fir. 23.

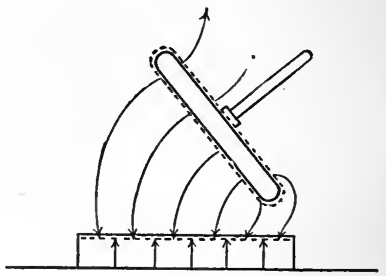

Fig. 24.

while under the influence of a charged body acquires thereby a charge of opposite sign.*

Since the electricity thus yielded by the electrophorus is not obtained at the expense of any part of the original charge, it is a matter of some interest to inquire what the source is from which the energy of this apparently unlimited supply is drawn; for it cannot be called into

* Priestley, in 1767, stated this principle in the following language:"The electric fluid, when there is a redundancy of it in any body, repels the electric fluid in any other body, when they are brought within the sphere of each other's influence, and drives it into the remote parts of the body; or quite out of the body, if there be any outlet for that purpose. In other words, bodies immerged in electric atmospheres always become possessed of the electricity, contrary to that of the body, in whose atmo. sphere they are immerged." 
existence without the expenditure of some other form of energy, any more than a steam-engine can work without fuel. As a matter of fact it is found that it is a little harder work to lift up the cover when it is charged than if it were not charged; for, when charged, there is the tension of the electric field to be overcome as well as the force of gravity. Slightly harder work is done at the expense of the muscular energies of the operator; and this is the real origin of the energy stored up in the separate charges. The purely mechanical actions of putting down the disk on the cake, touching it, and lifting it up, can be performed automatically by suitable mechanical arrangements, which render the production of these inductive charges practically continuous. Of such continuous electrophori, the latest is Wimshurst's machine, described in Lesson V.

27. "Free" and "Bound" Electriflcation.-We have spoken of a charge of electricity on the surface of a conductor, as being "bound" when it is attracted by the presence of a neighbouring charge of the opposite kind. The converse term "free" is sometimes applied to the ordinary state of electricity upon a charged conductor, not in the presence of a charge of an opposite kind. A "free" charge upon an insulated conductor flows away instantaneously to the earth, if a conducting channel be provided, as will be explained. It is immaterial what point of the conductor be touched. Thus, in the case represented in Fig. 17, wherein a + electrified body induces - electrification at the near end, and + electrification at the far end of an insulated conductor, the charge is "bound," being attracted, while the + charge at the other end, being repelled, is "free" ; and if the insulated conductor be touched by a person standing on the ground, the "free" charge will flow away through his body to the earth, or to the walls of the room, while the "bound" charge will remain, no matter whether he touch the conductor at the far end, or at the near end, or at the middle. 


\section{Method of charging the Gold-Leaf Electro-} scope by Influence.-The student will now be prepared to understand the method by which a Gold-Leaf Electroscope can be charged with the opposite kind of charge to that of the electrified body used to charge it. In Lesson II. it was assumed that the way to charge an electroseope was to place the excited body in contact with the knob, and thus permit, as it were, a small portion of the charge to flow into the gold leaves. A rod of glass rubbed on silk being + would thus obviously impart + electrification to the gold leaves.

Suppose, however, the rubbed glass rod to be held a few inches above the knob of the electroscope, as is indeed shown in Fig. 12. Even at this distance the gold leaves diverge, and the effect is due to influence. The gold leaves, and the brass wire and knob, form one continnous conductor, insulated from the ground by the glass jar. The presence of the + charge of the glass acts indnetively on this "insulated conductor," inducing electrification on the near end or knob, and inducing + at the far end, i.e. on the gold leaves, which diverge. Of these two induced charges, the - on the knob is "bound," while the + on the leaves is " free." If now, while the excited rod is still held above the electroscope, the knob be touched by a person standing on the ground, one of these two induced charges flows to the ground, namely, the free charge-not that on the knob itself, for it was "bound," but that on the gold leaves which was "free" - and the gold leaves instantly drop down straight. There now remains only the - charge on the knob, "bound" so long as the + charge of the glass rod is near to attract it. But if, finally, the glass rod be taken right away, the - charge is no longer "bound" on the knob, but is "free" to flow into the leaves, which once more diverge-but this time with a negative electrification.

29. The "Return-Shock."-It is sometimes noticed that, when a charged conductor is suddenly discharged, 
a discharge is felt by persons standing near, or may even affect electroscopes, or yield sparks. This action, known as the "return-shock," is due to influence. For in the presence of a charged conductor a charge of opposite sign will be induced in neighbouring bodies, and on the discharge of the conductor these neighbouring bodies may also suddenly discharge their induced charge into the earth, or into other conducting bodies. A "return-shock" is sometimes felt by persons standing on the ground at the moment when a flash of lightning has struck an object some distance away.

\section{Lesson IV.-Conduction and Distribution of Electricity}

30. Conduction. - Toward the close of Lesson I. we explained how certain bodies, such as the metals, conduct electricity, while others are non-conductors or insulators. This discovery is due to Stephen Gray; who, in 1729 , found that a cork, inserted into the end of a rubbed glass tube, and even a rod of wood stuck into the cork, possessed the power of attracting light bodies. He found, similarly, that metallic wire and packthread conducted electricity, while silk did not.

We may repeat these experiments by taking (as in Fig. 25) a glass rod, fitted with a cork and a piece of wood. If a bullet or a brass knob be hung to the end of this by a linen thread or a wire, it is found that when the glass tube is rubbed the bullet acquires the property of attracting light bodies. If a dry silk thread is used, however, no electricity will flow down to the bullet.

Gray even succeeded in transmitting a charge of electricity through a hempen thread over 700 feet long, suspended on silken loops. A little later Du Fay succeeded in sending electricity to no less a distance than 1256 feet through a moistened thread, thus proving the conducting power of moisture. From that time the 
classification of bodies into conductors and insulators has been observed.

This distinction cannot, however, be entirely maintained, as a large class of substances occupy an intermediate ground as partial conductors. For example, dry wood is a bad conductor and also a bad insulator; it is a good enough conductor to conduct away the highpotential electricity obtained by friction, but it is a bad conductor for the relatively low-potential electricity of small voltaic batteries. Substances that are very bad

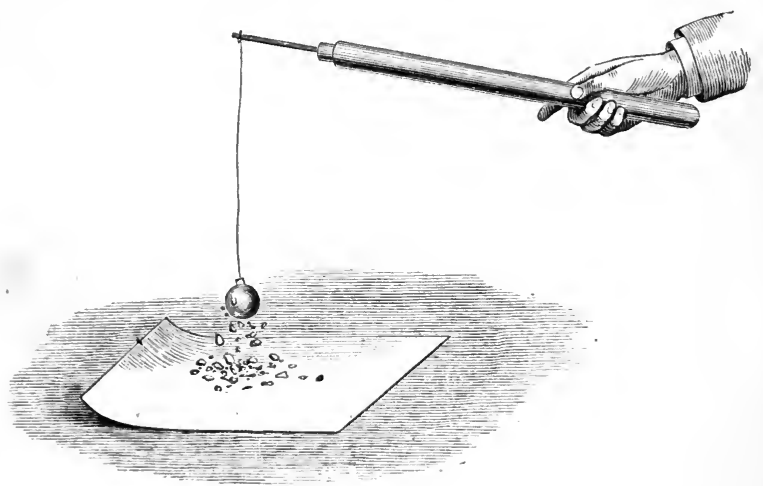

Fig. 25.

conductors are said to offer a great resistance to the flow of electricity through them. There is indeed no substance so good a conductor as to be devoid of resistance. There is no substance of so high a resistance as not to conduct a little. Even silver, which conducts best of all known substances, resists the flow of electricity to a small extent; and, on the other hand, such a non-conducting substance as glass, though its resistance is many million times greater than any metal, does allow a very small quantity of electricity to pass through it. In the 
following list, the substances named are placed in order, each conducting better than those lower down on the list.

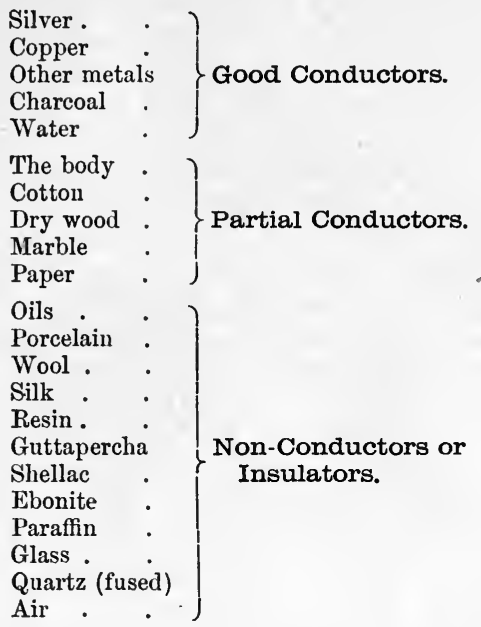

A simple way of observing experimentally whether a body is a conductor or not, is to take a charged goldleaf electroscope, and, holding the substance to be examined in the hand, touch the knob of the electroscope with it. If the substance is a conductor the electricity will flow away through it and through the body to the earth, and the electroscope will be discharged. Through good conductors the rapidity of the flow is so great that the discharge is practically instantaneous. Further information on this question is given in Lesson XXXIII.

31. Distribution of Charge on Bodies. - If electrification is produced at one part of a non-conducting body, it remains at that point and does not flow over the surface, or at most flows over it excessively slowly. 
Thus if a glass tube is rubbed at one end, only that one end is electrified. Hot glass is, however, a conductor. If a warm cake of resin be rubbed at one part with a piece of cloth, only the portion rubbed will attract light bodies, as may be proved by dusting upon it through a piece of muslin fine powders such as red lead, lycopodium, or verdigris, which adhere where the surface is electrified. The case is, however, wholly different when a charge of electricity is imparted to any part of a conducting body placed on an insulating support, for it instantly distributes itself all over the surface, though in general not uniformly over all points of the surface.

32. The Charge resides on the Surface.-A charge of electricity resides only on the surface of conducting bodies. This is proved by the fact that it is found to be immaterial to the distribution what the interior of a conductor is made of ; it may be solid metal, or hollow, or even consist of wood covered with tinfoil or gilt, but, if the shape be the same, the charge will distribute itself precisely in the same manner over the surface. There are also several ways of proving by direct experiment this very important fact. Let a hollow metal ball, having an aperture at the top, be taken (as in Fig. 26), and set upon an insulating stem, and charged by sending into it a few sparks from an electrophorus. The absence of any charge in the interior may be shown as follows:-In order to observe the nature of the electrification of a charged body, it is convenient to have some means of removing a small quantity of the charge as a sample for examination. To obtain such a sample, a little instrument known as a proof-plane is employed. It consists of a little disk of sheet copper or of gilt paper fixed at the end of a small glass rod. If this disk is laid on the surface of an electrified body at any point, part of the charge flows into it, and it may be then removed, and the sample thus obtained may be examined with a gold-leaf electroscope in the ordinary way. For some 
purposes a metallic bead, fastened to the end of a glass rod, is more convenient than a flat disk. If such a proofplane be applied to the outside of our electrified hollow ball, and then touched on the knob of an electroscope, the gold leaves will diverge, showing the presence of a

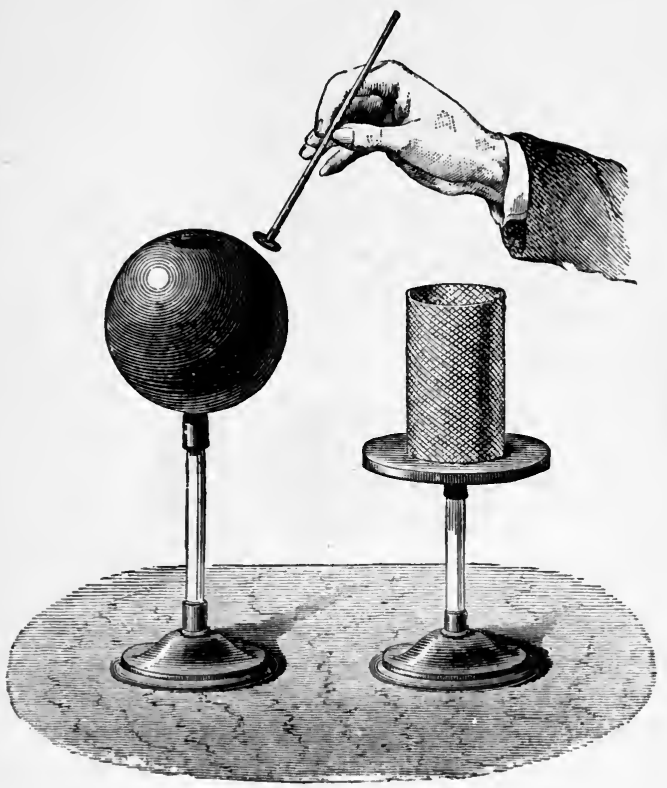

Fig. 26 .

charge. But if the proof-plane be carefully inserted through the opening, and touched against the inside of the globe and then withdrawn, it will be found that the inside is destitute of electrification. An electrified pewter mug will show a similar result, and so will even a cylinder of gauze wire. 
33. Biot's Experiment.-Biot proved the same fact in another way. A copper ball was electrified and insulated. Two hollow hemispheres of copper, of a larger size, and furnished with glass handles, were then placed together outside it (Fig. 27). So long as they did not come into contact the charge remained on the inner sphere; but if the outer shell touched the inner sphere for but an instant, the whole of the charge passed
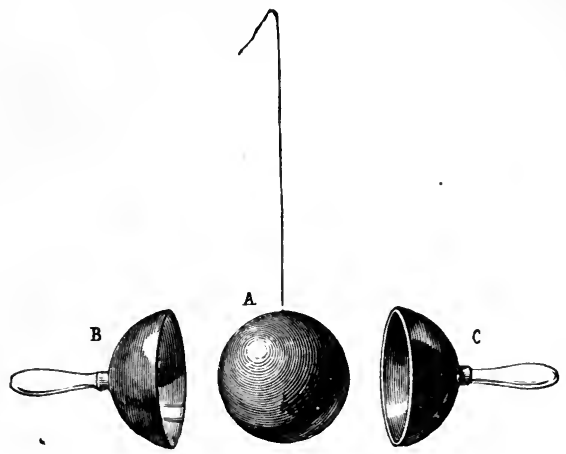

Fig. 27.

to the exterior; and when the hemispheres were separated and removed the inner globe was found to be completely discharged.

34. Further Explanation.-Doubtless the explanation of this behaviour of electricity is to be found in the property previously noticed as possessed by either kind of electrification, namely, that of repelling itself ; hence it retreats as far as can be from the centre and remains upon the surface. An important proposition concerning the absence of electric force within a closed conductor is proved in Lesson XXI.; meanwhile it must be noted that the proofs, so far, are directed to demonstrate the absence 
of a free charge of electricity in the interior of hollow conductors. Amongst other experiments, Terquem showed that a pair of gold leaves hung inside a wire cage could not be made to diverge when the cage was electrified. Faraday constructed a conical bag of linen-gauze, supported as in Fig. 28, upon an insulating stand, and to which silk strings were attached, by which it could be turned inside out. It was charged, and the charge was shown by the proof-plane and electroscope to be on the outside of the bag. On turning it inside out the elec-

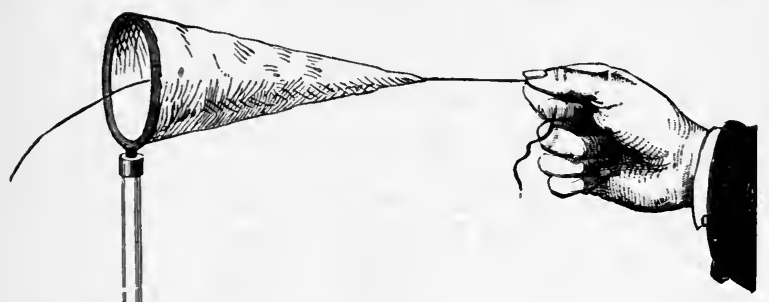

Fig. 28 .

tricity was once more found outside. Faraday's most striking experiment was made with a hollow cube, measuring 12 feet each way, built of wood, covered with tinfoil, insulated, and charged with a powerful machine, so that large sparks and brushes were darting off from every part of its outer surface. Into this cube Faraday took his most delicate electroscopes; but once within he failed to detect the least effect upon them.

35. Applications.-Advantage is taken of this in the construction of delicate electrometers and other 
instruments, which can be effectually screened from the influence of electrified bodies by enclosing them in a cover of thin metal, closed all round, except where apertures must be made for purposes of observation. Metal gauze answers excellently, and is nearly transparent. It was proposed by the late Professor Clerk Maxwell to protect buildings from lightning by covering them on the exterior with a network of wires.

36. Apparent Exceptions.-There are two apparent exceptions to the law that electrification resides only on the outside of conductors. (1) If there are electrified insulated bodies actually placed inside the hollow conductor, the presence of these electrified bodies acts inductively and attracts the opposite kind of charge to the inner side of the hollow conductor. (2) When electricity flows in a current, it flows through the substance of the conductor. The law is limited therefore to electricity at rest,- - that is, to statical charges.

37. Faraday's "Ice-pail " Experiment.-One experiment of Faraday deserves notice, as showing the part played by induction in these phenomena. $\mathrm{He}$ gradually lowered a charged metallic ball into a hollow conductor connected by a wire to a gold-leaf electroscope (Fig. 29), and watched the effect. A pewter icepail being convenient for his purpose, this experiment is continually referred to by this name, though any other hollow conductor - a tin canister or a silver mug, placed on a glass support-would of course answer equally well. The following effects are observed:- Suppose the ball to have a + charge: as it is lowered into the hollow conductor the gold leaves begin to diverge, for the presence of the charge acts inductively, and attracts a - charge into the interior and repels a + charge to the exterior. The gold leaves diverge more and more until the ball is right within the hollow conductor, after which no greater divergence is obtained. On letting the ball touch the inside the gold leaves still remain diverging as 
before, and if now the ball is pulled out it is found to have lost all its electrification. The fact that the gold leaves diverge no wider after the ball touched than they did just before, proves that when the charged ball is right inside the hollow conductor the induced charges are each of them precisely equal in amount to its own charge, and the interior negative charge exactly neutralizes the charge on the ball at the noment when they touch, leaving the equal exterior charge unchanged. An electric cage, such as this icepail, when connected with an electroscope

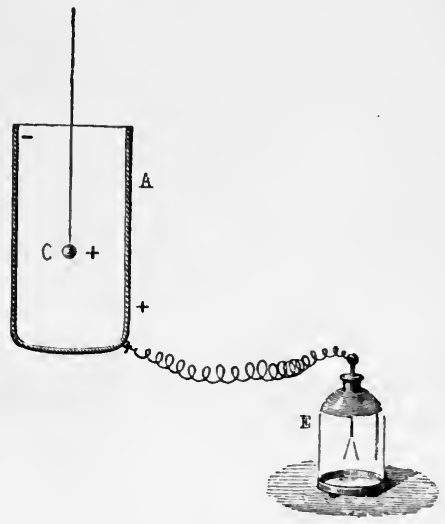

Fig. 29.

or electrometer, affords an excellent means of examining the charge on a body small enough to be hung inside it. For without using up any of the charge of the body (which we are obliged to do when applying the method of the proof-plane) we can examine the induced charge repelled to the outside of the cage, which is equal in amount and of the same sign. If two equal charges of opposite kinds are placed at the same time within the cage no effects are produced on the outside.

38. Distribution of Charge.-A charge of electricity is not usually distributed uniformly over the surfaces of bodies. Experiment shows that there is more electricity on the edges and corners of bodies than upon their flatter parts. This distribution can be deduced from the theory laid down in Lesson XXI., but meantime we will give some of the chief cases as they can be 
shown to exist. The term Electric Density is used to signify the amount of electricity at any point of a surface ; the electric density at a point is the number of units of electricity per unit of area (i.e. per square inch, or per square centimetre), the distribution being supposed uniform over this small surface.

(a) Sphere.-The distribution of a charge over an insulated sphere of conducting material is uniform, provided the sphere is also isolated, that is to say, is remote from the presence of all other conductors and all other electrified bodies. The density is uniform all over it. This is symbolized by the dotted line round the sphere in
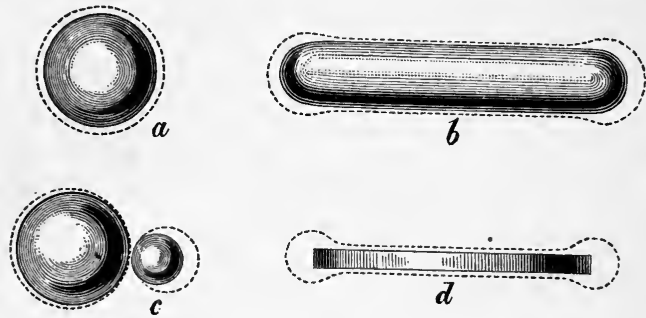

Fig. 30 .

Fig. $30 a$, which is at an equal distance from the sphere all round, suggesting an equal thickness of charge at every point of the surface. It must be remembered that the charge is not really of any perceptible thickness at all ; it resides on or at the surface, but cannot be said to form a stratum upon it.

(b) Cylinder with rounded Ends.-Upon an elongated conductor, such as is frequently employed in electrical apparatus, the density is greatest at the ends where the curvature of the surface is the greatest.

(c) Two Spheres in contact.-If two spheres in contact with each other are insulated and charged, it is found that the density is greatest at the parts farthest 
from the point of contact, and least in the crevice between them. If the spheres are of unequal sizes the density is greater on the smaller sphere, which has the surface more curved. On an egg-shaped or pear-shaped conductor the density is greatest at the small end. On a cone the density is greatest at the apex; and if the cone terminate in a sharp point the density there is very much greater than at any other point. At a point, indeed, the density of the collected electricity may be so great as to electrify the neighbouring particles of air, which then are repelled (see Art. 47), thus producing a continual loss of charge. For this reason points and sharp edges are always avoided on electrical apparatus, except where it is specially desired to set up a discharge.

(d) Flat Disk.-The density of a charge upon a flat disk is greater, as we should expect, at the edges than on the flat surfaces; but over the flat surfaces the distribution is fairly uniform.

These various facts are ascertained by applying a small proof-plane successively at various points of the electrified bodies and examining the amount taken up by the proofplane by means of an electroscope or electrometer. Coulomb, who investigated mathematically as well as experimentally many of the important cases of distribution, employed the torsion balance to verify his calculations. He investigated thus the case of the ellipsoid of revolution, and found the densities of the charges at the extremities of the axis to be proportional to the lengths of those axes. $\mathrm{He}$ also showed that the density of the charge at any other point of the surface of the ellipsoid was proportional to the length of the perpendicular drawn from the centre to the tangent at that point. Riess also investigated several interesting cases of distribution. He found the density at the middle of the edges of a cube to be nearly two and a half times as great as the density at the middle of a face ; while the density at a corner of the cube was more than four times as great. 
39. Redistribution of Charge.--If any portion of the charge of an insulated conductor be removed, the remainder of the charge will immediately redistribute itself over the surface in the same manner as the original charge, provided it be also isolated, i.e. that no other conductors or charged bodies be near to perturb the distribution by complicated effects of influence.

If a conductor be charged with any quantity of electricity, and another conductor of the same size and shape (but uncharged) be brought into contact with it for an instant and then separated, it will be found that the charge has divided itself equally between them. In the same way a charge may be divided equally into three or more parts by being distributed simultaneously over three or more equal and similar conductors brought into contact and symmetrically placed.

If two equal metal balls, suspended by silk strings, charged with unequal quantities of electricity, are brought for an instant into contact and then separated, it will be found that the charge has redistributed itself fairly, half the sum of the two charges being now the charge of each. This may even be extended to the case of charges of opposite signs. Thus, suppose two similar conductors to be electrified, one with a positive charge of 5 units and the other with 3 units of negative charge, when these are made to tonch and separated, each will have a positive charge of 1 unit; for the algebraic sum of +5 and -3 is +2 , which, shared between the two equal conductors, leaves +1 for each.

40. Capacity of Conductors.-If the conductors be unequal in size, or unlike in form, the shares taken by each in this redistribution will not be equal, but will be proportional to the electric capacities of the conductors. The definition of capacity in its relation to electric quantities is given in Lesson XXI., Art. 271. We may, however, make the remark, that two insulated conductors of the same form, but of different sizes, differ in their 
electrical capacity; for the larger one must have a larger amount of electricity imparted to it in order to electrify its surface to the same degree. The term potential is employed in this connection, in the following way :-A given quantity of electricity will electrify an isolated body up to a certain "potential" (or power of doing electric work) depending on its capacity. A large quantity of electricity imparted to a conductor of small capacity will electrify it up to a very high potential; just as a large quantity of water poured into a vessel of narrow capacity will raise the surface of the water to a high level in the vessel. The exact definition of Potential, in terms of energy spent against the electrical forces, is given in the lesson on Electrostatics (Art. 263).

It will be found convenient to refer to a positively electrified body as one electrified to a positive or high potential; while a negatively electrified body may be looked upon as one electrified to a low or negative potential. And just as we take the level of the sea as a zero level, and measure the heights of mountains above it, and the depths of mines below it, using the sea level as a convenient point of reference for differences of level, so we take the potential of the earth's surface (for the surface of the earth is always electrified to a certain degree) as zero potential, and use it as a convenient point of reference from which to measure differences of electric potential.

\section{Lesson V.-Electric Machines}

41. For the purpose of procuring larger supplies of electricity than can be obtained by the rubbing of a rod of glass or shellac, electric machines have been devised. All electric machines consist of two parts, one for producing, the other for collecting, the electric charges. Experience has shown that the quantities of + and - 
electrification developed by friction upon the two surfaces rubbed against one another depend on the amount of friction, upon the extent of the surfaces rubbed, and also upon the nature of the substances used. If the two substances employed are near together on the list of electrics given in Art. 6, the electrical effect of rubbing them together will not be so great as if two substances widely separated in the series are chosen. To obtain the highest effect, the most positive and the most negative of the substances convenient for the construction of a machine should be taken, and the greatest available surface of them should be subjected to friction, the moving parts having a sufficient pressure against one another compatible with the required velocity.

The earliest form of electric machine was devised by Otto von Guericke of Magdeburg, and consisted of a globe of sulphur fixed upon a spindle, and pressed with the dry surface of the hands while being made to rotate; with this he discovered the existence of electric sparks and the repulsion of similarly electrified bodies. Sir Isaac Newton replaced Von Guericke's globe of sulphur by a globe of glass. A little later the form of the machine was improved by various German electricians; Von Bose added a collector or "prime conductor," in the shape of an iron tube, supported by a person standing on cakes of resin to insulate him, or suspended by silken strings; Winckler of Leipzig substituted a leathern cushion for the hand as a rubber ; and Gordon of Erfurt rendered the machine more easy of construction by using a glass cylinder instead of a glass globe. The electricity was led from the excited cylinder or globe to the prime conductor by a metallic chain which hung over against the globe. A pointed collector was not employed until after Franklin's famous researches on the action of points. About 1760 De la Fond, Planta, Ramsden, and Cuthbertson, constructed machines having glass plates instead of cylinders. All frictional machines are, however, now 
obsolete, having in recent years been quite superseded by the modern Influence Machines.

42. The Cylinder Electric Machine. - The Cylinder Electric Machine consists of a glass cylinder mounted on a horizontal axis capable of being turned by a handle. Against it is pressed from behind a cushion of leather stuffed with horsehair, the surface of which is covered with a powdered amalgam of zinc or tin. A flap of silk attached to the cushion passes over the cylinder, covering its upper half. In front of the cylinder stands the "prime conductor," which is made of metal, and

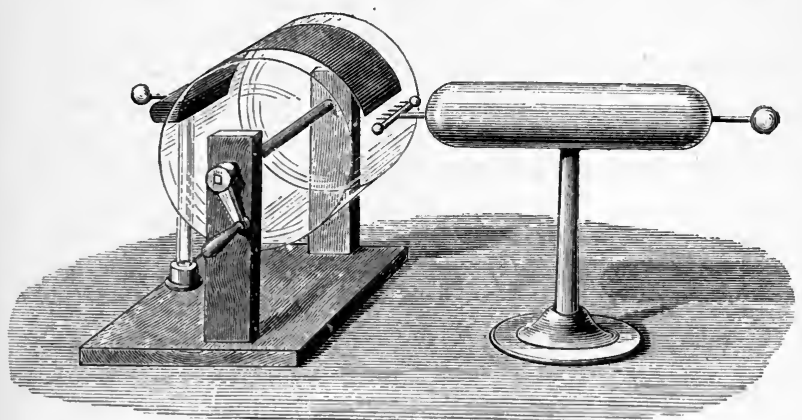

Fig. 31.

usually of the form of an elongated cylinder with hemispherical ends, mounted upon a glass stand. At the end of the prime conductor nearest the cylinder is fixed a rod bearing a row of fine metallic spikes, resembling in form a rake; the other end usually carries a rod terminated in a brass ball or knob. The general aspect of the machine is shown in Fig. 31. When the handle is turned the friction between the glass and the amalgam-coated surface of the rubber produces a copious electrical action, electricity appearing as a + charge on the glass, leaving the rubber with a - charge. The prime conductor collects 
this charge by the following process:-The + charge being carried round on the glass acts inductively on the long insulated conductor, repelling a + charge to the far end; leaving the nearer end - ly charged. The effect of the row of points is to emit a - ly electrified wind (see Art. 47) towards the attracting + charge upon the glass, which is neutralized thereby ; the glass thus arriving at the rubber in a neutral condition ready to be again excited. This action of the points is sometimes described, though less correctly, by saying that the points collect the + charge from the glass. If it is desired to collect also the - charge of the rubber, the cushion must be supported on an insulating stem and provided at the back with a metallic knob. It is, however, more usual to use only the + charge, and to conmect the rubber by a chain to "earth," so allowing the - charge to be neutralized.

43. The Plate Electric Machine. - The Plate Machine, as its name implies, is constructed with a

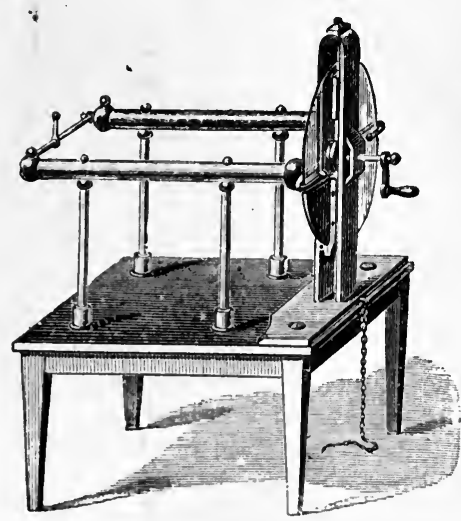

Fig. 32. circular plate of glass or of ebonite, and is usually provided with two pairs of rubbers formed of double cushions, pressing the plate between them, placed at its highest and lowest point, and provided with silk flaps, each extending over a quadrant of the circle. The prime conductor is either double or curved round to meet the plate at the two ends of its horizontal diameter, and is furnished with two sets of spikes, for the same purpose as the row of 
points in the cylinder machine. A common form of plate machine is shown in Fig. 32. The action of the machine is, in all points of theoretical interest, the same as that of the cylinder machine. Its advantages are that a large glass plate is more easy to construct than a large glass cylinder of perfect form, and that the length along the surface of the glass between the collecting row of points and the edge of the rubber cushions is greater in the plate than in the cylinder for the same amount of surface exposed to friction ; for, be it remarked, when the two charges thus separated have collected to a certain extent, a discharge will take place along this surface, the length of which limits therefore the power of the machine. In a more modern form, due to Le Roy, and modified by Winter, there is but one rubber and flap, occupying a little over a quadrant of the plate, and one collector or double row of points, while the prime conductor consists of a ring-shaped body.

44. Electric Amalgam.-Canton, finding glass to be highly electrified when dipped into dry mercury, suggested the employment of an amalgam of tin with mercury as a suitable substance wherewith to cover the surface of the rubbers. Still better is Kienmayer's amalgam, consisting of equal parts of tin and zinc, mixed while molten with twice their weight of mercury. Bisulphide of tin ("mosaic gold") may also be used. These amalgams are applied to the cushions with a little stiff grease. They serve the double purpose of conducting away the negative charge separated upon the rubber during the action of the machine, and of affording as a rubber a substance which is more powerfully negative (see list in Art. 6) than the leather or the silk of the cushion itself. Powdered graphite is also good.

45. Precautions in using Frictional Machines. - Several precautions must he observed in the use of electrical machines. Damp and dust must be scrupulously avoided. The surface of glass is hygroscopic, 
hence, except in the driest climates, it is necessary to warm the glass surfaces and rubbers to dissipate the film of moisture which collects. Glass stems for insulation may be varnished with a thin coat of shellac varnish, or with paraffin (solid). A few drops of anhydrous paraffin (obtained by dropping a lump of sodium into a bottle of paraffin oil), applied with a bit of flannel to the previously warmed surfaces, hinders the deposit of moisture. A frictional machine which has not been used for some months will require a fresh coat of amalgam on its rubbers. These should be cleaned and warmed, a thin uniform layer of tallow or other stiff grease is spread upon them, and the amalgam, previously reduced to a fine powder, is sifted over the surface. In spite of all precautions friction machines are uncertain in their behaviour in damp weather. This is the main reason why they have been superseded by influence machines, which do not need to be warmed.

All points should be avoided in apparatus for frictional electricity except where they are desired, like. the "collecting" spikes on the prime conductor, to let off a charge of electricity. All the rods, etc., in frictional apparatus are therefore made with rounded knobs.

46. Experiments with the Electric Machine. -With the electric machine many pleasing and instructive experiments are possible. The phenomena of attraction and repulsion can be shown upon a large scale. Fig. 33 represents a device known as the electric chimes,* in which two small brass balls hung by silk strings are set in motion and strike against the bells between which they are hung. The two outer bells are hung by metallic wires or chains to the knob of the machine. The third bell is hung by a silk thread, but communicates with the ground by a brass chain. The balls are first attracted to

* Invented in 1752 by Franklin, for the purpose of warning him of the presence of atmospheric electricity, drawn from the air above his house by a pointed iron rod. 
the electrified outer bells, then repelled, and, having discharged themselves against the uninsulated central bell, are again attracted, and so vibrate to and fro.

By another arrangernent small figures or dolls cut out of pith can be made to dance up and down between a metal plate hung horizontally from the knob of the machine, and another flat plate an inch or two lower and communicating with "earth."

Another favourite way of exhibiting electric repulsion is by means of a doll with long hair placed on the machine ; the individual hairs stand on end when the machine is worked, being repelled from the head, and from one another. A. paper tassel will behave similarly if hung to the prime con-

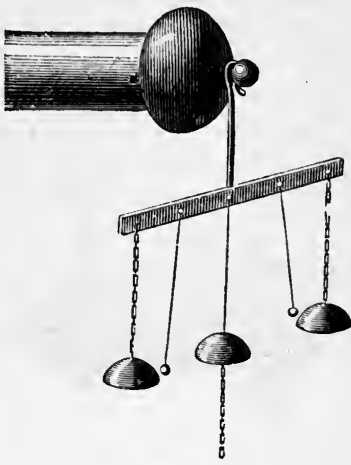

Fig. 33. ductor. The most striking way of showing this phenomenon is to place a person upon a glass-legged stool, making him touch the knob of the machine; when the machine is worked, his hair, if dry, will stand on end. Sparks will pass freely between a person thus electrified and one standing upon the ground.

The sparks from the machine may be made to kindle spirits of wine or ether, placed in a metallic spoon, connected by a wire with the nearest metallic conductor that runs into the ground. A gas jet may be lit by passing a spark to the burner from the finger of the person standing, as just described, upon an insulating stool.

47. Effect of Points; Flectric Wind.-The effect of points in discharging electricity from the surface of a conductor may be readily proved by numerous experiments. 
If the machine be in good working order, and capable of giving, say, sparks 4 inches long when the knuckle is presented to the knob, it will be found that, on fastening a fine-pointed needle to the conductor, it discharges the electricity so effectually at its point that only the shortest sparks can be drawn at the knob, while a fine jet or brush of pale blue light will appear at the point. If a lighted taper be held in front of the point, the flame will be visibly blown aside (Fig. 34) by the streams of electrified air repelled from the point. These air-currents can be

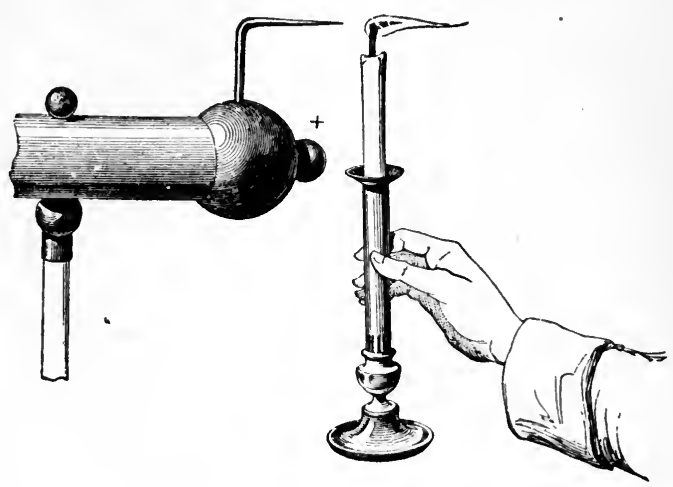

Fig. 34.

felt with the hand. They are due to a mutual repulsion between the elestrified air particles near the point and the electricity collected on the pcint itself. That this nutual reaction exists is proved by the electric fly or electric reaction-mill of Hamilton (Fig. 35), which consists of a light cross of brass or straw, suspended on a pivot, and having the pointed ends bent round at right angles. When placed on the prime conductor of the machine, or joined to it by a chain, the force of repulsion between the electricity of the points and that on the air 
immediately in front of them drives the mill round in the direction opposite to that in which the points are bent. It will even rotate if immersed in turpentine or petroleum. If the points of the fly are covered with small round lumps of wax it will not rotate, as the presence of the wax prevents the formation of any wind or stream of electrified particles.

The electric wind from a point will produce a charge upon the surface of any insulating borly, such as a plate of ebonite or glass, held a few inches away. The charge may be examined by dusting red lead or lycopodium powder upon the surface. If a slip of

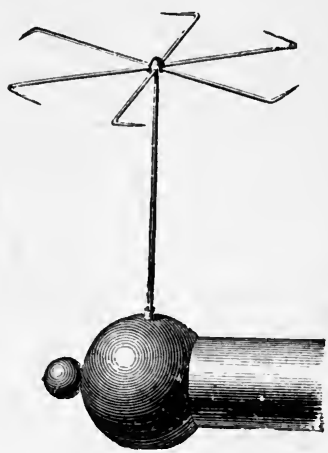

Fig. 35. glass or mica be interposed between the point and the surface against which the wind is directed, an electric shadow will be formed on the surface at the part so screener.

48. Armstrong's Hydro-Electrical Machine.The friction of a jet of steam issuing from a boiler, through a wooden nozzle, generates electricity. In reality it is the particles of condensed water in the jet which are directly concerned. Lord Armstrong, who investigated this source of electricity, constructed.a powerful apparatus, known as the hydro-electrical machine, capable of producing enormous quantities of electricity, and yielding sparks 5 or 6 feet long. The collector consisted of a row of spikes, placed in the path of the steam jets issuing from wooden nozzles, and was supported, together with a brass ball which served as prime conductor, upon a glass pillar.

49. Influence Machines.-There is another class 
of electrical machine, differing entirely from those we have been describing, and depending upon the principle of influence. They also have been termed convection-induction machines, because they depend upon the employment of a minute initial charge which, acting by influence, induces other charges, which are then conveyed by the moving parts of the machine to some other part, where they can be used either to increase the initial charge or to furnish a supply of electrification to a suitable collector. Of such instruments the oldest is the Electrophorus, explained fully in Lesson III. Bennet, Nicholson, Erasmus Darwin, and others devised pieces of apparatus for accomplishing by mechanisn that which the electrophorus accomplishes by hand. Nicholson's revolving doubler, invented in 1788, consists of a revolving apparatus, in which an insulated carrier can be brought into the presence of an electrified body, there touched for an instant while under influence, then carried forward with its acquired charge towards another body, to which it imparts its charge, and which in turn acts inductively on it, giving it an opposite charge, which it can convey to the first body, thus increasing its initial charge at every rotation.

In the modern influence machines two principles are embodied : (1) the principle of influence, namely, that a conductor touched while under influence acquires a charge of the opposite kind; (2) the principle of reciprocal accumulation. This principle must be carefully noted. Let there be two insulated conductors $\mathrm{A}$ and $\mathrm{B}$ electrified ever so little, one positively, the other negatively. Let a third insulated conductor C, which will be called a carrier, be arranged to move so that it first approaches $\mathrm{A}$ and then $\mathrm{B}$, and so forth. . If touched while under the influence of the small positive charge on $\mathrm{A}$ it will acquire a small negative charge; suppose that it then moves on and gives this negative charge to $\mathrm{B}$. Then let it be touched while under the influence of $B$, so aequiring a small positive charge. When it returns towards $\mathrm{A}$ let it give 
up this positive charge to $A$, thereby increasing its positive charge. Then $\mathrm{A}$ will act more powerfully, and on repeating the former operations both $\mathrm{B}$ and $\mathrm{A}$ will become more highly charged. Each accumulates the charges derived by influence from the other. This is the fundamental action of the machines in question. The modern influence machines date from 1860, when C. F. Varley produced a form with six carriers mounted on a rotating disk of glass. This was followed in 1865 by

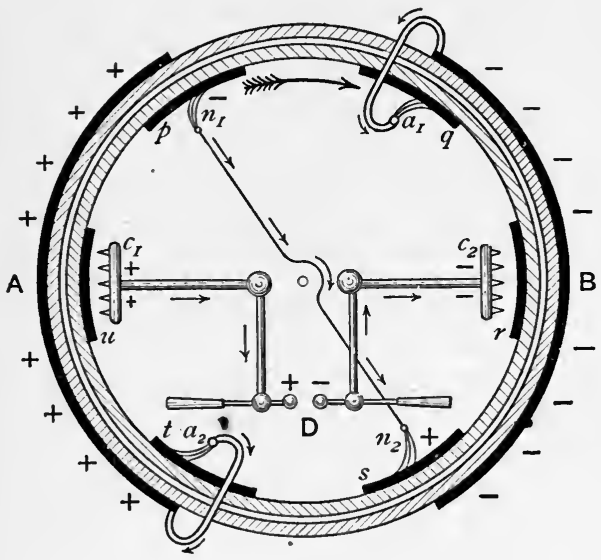

Fig. 36.

the machine of Holtz and that of Toepler, and in 1867 by those of Lord Kelvin (the "replenisher" and the "mouse-mill"). The latest forms are those of Mr. James Wimshurst.

50. Typical Construction. - Before describing some special forms we will deal with a generalized type of machine having two fixed field-plates, $\mathrm{A}$ and $\mathrm{B}$, which are to become respectively + and - , and a set of carriers, attached to a rotating disk or armature. Fig. 36 gives in 
a diagrammatic way a view of the essential parts. For convenience of drawing it is shown as if the metal fieldplates $A$ and $B$ were affixed to the outside of an outer stationary cylinder of glass; the six carriers $p, q, r, s, t$, and $u$ being attached to the inside of an inner rotating cylinder. The essential parts then are as follows :-

(i.) A pair of field-plates A and B.

(ii.) A set of rotating carriers $p, q, r, s, t$, and $u$.

(iii.) A pair of neutralizing brushes $n_{1}, n_{2}$ made of flexible metal wires, the function of which is to touch the carriers while they are under the influence of the field-plates. They are connected together by a diagonal conductor, which need not be insulated.

(iv.) A pair of appropriating brushes $a_{1}, a_{2}$, which reach over from the field-plates to appropriate the charges that are conveyed around by the carriers, and impart them to the field-plates.

(v.) In addition to the above, which are sufficient to - constitute a complete self-exciting machine, it is usual to add a discharging apparatus, consisting of two combs $c_{1}, c_{2}$ to collect any unappropriated charges from the carriers after they have passed the appropriating. brushes; these combs being connected to the adjustable discharging balls at $\mathrm{D}$ :

The operation of the machine is as follows. The neutralizing brushes are set so as to touch the moving carriers just before they pass out of the influence of the field-plates. Suppose the field-plate A to be charged ever so little positively, then the carrier $p$, touched by $n_{1}$ just as it passes, will acquire a slight negative charge, which it will convey forward to the appropriating brush $a_{1}$, and will thus make $B$ slightly negative. Each of the carriers as it passes to the right over the top will do the same thing. Similarly each of the carriers as it passes from 
right to left at the lower side will be touched by $n_{2}$ while under the influence of the - charge on $\mathrm{B}$, and will convey a small + charge to A through the appropriating brush $a_{2}$. In this way $\mathrm{A}$ will rapidly become more and more +, and $\mathrm{B}$ more and more - ; and the more highly charged they become, the more do the collecting combs $c_{1}$ and $c_{2}$ receive of. unappropriated charges. Sparks will snap across between the discharging knobs at $\mathrm{D}$.

The machine will not be self-exciting unless there is a good metallic contact made by the neutralizing brushes and by the appropriating brushes. If the discharging apparatus were fitted at $c_{1}, c_{2}$ with contact brushes instead of spiked combs, the machine would be liable to lose the charge of the field-plates, or even to have their charges reversed in sign whenever a large spark was taken from the knobs.

It will be noticed that there are two thicknesses of glass between the fixed field-plates and the rotating carriers. The glass serves not only to hold the metal parts, but prevents the possibility of back-discharges (by sparks or winds) from the carriers to the field-plates as they pass.

The essential features thus set forth will be found in Varley's machine of 1860, in Lord Kelvin's "replenisher" (which had only two carriers), and in many other machines, including the apparatus known as Clarke's "gas-lighter."

51. 'Toepler's Influence Machine. - In this machine, as constructed by Voss, are embodied various points due to Holtz and others. Its construction follows almost literally the diagram already explained, but instead of having two cylinders, one inside the other, it has two flat disks of varnished glass, one fixed, the other slightly smaller rotating in front of it (Fig. 37). The ficld-plates $A$ and $B$ consist of pieces of tinfoil, cemented on the back of the back disk, each protected by a coating of varnished paper. The carriers are small disks or sectors of tinfoil, to the number of six or eight, cemented to the front of the front disk. To prevent them from being worn away by rubbing against the brushes a small 
metallic button is attached to the middle of each. The neutralizing brushes $n_{1}, n_{2}$ are small whisps of fine springy brass wire, and are mounted on the ends of a diagonal conductor $\mathrm{Z}$. The appropriating brushes $a_{1}, a_{2}$ are also of thin brass wire, and are fastened to clamps projecting from the edge of the fixed disk, so that they communicate metallically with the two field-plates. The collecting combs, which have brass spikes so short as not to touch the carriers, are mounted on insulating pillars and are connected to the adjustable discharging knobs

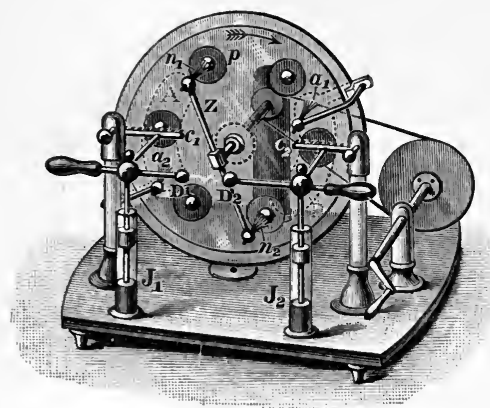

Fig. 37.
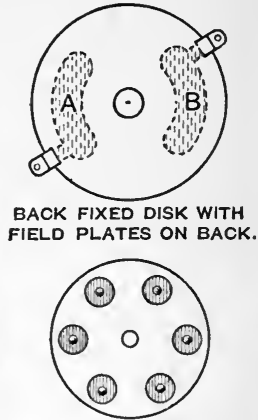

FRONT ROTATING DISK WITH CARRIERS ON FRONT.

$\mathrm{D}_{1}, \mathrm{D}_{2}$. These also communicate with two small Leyden jars $J_{1}, J_{2}$, the function of which is to accumulate the charges before any discharge takes place. These jars are separately depicted in Fig. 38. Without them, the discharges between the knobs take place in frequent thin blue sparks. With them the sparks are less numerous, but very brilliant and noisy.

To use the Toepler (Voss) machine first see that all the four brushes are so set as to make good metallic contact with the carriers as they move past, and that the 
neutralizing brushes are set so as to touch the carriers while under influence. Then see that the discharging knobs are drawn widely apart. Set the machine in rotation briskly. If it is clean it should excite itself after a couple of turns, and will emit a gentle hissing sound, due to internal discharges (visible as blue glimmers in the dark), and will offer more resistance to turning. If then the knobs are pushed nearer together sparks will pass across between them. The jars (the addition of which we owe to Holtz) should be kept free from dust. Sometimes a pair of terminal screws are added at $\mathrm{S}_{1}, \mathrm{~S}_{2}$ (Fig. 38), connected respectively with the outer coatings

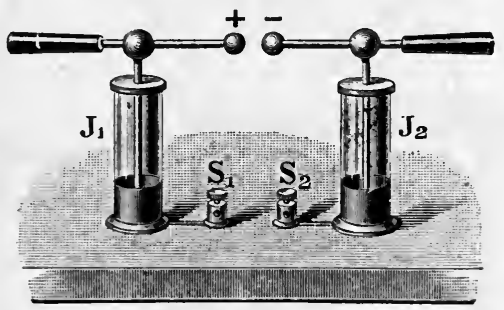

Fig. 38.

of the jars. These are convenient for attaching wires to lead away discharges for experiments at a distance. If not so used they should be joined together by a short wire, as the two jars will not work properly unless their outer coatings are connected.

52. Wimshurst's Influence Machine.--In this, the most widely used of influence machines, there are no fixed field-plates. In its simplest form it consists (Fig. 39) of two circular plates of varnished glass, which are geared to rotate in opposite directions. A number of sectors of metal foil are cemented to the front of the front plate and to the back of the back plate; these sectors serve both as carriers and as inductors. Across 
the front is fixed an uninsulated diagonal conductor, carrying at its ends neutralizing brushes, which touch the front sectors as they pass. Across the back, but sloping the other way, is a second diagonal conductor, with brushes that touch the sectors on the hinder plate. Nothing more than this is needed for the machine to excite itself when set in rotation; but for convenience

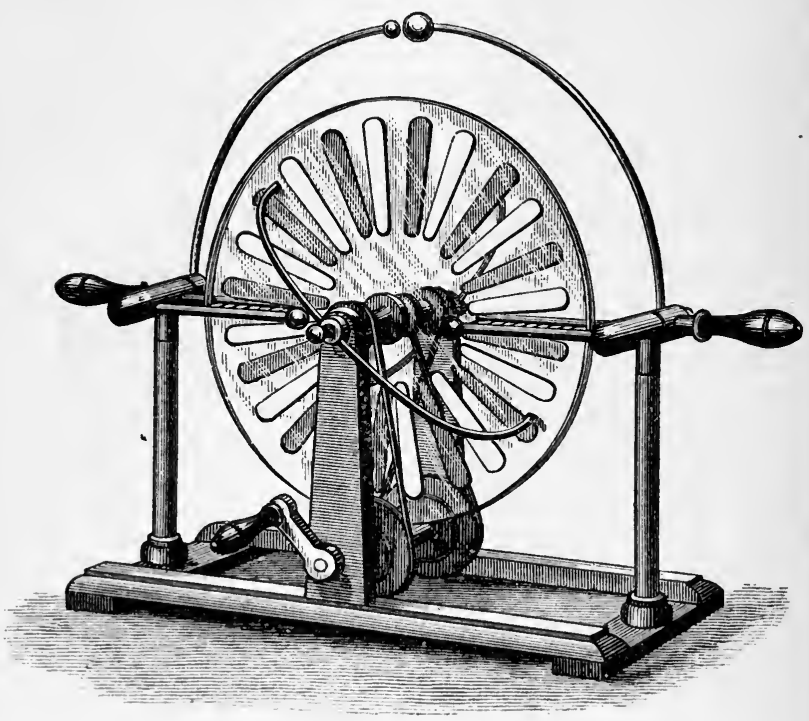

Fig. 39.

there is added a collecting and discharging apparatus. This consists of two pairs of insulated combs, each pair having its spikes turned inwards toward the revolving disks, but not touching them; one pair being on the right, the other on the left, mounted each on an insulating pillar of ebonite. These collectors are furnished with a pair of adjustable discharging knobs overhead; and 
sometimes a pair of Leyden jars are added, to prevent the sparks from passing until considerable quantities of charge have been collected.

The processes that occur in this machine are best explained by aid of a diagram (Fig. 40), in which, for greater clearness, the two rotating plates are represented

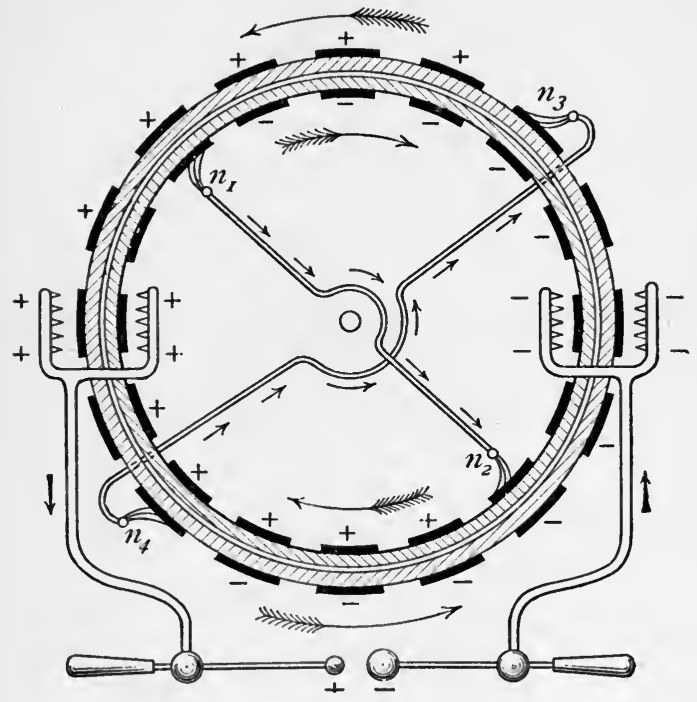

Fig. 40.

as though they were two cylinders of glass, rotating opposite ways, one inside the other. The inner cylinder will represent the front plate, the outer the back plate. In Figs. 39 and 40 the front plate rotates righthandedly, the back plate left-handedly. The neutralizing brushes $n_{1}, n_{2}$ touch the front sectors, while $n_{3}, n_{4}$ touch against the back sectors. 
Now suppose any one of the back sectors represented near the top of the diagram to receive a slight positive charge. As it is moved onward toward the left it will come opposite the place where one of the front sectors is moving past the brush $n_{1}$. The result will be that the sector so touched while under influence by $n_{1}$ will acquire a slight negative charge, which it will carry onwards toward the right. When this negatively-charged front sector arrives at a point opposite $n_{3}$ it acts inductively on the back sector which is being touched by $n_{3}$; hence this back sector will in turn acquire a positive charge, which it will carry over to the left. In this way all the sectors will become more and more highly charged, the front sectors carrying over negative charges from left to right, and the back sectors carrying over positive charges from right to left. At the lower half of the diagram a similar but inverse set of operations will be taking place. For when $n_{1}$ touches a front sector under the influence of a positive back sector, a repelled charge will travel along the diagonal conductor to $n_{2}$, helping to charge positively the sector which it touches. The front sectors, as they pass from right to left (in the lower half), will carry positive charges, while the back sectors, after tonching $n_{4}$, will carry negative charges from left to right. The metal sectors then act both as carriers and as inductors. It is clear that there will be a continual carrying of positive charges toward the right, and of negative charges to the left. At these points, toward which the opposite kinds of charges travel, are placed the collecting-combs communicating with the discharging knobs. The latter ought to be opened wide apart when starting the machine, and moved together after it has excited itself.

In larger Wimshurst influence machines two, three, or more pairs of oppositely -rotating plates are mounted within a glass case to keep off the dust. If the neutralizing brushes make good metallic contact these machines are all self-exciting in all weathers. Machines with only 
six or eight sectors on each plate give longer sparks, but less frequently than those that have a greater number. Mr. Wimshurst has designed many influence machines, from small ones with disks 2 inches across up to that at South Kensington, which has plates 7 feet in diameter.

Prior to Wimshurst's machine Holtz had constructed one with two oppositely-rotating glass disks; but they had no metal carriers upon them. It was not self-exciting.

53. Holtz's Influence Machine.--The Holtz machine in its typical form had the following peculiarities. There were no metal carriers upon the rotating plate, hence another mode of charging it had to be adopted in lieu of touching conductors while under influence, as will be seen. The field-plates A and B (Fig. 41) were of varnished paper - a poor conductor -fastened upon the back of the fixed disk. In the fixed disk of glass, on which the field-plates were mounted, there were cut two windows or openings, through which there pro-

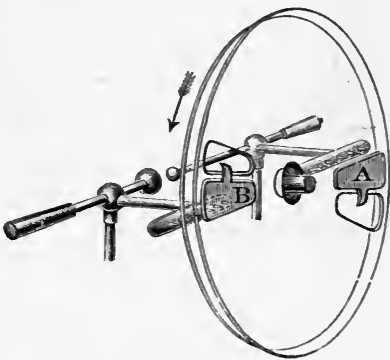

Fig. 41. jected from the field-plates two pointed paper tongues, which took the place of appropriating brushes. The discharging knobs were inserted in the neutralizing circuit which united two metal combs with pointed spikes, situated in front of the rotating front disk, opposite the two field-plates. There was (at first) no diagonal conductor. It will be noted that while the combs, which served both as neutralizing and collecting combs, were in front of the rotating plate, the appropriating tongues were situated at the back of the same. Fig. 41 is a view of the machine from behind. The machine was not self-exciting. In operating it the following procedure 
was used: first the two discharging knobs were put together, then the front disk was set into rapid rotation. While so rotating a small initial charge was communicated to one of the field-plates by holding to it a rubbed piece of ebonite or glass, or by sending into it a spark from a Leyden jar. Thereupon the machine charged itself, and began to emit pale blue sparks from the points of the combs and tongues with a hissing sound. On then drawing apart the discharging knobs, a torrent of sparks rushed across.

These arrangements being known, it is not difficult to follow the action of the machine, provided it is once understood that the whole operation depends upon the circumstance that the surface of a non-conducting body such as glass can be electrified by letting off against it an electric wind from a point placed near it (see Art. 47). Suppose that a small initial + charge is given to A. This will operate by influence upon the metal parts immediately opposite it, and cause the spikes to become electrified negatively, and to give off a negatively electrified wind, which will charge the face of the rotating plate, these charges being then carried over to the other side, where the spikes of the other comb will be emitting a positively electrified wind. The pointed tongues which project towards the back of the rotating disk also let off winds, the tendency being always for them to charge the back of the plate with a charge of opposite sign from that which is coming toward them on the front. If negative charges are being carried over the top on the front, then the tongue of $\mathrm{B}$ will tend to let off a positive charge against the back, thereby leaving $B$ more negative. In the same way the tongue of $\mathrm{A}$ will let off a negatively electrified wind, making A more positive, so building up or accumulating two opposite kinds of charges on the two field-plates. This action will not occur unless the moving plate rotates in the direction opposite to that in which the two tongues point. 
The defects of the Holtz machine were that it was so sensitive to damp weather as to be unreliable, that it was apt suddenly to reverse its charges, and that the electric winds by which it operated could not be produced without a sufficiently great initial charge.

In later Holtz machines a number of rotating disks fixed upon one common axis were employed, the whole being enclosed in a glass case to prevent the access of damp. A small disk of ebonite was sometimes fixed to the same axis, and provided with a rubber, in order to keep up the initial charge by friction. Holtz constructed many forms of machine, including one with thirty-two plates, besides machines of a second kind having two glass plates rotating in opposite directions.

The Holtz machine, as indeed every kind of influence machine, is reversible in its action ; that is to say, that if a continuous supply of the two electricities (furnished by another machine) be communicated to the armatures, the movable plate will be thereby set in rotation and, if allowed to run quite freely, will turn in an opposite sense.

Righi showed that a Holtz machine can yield a continuous current like a voltaic battery, the strength of the current being nearly proportional to the velocity of rotation. It was found that the electromotive-force of a machine was equal to that of 52,000 Daniell's cells, or nearly 53,000 volts, at all speeds. The resistance when the machine made 120 revolutions per minute was 2810 million ohms; but only 646 million ohms when making 450 revolutions per minute.

54. Experiments with Influence Machines.The experiments described in Art. 43, and indeed all those usually made with the old frictional machines, including the charging of Leyden jars, can be performed by the aid of influence machines. In some cases it is well to connect one of the two discharging knobs to the earth by a wire or chain, and to take the discharge from the other knob. To illuminate small vacuum tubes they 
may be connected by guttapercha-covered wires to the two discharging knobs, or to the terninals $\mathrm{S}_{1}, \mathrm{~S}_{2}$ of Fig. 38. The curious property of the electric discharge from a point in collecting dust or fumes is readily shown by connecting by a wire a needle which is introduced into a bell-jar of glass. The latter is filled with fumes by burning inside it a bit of magnesium wire or brown paper. Then on turning the handle of the influence machine the fumes are at once deposited, and the air left clear.

\section{Lesson VI.-The Leyden Jar and other Condensers}

55. It was shown in previous lessons that the opposite charges of electricity attract one another; that electricity cannot flow through glass; and that yet electrictiy can act across glass by influence. Two suspended pith-balls, one electrified positively and the other negatively, will attract one another across the intervening air. If a plate of glass be put between them they will still attract one another, though neither they themselves nor the electric charges on them can pass through the glass. If a pith-ball electrified with a-charge be hung inside a dry glass bottle, and a rubbed glass rod be held outside, the pithball will rush to the side of the bottle nearest to the glass rod, being attracted by the + charge thus brought near it. If a pane of glass be taken, and a piece of tinfoil be stuck upon the middle of each face of the pane, and one piece of tinfoil be charged positively, and the other negatively, the two clarges will attract one another across the glass, and will no longer be found to be free. If the pane is set up on elge, so that neither piece of tinfoil touches the table, it will be found that hardly any electricity can be got by merely touching either of the foils, for the charges are "bound," so to speak, by each other's attractions; each charge is inducing the other. In fact it will be 
found that these two pieces of tinfoil may be, in this manner, charged a great deal more strongly than either of them could possibly be if it were stuck to a piece of glass alone, and then electrified. In other words, the capacity of a conductor is greatly increased when it is placed near to a conductor electrified with the opposite kind of charge. If its capacity is increased, a greater quantity of electricity may be put into it before it is charged to an equal degree of potential. Hence, such an arrangement for holding a large quantity of electrification may be called a condenser of electricity.

56. Condensers.-Next, suppose that we have two brass disks, A and B (Fig. 42), set upon insulating stems, and that a glass plate is placed between them. Let $\mathrm{B}$ be connected by a wire to the knob of an electrical machine, and let $\mathrm{A}$ be joined by a wire to "earth." The + charge upon $B$ will act inductively across the glass plate on $A$, and will repel electricity in to the earth,

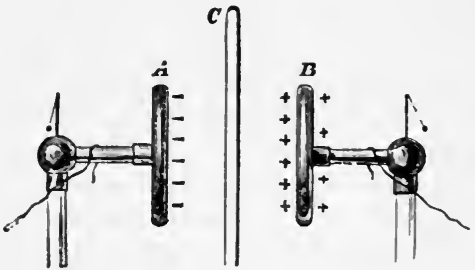

Fig. 42. leaving the nearest face of $\mathrm{A}$ negatively electrified. This - charge on A will attract the + charge of B to the side nearest the glass, and a fresh supply of electricity will come from the machine. Thus this arrangement will become a condenser. If the two brass disks are pushed up close to the glass plate there will be a still stronger attraction between the + and - charges, because they are now nearer one another, and the inductive action will be greater; hence a still larger quantity can be accumulated in the plates. We see then that the capacity of a condenser is increased by bringing the plates near together. If now, while the disks are strongly charged, the wires are removed and the 
disks are drawn backwards from one another, the two charges will not hold one another bound so strongly, and there will be more free electrification than before over their surfaces. This would be rendered evident to the experimenter by the little pith-ball electroscopes fixed to them (see the Fig.), which would fly out as the brass disks were moved apart. We have put no further charge on the lisk $\mathrm{B}$, and yet, from the indications of the electroscope, we should conclude that by moving it away from disk A it has become electrified to a higher degree. The fact is, that while the conductor $\mathrm{B}$ was near the - charge of $\mathrm{A}$ the capacity of B was greatly increased, but on moving it away from A its capacity has diminished, and hence the same quantity of electricity now electrifies it to a higher degree than before. The presence, therefore, of an earthconnected plate near an insulated conductor increases its capacity, and permits it to accumulate a greater charge by attracting and condensing the electricity upon the face nearest the earth-plate, the surface-density on this face being therefore very great; hence the appropriateness of the term condenser as applied to the arrangement. It was formerly also called an accumulator; but the term accumulator is now reserved for the special kind of battery for storing the energy of electric currents (Art. 492).

The stratum of air between the two disks will suffice to insulate the two charges one from the other. The brass disks thus separated by a stratum of air constitute an air-condenser, or air-leyden. Such condensers were first devised by Wilcke and Aepinus. In these experiments the sheet of glass or layer of air acts as a dielectric (Art. 295) conveying the inductive action through its substance. All dielectrics are insulators, but equally good insulators are not necessarily equally good dielectrics. Air and glass are far better insulators than ebonite or paraffin in the sense of being much worse conductors. But influence acts more strongly across a slab of glass than across a slab of ebonite or paraffin of equal thickness, and 
better still across these than across a layer of air. In other words, glass is a better dielectric than ebonite, or paraffin, or air, as it possesses a higher inductive capacity.

It will then be seen that in the act of charging a condenser, as much electricity flows out at one side as flows in at the other.

57. Displacement.-Whenever electric forces act on a dielectric, tending to drive electricity in at one side and out at the other, we may draw lines of force through the dielectric in the direction of the action, and we may consider tubular spaces mapped out by such lines. We may consider a tube of electric force having at one end a definite area of the positively charged surface, and at the other end an area of the negatively charged surface. These areas may be of different size or shape, but the quantities of + and - electrification over them will be equal. The quantity of electricity which has apparently been transferred along the tube was called by Maxwell " the displacement." In non-conductors it is proportional to the electromotive-force. In conductors electromotive forces prorluce currents, which may be regarded as displacements which increase continuously with time. In certain crystalline media the displacement does not take place exactly in the direction of the electric force : in this case we should speak of tubes of influence rather than tubes of force. A unit tube will be bounded at its two ends by unit charges + and - We may consider the whole electric field between positively and negatively charged bodies as mapped out into such tubes.

58. Capacity of a Condenser.-It appears, therefore, that the capacity of a condenser will depend upon-

(1) The size and form of the metal plates or coatings.

(2) The thinness of the stratum of dielectric between them ; and

(3) The dielectric capacity of the material.

59. The Leyden Jar.-The Leyden Jar, called after the city where it was invented, is a convenient form of 
condenser. It usually consists (Fig. 43) of a glass jar coated up to a certain height on the inside and outside with tinfoil. A brass knob fixed on the end of a stout brass wire passes downward through a lid or top of dry well-varnished wood, and communicates by a loose bit of brass chain with the inner coating of foil. To charge the jar the knob is held to the prime conductor of an electrical

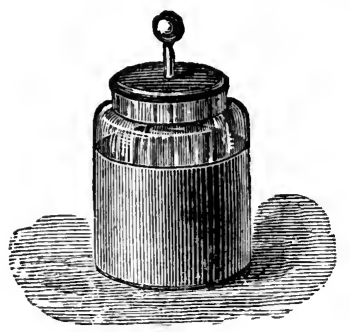

Fig. 43. machine, the outer coating being either held in the hand or connected to "earth" by a wire or chain. When a + charge of electricity is imparted thus to the inner coat. ing, it acts inductively on the onter coating, attracting acharge into the face of the outer coating nearest the glass, and repelling a + charge to the outside of the outer coating, and thence through the hand or wire to earth. After a few moments the jar will have acquired its full charge, the outer coating being - and the inner + . If the jar is of good glass, and dry, and free from dust, it will retain its charge for many hours or days. But if a path be provided by which the two mutually attracting electricities can flow to one another, they will do so, and the jar will be instantaneously discharged. If the outer coating be grasped with one hand, and the knuckle of the other hand be presented to the knob of the jar, a bright spark will pass between the knob and the knuckle with a sharp report, and at the same moment a convulsive "shock" will be communicated to the muscles of the wrists, elbows, and shoulders. A safer means of discharging the jar is afforded by the discharging tongs or discharger (Fig. 44), which consists of a jointed brass rod provided with brass knobs and a glass handle. One knob is laid against the outer coating, the other is then 
brought near the knob of the jar, and a bright snapping spark leaping from knob to knob announces that the two accumulated charges have flowed together, completing the discharge. Sometimes a jar discharges itself by a spark climbing over the top edge of the jar. Often when a jar is well charged a hissing sound is heard, due to partial discharges creeping over the edge. They can be seen in the dark as pale phosphorescent streans.

60. Discovery of the Leyden Jar.-The discovery of the Leyden jar arose from the attempt of Musschenbroek and his pupil Cuneus * to

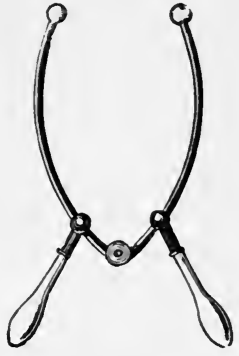

Fig. 44. collect the supposed electric "fluid" in a bottle half filled with water, which was held in the hand and was provided with a nail to lead the "fluid" down through the cork to the water from the electric machine. Here the water served as an inner coating and the hand as an outer coating to the jar. Cuneus on touching the nail received a shock. This accidental discovery created the greatest excitement in Europe and America.

61. Residual Charges. - If a Leyden jar be charged and discharged and then left for a little time to itself, it will be found on again discharging that a small second spark can be obtained. There is in fact a residual charge which seems to have soaked into the glass or been absorbed. The return of the residual charge is hastened by tapping the jar. The amount of the residual charge varies with the time that the jar has been left charged; it also depends on the kind of glass of which the jar is made. There is no residual charge discoverable in an air-leyden after it has once been discharged.

* The honour of the invention of the jar is also claimed for Kleist, Bishop of Pomerania. 
62. Batteries of Leyden Jars. - A large Leyden - jar will give a more powerful shock than a small one, for a larger charge can be put into it; its capacity is greater. A Leyden jar made of thin glass has a greater capacity as a condenser than a thick one of the same size ; but if it is too thin it will be destroyed when powerfully

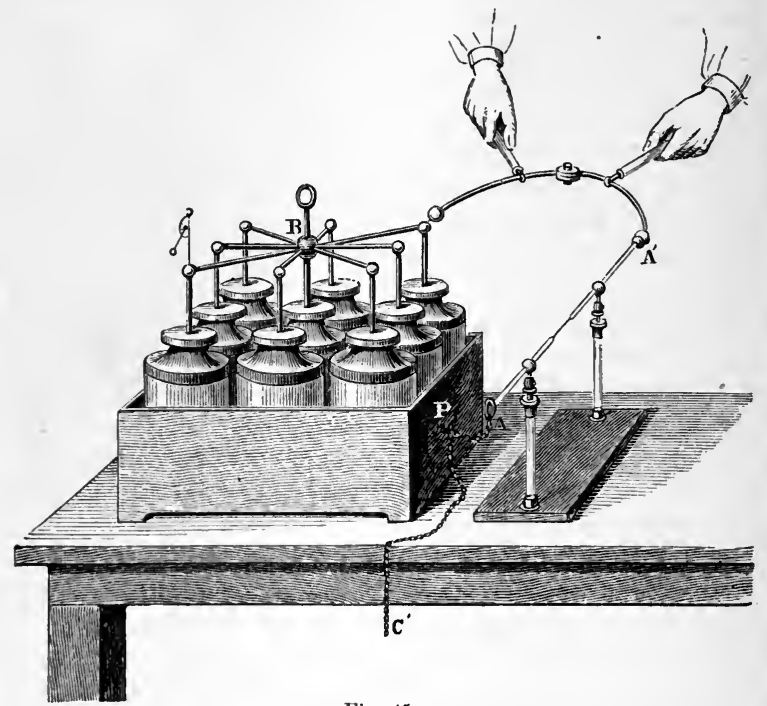

Fig. 45.

charged by a spark actually piercing the glass. "Toughened" glass is less easily pierced than ordinary glass, and hence Leyden jars made of it may be made thinner, and so will hold a greater charge. To prevent jars from being pierced by a spark, the highest part of the inside coating should be connected across by a strip of foil or a metallic disk to the central wire.

If a jar is desired to give long sparks, there must be 
left a long space of varnished glass above the top of the coatings.

If it is desired to accumulate a very great charge of electricity, a number of jars must be employed, all their inner coatings being connected together, and all their outer coatings being united. This arrangement is called a battery of Leyden jars, or Leyden battery (Fig. 45). As it has a large capacity, it will require a large quantity of electricity to charge it fully. When charged it produces very powerful effects; its spark will pierce glass readily, and every care must be taken to avoid a shock from it passing through the person, as it might be fatal. The "Universal Discharger" as employed with the Leyden battery is shown at the right of the figure.

63. Seat of the Charge.Benjamin Franklin discovered that the charges of the Leyden jar really reside on the surface of the glass, not on the metallic coatings. This he proved by means of a jar whose coatings could be removed (Fig. 46). The jar was charged and placed upon an insulating stand. The inner coating was then lifted out, and the glass jar was then taken out of the outer coating. Neither coating was found to be electrified to any extent, but on again putting the jar together it was found to

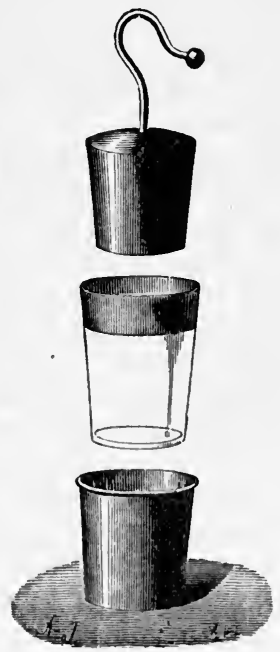

Fig. 46. be highly charged. The charges had all the time remained upon the imner and outer surfaces of the glass dielectric.

64. Dielectric Strain.-Faraday proved that the medium across which influence takes place really plays 
- an important part in the phenomena. It is now known that all dielectrics across which inductive actions are at work are thereby strained.* Inasmuch as a good vacuum is a good dielectric, it is clear that it is not necessarily the material particles of the dielectric substance that are thus affected; hence it is believed that electrical phenomena are due to stresses and strains in the so-called "ether," the thin medium pervading all matter and all space, whose highly elastic constitution enables it to convey to us the vibrations of light though it is millions of times less dense than air. As the particles of bodies are intimately surrounded by ether, the strains of the ether are also communicated to the particles of bodies, and they too suffer a strain. The glass between the two coatings of tinfoil in the Leyden jar is actually strained or squeezed, there being a tension along the lines of electric force. When an insulated charged ball is hung up in a room an equal amount of the opposite kind of charge is attracted to the inside of the walls, and the air between the ball and the walls is strained (electrically) like the glass of the Leyden jar. If a Leyden jar is made of thin glass it may give way under the stress; and when a Leyden jar is discharged the layer of air between the knob of the jar and the knob of the discharging tongs is more and more strained as they are approached towards one another, till at last the stress becomes too great, and the layer of air gives way, and is "perforated" by the spark that discharges itself across. The existence of such stresses enables us to understand the residual charge of Leyden jars in which the glass does not recover itself all at once, by reason of its viscosity, from the strain to which it has been subjected. It must never be forgotten that electric force acts across space in consequence of the transmission of stresses and

* In the exact sciences a strain means an alteration of form or volume due to the application of a stress. A stress is the force, pressure, or other ageucy which produces a strain. 
strains in the medium with which space is filled. In every case we store not electricity but energy. Work is done in pushing electricity from one place to another against the forces which tend to oppose the movement. The charging of a Leyden jar may be likened to the operation of bending a spring, or to purnping up water from a low level to a high one. In charging a jar we pump exactly as much electricity ont of the negative side as we pump into the positive side, and we spend energy in so doing. It is this stored energy which afterwards reappears in the discharge.

\section{LEsson VII.-Other Sources of Electrification}

65. It was remarked at the close of Lesson I. (p. 13) that friction was by no means the only sonrce of electricity. Some of the other sources will now be named.

66. Percussion.-A violent blow struck by one substance upon another produces opposite electrical states on the two surfaces. It is possible indeed to draw up a list resembling that of Art. 6, in such an order that each substance will take a + charge on being struck with one lower on the list.

67. Vibration.-Volpicelli showed that vibrations set up within a rod of metal coated with sulphur or other insulating substance, produced a separation of electricities at the surface separating the metal from the non-conductor.

68. Disruption and Cleavage.-If a card be torn asunder in the dark, sparks are seen, and the separated portions, when tested with an electroscope, will be found to be electrical. The linen faced with paper used in making strong envelopes and for paper collars, shows this very well. Lumps of sugar, crunched in the dark between the teeth, exhibit pale flashes of light. 
- The sudden cleavage of a sheet of mica also produces sparks, and both laminæ are found to be electrified.

69. Crystallization and Solidiflcation.-Many substances, after passing from the liquid to the solid state, exhibit electrical conditions. Sulphur fused in a glass dish and allowed to cool is violently electrified, as may be seen by lifting out the crystalline mass with a glass rod. Chocolate also becomes electrical during solidification. When arsenic acid crystallizes out from its solution in hydrochloric acid, the formation of each crystal is accompanied by a flash of light, doubtless due to an electrical discharge. A curious case occurs when the sulphate of copper and potassium is fused in a crucible. It solidifies without becoming electrical, but on cooling a little further the crystalline mass begins to fly to powder with an instant evolution of electricity.

70. Combustion.- Volta showed that combustion generated electricity. A piece of burning charcoal, or a burning pastille, such as is used for fumigation, placed in connexion with the knob of a gold-leaf electroscope, will cause the leaves to diverge.

71. Evaporation. - The evaporation of liquids is often accompanied by electrification, the liquid and the vapour assuming opposite states, though apparently only when the surface is in agitation. A few drops of a solution of sulphate of copper thrown into a hot platinum crucible produce violent electrification as they evaporate.

72. Atmospheric Electricity.-The atmosphere is found to be always electrified relatively to the earth : this is due, in part possibly, to evaporation going on over the oceans. The subject of atmospheric electricity is treated of separately in Lesson XXV.

73. Pressure.-A large number of substances when compressed exhibit electrification on their surface. Thus cork becomes + when pressed against amber, guttapercha, and metals; while it takes a - charge when pressed 
against spars and animal substances. Péclet found the degree of electrification produced by rubbing two substances together to be independent of the pressure and of the size of the surfaces of contact, but depended upon the materials and on the velocity with which they moved over one another. Rolling contact and sliding friction produced equal effects.

74. Pyro-electricity.-There are certain crystals which, while being heated or cooled, exhibit electrical charges at certain regions or poles. Crystals thus electrified by heating or cooling are said to be pyroelectric. Chief of these is the Tourmaline, whose power of attracting light bodies to its ends after being heated has been known for some centuries. It is alluded to by Theophrastus and Pliny under the name of Lapis Lyncurius. Tourmaline is a hard mineral, semi-transparent when cut into thin slices, and of a dark green or brown colour, but looking perfectly black and opaque in its natural condition, and possessing the power of polarizing light. It is usually found in slightly irregular three-sided prisms which, when perfect, are pointed at both ends. It belongs to the "hexagonal" system of crystals, but is only hemihedral, that is to say, has the alternate faces only developed. Its form is given in Fig. 47 , where a general view is first shown, the two ends A and $B$ being depicted in separate plans. These two ends differ slightly in shape. Each is made up of three sloping faces terminating in a point. But at $\mathrm{A}$ the edges between these faces run down to the comers of the prism, while in $B$ the edges between the terminal faces run down to the middle points of the long faces of the prism. The end $A$ is known as the analogous pole, and $B$ as the antilogous pole. While the crystal is rising in temperature A exhibits + electrification, $\mathrm{B}$ - ; but if, after having been heatel, it is allowed to cool, the polarity is reversed; for during the time that the temperature is falling $\mathrm{B}$ is + and $\mathrm{A}$ is -. If the 
temperature is steady no such electrical effects are observed either at high or low temperatures; and the phenomena cease if the crystal be warmed above $150^{\circ} \mathrm{C}$. This is not, however, due to the crystal becoming a conductor at that temperature; for its resistance at even higher temperatures is still so great as to make it practically a non-conductor. A heated crystal of tourmaline suspended by a silk fibre may be attracted and repelled by electrified bodies, or by a second heated tourmaline; the two similar poles repelling one another,

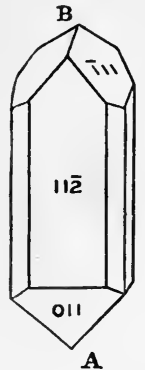

Fig. 47.

A

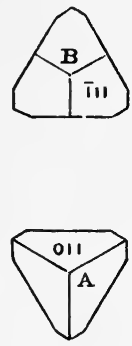

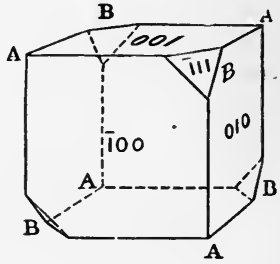

Fig. 48.

while the two poles of opposite form attract one another. If a crystal be broken up, each fragment is found to possess also an analogous and an antilogous pole.

Many other crystals beside the tourmaline are more or less pyro-electric. Amongst these are silicate of zinc (" electric calamine"), boracite, cane-sugar, quartz, tartrate of potash, sulphate of quinine, and several others. Boracite crystallizes in the form shown in Fig. 48, which represents a cube having four alternate corners truncated. The corners not truncated behave as analogous poles, the truncated ones as antilogous. When a natural hexagonal prism of quartz is heated its six edges are found to be + and - in alternate order. 
75. Piezo-electricity.-In certain crystals pressure in a particular direction may produce electrification. Haüy found that a crystal of calcspar pressed between the dry fingers, so as to compress it along the blunt edges of the crystal, became electrical, and that it retained its electricity for some days. He even proposed to employ a squeezed suspended crystal as an electroscope. A similar property is alleged of mica, topaz, and fluorspar. If two opposite edges of a hexagonal prism of quartz are pressed together, one becomes + , the other -. Pressure also produces opposite kinds of electrification at opposite ends of a crystal of tourmaline, and of other crystals of the class already noticed as possessing the peculiarity of skew-symmetry or hemihedry in their structure. Piezo-electricity is the name given to this branch of the science. It is known that skew-symmetry of structure is dependent on molecular constitution ; and it is doubtless the same peculiarity which determines the pyro-electric and piezo-electric properties, as well as the optical behaviour of these crystals in polarized light.

\section{Animal Electricity.} - Several species of creatures

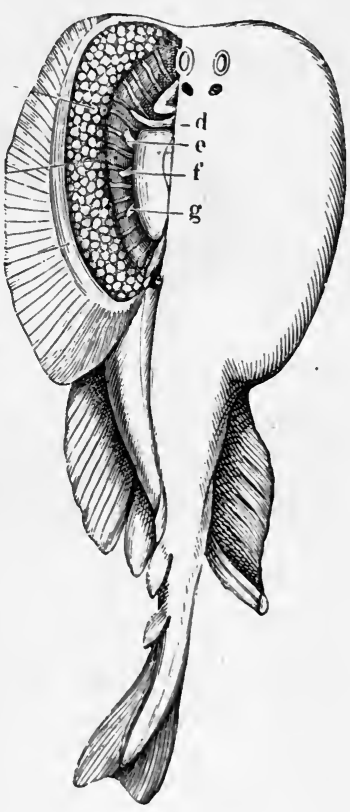

Fig. 49. inhabiting the water have the power of producing electric discharges physiologically. The best known of these creatures are the Torpedo, the Gymnotus, and the 
- Silurus. The Raia Torpedo,* or electric ray, of which there are three species inhabiting the Mediterranean and Atlantic, is provided with an electric organ on the back of its head, as shown in Fig. 49. This organ consists of lamine composed of polygonal cells to the number of 800 or 1000, or more, supplied with four large bundles of nerve fibres; the under surface of the fish is -, the upper + . In the Gymnotus electricus, or Surinam eel (Fig. 50), the electric organ goes the whole length of the body from tail to head. Humboldt gives a lively account

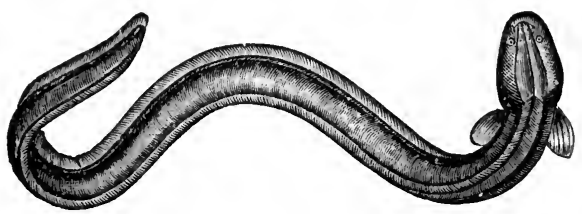

Fig. 50.

of the combats between the electric eels and the will horses, driven by the natives into the swamps inhabited by the Gymnotus. It is able to give a most terrible shock, and is a formidable antagonist when it has attained its full length of 5 or 6 feet. In the Silurus the current flows from head to tail.

Nobili, Matteucci, and others, have shown that nerveexcitations and muscular contractions of human beings also give rise to feeble discharges of electricity.

77. Electricity of Vegetables.-Buff thought he detected electrification produced by plant life ; the roots and juicy parts being negatively, and the leaves positively, electrified. The subject has, lowever, been little investigated.

* It is a curious point that the Arabian name for the torpedo, ra-ad, signifies lightning. This is pernaps not so eurious as that the Electra of the Homeric legends should possess certain qualities that would tend to suggest that she is a personification of the lightning. The resemblance between the names electra and electron (amber) eannot be accidental. 
78. Thermo-electricity. - Heat applied at the junction of two dissimilar metals produces a flow of electricity across the junction. This subject is discussed in Lesson XXXV. on Thermo-electric Currents.

79. Contact of Dissimilar Metals. - Volta showed that the contact of two dissimilar metals in air produced opposite kinds of electrification, one becoming positively, and the other negatively, electrified. This he proved in several ways, one of the most conclusive proofs being that afforded by his condensing electroscope. This consisted of a gold-leaf electroscope combined with a small condenser. A metallic plate formed the top of the electroscope, and on this was placed a second metallic plate fur-

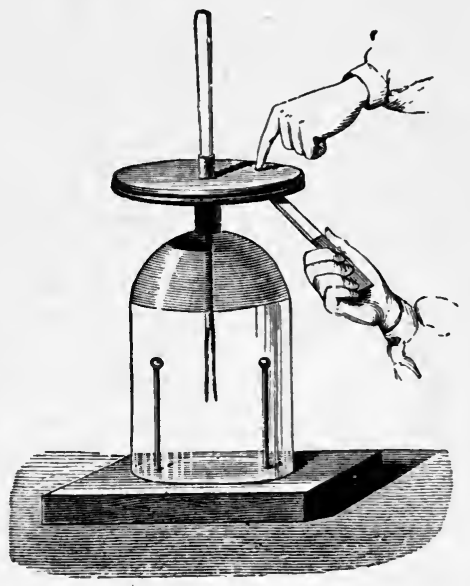

Fig. 51. nished with a handle, and insulated from the lower one by being well varnished at the surface (Fig. 51). As the capacity of such a condenser is considerable, a very feeble source may supply a quantity of electricity to the condenser without materially raising its potential, or causing the gold leaves to diverge. But if the upper plate be lifted, the capacity of the lower plate diminishes enormously, and the potential of its charge rises as shown by the divergence of the gold leaves.* To prove by the con-

* Formerly, this action was accounted for by saying that the electricity which was "bound" when the plates of the condenser were close together, 
densing electroscope that contact of dissimilar metals does produce electrification, a small compound bar made of two dissimilar metals - say zinc and copper-soldered together, is held in the moist hand, and one end of it is touched against the lower plate, the upper plate being placed in contact with the ground or touched with the finger. When the two opposing charges have thus collected in the condenser the upper plate is removed, and the diverging of the gold leaves shows the presence of a free charge, which can afterwards be examined to see whether it be + or - . Instead of employing the copperzinc bar, a single voltaic cell may be connected by copper wires to the two plates. For a long time the existence of this electrification by contact was denied, or rather it was declared to be due (when occurring in voltaic combina-

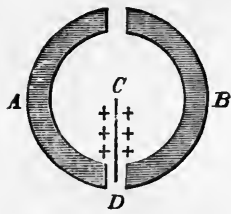

Fig. 52. tions such as are described in Lesson XIII.) to chemical actions going on; whereas the real truth is that the electricity of contact and the chemical action are both due to molecular conditions of the substances which come into contact with one another, though we do not yet know the precise nature of the molecular conditions which give rise to these two effects. Later experiments, especially those made with the modern delicate electrometers of Lord Kelvin, put beyond doubt the reality of Volta's discovery. One simple experiment explains the method adopted. A thin strip or needle of metal is suspended so as to turn about a point $C$. It is electrified from a known source. Under it are placed (Fig. 52) two semicircular disks, or half-rings of dissimilar

becomes "free" when the top plate is lifted up; the above is, however, a more scientific and more accurate way of saying the same thing. The student who is unable to reconcile these two ways of stating the matter should read again Articles 40 and 55, on pp. 46 and 68. A much more sensitive apparatus to show the effect is the quadrant electrometer (Art. 288). 
metals. Neither attracts or repels the electrified needle until the two are brought into contact, or connected by a third piece of metal, when the needle immediately turns, being attracted by the one that is oppositely electrified, and repelled by the one that is electrified similarly with itself.

80. Contact Series of Metals (in Air). - Volta found, moreover, that the differences of electric potential between the different pairs of metals were not all equal. Thus, while zinc and lead were respectively + and - to a slight degree, he found zinc and silver to be respectively + and - to a much greater degree. $\mathrm{He}$ was able to arrange the metals in a series such that each one enumerated became positively electrified when placed in contact in air with one below it in the series. Those in italics are added from observations made since Volta's time-

+ Sodium,
Magnesium,
Zinc,
Lead,
Tin,
Iron,

Copper,

Silver,

Gold,

Platinum,

- Graphite (Carbon).

Though Volta gave rough approximations, the actual numerical values of the differences of potential in air for different pairs of metals have only lately been measured by Ayrton and Perry, a few of whose results are tabulated here-

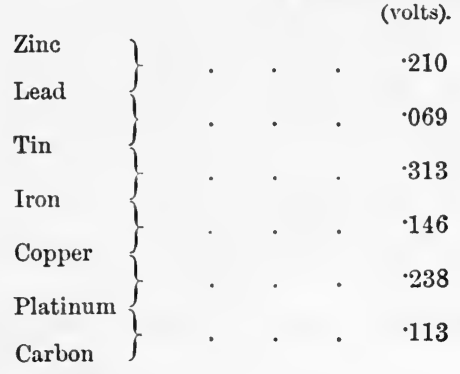


The difference of potential between zine and carbon is the same as that obtained by adding the successive differences, or 1.09 volts. * Volta's observations may therefore be stated in the following generalized form, known as Volta's Law. The difference of potential between any two metals is equal to the sum of the differences of potentials between the intervening metals in the contact-series.

It is most important to notice that the order of the metals in the contact-series in air is almost identical with that of the metals arranged according to their electro-chemical power, as calculated from their chemical equivalents and their heat of combination with oxygen (see Table, Art. 489). From this it would appear that the difference of potentials between a metal and the air that surrounds it measures the tendency of that metal to become oxidized by the air. If this is so, and if (as is the case) the air is a bad conductor while the metals are good conductors, it ought to follow that when two different metals touch they equalize their own potentials by conduction but leave the films of air that surround them at different potentials. All the exact experiments yet made have measured the difference of potentials not between the metals themselves, but between the air near one metal and that near another metal. Mr. John Brown has shown that while in air iron is positive to copper, but in an atmosphere of sulphuretted hydrogen, iron is negative to copper. He has also demonstrated the existence on freshly-cleaned metal surfaces of films of liquid or condensed gases, and has shown that polished zinc and copper, when brought so near that their films touch, will act as a battery.

81. Contact Actions. - A difference of potential is also produced by the contact of two dissimilar liquids with one another.

* For the definition of the volt, or unit of difference of potential, see Art. 254. 
A liquid and a metal in contact with one another also exhibit a difference of potential, and if the metal tends to dissolve into the liquid chemically there will be an electromotive force acting from the metal toward the liquid.

The thermo-electric difference of potential at a junction of two metals is a true contact difference. It is measured by the amount of heat produced (see Peltiereffect, Art. 420) by passing a current of electricity in the reverse direction through the junction.

A hot metal placed in contact with a cold piece of the same metal also produces a difference of potential, electrical separation taking place across the surface of contact.

Lastly, it has been shown by Professor J. J. Thomson that the surface of contact between two non-conducting substances, such as sealing-wax and glass, is the seat of a permanent difference of potentials.

82. Magneto-electricity.-Electric currents flowing along in wires can be obtained from magnets by moving closed conducting circuits in their neighbourhood. This source is dealt with in Art. 222, Lesson XVIII.

83. Summary. - We have seen in the preceding paragraphs how almost all conceivable agencies may produce electrification in bodies. The most important of these are friction, heat, chemical action, magnetism, and the contact of dissimilar substances. We noted that the production of electricity by friction depended largely upon the molecular condition of the surfaces. We may here add that the difference of potentials produced by contact of dissimilar substances also varies with the temperature and with the nature of the medium (air, vacuum, etc.) in which the experiments are made. Doubtless this source also depends upon the molecular conditions of dissimilar substances being different; the particles at the surfaces being of different sizes and 
shapes, and vibrating with different velocities and with different forces. There are (see Art. 10) good reasons for thinking that the electricity of friction is really due to electricity of contact, excited at successive portions of the surfaces as they are moved over one another. But of the molecular conditions of bodies which determine the production of electrification where they come into contact, little or nothing is yet known. 


\title{
CHAPTER II
}

\author{
MAGNETISM
}

Lessox VIII._Magnetic Attraction and Repulsion

84. Lodestones or Natural Magnets. - The name Magnet (Magnes Lapis) was given by the ancients to certain hard black stones found in various parts of the world, notably at Magnesia in Asia Minor, which possessed the property of attracting to them small pieces of iron. This magic property, as they deemed it, made the magnet-stone famous; but it was not until the tenth or twelfth century that such stones were discovered to have the still more remarkable property of pointing north and south when hung up by a thread. This property was turned to advantage in navigation, and from that time the magnet received the name of Lodestone* (or "leading-stone"). The natural magnet or lodestone is an ore of iron, known to mineralogists as magnetite and having the chemical composition $\mathrm{Fe}_{3} \mathrm{O}_{4}$. This ore is found in quantities in Sweden, Spain, the Isle of Elba, Arkansas, and other parts of the world, though not always in the magnetic condition. It frequently occurs in crystals; the usual form being the regular octahedron.

85. Artificial Magnets. - If a piece of hard iron be rubbed with a lodestone, it will be found to have also

* The common spelling loadstone is due to misapprehension. 
acquired the properties characteristic of the stone; it will attract light bits of iron, and if hung up by a
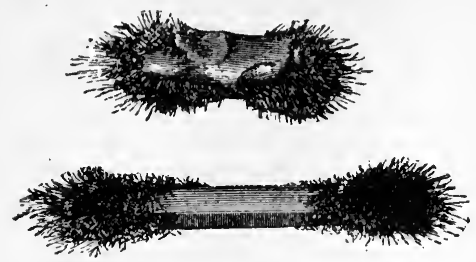

Figs. 53 and 54. thread it will point north and south. Savery, in 1729 , first showed how much more retentive of magnetism hardened steel is than mere iron. Figs. 53 and 54 represent a natural lodestone and an artificial magnet of steel, each of which has been dipped into iron-filings; the filings are attracted and adhere in tufts.

86. Writings of Dr. Gilbert.-This was all, or nearly all, that was known of the magnet until 1600 , when Dr. Gilbert published a large number of magnetic discoveries in his famous work De Magnete. He observed that the attractive power of a magnet appears to reside at two regions, and in a long-shaped magnet these regions, or poles, are usually at the ends (see Figs. 53 and 54). The portion of the magnet which lies between the two poles is apparently less magnetic, and does not attract iron-filings so strongly; and all round the magnet, halfway between the poles, there is no attraction at all. This region Gilbert ealled the equator of the magnet, and the imaginary line joining the poles he termed the axis.

87. Magnetic Needle.-To investigate more fully the magnetic forces a magnetic needle is employed. This consists (Fig. 55) of a light needle cut out of steel, and fitted with a little cap of brass, glass, or agate, by means of which it can be hung upon a sharp point, so as to turn with very little friction. It is rendered magnetic hy being rubbed upon a magnet; and when thus magnetized it will turn into the north-and-south 
position, or, as we should say, will set itself in the "magnetic meridian" (Art. 151). The compass sold by opticians consists of such a needle balanced above a card narked with the "points of the compass."

88. Magnetic Attractions and Repulsions.-If we take a magnet (either natural or artificial) in our hand and present the two "poles" of it successively to the northpointing end of a magnetic needle, we shall observe that

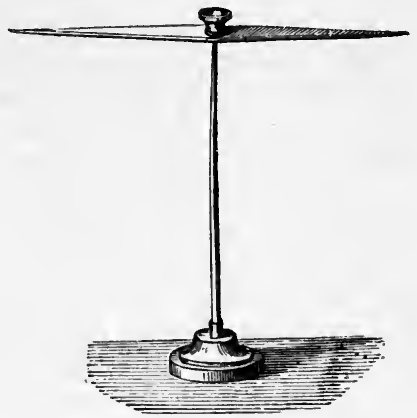

Fig. 55 . one pole of the magnet attracts it, while the other repels it (Fig. 56). Repeating the experiment on the south-

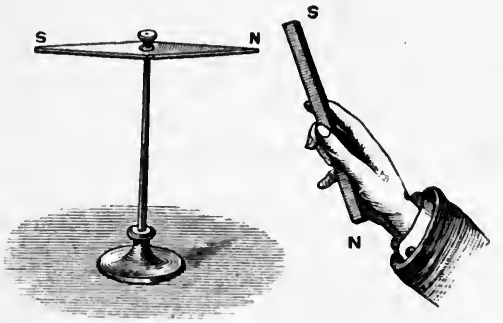

Fig. 56 . pointing end of the magnetic needle, we find that it is repelled by one pole and attracted by the other; and that the same pole which attracts the north-pointing end of the needle repels the southpointing end.

If we try a similar experiment on the magnetic needle, using for a magnet a second magnetized needle which has 
previously been suspended, and which has its north-pointing end marked to distinguish it from the south-pointing end, we shall discover that the $\mathrm{N}$-pointing pole repels the N-pointing pole, and that the S-pointing pole repels the S-pointing pole; but that a $\mathrm{N}$-pointing pole attracts and is attracted by a S-pointing pole.

89. Two Kinds of Magnetic Poles.-There would therefore appear to be two opposite kinds of magnetism, or at any rate two opposite kinds of magnetic poles, which attract or repel one another in very much the same fashion as the two opposite kinds of electrification do ; and one of these kinds of magnetism appears to have a tendency to move toward the north and the other to move toward the south. It has been proposed to call these two kinds of magnetism "north-seeking magnetism " and "south-seeking magnetism", but for our purpose it is sufficient to distinguish between the two kinds of poles. In common parlance the poles of a magnet are called the "North Pole" and "South Pole" respectively, and it is usual for the makers of magnets to mark the N-pointing pole with a letter N. It is therefore sometimes called the "marked" pole, to distinguish it from the S-pointing or "unmarked" pole. We shall, to avoid any doubt,* call that pole of a magnet which would, if the magnet were suspended, tend to turn to the north, the "North-seeking" pole, and the other the "Southseeking" pole.

We may therefore sum up our observations in the con-

* It is necessary to be precise on this point, as there is some confusion in the existing text-books. The cause of the confusion is this :-If the north-pointing pole of a needle is attracted by magnetism residing near the North Pole of the earth, the law of attraction (that unlike poles attract) shows us that these two poles are really magnetically of opposite kinds. Which are we then to call north magnetism? That which is at the $\mathbf{N}$ pole of the earth? If so, we must say that the N-pointing pole of the needle contains south magnetism. And if we call that north magnetism which points to the north, then we inust suppose the magnetic pole at the north pole of the earth to have south magnetism in it. In either case there is 
cise statement: Like magnetic poles repel one another; unlike poles attract one another. This we may call the first law of magnetism. As with the electric attractions and repulsions of rubbed bodies, so with these magnetic attractions and repulsions the effects are due, as we shall see, to stresses in the intervening medium.

90. The two Poles inseparable.-It is impossible to obtain a magnet with only one pole. If we magnetize a piece of steel wire, or watch spring, by rubbing it with one pole of a magnet, we shall find that still it has two poles-one N-seeking, the other S-seeking. And if we break it into two parts, each part will still have two poles of opposite kinds.

91. Magnetic Force.-The force with which a magnet attracts or repels another magnet, or any piece of iron or steel, we shall call magnetic force. ${ }^{*}$ The force exerted by a magnet upon a bit of iron or on another magnet is not the same at all distances, the force being greater when the magnet is nearer, and less when the magnet is farther off. (See Art. 128, on laws of magnetic force.)

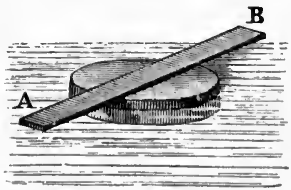

Fig. 5\%.

Whenever a force acts thus between two bodies, it acts on both of them, tending to move both. A magnet will attract a piece of iron, and a piece of iron will attract a magnet. This was shown by Sir Isaac Newton, who

then a difficulty. The Chinese and the French call the N-pointing pole of the needle a south pole, and the S-pointing pole a north pole. Lord Kelvin also calls the N-pointing pole a "True South" pole. But common practice goes the other way, and calls the N-pointing pole of a magnet its "North" pole. For experimental purposes it is usual to paint the two poles of a magnet of different colours, the N-seeking pole being coloured red and the S-seeking pole blue; but here again, strangely enough, authorities differ, for in the collections of apparatus at the Royal Institution and Royal School of Mines, the colours are used in exactly the opposite way to this, which is due to Airy.

* See footnote on "Force," Art. 169. 
fixed a magnet upon a piece of cork and floated it in a basin of water (Fig. 57), and found that it moved across the basin when a piece of iron was held near. A compass needle thus floated turns round and points north and south ; but it does not rush towards the north as a whole, nor towards the sonth. The reason of this will be explained later, in Art. 129.

Gilbert suggested that the force of a magnet might be measured by making it attract a piece of iron hung to one arm of a balance, weights being placed in the scalepan hanging to the other arm ; and he found, by hanging the magnet to the balance and placing the iron beneath it, that the effect produced was the same. The action and reaction are then equal for magnetic forces.

92. Magnetic Substances. - A distinction was drawn by Gilbert between magnets and magnetic substances. A magnet attracts only at its poles, and they possess opposite properties. But a lump of iron will attract either pole of the magnet, no matter what part of the lump be presented to the magnet. It has no distinguishable fixed "poles," and no magnetic "equator." A true magnet has poles, one of which is repelled by the pole of another magnet.

93. Other Magnetic Metals._Later experimenters have extended the list of substances which are attracted by a magnet. In addition to iron (and steel) the following metals are recognized as magnetic, viz., nickel and cobalt. Some of their alloys with iron are also nagnetic. It has also been supposed that chromium, cerium, and palladium are slightly magnetic, but further investigation has shown this to be erroneous. But only nickel and cobalt are at all comparable with iron and steel in magnetic power, and even they are very far inferior. Other bodies, sundry salts of iron and other metals, paper, porcelain, and oxygen gas are also very feebly attracted by a powerful magnet. Liquid oxygen is attracted to the poles of magnets. 
94. Diamagnetism.-A number of bodies, notably bismuth, antimony, phosphorus, and copper, are apparently repelled from the poles of a magnet. Such bodies are called diamagnetic bodies; a fuller account of them will be found in Lesson XXIX.

95. The Earth a Magnet. - The greatest of Gilbert's discoveries was that of the inherent magnetism of the earth. The earth is itself a great magnet, whose "poles" coincide nearly, but not quite, with the geographical north and south poles, and therefore it causes a freely-suspended magnet to turn into a northand-south position. Gilbert had some lodestones cut to the shape of spheres to serve as models of the globe of the earth. Such a globular magnet he called a terrella. $\mathrm{He}$ found that small magnets turned toward the poles of the terrella, and dip, as compass-needles do, toward the earth.

The subject of Terrestrial Magnetism is treated of in Lesson XII. It is evident from the first law of magnetism that the magnetic condition of the northern regions of the earth must be the opposite to that of the north-seeking pole of a magnetized needle. Hence arises the difficulty alluded to on page 92 .

96. Induction of Magnetism.--Magnetism may be communicated to a piece of iron without actual contact

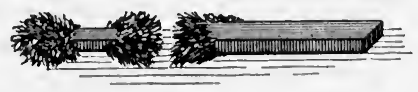

Fig. 58.

with a magnet. If a short, thin unmagnetized bar of iron be placed near some iron filings, and a magnet be brought near to the bar, the presence of the magnet will induce magnetism in the iron bar, and it will now attract the iron filings (Fig. 58). This inductive action is very similar to that observed in Lesson III. to take place when a non-electrified body was brought under the influence of 
an electrified one. The analogy, indeed, goes further than this, for it is found that the iron bar thus magnetized by induction will have two poles; the pole nearest to the pole of the inducing magnet being of the opposite kind, while the pole at the farther end of the bar is of the same kind as the inducing pole. Those bodies in which a magnetizing force produces a high degree of magnetization are said to possess a high permeability. It will be shown presently that magnetic induction takes place along certain directions called lines of magnetic induction, or lines of magnetic force, which may pass either through iron and other magnetic media, or through air, vacuum, glass, or other non-magnetic media: and, since induction goes on most freely in bodies of high magnetic permeability, the magnetic lines are sometimes (though not too accurately) said to "pass by preference through magnetic matter," or, that "magnetic matter conducts the lines of force."

97. Attraction across Bodies.-If a sheet of glass, or wood, or paper, be interposed between a magnet and the piece of iron or steel it is attracting, it will still attract it as if nothing were interposed. A magnet sealed up in a glass tube still acts as a magnet. Lucretius found a magnet put into a brass vase attracted iron filings through the brass. Gillert surrounded a magnet by a ring of flames, and found it still to be subject to magnetic attraction from without. Across water, vacuum, and all known substances, the magnetic forces will act; with the single apparent exception, however, that magnetic force will not act across a screen of iron or other magnetic material, if sufficiently thick. If a small magnet is suspended inside a hollow ball made of iron, no outside magnet will affect it, the reason being that the magnetic lines of force are conducted off laterally through the iron instead of penetrating through it. A hollow shell of iron will therefore act as a magnetic cage, and shield the space inside it from magnetic influences. 
Fig. 59 illustrates the way in which a cylinder of soft iron shields the space interior to it from the influence of an exterual magnet. A compass needle placed at $\mathrm{P}$ inside the cylinder is not affected by the presence of the magnet outside, for its lines of magnetic force are drawn off laterally. Similarly a magnet inside is shielded from affecting outside space.

Although magnetic induction takes place at a distance across an intervening layer of air, glass, or vacuum, there is no doubt that the intervening medium is directly concerned in the transmission of the magnetic force, though

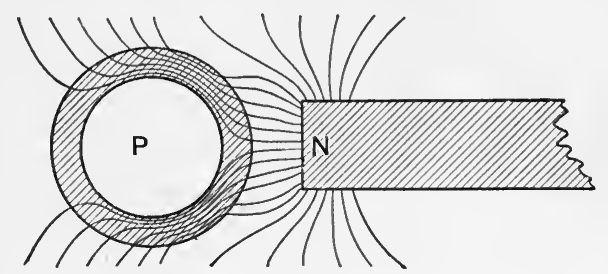

Fig. 59.

the true medium is probably the "ether" of space surrounding the molecules of matter, not the molecules themselves.

We now can see why a magnet should attract a notpreviously-magnetized piece of iron; it first magnetizes it by induction and then attracts it : for the nearest end will have the opposite kind of magnetism induced in it, and will be attracted with a force exceeding that with which the more distant end is repelled. But induction precedes attraction.

98. Retention of Magnetization.-Not all magnetic substances can become magnets permanently. Lodestone, steel, and nickel retain permanently the greater part of the magnetism imparted to them. Cast iron and many impure qualities of wrought iron also 
retain magnetism imperfectly. The softer and purer a specimen of iron is, the more lightly is its residual magnetism retained. The following experiment illustrates the matter:-Let a few pieces of iron rod, or a few soft iron nails be taken. If one of these (see Fig. 60) be placed in contact with the pole of a permanent steel magnet, it is attracted to it, and becomes itself a temporary magnet. Another bit of iron may then be hung to it, and another, until a chain of four or five pieces is built

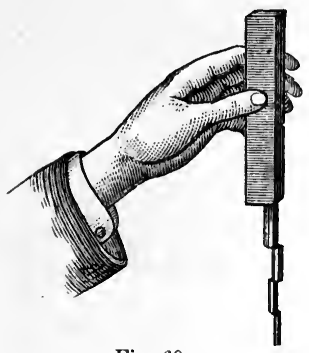

Fig. 60 .

up. But if the steel magnet be removed from the top of the chain, all the rest dirop off, and are found to be no longer magnetic. A similar chain of steel needles may be formed, but they will retain permanently most of their magnetism.

It will be found, however, that a steel needle is more difficult to magnetize than an iron needle of the same dimensions. It is harder to get the magnetism into steel than into iron, and it is harder to get the magnetism out of steel than out of iron; for the steel retains the magnetism once put into it. This power of resisting magnetization or demagnetization is sometimes called coercive force; a much better term, due to Lamont, is retentivity. The retentivity of hard-tempered steel is great; that of soft wrought iron is very small. The harder the steel, the greater its retentivity. Form affects retentivity. Elongated forms and those shaped as closed or nearly closed circuits retain their magnetism better than short rods, balls, or cubes.

99. Theories of Magnetism.-The student will not have failed to observe the striking analogies between the phenomena of attraction, repulsion, induction, etc., of magnetism and those of electricity. Yet the two sets 
of phenomena are quite distinct. A positively electrifierl body does not attract either the North-pointing or the South-pointing pole of the magnet as such; in fact, it attracts either pole quite irrespective of its magnetism, just as it will attract any other body. There does exist, indeed, a direct relation between magnets and currents of electricity, as will be later explained. There is none known, however, between magnets and stationary charges of electricity.

In many treatises it is the fashion to speak of a magnetic fluid or fluids; it is, however, absolutely certain that magnetism is not a fluid, whatever else it may be. The term is a relic of bygone times. A magnet when rubbed upon a piece of steel magnetizes it without giving up or losing any of its own magnetism. A fluid cannot possibly propagate itself indefinitely without loss. The arguments to be derived from the behaviour of a magnet on breaking, and from other experiments narrated in Lesson $\mathrm{X}$., are even stronger. No theory of magnetism will therefore be propounded until these facts have been placed before the student.

\section{Lesson IX.-Methods of making Magnets}

100. Magnetization by Single Touch.-It has been so far assumed that bars or needles of steel were to be magnetized by simply touching them, or stroking them from end to end with the pole of a permanent magnet of lodestone or steel. In this case the last touched point of the bar will be a pole of opposite kind to that used to touch it; and a more certain effect is produced if one pole of the magnet be rubbed on one end of the steel needle, and the other pole upon the other end. There are, however, better ways of magnetizing a bar or needle.

101. Magnetization by Divided Touch.-In this method the bar to be magnetized is laid down 
horizontally ; two bar magnets are then placed down upon it, their opposite poles being together. They are then drawn asunder from the middle of the bar towards its

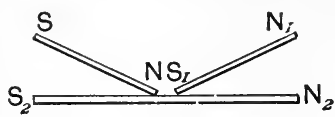

Fig. 61. ends, and back, several times. The bar is then turned over, and the operation repeated, taking care to leave off at the middle (see Fig. 61). The process is more effectual if the ends of the bar are meantime supported on the poles of other bar magnets, the poles being of the same names as those of the two magnets above them used for stroking the steel bar.

102. Magnetization by Double Touch.-Another method, known as double touch, differs slightly from that last described. A piece of wood or cork is interposed between the ends of the two bar magnets employed, and they are then both moved backwards and forwards along the bar that is to be magnetized. By none of these methods, however, can a steel bar be magnetized beyond a certain degree of intensity.

103. Forms of Magnets.-Natural magnets are usually of irregular form, though they are sometimes reduced to regular shapes by cutting or grinding. Formerly it was the fashion to mount them with soft iron cheeks or "armatures" to serve as pole-pieces.

For scientific experiments bar magnets of hardened steel are commonly used; but for many purposes the horse-shoe shape is preferred. In the horse-shoe magnet the poles are bent round so as to approach one another, the advantage here being that so both poles can attract one piece of iron. The "armature," or "keeper," as the piece of soft iron placed across the poles is named, is itself rendered a magnet by induction when placed across the poles; hence, when both poles magnetize it, the force with which it is attracted to the magnet is the greater.

104. Laminated Magnets.-It is found that long 
thin steel magnets are more powerful in proportion to their weight than thicker ones. Hence it was proposed by Scoresby * to construct compound magnets, consisting of thin laminæ of steel separately magnetized, and after-

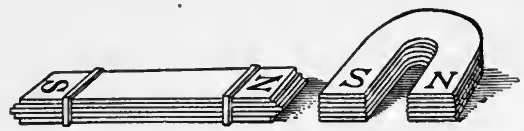

Fig. 62.

Fig. 63.

wards bound together in bundles (Fig. 62). These laminated magnets are more powerful than simple bars of steel. Compound horse-shoe magnets are sometimes used : the plates separately magnetized are assembled as in Fig. 63.

105. Magnetization derived from the Earth. - The magnetism of the earth may be utilized where no other permanent magnet is available to magnetize a bar of steel. Gilbert states that iron bars set upright for a long time acquire magnetism from the earth. If a steel poker be held in the magnetic meridian, with the north end dipping down, and in this position be struck with a wooden mallet, it will be found to have acquired magnetic properties. All vertical iron columns in our northern latitudes are found to have their lower ends $\mathrm{N}$-poles and their upper ends S-poles. In Australia and the southern hemisphere the tops of iron columns are $\mathrm{N}$-poles. Wires of steel subjected to torsion, while in the magnetic meridian, are also found to be thereby magnetized.

106. Magnetization after Heating.-Gilbert discovered also that if a bar of steel be heated to redness, and cooled, either slowly or suddenly, while lying in the magnetic meridian, it acquires magnetic polarity. No

* A similar suggestion was made by Geuns of Venlo in 1768, using horse-shoe magnets. Similar magnets have been constructed in recent years by Jamin. 
such property is acquired if it is cooled while lying east and west. It has been proposed to make powerful magnets by placing hot bars of steel to cool between the poles of very powerful electromagnets; and Carré produced strong magnets of iron cast in moulds lying in an intense magnetic field.

107. Magnetization by Currents of Electricity.-A current of electricity caused to circulate in a spiral wire wound around a core of iron or steel magnetizes it more powerfully than in any of the preceding

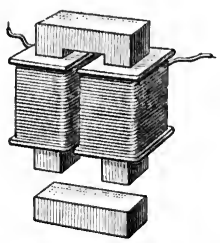

Fig. 64 . operations. In the case of a soft iron core, it is only a magnet while the current continues to flow. Such a combination is termed an Electromagnet; it is fully described in Lesson XXXI. Fig. 64 depicts a common form of electromagnet having two coils of insulated copper wire wound upon bobbins that are placed upon the limbs of a soft iron core. The armature is also of soft iron of sufficient thickness. Steel bars may be magnetized by drawing them over the poles of such an electromagnet while the latter is excited by the circulation of the electric current. Elias of Haarlem proposed to magnetize steel bars by passing them through a wire coiled up into a compact ring of many turns, through which a strong current was sent by a voltaic battery.

108. Hardening and Tempering of Steel for Magnets. - There are two ways of hardening steel : (1) by suddenly cooling it from a bright red temperature ; (2) by compressing it under hydranlic pressure while it cools slowly. If rods of steel are heated brilliantly red, and then quenched in water, oil, or mercury, they become intensely brittle and glass-hard. To temper hard steel it is then gently reheated to near a very dull red heat and softens slightly while acquiring a straw tint. If let down still further by continuing the reheating it becomes 
a blue tint, and is springy and flexible. Short bar magnets retain most magnetism if left glass-hard without tempering. But magnets whose length is more than twenty times their thickness retain more magnetism if tempered down to a straw or even to a blue tirt.

109. Destruction of Magnetism.-A steel magnet loses its magnetism partially or wholly if subjected to rough usage, or if purposely hit or knocked about. Newly magnetized magnets lose more strength by rough treatment than those which have been long magnetized. A magnet loses its magnetism, as Gilbert showed, on being raised to a bright red heat. The slightest vibration will destroy any magnetism remaining in annealed soft iron.

110. Magnets of Unvarying Strength.-Ordinary steel magnets have by no means a permanent or constant magnetism. They soon lose a considerable percentage of their magnetism, and the decay continues slowly for months and years. Every shock or jolt to which they are subjected, every contact with iron, every change of temperature weakens them. Every time that the keeper is slammed on to a horse-shoe magnet it is weakened. For the purpose of making magnetic measurements, and for use as controlling magnets of galvanometers, magnets are, however, required that shall possess the utmost constancy in their strength. Magnets of unvarying strength may be made by attention to the following points. Choose a form either of a nearly closed circuit or of a very long rod. Let the steel be hardened as much as possible (see Art. 108 above), then placed in steam at $100^{\circ}$ for twenty or thirty hours or more. Then magnetize as fully as possible, and then heat again for five hours in steam. Magnets of a shape constituting a nearly closed circuit are more constant than short straight magnets.

111. Effects of Heat on Magnetization.-If a permanent steel magnet be warmed by placing it in hot or boiling water, its strength will be thereby lessened, though it recovers partially on cooling. Chilling a magnet 
increases its strength. Cast-iron ceases to be attracted by a magnet at a bright red heat. Pure iron is transformed at $870^{\circ} \mathrm{C}$. to an almost non-magnetic state; the transformation-point of nickel is $320^{\circ} \mathrm{C}$, that of cobalt $1120^{\circ}$ C. Manganese is magnetic only when cooled to $-20^{\circ} \mathrm{C}$. It has been surmised that other metals would become magnetic if cooled to a low enongh temperature. Dewar observed that the magnetic susceptibility of iron, when cooled to near $-180^{\circ}$ C. in liquid oxygen, is nearly twice as high as at $0^{\circ} \mathrm{C}$. The magnetic metals at high temperatures do not become diamagnetic, but are still feebly magnetic; their susceptibility at temperatures above the transformation-point varying inversely as the absolute temperature.

112. Magnetic Saturation.-A magnet to which as powerful a degree of magnetization as it can attain to has been given is said to be saturated. A recently magnetized magnet will occasionally appear to be supersaturated, possessing even after the application of the magnetizing force has ceased a higher degree of magnetism than it is able to retain permanently. Thus a horseshoe-shaped steel magnet will support a greater weight immediately after being magnetized than it will do after its armature has been once removed from its poles. Even soft iron after being magnetized retains a small amount of magnetism when its temporary magnetism has disappeared. This small remaining magnetic charge is spoken of as residual magnetism.

113. Strength of a Magnet.- The "strength" of a magnet is not the same thing as its "lifting power." Its lifting power is a very uncertain quantity depending not only on the shape of its polar surfaces, but on the shape and quality of the mass of iron used as load. Consequently the "strength" of a magnet pole must be measured by the magnetic force which it exerts at a distance on other magnets. Thus, suppose there are two magnets, $\dot{A}$ 
and $\mathrm{B}$, whose strengths we compare by making them each act upon the $\mathrm{N}$ pole of a third magnet $\mathrm{C}$. If the $\mathrm{N}$ pole of $\mathrm{A}$ repels $\mathrm{C}$ with twice as much force as that with which the $\mathrm{N}$ pole of $\mathrm{B}$ placed at the same distance would repel C, then we should say that the "strength" of A was twice that of B. Another way of putting the matter is to say that the "strength" of a pole is the amount of free magnetism at that pole. By adopting the unit of strength of magnet poles as defined in Art. 141, we can express the strength of any pole in numbers as so many "units" of strength.

114. Lifting Power.-The lifting power of a magnet (also called its portative force) depends both upon the form of the magnet and on its magnetic strength. A horse-shoe magnet will lift a load three or four times as great as a bar magnet of the same weight will lift. A long bar magnet will lift more than a short bar magnet of equal strength. A bar magnet with a rounded or chamfered end will lift more than a similar bar with a flat or expanded end, though both may be equally strongly magnetized. Also the lifting power of a magnet grows in a very curious and unexplained way by gradually increasing the load on its armature day by day until it bears a load which at the outset it could not have done. Nevertheless, if the load is so increased that the armature is torn off, the power of the magnet falls at once to its original value. The attraction between a powerful electromagnet and its armature may amount to $230 \mathrm{lbs}$. per square inch, or 16,000 grammes per square centimetre (see Art. 384). Small magnets lift a greater load in proportion to their own weight than large ones, *

* Bernoulli gave the following rule for finding the lifting power $p$ of a magnet whose weight was $w$ :-

$$
p=a \sqrt[\frac{3}{3}]{w}
$$

where $a$ is a constant depending on the goodness of the steel and the 
- because the lifting power is proportional to the polar surface, other things being equal. Steel magnets seldom attain a tractive force as great as $40 \mathrm{lbs}$. per square inch of polar surface. A good steel horse-shoe magnet weighing itself $1 \mathrm{lb}$. ought to lift $25 \mathrm{lbs}$. weight. Sir Isaac Newton is said to have possessed a little lodestone mounted in a signet ring which would lift a piece of iron 200 times its own weight.

\section{Lesson X.—Distribution of Magnetism}

115. Magnetic Field.-The space all round a magnet pervaded by the magnetic forces is termed the "field" of that magnet. It is most intense near the poles of the magnet, and is weaker and weaker at greater distances away. At every point in a magnetic field the force has a particular strength, and acts in a particular direction. It is possible at any point in a magnetic field to draw a line in the direction of the resultant magnetic force acting at that point. The whole field may in this way be mapped out with magnetic lines (Art. 119). For a horse-shoe magnet the field is most intense between the two poles, and the lines of magnetic force are curves which pass from one pole to the other across the field. A practical way of investigating the distribution of the magnetic lines in a field is given in Art. 119, under the title "Magnetic Figures." When the armature is placed upon the poles of a horse-shoe magnet, the force of the magnet on all the external regions is weakened, for the induction now goes on through the iron of the keeper, not through the surrounding space. In fact a closed system of masnets - such as that made by placing four bar magnets along the sides of a square, the $\mathrm{N}$ pole of one touching the $\mathrm{S}$

method of magnetizing it. In the best steel magnets made at Haarlem by Van Wetteren this coefficient was from $19^{\circ} 5$ to 23 , the welghts being ex. pressed in kilogrammes. 
pole of the next-has no external field of force. A ring of steel may thus be magnetized so as to have neither external field nor poles; or rather any point in it may be regarded as a $\mathrm{N}$ pole and a $\mathrm{S}$ pole, so close together that they neutralize one another's forces.

That poles of opposite name do neutralize one another may be shown by the well-known experiment of hanging a small object-a steel ring or a key-to the $\mathrm{N}$ pole of a bar magnet. If now the $\mathrm{S}$ pole of another bar magnet be made to tonch the first the two poles will neutralize each other's actions, and the ring or key will drop down.

116. Breaking a Magnet.-We have already stated that when a magnet is broken into two or more parts, each is a complete magnet, possessing poles, and each is nearly as strongly magnetized as the original magnet.

[N

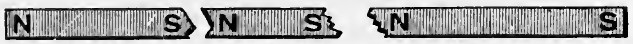

Fig. 65 .

Fig. 65 shows this. If the broken parts be closely joined these adjacent poles neutralize one another and disappear, leaving only the poles at the ends as before. If a magnet be ground to powder each fragment will still act as a

\begin{tabular}{|c|c|c|c|c|c|c|c|c|c|}
\hline $\mathbf{N}$ & & & & $S^{\prime} N$ & & & & & $S$ \\
\hline 32 & $\therefore n$ & 510 & $s n$ & ${ }_{s}$ & & 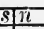 & 817 & 57 & $s$ \\
\hline$n$ & $s i n$ & 817 & $s 12$ & \begin{tabular}{l|l}
8 \\
2
\end{tabular} & & si & $s i$ & $s$ & $n$ \\
\hline$\pi$ & $s n$ & $s n$ & $s \bar{n}$ & $s]$ & & $s \sqrt{n}$ & $s \mid n$ & 81 & $s$ \\
\hline$\pi$ & sin & sin & $8 n$ & $s$ & & $s i n$ & $\sin$ & $s i$ & \\
\hline
\end{tabular}

Fig. 66 .

little magnet and exhibit polarity. A magnet may therefore be regarded as composed of many little magnets put together, so that their like poles all face one way. Such an arrangement is indicated in Fig. 66, from which it will be seen that if the magnet be broken asunder across 
- any part, one face of the fracture will present only $\mathrm{N}$ poles, the other only $\mathrm{S}$ poles. This would be true no matter how small the individual particles.

117. Normal Distribution.-In an ordinary bar magnet the poles are not quite at the ends of the bar, but a little way from it; and it can be shown that this is a result of the way in which the surface magnetism is distributed in the bar. A very long, thin, uniformly magnetized bar has its poles at the ends; but in ordinary thick magnets the "pole" occupies a considerable region, the "free magnetism" falling off gradually from the ends of the bar. In each region, however, a point can be generally determined at which the resultant magnetic forces act, and which may for most purposes be considered as the "pole." In certain cases of irregular magnetization it is possible to have one or more poles between those at the ends. Such poles are called corsequent poles (see Fig. 70).

118. Lamellar Distribution of Magnetism. Magnetic Shells. - Up to this point the ordinary distribution of magnetism along a bar has been the only distribution considered. It is theoretically possible to have nnagnetism distributed over a thin sheet so that the whole of one face of the sheet shall have one kind of magnetism, and the other face the other kind of magnetism ; such distribution is, however, unstable. If an immense number of little magnets were placed together side by side, like the cells in a honeycomb, all with their $\mathrm{N}$-seeking ends upwards, and S-seeking ends downwards, the whole of one face of the slab would be one large flat $\mathrm{N}$-seeking pole, and the other face S-seeking. Such a distribution as this over a surface or sheet is termed a lamellar distribution, to distinguish it from the ordinary distribution along a line or bar, which is termed, for distinction, the solenoidal, or circuital, distribution. A lamellarly magnetized magnet is sometimes spoken of as a magnetic shell. 
119. Magnetic Figures.-Gilbert showed * that if a sheet of paper or card be placed over a magnet, and iron filings are dusted over the paper, they settle down in curving lines, forming a magnetic figure, the general form of which for a bar magnet is shown in Fig. 67. The filings should be fine, and sifted through a bit of muslin; to facilitate their settling in the lines, the sheet of paper should be lightly tapped. The figures thus obtained can be fixed permanently by several processes. The best of these consists in employing a sheet of glass which has

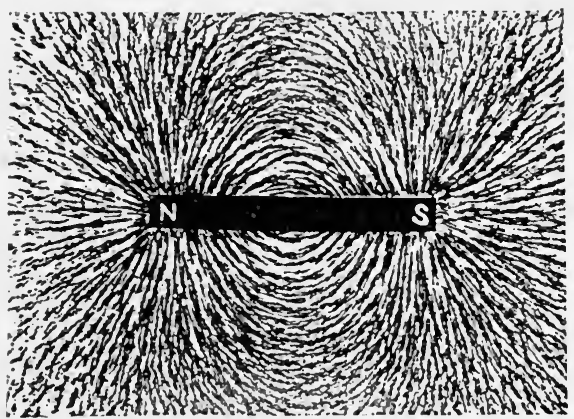

Fig. 6i.

been previously gummed and dried, instead of the sheet of paper; after this has been placed above the magnet the filings are sifted evenly over the surface, and then the glass is tapped; then a jet of steam is caused to play gently above the sheet, softening the surface of the gum, which, as it hardens, fixes the filings in their places. Inspection of the figure will show that the lines diverge nearly radially from each pole, and curve round to meet these from the opposite pole. Fig. 68, produced from a horse-shoe magnet, shows how the magnetic field is most

* The magnetic figures were known to Lucretius. 
intense between the poles, but spreads beyond them in wide curves. Faraday, who made a great use of this

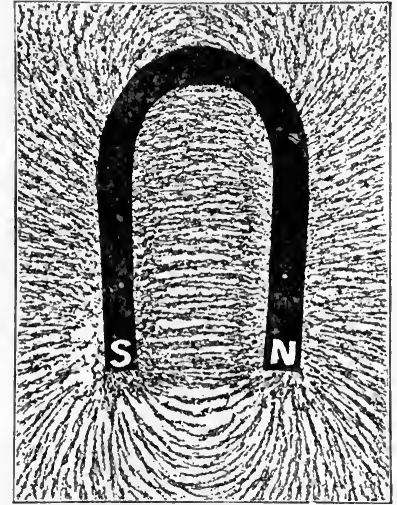

Fig. 6S.

method of investigating the distribution of magnetism in various "fields," gave to the lines the name of lines of force. They represent, as shown by the action on little magnetic particles which set themselves thus in obedience to the attractions and repulsions in the field, the resultant direction of the forces at every point ; for each particle tends to assume the direction of the force jointly due to the simultaneous action of both poles; hence the curves of filings may be taken to represent visibly the invisible lines of magnetic force.* Farallay pointed out that these "lines of force" map out the magnetic field, showing by their position the direction of the magnetic force, and by their number its intensity. If a small $\mathrm{N}$ seeking pole conld be obtained alone, and put down on any one of these lines of force, it would tend to move along

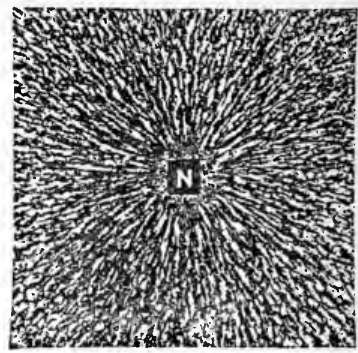

Fig. 69. that line from $\mathrm{N}$ to $\mathrm{S}$; a single $\mathrm{S}$-seeking pole would

* Or rather the component part of the magnetic force resolved into the plane of the figure; which is not quite the same thing, for above the poles the filings stand up nearly vertically to this plane. 
tend to move along the line in an opposite direction. In Fig. 69, which is the field about one end of a bar magnet, the magnetic lines are simply radial. Faraday also pointed out that the actions of attraction or repulsion in the field are always related to the directions in the field of the magnetic lines. He assigned to these lines of force certain physical properties (which are, however, only true of them in a secondary sense), viz. that they tend to shorten themselves from end to end, and that they repel one another as they lie side by side. The modern way of stating the matter is, that in every magnetic field there are certain stresses, consisting of a tension along the lines of force, and a pressure across them.

120. Consequent Poles.-The method of sprinkling filings may be applied to ascertain the presence of consequent poles in a bar of steel, the figure obtained re-

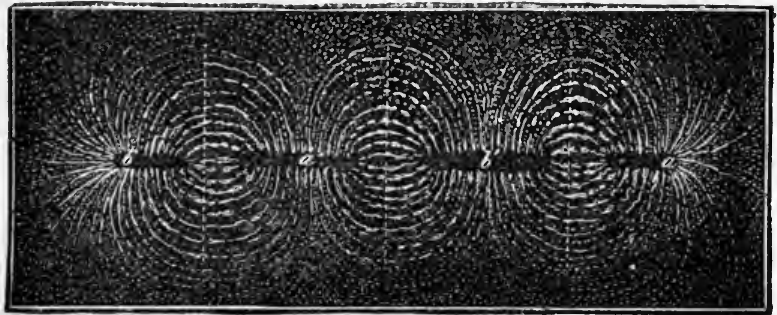

Fig. 70.

sembling that depicted in Fig. 70. Such a state of things is produced when a strip of very hard steel is purposely irregularly magnetized by touching it with strong magnets at certain points. A strip thus magnetized virtually consists of several magnets put end to end, but in reverse directions, NS, SN, etc. Consequent poles can also be prorluced in an electromarnet by reversing the direction in which the wire is coiled around part of the core.

121. Fields mapped by Filings. - The forces 
producing attraction between unlike poles, and repulsion between like poles, are beautifully illustrated by the magnetic figures obtained in the fields between the poles in the two cases, as given in Figs. 71 and 72. In Fig. 71 the poles are of opposite kinds, and the lines of force curve across out of one pole into the other; while in

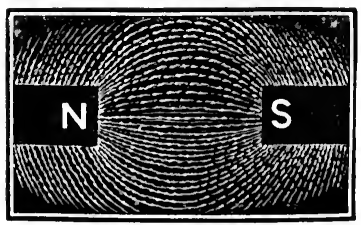

Fig. 71.

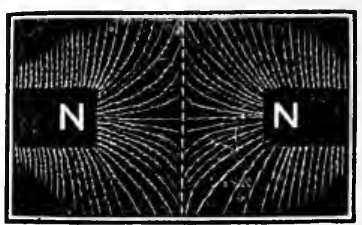

Fig. 72.

Fig. 72, which represents the action of two similar poles, the lines of force curve away as if repelling one another, and turn aside at right angles.

122. Magnetic Writing.-Another kind of magnetic figures was discovered by De Haldat, who wrote with the pole of a magnet upon a thin steel plate (such as a saw-blade), and then sprinkled filings over it. The writing, which is quite invisible in itself, comes out in the lines of filings that stick to the magnetized parts; this magic writing will continue in a steel plate many months.

123. Surface Magnetization.-In many cases the magnetism imparted to magnets is ' confined chiefly to the outer layers of stecl. If a short bar magnet be put into acid so that the onter layers are dissolved away, it is found that it has lost its magnetism when only a thin film has been thus removed. A short hollow steel tube when magnetized is nearly as strong a magnet as a solid rod of the same size. Long thin magnets, and those that are curved so as nearly to form a closed circuit, can be much more thoroughly magnetized. If a bundle of steel plates are magnetized while bound together, it will be 
found that only the outer ones are strongly magnetized. The inner ones may even exhibit a reversed magnetization.

\section{Mechanical Effects of Magnetization.-} Joule found an iron bar to increase by $\frac{1}{720000}$ of its length when strongly magnetized. Bidwell found that with still stronger magnetizing forces iron contracts again ; and rods stretched by a weight contract more when magnetized than unstretched rods do. Barrett observed that nickel shows a slight contraction when magnetized. These are proofs that magnetization is an action affecting the arrangement of the molecules. This supposition is confirmed by the observation of Page, that at the moment when a bar is magnetized or demagnetized, a faint metallic clink is heard in the bar. Sir W. Grove showed that when a tube containing water rendered muddy by stirring up in it finely-divided magnetic oxide of iron was magnetized; the liquid became clearer in the direction of magnetization, the particles apparently setting themselves end-on, and allowing more light to pass between them. A twisted iron wire tends to untwist itself when magnetized. A piece of iron, when powerfully magnetized and demagnetized in rapid succession, grows hot, as if magnetization were accompanied by interual friction.

125. Action of Magnetism on Light.-Faraday discovered that a ray of polarized light passing through certain substances in a powerful magnetic field has the direction of its vibrations changed. This phenomenon, which is sometimes called "The Magnetization of Light," is better clescribed as "The Rotation of the Plane of Polarization of Light by Magnetism." The amount of rotation differs in different media, and varies with the magnetizing force. More recently Kerr has shown that a ray of polarized light is also rotated by reflexion at the end or side of a powerful magnet. Further mention is made of these discoveries in the chapter on Electro-optics, Lesson LVI.

126. The Act of Magnetizing.-All these various 
phenomena point to a theory of magnetism very different from the old notion of fluids. It appears that every particle of a magnet is itself a magnet, and that the magnet only becomes a magnet as a whole by the particles being so turned as to point one way. The act of magnetizing consists in turning the molecules more or less into one particular direction. This conclusion is supported by the observation that if a

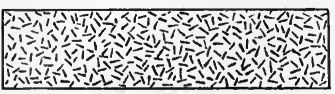

Fig. 73. glass tube full of iron filings is magnetized, the filings can be seen to set themselves endways, and that, when thus once set, they act as a magnet until shaken up. It appears to be harder to turn the individual molecules of solid steel than those of soft iron; but, when once so set, they remain end-on unless violently struck or heated. As Weber, who propouncled this notion of molecular magnetism, pointed out, it follows from this theory that when all the particles are turned end-on the limits of possible magnetization woull have been attained. Some careful experiments of Beet: on iron deposited by electrolysis entirely confirm this conclusion, and add weight

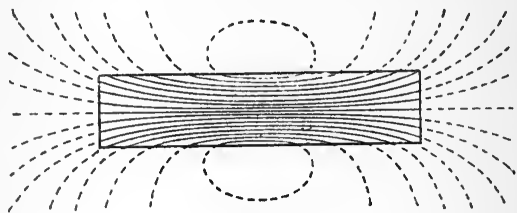

Fig. 74 .

to the theory. Fig. 73 may be taken to represent a .nonmagnetized piece of iron or steel in which the arrangement of the particles is absolutely miscellaneous : they do not point in any one direction more than another. When magnetized slightly, there will be a greater percentage pointing in the direction of the magnetizing force. When fully magnetized-if that were possible-they would all point in the same direction as in Fig. 74 . 
In very few cases, however, is the magnetization uniform throughout the whole length of a bar: the particles are more fully completely turned into line at the middle part of the bar than at the ends.

If the intrinsic magnetization of the steel at every part of a magnet were equal, the free poles would be found only at the end surfaces; but the fact that the free magnetism is not at the ends merely, but diminishes from the ends towards the niddle, shows that the intensity of the intrinsic magnetization must be less towards and at the ends than it is at the middle of the bar. In Fig. 74 an attempt is made to depict this. It will be noticed that the magnetic lines run through the steel and emerge into the air in curves. Some of the lines do not run all the length of the bar but leak out at the sides. If the bar were uniformly magnetized the lines would emerge at the ends only. It is clear that the middle piece is more thoroughly magnetized than any other part. Magnetism in fact consists of a sort of grain or structure conferred upon the steel. Wherever this structure comes up at a surface, there the surface properties of magnetism are found. A pole is simply a region where the magnetic lines pass through the surface of the steel or iron.

The optical phenomena led Clerk Maxwell to the further conclusion that these longitudinally-set molecules are rotating round their long axes, and that in the "ether" of space there is also a vortical motion along the lines of magnetic induction; this motion, if occurring in a perfect medium (as the "ether" may be considered), producing tensions along the lines of the magnetic field, and pressures at right angles to them, would afford a satisfactory explanation of the magnetic attractions and repulsions which apparently act across empty space.

Hughes, Barus, and others have lately shown that the magnetism of iron and steel is intimately connected with the molecular rigidity of the material. Hughes's researches with the "induction balance" (Art. 514) and 
"magnetic balance" (Art. 140) tended to prove that each molecule of a magnetic metal has an absolutely constaut inherent magnetic polarity; and that when a piece of iron or steel is apparently neutral, its molecules are internally arranged so as to satisfy each other's polarity, forming closed magnetic circuits amongst themselves.

127. Ewing's Theory of Molecular Magnetism.-Weber supposed that there was in hard steel some sort of friction which prevented the molecules when once magnetized from turning back into higgledy-piggledy positions. Ewing, however, showed that a complete explanation was afforded by supposing the particles to be subject to mutual forces. In any group not subjected to an external magnetizing force the particles will arrange themselves so as to satisfy one another's polarity. Of the

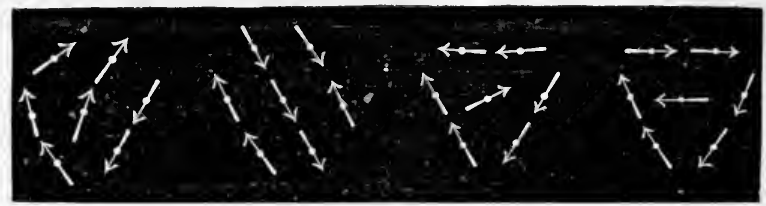

Fig. 75.

possible groupings some are, however, unstable. Four possible stable groupings of seven pivoted needles are shown in Fig. 75. Ewing constructed a model consisting of a large number of pivoted magnetic needles arranged in one layer. When these needles were simply agitated and allowed to come to rest they settled down in miscellaneous groups; but when acted upon by a gradually increasing magnetic force they turned round, the operation showing three stages-(i.) with very small magnetizing force the needles merely turned through a small angle; (ii.) when a certain force was applied the groupings became unstable, some of the needles suddenly swinging round to a new position, with the result that the majority of the needles 
point nearly but not quite along the direction of the force ; (iii.) a further increase of the magnetizing force cannot produce much more effect; it can only pull the needles a little more perfectly into line. All these things correspond to the three stages observed (see Art. 364) in the gradual magnetization of iron or steel.

\section{Lesson XI.-Laws of Magnetic Force}

\section{Laws of Magnetic Force.}

Finst Law. - Like magnetic poles repel one another; unlike magnetic poles attract one another.

Second Law.- The force exerted between two magnetic poles is proportional to the product of their strengths, and is inversely proportional to the square of the distance betwcen them, provided that the distance is so great that the poles may be regarded as mere points.

129. The Law of Inverse Squares.-The second of the above laws is commonly known as the law of inverse squares; it is essentially a law of point-action, and is not true for poles of elongated or extended surface. The similar law of electrical attraction has al-

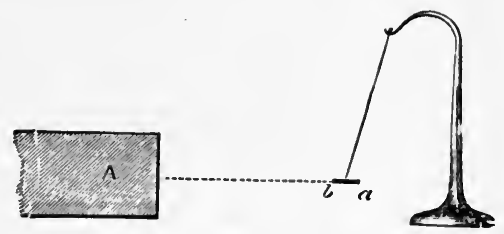

Fig. 76. ready been explained and illustrated (Art. 19). This law furnishes the explanation of a fact mentioned in an carlier lesson, Art. 91, that small pieces of iron are drawn bodily up to a magnet pole. If a small piece of iron wire, $a, b$ (Fig $76)$, be suspended by a thread, and the $\mathrm{N}$-pointing pole 
A of a magnet be brought near it, the iron is thereby inductively magnetized; it turns round and points towards the magnet pole, setting itself as nearly as possible along a line of force, its near end $b$ becoming a S-seeking pole, and its farther end $a$ becoming a $\mathrm{N}$-seeking pole. Now the pole $b$ will be attracted and the pole $a$ will be repelled. But these two forces do not exactly equal one another, since the distances are unequal. The repulsion will (by the law of inverse squares) be proportional to $\frac{1}{(\mathrm{~A} a)^{2}}$; and the attraction will be proportional to $\frac{1}{(\mathrm{~A} b)^{2}}$. Hence the bit of iron $a, b$ will experience a pair of forces, turning it into a certain direction, and also a total force drawing it bodily toward A. Only those bodies are attracted by magnets in which magnetism can thus be induced; and they are attracted only because of the magnetism induced in them.

We mentioned, Art. 91, that a magnet needle floating freely on a bit of cork on the surface of a liquid is acted upon by forces that give it a certain direction, but that, unlike the last case, it does not tend to rush as a whole either to the north or to the south. It experiences a rotation, because the attraction and repulsion of the magnetic poles of the earth act in a certain direction; but since the magnetic poles of the earth are at a distance enormously great as compared with the length from one pole of the floating magnet to the other, we may say that, for all practical purposes, the poles of the magnet are at the same distance from the $\mathrm{N}$ pole of the earth. The attracting force on the $\mathrm{N}$-pointing pole of the needle is therefore practically no greater than the repelling force acting on the S-pointing pole, hence there is no motion of translation given to the floating needle as a whole: it is directed, not attracted.

130. Measurement of Magnetic Forces.-The truth of the law of inverse squares can be demonstrated by experiment. But this implies that we have some 
means of measuring accurately the amount of the magnetic forces of attraction or repulsion. Magnetic force may be measured in any one of the four following ways: (1) by observing the time of swing of a magnetic needle oscillating under the influence of the force; (2) by observing the deflexion it produces upon a magnetic needle which is already attracted into a different direction by a force of known intensity ; (3) by balancing it against the torsion of an elastic thread; (4) by balancing it against the force of gravity as brought into play in attempting to deflect a magnet hung by two parallel strings (called the bifilar suspension), for these strings cannot be twisted out of their parallel position without raising the centre of gravity of the magnet.

131. Deflexion Experiment.-Fig. 77 shows an apparatus in which a compass-needle can be deflecterl by one pole of a magnet made of a long thin bar of steel, so mounted that its upper pole is always over the centre of the needle, and therefore has no tendency to turn it. So set, it acts as a onepole magnet, the pole of which can be placed at different distances from the compass-needle. It is found, using a proper tangent-scale (see Art. 211) for the compass-needle, that

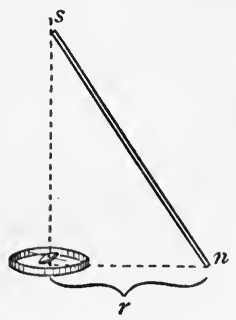

Fig. 7 . when the distance is doubled the deflecting force is reduced to one quarter, and so forth.

132. The Torsion Balance.-Coulomb applied the Torsion Balance to the measurement of magnetic forces. The main principles of this instrument (as used to measure forces of electrostatic repulsion) were described on p. 20 . Fig. 78 shows how it is arranged for measuring magnetic repulsions.

To prove the law of inverse squares, Coulomb made the following experiment :-The instrument was first adjusted 
so that a magnetic needle, hung in a copper stirrup to the fine silver thread, lay in the magnetic meridian without the wire being twisted. This was done by first putting in the magnet and adjusting roughly, then replacing it by a copper bar of equal weight, and once more adjusting, thus diminishing the error by repeated trials. The next

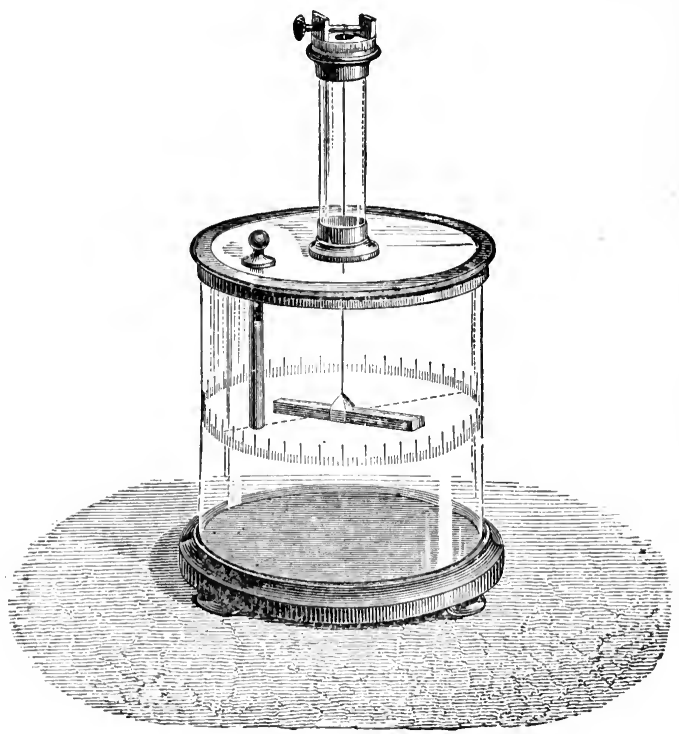

Fig. 78.

step was to ascertain through what number of degrees the torsion-head at the top of the thread must be twisted in order to drag the needle $1^{\circ}$ out of the magnetic meridian. In the particular experiment cited it was found that $35^{\circ}$ of torsion corresponded to the $1^{\circ}$ of deviation of the magnet; then a magnet was introduced through the lid, that pole being downwards which repelled the pole of the 
suspended needle. It was found (in this particular experiment) to repel the pole of the needle through $24^{\circ}$. From the preliminary trial we know that this directive force corresponds to $24^{\circ} \times 35^{\circ}$ of the torsion-head, and to this we must add the actual torsion on the wire, viz. the $24^{\circ}$, making a total of $864^{\circ}$, which we will call the "torsion equivalent" of the repelling force when the poles are thus $24^{\circ}$ apart. Finally, the torsion-head was turned round so as to twist the suspended magnet round, and force it nearer to the fixed pole, until the distance between the repelling poles was reduced to half what it was at first. It was found that the torsion-head had to be turned round 8 complete rotations to bring the poles to $12^{\circ}$ apart. These 8 rotations were an actual twist of $8^{\circ} \times 360^{\circ}$, or $2880^{\circ}$. But the bottom of the torsion thread was still twisted $12^{\circ}$ as compared with the top, the force producing this twist corresponding to $12 \times 35$ (or $420^{\circ}$ ) of torsion ; and to these the actual torsion of $12^{\circ}$ must be added, making a total of $2880^{\circ}+420^{\circ}+12^{\circ}=3312$. The result then of halving the distance between the magnet poles was to increase the force fourfold, for 3312 is very nearly four times 864. Had the distance between the poles been reduced to one-third the force would have been nine times as great.

We may also, assuming this law proved, employ the balance to measure the strengths of magnet poles by measuring the forces they exert at known distances.

133. Method of Oscillations. *-If a magnet suspended by a fine thread, or poised upon a point, be pushed aside from its position of rest, it will vibrate backwards and forwards, performing oscillations which, although they gradually decrease in amplitude, are executed in very

* It is possible, also, to measure electrical forces by a " method of oscillations"; a small charged ball at the end of a horizontally-suspended arm being caused to oscillate under the attracting force of a charged condnctor near it, whose " force" at that distance is proportional to the square of the number of oscillations in a given time. 
nearly equal times. In fact, they follow a law similar to that of the oscillations executed by a pendulum swinging under the influence of gravity. The law of pendular vibrations is, that the square of the number of oscillations executed in a given time is proportional to the force. Hence we can measure magnetic forces by counting the oscillations made in a minute by a magnet. It must be remembered, however, that the actual number of oscillations made by any given magnet will depend on the weight of the magnet and on its leverage around its centre, as well as upon the strength of its poles, and on the intensity of the field in which it may be placed (see calculations, Art. 361).

We can use this method to compare the intensity of the force of the earth's magnetism * at any place with that at any other place on the earth's surface, hy oscillating a magnet at one place and then taking it to the other place and oscillating it there. If, at the first, it makes $a$ oscillations in one minute, and at the second $b$ oscillations a minute, then the magnetic forces at the two places will be to one another in the ratio of $a^{2}$ to $b^{2}$.

Again, we may use the method to compare the force exerted at any point by a magnet near it with the force of the earth's magnetism at that point. For, if we swing a small magnetic needle there, and find that it makes $m$ oscillations a minute under the joint action † of the earth's magnetism, and that of the neighbouring magnet, and that, when the magnet is removed, it makes $n$ oscillations a minute under the influence of the earth's magnetism alone, then $m^{2}$ will be proportional to the joint forces, $n^{2}$ to the force due to the earth's magnetism, and the difference of these, or $m^{2}-n^{2}$ will be proportional to the force due to the neighbouring magnet.

* Or, more strictly, of its horizontal component.

+ We are here assuming that the magnet is so placed that its force is in a line with that of the earth's magnetism at the point, and that the other pole of the magnet is so far away as not to affect the oscillating needle. 
134. Surface Distribution.-We will now apply the method of oscillations to measure the relative quantities of surface magnetism at different points along a bar magnet. The magnet to be examined is set up vertically (Fig. 79). A small magnet, capable of swinging horizontally, is brought near it and set at a short distance away from its extremity, and then oscillated, while the rate of its oscillations is counted. Suppose the needle were such that, when exposed to the earth's magnetism alone, it would perform 3 complete oscillations a minute, and that, when vibrating at its place near the end of the vertical magnet it oscillated 14 times a minute, then the force due to the magnet will be proportional to $14^{2}-3^{2}=196-9=187$. Nextly, let the oscillating magnet be brought to an equal distance opposite a point a little away from the end of the vertical magnet. If, here, it oscillated 12 times a minute, we know that the force will be proportional to $12^{2}-3^{2}=144-9=135$. So

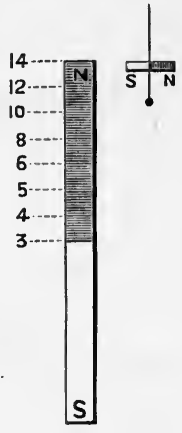

Fig. 79. we shall find that as the force falls off the oscillations will be fewer, until, when we put the oscillating nagnet opposite the middle of the vertical magnet, we shall find that the number of oscillations is 3 per minute, or that the earth's force is the only force affecting the oscillations. In Fig. 80 we have indicated the number of oscillations at successive points, as $14,12,10,8,6,5,4$, and 3 . If we square these numbers and subtract 9 from each, we shall get for the forces at the various points the following:- $-187,135,91,55,27,16,7$, and 0 . These forces may be taken to represent the strength of the free magnetism at the various points, and it is convenient to plot them ont graphically in the manner shown in Fig. 80, where the heights of the dotted lines are chosen to a scale to represent proportionally the forces. The curve which 
joins the tops of these orlinates shows graphically how the force, which is greatest at the end, falls off toward the middle. On a distant magnet pole these forces, thus represented by this curvilinear triangle, would act as if

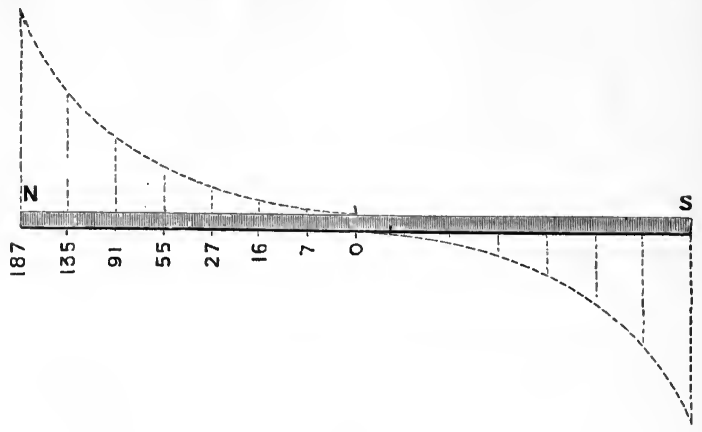

Fig. 80.

concentrated at a point in the magnet opposite the "centre of gravity" of this triangle; or, in other words, the "pole," which is the centre of the resultant forces, is not at the end of the magnet. In thin bars of magnetized steel it is at about $\frac{1}{10}$ of the magnet's length from the end.

135. Magnetic Moment.-It is found that the tendency of a magnet to turn or to be turned by another magnet depends not only on the strength $m$ of its poles, but the length $l$ between them. The product of these two quantities $m \times l$ is called the magnetic moment of the magnet, and is sometimes denoted by the symbol M. As the exact position of a magnet's poles are often unknown, it is easier to determine $\mathrm{M}$ than to measure either $m$ or $l$ separately.

136. Method of Deflexions.-There are a number of ways in which the deflexion of a magnet by another magnet may be made use of to measure magnetic forces.*

* The student desirous of mastering these methods of measuring magnetic forces should consult Professor Andrew Gray's Absolute Measurements in Electricity and Magnetism. 
We cannot here give more than a glance at first principles. When two equal and opposite forces act on the ends of a rigid bar they simply tend to turn it round. Such a pair of forces form what is called a "couple," and the torque, or tendency to turn (formerly called the "moment" of the couple), is obtained by multiplying one of the two forces by the perpendicular distance between the directions of the forces. Such a couple tends to produce a motion of rotation, but not a motion of translation. Now a magnetic needle placed in a magnetic field across the lines of force experiences a torque, tending to rotite it round into the magnetic meridian, for the $\mathrm{N}$-seeking pole is urged northwards, and the S-seeking pole is urged southwards, with an equal and opposite force. The force acting on each pole is the product of the strength of the pole and the intensity of the "field," that is to say, of the horizontal component of the force of the earth's magnetism at the place. We will call the

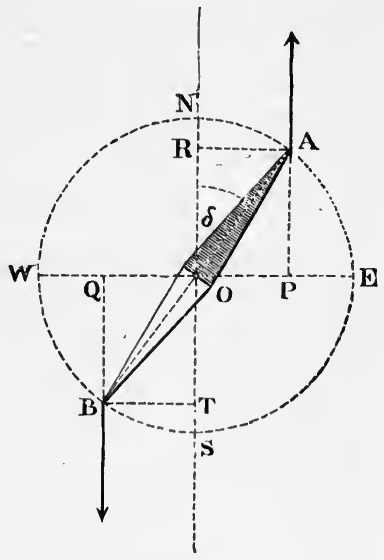

Fig. 81 . strength of the $\mathrm{N}$-seeking pole $m$; and we will use the symbol $H$ to represent the force which the earth's magnetism would exert in a horizontal direction on a unit of magnetism. (The value of $\mathrm{H}$ is different at different regions of the globe.) The force on the pole A (see Fig. 81) will be then $m \times \mathrm{H}$, and that on pole $\mathrm{B}$ will be equal and opposite. We take $\mathrm{NS}$ as the direction of the magnetic meridian: the forces will be parallel to this direction. Now, the needle $\mathrm{AB}$ lies 
obliquely in the field, while the magnetic force acting on $\mathrm{A}$ is in the direction of the line PA, and that on $\mathrm{B}$ in the direction $\mathrm{QB}$, as shown by the arrows. $\mathrm{PQ}$ is the perpendicular distance between these forces; hence the "moment" of the couple, or torque, will be got by multiplying the length $P Q$ by the force exerted on one of the poles. Using the symbol $\mathrm{Y}$ for the torque, we may write

$$
\mathrm{Y}=\mathrm{PQ} \times m \cdot \mathrm{H} .
$$

But $\mathrm{PQ}$ is equal to the length of the magnet multiplied by the sine ${ }^{*}$ of the angle AOR, which is the angle of deflexion, and which we will call $\delta$. Hence, using $l$ for the length between the poles of the magnet, we may write the expression for the moment of the couple.

$$
\mathrm{Y}=m l \mathrm{H} \cdot \sin \delta .
$$

In words this is : the torque acting on the needle is proportional to its "magnetic moment" $(m \times l)$, to the horizontal force of the earth's magnetism, and to the sine of the angle of deflexion.

The reader will not have failed to notice that if the needle were turned more obliquely, the distance $P Q$ would be longer, and would be greatest if the needle were turned round east-and-west, or in the direction EW. Also the torque tending to rotate the magnet will be less and less as the needle is turned more nearly into the direction NS.

137. Law of Tangents.-Now, let us suppose that the deflexion $\delta$ were produced by a magnetic force applied at right angles to the magnetic meridian, and tending to draw the pole $\mathrm{A}$ in the direction RA. The length of the line RT multiplied by the new force will be the leverage of the new couple tending to twist the magnet into the direction

* If any reader is unacquainted with trigonometrical terms he should consult the note at the end of this lesson, on "Ways of Reckoning Angles." 
EW. Now, if the needle has come to rest in equilibrium between these two forces, it is clear that the two opposing twists are just equal and opposite in power, or that the torque due to one couple is equal to that of the other couple. Hence the force in the direction WE will be to the force in the direction $\mathrm{SN}$ in the same ratio as $\mathrm{PQ}$ is to $\mathrm{RT}$, or as $\mathrm{PO}$ is to RO.

Or, calling this force $f$,

$$
f: \mathrm{H}=\mathrm{PO}: \mathrm{RO} .
$$

Or

$$
f=\mathrm{H} \frac{\mathrm{PO}}{\mathrm{RO}} \text {. }
$$

But $P O=A R$ and $\frac{A R}{R O}=\tan \delta$, hence

$$
f=\mathrm{H} \tan \delta ;
$$

or, in other words, the magnetic force which, acting at right angles to the meridian, produces on a magnetic needle the deflexion $\delta$, is equal to the horizontal force of the earth's magnetism at that point, multiplied by the tangent of the angle of deflexion. Hence, also, two different magnetic forces acting at right angles to the meridian would severally deflect the needle through angles whose tangents are. proportional to the forces.

This very important theorem is applied in the construction of certain galvanometers (see Art. 212).

138. Magnetometers. The name Magnetometer is given to any magnet specially arranged as an instrument for the purpose of measuring magnetic forces. The methods of observ-

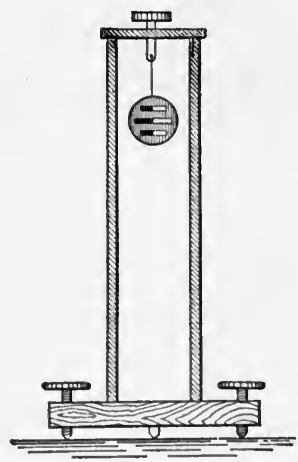

Fig. 82. ing the absolute values of magnetic forces in dyne-units (units in the "C.G.S." system) will be explained in Art. 361 
at the end of Lesson XXVII. Very simple magnetometers, consisting of small needles pivoted, or suspended by a fibre, are commonly used for measuring the relative values of magnetic forces. One very sensitive form (Fig. 82), to be used, like the reflecting galvanometer (Art. 215), with a beam of light as a pointer, consists of a small thin silvered glass

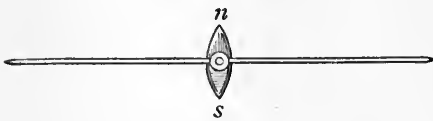

Fig. S3.

nuirror, a half-inch or less in diameter, having two or three very light magnets cemented at its back, suspended by a single thread of cocoon silk, and enclosed in a suitable case. Another useful form (Fig. 83) consists of a short compass-needle poised on a pivot having a light index of aluminium long enough to move over a scale divided into tangent values (see Art. 212).

A convenient deflexion magnetometer for comparing the magnetic moments (Art. 135) of two magnets is

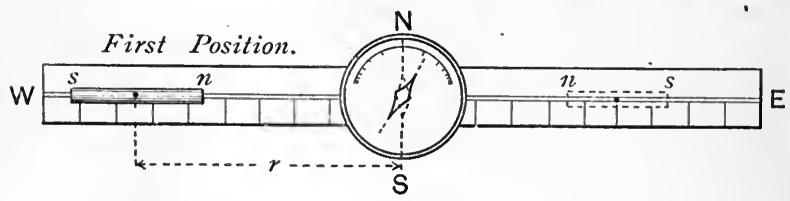

Fig. S4.

afforded by such a tangent compass placed in the middle of a graduated platform (Fig. 84). There are two methods of using this apparatus.

First Position: End-on Method.-The platform being set magnetically east and west, the deflecting magnet is set end-on. Under these circumstances the force is found to vary directly as the magnetic moment (Art. 135), and inversely as the cube of the distance between the centres of the magnets, or in symbols :-

$$
f=2 \mathrm{M} / r^{3} \text {. }
$$


But we have seen above that where magnetic force is measured by a deflexion $\delta$ at a place where the $\mathrm{H}$ is earth's horizontal magnetic force, $f$ is equal to $\mathrm{H} \tan \delta$; so that

whence

$$
2 \mathrm{M} / r^{3}=\mathrm{H} \tan \delta,
$$

$$
\mathrm{M}=\frac{1}{2} r^{3} \mathrm{H} \tan \delta .
$$

Second Position: Broadside-on.-The platform being turned into the north-south position, the deflecting magnet is set broadside-on. In this case the magnet deflects the needle in the other direction and with half the force that it would have exerted at an equal distance in the end-on position. But the force still varies inversely as the cube of the distance: the formula being now

whence

$$
f=\mathrm{M} / r^{3},
$$

$$
\mathrm{MI}=r^{3} \mathrm{H} \tan \delta .
$$

139. Balance Methods. - In either position of the magnetometer platform two magnets can be placed on the two sides of the board so as to balance one another's effects by adjusting them to proper distances. This gives a comparison of their magnetic moments in terms of their respective distances, or

$$
\mathrm{M}_{1}: \mathrm{M}_{2}=r_{1}{ }^{3}: r_{2}{ }^{3} \text {. }
$$

140. Hughes's Magnetic

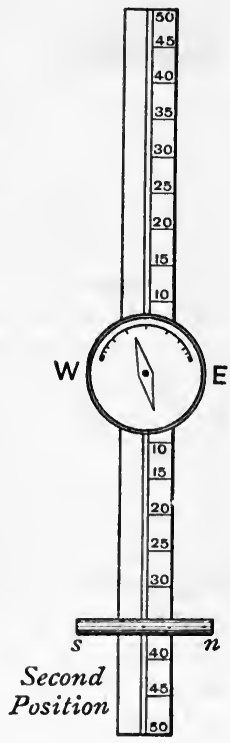

Fig. 85. Balance.-A very convenient instrument for testing the magnetic properties of different specimens of iron and steel was devised by Hughes in 1884 . The sample to be tested 
is placed in a magnetizing coil A (Fig. 86), and a current is sent round it. It deflects a lightly-suspended indicating needle $B$, which is then brought to zero by turning a large compensating magnet $\mathrm{M}$ upon its centre. A small coil $\mathrm{C}$ is added to balance the direct deflecting effect due

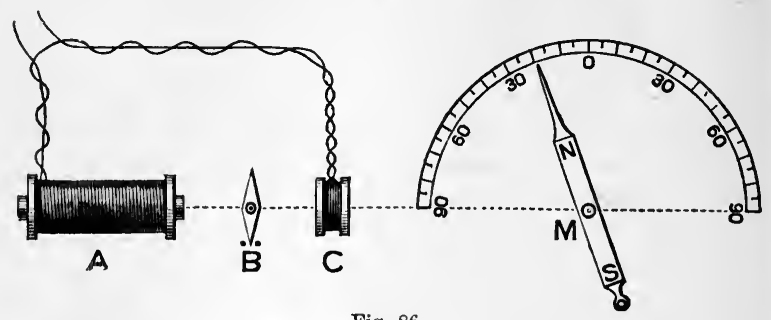

Fig. 86 .

to coil A. The author of this book has shown that if the distance from $M$ to $B$ is $2 \cdot 3$ times the length of $M$, the angle through which $M$ is turned is proportional to the magnetic force due to the iron core at $A$, provided the angle is less than $60^{\circ}$.

141. Unit Strength of Pole. - The Second Law of Magnetic Force (see Art. 128) stated that the force exerted between two poles was proportional to the product of their strengths, and was inversely proportional to the square of the distance between them. It is possible to choose such a strength of pole that this proportionality shall become numerically an equality. In order that this may be so, we must adopt the following as our unit of strength of a pole, or unit magnetic pole: A Unit Magnetic Pole is one of such a strength that, when placed at a distance of one centimetre from a similar pole of equal strength it repels it with a force of one dyne (see Art. 352). If we arlopt this definition we may express the second law of magnetic force in the following equation :-

$$
f=\frac{m \times m^{\prime}}{d^{2}},
$$


where $f$ is the force (in dynes), $m$ and $m^{\prime}$ the strengths of the two poles, and $d$ the distance between them (in centimetres). From this definition is derived the arbitrary convention about magnetic lines. If at any place in a magnetic field we imagine a unit magnetic pole to be set it will be acted upon, tending to move along the lines of the field. Then if at that place we find the force on the pole to be $\mathrm{H}$ dynes, we may conceive that there are $\mathrm{H}$ lines drawn per square centimetre. For example, if we describe the field as having 50 lines side by side per square centimetre, we mean that a unit pole placed there will be acted on with a force of 50 dynes. Or we may say simply: the intensity of the field is 50 gausses (see Art. 338).

142. Theory of Magnetic Curves.-We saw (Art. 119) that magnetic figures are produced by iron filings setting themselves in certain directions in the field of force around a magnet. We can now apply the law of inverse squares to aid us in determining the direction in which a filing will set itself at any point in the field. Let NS (Fig. 87) be a long thin magnet, and $\mathrm{P}$ any point in the field due to its magnetism. If the $\mathrm{N}$ seeking pole of a small magnet be put at $\mathrm{P}$, it will be attracted by $\mathrm{S}$ and repelled by $\mathrm{N}$; the directions of these two forces will be along the lines PS and PN. The amounts of the forces may be represented by certain lengths marked out along these lines. Suppose the distance PN is twice as great as PS, the repelling force along PN will be $\frac{1}{4}$ as strong as the attracting force along PS. So measure a distance out, $\mathrm{PA}$ towards $\mathrm{S}$ four times as long as the length $\mathrm{PB}$ measured along $\mathrm{PN}$ away from N. Find the resultant force in the usual way of compounding mechanical forces, by completing the parallelogram PARB; the diagonal PR represents by its length and direction the magnitude and the direction of the resultant magnetic force at the point $P$. In fact the line $\mathrm{PR}$ represents the line along which a small magnet or an 
iron filing would set itself. In a similar way we might ascertain the direction of the lines of force at any point of the field. The little arrows in Fig. 87 show how the lines of force start out from the $\mathrm{N}$ pole and curve round to meet in the $\mathrm{S}$ pole. The student should compare this

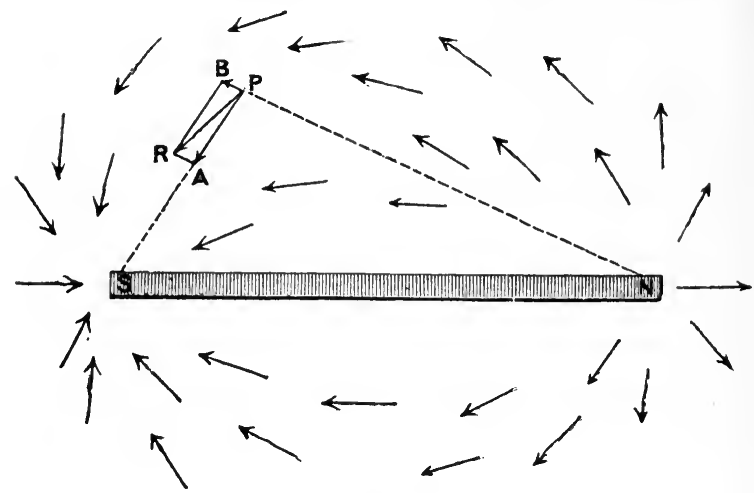

Fig. 87.

figure with the lines of filings of Fig. 67. Henceforth we must think of every magnet as being permeated by these magnetic lines which extend out into the surrounding space. The whole number of magnetic lines which run through a magnet is termed its magnetic flux (Art. 337).

143. A Magnetic Paradox. - If the N-seeking pole of a strong magnet be held at some distance from the $\mathrm{N}$-seeking pole of a weak magnet, it will repel it; but if it is pushed up quite close it will be found now to attract it. This paradoxical experiment is explained by the fact that the magnetism induced in the weak magnet by the powerful one will be of the opposite kind, and will be attracted; and, when the powerful magnet is near, this induced magnetism may overpower and mask the original magnetism of the weak magnet. The 
student must be cautioned that in most of the experiments on magnet poles similar perturbing causes are at work. The ntagnetism in a magnet is not quite fixed, but is liable to be disturbed in its distribution by the near presence of other magnet poles, for no steel is so hard as not to be temporarily affected by magnetic induction.

\section{Note on Ways of Reckoning Angles and Solid ANgles}

144. Reckoning in Degrees.-When two straight lines cross one another they form an angle between them; and this angle may be defined as the amount of rotation which one of the lines has performed round a fixed point in the other line. Thus we may suppose the line CP in Fig. 88 to have originally lain along $\mathrm{CO}$, and then turued round to its present position. The amount by which it has been rotated is clearly a certain fraction of the whole way round; and the amount of rotation round $\mathrm{C}$ we call "the angle which PC makes with OC," or more simply "the angle PCO." But there are a number of different ways of reckoning this angle. The common way is to reckon the angle by "degrees" of

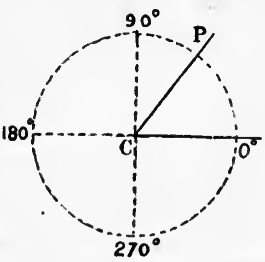

Fig. ss. arc. Thus, suppose a circle to be drawn round $C$, if the circumference of the circle were divided inte 360 parts each part would be called "one degree" $\left(1^{\circ}\right)$, and the angle would be reckoned by naming the number of such degrees along the curved arc OP. In the figure the arc is about $57 \frac{1}{4}^{\circ}$, or $\frac{57 \frac{1}{4}}{360}$ of the whole way round, no matter what size the circle is drawn.

145. Reckoning in Radians.-A more sensible but less usual way to express an angle is to reckon it by the ratio between the length of the curved arc that "subtends" the angle and the length of the radius of the circle. Suppose we have drawn round the centre $\mathrm{C}$ a circle whose radius is one centimetre, the diameter will be two centimetres. The length of the circumference all round is known to be about $3 \frac{1}{7}$ times the length of the diameter, or more exactly $3 \cdot 14159 \ldots$ This number is so awkward 
that, for convenience, we always use for it the Greek letter $\pi$. Hence the length of the circumference of our circle, whose radius is one centimetre, will be $6 \cdot 28318$. . centimetres, or $2 \pi$ centimetres. We can then reckon any angle by naming the length of arc that subtends it on a circle one centimetre in radius. If we choose the angle $\mathrm{PCO}$, such that the curved arc OP shall be just one centimetre long, this will be the angle one, or unit of angular measure, or, as it is sometimes called, the angle PCO will be one "radian." In degree-measure one radian $=\frac{360^{\circ}}{2 \pi}=57^{\circ} 17^{\prime}$ nearly. All the way round the circle will be $2 \pi$ radians. A right angle will be $\frac{\pi}{2}$ radians.

146. Reckoning by Sines or Cosines.-In trigonometry other ways of reckoning angles are used, in which, however, the

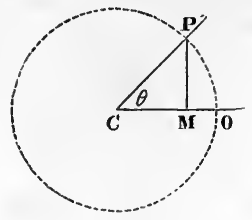

Fig. 89. angles themselves are not reckoned, but certain "functions" of them called "sines," " cosines," "tangents," etc. For readers not accustomed to these we will briefly explain the geometrical nature of these "functions." Suppose we draw (Fig. 89) our circle as before round centre $\mathrm{C}$, and then drop down a plumb-line PM, on to the line $\mathrm{CO}$; we will, instead of reckoning the angle by the curved arc, reckon it by the length of the line PM. It is clear that if the angle is small PM will be short; but as the angle opens out towards a right angle, PM will get longer and longer (Fig. $90)$. The ratio between the length of this line and the radius of the circle is called the "sine" of the angle, and if the radius is 1 the length of PM will be the value of the sine. It can never be greater than 1, though it may have all values between 1 and -1 . The length of the line CM will also depend upon the amount of the angle. If the angle is small CM will be nearly as long as $\mathrm{CO}$; if the angle open out to nearly a right angle

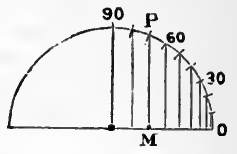

Fig. 90 . CM will be very short. The length of CM (when the radius is 1 ) is called the "cosine" of the angle. If the angle be called $\theta$, then we may for shortness write these functions:

$$
\begin{aligned}
& \operatorname{Sin} \theta=\frac{\mathrm{PM}}{\mathrm{CP}} \\
& \operatorname{Cos} \theta=\frac{\mathrm{CM}}{\mathrm{CP}} .
\end{aligned}
$$


147. Reckoning by Tangents.-Suppose we draw our circle as before (Fig. 91), but at the point $\mathrm{O}$ draw a straight line touching the circle, the tangent line at $\mathrm{O}$; let us also prolong CP until it meets the tangent line at $\mathrm{T}$. We may measure the angle between $\mathrm{OC}$ and $\mathrm{OP}$ in terms of the length of the tangent $\mathrm{OT}$ as compared with the length of the radius. Since our radius is 1 , this ratio is numerically the length of OT, and we may therefore call the length of OT the "tangent" of the angle OCP. It is clear that smaller angles will have smaller tangents, but that larger angles may have very large tangents; in fact, the length of the tangent when $\mathrm{PC}$ was moved round to a right angle would be infinitely great. It can be shown that the ratio between the lengths of the sine and of the cosine of the angle is the same as

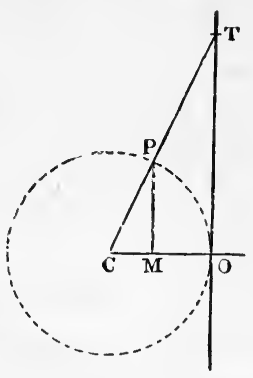

Fig. 91. the ratio between the length of the tangent and that of the radius; or the tangent of an angle is equal to its sine divided by its cosine. The formula for the tangent may be written :

$$
\tan \theta=\frac{\mathrm{TO}}{\mathrm{OC}}=\frac{\mathrm{PM}}{\mathrm{MC}} \text {. }
$$

148. Solld Angles.-When three or more surfaces intersect at a point they form a solid angle: there is a solid angle, for

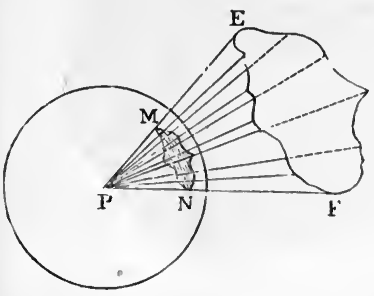

Fig. 9.. example, at the top of a pyramid, or of a cone, and one at every corner of a diamond that has been cut. If a surface of any given shape be near a point, it is said to subtend a certain solid angle at that point, the solid angle being mapped ont by drawing lines from all points of the edge of this surface to the point $\mathrm{P}$ (Fig. 92). An irregular cone will thus be generated whose solid angle is the solid angle snb. tended at $\mathrm{P}$ by the surface $\mathrm{EF}$. To reckon this solid angle we adopt an expedient similar to that adopted when we wished to reckon a plane angle in radians. About the point $P$, with radius of 1 centimetre, describe a sphere, which will intercept 
the cone over an area $\mathrm{MN}$ : the area thus intercepted measures the solid angle. If the sphere have the radius 1, its total surface is $4 \pi$. The solid angle subtended at the centre by a hemisphere would be $2 \pi$. It will be seen that the ratio between the area of the surface EF and the area of the surface MN is the ratio between the squares of the lines EP and MP. The solid angle subtended by a surface at a point (other things being equal) is inversely proportional to the square of its distance from the point. This is the basis of the law of inverse squares.

A table of radians, sines, tangents, etc., is given at the end of this book as Appendix A.

\section{Lesson XII.-Terrestrial Magnetism}

149. The Mariner's Compass.-It was mentioned in Art. 87 that the compass sold by opticians consists of

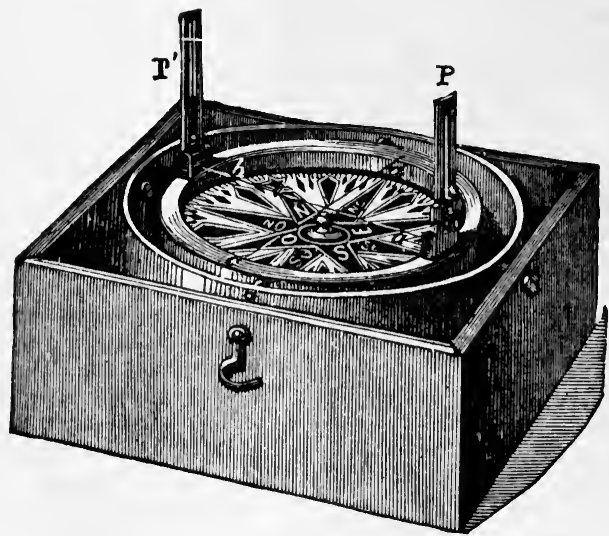

Fig. 93.

a magnetized steel needle balanced on a fine point above a card marked out $\mathrm{N}, \mathrm{S}, \mathrm{E}, \mathrm{W}$, etc. The Mariner's Compass is, lowever, somewhat difierently arranged. In Fig. 93 one of the forms of a Mariner's Compass, 
used for nautical observations, is shown. Here the card, divided out into the 32 "points of the compass," is itself attached to the needle, and swings round with it so that the point marked $\mathrm{N}$ on the card always points to the north. In the best modern ships' compasses, such as those of Lord Kelvin, several magnetized needles are placed side by side, as it is found that the indications of such a compound needle are more reliable. The iron fittings of wooden vessels, and, in the case of iron vessels, the ships themselves, affect the compass, which has therefore to be corrected by placing compensating masses of iron near it, or by fixing it high upon a mast. The error of the compass due to magnetism of the ship is known as the deviation.

150. The Earth a Magnet.-Gilbert made the great discovery that the compass-needle points north and south because the earth is itself also a great magnet. The magnetic poles of the earth are, however, not exactly at the geographical north and south poles. The magnetic north pole of the earth is more than 1000 miles away from the actual pole, being in lat. $70^{\circ} 5^{\prime} \mathrm{N}$., and long. $96^{\circ} 46^{\prime} \mathrm{W}$. In 1831 it was found by Sir J. C. Ross to be situated in Boothia Felix, just within the Arctic Circle. The south magnetic pole of the earth has never been reached; and by reason of irregularities in the distribution of the magnetism there appear to be two south magnetic polar regions.

151. Declination.-In consequence of this natural distribution the compass-needle does not at all points of the earth's surface point truly north and south. Thus, in 1894, the compass-needle at London pointed at an angle of about $17^{\circ}$ west of the true north; in 1900 it was $16^{\circ} 16^{\prime}$. This angle between the magnetic meridian ${ }^{*}$ and

* The Magnetic Meridian of any place is an imaginary plane drawn through the zenith, and passing throngh the magnetic north point and magnetic south point of the horizon, as observed at that place by the pointing of a horizontally-suspended compass-needle. 
the geographical meridian of a place is called the magnetic Declination of that place. The existence of this declination was discovered by Columbus in 1492, though it appears to have been previously known to the Chinese, and is said to have been noticed in Europe in the early part of the thirteenth century by Peter Peregrinus. The fact that the declination differs at different points of the earth's surface, is the undisputed discovery of Columbus.

In order that ships may steer by the compass, magnetic charts (Art. 154) must be prepared, and the declination at different places accurately measured. The upright pieces $\mathrm{P}, \mathrm{P}^{\prime}$, on the "azimuth compass" drawn in Fig. 93, are for the purpose of sighting a star whose position may be known from astronomical tables, and thus affording

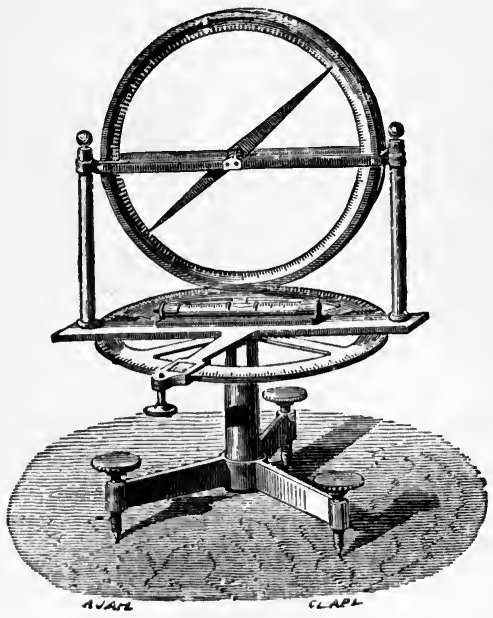

Fig. 94 . a comparison between the magnetic meridian of the place and the geographical meridian, and of measuring the angle between them.

152. Inclination or Dip.Norman, an instrument-maker, discovered in 1576 that a balanced needle, when magnetized, tends to dip lownwards toward the north. He therefore constructed a Dipping - Needle, capable of turning in a vertical plane about a horizontal axis, with which he found the "dip" to be (at London) an 
angle of $71^{\circ} 50^{\prime}$. A simple form of Dipping-needle is shown in Fig. 94. The dip-circles used in the magnetic observatory at Kew are much more exact and delicate instruments. It was, however, found that the dip, like the declination, differs at different parts of the earth's surface, and that it also undergoes changes from year to year. The "dip" in London for the year 1894 is $67^{\circ}$ $18^{\prime}$; in 1900 it was $67^{\circ} 9^{\prime}$. At the north magnetic pole the needle dips straight down. The following table gives particulars of the Declination, Inclination, and total magnetic force at a number of important places, the values being approximately true for the year 1900 .

Table of Magnetic Declination and Inchination (for Year 1900)

\begin{tabular}{|c|c|c|c|c|c|}
\hline \multicolumn{2}{|c|}{ Locality. } & & Declination. & Dip. & $\begin{array}{l}\text { Total Force } \\
\text { (C.G.S.) }\end{array}$ \\
\hline London & & . & $16^{\circ} 16^{\prime} \mathrm{W}$. & $67^{\circ} 9^{\prime} \quad \mathrm{N}$. & 0.47 \\
\hline St. Petersbu & urg & . & $0^{\circ} 30^{\prime} \mathrm{E}$ & $70^{\circ} 46^{\prime} \mathrm{N}$. & 0.48 \\
\hline Berlin . & & . & $9^{\circ} 30^{\prime} \mathrm{W}$. & $66^{\circ} 43^{\prime} \mathrm{N}$. & 0.48 \\
\hline Paris . & • & & $14^{\circ} 30^{\prime} \mathrm{W}$. & $64^{\circ} 55^{\prime} \mathrm{N}$. & 0.47 \\
\hline Rome . & & & $10^{\circ} 0^{\prime} \quad$ W. & $58^{\circ} 0^{\prime} \mathrm{N}$. & 0.45 \\
\hline New York & & . & $9^{\circ} 12^{\prime} \mathrm{W}$ & $70^{\circ} 6^{\prime} \quad \mathrm{N}$. & $0 \cdot 61$ \\
\hline Washington & & & $4^{\circ} 35^{\prime} \mathrm{W}$ & $70^{\circ} 18^{\prime} \mathrm{N}$. & $0 \cdot 60$ \\
\hline San Francise & & . & $16^{\circ} 42^{\prime} \mathrm{E}$. & $62^{\circ} 20^{\prime} \mathrm{N}$. & 0.54 \\
\hline Mexico & . & . & $8^{\circ} 0^{\prime} \quad$ E. & $45^{\circ} 1^{\prime} \mathrm{N}$. & 0.48 \\
\hline St. Helena & . & . & $25^{\circ} 0^{\prime} \mathrm{W}$. & $32^{\circ} 12^{\prime} \mathrm{S}$. & $0 \cdot 31$ \\
\hline Cape Town & . & . & $29^{\circ} 24^{\prime} \mathrm{W}$. & $58^{\circ} 2^{\prime} \mathrm{S}$. & 0.36 \\
\hline Sydney & . & . & $9^{\circ} 36^{\prime} \mathbf{E}$ & $62^{\circ} 45^{\prime} \mathrm{S}$. & 0.57 \\
\hline Hobarton & . & . & $25^{\circ} 0^{\prime}$ E. & $71^{\circ} 12^{\prime} \mathrm{S}$. & $0 \cdot 64$ \\
\hline Bombay & . & . & $0^{\circ} 36^{\prime} \mathrm{E}$. & $20^{\circ} 38^{\prime} \mathrm{N}$. & 0.37 \\
\hline Tokio . & & . & $4^{\circ} 6^{\prime} \quad W$. & $49^{\circ} 52^{\prime} \mathrm{N}$. & 0.45 \\
\hline
\end{tabular}

153. Intensity.-Three things must be known in order to specify exactly the magnetism at any place; these three elements are : 


\section{The Declination; \\ The Inclination, and \\ The Intensity of the Magnetic Force.}

The magnetic force is measured by one of the methods mentioned in the preceding lesson. Its direction is in the line of the dipping-needle, which, like every magnet, tends to set itself along the lines of force. It is, however, more convenient to measure the force not in its total intensity in the line of the dip, but to measure the horizontal component of the force,- that is to say, the force in the direction of the horizontal compass-neelle, from which the total force can be calculated if the dip is known.* Or if the horizontal and vertical components of the force are known, the total force and the angle of the dip can both be calculated. $\dagger$ The horizontal component of the force, or "horizontal intensity," can be ascertained either by the method of Vibrations or by the method of Deflexions. The mean horizontal force of the earth's magnetism at London in 1890 was $\cdot 1823$ garisses, the mean vertical force 4377 , the total force (in the line of dip) was 4741 gausses. The distribution of the magnetic force at different points of the earth's surface is irregular, and varies in different latitudes according to an approximate law, which, as given by Biot, is that the force is proportional to $\sqrt{1+3 \sin ^{2} l}$, where $l$ is the magnetic latitude.

154. Magnetic Maps.-For purposes of convenience it is usual to construct magnetic maps, on which such data as those given in the Table on p. 139 can be marked down. Such maps may be constructed in several ways. Thus, it would be possible to take a map of England, or of the world, and mark it over with lines such as to represent by their direction the actual direction in which the compass points; in fact to draw the lines of force or

* For if $\mathbf{H}=$ Horizontal Component of Force, and $\mathbf{I}=$ Total Force, and $\theta=$ angle of $\operatorname{dip}, \mathrm{I}=\mathrm{H} \div \cos \theta$.

$\uparrow$ For $\mathrm{H}^{2}+\mathrm{V}^{2}=\mathrm{I}^{2}$, where $\mathrm{V}=$ Vertical Component of Force. 
magnetic meridians. A more useful way of marking the map is to find out those places at which the declination is the same, and to join these places by a line. The Magnetic Map of Great Britain, which forms the Frontispiece to these lessons, is constructed on this plan from the magnetic survey lately made by Rücker and Thorpe. At Plymouth the compass-needle in 1900 will point $18^{\circ}$ to the west of the geographical north. The declination at Lynton, at Shrewsbury, and at Berwick will in that year be the same as at Plymouth. Hence a line joining these towns may be called a line of equal declination, or an Isogonic line. It will be seen from this map that the declination is greater in the north-west of England than in the south-east. We might similarly construct a magnetic map, marking it with lines joining places where the dip was equal; such lines would be called Isoclinic lines. In England they run across the map from west-south-west to east-north-east. For example, in 1900 the needle will dip about $67^{\circ}$ at London, Southampton, and Plymouth. Through these places then the isoclinic of $67^{\circ}$ may be drawn for that epoch. On the globe the isogonic lines run for the most part from the north magnetic pole to the south magnetic polar region, but, owing to the irregularities of distribution of the earth's magnetism, their forms are not simple. The isoclinic lines of the globe run round the earth like the parallels of latitude, but are irregular in form. Thus the line joining places where the northseeking pole of the needle dips down $70^{\circ}$ runs across England and Wales, passes the south of Ireland, then crosses the Atlantic in a south-westerly direction, traverses the United States, swerving northwards, and just crosses the southern tip of Alaska. It drops somewhat southward again as it crosses China, but again curves northwards as it enters Russian territory. Finally it crosses the southern part of the Baltic, and reaches England across the German Ocean. The magnetic chart of the United States, which is also given at the front of this book, is for the year 
1900. It has been prepared from data furnished by Professor Mendenhall of the U.S. Geodetic Survey. It will be noticed that in the year 1900 the magnetic declination will be zero at Lansing (Mich.), Columbus (Ohio), and Charleston (S. Carolina).

The line passing through places of no declination is called the agonic line. It passes across both hemispheres, crossing Russia, Persia, and Australia. There is another agonic line in eastern Asia enclosing a region around Japan, within which there is a westerly declination.

155. Variations of Earth's Magnetism. - We have already mentioned that both the declination and the inclination are subject to changes; some of these changes take place very slowly, others occur every year, and others again every day.

Those changes which require many years to run their course are called secular changes.

The variations of the declination previous to 1580 are not recorded; the compass at London then pointed $11^{\circ}$ east of true north. This easterly declination gradually decreased, until in 1657 the compass pointed true north. It then moved westward, attaining a maximum of $24^{\circ}$ $27^{\prime}$ about the year 1816, from which time it has slowly diminished to its present value $\left(16^{\circ} 9^{\prime}\right.$ in 1901); it diminishes (in England) at about the rate of $7^{\prime}$ per year. At about the year 1976 it will again point truly nortl, making a complete cycle of changes in about 320 years.

The Inclination in 1576 was $71^{\circ} 50^{\prime}$, and it slowly increased till 1720, when the angle of dip reached the maximum value of $74^{\circ} 42^{\prime}$. It has since steadily diminished to its present (1901) value of $67^{\circ} 8^{\prime}$. The period in which the cycle is completed is not known, but the rate of variation of the dip is less at the present time than it was fifty years ago. In all parts of the earth both declination and inclination are slowly changing. The following table gives the data of the secular changes at London. 
Table of Secular Magnetic Variations

\begin{tabular}{|l|l|l|}
\hline Year. & Declination. & Inclination. \\
\hline 1576 & & $71^{\circ} 50^{\prime}$ \\
1580 & $11^{\circ} 17^{\prime} \mathrm{E.}$ & $72^{\circ} 0^{\prime}$ \\
1630 & $6^{\circ} 12^{\prime}$ & \\
1622 & $4^{\circ} 0^{\prime}$ & \\
1634 & $0^{\circ} 0^{\prime}$ min. & \\
1657 & $3^{\circ} 0^{\prime} \mathrm{W}$. & $73^{\circ} 30^{\prime}$ \\
1676 & $9^{\circ} 0^{\prime}$ & \\
1705 & $13^{\circ} 0^{\prime}$ & $74^{\circ} 42^{\prime} \max$. \\
1720 & $19^{\circ} 30^{\prime}$ & \\
1760 & $24^{\circ} 6^{\prime}$ & $72^{\circ} 8^{\prime}$ \\
1780 & $24^{\circ} 30^{\prime} \max$. & $70^{\circ} 35^{\prime}$ \\
1800 & $24^{\circ} 2^{\prime}$ & $69^{\circ} 3^{\prime}$ \\
1816 & $23^{\circ} 0^{\prime}$ & \\
1830 & $20^{\circ} 33^{\prime}$ & $68^{\circ} 2^{\prime}$ \\
1855 & $19^{\circ} 14^{\prime}$ & $67^{\circ} 43^{\prime}$ \\
1868 & $18^{\circ} 40^{\prime}$ & $67^{\circ} 40^{\prime}$ \\
1878 & $17^{\circ} 26^{\prime}$ & $67^{\circ} 23^{\prime}$ \\
1880 & $16^{\circ} 16^{\prime}$ & $67^{\circ} 9^{\prime}$ \\
1890 & $15^{\circ} 3^{\prime}$ & $66^{\circ} 54^{\prime}$ \\
1900 & & \\
\hline 1910 & &
\end{tabular}

The Total Magnetic force, or "Intensity," also slowly changes in value. As measured near London, it was equal to 4791 gausses in $1848, \cdot 4740$ in 1866 , in 1880 .4736 gausses, in 1890.4741 . $^{*}$ Owing to the steady decrease of the angle at which the needle dips, the horizontal component of this force (i.e. the "Horizontal Intensity ") is slightly increasing. It was 1716 gausses in $1814, \cdot 1797$ gausses at the beginning of 1880 , and $\cdot 1823$ gausses in 1890 .

156. Daily Variations.-Both compass and dipping-needle, if minutely observed, exhibit slight daily

* That is to say, a north magnet pole of unit strength is urged in the line of dip, with a mechanical force of a little less than half a dyne. 
motions. About 7 A.M. the compass-needle begins to travel westward with a motion which lasts till about 1 P.s. ; during the afternoon and evening the needle slowly travels back eastward, until about 10 P.M. ; after this it rests quiet; but in summer-time the needle begins to move again slightly to the west at about midnight, and returns again eastward before 7 A.M. These delicate variations-never more than $10^{\prime}$ of arc-appear to be connected with the position of the sun; and the moon also exercises a minute influence upon the position of the needle.

157. Annual Variations.-There is also an annual variation corresponding with the movement of the earth around the sun. In the British Islands the total force is greatest in June and least in February, but in the Southern Hemisphere, in Tasmania, the reverse is the case. The dip also differs with the season of the year, the angle of dip being (in England) less during the four summer months than in the rest of the year.

158. Eleven-Year Period. - General Sabine discovered that there is a larger amount of variation of the declination occurring about once every eleven years. Schwabe noticed that the recurrence of these periods coincided with the eleven-year periods at which there is a maximum of spots on the sun. Professor Balfour Stewart and others have endeavoured to trace a similar periodicity in the recurrence of auroræ* and of other phenomena.

159. Magnetic Storms.-It is sometimes observed that a sudden (though very minute) irregular disturbance will affect the whole of the compass-needles over a considerable region of the globe. Such occurrences are known as magnetic storms; they frequently occur at the time when an aurora is visible.

160. Self-recording Magnetic Apparatus.-At Kew and other magnetic observatories the daily and

* See Tesson XXIV., on Atmospheric Electricity. 
hourly variations of the magnet are recorded on a continuous register. The means employed consists in throwing a beam of light from a lamp on to a light mirror attached to the magnet whose motion is to be observed. A spot of light is thus reflected upon a ribbon of photographic paper prepared so as to be sensitive to light. The paper is moved continuously forward by a clockwork train; and if the magnet be at rest the dark trace on the paper will be simply a straight line. If, however, the magnet moves aside, the spot of light reflected from the mirror will be displaced, and the photographed line will be curved or crooked. Comparison of such records, or magnetographs, from stations widely apart on the earth's surface, promises to afford much light upon the cause of the changes of the earth's magnetism, to which hitherto no reliable origin has been with certainty assigned. Schuster has shown that these changes generally come from without, and not from within.

161. Theory of Earth's Magnetism.-The phenomenon of earth - currents (Art. 233) appears to be connected with that of the changes in the earth's magnetism, and can be observed whenever there is a display of aurora, and during a magnetic storm; but it is not yet determined whether these currents are due to the variations in the magnetism of the earth, or whether these variations are due to the currents. It is known that the evaporation (see Art. 71) always going on in the tropics causes the ascending currents of heated air to be electrified positively relatively to the earth. These aircurrents travel northward and southward toward the colder polar regions, where they descend. These streams of electrified air will act (see Art. 397) like true electric currents, and as the earth rotates within them it will be acted upon magnetically. The author has for twelve years upheld the view that this thermodynamic production of polar currents in conjunction with the earth's diurnal rotation affords the only rational means yet 
suggested for accounting for the growth of the earth's magnetism to its present state. The action of the sun and moon in raising tides in the atmosphere might account for the variations mentioned in Art. 155. It is important to note that in all magnetic storms the intensity of the perturbations is greatest in the regions nearest the poles; also, that the magnetic poles coincide very nearly with the regions of greatest cold; that the region where auroræ (Art. 336) are seen in greatest abundance is a region lying nearly symmetrically round the magnetic pole. It may be added that the general direction of the feeble daily earth-currents (Art. 233) is from the poles toward the equator. 


\title{
CHAPTER III
}

\author{
CURRENT ELECTRICITY
}

\section{Iuesson XIII.—Simple Voltaic Cells}

162. Flow of Currents. - It has been already mentioned, in Lesson IV., how electricity flows away from a charged body through any conducting substance, such as a wire or a wetted string. If, by any arrangement, electricity could be supplied to the body just as fast as it flowed away, a continuous current would be produced. Such a current always flows through a conducting wire, if the ends are kept at different electric potentials. In like manner, a current of heat flows through a rod of metal if the ends are kept at different temperatures, the flow being always from the high temperature to the lower. No exact evidence exists as to the direction in which the current in a wire really "flows." It is convenient to regard the electricity as flowing from positive to negative; or, in other words, the natural direction of an electric current is from the high potential to the low. It is obvious that such a flow tends to bring both to one level of potential. In order that a continuous flow may be kept up there must be a circuit provided. The "current" has sometimes been regarded as a double transfer of positive electricity in one direction, and of negative electricity in the opposite direction. The only evidence to support this very un- 
necessary supposition is the fact that, in the decomposition of liquids by the current, some of the elements are liberated at the place where the current enters, others at the place where it leaves the liquid.

The quantity of electricity conveyed by a current is proportional to the current and to the time that it continues to flow. The practical unit of current is called the ampere (see Arts. 207 and 254). The quantity of electricity conveyed by a current of one ampere in one second is called one ampere-second or one coulomb. One amperehour equals 3600 coulombs. If $\mathrm{C}$ is the number of amperes of current, $t$ the number of seconds that it lasts, and $\mathrm{Q}$ the number of coulombs of electricity thereby conveyed, the relation between them is expressed by the formula :-

$$
\mathrm{Q}=\mathrm{C} \times t .
$$

Example. - If a current of 80 amperes flows for 15 minutes the total quantity of electricity conveyed will be $80 \times 15 \times 60=72,000$ coulombs. This is equal to 20 ampere-hours.

Currents are called continuous if they flow, without stopping, in one direction. They are called alternate currents if they continually reverse in direction in a regular periodic manner, flowing first in one direction round the circuit and then in the other.

Continuous currents of electricity, such as we have described, are produced by voltaic cells, and batteries of such cells, or else by dynamos driven toy power, though there are other sources of currents hereafter to be mentioned. Alternate currents are produced by special alternate current dynamos or alternators, and are separately treated of in Art. 470.

163. Discoveries of Galvani and of Volta. The discovery of electric currents originated with Gulvani, a physician of Bologna, who, about the year 1786, made a series of curious and important observations upon the 
convulsive motions produced by the "return-shock" (Art. 29) and other electric discharges upon a frog's leg. He was led by this to the discovery that it was not necessary to use an electric machine to produce these effects, but that a similar convulsive kick was produced in the frog's leg when two dissimilar metals, iron and copper, for example, were placed in contact with a nerve and a muscle respectively, and then brought into contact with each other. Galvani imagined this action to be due to electricity generated by the frog's leg itself. It was, however, proved by Volta, Professor in the University of Pavia, that the electricity arose not from the muscle or nerve, but from the contact of the dissimilar metals. When two metals are placed in contact with one another in the air, one becomes positive and the other negative, as we have seen near the end of Lesson VII., though the charges are very feeble. Volta, however, proved their reality by two different methods.

164. The Voltaic Pile. - The second of Volta's proofs was less direct, but even more convincing; and consisted in showing that when a number of such contacts of dissimilar metals could be arranged so as to add their electrical effects together, those effects were more powerful in proportion to the number of the contacts. With this view he constructed the apparatus known (in honour of the discoverer) as the Voltaic Pile (Fig. 95). It is made by placing a pair of disks of zinc and copper in contact with one another, then laying on the copper disk a piece of flannel or blottingpaper moistened with brine, then another pair of disks of zinc and copper, and so on, each pair of disks in the pile being

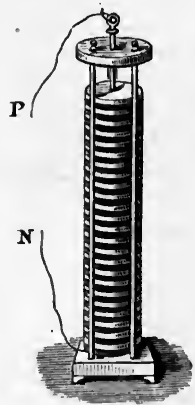

Fig. 95 . separated by a moist conductor. Such a pile, if composed of a number of such pairs of disks, will produce electricity 
enough to give quite a perceptible shock, if the top and bottom disks, or wires connected with them, be touched simultaneously with the moist fingers. When a single pair of metals are placed in contact, one becomes + ly electrical to a certain small extent, and the other - ly electrical, or, in other words, there is a certain difference of electric potential (see Art. 265) between them. But when a number are thus set in series with moist conductors between the successive pairs, the difference of potential between the first zinc and the last copper disk is increased in proportion to the number of pairs; for now all the successive small differences of potential are added together.

165. The Crown of Cups.-Another combination devised by Volta was his Couronne de Tasses or Crown of Cups. It consisted of a number of cups (Fig. 96), filled either with brine or dilute acid, into which dipped a number of compound strips, half zinc half copper, the zinc portion of one strip dipping into one cup, while

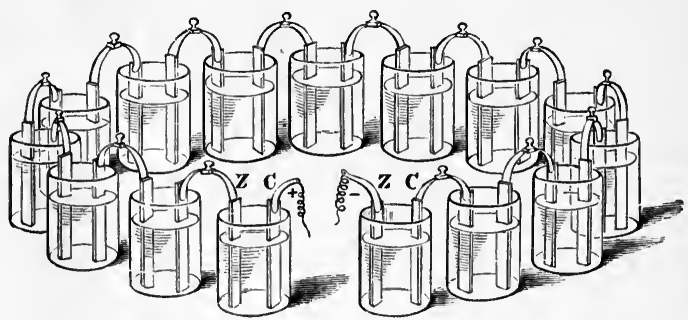

Fig. 96 .

the copper portion dipped into the other cup. The difference of potential between the first and last cups is again proportional to the number of pairs of metal strips. This arrangement, though badly adapted for such a purpose, is powerful enough to ring an electric bell, the wires of which are joined to the first zinc and the last copper strip. The electrical action of these 
combinations is, however, best understool by studying the phenomena of one single cup or cell.

166. Simple Voltaic Cell._Place in a glass jar some water having a little sulphuric acid or any other oxidizing acid added to it (Fig. 97). Place in it separately two clean strips, one of zinc Z, and one of copper C. This cell is capable of supplying a continuous flow of electricity through a wire whose ends are brought into connexion with the two strips. When the current flows the zinc strip is observed to waste away ; its consumption in fact furnishes the energy required to drive the current through the cell and the connecting wire. The cell may there-

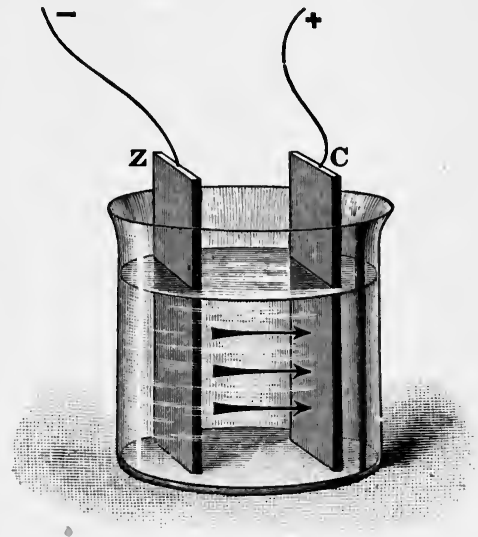

Fig. 97. fore be regarded as a sort of chemical furnace in which fuel is consumed to drive the current. The zinc is the fuel,* the acid is the aliment, whilst the copper is merely a metallic hand let down into the cell to pick up the current, and takes no part chemically. Before the strips are connected by a wire no appreciable difference of potential between the copper and the zinc will be observed by an electrometer; because the electrometer only measures the potential at a point in the air or oxidizing medium outside the zinc or the copper, not the

* Zinc, as is well known, will burn with a blue flame in air or oxygen, giving out heat. Zinc foil is easily kindled. 
potentials of the metals themselves. The zinc is trying to dissolve and throw a current across to the copper; while the copper is trying (less powerfully) to dissolve and throw a current across the other way. The zinc itself is at about 1.86 volts higher potential than the surrounding oxidizing media (see Art. 489); while the copper is at only about 81 volts higher, having a less tendency to become oxidized. There is then a latent difference of potential of about 1.05 volts between the zinc and the copper; but this produces no current as long as there is no metallic circuit. If the strips are made to touch, or are joined by a pair of metal wires, immediately there is a rush of electricity through the acid from the zinc to the copper, as indicated by the arrows in Fig. 97 , the current returning by the metal circuit from the copper to the zinc. A small portion of the zinc is at the same time dissolved away; the zinc parting with its latent energy as its atoms combine with the acid. This energy is expended in forcing electricity through the acid to the copper strip, and thence through the wire circuit back to the zinc strip. The copper strip, whence the current starts on its journey through the external circuit, is called the positive pole, and the zinc strip is called the negative pole. If two copper wires are united to the tops of the two strips, though no current flows so long as the wires are kept separate, the wire attached to the zinc will be found to be negative, and that attached to the copper positive, there being still a tendency for the zinc to oxidize and drive electricity through the cell from zinc to copper. This state of things is represented by the + and - signs in Fig. 97 ; and this distribution of potentials led some to consider the junction of the zinc with the copper wire as the starting point of the current. But the real starting point is in the cell at the surface of the zinc where the chemical action is furnishing energy ; for from this point there are propagated through the liquid certain electro-chemical actions (more fully ex- 
plained in Chap. XI.) which have the result of constantly renewing the difference of potential. At the same time it will be noticed that a few bubbles of hydrogen gas appear on the surface of the copper plate. Both these actions go on as long as the wires are joined to form a complete circuit. The metallic zinc may be considered as a store of energy. We know that if burned as a fuel in oxygen or air it will give out that store of energy as heat. If burned in this quiet chemical manner in a cell it gives out its store not as heat - any heat in a cell is so much waste-but in the form of electric energy, i.e. the energy of an electric current propelled by an electromotive force.

167. Effects produced by Current.-The current itself cannot be seen to flow through the wire circuit; hence to prove that any particular cell or combination produces a current requires a knowledge of some of the effects which currents can produce. These are of various kinds. A current flowing through a thin wire will heat it; flowing near a magnetic needle it will cause it to turn aside; flowing through water and other liquids it decomposes them ; and, lastly, flowing through the living body or any sensitive portion of it, it produces certain sensations. These effects, thermal, magnetic, chemical, and physiological, will be considered in special lessons.

168. Voltaic Battery.-If a number of such simple cells are united in series, the zinc plate of one joined to the copper plate of the next, and so on, a greater difference of potentials will be produced between the copper "pole" at one end of the series and the zine "pole" at the other end. Hence, when the two poles are joined by a wire there will be a more powerful flow of electricity than one cell would cause. Such a combination of Voltaic Cells is called a Voltaic Battery.* There are

* By some writers the name Galvanic Battery is given in honour of Galvani; but the honour is certainly Volta's. The electricity that flows thus in currents is sometimes called Voltaic Electricity, or Galvanic 
many ways of grouping a battery of cells, but two need special notice. If the cells are joined up in one row, as in Fig. 96 or Fig. 98, they are said to be in series. Electricians often represent a cell by a symbol in which

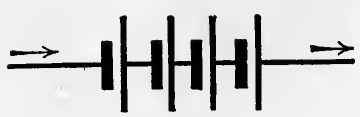

Fig. 98. a short thick line stands for the zinc and a longer thin line for the copper (or carbon). Thus Fig. 98 represents four cells joined in series. The maximum current (amperes) which so grouped they will yield is not more than a single cell would yield on short circuit; but they yield that current with a fourfold electromotive-force (volts.)

The other chief way of grouping cells is to join all the zincs together and all the coppers (or carbons) together; and they are then said to be in parallel, or are joined "for quantity." So joined they have no greater electromotive-force than one cell. The zincs act like one big zinc, the coppers

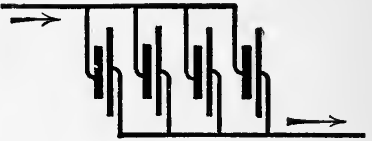

Fig. 99 . like one big copper. But they will yield more current. Fig. 99 shows the four cells grouped in parallel; they would yield thus a current four times as great as one cell alone would yield.

169. Flectromotive-Force-The term electromotive-force is employed to denote that which moves or tends to move electricity from one place to another.*

Electricity, or sometimes even Galvanism (!), but, as we shall see, it differs only in degree from Frictional or any other Electricity, and both can flow along wires, and magnetize iron, and decompose chemical compounds. The word Battery means an arrangement of one or more cells; just as in warfare a battery of guns means an arrangement of one or more.

* The beginner must not confuse Electromotive-force, or that which tends to move electricity, with Electric "force," or that force with which electricity tends to move matter. Newton has virtually defined "force," once for all, as that which moves or tends to move matter. When matter is moved by a magnet we speak rightly of magnetic force; when electricity 
For brevity we sometimes write it E.M.F. In this particular case it is obviously the result of the difference of potential, and proportional to it. Just as in waterpipes a difference of level produces a pressure, and the pressure produces a flow so soon as the tap is turned on, so difference of potential produces electromotive-force, and electromotive-force sets up a current so soon as a circuit is completed for the electricity to flow through. Electromotive-force, therefore, may often be conveniently expressed as a difference of potential, and vice versî ; but the student must not forget the distinction. The unit in which electromotive-force is measured is termed the volt (see Art. 354). The terms pressure and voltage are sometimes used for difference of potential or electromotive-force.

170. Volta's Laws. - Volta showed (Art. 79) that the difference of potential between two metals in contact (in air) depended merely on what metals they were, not on their size, nor on the amount of surface in contact. $\mathrm{He}$ also showed that when a number of metals touched one another the difference of potential between the first and last of the row is the same as if they touched one another directly. A quantitative illustration from the researches of Ayrton and Perry was given in Art. 80. But the case of a series of cells is different from that of a mere row of metals in contact. If in the row of cells the zincs and coppers are all arranged in one order, so that all of them set up electromotive-forces in the same direction, the total electromotive-force of the series will be equal to the electromotive-force of one cell multiplied by the number of cells.

Hitherto we have spoken only of zinc and copper as the materials for a cell ; but cells may be made of any two metals. The effective electromotive-force of a cell depends on the difference between the two. If zinc was

moves matter we may speak of electric force. But E.M.F. is quite a different thing, not "force" at all, for it acts not on matter but on electricity, and tends to move it. 
used for both metals in a cell it would give no current, for each plate would be trying to dissolve and to throw a current across to the other with equal tendency. That cell will have the greatest electromotive-force, or be the most "intense," in which those materials are used which have the greatest difference in their tendency to combine chemically with the acid, or which are widest apart on the "contact-series" given in Art. 80. Zinc and copper are convenient in this respect; and zinc and silver would be better but for the expense. For nore powerful batteries a zinc-platinum or a zinc-carbon combination is preferable. That plate or piece of metal in a cell by which the current enters the liquid is called the anode; it is that plate which dissolves away. The plate or piece of metal by which the current leaves the cell is called the kathode; it is not dissolved, and in some cases receives a deposit on its surface.

171. Resistance. - The same electromotive-force does not, however, always produce a current of the same strength. The amount of current depends not only on the force tending to drive the electricity round the circuit, but also on the resistance which it has to encounter and overcome in its flow. If the cells be partly choked with sand or sawdust (as is sometimes done in so-called "Sawdust Batteries" to prevent spilling), or, if the wire provided to complete the circuit be very long or very thin, the action will be partly stopped, and the current will be weaker, although the E.M.F. may be unchanged. The analogy of the waterpipes will again help us. The pressure which forces the water through pipes depends upon the difference of level between the cistern from which the water flows and the tap to which it flows; but the amount of water that runs through will depenil not on the pressure alone, but on the resistance it meets with; for, if the pipe be a very thin one, or choked with sand or sawdust, the water will only run slowly through. 
Now the metals in general conduct well : their resistance is small; but metal wires must not be too thin or too long, or they will resist too much, and permit only a feeble current to pass through them. The liquids in the cell do not conduct nearly so well as the metals, and different liquids have different resistances. Pure water will hardly conduct at all, and is for the feeble electricity of the voltaic battery almost a perfect insulator, though for the high-potential electricity of the frictional machines it is, as we have seen, a fair conductor. Salt and saltpetre dissolved in water are good conductors, and so are dilute acids, though strong sulphuric acid is a bad conductor. The resistance of the liquid in the cells may be reduced, if desired, by using larger plates of metal and putting them nearer together. Gases are bad conductors; hence the bubbles of hydrogen gas which are given off at the copper plate during the action of the cell, and which stick to the surface of the copper plate, increase the internal resistance of the cell by diminishing the effective surface of the plates.

\section{Lesson XIV.-Chemical Actions in the Cell}

172. Chemical Actions.-The production of a current of electricity by a voltaic cell is always accompanied by chemical actions in the cell. One of the metals at least must be readily oxidizable, and the liquid must be one capable of acting on the metal. As a matter of fact, it is found that zine and the other metals which stand at the electropositive end of the contact-series (see Art. 80) are oxidizable ; whilst the electronegative substances-copper, silver, gold, platinum, and graphiteare less oxidizable, and the last three resist the action of every single acid. There is no proof that their electrical behaviour is due to their chemical behaviour; nor that their chemical behaviour is due to their electrical. 
Probably both result from a common cause (see Art. 80 , and also 489). A piece of quite pure zinc when dipped alone into dilute sulphuric acid is not attacked by the liquid. But the ordinary commercial zinc is not pure, and when plunged into dilute sulphuric acid dissolves away, a large quantity of bubbles of hydrogen gas being given off from the surface of the metal. Sulphuric acid is a complex substance, in which every molecule is made up of a group of atoms-2 of Hydrogen, 1 of Sulphur, and 4 of Oxygen ; or, in symbols, $\mathrm{H}_{2} \mathrm{SO}_{4}$. The chemical reaction by which the zinc enters into combination with the radical of the acid, turning out the hydrogen, is expressed in the following equation :-

$$
\mathrm{Zn}+\mathrm{H}_{2} \mathrm{SO}_{4}=\mathrm{ZnSO}_{4}+\mathrm{H}_{2}
$$

Zine and Sulphurie Acid produce Sulphate of Zine and Hydrogen.

The sulphate of zinc produced in this reaction remains in solution in the liquid.

Now, when a plate of pure zinc and a plate of some less-easily oxidizable metal-copper or platinum, or, best of all, carbon (the hard carbon from gas retorts) - are put side by side into the cell containing acid, no appreciable chemical action takes place until the circuit is completed by joining the two plates with a wire, or by making them touch one another. Directly the eircuit is completed a current flows and the chemical actions begin, the zinc dissolving in the acid, and the acid giving up its hydrogen in streams of bubbles. But it will be noticed that these bubbles of hydrogen are evolved not at the zinc plate, nor yet throughout the liquid, but at the surface of the copper plate (or the carbon plate if carbon is employed). This apparent transfer of the hydrogen gas through the liquid from the surface of the rinc plate to the surface of the copper plate where it appears is very remarkable. The ingenious theory framed by Grotthuss to account for it is explained in Lesson XLVII. on Electro-Chemistry. 
These chemical actions go on as long as the current passes. The quantity of zinc used up in each cell is proportional to the amount of electricity which flows round the circuit while the battery is at work; or, in other words, is proportional to the current. The quantity of hydrogen gas evolved is also proportional to the amount of zinc consumed, and also to the current. After the acid has thus dissolved zinc in it, it will no longer act as a corrosive solvent ; it has been "killed," as workmen say, for it has been turned into sulphate of zinc. The battery will cease to act, therefore, either when the zinc has all dissolved away, or when the acid has become exhausted, that is to say, when it is all turned into sulphate of zinc. Stout zinc plates will last a long time, but the acids require to be renewed frequently, the spent liquor being emptied out.

173. Local Action.-When the circuit is not closed the current cannot flow, and there should be no chemical action so long as the battery is producing no current. The impure zinc of commerce, however, does not remain quiescent in the acid, but is continually dissolving and giving off hydrogen bubbles. This local action, as it is termed, is explained in the following manner :The impurities in the zinc consist of particles of iron, arsenic, and other metals. Suppose a particle of iron to be on the surface anywhere and in contact with the acid. It will behave like the copper plate of a battery towards the zinc particles in its neighbourhood, for a local difference of potential will be set up at the point where there is metallic contact, causing a local or parasitic current to run from the particles of zinc through the acid to the particle of iron, and so there will be a continual wasting of the zinc, both when the battery circuit is closed and when it is open.

174. Amalgamation of Zinc.-We see now why a piece of ordinary commercial zinc is attacked on being placed in acid. There is local action set up all over its 
surface in consequence of the metallic impurities in it. To do away with this local action, and abolish the wasting of the zinc while the battery is at rest, it is usual to amalgamate the surface of the zinc plates with mercury. The surface to be amalgamated should be cleaned by dipping into acid, and then a few drops of mercury should be poured over the surface and rubbed into it with a bit of linen rag tied to a stick. The mercury unites with the zinc at the surface, forming a pasty amalgam. The iron particles do not dissolve in the mercury, but float up to the surface, whence the hydrogen bubbles which may form speedily carry them off. As the zinc in this pasty amalgam dissolves into the acid the film of mercury unites with fresh portions of zinc, and so presents always a clean bright surface to the liquid.

A newer and better process is to add about 4 per cent of mercury to the molten zinc before casting into plates or rods. If the zinc plates of a battery are well amalgamated there should be no evolution of hydrogen bubbles when the circuit is open. Nevertheless there is still always a little wasteful local action during the action of the battery. Jacobi found that while one part of hydrogen was evolved at the kathode, 33.6 parts of zinc were dissolved at the anode, instead of the 32.5 parts which are the chemical equivalent of the hydrogen.

175. Polarization.-The bubbles of hydrogen gas liberated at the surface of the copper plate stick to it in great numbers, and form a film over its surface; hence the effective amount of surface of the copper plate is very seriously reduced in a short time. When a simple cell, or battery of such cells, is set to produce a current, it is found that the current after a few minutes, or even seconds, falls off very greatly, and may even be almost stopped. This immediate falling off in the current, which can be observed with any galvanometer and a pair of zinc and copper plates dipping into acid, is almost entirely due 
to the film of hydrogen bubbles sticking to the copper pole. A battery which is in this condition is said to be "polarized."

176. Effects of Polarization.-The film of hydrogen bubbles affects the strength of the current of the cell in two ways.

Firstly, it weakens the current by the increased resistance which it offers to the flow, for bubbles of gas are bad conductors ; and, worse than this,

Secondly, it weakens the current by setting up an opposing electromotive-force; for hydrogen is almost as oxidizable a substance as zinc, especially when it is being deposited (or in a "nascent" state), and is electropositive, standing high in the series on p. 85 . Hence the hydrogen itself produces a difference of potential, which would tend to start a current in the opposite direction to the true zinc-to-copper current. No cell in which the polarization causes a rapid falling off in power can be used for closed circuit work.

It is therefore a very important matter to abolish this polarization, otherwise the currents furnished by batteries would not be constant.

177. Remedies against Internal Polarization. - Various reniedies have been practised to reduce or prevent the polarization of cells. These may be classed as mechanical, chemical, and electrochenical.

1. Mechanical Means.-If the hydrogen bubbles be simply brushed away from the surface of the kathode, the resistance they caused will be diminished. If air be blown into the acid solution through a tube, or if the liquid be agitated or kept in constant circulation by siphons, the resistance is also diminished. If the surface be rongh or covered with points, the bubbles collect more freely at the points and are quickly carried up to the surface, and so got rid of. This remedy was applied in Smee's Cell, which consisted of a zinc and a platinized silver plate dipping into dilute sulphuric acid; the silver 
plate, having its surface thus covered with a rough coating of finely divided platinum, gave up the hydrogen bubbles freely; nevertheless, in a battery of Smee cells the current diminishes greatly after a few minutes.

2. Chemical Means.-If a highly-oxidizing substance be added to the acid it will destroy the hydrogen bubbles whilst they are still in the nascent state, and thus will prevent both the increased internal resistance and the opposing electromotive-force. Such substances are bichromate of potash, nitric acid, and chlorine.

3. Electrochemical Means.-It is possible by employing double cells, as explained in the next lesson, to so arrange matters that some solid metal, such as copper, shall be liberated instead of hydrogen bubbles, at the point where the current leaves the liquid. This electrochemical exchange entirely obviates polarization.

178. Simple Laws of Chemical Action in the Cell.-We will conclude this section by enumerating the two simple laws of chemical action in the cell.

I. The amount of chemical action in the cell is proportional to the quantity of electricity that passes through it - that is to say, is proportional to the current while it passes.

A current of one ampere flowing through the cell for one second causes 0.00033698 (or $\frac{1}{296}$ ) of a gramme of zinc to dissolve in the acid, and liberates 0.000010384 (or $\frac{1}{96302}$ ) of a gramme of hydrogen.

II. The amount of chemical action is equal in each cell of a battery consisting of cells joined in series.

The first of these laws was thought by Faraday, who discovered it, to disprove Volta's contact theory. He foresaw that the principle of the conservation of energy would preclude a mere contact force from furnishing a continuous supply of current, and hence ascribed the current to the chemical actions which were proportional in quantity to it. How the views of Volta and Faraday are to be harmonized has been indicated in the last 
paragraph of Art. 80. These laws only relate to the useful chemical action, and do not include the waste of "local" actions (Art. 166) due to parasitic currents set up by impurities.

\section{Lesson XV._-Voltaic Cells}

179. A good Voltaic cell should fulfil all or most of the following conditions :-

1. Its electromotive-force should be high and constant.

2. Its internal resistance should be small.

3. It should give a constant current, and therefore must be free from polarization, and not liable to rapid exhaustion, requiring frequent renewal of the acid.

4. It should be perfectly quiescent when the circuit is open.

5. It should be cheap and of durable materials.

6. It should be manageable, and if possible, should not emit corrosive fumes.

No single cell fulfils all these conditions, however, and some cells are better for one purpose and some for another. Thus, for telegraphing through a long line of wire a considerable internal resistance in the battery is no great disadvantage ; while, for producing an electric light, much internal resistance is absolutely fatal. The electromotive-force of a battery depends on the materials of the cell, and on the number of cells linked together, and a high E.M.F. can therefore be gained by choosing the right substances and by taking a large number of cells. The resistance within the cell can be diminished by increasing the size of the plates, by bringing them near together, so that the thickness of the liquid between them may be as small as possible, and by choosing liquids that are good conductors. 
180. Classification of Cells. - Of the innumerable forms of cell that have been invented, only those of first importance can be described. Cells are sometimes classified into two groups, according as they contain one or two fluids, or electrolytes, but a better classification is that adopted in Art. 177, depending on the means of preventing polarization.

\section{Class I.-With Mechanical Depolarization} (Single Fluid)

The simple cell of Volta, with its zinc and copper plates, has been already described. The larger the copper plate, the longer time does it take to polarize. Cruickshank suggested to place the plates vertically in a trough, producing a more powerful combination. Dr. Wollaston proposed to use a plate of copper of double size, bent round so as to approach the zinc on both sides, thus diminishing the resistance, and allowing the hydrogen more surface to deposit upon. Smee, as we have seen, replaced the copper plate by platinized silver, and Walker suggested the use of plates of hard carbon instead of copper or silver, thereby saving cost, and at the same time increasing the electromotive-force. The roughness of the surface facilitates the escape of hydrogen bubbles. By agitating such cells, or raising their kathode plates for a few moments into the air, their power is partially restored. The Law cell, used in the United States for open-circuit work, is of this class: it has a small rod of zinc and a cleft cylinder of carbon of large surface immersed in solution of salammoniac.

\section{Class II. - Witu Chemical Depolarization}

In these cells, in addition to the dilute acid or other excitant to dissolve the zinc, there is added some more 
powerful chemical agent as a depolarizer. Amongst depolarizers the following are chiefly used :-Nitric acid, solutions of chromic acid, of bichromate of potash, of bichromate of soda, of nitrate of potash, or of ferric chloride; chlorine, bromine, black oxide of manganese, sulphur, peroxide of lead, red lead, oxide of copper. Most of these materials would, however, attack the copper as well as the zinc if used in a zinc-copper cell. Hence they can oniy be made use of in zinccarbon or zinc-platinum cells. Nitric acid also attacks zinc when the circuit is open. Hence it cannot be employed in the same single cell with the zinc plate. In the Bichromate Cell, invented by Poggendorff, bichromate of potash is added to the sulphuric acid. This cell is most conveniently made up as shown in Fig. 100, in which a plate of zinc is the anode, and a pair

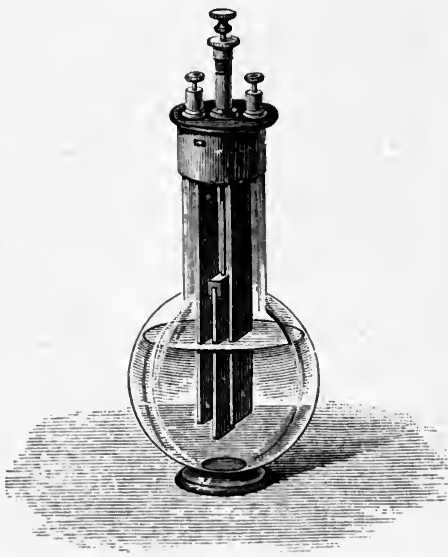

Fig. 100. of carbon plates, one on each side of the zinc, joined together at the top serve as a kathode. As this solution would attack the zinc even when the circuit is open, the zinc plate is fixed to a rod by which it can be drawn up out of the solution when the cell is not being worked.

To obviate the necessity of this operation the device is adopted of separating the depolarizer from the liquid into which the zinc dips. In the case of liquid depolarizers this is done by the use of an internal porous cell or partition. Porous cells of earthenware or of parchment paper 
allow the electric current to flow while keeping the liquids apart. In one compartment is the zinc anode dipping into its aliment of dilute acid : in the other conpartment the carbon (or platinum) kathode dipping into the depolarizer. Such cells are termed two-fluid cells. In the case of solid depolarizers such as black oxide of manganese, oxide of copper, etc., the material merely needs to be held up to the kathode. All solid depolarizers are slow in acting.

\section{Class III.-With Electrochemical Depolarization}

When any soluble metal is immersed in a solution of its own salt-for example, zinc dipped into sulphate of zinc, or copper into sulphate of copper-there is a definite electromotive-force between it and its solution, the measure of its tendency to dissolve. If a current is sent from metal to solution some of the metal dissolves; if, however, the current is sent from solution to metal some more metal will be deposited (or "plated") out of the solution. But as long as the chemical nature of the surface and of the liquid is unchanged there will be no change in the electromotive-force at the surface. It follows that if a cell were made with two metals, each dipping into a solution of its own salt, the two solutions being kept apart by a porous partition, such a cell would never change its electromotive-force. The anode would not polarize where it dissolves into the excitant; the kathode would not polarize, since it receives merely an additional thickness of the same sort as itself. This electrochemical method of avoiding polarization was discovered by Daniell. It is the principle not only of the Daniell cell, but of the Clark cell and of others. For perfect constancy the two salts used should be salts of the same acid, both sulphates, or both chlorides, for example.

181. Daniell's Cell.-Each cell or "element" of 
Daniell's battery has an inner porous cell or partition to keep the separate liquids from mixing. The outer cell (Fig. 101) is usually of copper, and serves also as a copper kathode. Within it is placed a cylindrical cell of unglazed porous ware (a cell of parchment, or even of brown paper, will answer), and in this is a rod of amalgamated zinc as anode. The liquid in the inner cell is dilute sulphuric acid or dilute sulphate of zinc; that in the outer cell is a saturated solution of sulphate of copper ("blue vitriol"), some spare crystals of the same substance being contained in a perforated shelf at the top of the cell, in order that they may dissolve and replace that which is used up while the battery is in action.

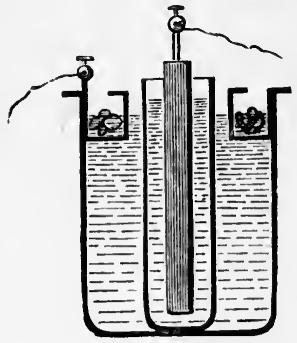

Fig. 101.

When the circuit is closed the zinc dissolves in the dilute acid, forming sulphate of zinc, and liberating hydrogen ; but this gas does not appear in bubbles on the surface of the copper cell, for, since the inner cell is porous, the molecular actions (by which the freed atoms of hydrogen are, as explained by Fig. 266, handed on through the acid) traverse the pores of the inner cell, and there, in the solution of sulphate of copper, the hydrogen atoms are exchanged for copper atoms, the result being that pure copper, and not hydrogen gas, is deposited on the outer copper plate. Chemically these actions may be represented as taking place in two stages.

$$
\mathrm{Zn}+\mathrm{H}_{2} \mathrm{SO}_{4}=\mathrm{ZnSO}_{4}+\mathrm{H}_{2}
$$

Zinc and Sulphuric Acid produce Sulphate of Zinc and Hydrogen. And then
$\mathrm{H}_{2}+\mathrm{CuSO}_{4}$
$=\mathrm{H}_{2} \mathrm{SO}_{4}+$
Cu.

Hydrogen and Sulphate of Copper produce Sulphuric Acid and Copper. 
The hydrogen is, as it were, translated electrochemically into copper during the round of changes, and so while the zinc dissolves away the copper grows, the dilute sulphuric acid gradually changing into sulphate of zinc, and the sulphate of copper into sulphuric acid. In the case in which a solution of sulphate of zine is used there is no need to consider any hydrogen atoms, copper being exchanged chemically for zinc. There is therefore no polarization so long as the copper solution is saturated ; and the cell is very constant, though not so constant in all cases as Clark's standard cell described in Art. 188, owing to slight variations in the electromotive-force as the composition of the other fluid varies. When sulphuric acid diluted with twelve parts of water is used the E.M.F. is 1.178 volts. The E.M.F. is 1.07 volts when concentrated zinc sulphate is used; 1.1 volts when a halfconcentrated solution of zinc sulphate is used; and, in the common cells made up with water or dilute acid, $1 \cdot 1$ volts or less. Owing to its constancy, this battery, made up in a convenient flat form (Fig. 106), has been much used in telegraphy. It is indispensable in those "closed circuit" methods of telegraphy (Art. 500), where the current is kept always flowing until interrupted by signalling.

182. Grove's Cell. - Sir William Grove devised a form of cell having both higher voltage and smaller internal resistance than Daniell's cell. In Grove's element there is an outer cell of glazed ware or of ebonite, containing the amalgamated zinc plate and dilute sulphuric acid. In the inner porous cell a piece of platinum foil serves as the negative pole, and it dips into the strongest nitric acid. There is no polarization in this cell, for the hydrogen liberated by the solution of the zinc in dilute sulphuric acid, in passing through the nitric acid in order to appear at the platinum pole, decomposes the nitric acid and is itself oxidized, producing water and the red fumes of nitric peroxide gas. This 
gas does not, however, produce polarization, for as it is very soluble in nitric acid, it does not form a film upon the face of the platinum plate, nor does it, like hydrogen, set up an opposing electromotive-force with the zinc. The Grove cells may be made of a flat shape, the zinc being bent up so as to embrace the flat porous cell on both sides. This reduces the internal resistance, which is already small on account of the good conducting powers of nitric acid. Hence the Grove's cell will furnish for three or four hours continuously a strong current. The E.M.F. of one cell is about 1.9 volts, and its internal resistance is very low (about $0.1 \mathrm{ohm}$ for the quart size). A single cell will readily raise to a bright red heat two or three inches of thin platinum wire, or drive a small electromagnetic engine. For producing larger power a number of cells must be joined up in series, the platinum of one cell being clamped to the zinc of the next to it. Fifty such cells, each holding about a quart of liquid, amply suffice to produce an electric arc light, as will be explained in Lesson XXXIX.

183. Bunsen's Cell.--The cell which bears Bunsen's name is a modification of that of Grove, and was indeed originally suggested by him. In the Bunsen cell the expensive * platinum foil is replaced by a rod or slab of hard gas carbon. A cylindrical form of cell, with a rod of carbon, is shown in Fig. 102. The voltage for a zinccarbon combination is a little higher than for a zincplatinum one, which is an advantage; but the Bunsen cell is troublesome to keep in order, and there is some difficulty in making a good contact between the rough surface of the carbon and the copper strap which connects

* Platinum costs about 30 shillings an ounce-nearly half as much as gold ; while a hundredweight of the gas carbon may be had for a mere trifle, often for nothing more than the cost of carrying it from the gasworks. An artificial carbon prepared by grinding up gas carbon with some carbonaceous matter such as tar, sugar residues, etc., then pressing into moulds, and baking in a furnace, is used both for battery plates and for the carbon rods used in arc lamps. 
the carbon of one cell to the zinc of the next. The top

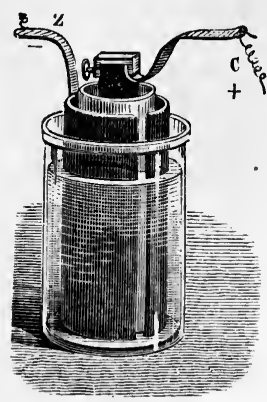

Fig. 102.

part of the carbon is sometimes impregnated with paraffin wax to keep the acid from creeping up, and electrotyped with copper. Fig. 103 shows the usual way of coupling up a series of five such cells. The Bunsen's battery will continue to furnish a current for a longer time than the flat Grove's cells, on account of the larger quantity of acid contained by the cylindrical pots.*

Chromic solutions, formed by adding strong sulphuric acid to solutions of bichromate of potash or of soda, are often used instead of nitric reid, in cells of this form.

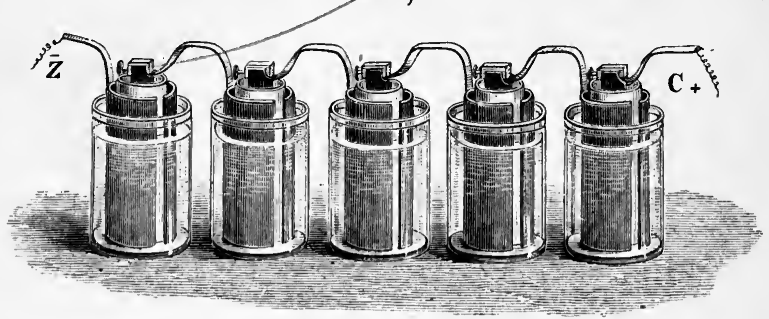

Fig. 103.

Soluble depolarizers in the form of chromic powders are made by heating strong sulphuric acid and gradually stirring into it powdered bichromate of soda. The pasty mass is then cooled and powdered.

* Callan constructed a large battery in which cast iron formed the positive pole, being inmersed in strong nitric acid, the zincs dipping into dilute acid. The iron under these circunnstances is not acted upon by the acid, but assumes a so-called "passive state." In this condition its surface appears to be inpregnated with a tilm of magnetic peroxide, or of oxygen. 
184. Leclanché's Cell.-For working electric bells and telephones, and also to a limited extent in telegraphy, a zinc-carbon cell is employed, invented by Leclanché, in which the exciting liquid is not dilute acid, but a solution of salammoniac. In this the zinc dissolves, forming a double chloride of zinc and ammonia, while ammonia gas and hydrogen are liberated at the carbon pole. The depolarizer is the black binoxide of manganese, fragments of which, mixed with powdered carbon, are held up to the carbon kathode either by packing them together inside a porous pot or by being attached as an agglomerated block. The oxide of manganese will slowly yield up oxygen as

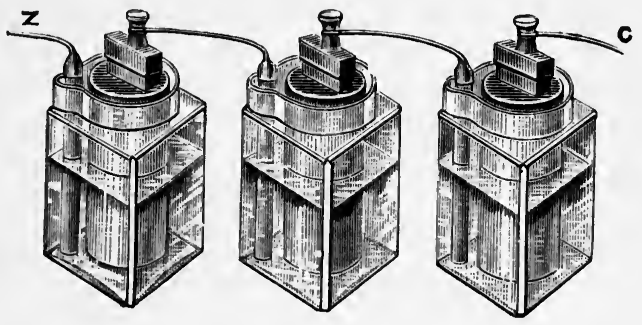

Fig. 104.

required. If used to give a continuous current for many minutes together, the power of this cell falls off owing to the accumulation of the hydrogen bubbles; but if left to itself for a time the cell recovers itself, the binoxide gradually destroying the polarization. As the cell is in other respects perfectly constant, and does not require renewing for months or years, it is well adapted for domestic purposes. It has the advantage of not containing corrosive acids. Millions of these cells are in use on "open-circuit" service-that is to say, for those cases in which the current is only required for a few moments at a time, and the circuit usually left open. Three 
Leclanché cells are shown joined in series, in Fig. 104. Walker used sulphur in place of oxide of manganese. Niaudet employed bleaching powder (so-called chloride of lime) as depolarizer, it being rich in chlorine and oxygen. Common salt may be used instead of salammoniac.

Modifications of the Leclanché cell in which the excitant cannot be spilled are used for portability. The space inside the cell is filled up with a spongy or gelatinous mass, or even with plaster of Paris, in the pores of which the salammoniac solution remains. They are known as dry cells.

185. Lalande's Cell.--This cell belongs to Class II., having as depolarizer oxide of copper mechanically attached to a kathode of copper or iron. The anode is zinc, and the exciting liquid is a 30 per cent solution of caustic potash into which the zinc dissolves (forming rincate of potash), whilst metallic copper is reduced in a granular state at the kathode. It has only 0.8 to 0.9 volts of E.M.F., but is capable of yielding a large and constant current.

186. De la Rue's Battery.-De la Rue constructed a constant cell belonging to Class III., in which zinc and silver are the two metals, the zinc being immersed in chloride of zinc, and the silver embedded in a stick of fused chloride of silver. As the zinc dissolves away, metallic silver is deposited upon the kathode, just as the copper is in the Daniell's cell. De la Rue constructed an enormous battery of over 11,000 little cells. The difference of potential between the first zinc and last silver of this battery was over 11,000 rolts, yet even so no spark would jump from the + to the - pole until they were brought to within less than a quarter of an inch of one another. With 8040 cells the length of spark was only 0.08 of an inch, or $0.2 \mathrm{~cm}$.

187. Gravity Cells.-Instead of employing a porous cell to keep the two liquids separate, it is possible, where one of the liquids is heavier than the other, to arrange 
that the heavier liquid shall form a stratum at the bottom of the cell, the lighter floating upon it. Such arrangements are called gravity cells; but the separation is never perfect, the leavy liquid slowly diffusing upwards. Daniell's cells arranged as gravity cells have been contrived by Meidinger, Minotto, Calland, and Lord Kelvin. In Siemens' modification paper-pulp is used to separate the two liquids. The "Sawdust Battery" of Kelvin is a Daniell's battery, having the cells filled with sawdust, to prevent spilling and make them portable.

188. Clark's Standard Cell.-A standard cell whose E.M.F. is even more constant than that of the Daniell was suggested by Latimer Clark. This cell, which is now adopted as the international standard cell, consists of an anode of pure zinc in a concentrated solution of zinc-sulphate, whilst the kathode is of pure mercury in contact with a paste of mercurous sulphate. Precise instructions for setting up Clark cells are given in Appendix B at the end of this book. Fig. 105 shows, in actual size, the

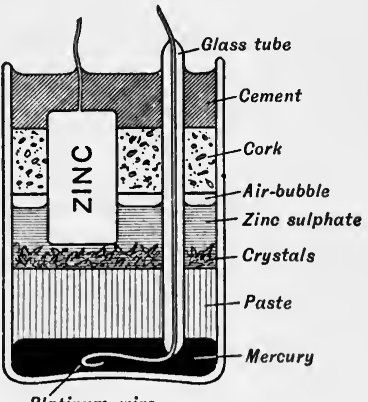

Platinum wire

Fig. 105. form of the Clark cell. Its E.M.F. is $1 \div 34$ volts at $15^{\circ} \mathrm{C}$.

Weston uses a cadmium anode immersed in sulphate of calmium and finds the cell so modified to give 1.025 volts at all ordinary temperatures.

Von Helmholtz has used mercurous chloride (calomel) and chloride of zinc, in place of sulphates, in a standard cell. Carhart finds its E.M.F. (a little over 1 volt) to vary with the dilution of the chloride of zinc. 
189. Statistics of Cells.-The following table gives the electromotive-forces of the various batteries enumerated :-

\begin{tabular}{|c|c|c|c|c|c|}
\hline Name. & Anode. & Excitant. & Depolarizer. & Kathode. & $\begin{array}{l}\text { Approxi- } \\
\text { mate } \\
\text { Volts. }\end{array}$ \\
\hline Class $I$. & & (Solution of) & & & \\
\hline $\begin{array}{l}\text { Volta (Wollaston, } \\
\text { etc.). }\end{array}$ & Zine & $\mathrm{H}_{2} \mathrm{SO}_{4}$ & none & Copper & 1.0 to 0.5 \\
\hline Smee . . & Zine & $\mathrm{H}_{2} \mathrm{SO}_{4}$ & none & $\begin{array}{l}\text { Platinized } \\
\text { Silver }\end{array}$ & 1.0 to 0.5 \\
\hline $\begin{array}{l}\text { Law } \\
\text { Class II. }\end{array}$ & Zine & $\mathrm{H}_{2} \mathrm{SO}_{4}$ & none & Carbon & 1.0 to 0.5 \\
\hline $\begin{array}{l}\text { Poggendorff (Gre- } \\
\text { net, Fuller, etc.). }\end{array}$ & Zine & $\mathrm{H}_{2} \mathrm{SO}_{4}$ & $\mathrm{~K}_{2} \mathrm{Cr}_{2} \mathrm{O}_{7}$ & Carbon & $2 \cdot 1$ \\
\hline Grove . & Zine & $\mathrm{H}_{2} \mathrm{SO}_{4}$ & $\mathrm{HNO}_{3}$ & Platinum & $1 \cdot 9$ \\
\hline$\underset{\text { Bunsen }}{\text { Bunché }}$ & $\underset{\text { Zine }}{\text { Zine }}$ & $\mathrm{NH}_{4} \mathrm{SO}_{4}$ & $\mathrm{HNO}_{3}$ & $\begin{array}{l}\text { Carbon } \\
\text { Carbon }\end{array}$ & $\begin{array}{l}1 \cdot 9 \\
1 \cdot 4\end{array}$ \\
\hline $\begin{array}{l}\text { Leclanche } \\
\text { Lalande. }\end{array}$ & $\begin{array}{l}\text { Zine } \\
\text { Zine }\end{array}$ & $\begin{array}{l}\mathrm{NH}_{4} \mathrm{Cl} \\
\mathrm{KHO}\end{array}$ & $\mathrm{CuO}^{\mathrm{MnO}}$ & $\begin{array}{l}\text { Carbon } \\
\text { Carbon }\end{array}$ & $\begin{array}{l}1.4 \\
0.8\end{array}$ \\
\hline Upward . & Zine & $\mathrm{ZnCl}_{2}$ & $\mathrm{Cl}$ & Carbon & $2 \cdot 0$ \\
\hline Fitch . . & Zinc & $\mathrm{NH}_{4} \mathrm{Cl}$ & $\begin{array}{c}\mathrm{KClO}_{3}+\mathrm{Na} \\
\mathrm{ClO}_{3}\end{array}$ & Carbon & $1 \cdot 1$ \\
\hline $\begin{array}{l}\text { Papst } \\
\text { Obach (dry) : }\end{array}$ & $\begin{array}{l}\text { Iron } \\
\text { Zinc }\end{array}$ & $\begin{array}{c}\mathrm{Fe}_{2} \mathrm{Cl}_{6} \\
\mathrm{NH}_{4} \mathrm{Cl} \\
\text { in } \mathrm{CaSO}_{4}\end{array}$ & $\begin{array}{c}\mathrm{Fe}_{2} \mathrm{Cl}_{6} \\
\mathrm{MnO}_{2}\end{array}$ & $\begin{array}{l}\text { Carbon } \\
\text { Carbon }\end{array}$ & $\begin{array}{l}0.4 \\
1 \cdot 46\end{array}$ \\
\hline \multicolumn{6}{|l|}{ Class III. } \\
\hline $\begin{array}{l}\text { Daniell (Meidinger, } \\
\text { Minotto, etc.). }\end{array}$ & Zinc & $\mathrm{ZnSO}_{4}$ & $\mathrm{CuSO}_{4}$ & Copper & 1.07 \\
\hline De la Rue & Zine & $\mathrm{ZnCl}_{2}$ & $\mathbf{A g C l}$ & Silver & $1 \cdot 42$ \\
\hline Clark (Standard) & Zine & $\mathrm{ZnSO}_{4}$ & $\mathrm{Hg}_{2} \mathrm{SO}_{4}$ & Mercury & $1 \cdot 434$ \\
\hline Weston - & Cadminm & $\mathrm{CdSO}_{4}$ & $\mathrm{Hg}_{2} \mathrm{SO}_{4}$ & Mercury & 1.025 \\
\hline $\begin{array}{l}\text { Vọ Helmholtz } \\
\text { Class IV. }\end{array}$ & Zine & $\mathrm{ZnCl}_{2}$ & $\mathrm{Hg}_{2} \mathrm{Cl}_{2}$ & Mercury & $1 \cdot 0$ \\
\hline $\begin{array}{c}\text { Accumulators. } \\
\text { (Planté, Faure, etc.) }\end{array}$ & Lead & $\mathrm{H}_{2} \mathrm{SO}_{2}$ & $\mathrm{PbO}_{2}$ & Lead & $2 \cdot 1$ to $1 \cdot 85$ \\
\hline
\end{tabular}

190. Strength of Current.-The student must not mistake the figures given in the above table for the strength of current which the various batteries will yield ; the current depends, as was said in Lesson XIII., on the internal resistance of the cells and on that of their 
circuit, as well as on their E.M.F. The E.M.F. of a cell is independent of its size, and is determined solely by the materials chosen and their condition. The resistance depends on the size of the cell, the conducting qualities of the liquid, the thickness of the liquid which the current must traverse, etc.

The definition of the strength of a current is as follows: The strength of a current is the quantity of electricity which flows past any point of the circuit in one second.* Suppose that at the end of 10 seconds 25 coulombs of electricity have passed through a circuit, then the average current during that time has been $2 \frac{1}{2}$ coulombs per second, or $2 \frac{1}{2}$ amperes. The usual strength of currents used in telegraphing over main lines is only from five to ten thousandths of an ampere.

If in $t$ seconds a quantity of electricity $\mathrm{Q}$ has flowed through the circuit, then the current $\mathrm{C}$ during that time is represented by the equation

$$
\mathrm{C}=\frac{\mathrm{Q}}{t} \text {. }
$$

This should be compared with Art. 162.

The laws which determine the strength or quantity of a current in a circuit were first enunciated by Dr. G. S. Ohm, who stated them in the following law :

191. Ohm's Law.- The current varies directly as the electromotive-force, and inversely as the resistance of the circuit; or, in other words, anything that makes the

* The terms "strength of current," " intensity of current," are oldfashioned, and mean no more than "current" means-that is to say, the number of amperes that are flowing. The terms "strong," "great," and "intense," as applied to currents, mean precisely the same thing. Formerly, before Ohm's Law was properly understood, electricians used to talk about "quantity currents" and "intensity currents," meaning by the former term a current flowing through a circuit in which there is very small resistance inside the battery or out; and by the latter expression they designated a current due to a high electromotive-force. The terms were convenient, but should be avoided as misleading. 
E.M.F. of the cell greater will increase the current, while anything that increases the resistance (either the internal resistance in the cells themselves or the resistance of the external wires of the circuit) will diminish the current.

In symbols this becomes

$$
\frac{\mathrm{E}}{\mathrm{R}}=\mathrm{C},
$$

where $\mathrm{E}$ is the number of volts, $\mathrm{R}$ the number of ohms of the circuit, and $\mathrm{C}$ the number of amperes of current.

Example.-To find the current that can be sent through a resistance of $5 \mathrm{ohms}$ by an E. M.F. of 20 rolts. $20 \div 5=4$ amperes.

(See further concerning Ohm's Law in Lesson XXXIII.) Ohm's Law says nothing about the energy or power conveyed by a current. The pover of a current is proportional both to the current and to the electromotive-force which drives it (see Art. 435).

192. Resistance and Grouping of Cells.-The internal resistances of the cells we have named differ very greatly, and differ with their size. Roughly speaking, we may say that the resistance in a Daniell's cell is about five times that in a Grove's cell of equal size. The Grove's cell has indeed both a higher E.M.F. and less internal resistance. It would in fact send a current about eight times as strong as the Daniell's cell of equal size through a short stout wire.

We may then increase the strength of a battery in two ways :-

(1) By increasing its E.M.F.

(2) By diminishing its internal resistance.

The electromotive-force of a cell being determined by the materials of which it is made, the only way to increase the total E.M.F. of a battery of given materials is to increase the number of cells joined "in series." It is 
frequent in the telegraph service to link thus together two or three hundred of the flat Daniell's cells ; and they are usually made up in trough-like boxes, containing a series of 10 cells, as shown in Fig. 106.

To diminish the internal resistance of a cell the following expedients may be resorted to :-

(1) The plates may be brought nearer together, so that the current shall not have to traverse so thick a stratum of liquid.

(2) The size of the plates may be increased, as this

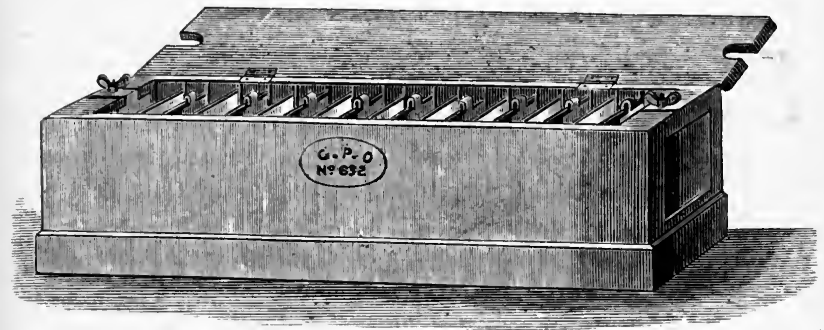

Fig. 106.

affords the current, as it were, a greater number of possible jaths through the stratum of liquid.

(3) The zincs of several cells may be joined together, to form, as it were, one large zinc plate, the coppers being also joined to form one large copper plate. Suppose four similar cells thus joined "in parallel," the current has four times the available number of paths by which it can traverse the liquid from zinc to copper; hence the internal resistance of the whole will be only $\frac{1}{4}$ of that of a single cell. But the E.M.F. of them will be no greater thus than that of one cell.

It is most important for the student to remember that the current is also affected by the resistances of the wires of the external circuit; and if the external resistance be 
already great, as in telegraphing through a long line, it is little use to diminish the internal resistance if this is already much smaller than the resistance of the line wire. It is, on the contrary, advantageous to increase the number of cells in series, though every cell adds a little to the total resistance.

Example. - If the line has a resistance of $1000 \mathrm{ohms}$, and five cells are used each of which has an E.M.F. of $1 \cdot 1$ volt and an internal resistance of 3 ohms, by Ohm's Law the current will be $5.5 \div 1015$; or 0.0054 ampere. If now eight cells are used, though the total resistance is thereby increased from 1015 to $1040 \mathrm{ohms}$, yet the E.M.F. is increased from $5 \cdot 5$ to $8 \cdot 8$ volts, and the current to 0.0085 ampere.

The E.M.F. of the single-fluid cells of Volta and Smee is marked in the table as doubtful, for the opposing E.M.F. of polarization sets in almost before the true E.M.F. of the cell can be measured. The different values assigned to other cells are accounted for by the different degrees of concentration of the liquids. Thus in the Daniell's cells used in telegraphy, water only is supplied at first in the cells containing the zincs; and the E.M.F. of these is less than if acid or sulphate of zinc were added to the water.

193. Other Batteries.-Numerous other forms of battery have been suggested by different electricians. There are three, of theoretical interest only, in which, instead of using two metals in one liquid which attacks them unequally, two liquids are used having unequal chemical action on the metal. In these there is no contact of dissimilar metals. The first of these was invented by the Emperor Napoleon III. Both plates were of copper dipping respectively into solutions of dilute sulphuric acid and of cyanide of potassium, separated by a porous cell The second of these combinations, due to Wöhler, employs plates of aluminium only, dipping respectively into strong nitric acid and a solution of caustic soda. In the third, 
invented by Dr. Fleming, the two liquids do not even touch one another, being joined together by a second metal. In this case the liquids chosen are sodium persulphide and nitric acid, and the two metals copper and lead. A similar battery might be made with copper and zinc, using solutions of ordinary sodium sulphide, and dilute sulphuric acid in alternate cells, a bent zinc plate dipping into the first and second cells, a bent copper plate dipping into second and third, and so on ; for the electronotive-force of a copper-sodium-sulphide-zinc combination is in the reverse direction to that of a copper-sulphuricacid-zinc combination.

Upward proposed a chlorine battery, having slabs of zinc immersed in chloride of zinc and kathodes of carbon surrounded by crushed carbon in a porous pot, gaseous chlorine being pumped into the cells, and dissolving into the liquids to act as a depolarizer. It has an E.M.F. of 2 volts.

Bennett described a cheap and most efficient battery, in which old meat-canisters packed with iron filings answer for the positive element, and serve to contain the exciting liquid, a strong solution of caustic soda. Serap zinc thrown into mercury in a shallow inner cup of porcelain forms the anode.

Marié Davy employed a cell in which the zine dipped into sulphate of zinc, while a carbon plate dipped into a pasty solution of mercurous sulphate. When the cell is in action mercury is deposited on the surface of the carbon, so that the cell is virtually a zinc-mercury cell. It was largely used for telegraphy in France before the introduction of the Leclanché cell.

Obach's dry cell has an outer cylinder of zinc which serves as a case, lined with plaster of Paris soaked in salammoniac; with a central carbon kathode surrounded with binoxide of manganese mixed with graphite.

The Fitch cell, used in the United States, is a zinccarbon cell with an excitant composed of salammoniac 
solution to which the chlorates of potash and soda have been added.

Papst used an iron-carbon cell with ferric chloride solution as excitant. The iron dissolves and chlorine is at first evolved, but without polarization; the liquid regenerating itself by absorbing moisture from the air. It is very constant but of low E.M.F.

Jablochkoff described a battery in which plates of carbon and iron are placed in fused nitre; the carbon is here the electropositive element, being rapidly consumed in the liquid.

Planté's and Faure's Secondary Batteries, and Grove's Gas Battery, are described in Arts. 492, 493.

The so-called Dry Pile of Zamboni deserves notice. It consists of a number of paper disks, coated with zincfoil on one side and with binoxide of manganese on the other, piled upon one another, to the number of some thousands, in a glass tube. Its internal resistance is enormous, as the internal conductor is the moisture of the paper, and this is slight; but its electromotive-force is very great, and a good dry pile will yield sparks. Many years may elapse before the zinc is completely oxidized or the manganese exhausted. In the Clarendon Laboratory at Oxford there is a dry pile, the poles of which are two metal bells : between them is hung a small brass ball, which, by oscillating to and fro, slowly discharges the electrification. It has now been continuously ringing the bells for more than filty years.

194. Effect of Heat on Cells. - If a cell be warmed it yields a stronger current than when cold. This is chiefly due to the fact that the liquids conduct better when warm, the internal resistance being thereby reduced. A slight change is also observed in the E.M.F. on heating; thus the E.M.F. of a Daniell's cell is about $1 \frac{1}{2}$ per cent higher when warmed to the temperature of boiling water, while that of a bichroniate battery falls off nearly 2 per cent under similar circumstances. In the 
Clark standard cell the E.M.F. decreases slightly with temperature, the coefficient being 0.00077 per degree centigrade. Its E.M.F. at any temperature $\theta$ may be calculated by the formula,

$$
\text { E.M.F. }=1.434[1-0.00077(\theta-15)] \text { volt. }
$$

\section{Lesson XVI.-Magnetic Actions of the Current}

195. Oersted's Discovery. - A connexion of some kind between magnetism and electricity had long been suspected. Lightning had been known to magnetize knives and other objects of steel ; but almost all attempts to imitate these effects by powerful charges of electricity, or by sending currents of electricity through steel bars, had failed.* About 1802 Romagnosi, of Trente, vaguely observed that a voltaic pile affects a compass-needle. The true connexion between magnetism and electricity remained, however, to be discovered.

In 1819, Oersted, of Copenhagen, showed that a magnet tends to set itself at right angles to a wire carrying an electric current. He also found that the way in which the needle turns, whether to the right or the left of its usual position, depends upon the position of the wire that carries the current-whether it is above or below the needle,- and on the direction in which the current flows through the wire.

196. Oersted's Experiment. - Very simple apparatus suffices to repeat the fundamental experiment. Let a magnetic needle be suspended on a pointed pivot, as in Fig. 107. Above it, and parallel to it, is held a stout

* Down to this point in these lessons there has been no connexion between magnetism and electricity, though something has been said about each. The student who cannot remember whether a charge of electricity does or does not affect a magnet, should turn back to what was said in Art. 99. 
copper wire, one end of which is joined to one pole of a battery of one or two cells. The other end of the wire is then brought into contact with the other pole of the battery. As soon as the circuit is completed the current flows through the wire and the needle turns briskly aside. If the current be flowing along the wire above the needle in the direction from north to south, it will cause the

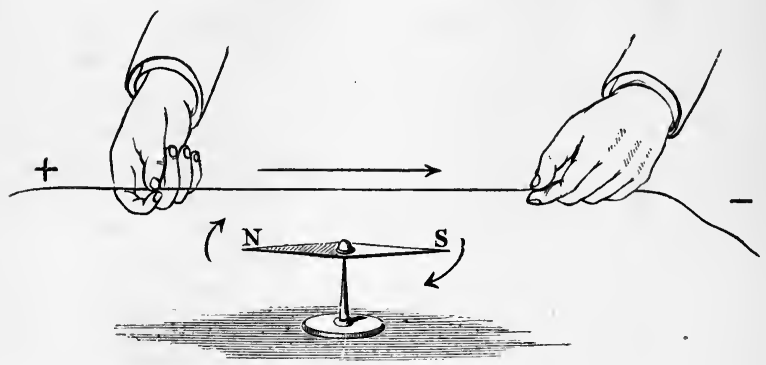

Fig. 107 .

$\mathrm{N}$-seeking end of the needle to turn eastwards; if the current flows from south to north in the wire the $\mathrm{N}$-seeking end of the needle will be deflected westwards. If the wire is, however, below the needle, the motions will be reversed, and a current flowing from north to south will cause the $\mathrm{N}$-seeking pole to turn westwards.

197. Ampère's Rule.-To keep these movements in memory, Ampère suggested the following fanciful but useful rule. Suppose a man swimming in the wire with the current, and that he turns so as to face the needle, then the $N$-seeking pole of the needle will be deflected towards his left hand. In other words, the deflexion of the N-seeking pole of a magnetic needle, as viewed from the conductor, is towards the left of the eurrent.

For certain particular cases in which a ficed magnet pole acts on a movable circuit, the following converse to 
Ampère's Rule will be found convenient. Suppose a man swimming in the wire with the current, and that he turns so as to look along the direction of the lines of force of the pole (i.e. as the lines of force run, from the pole if it be. $\mathrm{N}$-seeking, towards the pole if it be S-seeking), then he and the conducting wire with him will be urged toward his left.

198. Corkscrew Rule.-More convenient is the following rule suggested by Maxwell. The direction of the current and that of the resulting magnetic force are related to one another, as are the rotation and the forward travel of an ordinary (righthanded) corkscrew. In Fig. 108, if the circle represents the circulation of current, the arrow gives the direction of the resulting magnetic force. One advantage of this rule is, that it is equally applicable

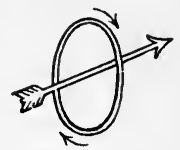

Fig. 108. in the other case. If the arrow represents the direction of the current along a straight wire, the circle will represent the direction of the resulting magnetic force around it.

199. Galvanoscope. - A little consideration will show that if a current be carried below a needle in one

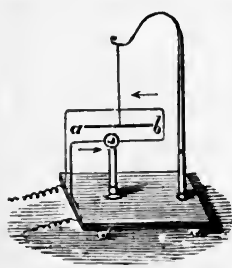

Fig. 109. direction, and then back in the opposite direction above the needle, by bending the wire round, as in Fig. 109, the forces exerted on the needle by both portions of the current will be in the same direction. For let $a$ be the $\mathrm{N}$-seeking, and $b$ the S-seeking, pole of the suspended needle, then the tendency of the current in the lower part of the wire will be to turn the needle so that $a$ comes towards the observer, while $b$ retreats; while the current flowing above, which also deflects the $\mathrm{N}$-seeking pole to its left, will equally urge $a$ towards the observer, and $b$ from him. The needle 
will not stand out completely at right angles to the direction of the wire conductor, but will take an oblique position. The directive forces of the earth's magnetism are tending to make the needle point north-and-south. The electric current is acting on the needle, tending to make it set itself west-and-east. The resultant force will be in an oblique direction between these, and will depend upon the relative strength of the two conflicting forces. If the current is very strong the needle will turn widely round ; but could only turn completely to a right angle if the current were infinitely strong. If, however, the current is feeble in comparison with the directive magnetic force, the needle will turn very little.

This arrangement will, therefore, serve roughly as a Galvanoscope or indicator of currents ; for the movement of the needle shows the direction of the current, and indicates whether it is a strong or a weak one. This apparatus is too rough to detect very delicate currents. To obtain a more sensitive instrument there are two possible courses: (i.) increase the effective action of the current by carrying the wire more than once round the needle; (ii.) decrease the opposing directive force of the earth's magnetism by some compensating contrivance.

200. Schweigger's Multiplier.-The first of the above suggestions was carried out by Schweigger, who constructed a multiplier of many turns of wire. A suitable frame of wood, brass, or ebonite, is prepared to receive the wire, which must be "insulated," or covered with silk, or cotton, or guttapercha, to prevent the separate turns of the coil from coming into contact with each other. Within this frame, which may be circular, elliptical, or more usually rectangular, as in Fig. 110, the needle is suspended, the frame being placed so that the wires lie in the magnetic meridian. The greater the number of turns the more powerful will be the magnetic 
deflexion produced by the passage of equal quantities of current. But if the wire is thin, or the number of turns of wire numerous, the resistance thereby offered to the flow of electricity may very greatly reduce the strength of the current. The student will grasp the importance of this observation when he has read the chapter on Ohm's Law. Cumming, of Cambridge, appears to have been the first to use a coil surrounding a pivoted needle to

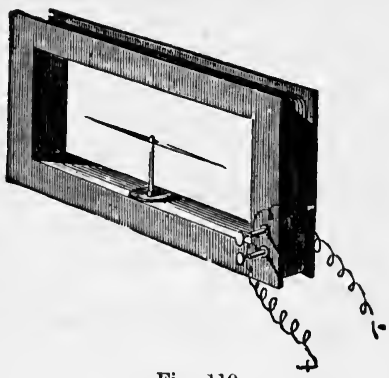

Fig. 110.

measure the current. To him we owe the term Galvanometer.

201. Astatic Combinations. - The directive force exercised by the earth's magnetism on a magnetic needle may be reduced or obviated by one of two methods :-

(a) [Haïy's Method]: By employing a compensating magnet. An ordinary long bar magnet laid in the magnetic meridian, but with its $\mathrm{N}$-seeking pole directed towards the north, will, if placed horizontally above or below a suspended magnetic needle, tend to make the needle set itself with its S-seeking pole northwards. If near the needle it may overpower the directive force of the earth, and cause the needle to reverse its usual position. If it is far away, all it can do is to lessen the directive force of the earth. At a certain distance the magnet will just compensate this force, and the needle will be neutral. This arrangement for reducing the carth's directive force is applied in the reflecting galvanometer shown in Fig. 122, in which the magnet at the top, curved in form and capable of adjustment to any height, affords a means of adjusting the instrument to the desired degree of sensitiveness by raising or lowering it. 
(b) [Nobili's Method]. By using an astatic pair of magnetic needles. If two magnetized needles of equal strength and size are bound together by a light wire of

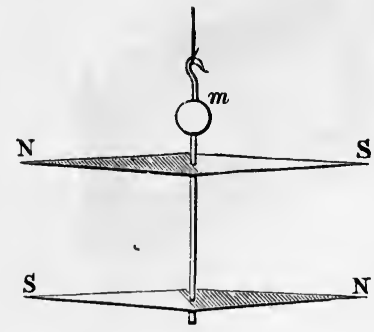

Fig. 111. brass, or aluminium, in reversed positions, as shown in Fig. 111, the force urging one to set itself in the magnetic meridian is exactly counterbalanced by the force that acts on the other. Consequently this pair of needles will remain in any position in which it is set, and is independent of the earth's magnetism. Such a combination is known as an astatic pair. It is, however, difficult in practice to obtain a perfectly astatic pair, since it is not easy to magnetize two needles exactly to equal strength, nor is it easy to fix them perfectly parallel to one another. Such an astatic pair is, however, readily deflected by a current flowing in a wire coiled around one of the needles ; for, as shown in Fig. 112, the current which flows above one needle and below the other will urge both in the same direction, because they are already in reversed positions. It is even possible to go further, and to carry the

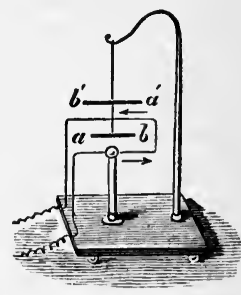

Fig. 112. wire round both needles, winding the coil around the upper in the opposite sense to that in which the coil is wound round the lower needle. Several other astatic combinations are possible. For example, two needles may be set vertically, with similar poles upward, at the ends of a pivoted horizontal strip of wood or brass.

Nobili applied the astatic arrangement of needles to the multiplying coils of Schweigger, and thus constructed 
a very sensitive instrument, the Astatic Galvanometer, shown in Fig. 119. The special forms of galvanometer adapted for the measurement of currents are described in the next lesson.

202. Magnetic Field due to Current: Magnetic Whirls.-Arago found that if a current be passed through a piece of copper wire it becomes capable of attracting iron filings to it so long as the current flows. These filings set theniselves at right angles to the wire, and cling around it, but drop off when the circuit is broken. There is, then, a magnetic "field," around the wire which carries the current; and it is important to know how the lines of force are distributed in this field.

Let the central spot in Fig. 113 represent an im-

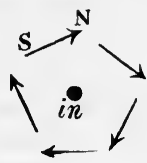

Fig. 113.

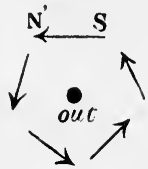

Fig. 114 . aginary cross-section of the wire, and let us suppose the current to be flowing in through the paper at that point. Then by Ampère's rule a magnet needle placed below will tend to set itself in the position shown, with its $\mathrm{N}$ pole pointing to the left.* The current will urge a needle above the wire into the reverse position. A needle on the right of the current will set itself at right angles to the current (i.e. in the plane of the paper), and with its $\mathrm{N}$ pole pointing down, while the $\mathrm{N}$ pole of a needle on the left would be urged $u p$. In fact the tendency would be to urge the $\mathrm{N}$ pole round the conductor in the same

* If the student has any difficulty in applying Ampere's rule to this case and the others which succeed, he should carefully follow out the following mental operation. Consider the spot marked " in" as a hole in the ground into which the current is flowing, and into which he dives head-foremost. While in the hole he must turn round so as to face each of the magnets in succession, and remember that in each case the $\mathrm{N}$ seeking pole will be urged to his left. In diagram 114 he must conceive himself as coming up out of the hole in the ground where the current is flowing out. 
way as the hands of a watch move; while the $\mathrm{S}$ pole would be urged in the opposite cyclic direction to that of the hands of a watch. If the current is reversed, and is regarded as flowing towards the reader, i.e. coming up out of the plane of the paper, as in the diagram of Fig. 114 , then the motions would be just in the reverse sense. It would seem from this as if a $\mathrm{N}$-seeking pole of a magnet ought to revolve contimuously round and round a current; but as we cannot obtain a magnet with one pole only, and as the S-seeking pole is urged in an opposite direction, all that occurs is that the needle sets itself

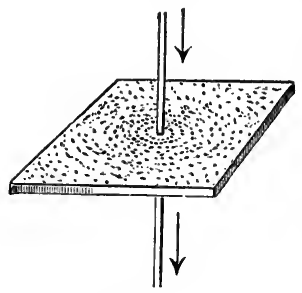

Fig. 115.

as a tangent to a circular curve surrounding the conductor. The field surrounding the conductor consists in fact of a sort of enveloping magnetic whirl all along it, the whirl being strong near the wire and weaker farther away. This is what Oersted meant when he described the electric current as acting " in a revolving manner" upon the magnetic needle. The field of force, with its circular lines surrounding a current flowing in a straight conductor, can be examined experimentally with iron filings in the following way : A card is placed horizontally and a stout copper wire is passed vertically through a hole in it (Fig. 115). Iron filings are sifted over the card (as described in Art. 119), and a strong current from three or four large cells is passed through the wire. On tapping the card gently the filings near the wire set themselves in concentric circles round it. It is because of this surrounding field that two conductors can apparently act on one another at a distance. If both currents are flowing in the same direction, their magnetic fields tend to merge, and the resulting stress in the medium tends to drag them together with an apparent attraction. If the currents are flowing in opposite 
directions the stresses in the intervening magnetic field tend to thrust them apart (see also Art. 389).

It is known that energy has to be spent in producing any magnetic field. When a current is turned on in a wire the magnetic field grows around the wire, some of the energy of the battery being used during the growth of the current for that purpose. One reason why electric currents do not instantly rise to their final value is because of the reactive effect of this surrounding magnetic field. No current can exist without this surrounding magnetic field. Indeed it is impossible to refute the proposition that what we commonly call an electric current in a wire really is this external magnetic whirl.

203. Equivalent Magnetic Shell : Ampère's Theorem.-For many purposes the following way of regarding the magnetic action of electric currents is more convenient than the preceding. Suppose we take a battery and connect its terminals by a circuit of wire,

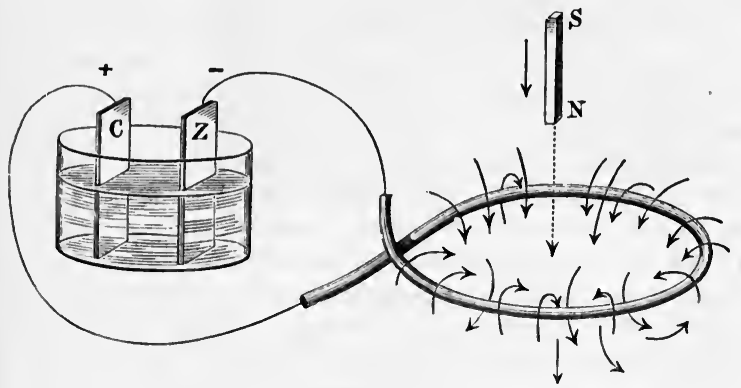

Fig. 116.

and that a portion of the circuit be twisted, as in Fig. 116, into a looped curve, it will be found that the entire space enclosed by the loop possesses magnetic properties. In our figure the current is supposed to be flowing round 
the loop, as viewed from above, in the same direction as the hands of a clock move round; an imaginary man swimming round the circuit and always facing towards the centre would have his left side down. By Ampère's rule, then, a $\mathrm{N}$ pole would be urged downwards through the loop, while a $\mathbf{S}$ pole would be urged upwards. In fact the space enclosed by the loop of the circuit behaves like a magnetic shell (see Art. 118), having its upper face of S-seeking magnetism, and its lower face of N-seeking magnetism. It can be shown in every case that a closed voltaic circuit is equivalent to a magnetic shell whose edges coincide in position with the circuit, the shell being of such a strength that the number of its lines of force is the same as that of the lines of force due to the current in the circuit. The circuit acts on a magnet attracting or repelling it, and being attracted or repelled by•it, just exactly as its equivalent magnetic shell would do. Also, the circuit itself, when placed in a magnetic field, experiences the same force as its equivalent magnetic shell would 'do.

204. Maxwell's Rule.-Professor Clerk Maxwell, who developed this method of treating the subject, has given the following elegant rule for determining the mutual action of a circuit and a magnet placed near it. Every portion of the circuit is acted upon by a force urging it in such a direction as to make it enclose within its embrace the greatest possible number of lines of force. If the circuit is fixed and the magnet movable, then the force acting on the magnet will also be such as to tend to make the number of lines of force that pass through the circuit a maximum (see also Art. 349).

This is but one case of the still more general law governing every part of every electromagnetic systen, viz. : Every electromagnetic system tends so to change the configuration of its parts as to make the flux of magnetic lines through the exciting circuit a maximum (Art. 379).

205. De la Rive's Floating Battery.-The pre. 
ceding remarks may be illustrated experimentally by the aid of a little floating battery. A plate of zinc and one of copper (see Fig. 117) are fixed side by side in a large cork, and connected above by a coil of several windings of covered copper wire. This is floated upon a dish containing dilute sulphuric acid. If one pole of a bar magnet be held towards the ring it will be attracted or repelled according to the pole employed. The floating circuit will so move as to make the flux of magnetic lines through the

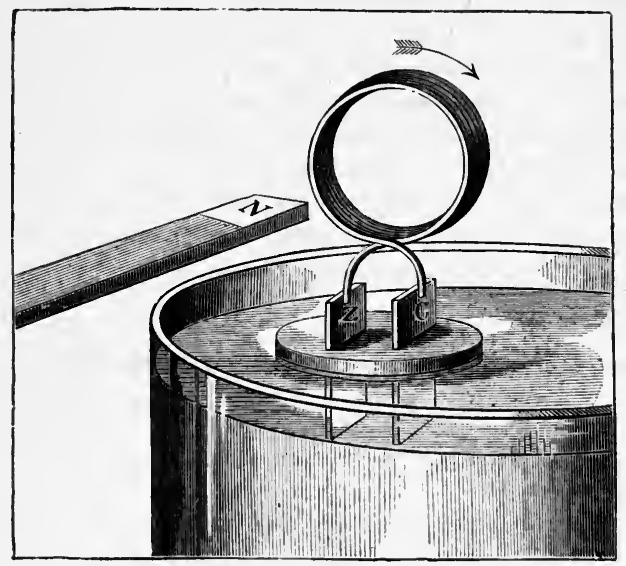

Fig. 117.

coil a maximum. If the $\mathrm{S}$ pole of the magnet be presented to that face of the ring which acts as a S-seeking pole (viz. that face round which the current is flowing in a clockwise direction), it will repel it. If the pole be thrust right into the ring, and then held still, the battery will be strongly repelled, will draw itself off, float away, turn round so as to present toward the $\mathrm{S}$ pole of the magnet its $\mathrm{N}$-seeking face, will then be attracted up, and will thread itself on to the magnet up to the middle, in 
which position as many magnetic lines of force as possible cross the area of the ring.

It can be shown also that two circuits traversed by currents attract and repel one another just as two magnetic shells would do.

It will be explained in Lesson XXXI. on Electromagnets how a piece of iron or steel can be magnetized by causing a current to flow in a spiral wire round it.

206. Strength of the Current in Magnetic Measure.-When a current thus acts on a magnet pole near it, the force $f$ which it exerts will be proportional to the strength $\mathrm{C}$ of the current, and proportional also to the strength $m$ of the magnet pole, and to the length $l$ of the wire employed : the force exerted between each element of the circuit and the pole will also vary inversely as the square of the distance $r$ between them. If the wire is looped into a circular coil with the magnet pole at the centre, so that each portion of the circuit is approximately at the same distance from the pole, $f=\frac{\mathrm{C} l m}{r^{2}}$ dynes. Suppose the wire looped up into a circle round the magnet pole, then $l=2 \pi r$, and $f=\frac{2 \pi \mathrm{C}}{r} n$ dynes. Suppose also that the circle is of one centimetre radius, and that the magnet pole is of strength of one unit (see Art. 352), then the force exerted by the current of strength $\mathrm{C}$ will be $\frac{2 \pi \mathrm{C}}{1} \times 1$, or $2 \pi \mathrm{C}$ dynes. In order, therefore, that a current of strength $\mathrm{C}$ should exert a force of $\mathrm{C}$ dynes on the unit pole, one must consider the current as travelling round only $\frac{1}{2 \pi}$ part of the circle, or round a portion of the circumference equal in length to the radius.

207. Unit of Current. - A current is said to have a strength of one "absolute" unit when it is such that if one centimetre length of the circuit is bent into an arc of one centimetre radius, the current in it exerts a force of one dyne on a magnet-pole of unit strength placed at the 
centre of the arc. The practical unit of "one ampere" is only $\frac{1}{10}$ of this theoretical unit (see also Art. 354).

If the wire, instead of being looped into a coil, is straight and of indefinite length, the force which the current in it exerts upon a pole of strength $m$ placed at point $\mathrm{P}$ near it will be found to vary inversely as the simple distance (not as the square), and the pole will tend to move at right angles both to the wire and to the line OP. In Fig. 118 the descending current will (according to

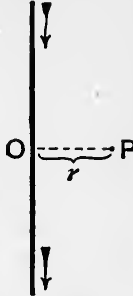

Fig. 118. the corkscrew rule above) tend to drive a $\mathrm{N}$ pole at $\mathrm{P}$ towards the spectator. If the current is $\mathrm{C}$ amperes the force (in dynes) on the pole of $m$ units will (see Art. 343) be

$$
f=2 m \mathrm{C} / 10 r .
$$

EXAMPLE.-The force exerted by a current of 60 amperes in a long straight conductor upon a pole of 200 units placed 2 centimetres away from it will be 1200 dynes, or (dividing by $g=981$ ) about $1 \cdot 22$ grammes' weight.

\section{LESsON XVII.-Galvanometers}

208. The term Galvanometer is applied to an instrument for measuring the strength of electric currents by means of their electromagnetic action. There are two general classes of Galvanometers: (1) those in which the current flowing in a fixed coil of wire causes the deflexion of a pivoted or suspended magnetic needle ; (2) those in which the current flowing in a movable coil suspended between the poles of a fixed magnet causes the coil to turn. There is a third kind of instrument (called for distinction electrodynamometer, see Art. 394), in which both the moving part and the fixed part are coils. These last are used chiefly for alternating-currents. 
The simple arrangement described in Art. 199 was termed a "Galvanoscope," or current indicator, but it could not rightly be termed a "galvanometer" * or current measurer, because its indications were only qualitative, not quantitative. The indications of the needle did not afford accurate knowledge as to the exact strength of current flowing through the instrument. A good galvanometer must fulfil the essential condition that its readings shall really measure the strength of the current in some certain way. It should also be sufficiently sensitive for the currents that are to be measured to affect it. The galvanometer adapted for measuring very small currents (say a current of only one or two millionth parts of an ampere) will not be suitable for measuring very strong currents, such as are used in electric lighting or electroplating. Large currents need thick wires ; and a coil of few turns will suffice. If very small currents are to turn the needle they must circulate hundreds or thousands of times around it, and therefore a coil of many turns is appropriate, and the wire may be a very fine one. Moreover, if the current to be measured has already passed through a circuit of great resistance (as, for example, some miles of telegraph wire), a galvanometer whose coil is a short one, consisting only of a few turns of wire, will be of no use, and a long-coil galvanometer must be employed with many hundreds or even thousands of turns of insulated wire round the needle. The reason of this is explained hereafter (Art. 408). Hence it will be seen that different styles of instrument are needed for different kinds of works; but of all it is required that they should afford quantitative measurements, that they should be sufficiently sensitive for the current that is to be measured, and carry that current without overheating.

* The terms Rheoscope and Rheometer are still occasionally applied to these instruments. A current interrupter is sometimes called a Rheotome, and the Commutator or Current Reverser, shown in Fig. 136, is in some books called a Rheotrope; but these terms are dropping out of use. 
209. Methods of Control.-In all instruments, whether the moving part be a magnet or a coil, some controlling force is needful, otherwise the very smallest current would turn the index completely about. If small currents are to produce a small deflexion, and larger currents a larger, there must be forces tending to control. Several means of control may be used. These are :-

(a) Earth's Magnetic Force.-When the needle is hung on pivot or fibre, the earth's magnetic force tries to bring it back into the magnetic meridian. This is the commonest method in galvanometers with moving needles.

(b) Torsion of Wire.-Moving part in turning twists the suspending wire, which then tries to untwist, with a force which increases as the angle of deflexion. This method is commonest in galvanometers with suspended coils.

(c) Gravity. - If needle is pivoted on trunnions to move in vertical plane, it may be weighted at one end.

(d) Permanent Magnet Control.-To render a needle instrument independent of position, it may be arranged with a powerful external steel magnet to bring the needle back to zero.

(e) Bifilar Suspension.-A needle or coil hung by two parallel threads tends by gravity to return to its initial position.

To make an instrument very sensitive the control must be weakened as much as possible.

210. Methods of Observation.-There are the following methods of using galvanometers in making observations :-

(i.) Deflexion Method.-The angle through which the moving part (whether needle or coil) is deflected is read off on a scale, by pointer or reflected beam of light, when the moving part has come to rest. This is the commonest method.

(ii.) Torsion Method.- The moving part is suspended by a wire from a torsion head, which is turned round until the index is brought back to zero; the 
controlling force then balancing the deflecting force. This very accurate method, due to $\mathrm{Ohm}$, is used in Siemens' electrodynamometer (Art. 394).

(iii.) First Swing Method. - Instead of waiting for moving part to come to rest the first swing may be observed. This method, which is the only one practicable for sudden discharges, or for transient currents, is called the ballistic method (see Art. 218). If the moving part is not damped in its motion the first swing on turning on a battery current is exactly twice the angle at which the deflexion settles down.

(iv.) Oscillation Method.-Instead of observing deflexion, the time of oscillation of the needle may be observed, the coil being in this method set at right angles to the magnetic meridian. Allowance must be made, as in Art. 133, for the earth's magnetism.

(v.) Cumulative Method.-For very minute currents a method is sometimes adopted to get up a measurable swing by reversing the current (by hand) as the needle swings through zero. Sometimes a rotating commutator of special construction is employed to produce, and accumulate, the successive impulses.

(vi.) Null Methods.-In many cases combinations are used (Wheatstone's “ Bridge," “Differential Galvanometers," etc.) of such a kind that when the conditions of electrical equilibrium are attained no current will flow through the galvanometer in the circuit. Such methods, which are generally exceedingly accurate, are known as null methods. For such methods sensitive galvanometers are applicable, but the graduation of their scale is unimportant.

211. Nobili's Astatic Galvanometer. - The 
instrument constructed by Nobili, consisting of an astatic pair of needles delicately hung, so that the lower one lay within a coil of wire wound upon an ivory frame (Fig. 119), was for long the favourite form of sensitive galvanometer. The needles of this instrument, being independent of the earth's magnetism, take their position in obedience to the torsion of the fibre by which they are hung. The frame on which the coil is wound must be set carefully parallel to the needles; and three screw feet serve to adjust the base of the instrument level. Protection against currents of air is afforded by a glass shade. When a current is sent through the wire coils the needles move to right or left over a graduated circle. When the deflexions are small (i.e. less than $10^{\circ}$ or $\left.15^{\circ}\right)$ they are very nearly proportional to the strength of the currents that produce

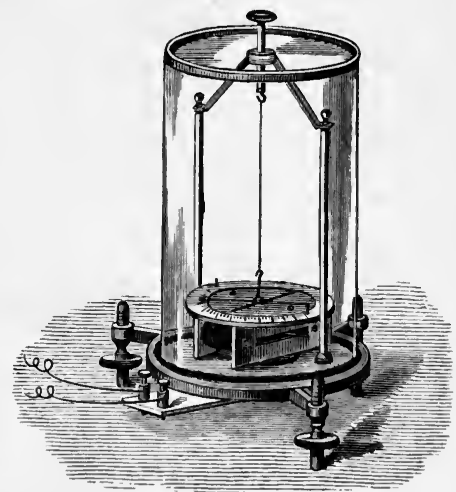

Fig. 119. them. Thus, if a current produces a deflexion of $6^{\circ}$ it is known to be approximately three times as strong as a current which only turns the needle through $2^{\circ}$. But this approximate proportion ceases to be true if the deflexion is more than $15^{\circ}$ or $20^{\circ}$; for then the needle is not acted upon so advantageously by the current, since the poles are no longer within the coils, but are protruding at the side, and, moreover, the needle being oblique to the force acting on it, part only of the force is turning it against the directive force of the fibre; the other part of the force is uselessly pulling or pushing 
the needle along its length. It is, however, possible to calibrate the galvanometer - that is, to ascertain by special measurements, or by comparison with a standard instrument, to what strengths of current particular amounts of deflexion correspond. Thus, suppose it once known that a deflexion of $32^{\circ}$ on a particular galvanometer is produced by a current of $\frac{1}{100}$ of an ampere, then a current of that strength will always produce on that instrument the same deflexion, unless from any accident the controlling force has been altered.

212. The Tangent Galvanometer.-It is notfor the reasons mentioned above-possible to construct a

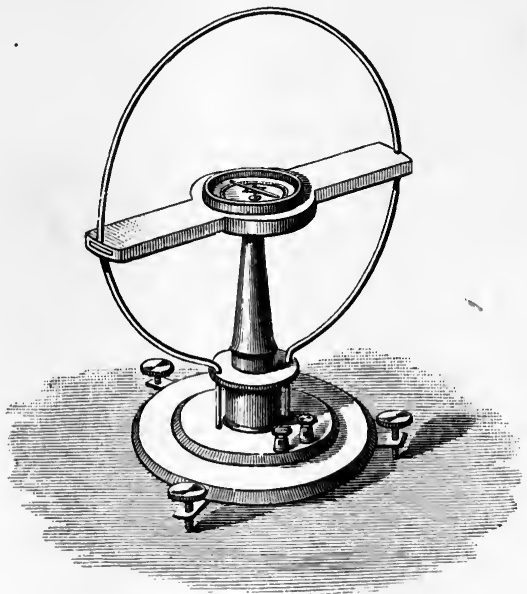

Fig. 120.

galvanometer in which the angle (as measured in degrees of arc) through which the needle is deflected is proportional throughout its whole range to the strength of the current. But it is possible to construct a very simple galvanometer 
in which the tangent ${ }^{*}$ of the angle of deflexion shall be accurately proportional to the strength of the current. The essential feature of all tangent galvanometers is that while the coil is a large open ring the needle is relatively very small. Fig. 120 shows a form of Tangent Galvanometer suitable for large currents. The coil of this instrument consists of a simple circle of stout copper wire from 10 to 15 inches in diameter. Other tangent galvanometers have many turns of fine wire wound upon a large open ring. At the centre is delicately suspended a magnetized steel needle not exceeding 1 inch in length, and usually furnished with a light index of aluminium. The instrument is adjusted by setting the coil in the magnetic meridian, the small needle lying then in the plane of the coil.

The "field" due to a current passing round the circle is very uniform at and near the centre, and the lines of force are there truly normal to the plane of the coil. This is not true of other parts of the space inside the ring, the force being neither uniform nor normal in direction, except centrally in the plane of the coil and along the axis. The needle being small, its poles are never far from the centre, and hence never protrude into the regions where the field is irregular. $\dagger$ Whatever magnetic force the current in the coil can exert on the needle is exerted normally to the plane of the ring, and therefore at right angles to the magnetic meridian. As the two forces-that due to the current and that due to the controlling magnetism of the earth-act squarely to

* See note on Ways of Reckoning Angles, p. 133.

t In order to ensure uniformity of field, Gaugain proposed to hang the needle at a point on the axis of the coil distant from its centre by a distance equal to half the radius of the coils. Helmholtz's arrangement of two parallel coils, symmetrically set on either side of the needle, is better ; and a three-coil galvanometer, having the central coil larger than the others, so that all three may lie in the surface of a sphere having the small needle at its centre, is the best arrangement of all for ensuring that the field at the centre is uniform. 
one another, the action of the current will not be measured by equal degrees marked out around a circle, but will be measured by equal divisions along a tangent line, as shown below. Now, it was proved in Art. 137 that the magnetic force which, acting at right angles to the meridian, produces on a magnetic needle the deflexion $\delta$ is equal to the horizontal force of the earth's magnetism at that place multiplied by the tangent of the angle of deflexion. Hence a current flowing in the coil will turn the needle aside through an angle such that the tangent of the angle of deflexion is proportional to the strength of the current.

Example. - Suppose a certain battery gave a deflexion of $15^{\circ}$ on a tangent galvanometer, and another battery yielding a stronger current gave a deflexion of $30^{\circ}$. The strengths currents are not in the proportion of $15: 30$, but in the proportion of $\tan 15^{\circ}$ to $\tan 30^{\circ}$. These values must be obtained from a table of natural tangents like that given in Appendix A, from which it will be seen that the ratio between the strengths of the currents is $\cdot 268: 577$, or about $10: 22$.

Or, more generally, if current $\mathrm{C}$ produces deflexion $\delta$, and current $\mathrm{C}^{\prime}$ deflexion $\delta^{\prime}$, then

$$
\mathrm{C}: \mathrm{C}^{\prime}=\tan \delta: \tan \delta^{\prime} .
$$

To obviate reference to a table of figures, the circular scale of the instrument is sometimes graduated into

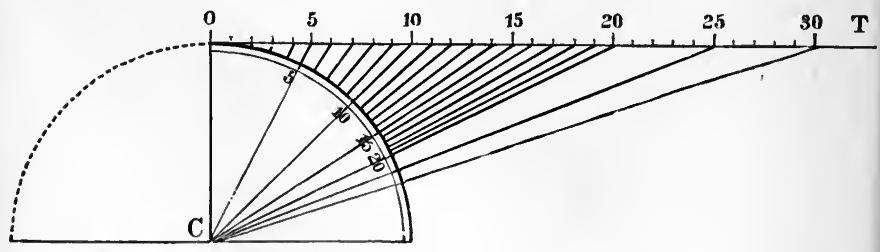

Fig. 121.

tangent values instead of being divided into equal degrees of arc. Let a tangent OT be drawn to the 
circle, as in Fig. 121, and along this line let any number of equal divisions be set off, beginning at $O$. From these points draw back to the centre. The circle will thus be divided into a number of pieces, of which those near $O$ are nearly equal, but which get snaller and smaller away from $O$. These unequal pieces correspond to equal increments of the tangent. If the scale were divided thus, the readings would be proportional to the tangents. It is, however, harder to divide an arc into tangent lines with accuracy than to divide it into equal degrees ; hence this graduation, though convenient, is not used where great accuracy is needed.

\section{Absolute Measure of Current by Tangent} Galvanometer.-The strength of a current may be determined in "absolute" units by the aid of the tangent galvanometer if the "constants" of the instrument are known. The tangent of the angle of deflexion represents (see Art. 137) the ratio between the magnetic force due to the current and the horizontal component of the earth's magnetic force. Both these forces act on the needle, and depend equally upon the magnetic moment of the needle, which, therefore, we need not know for this purpose. We know that the force exerted by the current at centre of the coil is proportional to the horizontal force of the earth's magnetism multiplied by the tangent of the angle of deflexion. These two quantities can be found from the tables, and from them we calculate the absolute value of the current as follows:-Let $\dot{r}$ represent the radius of the galvanometer coil (measured in centimetres); its total length (if of one turn only) is $2 \pi r$. The distance from the centre to all parts of the coil is of course $r$. From our definition of the unit of strength of current (Art. 207), it follows

that

$\mathrm{C} \times \frac{2 \pi r}{r^{2}}=$ force (in dynes) at centre,

or

$\mathrm{C} \times \frac{2 \pi}{r}=\mathrm{H} \cdot \tan \delta$; 
hence

$$
\mathrm{C}=\frac{r}{2 \pi} \cdot \mathrm{H} \cdot \tan \delta .
$$

The quantity $2 \pi / r$, or $2 \pi n / r$ if the coil has $n$ turns, is sometimes called the "constant" or the "principal constant" of the galvanometer and denoted by the symbol G. Hence the value of the current in absolute (electromagnetic) units * will be expressed as

$$
\mathrm{C}=\frac{\mathrm{H}}{\mathrm{G}} \cdot \tan \delta \text {. }
$$

The constant $G$ represents the strength of field produced at the centre of the coil by unit current.

214. Sine Galvanometer.-The disadvantage of the tangent galvanometer just described is that it is not very sensitive, because the coil is necessarily very large as compared with the needle, and therefore far away from it. A galvanometer with a smaller coil or a larger needle could not be used as a tangent galvanometer, though it would be more sensitive. Any sensitive galvanometer in which the needle is directed by the earth's magnetism can, however, be used as a Sine Galvanometer, provided the frame on which the coils are wound is capable of being turned round a central axis. When the instrument is so constructed, the following method of measuring currents is adopted. The coils are first set parallel to the needle (i.e. in the magnetic meridian); the current is then sent through it, producing a deflexion; the coil itself is rotated round in the same sense, and, if turned round through a wide enough angle, will overtake the needle, which will once more lie parallel to the coil. In this position two forces are acting on the needle: the directive force of the earth's magnetism acting along the magnetic meridian, and the force due to the current passing in the coil, which tends to thrust the poles of the needle out at right angles;

* The student will remember (Arts. 20i and 354) that the practical unit of current which we call "one ampere" is only $3^{16}$ of one "absolute " unit of the centimetre-gramme-second system. 
in fact there is a "couple" which exactly balances the "couple" due to terrestrial magnetism. Now it was shown in the Lesson on the Laws of Magnetic Force (Art. 136) that when a needle is deflected the "moment" of the couple is proportional to the sine of the angle of deflexion. Hence in the sine galvanometer, when the coil has been turned round so that the needle once more lies along it, the strength of the current in the coil is proportional to the sine of the angle through which the coil has been turned.*

215. The Mirror Galvanometer.-When a galvanometer of great delicacy is needed, the moving parts must be made very light and small. To watch the movements of a very small needle an index of some kind must be used ; indeed, in the tangent galvanometer it is usual to fasten to the short stout needle a delicate stiff pointer of aluminium. A far better method is to fasten to the needle a very light mirror of silvered glass, by means of which a beam of light can be reflected on to a scale, so that every slightest motion of the needle is magnified and made apparent. The mirror galvanometers devised by Sir W. Thomson (Lord Kelvin) for signalling through submarine cables, are admirable examples of this class of instrument. In Fig. 122 the general arrangements of this instrument are shown. The body of the galvanometer, consisting of a bobbin

* Again the student who desires to compare tle strength of two currents will require the help of a table of natural sines, like that given in Appendix A. Suppose that with current $\mathbf{C}$ the coils had to be turned through an angle of $\theta$ degrees; and that with a different current $C^{\prime}$ the coils had to be turned through $\theta^{\prime}$ degrees, then

$$
\mathrm{C}: \mathrm{C}^{\prime}=\sin \theta: \sin \theta^{\prime} .
$$

It is of course assumed that the instrument is provided with a scale of degrees on which to read off the angle through which the coils have been turned. It is possible here also, for rough purposes, to graduate the circle not in degrees of arc, but in portions corresponding to equal additional values of the sine. The student should try this way of dividing a circle after reading the note On Ways of Reckoning Angles, p. 133. 
on which is wound the coil, is supported on three screw feet by which it can be adjusted. The magnet consists of one or more small pieces of steel watch-spring attached to the back of a light concave silvered glass mirror about as large as a threepenny piece, weighing altogether only two or three grains. This mirror is hung by a single fibre of cocoon silk within the coil, and a curved magnet, which

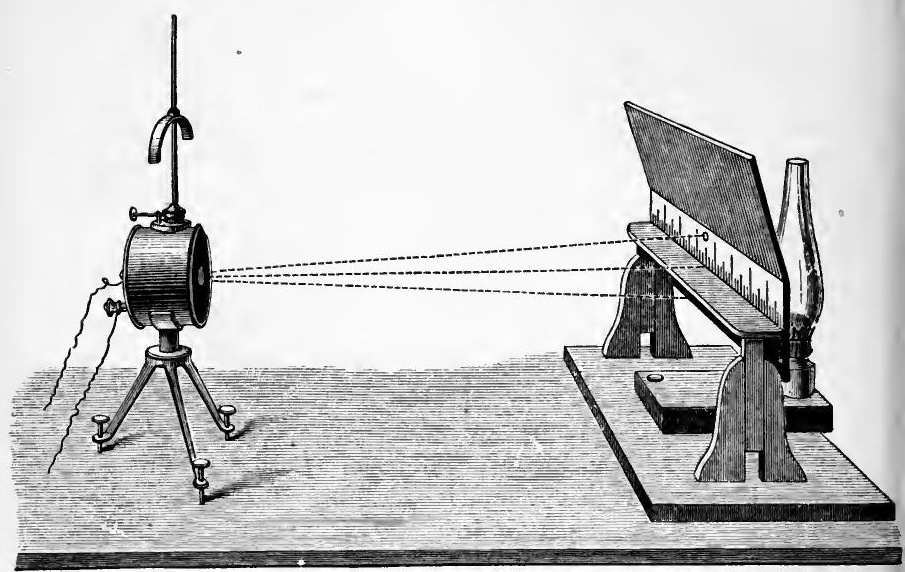

Fig. 122.

serves to counteract the magnetism of the earth, or to direct the needle, is carried upon a vertical support above. Another view of the suspended mirror and magnets is shown in Fig. 123. Opposite the galvanometer is placed the scale. A beam of light from a paraffin lamp passes through a narrow aperture under the scale and falls on the mirror, which reflects it back on to the scale. The mirror is slightly concave, and gives a well-defined spot of light if the scale is adjusted to suit the focus of the mirror. The adjusting magnet enables the operator to 
bring the reflected spot of light to the zero point at the middle of the scale. The feeblest current passing through the galvanometer will cause the spot of light to shift to right or left. The tiny current generated by dipping into a drop of salt water the tip of a brass pin and a steel needle (connected by wires to the terminals of the galvanometer) will send the spot of light swinging right across the scale. If a powerful limelight is used, the movement of the needle can be shown to a thousand persons at once. For still more delicate work an astatic pair of needles can be used, each being surrounded by

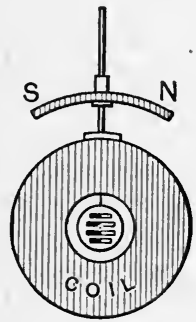

Fig. 123.

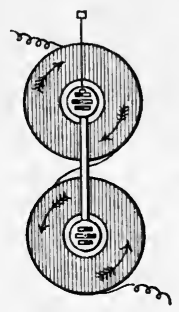

Fig. 124.

its coil, and having the mirror rigidly attached to one of the needles. Such a form, with two bobbins, wound so as to be traversed by the current in opposite senses, is represented diagrammatically in Fig. 124. Such an instrument, made with four bobbins, two in front and two behind the suspended needle system, and having on each bobbin about 2 miles of a wire about $\frac{1}{1000}$ inch in thickness, insulated by a coating of silk, is capable of showing by a deflexion of one division on its scale an exceedingly minute current, even down to one fifty-four thousand millionth part of one ampere.

216. Suspended Coil Galvanometers.-These have been used by Sturgeon (1836), Varley (1860), and others, and the principle was also applied in Lord 
Kelvin's "Siphon Recorder." The best known is that of D'Arsonval depicted in Fig. 125. Between the poles of a

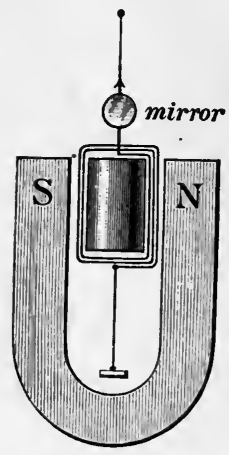

Fig. 125. compound permanent steel magnet of $\mathrm{U}$-shape is suspended by very thin hard-drawn silver wires an open coil of very fine wire wound on a light rectangular frame. The current is led to and from the coil by the suspending wires. Within the suspended coil is a cylinder of soft iron, supported from behind, to concentrate the magnetic field. The vertical parts of the coil then hang freely in the two narrow gaps where the magnetic field is very intense. The force tending to turn the coil is proportional to the current, to the number of windings, and to the intensity of the magnetic field, so that by making the magnet very powerful the instrument becomes very sensitive. The elasticity of the suspending wires controls the position of the coil and tends to bring it back to its initial position. These galvanometers are independent of the earth's magnetic field, and are not affected by magnets in their neighbourhood, so that they can be used in many places where other galvanometers could not. They are also remarkably dead-beat. Some are provided with a pointer and a horizontal dial ; others more usually have a mirror attached to the coil to reflect a spot of light.

Most recent is the suspended-coil

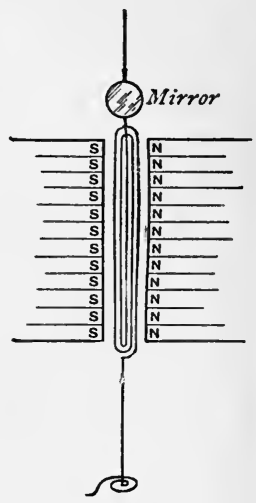

Fig. 126. galvanometer of Ayrton and Mather (Fig. 126). Here the suspended coil is formed as an elongated loop with no 
aperture between its sides. Consequently the poles of the magnets may be brought very close together; and these are made up of a number of flat steel magnets of nearly circular form piled up on one another. One of these instruments, with mirror and scale, will show a deflexion of one scale division, with a current less than one ninety-millionth part of 1 ampere.

Strong currents must not be passed through very sensitive galvanometers, for, even if they are not spoiled, the deflexions of the needle will be too large to give accurate measurements. In such cases the galvanometer is used with a shunt, or coil of wire arranged so that the greater part of the current shall flow through it, and pass the galvanometer by, only a small portion of the current actually traversing the coils of the instrument. The resistance of the shunt must bear a known ratio to the resistance of the instrument, according to the principle laid down in Art. 409 about branched circuits.

216a. Oscillographs.-For the purpose of studying exceedingly rapid changes of current, or rapidly alternating currents, instruments called Oscillographs have been devised by Blondel, and by Duddell. In Duddell's Oscillograph the current is sent through two thin parallel wires forming a single loop stretched vertically in a narrow gap between the poles of an excessively strong electromagnet. The loop tends to turn and moves a light mirror. As its moment of inertia is excessively small it can respond to currents that oscillate with a frequency of several hundreds or even thousands per second.

217. Differential Galvanometer.-For the purpose of comparing two currents a galvanometer is sometimes employed, in which the coil consists of two separate wires wound side by side. If two equal currents are sent in opposite directions through these wires, the needle will not move. If the currents are unequal, then the needle will be moved with an intensity corresponding to the difference of the strengths of the two currents. 
218. Ballistic Galvanometer.-In order to measure the strength of currents which last only a very short time, galvanometers are employed in which the needle takes a relatively long time to swing. This is the case with long or heavy needles; or the needles may be weighted by enclosing them in leaden cases. As the needle swings slowly round, it adds up, as it were, the varying impulses received during the passage of a transient current. The sine of half the angle of the first swing is proportional to the quantity of electricity that has flowed through the coil. The charge of a condenser may thus be measured by discharging it through a ballistic galvanometer (see Art. 418b). The needle must not be damped.

219. Methods of Damping: Aperiodic Galvanometers. -To prevent the needle from swinging to and fro for a long time devices are used to damp the motion. These are :-

(a) Air Damping.-A light vane attached to needle beats against the air and damps the motion. In mirror instruments the mirror itself damps, particularly if confined in a narrow chamber.

(b) Oil Damping. - A vane dips into oil.

(c) Magnetic Damping. - If the needle swings close to or inside a mass of copper, it will soon come to rest by reason of the eddycurrents (Art. 457) induced in the copper. Eddy-currents damp the motion of the suspended coil in instruments of that class.

The period of swing can be reduced by diminishing the weiglit and leverage of the moving parts so as to lessen their moment of inertia. It can also be lessened (at the expense of the sensitiveness of the instrument) by increasing the controlling forces. An instrument so well damped as to come to rest without getting up a periodic swing is called an aperiodic or dead-beat instrument.

220. Voltmeters, or Potential Galvanometers.-If any galvanometer be constructed with a very long thin wire of high resistance as its coil, very little current will flow through it, but what little current flows will be exactly proportional to the potential difference that may be applied to the two ends of its circuit. Such a galvanometer, suitably provided with a scale, will indicate the number of volts between its terminals. Many forms of voltmeter-galvanometers exist, but they all agree in the essential of having a coil of a high resistance-sometimes several thousand ohms. The suspendedcoil galvanometers described in Art. 216 make excellent voltmeters. Weston's voltmeter, largely used in America, 
is of this class, the coil being delicately pivoted, and controlled by a spiral spring. Any sensitive mirror galvanometer can be used as a voltmeter by simply adding externally to its circuit a resistance sufficiently great. There are also other voltmeters that depend on electrostatic actions; they are a species of electrometer and are described in Art. 290. Cardew's voltmeter (see Art. 430) differs from the above class of instrument, and consists of a long thin platinum wire of high resistance, which expands by heating when it is comnected across a circuit. All voltmeters are placed as shunts across between the two points the potential difference of which is to be measured. They are never joined up in circuit as amperemeters are.

221. Amperemeters, or Ammeters.-A galvano- meter graduated so that its index reads directly on the scale the number of amperes (Art. 207) flowing through the coil is called an Amperemeter. Snch instruments were introduced in form for industrial use in 1879 by Ayrton and Perry. Many other forms were subsequently invented. In Ayrton and Perry's instruments (Fig. 127), which are portable and "dead-beat" in action, the needle, which is oval in shape, is placed between the poles of a powerful permanent magnet to control its direction and make it independent of the

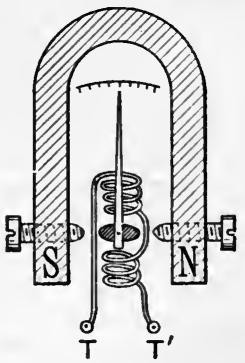

Fig. 127 . earth's magnetism. By a peculiar shaping of the polepieces, needle, and coils, the angular deflexions are proportional to the strength of the deflecting current. These amperemeters are made with short coils of very low resistance and few turns of wire. Ayrton and Perry also arranged voltmeters (Art. 220) in a similar form, but with long coils of high resistance.

Among the innumerable forms of amperemeter in 
commerce there are a number in which there is neither magnet nor iron, but which depend upon the mutual force between a fixed and a movable coil traversed by the current. These are dealt with in

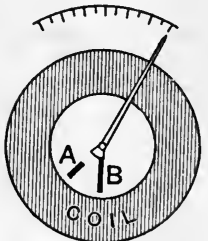

Fig. 128. Art. 394, and are suitable for alternate currents as well as continuous currents. Of this kind are Siemens' electrodynamometer and the Kelvin balances.

Other instruments depend upon the magnetic properties of iron under the influence of the current. Of this class are the Schuckert instruments represented in Fig. 128. An index pivoted in the axis of an open coil carries a light strip of soft iron seen endways at B. Another strip A is fixed within the coil. The current flowing round the coil magnetizes these strips and they repel one another. Gravity is here the controlling force.

\section{Lesson XVIII.-Currents produced by Induction}

222. Faraday's Discovery.-In 1831 Faraday discovered that currents can be induced in a closed circuit by moving magnets near it, or by moving the circuit across the magnetic field; and he followed up this discovery by finding that a current whose strength is changing may induce a secondary current in a closed circuit near it. Such currents, whether generated by magnets or by other currents, are known as-Induction Currents. And the action of a magnet or current in producing such induced currents is termed magnetoelectric (or electromagnetic) induction,* or simply in-

* The sturlent must not confuse this electronagnetic induction with the phenomenon of the electrostatic induction of one charge of eleetricity by another charge, as explained in Lesson III., and which has nothing to do with currents. Formerly, before the identity of the electricity derived from different sources was understood (Art. 246), electricity derived thus 
duction. Upon this principle are based the modern dynamo machines for generating electric currents mechanically, as well as induction coils, alternate-current transformers, and other appliances.

223. Induction of Currents by Magnets.-If a coil of insulated wire be connected in circuit with a sufficiently delicate galvanometer, and a magnet be inserted rapidly into the hollow of the coil (as in Fig. 129), a momentary current is observed to flow round the circuit while the magnet is heing moved into the coil. So long as the magnet lies motionless in the coil it induces no currents. But if it be rapidly pulled out of the coil another momentary current will be observed to flow, and in the opposite direction to the former. The in luced current caused by inserting the magnet is an inverse current, or is in the opposite direction to that which-world magnetize the magnet with its

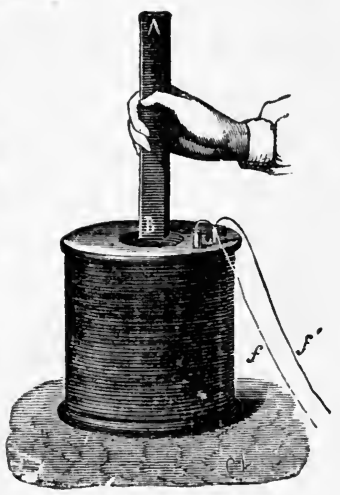

Fig. 129. existing polarity. The induced current caused by withdrawing the magnet is a direct current:

Precisely the same effect is produced if the coil be moved towards the magnet as if the magnet were moved towards the coil. The more rapid the motion is, the stronger are the induced currents.

The magnet does not grow any weaker by being so used, for the real source of the electrical energy generated is the mechanical energy spent in the motion.

from the motion of magnets was termed magneto-electricity. For most purposes the adjectives magneto-electric and electro-magnetic are synonymous. The production of electricity from magnetism, and of magnetism from electricity, are, it is true, two distinct operations; but both are included in the branch of science denominated Eleetromagnetics. 
If the circuit is not closed, no currents are produced; but the relative motion of coil and magnet will still set up electromotive-forces, tending to produce currents.

Faraday discovered these effects to be connected with the magnetic field surrounding the magnet. He showed that no effect was produced unless the circuit cut across the invisible magnetic lines of the magnet.

224. Induction of Currents by Currents.Faraday also showed that the approach or recession of a current might induce a current in a closed circuit near it. This may be conveniently shown as an experiment by the apparatus of Fig. 130.

A coil of insulated wire $\mathrm{P}$ is connected in circuit with a battery $\mathrm{B}$ of two or three cells, and a key $\mathrm{K}$ to turn the

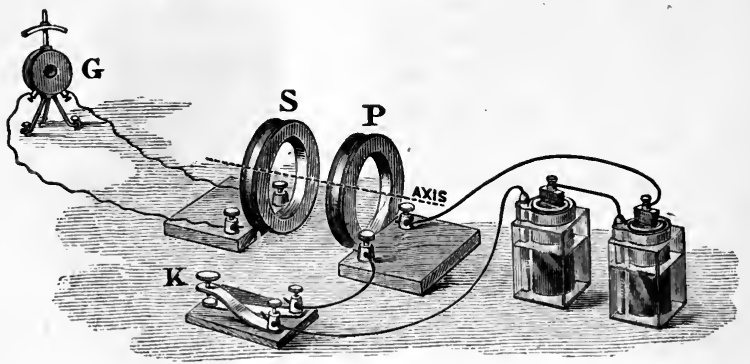

Fig. 130.

current on or off. A second coil S, entirely unconnected with the first, is joined up with wires to a sensitive galvanometer G. We know (Art. 202) that a coil of wire in which a current is circulating acts like a magnet. And we find that if while the current is flowing in $\mathrm{P}$, the coil is suddenly moved up toward S, a momentary current will be induced in $\mathrm{S}$. If $\mathrm{P}$ is suddenly moved away from $S$ another momentary current will be observed in the second circuit. The first of these two momentary currents is an "inverse" one, while the second one is 
found to be a "direct" one (i.e. one which runs the same way round the coil $\mathrm{S}$ as the battery current runs round the coil P). The coil $\mathrm{P}$ is called the primary coil, and the current in it the primary current. The other coil S is called the secondary coil, and the'momentary currents induced in it are sometimes called secondary currents.

Let $\mathrm{P}$ now be placed close to $\mathrm{S}$, no current flowing in either coil. Then on pressing the key $K$ to turn on the primary current, it will be noticed that during the moment while the current in $\mathrm{P}$ is growing there will be a transient inverse current in $\mathrm{S}$. The effect of turning on the current is just as if the current had been turned on while $\mathrm{P}$ was far away and then $\mathrm{P}$ suddenly brought up to $\mathrm{S}$. Breaking the battery circuit while the primary coil lies close to the secondary coil produces the same effect as if the primary coil were suddenly removed to an infinite distance. Making the battery circuit while the primary coil lies close to the secondary produces the same effect as bringing it up suddenly from a distance.

So long as a steady current traverses the primary circuit there are no induced currents in the-secondary circuit, unless there is relative motion between the two circuits : but moving the secondary circuit towards the primary has just the same effect as moving the primary crircuit towards the secondary, and vice versâ.

We may tabulate these results as follows:

\begin{tabular}{|c|l|l|}
\hline $\begin{array}{c}\text { By } \\
\text { of } \\
\text { of }\end{array}$ & $\begin{array}{c}\text { Momentary Inv́erse } \\
\text { currents are induced } \\
\text { in the secondary circuit }\end{array}$ & $\begin{array}{c}\text { Momentary Direct } \\
\text { currents are induced } \\
\text { in the secondary circuit }\end{array}$ \\
\hline Magnet & while approaching. & while receding. \\
\hline Current & $\begin{array}{l}\text { while approaching, } \\
\text { or beginning, } \\
\text { or increasing in strength. }\end{array}$ & $\begin{array}{l}\text { or decreasing in strength. } \\
\text { or ending, } \\
\text { whileceding, }\end{array}$ \\
\hline
\end{tabular}


225. Fundamental Laws of Induction.-When we reflect that every circuit traversed by a current has a magnetic field of its own in which there are magnetic lines running through the circuit (Arts. 202 and 389), we shall see that the facts tabulated in the preceding paragraph may be summed up in the following fundamental laws :-

(i.) A decrease in the number of lines which pass through a circuit induces a current round the circuit in the positive direction (i.e. produces a "direct" current); while an increase in the number of lines which pass through the circuit induces a current in the negative direction round the circuit (i.e. an "inverse" current).

Here we suppose the positive direction along lines to be the direction along which a free $\mathrm{N}$-pole would tend to move, and the positive direction of the current that in which the current must flow to increase the magnetic flux. Compare the "corkscrew" rule given on p. 183.

(ii.) The total induced electromotive-force acting round a closed circuit is equal to the rate of decrease in the number of lines which pass through the circuit.

Suppose at first the number of magnetic lines (Art. 119) passing through the circuit to be $\mathrm{N}_{1}$, and that after a very short interval of time $t$ the number becomes $\mathrm{N}_{2}$, the average induced electromotive-force $\mathrm{E}$ is

$$
\mathrm{E}=\frac{\mathrm{N}_{1}-\mathrm{N}_{2}}{t} .
$$

By Ohm's law,

$$
\begin{aligned}
& \mathrm{C}=\mathrm{E} \div \mathrm{R}, \\
& \mathrm{C}=\frac{\mathrm{N}_{1}-\mathrm{N}_{2} .}{t \mathrm{R}} .
\end{aligned}
$$

If $\mathrm{N}_{2}$ is greater than $\mathrm{N}_{1}$, and there is an increase in the number of lines, then $\mathrm{N}_{1}-\mathrm{N}_{2}$ will be a negative quantity, and $\mathrm{C}$ will have a negative sign, showing that the E.M.F. is an inverse one. A coil of 50 turns of wire cutting 1000 lines will produce the same effect as a coil of 5 turns 
cutting 10,000 lines, or of 1 turn cutting 50,000 lines.

To induce an electromotive-force equal to that of a single Daniell's cell would require that $110,000,000$ lines should be cut in one second. As such large numbers are inconvenient to express the facts, the unit of E.M.F., the volt, has been chosen to correspond to the cutting of 100,000,000 lines per second.

Exampie. - Suppose the number of magnetic lines to diminish from 800,000 to 0 in the $\frac{1}{50}$ of a second, the rate of diminution is 40,000,000 lines per second. And since 1 volt is taken as $10^{8}$ lines per second, the average induced E.M.F. during that time will be 0.4 volt.

A reference to Fig. 176 will make this important law clearer. Suppose ABCD to be a wire circuit of which the piece $\mathrm{AB}$ can slide along $\mathrm{DA}$ and $\mathrm{CB}$ towards $\mathrm{S}$ and $\mathrm{T}$. Let the vertical arrows represent vertical lines of force in a uniform magnetic field, and show (as is the case with the rertical components of the earth's lines of force in the northern hemisphere) the direction in which a $\mathrm{N}$-pointing pole would move if free. The positive direction of these magnetic lines is therefore vertically downwards through the circuit. Now if AB slide towards ST with a uniform velocity it will cut a certain number of lines every second, and a certain number will be added during every second of time to the total number passing through the circuit. If $\mathrm{N}_{1}$ be the number at the beginning, and $\mathrm{N}_{2}$ that at the end of a circuit, $\mathrm{N}_{1}-\mathrm{N}_{2}$ will be a negative quantity, and there will be generated an electromotive-force whose direction throngh the sliding piece is from A towards B.

It is important to note that all these inductive operations are really magnetic. In the experiment with the two coils $\mathrm{P}$ and $\mathrm{S}$ it is the magnetic lines of coil $\mathrm{P}$ which pass through coil $\mathrm{S}$ and set up the induced E.M.F. This is proved by the following further experiment. Take a bar of iron-a poker, or better still, a bundle of iron wires - and lay it along the dotted line so that its ends pass 
through $\mathrm{P}$ and $\mathrm{S}$. It will by its great magnetic permeability help to conduct the magnetic lines from $\mathrm{P}$ through S. And when it is so placed it will be found greatly to intensify the actions. In fact if $\mathrm{P}$ is many inches away from $\mathbf{S}$, and the iron core is present, the inductive effects of turning the current on and off may be as great as if, in the absence of the core, $\mathrm{P}$ were pushed up close to $\mathrm{S}$.

226. Direction of Induced E.M.F.-It is convenient to have rules for remembering the relations in direction between the magnetism, the motion, and the

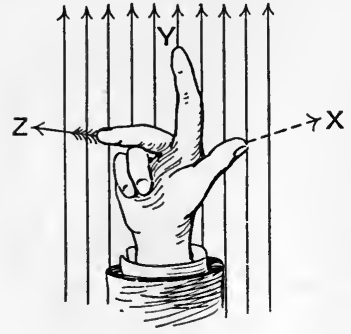

Fig. 131. induced electromotive - force. Of such rules the following, due to Fleming, is most useful: Let the forefinger of the right hand (Fig. 131) point in the direction of the magnetic lines; then turn the thumb in the direction of the motion: the middle finger bent at right angles to both thumb and forefinger will show the direction of the induced E.M.F.

Another often given is an adaptation of Ampère's rule: Suppose a figure swimming in any conductor to turn so as to look along the (positive direction of the) lines, then if he and the conductor be moved towards his right hand he will be swimming with the current induced by this motion; if he be moved towards his left hand, the current will be against him.

227. Faraday's Disk Machine. - Faraday constructed several magneto-electric machines, one of them consisting of a copper disk (Fig. 132) which he rotated between the poles of a steel magnet. The current flowed from shaft to rim or vice versû, according to the sense of the rotation. It was conducted away by wires having sliding contacts. In other machines copper wire coils 
were spun so as to cut magnetic lines. The same induction principle is applied in modern dynamo-electric machines (Lesson XLII.). In all cases power must be employed to produce the motion. They are all contrivances for converting mechanical energy into electrical energy.

228. F a r aday's Ring : Principle of Transformation.-Amongst

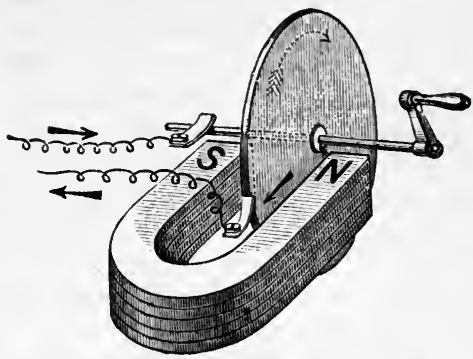

Fig. 132. Faraday's earliest experiments he took an iron ring about 8 inches in diameter (Fig. 133) and wound upon it two insulated coils of wire $\mathrm{P}$ and $\mathrm{S}$, each of many turns. If coil $P$ was connected to a battery circuit, and coil $S$. to a galvanometer, he found that whenever a current was turned on or off in coil

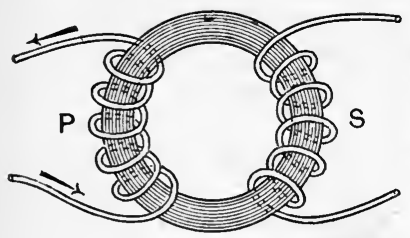

Fig. 133.

$P$, secondary currents were generated in coil s. In fact the currents in $P$ magnetized the iron-ring, and the magnetic lines created by $P$-passed through $\mathrm{S}$, setting -up induction currents. If $\mathrm{S}$ is used as the primary then $\mathrm{P}$ will work as secondary; in fact the induction between $\mathrm{P}$ and $\mathrm{S}$ is mutual. The Faraday ring, with its two coils wound upon a closed circuit of iron, may be regarded as the very type of all transformers or induction coils. Faraday also employed some induction-coils in which the two coils A and B (Fig. 134) were wound cylindrically outside one another upon a straight core $\mathrm{C}$ of iron. 
In all transformers the electromotive-forces generated in the secondary circuit are to those employed in the

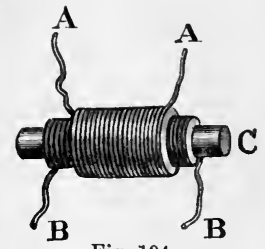

Fig. 134. primary circuit, nearly in the same proportion as the relative numbers of turns in the two coils. For example, if the primary coil has 100 turns and the secondary has 2500 turns, the electromotive-force in the secondary circuit will be nearly twenty-five times as great as that used in the primary. By choosing the proper number of turns, the electromotive-force can be transformed either up or down.

229. The Induction Coil.-In order to generate enormously high electromotive-forces which shall be able to send sparks across air spaces that ordinary batteries working at under 100 volts could not possibly pierce, advantage is taken of the transformer principle. To produce spark discharges there is used the apparatus depicted in Fig. 135, as improved by Callan, Sturgeon, Ruhmkorff, and others, and termed the Induction Coil or Inductorium. The induction coil consists of a cylindrical bobbin having a central iron core surrounded by a short inner or "primary". coil of stout wire, and by an outer "secondary" coil consisting of many thousand turns of very fine wire, very carefully insulated between its different parts. The primary circuit is joined to the terminals of a few powerful Grove's or Bunsen's cells, and in it are also included an interrupter, and a commutator or key. The object of the interrupter is to make and break the primary circuit in rapid succession. The result of this is at every "make" to induce in the outer "secondary" circuit a momentary inverse current, and at every "break" a powerful momentary direct current. As the number of magnetic lines created and destroyed at each "make" and "break" is the same, the two electromotive impulses are equal ; but by the use of a condenser 
the current at "make" is caused to take a considerable fraction of time to grow, whilst at "break" the cessation is instantaneous. The rate of cutting of the magnetic lines is therefore much greater at "break" than at "make." The induced electromotive-forces at "make" last longer, but are feebler, and do not suffice to send sparks. The currents at "break" manifest themselves as a brilliant torrent of sparks between the ends of the

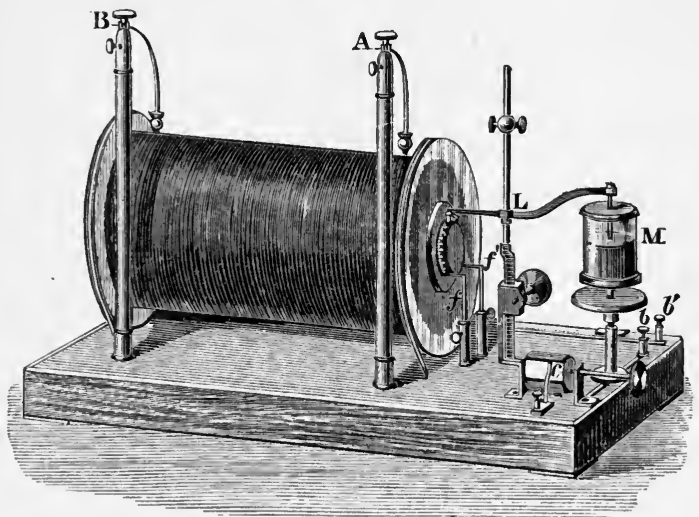

Fig. 135.

secondary wires when brought near enough together. The primary coil is made of stout wire, that it may carry strong magnetizing currents, and consists of few turns to keep the resistance low, and to avoid self-induction of the primary current on itself. The central iron core is for the purpose of increasing, by its great magnetic permeability, the number of lines of force that pass through the coils : it is usually made of a bundle of fine wires to avoid the induced currents which if it were a solid bar would be set circulating in it, and which would retard its rapidity of magnetization or demagnetization. 
The secondary coil is made with many turns, in order that the coefficient of transformation may be large ; and as the induced electromotive-force will be thousands of volts, the resistance of this coil will be immaterial, and it may be made of the thinnest wire that can conveniently be wound. In Mr. Spottiswoode's giant Induction Coil (which yields a spark of $42 \frac{1}{2}$ inches' length in air, when worked with 30 Grove's cells), the secondary coil contains 280 miles of wire, wound in 340,000 turns, and has a resistance of over 100,000 ohms.

The interrupters of induction coils are usually selfacting. That of Foucault, shown with the coil in Fig. 135, consists of an arm of brass $\mathrm{L}$, which dips a platinum wire into a cup of mercury $\mathrm{M}$, from which it draws the point out, so breaking circuit, in consequence of its other end being attracted toward the core of the coil whenever it is magnetized; the arm being drawn back again by a spring when, on the breaking of the circuit, the core ceases to be a magnet. A more common interrupter on small coils is a "break," consisting of a piece of thin steel which makes contact with a platinum point, and which is drawn back by the attraction of the core on the passing of a current; and so makes and breaks circuit by vibrating backwards and forwards just as does the hammer of an ordinary electric bell.

Associated with the primary circuit of a coil is usually a small condenser (see Art. 303), made of alternate layers of tinfoil and paraffined paper, into which the current flows whenever circuit is broken. The effect of the condenser is, as stated above, to suppress the "inverse" current at "make" and to increase greatly the direct, electromotive-force at "lureak." The sparks are longer, and only pass one way. The condenser does this by the action known as electric resonance (see Art. 517).

230. Ruhmkorff's Reverser.-In order to cut off or reverse the direction of the battery current at will, Ruhmkorff applied the current-reverser, or reversingswitch ("commutator") shown in Fig. 136. In this 
instrument the battery poles are connected through the ends of the axis of a small ivory or ebonite cylinder to two cheeks of brass $\mathrm{V}$ and $\mathrm{V}^{\prime}$, which can be turned so as to place them either way in contact with two vertical springs $\mathrm{B}$ and $\mathrm{C}$, which are joined to the ends of the primary coil. Many other forms of reversing-switch have been devised; one, much used as a key for telegraphic signalling, is drawn in Fig. 271.

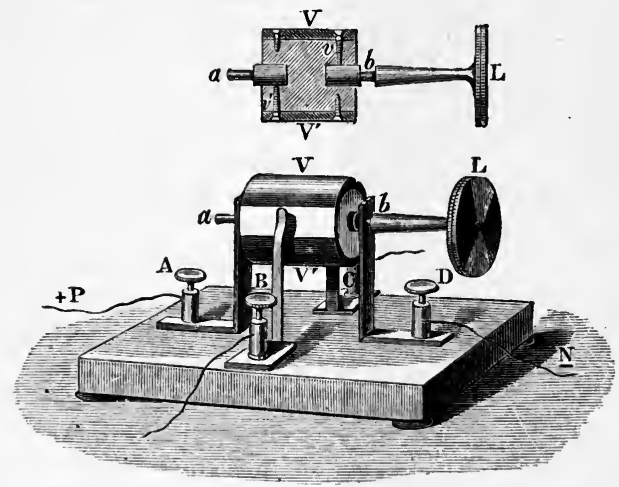

Fig. 136.

231. Luminous Effects of Induction Sparks.The induction coil furnishes a rapid succession of sparks with which all the effects of disruptive discharge may be studied. These sparks differ only in degree from those furnished by friction machines and by Leyden jars (see Lesson XXIV. on Phenomena of Discharge).

For studying discharge through glass vessels and tubes from which the air has been partially exhausted, the coil is very useful. Fig. 137 illustrates one of the many beantiful effects which can be obtained, the spark expanding in the rarefied gas into flickering sheets of light, exhibiting striæ and other phenomena. 


\section{Induction Currents from Earth's Mag-} netism.-It is easy to obtain induced currents from the

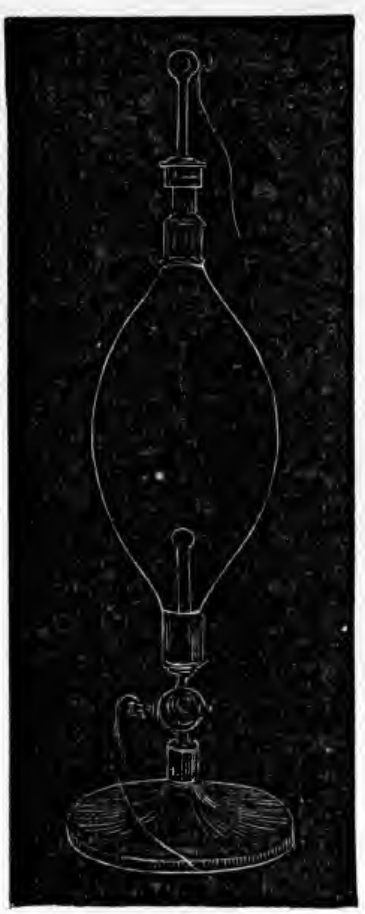

Fig. 137. earth's magnetism. A coil of fine wire joined to a sensitive galvanometer, when suddenly inverted, cuts the lines of the earth's magnetism, and induces a current.

Faraday, indeed, applied this method to investigate the direction and number of magnetic lines. If a small wire coil be joined in circuit with a suitable galvanometer having a heavy needle, and the little coil be suddenly inverted while in a magnetic field, it will cut twice all the lines that pass through its own area, and the sine of half the angle of the first swing (Art. 418) will be proportional to the number of lines cut; for with a slowmoving needle, the total quantity of electricity that flows through the coils will be the integral whole of all the separate quantities conveyed by the induced currents, strong or weak, which flow zound the circuit during the rapid process of cutting the lines. The little exploring coil acts therefore as a magnetic proof-plane. For small deflexions the first swing may be taken as a sufficient approxinution instead of the sine of half the angle (see Art. 418).

If the circuit be moved parallel to itself across a uniform magnetic field there will be no induction currents, 
for just as many magnetic lines will be cut in moving ahead in front as are left behind. There will be no current in a wire moved parallel to itself along a line of force; nor, if it lie along such a line while a current is sent through it, will it experience any mechanical force.

233. Earth Currents. - The variations of the earth's magnetism, mentioned in Lesson XII., alter the number of magnetic lines which pass through the telegraphic circuits, and hence induce in them disturbances which are known as "earth currents." During magnetic storms the earth currents on the British lines of telegraph have been known to attain a strength of 40 milliamperes, which is stronger than the usual working currents. Feeble earth currents are observed every day, and are more or less periodic in character.

\section{Lesson XIX.-Chemical Actions of Currents}

234. Conducting Properties of Liquids. - In addition to the chemical actions inside the cells of the battery, which always accompany the production of a current, there are also chemical actions produced outside the battery when the current is cansed to pass through certain liquids. Liquids may be divided into three classes-(1) those which do not conduct at all, such as turpentine and many oils, particularly petroleum; (2) those which conduct without decomposition, viz. mercury and other molten metals, which conduct just as solid metals do ; (3) those which are decomposed when they conduct a current, viz. the dilute acids, solutions of metallic salts, and certain fused solid compounds.

235. Decomposition of Water. - In the year 1800 Carlisle and Nicholson discovered that the voltaic current could be passed through water, and that in passing through it decomposed a portion of the liquid into 
its constituent gases. These gases appeared in bubbles on the ends of the wires which led the current into and out of the liquid ; bubbles of oxygen gas appearing at the point where the current entered the liquid, and hydrogen bubbles where it left the liquid. It was soon found that a great many other liquids, particularly dilute acids and solutions of metallic salts, could be similarly decomposed by passing a current through them.

236. Electrolysis.-To this process of decomposing a liquid by means of an electric current Faraday gave the name of electrolysis (i.e. electric analysis); and those substances which are capable of being thus decomposed or " electrolyzed" he termed electrolytes.

The ends of the wires leading from and to the battery are called electrodes ; and to distinguish them, that by which the current enters is called the anode, that by which it leaves the kathode. The vessel in which a liquid is placed for electrolysis is termed an electrolytic cell.

237. Electrolysis of Water.-Returning to the decomposition of water; we may remark that perfectly pure water appears not to conduct, but its resistance is greatly reduced by the addition of a few drops of sulphuric or hydrochloric acid. The apparatus shown in Fig. 138 is suitable for this purpose. Here a battery of two cells (those shown are circular Bunsen's cells) is seen with its poles comnected to two strips of metallic platinum as electrodes, which project up into a vessel containing the acidulated water. Two tubes closed at one end, which have been previously filled with water and inverted, receive the gases evolved at the electrodes. Platinum is preferred to other metals such as copper or iron for electrodes, since it is less oxidizable and resists every acid. It is found that there is almost exactly twice as much hydrogen gas (by volume) evolved at the kathode as there is of oxygen at the anode. This fact corresponds with the known chemical composition of water, which is 
produced by combining together these two gases in the proportion of two volumes of the former to one of the latter. The proportions of gases evolved, however, are not exactly two to one, for at first a very small quantity of the hydrogen is absorbed or "occluded" by the platinum surface, while a more considerable proportion of the oxygen-about 1 per cent-is given off in the

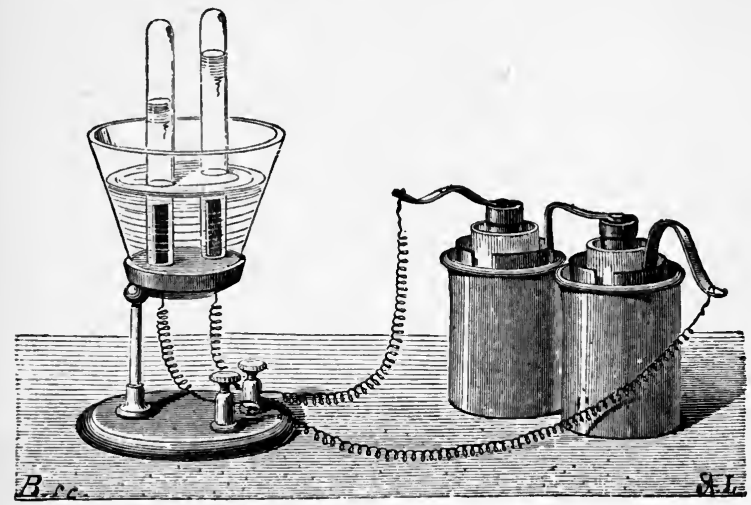

Fig. 138.

denser allotropic form of ozone, which occupies less space and is also slightly soluble in the water. When a sufficient amount of the gases has been evolved and collected they may be tested; the hydrogen by showing that it will burn, the oxygen by its causing a glowing spark on the end of a splinter of wood to burst into flame. If the two gases are collected together in a common receiver, the mixed gas will be found to possess the well-known explosive property of mixed hydrogen and oxygen gases. The chemical decomposition is expressed in the following equation:
$\mathrm{H}_{2} \mathrm{O}$
$=$
$\mathrm{H}_{2}$
$+$
O
Water yields
2 vols. of Hydrogen
and 1 vol. of Oxygen. 


\section{Electrolysis of Sulphate of Copper.-We} will take as another case the electrolysis of a solution of the well-known "blue vitriol" or sulphate of copper. If a few crystals of this substance are dissolved in water a blue liquid is obtained, which is easily electrolyzed between two electrodes of platinum foil, by the current from a single cell of any ordinary battery. The chemical formula for sulphate of copper is $\mathrm{CuSO}_{4}$. The result of the electrolysis is to split it up into two parts. Metallic copper is carried forward by the current and deposited in a film upon the kathode, leaving behind at the anode "sulphion," an easily decomposed compound of sulphur and oxygen, which is immediately acted upon by the water forming sulphuric acid and oxygen. This oxygen is liberated in bubbles at the anode. The chemical changes are thus expressed:

\begin{tabular}{|c|c|c|c|c|c|c|c|}
\hline $\begin{array}{r}\mathrm{C} \\
\text { Sulpha }\end{array}$ & $\begin{array}{l}\mathrm{SO}_{4} \\
\text { of } \mathrm{C}\end{array}$ & per & becomes & $\begin{array}{c}\mathrm{Cu} \\
\text { Copper }\end{array}$ & $\begin{array}{c}+ \\
\text { and }\end{array}$ & \multicolumn{2}{|c|}{$\begin{array}{c}\mathrm{SO}_{4} \\
\text { Sulphion }\end{array}$} \\
\hline $\begin{array}{l}\mathrm{SO}_{4} \\
\text { alphion }\end{array}$ & $\begin{array}{c}+ \\
\text { and }\end{array}$ & $\begin{array}{l}\mathrm{H}_{2} \mathrm{O} \\
\text { water }\end{array}$ & $\begin{array}{c}= \\
\text { produce }\end{array}$ & $\begin{array}{r}\mathrm{H}_{2} \\
\text { Sulphu }\end{array}$ & & $\begin{array}{c}+ \\
\text { and }\end{array}$ & $\begin{array}{c}\mathrm{O} \\
\text { Oxygen }\end{array}$ \\
\hline
\end{tabular}

In this way, as the current continues to flow, copper is continually withdrawn from the liquid and deposited on the kathode, and the liquid gets more and more acid. If copper electrodes are used, instead of platinum, no oxygen is given off at the anode, but the copper anode itself dissolves away into the liquid at exactly the same rate as the copper of the liquid is deposited on the kathode.

239. Anions and Kations.-The atoms which thus are severed from one another and carried invisibly by the current to the electrodes, and there deposited, are obviously of two classes; some are left behind at the anode, others are carried forward to the kathode. Faraday gave the name of ions to these wandering atoms: those left at the anode being anions, and those going to the kathode being kations. Anions are sometimes 
regarded as "electronegative," because they move as if attracted toward the + pole of the battery, while the kations are regarded as "electropositive." Hydrogen and the metals are kations, moving apparently with the direction assumed as that of the current, and are deposited where the current leaves the electrolytic cell. The anions are oxygen, chlorine, etc. When, for example, chloride of tin is electrolyzed, metallic tin is deposited on the kathode, and chlorine gas is evolved at the anode.

\section{Quantitative Laws of Electrolysis.}

(i.) The amount of chemical action is equal at all points of a circuit. If two or more electrolytic cells are placed at different points of a simple circuit the amount of chemical action will be the same in all, for the same quantity of electricity flows past every point of the circuit in the same time. If all these cells contain acidulated water, the quantity, for example, of hydrogen set free in each will be the same; or, if they contain a solution of sulphate of copper, identical quantities of copper will be deposited in each. If some of the cells contain acidulated water, and others contain sulphate of copper, the weights of hydrogen and of copper will not be equal, but will be in chemically equivalent quantities.

(ii.) The amount of an ion liberated at an electrode in a given time is proportional to the strength of the current. A current of two amperes will cause just twice the quantity of chemical decomposition to take place as a current of one ampere would do in the same time.

(iii.) The amount of an ion liberated at an electrode in one second is equal to the strength of the current multiplied by the "electro-chemical equivalent" of the ion. It has been found by experiment that the passage of one coulomb of electricity through water liberates 000010384 gramme of hydrogen. Hence, a current the strength of which is $\mathrm{C}$ (amperes) will liberate $\mathrm{C} \times \cdot 000010384$ grammes of hydrogen per second. The quantity 000010384 is 
called the electrochemical equivalent of hydrogen. The "electrochemical equivalents" of other elements can be easily calculated if their chemical "equivalent" is known. Thus the chemical "equivalent" * of copper is 31.59 ; multiplying this by 000010384 we get as the electrochemical equivalent of copper the value 0003281 (gramme).

Table of Electrochemical Equivalents, etc.

\begin{tabular}{|c|c|c|c|c|c|c|c|c|}
\hline \multicolumn{5}{|c|}{ Element. } & $\begin{array}{l}\text { Atomic } \\
\text { Weight. }\end{array}$ & $\begin{array}{l}\text { Val- } \\
\text { ency. }\end{array}$ & $\begin{array}{c}\text { Chemical } \\
\text { Equivalent. }\end{array}$ & $\begin{array}{l}\text { Electrochemical } \\
\text { Equivalent } \\
\text { (grammes } \\
\text { per coulomb). }\end{array}$ \\
\hline \multicolumn{9}{|c|}{ Electropositive- } \\
\hline Hydrogen & & & & . & 1 & 1 & 1 & 0.000010384 \\
\hline Potassium & & & & . & $39 \cdot 03$ & 1 & $39 \cdot 03$ & 0.0004053 \\
\hline Sodium . & & & & . & $23^{\circ}$ & 1 & 23 . & 0.0002388 \\
\hline Gold . & & & & . & $196 \cdot 2$ & 3 & $65 \cdot 4$ & 0.0006791 \\
\hline Silver . & & & & . & $107 \cdot 67$ & 1 & $107 \cdot 67$ & 0.0011181 \\
\hline \multirow{2}{*}{\multicolumn{4}{|c|}{$\begin{array}{c}\text { Copper (Cupric) } \\
,,\end{array}$}} & & $63 \cdot 18$ & 2 & $31 \cdot 59$ & 0.0003281 \\
\hline & & & & . & $63 \cdot 18$ & 1 & $63 \cdot 18$ & 0.0006562 \\
\hline \multirow{2}{*}{\multicolumn{5}{|c|}{$\begin{array}{l}\text { Mercury (Mercuric) } \\
\text { (Mercurous) }\end{array}$}} & $199 \cdot 8$ & 2 & $99 \cdot 9$ & 0.0010374 \\
\hline & & & & & $199 \cdot 8$ & 1 & $199 \cdot 8$ & 0.0020748 \\
\hline \multicolumn{4}{|c|}{ Tin (Stannic). } & & $117 \cdot 8$ & 4 & $29 \cdot 45$ & 0.0003058 \\
\hline , (Stan & ous & & & & $117 \cdot 8$ & 2 & $58 \cdot 9$ & 0.0006116 \\
\hline \multicolumn{3}{|c|}{ Iron (Ferrous). } & & & $55 \cdot 9$ & 2 & $27 \cdot 95$ & 0.0002902 \\
\hline \multicolumn{3}{|c|}{ (Ferric) } & & & $55 \cdot 9$ & (3) & $18 \cdot 64$ & 0.0001935 \\
\hline \multicolumn{3}{|c|}{ Nickel (Ferric). } & & & $58 \cdot 6$ & 2 & $29 \cdot 3$ & 0.0003043 \\
\hline \multirow{2}{*}{\multicolumn{3}{|c|}{$\begin{array}{l}\text { Zinc . · : } \\
\text { Lead. }\end{array}$}} & & & $64 \cdot 9$ & 2 & $32 \cdot 45$ & 0.00033698 \\
\hline & & & & . & $206 \cdot 4$ & 2 & $103 \cdot 2$ & 0.0010716 \\
\hline \multicolumn{9}{|c|}{ Electronegative - } \\
\hline Oxygen & . & . & & . & $15 \cdot 96$ & 2 & $7 \cdot 98$ & 0.00008286 \\
\hline Chlorine & & & & & $35 \cdot 37$ & 1 & $35 \cdot 37$ & 0.0003673 \\
\hline Iodine. & & & & . & $126 \cdot 54$ & 1 & $126 \cdot 54$ & 0.0013140 \\
\hline Bromine & • & - & & • & $79 \cdot 76$ & 1 & $79 \cdot 76$ & 0.0008282 \\
\hline Nitrogen & . & • & $\cdot$ & . & $14 \cdot 01$ & 3 & $4 \cdot 67$ & $0 \cdot 00004849$ \\
\hline
\end{tabular}

* The chemical equivalent must not be confounded with the atomic weight. The atomic weight of copper is 63 , that is to say, its atoms are 63 times as heavy as atoms of hydrogen. But in chemical combinations one 
241. Weight of Element deposited. - The following equation embodies the rule for finding the weight of any given ion disengaged from an electrolytic solution during a known time by a current of known strength. Let $\mathrm{C}$ be the current (reckoned in amperes), $t$ the time (in seconds), $z$ the electrochemical equivalent, and $w$ the weight (in grammes) of the element liberated; then

$$
w=z \mathrm{C} t,
$$

or, in words, the weight (in grammes) of an element deposited by electrolysis is found by multiplying its electrochemical equivalent by the strength of the current (in amperes), and by the time (in seconds) during which the current continues to flow.

ExaMpLe.-A current from five Daniell's cells was passed through two electrolytic cells, one containing a solution of silver, the other acidulated water, for ten minutes. A tangent galvanometer in the circuit showed the strength of the current to be 5 amperes. The weight of silver deposited will be $0.001118 \times 5 \times 10 \times 60$ $=0.3354$ gramme. The weight of hydrogen evolved in the second cell will be $000010384 \times \cdot 5 \times 10 \times 60$ $=0.003115$ gramme.

242. Voltameters.-The second of the above laws, that the amount of an ion liberated in a given time is proportional to the current, is sometimes known as Faraday's Law, from its discoverer. Faraday pointed out that it affords a chemical means of measuring currents. He gave the name of voltameter to an electrolytic cell arranged for the purpose of measuring the current by the amount of chemical action it effects.

243. Water - Voltameter.-The apparatus shown in Fig. 138 might be appropriately termed a Water-

atom of copper replaces, or is " worth," two atoms of hydrogen; hence the weight of copper equivalent to 1 of hydrogen is $\frac{83}{2}=31 \frac{1}{2}$. In all cases the chemical "equivalent" is the quotient $\frac{\text { atomic weight }}{\text { valency }}$. The above table gives full statistical information. 
Voltameter, provided the tubes to collect the gases be graduated, so as to measure the quantities evolved. The weight of each measured cubic centimetre of hydrogen (at the standard temperature of $0^{\circ} \mathrm{C}$., and pressure of 760 millims.) is known to be 00008988 grammes. Hence, if the number of cubic centimetres liberated during a given time by a current of unknown strength be ascertained, the mean strength of the current can be calculated by first reducing the volume to weight, and then dividing by the electrochemical equivalent, and by the time. Each coulomb of electricity liberates in its flow $\cdot 1155$ cubic centimetres of hydrogen, and 0577 c.c. of oxygen. If these gases are collected together in a mixed-gas voltameter there will be $\cdot 1732$ c.c. of the mixed gases evolved for every coulomb of electricity which passes. To decompose 9 grammes of water, liberating 1 gramme of $\mathrm{H}$ and 8 grammes of $O$, requires 96,302 coulombs to be sent through the liquid with an electromotive force of at least 1.47 volts (see Art. 487 ).

244. Copper and Silver Voltameters. - As mentioned above, if sulphate of copper is electrolyzed between two electrodes of copper, the anode is slowly dissolved, and the kathode receives an equal quantity of copper as a deposit on its surface. One coulomb of electricity will cause 0003281 gramme to be deposited; and to deposit one gramme weight requires a total quantity of 3048 coulombs to flow through the electrodes. A current of one ampere deposits in one hour 1.177 grammes of copper, or 4.0248 grammes of silver.

By weighing one of the electrodes before and after the passage of a current, the gain (or loss) will be proportional to the quantity of electricity that has passed. In 1879 Edison, the inventor, applied this method for measuring the quantity of electricity supplied to houses for electric lights in them; a small copper voltameter being placed in a branch of the circuit which supplied the house, to serve as a meter. Various other kinds of 
supply meters have been proposed, having clockwork counters, rolling integrating disks, and other mechanical devices to add up the total quantity of electricity conveyed by the current (see Art. 442).

245. Comparison of Voltameters with Galvanometers. - It will be seen that both Galvanometers and Voltameters are intended to measure the strength of currents, one by magnetic, the other by chemical means. Faraday demonstrated that the magnetic and the chemical actions of a current are proportional to one another. In Fig. 139 is shown a circuit that is branched so that the current divides, part going through a branch of small resistance $r$ and part through a branch of larger resistance $R$. The current will divide, the greater part going by the path of lesser resistance. Three amperemeters are used. It will be found that the number of amperes

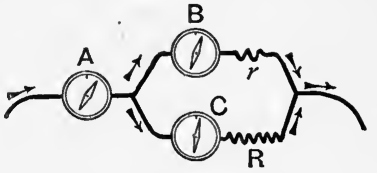

Fig. 139.

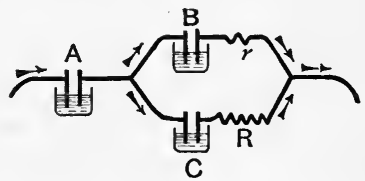

Fig. 140. in the main circuit is equal to the sum of the amperes in the two branches. In Fig. 140 the three amperemeters have been replaced by three copper voltameters. The weight of copper deposited in the voltameter $\mathrm{A}$ in the main circuit will be found to be equal to the sum of the weights deposited in $\mathrm{B}$ and $\mathrm{C}$ in the two branches. A galvanometer shows, however, the strength of the current at any moment, and its variations in strength from one moment to another, by the position of the needle. In a voltameter, a varying current may liberate the atoms of copper or the bubbles of gas rapidly at one moment, and slowly the next, but all the varying quantities will be simply added together in the total yield. 
In fact, the voltameter gives us the "time integral" of the current. It tells us what quantity of electricity has flowed through it during the experiment, rather than how strong the current was at any one moment.

246. Chemical Test for Weak Currents.-A very feeble current suffices to produce a perceptible amount of change in certain chemical substances. If a few crystals of the white salt iodide of potassium are dissolved in water, and then a little starch paste is added, a very sensitive electrolyte is obtained, which turns to a dark blue colour at the anode when a very weak current passes through it. The decomposition of the salt liberates iodine at the anode, which, acting on the starch, forms a coloured compound. White blottingpaper, dipped into the prepared liquid, and then laid on the kathode and touched by the anode, affords a convenient way of examining the discoloration due to a current. A solution of ferrocyanide of potassium affords when using an anode of iron the well-known tint of Prussian blue. Bain proposed to utilize this in a Chemical Writing Telegraph, the short and long currents transmitted along the line being thus recorded in blue marks on a strip of prepared paper, drawn along by clockwork under an iron stylus joined to the positive wire. Faraday showed that chemical discoloration of paper moistened with starch and iodide of potassium was produced by the passage of electricity from sources of all different kinds-frictional, voltaic, thermo-electric, and magneto-electric, - even by that evolved by the Torpedo and the Gymnotus. In fact, he relied on this chemical test as one proof of the identity of the different kinds.

247. Internal and External Actions.-In an earlier lesson it was shown that the quantity of chemical action inside the cells of the battery was proportional to the current. Hence, Law (i.) of Art. 240 applies both to the portion of the circuit within the battery and to that without it. 
Stppose 3 Daniell's cells are being employed to decompose water in a voltameter. Then while 1 gramme weight $(11,126 \mathrm{cub}$. centims.) of hydrogen and 8 grammes (5563 c.c.) of oxygen are set free in the voltameter, 31.5 grammes of copper will be deposited in each cell of the battery, and (neglecting loss by local action) 32.5 grammes of zinc will be dissolved in each cell.

248. Reversibility.-It will therefore be evident that the electrolytic cell is the converse of the voltaic cell. The chemical work done in the voltaic cell furnishes the energy of the current which that cell sets up in the circuit. In the electrolytic cell chemical work is performed, the necessary energy being furnished by the current of electricity which is sent into the cell from an independent battery or other source. It is important to note the bearing of this with respect to the energy of the circuit. Suppose a current of strength C to flow through a cell of which the electromotiveforce is $\mathrm{E}$, and which acts in the same direction as the current. The energy

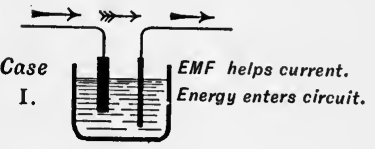
given to the circuit per second by this cell will be (Art. 435 ) the product of $\mathrm{C}$ and $\mathrm{E}$; the chemical energy of the voltaic cell entering the circuit at the place where the chemical action is going on. In Fig. 141 the current is indicated by the arrows with thick shafts, the electromotive-force by the feathered arrow. For example, if 10 amperes flow through a Daniell cell acting with $1 \cdot 1$ volts of electromotive-force, the power given ont by the cell is 11 watts (Art. 435). But if the cell be so connected into the circuit, as in Case II. of Fig. 141, that the E.M.F. of the cell opposes the current that is being driven along the circuit, then the energy per second 
will be the product of $\mathrm{C}$ and $-\mathrm{E}$, or $-\mathrm{CE}$, the negative sign indicating that the circuit is losing energy, part of its energy being absorbed in the cell in doing chemical work. If the current is sent backwards through a Daniell cell the chemical processes are reversed, copper is dissolved and zinc is deposited. But all cells are not reversible in their chemical action.

A theory of electrolysis, and some examples of its application, are given in Art. 488 on Electro-chemistry.

\section{Lesson XX.-Physical and Physiological Effects of the}

\section{Current}

249. Molecular Actions.--Metal conductors, when subjected to the prolonged action of currents, undergo slow molecular changes. Wires of copper and brass gradually become brittle under its influence. During the passage of the current through metallic wires their cohesion is temporarily lessened, and there also appears to be a decrease in their coefficient of elasticity. It was thought by Edlund that a definite elongation could be observed in strained wires when a current was passed through-them; but it has not yet been satisfactorily shown that this elongation is independent of the elongation due to the heating of the wire owing to the resistance it opposes to the current.

250. Electric Osmose.-Porret observed that if a strong current is led into certain liquids, as if to electrolyze them, a porous partition being placed between the electrodes, the current meehanically carries part of the liquid through the porous diaphragm, so that the liquid is forced up to a higher level on one side than on the other. This phenomenon, known as electric osmose, is most manifest when badly-conducting liquids, such as alcohol and bisulphide of carbon, are used. The transfer through the diaphragm takes place in the direction of 
the current; that is to say, the liquid is higher about the kathode than round the anode.

251. Electric Distillation.-Closely connected with the preceding phenomenon is that of the electric distillation of liquids. It was noticed by Beccaria that an electrified liquid evaporated more rapidly than one not electrified. Gernez has recently shown that in a bent closed tube, containing two portions of liquid, one of which is made highly + and the other highly -, the liquid passes over from + to - . This apparent distillation is not due to difference of temperature, nor does it depend on the extent of surface exposed, but is effected by a slow creeping of the liquid along the interior surface of the glass tubes. Bad conductors, such as turpentine, do not thus pass over.

252. Diaphragm Currents.-Professor Quincke discovered that a current is set up in a liquid when it is forced by pressure throngh a porous diaphragm. This phenomenon may be regarded as the converse of electric osmose. The E.M.F. of the current varies with the pressure and with the nature of the diaphragm. When water was forced at a pressure of one atmosphere through sulphur, the difference of potential was over 9 volts. With diaphragms of porcelain and bladder the differences were only $\cdot 35$ and $\cdot 01$ volts respectively.

253. Electro-Capillary Phenomena.-If a horizontal glass tube, turned up at the ends, be filled with dilute acid, and a single drop of mercury be placed at about the middle of the tube, the passage of a current through the tube will cause the drop to move along towards the negative pole. It is believed that the liberation of very small quantities of gas by electrolysis at the surface where the mercury and acid meet alters the surface-tension very considerably, and thus a movement results from the capillary forces. Lippmann, Dewar, and others have constructed upon this principle capillary electrometers, in which the pressure of a column of liquid 
is made to balance the electro-capillary force exerted at the surface of contact of mercury and dilute acid, the electro-capillary force being nearly proportional to the electromotive-force when this does not exceed one volt. Fig. 142 shows the capillary electrometer of Dewar. A glass tube rests horizontally between two glass dishes

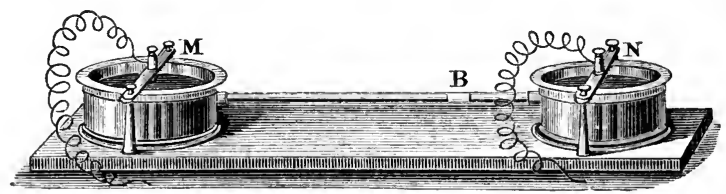

Fig. 142.

in which holes have been bored to receive the ends of the tube. It is filled with mercury, and a single drop of dilute acid is placed in the tube. Platinum wires to serve as electrodes dip into the mercury in the dishes. An E.M.F. of only $\frac{1}{300}$ volt suffices to produce a measurable displacement of the drop. The direction of the displacement varies with that of the current.

254. Physiologisal Actions.-Currents of electricity passed through the limbs affect the nerves with certain painful sensations, and cause the muscles to undergo involuntary contractions. The sudden rush of even a small charge of electricity from a Leyden jar charged to a high potential, or from an induction coil (see Fig. 135), gives a sharp and painful shock to the system. The current from a few strong Grove's cells, conveyed through the body by grasping the terminals with moistened hands, gives a very different kind of sensation, not at all agreeable, of a prickling in the joints of the arms and shoulders, but not producing any spasmodic contractions, except it be in nervous or weakly persons, at the sudden making or breaking of the circuit. The difference between the two cases lies in the fact that the tissues of the body offer a very con- 
siderable resistance, and that the difference of potential in the former case may be many thousands of volts; hence, though the actual quantity stored up in the Leyden jar is very small, its very high E.M.F. enables it at once to overcome the resistance. The battery, although it might, when working through a good conductor, afford in one second a thousand times as much electricity, cannot, when working through the high resistance of the body, transmit more than a small fraction, owing to its limited E.M.F.

After the discovery of the shock of the Leyden jar by Cunæus in 1745 many experiments were tried. Louis XV. of France caused an electric shock from a battery of Leyden jars to be administered to 700 Carthusian monks joined hand in hand, with prodigious effect. Franklin killed a turkey by a shock from a Leyden jar.

In 1752 Sulzer remarked that "if you join two pieces of lead and silver, and then lay them upon the tongue, you will notice a certain taste resembling that of green vitriol, while each piece apart produces no such sensation." This galvanic taste, not then suspected to have any connexion with electricity, may be experienced by placing a silver coin on the tongue and a steel pen under it, the edges of them being then brought into metallic contact. The same taste is noticed if the two wires from the poles of a single voltaic cell are placed in contact with the tongue.

Ritter discovered that a feeble current transmitted through the eyeball produces the sensation as of a bright flash of light by its sudden stimulation of the optic nerve. A stronger current transmitted by means of moistened conductors attached to the battery terminals gave a sensation of blue and green colours in flowing between the forehead and the hand. Von Helmholtz, repeating this experiment, observed only a wild rush of colour. Dr. Hunter saw flashes of light when a piece of metal placed under the tongue was touched against another which 
touched the moist tissues of the eye. Volta and Ritter heard musical sounds when a current was passed through the ears ; and Humboldt found a sensation to be produced in the organs of smell when a current was passed from the nostril to the soft palate. Each of the specialized senses can be stimulated into activity by the current. Man possesses no specialized sense for the perception of electrical forces, as he does for light and for sound ; but there is no reason for denying the possibility that some of the lower creatures may be endowed with a special electrical sense.

The following experiment shows the effect of feeble currents on cold-blooded creatures. If a copper (or silver) coin be laid on a piece of sheet zinc, and a coinmon garden snail be set to crawl over the zinc, directly it comes into contact with the copper it will suddenly pull in its horns, and shrink in its body. If it is set to crawl over two copper wires, which are then placed in contact with a feeble voltaic cell, it immediately announces the establishment of a current by a similar contraction.*

255. Muscular Contractions.-In 1678 Swammerdam showed to the Grand Duke of Tuscany that when a portion of muscle of a frog's leg hanging by a thread of nerve bound with silver wire was held over a copper support, so that both nerve and wire touched the copper, the muscle immediately contracted. More than a century later Galvani's attention was drawn to the subject by his observation of spasmodic contractions in the legs of freshly-killed frogs under the influence of the "returnshock" experienced every time a neighbouring electric machine was discharged. Unaware of Swammerdam's experiment, he discovered in 1786 the fact (alluded to in Art. 163 as leading ultimately to the discovery of the Voltaic Pile) that when nerve and muscle touch two dissimilar metals in contact with one another a contraction of the muscle takes place. The limbs of the frog,

\footnotetext{
* It will scarcely be credited that a certain Jules Alix once seriously pro-
} posed a system of telegraphy based on this physiological plenomenon. 
prepared as directed by Galvani, are shown in Fig. 143. After the animal has been killed the hind limbs are detached and skinned ; the crural nerves and their attachments to the lumbar vertebræ remaining. For some hours after death the limbs retain their contractile power. The frog's limbs thus prepared form an excessively delicate galvanoscope: with them, for example, the excessively

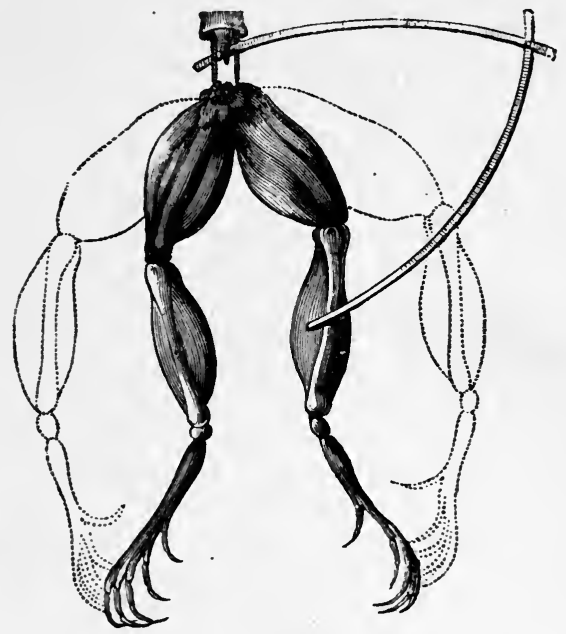

Fig. 143.

delicate induction-currents of the telephone (Lesson LIII.) can be shown, though the most sensitive galvanometers barely detect them. Galvani and Aldini proved that other creatures undergo like effects. With a pile of 100 pairs Aldini experimented on newly-killed sheep, oxen, and rabbits, and found them to suffer spasmodic muscular contractions. Humboldt proved the same on fishes; and Zanotti, by sending a current through a newly-killed grasshopper, caused it to emit its familiar chirp. Aldini, 
and later Dr. Ure of Glasgow, experimented on the bodies of executed criminals, with a success terrible to behold. The facial muscles underwent horrible contortions, and the chest heaved with the contraction of the diaphragm. The small muscles attached to the roots of the hairs of the head appear to be markedly sensitive to electrical conditions from the readiness with which electrification causes the hair to stand on end.

The resistance of the human body to the flow of electric current through it depends mainly on the dryness of the skin. It may vary from 10,000 down to 300 ohms when the skin is moist. From experiments made in America in connexion with the execution of criminals, it was found that the average resistance of the human body is $2500 \mathrm{ohms}$, and that 3000 (alternating) volts applied between the head and spine caused instantaneous death.

A current of as much as 20 milliamperes produces terrible muscular contractions, whilst a current of 2 amperes traversing a vital part is almost certainly fatal. The effect of the current is two-fold; in the first place it acts upon the nerves, causing spasms, secondly it destroys the tissue either by burning or by electrolysis, the blood becoming coagulated. To restore a person who has been rendered insensible by an electric shock, all the same restoratives should be used as for a person drowned.

256. Conditions of Muscular Contraction.-To produce muscular contraction the current must traverse a portion of the nerve longitudinally. In a freshly-prepared frog the current causes a contraction only momentarily when the circuit is made or broken. A rapidly interrupted current will induce a second contraction before the first his had time to pass off, and the muscle may exhibit thus a continuous contraction resembling tetanus. The prepared frog after a short time becomes less sensitive, and a "direct" current (that is to say, one passing along the nerve in the direction from the brain to the muscle) only produces an effect when circuit is made, while an "inverse" 
current only produces an effect when the circuit is broken. Matteucci, who observed this, also discovered by experi' ments on living animals that there is a distinction between the conductivity of sensory and motor nerves,-a "direct" current affecting the motor nerves on making the circuit, and the sensory nerves on breaking it; while an "inverse" current produced inverse results. Little is, however, yet known of the conditions of conductivity of the matter of the nerves; they conduct better than muscular tissue, cartilage, or bone; but of all substances in the body the blood conducts best. Powerful currents doubtless electrolyze the blood to some extent, coagulating it and the albumin it contains. The power of contracting under the influence of the current appears to be a distinguishing property of protoplasm wherever it occurs. The amœba, the most structureless of organisms, suffers contractions. Ritter discovered that the sensitive plant shuts up when electrified, and Burdon Sanderson has shown that this property extends to other vegetables, being exhibited by the carnivorous plant, the Dionæa or Venus's Fly Trap.

257. Animal Electricity.-Although, in his later writings at least, Galvani admitted that the electricity thus operating arose from the metals employed, he insisted on the existence of an animal electricity resident in the muscular and nervous structures. $\mathrm{He}$ showed that contractions could be produced without using any metals at all by merely touching a nerve at two different points along its length with a morsel of muscle cut from a living frog; and that a conductor of one metal when joining a nerve to a muscle also sufficed to cause contraction in the latter. Galvani and Aldini regarded these facts as a disproof of Volta's contact-theory. Volta regarded them as proving that the contact between nerve and muscle itself produced (as in the case of two dissimilar metals) opposite electrical conditions. Nobili, later, showed that when the nerve and the muscle of the frog are respec- 
tively connected by a water-contact with the terminals of a delicate galvanometer, a current is produced which lasts several hours: he even arranged a number of frogs' legs in series, like the cells of a battery, and thus increased the current. Matteucci showed that through the muscle alone there may be an electromotive-force. Du Bois Reyinond has shown that if the end of a muscle be cut across, the ends of the muscular fibres of the transverse section are negative, and the sides of the muscular fibres are positive, and that this difference of potential will produce a current even while the muscle is at rest. To demonstrate this he employed a fine astatic galvanometer with 20,000 turns of wire in its coils ; and to obviate errors arising from the contact of the ends of the wires with the tissues, unpolarizable electrodes were used, made by plunging terminal zine points into a saturated solution of sulphate of zinc, contained in a fine glass tube, the end of which was stopped with a porous plug of moistened china clay. Normal muscle at rest shows no current whatever between its parts. Injured muscle at rest shows a current from the injured toward the uninjured part (returning toward the injured part through the galvanometer). Normal muscle when active shows a current from the active part toward the resting part. Du Bois Reymond obtained currents from his own muscles by dipping the tips of his forefingers into two cups of salt water conmunicating with the galvanometer terminals. A sudden contraction of the muscles of either arm produced a current from the contracted toward the uncontracted muscles. Dewar has shown that when light falls upon the retina of the eye an electric current is set up in the optic nerve. In the skin, and especially in the skin of the common eel, there is an electromotive-force from without inwards.

258. Surgical Applications. - Electric currents have been successfully employed as an adjunct in restoring persons rescued from drowning; the contraction of the diaphragm and chest muscles serving to start respiration. 
Since the discovery of the Leyden jar many attempts have been made to establish an electrical medical treatment. Discontinuous currents, particularly those furnished by small induction-coils and magneto-electric machines, are employed by practitioners to stimulate the nerves in paralysis and other affections. Living muscle when stimulated by a single shock such as a single spark from an electric machine causes a single muscular twitch, the contraction lasting about $\frac{1}{10}$ second. A rapid succession of shocks, such as is given by an induction coil or an alternating current, causes a succession of contractions which blend into a permanent state of contraction which lasts as long as the stimulating cause is applied. When a continuous current is used, not exceeding 4 or 5 milliamperes, there is a twitch on making the circuit followed by a relaxation that lasts as long as the current lasts, but with another twitch when the circuit is broken. With currents of 16 to 25 milliamperes the contraction is permanent till the current is cut off. In certain kinds of paralysis all irritability of muscle disappears. Application of electric stimulus in health generally slightly increases muscular power, and in debility after illness may aid nutrition.

The usual pathological dose of current is from 2 to 10 milliamperes. Apparatus pretending to cure, and incapable of furnishing such currents, is worthless. Continuous currents appear to produce a sedative effect around the anode, which is of service in neuralgia and painful affections, and an increase in irritability around the kathode, useful in certain cases of paralysis. In cases of paralysis due to lesion of nerve connexions or degeneration of nerve centres electricity is unavailing. The continuous current is alsoemployed electrolytically to disperse tumours. Alternate currents, and rapidly interrupted uni-directional currents, stimulate the nerves. Bare metal electrodes must never be allowed to touch the skin unless the object is to produce sores. They should be covered with moist washleather. 


\title{
非art Secand
}

\author{
CHAPTER IV
}

\section{ELECTROSTATICS}

\section{Lesson XXI.-Theory of Potential}

259. By the lessons in Chapter I. the student will have obtained some elementary notions upon the existence and measurement of definite quantities of electricity. In the present lesson, which is both one of the hardest and one of the most important to the beginner, and which he must therefore study the more carefully, the laws which concern the magnitude of electrical quantities and their measurement are more fully explained. In no branch of knowledge is it more true than in electricity, that "science is measurement." That part of the science of electricity which deals with the measurement of charges of electricity is called Electrostatics. We shall begin by discussing first the simple laws of electric force, which were brought to light in Chapter I. by simple experimental means.

260. First Law of Electrostatics. - Electric charges of similar sign repel one another, but electric charges of opposite signs attract one another. The fundamental facts expressed in this Law were fully explained in 
Lesson I. Though familiar to the student, and apparently simple, these facts require for their complete explanation the aid of advanced mathematical analysis. They will here be treated as simple facts of observation.

261. Second Law of Electrostatics.-The force exerted between two charges of electricity (supposing them to be collected at points or on two small spheres) is directly proportional to their product, and inversely proportional to the square of the distance between them. This law, discovered by Coulomb, and called Coulomb's Law, was briefly alluded to (on p. 21) in the account of experiments made with the torsion-balance; and examples were there given in illustration of both parts of the law. We saw, too, that a similar law held good for the forces exerted between two magnetic point-poles. Coulomb applied also the method of oscillations to verify the indications of the torsion-balance and found the results entirely confirmed. We may express the two clauses of Coulomb's Law in the following symbolic manner. Let $f$ stand for the force, $q$ for the quantity of electricity in one of the two charges, and $q_{1}$ for that of the other charge, and let $r$ stand for the distance between them. Then,

(1) $f$ is proportional to $q \times q^{\prime}$,

and $\quad$ (2) $f$ is proportional to $\frac{1}{r^{2}}$.

These two expressions may be combined into one; and it is most convenient so to choose our units or standards of measurement that we may write our symbols as an equation :-

$$
f=\frac{q \times q^{\prime}}{r^{2}} .
$$

262. Unit of Electric Quantity.-If we are, however, to write this as an equality, it is clear that we must choose our unit of electricity in accordance with the units already fixed for measuring force and distance. 
Electricians of all nations have agreed in adopting a system which is based upon three fundamental units: viz. the Centimetre for a unit of length; the Gramme for a unit of mass; the Second for a unit of time. All other units can be derived from these, as is explained in the note at the end of this lesson. Now, amongst the derived units of this system is the unit of force, named the Dyne, which is that force which, acting for one second on a mass of one gramme, imparts to it a velocity of one centimetre per second. Taking the dyne then as the unit of force, and the centimetre as the unit of length (or distance), we must find a unit of electric quantity to agree with these in our equation. It is quite clear that if $q, q^{\prime}$, and $r$ were each made equal to 1 (that is, if we took two charges of value 1 each, and placed them one centimetre apart), the value of $\frac{q \times q^{\prime}}{r^{2}}$ would be $\frac{1 \times 1}{1 \times 1}$, which is equal to 1 . Hence we adopt, as our Definition of a Unit of Electricity, ${ }^{*}$ the following, which we briefly gave at the end of Lesson II. One Unit of Electricity is that quantity which, when placed at a distance of one centimetre (in air) from a similar and equal quantity, repels it with a force of one dyne.

An example will aid the student to understand the application of Coulomb's Law.

Example.-Two small spheres, charged respectively with 6 units and 8 units of + electricity, are placed 4 centimetres apart; find what force they exert on one another. By the formula, $f=\frac{q \times q^{\prime}}{r^{2}}$, we find $f=$ $\frac{6 \times 8}{4^{2}}=\frac{48}{16}=3$ dynes.

The force in the above example would clearly be a force of repulsion. Had one of these charges been

* That is one unit, in the electrostatic system. It is only 3000000000 of the quantity called 1 coulomb. 
negative, the product $q \times q^{\prime}$ would have had a - value, and the answer would have come out as minus 3 dynes. The presence of the negative sign, therefore, prefixed to a force, will indicate that it is a force of attraction, whilst the + sign would signify a force of repulsion.

The intensity of an electric field (Art. 266) being measured by the force it exerts on a unit charge, it at once follows that at a distance of $r$ (in air) from a charge $q$ the intensity of the electric field due to that charge will be $q / r^{2}$. If the intervening medium be not air, but have a specific dielectric capacity $k$, the field will be only $q / k r^{2}$.

263. Potential.-We must next define the term potential, as applied to electric forces; but to make the meaning plain a little preliminary explanation is necessary. Suppose we had a + charge on a small insulated sphere A (see Fig. 144), placed by itself far from all other electric charges and conductors. If we were to bring another positively-charged body B near it, A would repel B. But the repelling force would depend on the quantity of the new charge, and on the distance at which it was

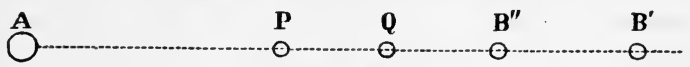

Fig. 144.

placed. Suppose the new charge thus brought near to be one + unit; when $B$ was a long way off it would be repelled with a very slight force, and very little work need be expended in bringing it up nearer against the repelling forces exerted by $A$; but as $B$ was brought nearer and nearer to $A$, the repelling force would grow greater and greater, and more and more work would have to be done against these opposing forces in bringing up B. Suppose that we had begun at an infinite distance away, and that we pushed up our little test charge $B$ from $B^{\prime}$ to $B^{\prime \prime}$ and then to $Q$, and so finally moved it up to the point $P$, against the opposing forces exerted by $A$, we 
should have had to spend a certain amount of work; that work represents the potential * at the point, $\mathrm{P}$ due to $\mathrm{A}$. For the following is the definition of electric potential :The potential at any point is the work that must be spent upon a unit of positive electricity in bringing it up to that point from an infinite distance. Had the charge on A been a - charge, the force would have been one of attraction, in which case we should have theoretically to measure the potential at $\mathrm{P}$, either by the opposite process of placing there $\mathrm{a}+$ unit, and then removing it to an infinite distance against the attractive forces, or else by measuring the amount of work which would be done by a + unit in being attracted up to $\mathrm{P}$ from an infinite distance.

It can be shown that where there are more electrified bodies than one to be considered, the potential due to them at any point is the sum of the potentials (at that point) of each one taken separately.

It can also be shown that the potential at a point $\mathrm{P}$, near an electrified particle $A$, is equal to the quantity of electricity at $\mathrm{A}$ divided by the distance between $\mathrm{A}$ and $\mathrm{P}$. Or, if the quantity be called $q$, and the distance $r$, the potential is $q \div r$. $\dagger$

Proof.-First determine the difference of potential between point $\mathrm{P}$ and point $\mathrm{Q}$ due to a charge of electricity $q$ on a small sphere at $\mathrm{A}$.

Call distance $\mathrm{AP}=r$, and $\mathrm{AQ}=r^{\prime}$. Then $\mathrm{PQ}=r^{\prime}-r$. The difference of potential between $Q$ and $P$ is the work done in moving $\mathrm{a}+$ unit from $\mathrm{Q}$ to $\mathrm{P}$ against the force ; and since

* In its widest meaning the term "potential" must be understood as "power to do work." For if we have to do a certain quantity of work against the repelling force of a charge in bringing up a unit of electricity from an infinite distance, just so much work has the charge power to do, for it will spend an exactly equal amount of work in pushing the unit of electricity back to an infinite distance. If we lift a pouncl five feet high against the force of gravity, the weight of the pound can in turn do five foot-pounds of work in falling back to the ground.

t The complete proof would require an elementary application of the integral calculus, but an easy geometrical demonstration, sufficient for present purposes, is given below. 
work $=($ average $)$ force $\times$ distance through which it is overcome

$\mathrm{V}_{\mathbf{P}}-\mathrm{V}_{\mathbf{Q}}=f\left(r^{\prime}-r\right)$.

Force at P exerted by $q$ on a + unit $=q / r^{2}$,

and the force at $\mathrm{Q}$ exerted by $q$ on a + unit $=q / r^{\prime 2}$.

Suppose now that the distance $\mathrm{PQ}$ be divided into any number (n) of equal parts $r r_{1}, r_{1} r_{2}, r_{2} r_{3}, \ldots \ldots r_{n-1} r^{\prime}$.

The force at $r=q / r^{2}$.

$, \quad, \quad r_{1}=q / r_{1}^{2} \ldots$. . etc.

Now since $r_{1}$ may be made as close to $r$ as we choose, if we only take $n$ a large enough number, we shall commit no serious error

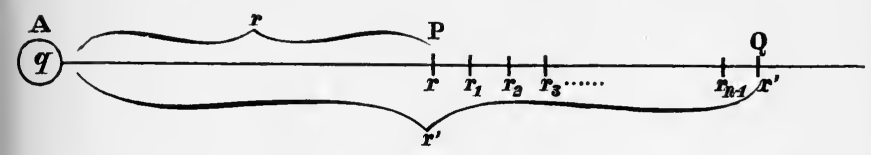

Fig. 145.

in supposing that $r \times r_{1}$ is a fair mean between $r^{2}$ and $r_{1}^{2}$; hence we may assume the average force over the short length from $r$ to $r_{1}$ to be $\frac{q}{r r_{1}}$.

Hence the work done in passing from $r_{1}$ to $r$ will be

$$
\begin{aligned}
& =\frac{q}{r r_{1}}\left(r_{1}-r\right) \\
& =q\left(\frac{1}{r}-\frac{1}{r_{1}}\right) .
\end{aligned}
$$

On a similar assumption, the work done in passing from $r_{2}$ to $r_{1}$ will be

$$
\begin{aligned}
& =q\left(\frac{1}{r_{1}}-\frac{1}{r_{2}}\right), \text { and that done from } r_{3} \text { to } r_{2} \text { will be } \\
& =q\left(\frac{1}{r_{2}}-\frac{1}{r_{2}}\right) \text {, etc., giving us } n \text { equations, of which the }
\end{aligned}
$$

last will be the work done in passing from $r^{\prime}$ to $r_{n-1}$

$$
=q\left(\frac{1}{r_{n-1}}-\frac{1}{r^{\prime}}\right) \text {. }
$$

Adding up all these portions of the work, the intermediate 
values of $r$ cancel out, and we get for the work done in passing from $\mathrm{Q}$ to $\mathrm{P}$

$$
\mathrm{V}_{\mathbf{P}}-\mathrm{V}_{\mathbf{Q}}=q\left(\frac{1}{r}-\frac{1}{r^{\prime}}\right) \text {. }
$$

Next suppose $Q$ to be an infinite distance from $A$. Here $r^{\prime}$ $=$ infinity, and $\frac{1}{r^{\prime}}=0$. In that case the equation becomes

$$
\mathrm{V}_{\mathbf{P}}=\frac{q}{r}
$$

If there are a number of electrified particles at different distances from $\mathrm{P}$, the separate values of the potential $q / r$ due to each electrified particle separately can be found, and therefore the potential at $P$ can be found by dividing the quantity of each charge by its distance from the point $P$, and then adding up together the separate amounts so obtained. The symbol V is generally used to represent potential. The potential at $P$ we will call $V_{P}$, then

$$
\begin{array}{ll}
\mathrm{V}_{\mathrm{P}} & =\frac{q^{\prime}}{r^{\prime}}+\frac{q^{\prime \prime}}{r^{\prime \prime}}+\frac{q^{\prime \prime \prime}}{r^{\prime \prime \prime}}+\ldots . \text { etc. } \\
\text { or } \quad \mathrm{V}_{\mathrm{P}} & =\Sigma \frac{q}{r} .
\end{array}
$$

This expression $\Sigma q / v$ represents the work done on or by a unit of + electricity when moved up to the given point $P$ from an infinite distance, according as the potential at $\mathrm{P}$ is positive or negative.

264. Zero Potential. - At a place infinitely distant from all electrified bodies there would be no electric forces and the potential would be zero. For purposes of convenience it is, however, usual to consider the potential of the earth as an arbitrary zero, just as it is convenient to consider "sea-level" as a zero from which to measure heights or depths (see Art. 269).

265. Difference of Potentials.-Since potential represents the work that must be done on a + unit in bringing it up from an infinite distance, the difference of 
potential between two points is the work to be done on or by a + unit of electricity in carrying it from one point to the other. Thus if $\mathrm{V}_{\mathrm{P}}$ represents the potential at $\mathrm{P}$, and $V_{Q}$ the potential at another point $Q$, the difference of potentials $V_{P}-V_{Q}$ denotes the work done in moving up the + unit from $Q$ to $P$. It is to be noted that since this value depends only on the values of the potential at $P$ and at $Q$, and not on the values at intermediate points, the work done will be the same, whatever the path along which the particle moves from $Q$ to $P$. In the same way it is true that the expenditure of energy in lifting a pound (against the earth's attraction) from one point to another on a higher level, will be the same whatever the path along which the pound is lifted.

266. Electric Force.-The definition of "work" is the product of the force overcome into the distance through which the force is overcome; or work $=$ force $\times$ distance through which it is overcome.

Hence, if the difference of potential between two points is the work done in moving up. our + unit from one point to the other, it follows that the average electric force between those points will be found by dividing the work so done by the distance between the points; or $\frac{\mathrm{V}_{\mathrm{P}}-\mathrm{V}_{\mathrm{Q}}}{\mathrm{PQ}}=f$ (the average electric force along the line $P Q$ ). The (average) electric force is therefore the rate of change of potential per unit of length. If $\mathrm{P}$ and $\mathrm{Q}$ are near together the force will be practically uniform between $\mathrm{P}$ and $\mathrm{Q}$. The term electromotive intensity is sometimes used for the force in an electric field.

We may represent this intensity of the electric field by supposing the number of electric lines per square centimetre to be drawn to represent the number of dynes of force on a + unit placed at the point.

267. Equipotential Surfaces.-A charge of electricity collected on a small sphere acts on external bodies as if the charge were all collected into one point at its 
centre.* We have seen that the force exerted by such a charge falls off at a distance from the ball, the force becoming less and less as the square of the distance increases. But the force is the same in amount at all points equally distant from the small charged sphere. And the potential is the same at all points that are equally distant from the charged sphere. If, in Fig. 145, the point A represents the sphere charged with $q$ units of
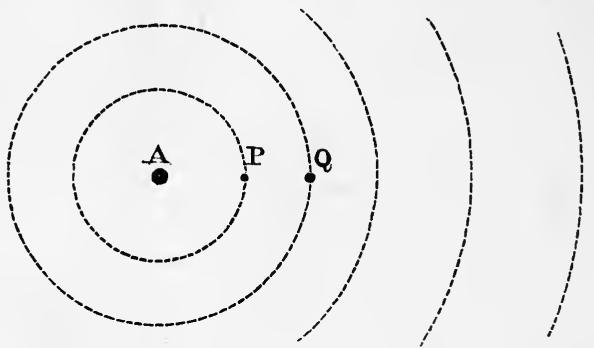

Fig. 146.

electricity, then the potential at $\mathrm{P}$, which we will call $\mathrm{V}_{P}$, will be equal to $q / r$, where $r$ is the distance from $A$ to $P$. But if we take any other point at the same distance from $\mathrm{A}$ its potential will also be $q / r$. Now all the points that are the same distance from $A$ as $P$ is, will be found to lie upon the surface of a sphere whose centre is at $A$, and which is represented by the circle drawn through $\mathrm{P}$, in Fig. 146. All round this circle the potential will have equal values; hence this circle represents an equipotential surface. The work to be done in bringing up a + unit from an infinite distance will be the same, no

* The student must be warned that this ceases to be true if other charges are brought very near to the sphere, for then the electricity will no longer be distributed uniformly over its surface. It is for this reason that we have said, in describing the measurement of electrical forces with the torsion balance, that " the balls inust be very small in proportion to t'ie distances between them." 
matter what point of this equipotential surface it is brought to, ancl to move it about from one point to another in the equipotential surface requires no further overcoming of the electrical forces, and involves therefore no further expenditure of work. At another distance, say at the point $Q$, the potential will have another value, and through this point $Q$ another equipotential surface may be drawn. Suppose we chose $Q$ so far from $P$ that to push up a unit of + electricity against the repelling force of $A$ required the expenditure of just one erg of work (for the definition of one $\mathrm{erg}$ see the Note on Units at the end of this lesson); there will be then unit difference of potential between the surface. drawn through $Q$ and that drawn through $P$, and it will require one erg of work to carry a + unit from any point on the one surface to any point on the other. In like manner we might construct a whole system of equipotential surfaces about the point $A$, cloosing them at such distances that there should be unit difference of potential between each one and the next. The widths between them would get wider and wider, for, since the force falls off as you go farther from A, you must, in doing one erg of work, bring up the + unit through a longer distance against the weaker opposing force.

The form of the equipotential surfaces about two small electrified bodies placed near to one another would not be spherical ; and around a number of electrified bodies placed near to one another the equipotential surfaces would be highly complex in form.

268. Lines of Force.-The electric force, whether of attraction or repulsion, always acts across the equipotential surfaces in a direction normal to the surface. The lines which mark the direction of the resultant electric forces are sometimes called lines of electric.force. In the case of the single electrified sphere the lines of force would be straight lines, radii of the system of equipotential spheres. In general, however, lines of force are 
curved; in this case the resultant force at any point would be in the direction of the tangent to the curve at that point. Two lines of force cannot cut one another, for it is impossible ; the resultant force at a point cannot act in two directions at once. The positive direction along a line of force is that direction in which a small positively-charged body would be impelled by the electric force if free to move. A space bounded by a number of lines of force is sometimes spoken of as a tube of force. All the space, for example, round a small insulated electrified sphere may be regarded as mapped out into a number of conical tubes, each having its apex at the centre of the sphere. The total electric force exerted across any section of a tube of force is constant wherever the section be taken.

269. Potential within a Closed Conductor.The experiments related in Arts. 32 to 36 prove most convincingly that there is no electric force inside a closed conductor due to charges outside or on the surface of the conductor. Now we have shown above that electric force is the rate of change of potential per unit of length. If there is no electric force there is no change of potential. The potential within a closed conductor (for example, a hollow sphere) due to charges outside or on the surface is therefore the same all over the interior; the same as the potential of the surface. The surface of a closed conductor is necessarily an equipotential surface. If it were not at one potential there would be a flow of electricity from the higher potential to the lower, which would instantaneously establish equilibrium and reduce the whole to one potential. The student should clearly distinguish between the surface-density at a point, and the potential at that point due to neighbouring charges of electricity. We know that when an electrified body is placed near an insulated conductor the nearer and farther portions of that conductor exhibit induced charges of opposite kinds. Yet all is at one potential. If the + 
and - charges on the conductor had not separated by a movement of electricity from one side to the other, a difference of potential would exist between those sides because they are at different distances from the electrified body. But that is a state of affairs which could not continue in the conductor, for the difference of potential would cause electricity to flow until the combined potential due to the electrified body and the charges at the opposite sides was the same at every point in the conductor.

The potential at any point in a conducting sphere (hollow or solid) due to an electrified particle A, sitnated at a point outside (Fig. 148), is equal to the quantity of electricity $q$ at $\mathrm{A}$ divided by the distance between $\mathrm{A}$ and the centre of the sphere. For if $\mathrm{B}$ be the centre of the sphere, the potential at $\mathrm{B}$ due to $q$ is $q / r$, where $r=\mathrm{AB}$; but all points in the sphere are at the same potential, therefore they are all at the potential $q / r$.

The earth is a large conducting sphere. Its potential, due to a positive charge $q$ near to its surface, is $q / r$, where $r$ may be taken as the radius of the earth; that is, $636,000,000$ centimetres. But it is impossible to produce a + charge $q$ without generating also an equal negative charge $-q$; so the potential of the earth due to both charges is $q / r-q / r=0$ (see Art. 264).

270. Law of Inverse Squares.-An important consequence follows from the absence of electric force inside a closed conductor due to a charge on its surface ; this fact enables us to demonstrate the necessary truth of the "law of inverse squares" which was first experimentally, though roughly, proved by Coulomb with the torsion balance. Suppose a point $\mathrm{P}$ anywhere inside a hollow sphere charged with electricity (Fig. 147). The charge is uniform all over, and the quantity of electricity on any small portion of its surface will be proportional to the area of that portion. Consider a small portion of the surface $\mathrm{AB}$. The charge on $\mathrm{AB}$ would repel a + unit 
placed at $\mathrm{P}$ with a certain force. Now draw the lines $\mathrm{AD}$ and $\mathrm{BC}$ through $\mathrm{P}$, and regard these as mapping out a small conical surface of two sheets, having its apex at $\mathrm{P}$;

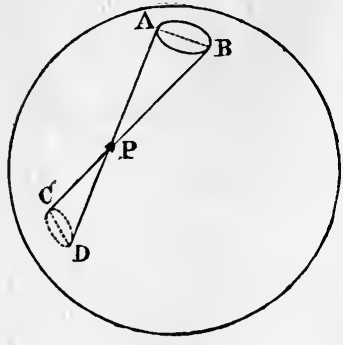

Fig. 147. the small area CD will represent the end of the opposed cone, and the electricity on CD will also act on the + unit placed at $P$, and repel it. Now these surfaces $A B$ and $C D$, and the charges on them, will be directly proportional to the squares of their respective distances from $P$. If, then, the forces which they exercise on $P$ exactly neutralize one another (as experiment shows they do), it is clear that the electric force must fall off inversely as the squares of the distances; for the whole surface of the sphere can be mapped out sinilarly by imaginary cones drawn through $P$. The reasoning can be extended also to hollow conductors of any form.

271. Capacity.- - In Lesson IV. the student was given some elementary notions on the subject of the Capacity of conductors. We are now ready to give the precise definition. The Electrostatic Capacity of a conductor is measured by the quantity of electricity which must be imparted to it in order to raise its potential from zero to unity. A small conductor, such as an insulated sphere of the size of a pea, will not want so much as one unit of electricity to raise its potential from 0 to 1 ; it is therefore of small capacity - while a large sphere will require a large quantity to raise its potential to the same degree, and would therefore be said to be of large capacity. If $\mathrm{K}$ stand for capacity, and $\mathrm{Q}$ for a quantity of electricity,

$$
\mathrm{K}=\frac{\mathrm{Q}}{\mathrm{V}} \text { and } \mathrm{KV}=\mathrm{Q} \text {. }
$$

This is equivalent to saying in words that the quantity 
of electricity necessary to charge a given conductor to a given potential is numerically equal to the product of the capacity into the potential through which it is raised. The capacity of an insulated body is affected by the presence of neighbouring conductors. Whenever we speak of the capacity of a body, we mean of that body when isolated as well as insulated.

272. Unit of Capacity.-A conductor that required only one unit of electricity to raise its potential from 0 to 1, would be said to possess unit capacity. A sphere one centimetre in radius possesses unit capacity; for if it be charged with a quantity of one unit, this charge will act as if it were collected at its centre. At the surface, which is one centimetre away from the centre, the potential, which is measured as $q / r$, will be 1 . Hence, as 1 unit of quantity raises it t" unit 1 of potential, the sphere possesses unit capacity. The capacities of spherrs (isolated in air) are proportional to their.

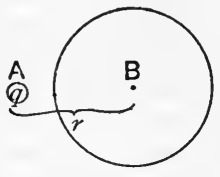

Fig. 148. radii. We may imagine the charge $q$ (Fig. 148) being brought nearer and nearer the sphere until it reaches the surface, then $r$ becomes the radius of the sphere. We may further inagine the surface completely covered with little quantities $q$, so as to have a total charge $Q$ uniformly distributed. Each little quantity would give to the sphere a potential $q / r$; the total potential of the sphere due to the charge $Q$ on its surface would be $Q / r$. The greater the sphere the less would be the potential at any point in it due to the same charge $Q$. Thus it would be necessary to give a charge of 100 units to a sphere of 100 centimetres' radius in order to raise its potential to unity. It therefore has a capacity of 100 . The earth has a capacity of about 630 millions (in electrostatic units).* It is almost impossible to calculate the capacities of conductors of other shapes. It nust be noted

* Or about 700 microfarads (see Art. 283). 
that the capacity of a sphere, as given above, means its capacity when far removed from other conductors or charges of electricity. The capacity of a conductor is increased by bringing near it a charge of an opposite kind; for the potential at the surface of the conductor is the sum of the potential due to its own charge, and of the potential of opposite sign due to the neighbouring charge. Hence, to bring up the resultant potential to unity, a larger quantity of electricity must be given to it; or, in other words, its capacity is greater. This is the true way of regarding the action of Leyden jars and other condensers, and must be remembered by the student when he advances to the consideration of the theory of condenser action, in Lesson XXIII. .

273. Surface-Density. * C Coulomb applied this term to denote the amount of electrification per unit of area at any point of a surface. It was mentioned in Lesson IV. that a charge of electricity was never disturbed uniformly over a conductor, except in the case of an insulated sphere. Where the distribution is unequal, the density at any point of the surface may be expressed by considering the quantity of electricity which exists upon a small unit of area at that point. If $\mathrm{Q}$ be the quantity of electricity on the small surface, and $\mathrm{S}$ be the area of that small surface, then the surface-density (denoted by the Greek letter $\rho$ ) will be given by the equation,

$$
\rho=\frac{\mathrm{Q}}{\mathrm{S}} \text {. }
$$

* The word Tension is sometimes used for that which is here precisely defined as Coulomb defined it. The term tension is, however, unfortunate; and it is so often misapplied in text-books to mean not only surfacedensity but also potential, and even electric force (i.e. the mechanical force exerted upon a material body by electricity), that we might well avoid its use altogether. The term would be invaluable if we might adopt it to denote only the mechanical stress across a dielectric, as in Art. 279. This was Maxwell's use of the word, denoting a pulling force distributed over an area, just as the word pressure means a distributed pushing force. 
In dry air, the limit to the possible electrification is reached when the density reaches the value of about 20 units of electricity per square centimetre. If charged to a higher degree than this, the electricity escapes in "sparks" and "brushes" into the air. In the case of uniform distribution over a surface (as with the sphere, and as approximately obtained on a flat disk by a particular device known as a guard-ring), the density is found by dividing the whole quantity of the charge by the whole surface.

274. Surface-Density on a Sphere.-The surface of a sphere whose radius is $r$, is $4 \pi r^{2}$. Hence, if a charge $Q$ be imparted to a sphere of radius $r$, the surfacedensity all over will be $\rho=\frac{Q}{4 \pi r^{2}}$; or, if we know the surface-density, the quantity of the charge will be $\mathrm{Q}=4 \pi r^{2} \rho$.

The surface-density on two spheres joined by a thin wire is an important case. If the spheres are unequal, they will share the charge in proportion to their capacities (see Art. 40), that is, in proportion to their radii. If the spheres are of radii 2 and 1 , the ratio of their charges will also be as 2 to 1 . But their respective densities will be found by dividing the quantities of electricity on each by their respective surfaces. But the surfaces are proportional to the squares of the radii, i.e. as $4: 1$; hence, the densities will be as $1: 2$, or inversely as the radii. - Now, if one of these spheres be very small-no bigger than a point-the density on it will be relatively immensely great, so great that the air particles in contact with it will rapidly carry off the charge by convection. This explains the action of points in discharging conductors, noticed in Chapter I., Arts. 38, 45, and 47.

275. Electric Images.-It can be slown mathematically that if $+q$ units of electricity are placed at a point near a non-insulated conducting sphere of radius 
$r$, at a distance $d$ from its centre, the negative induced charge will be equal.to $-q r / d$, and will be distributed over the nearest part of the surface of the sphere with a surface-density inversely proportional to the cube of the distance from that point. Lord Kelvin pointed out that, so far as all external points are concerned, the potential due to this peculiar distribution on the surface would be exactly the same as if this negative charge were all collected at an internal point at a distance of $r-r^{2} / d$ behind the surface. Such a point may be regarded as a virtual image of the external point, in the same way as in optics we regard certain points behind mirrors as the virtual images of the external points from which the rays proceed. Clerk Maxwell has given the following definition of an Electric Image :-An electric image is an electrified point, or system of points, on one side of a surface, which would produce on the other side of that surface the same electrical action which the actual electrification of that surface really does produce. If the sphere is not connected to earth, and were unelectrified before $+q$ was brought near it, we may find the surface-density at any point by the following convention. Imagine that there are coexisting on the sphere two charges, $-r q / d$ and $+r q / d$ respectively, the first being distributed so that its surface-density is inversely proportional to the cube of the distance from the electrified point, and the second being uniformly distributed. The actual surface-density is the algebraic sum of these two. A + charge of electricity placed ${ }^{*}$ 1 inch in front of a flat metallic plate induces on it a negative charge distributed over the neighbouring region of the plate (with a density varying inversely as the cube. of the distance from the point); but the electrical action of this distribution, so far as all points in front of the plate are concerned, would be precisely represented by its "image," namely, by an equal quantity of negative electricity placed at a point 1 inch behind the plate. Many beautiful mathematical applications of this 
method have been made, enabling the distribution to be calculated in difficult cases, as, for example, the distribution of the charge on the inner surface of a hollow bowl.

276. Force near a Charged Sphere. - It was shown above that the quantity of electricity $Q$ upon a sphere charged until its surface-density was $\rho$, was

$$
\mathrm{Q}=4 \pi r^{2} \rho .
$$

The problem is to find the force exercised by this charge upon a + unit of electricity, placed at a point infinitely near the surface of the sphere. The charge on the sphere acts as if at its centre. The distance between the two quantities is therefore $r$. By Coulomb's Law the force $f=\frac{Q \times 1}{r^{2}}=\frac{4 \pi r^{2} \rho}{r^{2}}=4 \pi \rho$.

This important result may be stated in words as follows :- The force (in dynes) exerted by a charged sphere upon a unit of electricity placed infinitely near to its surface, is numerically equal to $4 \pi$ times the surface-density of the charge.

277. Force near a Charged Plate of indefinite size. - Suppose a plate of indefinite extent to be charged so that it has a surface-density $\rho$. This surface-density will be uniform, for the edges of the plate are supposed to be so far off as to exercise no influence. It can be shown that the force exerted by such a plate upon $a+$ unit anywhere near it, will be expressed (in dynes) numerically as $2 \pi \rho$. This will be of opposite signs on opposite sides of the plate, being $+2 \pi \rho$ on one side, and $-2 \pi \rho$ on the other sile, since in one case the force tends to move the unit from right to left, in the other from left to right. It is to be observed, therefore, that the force changes its value by the amount of $4 \pi \rho$ as the point passes through the surface. The same was true of the charged sphere, where the force outside was $4 \pi \rho$, and inside was zero. The same is true of all charged 
surfaces. These two propositions are of the utmost importance in the theory of Electrostatics.

278. Proof of Theorem. - The elementary geometrical proof is as follows :-

Required the Electric Force at point at any distance from a plane of infinite extent charged to surface-density $\rho$.

Let $\mathrm{P}$ be the point, and PX or $a$ the normal to the plane. T'ake any small cone having its apex at P. Let the solid angle of this cone be $\omega$; let its length be $r$; and $\theta$ the angle its axis

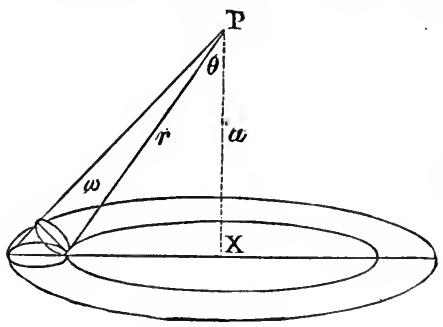

Fig. 149.

makes with $\alpha$. The cone meets the surface of the plane obliquely, and if an orthogonal section be made where it meets the plane, the angle between these sections will be $=\theta$.

Now solid angle $\omega$ is by definition $=\frac{\text { orthogonal area of section }}{r^{2}}$.

Hence, area of oblique section $=r^{2} \omega \times \frac{1}{\cos \theta}$;

$\therefore$ charge on oblique section $=\frac{r^{2} \omega \rho}{\cos \theta}$.

Hence if $a+u n i t$ of electricity were placed at $\mathrm{P}$, the force exerted on this by this small change $=\frac{r^{2} \omega \rho}{\cos \theta} \times 1 \div r^{2}$,

or

$$
=\frac{\omega \rho}{\cos \theta} \text {. }
$$

Resolve this force into two parts, one acting along the plane, the other along $a$, normal to the plane. The normal component along $a$ is $\cos \theta \times \frac{\omega \rho}{\cos \theta}=\omega \rho$. 
But the whole surface of the plane may be similarly mapped out into small surfaces, all forming small cones, with their summits at $P$. If we take an infinite number of such small cones meeting every part, and resolve their forces in a similar way, we shall find that the components along the plane will neutralize one another all round, while the normal components, or the resolved forces along $a$, will be equal to the sum of all their solid angles multiplied by the surface-density; or

\section{Total resultant force along $a=\Sigma \omega \rho$.}

But the total solid angle subtended by an indefinite plane at a point is $2 \pi$, for it subtends a whole hemisphere.

$\therefore$ Total resultant force $=2 \pi \rho$.

279. Electric Stress in Medium. - In every electric field (Art. 13) there exists a tension along the lines of electric force accompanied by an equal pressure in all directions at right angles to the lines. If $\mathrm{F}$ stands for the resultant electric force on a + unit placed at any point in the field (i.e. the "electromotive intensity" at that point), the tension will be equal to $\mathrm{F}^{2} / 8 \pi$ (dynes per square centimetre). In media having dielectric capacities greater than unity the tension is proportionately greater. For the optical effects of these stresses see Art. 525.

\section{NOTE ON FUNDAMENTAL AND DERIVED UNITS}

280. Fundamental Units.-All physical qualities, such as force, velocity, etc., can be expressed in ternis of the three fundamental quantities : length, mass, and time. Each of these quantities must be measured in terms of its own units.

The system of units, adopted by almost universal consent, and used throughout these lessons, is the so-called "Centimetre-Gramme-Second" system, in which the fundamental units are :-

The Centimetre as a unit of length;

The Gramme as a unit of mass;

The Second as a unit of time.

The Centimetre is equal to 0.3937 inch in length, and nominally 
represents one thousand-millionth part, or $\frac{1}{1,000,000,000}$ of a quadrant of the earth.

The Metre is 100 centimetres, or 39.37 inches.

The Kilometre is 1000 metres, or about $1093 \cdot 6$ yards.

The Millimetre is $\frac{1}{10}$ of a centimetre, or 0.03937 inch.

The Gramme represents the mass of a cubic centimetre of water at $4^{\circ} \mathrm{C}$., this is equal to 15.432 grains : the Kilogramme is 1000 grammes or about $2 \cdot 2$ pounds.

\section{Derived Units.-}

Area. - The unit of area is the square centimetre.

Volume. - The unit of volume is the cubic centimetre.

Velocity.-The unit of velocity is the velocity of a body which moves through unit distance in unit time, or the velocity of one centimetre per second.

Acceleration. - The unit of acceleration is that acceleration which imparts unit velocity to a body in unit time, or an acceleration of one centimetre-per-second per second. The acceleration due to gravity imparts in one second a velocity considerably greater than this, for the velocity it imparts to falling bodies is about 981 centimetres per second (or about $32 \cdot 2$ feet per second). The value differs slightly in different latitudes. At Greenwich the value of the acceleration of gravity is $g=981 \cdot 1$; at the Equator $g=978 \cdot 1$; at the North Pole $g=983 \cdot 1$.

Force.-The unit of force is that force which, acting for one second on a mass of one gramme, gives to it a velocity of one centimetre per second. It is called one Dyne. The force with which the earth attracts any mass is usually called the "weight" of that mass, and its value obviously differs at different points of the earth's surface. The force with which a botly gravitates, i.e. its weight (in dynes), is found by multiplying its mass (in grammes) by the value of $g$ at the particular place where the force is exerted. One pound force in England is about 445,000 dynes.

Work.-The unit of work is the work done in overcoming unit force through unit distance, i.e. in pushing a body through a distance of one centimetre against a force of one dyne. . It is called one Erg. Since the "weight" of one gramme is $1 \times 981$ or 981 dynes, the work of raising one gramme through the height of one centimetre against ihe force of gravity is 981 ergs.

Energy.-The unit of energy is also the erg; for the energy of a body is measured by the work it can do. 
Heat.-The unit of heat, the calorie, is the amount of heat required to warm one gramme niass of water from $0^{\circ}$ to $1^{\circ}$ (C.) ; and the dynamical equivalent of this amount of heat is 42 million ergs, which is the value of Joule's equivalent, as expressed in C.G.S. measure (see also Art. 439).

These units are sometimes called "absolute" units; the term absolute, introduced by Gauss, meaning that they are independent of the size of any particular instrument, or of the value of gravity at any particular place, or of any other arbitrary quantities than the three standards of length, mass, and time. It is, however, preferable to refer to them by the more appropriate name of "C.G.S. units," as being derived from the centimetre, the gramme, and the second.

282. Electrical Units.-There are two systems of electrical units derived from the fundamental "C.G.S." units, one set being based upon the force exerted between two quantities of electricity, and the other upon the force exerted between two magnet poles. The former set are termed electrostatic units, the latter electromagnetic units. The important relation between the two sets is explained in Chap. V., Art. 359.

283. Electrostatic Units. - No special names have been assigned to the electrostatic units of Quantity, Potential, Capacity, ete. The reasons for adopting the following values as units are given either in Chapter I. or in the present chapter.

Unit of Quantity. - The unit of quantity is that quantity of electricity which, when placed at a distance of one centimetre (in air) from a similar and equal quantity, repels it with a force of one dyne (Art. 262).

Potential.-Potential being measured by work done in moving a unit of + electricity against the electric forces, the unit of potential will be measured by the unit of work, the erg.

Unit Difference of Potential.-Unit difference of potential exists between two points, when it requires the expenditure of one erg of work 10 bring a + unit of electricity from one point to the other against the electric force (Art. 265).

Unit of Capacity. - That conductor possesses unit eapacity which requires a charge of one unit of electricity to bring it up to unit potential. A sphere of one centimetre radius possesses unit capacity (Art. 272).

Specific Inductive Capacity, or Dielectric Cocfficient, is defined in Art. 295 as the ratio between two quantities of electricity. The specific inductive capacity of the air is, in the absence of any knowledge of its absolute value, taken as unity.

Electromotive Intensity is the electric force or intensity of an 
electric field at any point, and is measured by the force which it exerts on a unit charge placed at that point.

It may be convenient here to append the rules for reducing to their corresponding values in terms of the practical (electro-magnetic) units values that may have been expressed in terms of the electrostatic units, as follows :-

Potential. To bring to volts multiply by 300 .

Capacity. To bring to microfarads divide by 900,000 .

Quantity. To bring to coulombs divide by $3 \times 10^{9}$.

Current. To bring to amperes divide by $3 \times 10^{9}$.

Resistance. To bring to ohms multiply by $9 \times 10 .^{11}$

Example.-Suppose two equally charged spheres whose centres are 40 centimetres apart are found to repel one another with a force of 630 dynes (= about the weight of 10 grains). By the law of inverse squares we find that the charge on each is 1004 (electrostatic) mits. Dividing by $3 \times 10^{9}$ we find that this amounts to 0.0000003347 coulomb.

284. Dimensions of Units. - It has been assumed above that a velocity can be expressed in centimetres per second ; for velocity is rate of change of place, and it is clear that if change of place may be measured as a length in centimetres, the rate of change of place will be measured by the number of centimetres through which the body moves in unit of time. It is impossible, indeed, to express a velocity without regarding it as the quotient of a certain number of units of length divided by a certain number of units of time. In other words, a velocity $=\frac{\text { a length }}{\text { a time }}$; or, adopting $\mathrm{L}$ as a symbol for length, and $\mathrm{T}$ as a symbol for time, $V=\frac{\mathrm{L}}{\mathrm{T}}$, which is still more conveniently written $\mathrm{V}=\mathrm{L} \times \mathrm{T}^{-1}$. In a similar way acceleration being rate of change of velocity, we have $A=\frac{V}{T}=\frac{L}{T \times T}=\frac{L}{T^{2}}=L \times T^{-2}$.

Now these physical quantities, "velocity" and " acceleration," are respectively always quantities of the same nature, no matter whether the centimetre, or the inch, or the mile, be taken as the unit of length, or the second or any other interval be taken as the unit of time. Hence we say that these abstract equations express the dimensions of those quantities with respect to the fundamental quantities length and time. A little consideration will show the student that the dimensions of the various units mentioned above will therefore be as given in the table opposite.

The dimensions of magnetic units are given in the Table in Art. 356, p. 348. 


\section{Table of Dimensions of Units}

\begin{tabular}{|c|c|c|c|c|}
\hline & \multicolumn{3}{|c|}{ Units. } & Dimensions. \\
\hline $\begin{array}{c}l \\
m \\
t\end{array}$ & $\begin{array}{l}\text { (Fundamental } \\
\text { Length } \\
\text { Mass } \\
\text { Time }\end{array}$ & & . & $\begin{array}{c}\mathrm{L} \\
\mathrm{M} \\
\mathrm{T}\end{array}$ \\
\hline $\begin{array}{l}a \\
f\end{array}$ & $\begin{array}{l}\text { (Derived) } \\
\text { Area } \\
\text { Volume } \\
\text { Velocity } \\
\text { Acceleration } \\
\text { Force } \\
\text { Work }\end{array}$ & $\begin{array}{l}=\quad \mathrm{I} \times \mathrm{L} \\
=\quad \mathrm{L} \times \mathrm{L} \times \mathrm{L} \\
=\quad \mathrm{L} \div \mathrm{T} \\
=\text { velocity } \div \text { time } \\
=\text { mass } \times \text { acceleration } \\
=\text { force } \times \text { length }\end{array}$ & $\begin{array}{l}= \\
= \\
= \\
= \\
= \\
=\end{array}$ & $\begin{array}{c}\mathrm{L}^{2} \\
\mathrm{~L}^{3} \\
\mathrm{LT}^{-1} \\
\mathrm{LT}^{-2} \\
\mathrm{MLT}^{-2} \\
\mathrm{ML}^{2} \mathrm{~T}^{-2}\end{array}$ \\
\hline $\begin{array}{l}q \\
i \\
\mathrm{~V} \\
\mathrm{R} \\
\mathrm{C} \\
k \\
\mathrm{~F}\end{array}$ & $\begin{array}{l}\text { (Electrostatic) } \\
\text { Quantity } \\
\text { Current } \\
\text { Potential } \\
\text { Resistance } \\
\text { Capacity } \\
\text { Sp. Ind. Cap } \\
\text { Electromotive }\end{array}$ & $\begin{aligned} &=\sqrt{ } \text { force } \times(\text { distance })^{2} \\
&=\text { quantity } \div \text { time } \\
&=\text { work } \div \text { quantity } \\
&=\text { potential } \div \text { current } \\
&=\text { quantity } \div \text { potential } \\
& \text { e Intensity }=\text { force } \div \text { quanti }\end{aligned}$ & $\begin{array}{l}= \\
= \\
= \\
= \\
= \\
\text { quantity } \\
\text { ty }=\end{array}$ & $\begin{array}{c}\mathrm{M}^{\frac{1}{2}} \mathrm{~L}^{\frac{3}{2}} \mathrm{~T}^{-1} \\
\mathrm{M}^{\frac{1}{2}} \mathrm{~L}^{\frac{3}{2}} \mathrm{~T}^{-2} \\
\mathrm{M}^{\frac{1}{2}} \mathrm{~J}^{\frac{1}{2}} \mathrm{~T}^{-1} \\
\mathrm{~L}^{-1} \mathrm{~T}^{1} \\
\mathrm{~L} \\
\text { a numeral } \\
\mathrm{M}^{\frac{1}{2}} \cdot \mathrm{L}^{-\frac{1}{2}} \mathrm{~T}^{-1}\end{array}$ \\
\hline
\end{tabular}

\section{Lesson XXII._Electrometers}

285. In Lesson II. we described a number of electroscopes or instruments for indicating the presence ard sign of a charge of electricity; some of these also served 
to indicate roughly the amount of these charges, but none of them save the torsion balance could be regarded as affording an accurate means of measuring either the quantity or the potential of a given charge. $A n$ instrument for measuring differences of electrostatic potential is termed an Electrometer. Such instruments can also be used to measure electric quantity indirectly, for the quantity of a charge can be ascertained by measuring the potential to which it can raise a conductor of known capacity. The earliest electrometers attempted to measure the quantities directly. Lane and Snow Harris constructed "Unit Jars" or small Leyden jars, which, in order to measure out a certain quantity of electricity, were charged and discharged a certain number of times.

286. Repulsion Electrometers. - The torsion balance, described in Art. 18, measures quantities by measuring the forces exerted by the charges given to the fixed and movable balls. It can only be applied to the measurement of repelling forces, for the equilibrium is unstable in the case of a force of attraction.

Beside the gold-leaf electroscope and others described in Lesson II., there exist several finer electrometers based upon the principle of repulsion, some of which resemble the torsion balance in having a movable arm turning about a central axis. Amongst these are the electrometers of Dellmann and of Peltier. In the latter a light arm of aluminium, balanced upon a point, carries also a small magnet to direct it in the magnetic meridian. A fixed arm, in metallic contact with the movable one, also lies in the magnetic meridian. A clarge imparted to this instrument produces a repulsion hetween the fixed and movable arms, causing an angular deviation. Here, however, the force is measured not by being pitted against the torsion of an elastic fibre, or against gravitation, but against the directive magnetic force of the earth acting on the small needle. Now this depends on the intensity 
of the horizontal component of the earth's magnetism at the place, on the magnetic moment of the needle, and on the sine of the angle of its deviation. Hence, to obtain quantitative values for the readings of this electrometer, it is necessary to make preliminary experiments and to "calibrate" the degree-readings of the deviation.

\section{Attracted-Disk Electrometers. - Snow} Harris was the first to construct an electrometer for measuring the attraction between an electrified and a non-electrified disk; and the instrument he devised may be roughly described as a balance for weighing a charge of electricity. More accurately speaking, it was an instrument resembling a balance in form, carrying at one end a light scale pan; at the other a disk was hung above a fixed insulated disk, to which the charge to be measured was imparted. The chief defect of this instrument was the irregular distribution of the charge on the disk. The force exerted by an electrified point falls off inversely as the square of the distance, since the lines of force emanate in radial lines. But in the case of a uniformly electrified plane surface, the lines of force are normal to the surface, and parallel to one another; and the force is independent of the distance. The distribution over a small sphere nearly fulfils the first of these conditions. The distribution over a flat disk would nearly fulfil the latter condition, were it not for the perturbing effect of the edges of the disk where the surface-density is much greater (see Art. 38); for this reason Snow Harris's electrometer was very imperfect.

Lord Kelvin introduced several very important modifications into the construction of attracted-disk electrometers, the chief of these being the employment of the "guardplate" and the providing of means for working with a definite standard of potential. It would be beyond the scope of these lessons to give a complete description of all 
the various forms of attracted-disk electrometer ; ${ }^{*}$ but the main principles of them all can be readily explained.

The disk C; whose attraction is to be measured, is suspended (Fig. 150) within a fixed guard-plate B, which surrounds it without touching it, and which is placed in metallic contact with it by a fine wire. A lever $\mathrm{L}$ supports the disk, and is furnished with a counterpoise. In order to know whether the disk is precisely level with the lower surface of the guard-plate a little gauge or index

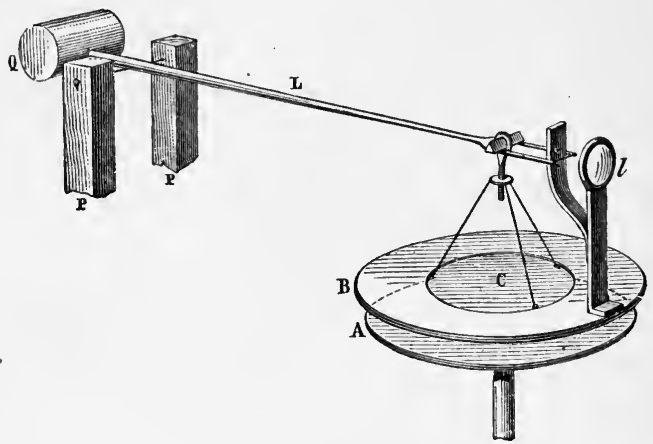

Fig. 150 .

is fixed above, and provided with a lens $l$ to observe its indications. Beneath the disk and guard-plate is a second disk A, supported on an insulating stand. This lower disk can be raised or lowered at will by a micrometer screw, great care being taken in the mechanical arrangements that it shall always be parallel to the plane of the guard-plate. Now, since the disk and guard-plate are in metallic connexion with one another, they form virtually part of one surface, and as the irregularities of distribution

* For these the student is referred to the volume of Iord Kelvin's papers, "On Electrostatics and Magnetism ;" or to Professor Andrew Gray's Absolute Measurements in Electricity and Magnetism. 
occur at the edges of the surface, the distribution over the area of the disk is practically uniform. Any attraction of the lower plate upon the disk might be balanced either by increasing the weight of the counterpoise, or by putting a torsion on the aluminium wire which serves as a fulcrum ; but in practice it is found most convenient to obtain a balance by altering the distance of the lower plate until the electric force of attraction exactly balances the forces (whether of torsion or of gravity acting on the counterpoise) which tend to lift the disk above the level of the guard-plate.

The theory of the instrument is simple also. Let $V_{1}$ represent the potential of the movable disk, which has a positive charge of surface-density $\rho$, and let $V_{2}$ be the potential of the fixed plate, upon which is a charge of surface-density - $\rho$. The difference of potential $V_{1}-V_{2}$ is the work which would have to be done upon a unit of positive charge in taking it from $V_{2}$ to $V_{1}$. Now the force upon such a unit placed between the two plates would be (an attraction of $2 \pi \rho$ due to the fixed plate, and a repulsion of $2 \pi \rho$ due to the movable plate, see Art. $2 \pi 8$ ) altogether $4 \pi \rho$, and if the distance between the plates were 1 , work $=$ force $\times$ distance.

$$
\mathrm{V}_{1}-\mathrm{V}_{2}=4 \pi \rho \mathrm{D} \text {. }
$$

If $\mathrm{S}$ is the area of the movable plate, $\mathrm{S} \rho$ is the total quantity of electricity on it; therefore it would be attracted by the fixed plate with a force $F=2 \pi \rho \times S \rho$. From this we get

$$
\rho=\sqrt{\frac{\mathrm{F}}{2 \pi \mathrm{S}}} .
$$

Substituting this value of $\rho$ in the above equation, we get

$$
\mathrm{V}_{1}-\mathrm{V}_{2}=\mathrm{D} \sqrt{\frac{8 \pi \mathrm{F}}{\mathrm{S}}} .
$$

If $\mathrm{F}$ is measured in dynes, $\mathrm{S}$ in square centimetres, and $\mathrm{D}$ in centimetres, the potentials will be in absolute electro. 
static units, and must be multiplied by 300 to bring to volts (see Art. 283).

From this we gather that, if the force $\mathrm{F}$ remain the same throughout the experiments, the difference of potentials between the disks will be simply proportional to the distance between them when the disk is in level equilibrium. And the quantity $\sqrt{\frac{8 \pi \mathrm{F}}{\mathrm{S}}}$ may be determined once for all as a "constant" of the instrument.

In the more elaborate forms of the instrument, such as the "absolute electrometer," and the "portable electrometer," the disk and guard-plate are covered with a metallic cage, and are together placed in communication with a condenser to keep them at a known potential. This obviates having to make measurements with zero readings, for the differences of potential will now be proportional to differences of micrometer readings,

or,

$$
\mathrm{V}_{1}-\mathrm{V}_{2}=\left(\mathrm{D}_{1}-\mathrm{D}_{2}\right) \sqrt{\frac{8 \pi \mathrm{F}}{\mathrm{S}}}
$$

The condenser is provided in these instruments with a gauge, itself an attracted disk, to indicate when it is charged to the right potential, and with a replenisher to increase or decrease the charge, the replenisher being a little influence machine (see Art. 50).

288. The Quadrant Electrometer.-The Quadrant Electrometer of Lord Kelvin is an example of a different class of electrometers, in which use is made of an auxiliary charge of electricity previously imparted to the needle of the instrument. The needle, which consists of a thin flat piece of aluminium hung horizontally by a fibre of thin wire, thus charged, say positively, will be attracted by a - charge, but repelled by a + charge. Such attraction or repulsion will be stronger in proportion to these charges, and in proportion to the charge on the needle. Four quadrant-pieces (Fig. 151) of brass are fixed 
horizontally below the needle without touching it or one another. Opposite quadrants are joined with fine wires. If quadrants 1 and 3 are ever so little + as compared with quadrants 2 and 4 , the needle will turn away from the former to a position more nearly over the latter.

If there is the slightest difference of potential between the pairs of quadrants, the needle, which is held in its zero position by the elasticity of the wire, will turn, and so indicate the difference of potential. When these deflexions are small, the scale readings will be very nearly proportional to the difference of potential. The instrument is sufficiently delicate to show a difference of potential between the quadrants as small as the $\frac{1}{70}$ of that

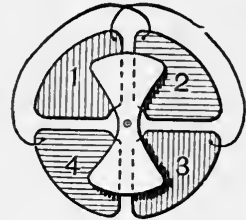

Fig. 151. of the Daniell's cell. If $\mathrm{V}_{1}$ be the potential of one pair of quadrants, $V_{2}$ that of the other pair, and $V_{3}$ the potential of the needle, the force tending to turn will be proportional to $\mathrm{V}_{1}-\mathrm{V}_{2}$, and will also be proportional to the difference between $\mathrm{V}_{3}$ and the average of $\mathrm{V}_{1}$ and $\mathrm{V}_{2}$. Or, in symbols,

$$
f=a\left(\mathrm{~V}_{1}-\mathrm{V}_{2}\right)\left(\mathrm{V}_{3}-\frac{\mathrm{V}_{1}+\mathrm{V}_{2}}{2}\right) ;
$$

where $a$ is a constant depending on the construction of the particular instrument.

Fig. 152 shows a very simple form of the Quadrant Electrometer, as arranged for qualitative experiments. The four quadrants are enclosed within a glass case, and the needle, which carries a light mirror $\mathbf{M}$ below it, is suspended from a torsion head $\mathrm{C}$ by a very thin metallic. wire $\mathrm{F}$. It is electrified to a certain potential by being connected, through a wire attached to $\mathrm{C}$, with a charged Leyden jar or other condenser. In order to observe the minutest motions of the needle, a reading-telescope and scale are so placed that the observer looking through the 
telescope sees an image of the zero of the scale reflected in the little mirror. The wires connecting quadrants 1 and 3,2 and 4, are seen above the top of the case.

For very exact measurements many additional refinements are introduced into the instrument. Two sets of quadrants are employed, an upper and a lower, having the needle between them. The torsion wire is replaced by a delicate bifilar suspension (Art. 130). To keep up the charge of the Leyden jar a "replenisher" is added ; and an "attracteddisk," like that of the Absolute Electrometer, is employed in order to act as a gange to indicate when the jar is charged to the right potential. In these forms the jar consists of $x$ glass vessel placed below the quadrants, coated externally with strips of tinfoil, and containing strong sulphuric acid, which serves the double function of

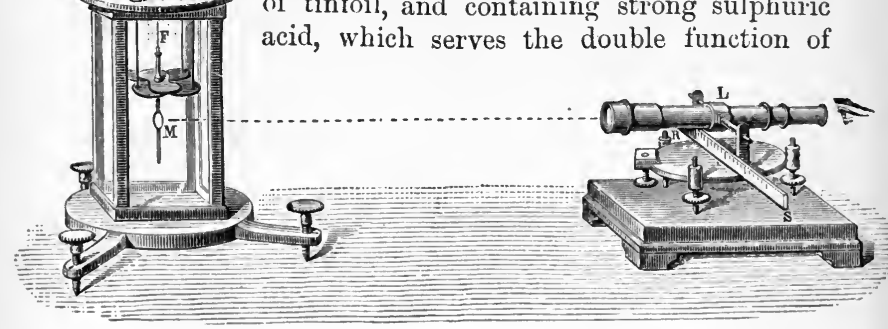

Fig. 152.

keeping the apparatus dry by absorbing the moisture and of acting as an internal coating for the jar. It is also more usual to throw a spot of light from a lamp upon a scale by means of the little mirror (as described in the case of the Mirror Galvanometer, in Art. 215), than to adopt the subjective method with the telescope, which only one person at a time can use. When the instrument is provided with replenisher and gauge, the measurements 
can be made in terms of absolute units, provided the "constant" of the particular instrument (depending on the suspension of the needle, size and position of needle and quadrants, potential of the gauge, etc.) is once ascertained.

289. Use of Quadrant Electrometer. -An example will illustrate the mode of using the instrument. It is known that when the two ends of a thin wire are kept at two different potentials a current flows through the wire, and that if the potential is measured at different points along the wire, it is found to fall off in a perfectly uniform manner from the end that is at a high potential down to that at the low potential. At a point one quarter along the potential will have fallen off one quarter of the whole difference. This could be proved by joining the two ends of the wire througl which the current was flowing to the terminals of the Quadrant Electrometer, when one pair of quadrants would be at the high potential and the other at the low potential. The needle would turn and indicate a certain deflexion. Now, disconnect one of the pairs of quadrants from the low potential end of the wire, and place them in communication with a point one quarter along the wire from the high potential end. The needle will at once indicate that the difference of potential is but one quarter of what it was before.

Often the Quadrant Electrometer is employed simply as a very delicate electroscope in systems of measurement in which a difference of electric potential is measured by being balanced against an equal and opposite difference of potential, exact balance being indicated by there being no deflexion of the Electrometer needle. Such methods of experimenting are known as Null Methods, or Zero Methods.

290. Electrostatic Voltmeter.-We have seer. that in the quadrant electrometer it is necessary to give the needle a high initial charge, the reason being that if there did not exist between the quadrants and the needle a much greater difference of potential than the small voltage we are measuring, the force tending to turn the needle would be too small to be conveniently observed. Where, however, we are dealng with high differences of potential a separately-charged needle is not requisite; we may simply join one conductor to the needle and the other to a set of quadrants, and the force of 
attraction, which, other things being equal, increases as the square of the difference of potential, is sufficiently

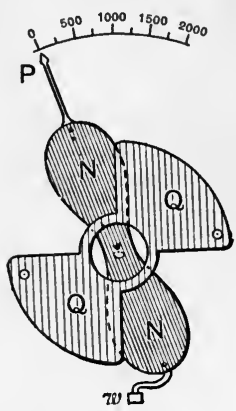

Fig. 153. great to give reliable readings. This is known as the idiostatic method of using the instrument.

A front view of the instrument as commonly used to measure differences of potential of 1000 volts or more, is shown in Fig. 153. The needle NN is a paddle-shaped plate of aluminium supported by knife edges at its centre ; its position is controlled by gravity, little weights being hung on a projection at its lower end. The quadrants $Q$ are both behind and in front of it, and so placed that when a difference of potential exists between the needle and them the needle is deflected from its normal position and moves its pointer over a graduated scale.

It will be seen that it does not matter whether the needle is positively charged and the quadrants negatively charged or vice versâ; an attraction between the two will always take place, so a deflexion will be given even when the difference of potential is rapidly alternating. This property of the instrument makes it exceedingly useful for the measurement of voltage when alternating currents are used.

Another advantage of this instrument over the high-resistance galvanometers that are used as voltmeters is, that it does not take any current, and consequently it does not waste any power.

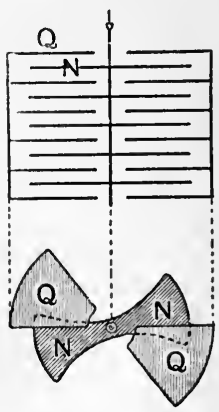

Fig. 154.

In order to make the electrostatic voltmeter sufficiently delicate to measure down to 100 volts or so, a number of 
needles are placed horizontally one above the other on a vertical aluminium wire, and attracted by a tier of quadrants symmetrically placed on each side ; this instrument is Lord Kelvin's multicellular voltmeter. It is shown in elevation and plan in Fig. 154.

291. Dry-Pile Electrometer. - The principle of symmetry observed in the Quadrant Electrometer was previously employed in the Electroscope of Bohnenberger -a much less accurate instrument-in which the charge to be examined was imparted to a single gold leaf, placed symmetrically between the poles of a dry-pile (Art. 193), toward one or other pole of which the leaf was attracted. Fechner modified the instrument by connecting the + pole of the dry-pile with a gold leaf hanging between two metal disks, from the more + of which it was repelled. The inconstancy of dry-piles as sources of electrification led Hankel to substitute a battery of a very large number of small Daniell's cells.

292. Capillary Electrometers.-The Capillary Electrometer of Lippmann, as modified by Dewar, was described in Art. 253.

\section{Lesson XXIII.-Dielectric Capacity, etc.}

293. A Leyden jar or other condenser may be regarded as a conductor, in which (owing to the particular device of bringing near together the two oppositelycharged surfaces) the conducting surface can be made to hold a very large charge without its potential (whether + or - ) rising very high. The capacity of a condenser, like that of a simple conductor, will be measured (see Art. 271) by the quantity of electricity required to produce unit rise of potential.

294. Theory of Spherical Condenser.-Suppose a Leyden jar made of two concentric metal spheres, one inside the other, the space between them being filled 
by air. The inner one, $A$, will represent the interior

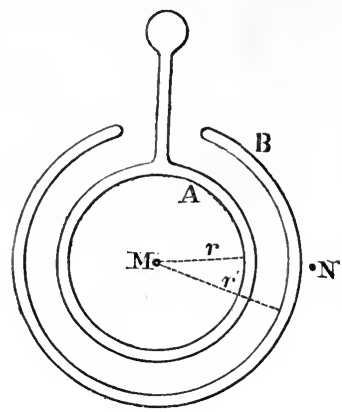

Fig. 155. coating of tinfoil, and the outer sphere, B (Fig. 155), will represent the exterior coating. Let the radii of these spheres be $r$ and $r^{\prime}$ respectively. Suppose a charge of $Q$ units to be imparted to $\mathrm{A}$; it will induce on the inner side of $B$ an equal negative charge $-Q$, and to the outer side of $B$ a charge $+Q$ will be repelled. This latter is removed by contact with " earth," and need be no further considered. The potential * at the centre M, calculated by the rule given in Art. 263, will be

$$
\mathrm{V}_{\mathrm{M}}=\frac{\mathrm{Q}}{r}-\frac{\mathrm{Q}}{r^{\prime}}
$$

At a point $\mathrm{N}$, outside the outer sphere and quite near to it, the potential will be the same as if these two charges, $+\mathrm{Q}$ and $-\mathrm{Q}$, were both concentrated at $\mathrm{M}$. Hence

$$
\mathrm{V}_{\mathrm{N}}=\frac{+\mathrm{Q}-\mathrm{Q}}{r^{\prime}}=0 \text {. }
$$

So then the difference of potentials will be

$$
\mathrm{V}_{\mathrm{M}}-\mathrm{V}_{\mathrm{N}}=\frac{\mathrm{Q}}{r}-\frac{\mathrm{Q}}{r^{\prime}}=\mathrm{Q}\left(\frac{r^{\prime}-r}{r r^{\prime}}\right) \text {; }
$$

whence $\frac{\mathrm{Q}}{\mathrm{V}_{\mathrm{M}}-\mathrm{V}_{\mathrm{N}}}=\frac{r r^{\prime}}{r^{\prime}-r}$.

But by Art. 270 the capacity $K=\frac{Q}{V_{M}-V_{N}}$, therefore $\mathrm{K}=\frac{r r^{\prime}}{r^{\prime}-r^{\prime}}$.

* The student must remember that as there is no electric foree within a closer conductor, the potential at the middle is just the same as at any other point inside. 
We see from this formula that the capacity of the condenser is proportional to the size of the metal globes, and that if the insulating layer is very thin,--that is, if $r$ be very nearly as great as $r^{\prime}, r^{\prime}-r$ will become 'very small, and the value of the expression $\frac{r r^{\prime}}{r^{\prime}-r}$ will become very great; which proves the statement that the capacity of a condenser depends upon the thinness of the layer of dielectric. If $r^{\prime}$ is very great compared with $r$, the expression for the eapacity becomes equal simply to $r$, that of the inner sphere when isolated.

295. Specific Inductive Capacity.-Cavendish was the first to discover that the capacity of a condenser depended not on its actual dimensions only, but upon the inductive power of the material used as the dielectric between the two surfaces. If two condensers (of any of the forms to be described) are made of exactly the same size, and in one of them the dielectric be a layer of air, and in the other a layer of some other insulating substance, it is found that equal quantities of electricity imparted to them do not produce equal differences of potentials ; or, in other words, it is found that they have not the same capacity. If the dielectric be mica, for example, it is found that the capacity is about six times as great; for mica possesses a high inductive power and allows the transmission across it of electrostatic influence six times as well as air does. The name specific inductive capacity, ${ }^{*}$ or dielectric capacity, is given to the ratio between the capacities of two condensers equal in size, one of them being an air condenser, the other filled with the specified dielectric. The specific inductive capacity of dry air at the temperature $0^{\circ}$ C., and pressure 76 centimetres, is taken as the standard, and, in the absence of any known way of finding its absolute value, is reckoned

* The name is not a very happy one,-inductivity would have been better, and is the analogous term, for dielectrics, to the term "conductivity" used for conductors. The term dielectric coefficient is also used by some modern writers. 
as unity. The symbol $k$ is used to denote the dielectric capacity of any material.

Cavendish, about the year 1775 , measured the dielectric capacity of glass, bees-wax, and other substances, by forming them into condensers between two circular metal plates, the capacity of these condensers being compared with that of an air condenser (resembling Fig. 42) and

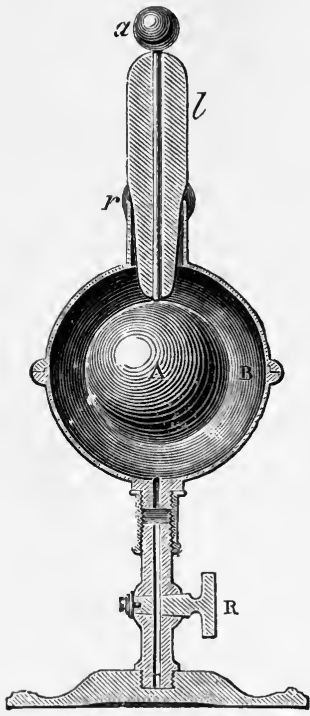

Fig. 156. with other condensers which he called "trial-plates." He even went so far as to compare the capacities of these "trial-plates" with that of an isolated sphere of $12 \frac{1}{2}$ inches diameter hung up in a room.

296. Faraday's Experiments. - In 1837 Faraday, who did not know of the then unpublished researches of Cavendish, independently discovered specific inductive capacity, and measured its value for several substances, using for this purpose two condensers of the form shown in Fig. 156. Each consisted of a brass ball A, enclosed inside a hollow sphere of brass $\mathrm{B}$, and insulated by a long plug of shellac, up which passed a wire terminating in a knob $a$. The outer sphere consisted of two parts which could be separated from each other in order to fill the hollow space with any desired material : the experimental process then was to compare their capacities when one was filled with the substance to be examined, the other containing only dry air. One of the condensers was charged with electricity. It was then made to share its 
charge with the other condenser, by putting the two inner coatings into metallic communication with one another; the outer coatings also being in communication with one another. If their capacities were equal they would share the charge equally, and the potential after contact would be just half what it was in the charged condenser before contact. If the capacity of one was greater than the other the final potential would not be exactly half the original potential, because they would not share the charge equally, but in proportion to their capacities. The potentials of the charges were measured before and after contact by means of a torsion balance. ${ }^{*}$ Faraday's results showed the following values: Sulphur, $2 \cdot 26$; shellac, $2 \cdot 0$; glass, $1 \cdot 76$ or more.

297. Recent Researches. - Since 1870 large additions to our knowledge of this subject have been made. Gibson and Barclay measured the inductivity of paraffin wax by comparing the capacity of an air condenser with one of paraffin by means of an arrangement of sliding condensers, using a sensitive quadrant electrometer to adjust the capacity of the condensers exactly to equality. Hopkinson has examined the dielectric power of glass of various kinds, using a constant battery to produce the required difference of potentials, and a condenser provided with a guard-ring for a purpose similar to that of the guard-ring in absolute electrometers. Gordon made a large number of observations, using a delicate apparatus known as a statical "inductivity balance," which is a complicated condenser, so arranged in connexion with a

* The value of the dielectric capacity $k$ could then be calculated as follows :-

$$
\mathbf{Q}=\mathbf{V} \mathbf{K}=\mathbf{V}^{\prime} \mathbf{K}+\mathbf{V}^{\prime} \mathbf{K} k
$$

(where $K$ is the capacity of the first apparatus and $V$ its potential, and $V^{\prime}$ the potential after communication with the second apparatus, whose capacity is $\mathbf{K} k$ ): hence

and

$$
\begin{aligned}
& \mathbf{V}=\mathbf{V}^{\prime}(1+k), \\
& k=\frac{\mathbf{V}-\mathbf{V}^{\prime}}{\mathbf{V}^{\prime}} .
\end{aligned}
$$


quadrant electrometer that when the capacities of the separate parts are adjusted to equality there shall be no deflexion in the electrometer, whatever be the amount or sign of the electrification at the moment. . This arrangement, when employed in conjunction with an induction coil (Fig. 135) and a rapid commutator, admits of the inductive capacity being measured when the duration of the actual charge is only very small, the electrification being reversed 12,000 times per second. Such an instrument, therefore, overcomes one great difficulty besetting these measurements, namely, that owing to the apparent absorption of part of the charge by the dielectric (as mentioned in Art. 61), the capacity of the substance, when measured slowly, is different from its "instantaneous capacity." This electric absorption is discussed further in Art. 299. For this reason the values assigned by different observers for the dielectric capacity of various substances differ to a most perplexing degree, especially in the case of the less perfect insulators. The following table summarizes Gordon's observations :-

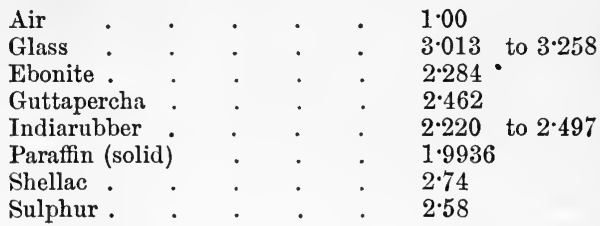

Hopkinson, whose method was a "slow" one, found for glass much higher inductive capacities, ranging from 6.5 to $10^{\cdot} 1$, the denser kinds having higher capacities. Mica has values ranging from 5.5 to 8 . Cavendish observed that the apparent capacity of glass became much greater at those temperatures at which it begins to conduct electricity. Boltzmann has announced that in the case of two crystalline substances, Iceland spar and sulphur, the inductive capacity is different in different 
directions, according to their position with respect to the axes of crystallization.

298. Dielectric Capacity of Liquids and Gases.-The dielectric capacity of liquids also has specific values, as follows :-

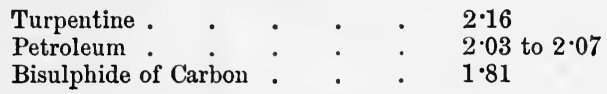

Faraday examined the inductive capacity of several gases by means of his apparatus (Fig. 156), one of the condensers being filled with air, the other with the gas which was let in through the tap below the sphere after exhaustion by an air pump. The method was too rough, however, to enable him to detect any difference between them. More recently Boltzmann, and independently Ayrton and Perry, have measured the dielectric capacities of different gases by very exact methods ; and their results agree very fairly.

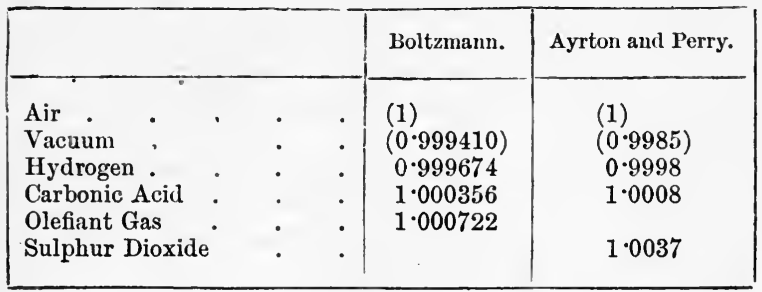

The effect of using instead of air a medium of higher dielectric power $k$ is to change the forces exerted between charged bodies. For given fixed charges the forces vary inversely as $k$; while for given differences of potential between the bodies the forces vary directly as $k$.

299. Mechanical Effects of Dielectric Stress.That different insulating substances have specific inductive power sufficiently disproves the idea that influence is 
merely an "action at a distance," for it is evident that the dielectric medium is itself concerned in the propagation of influence, and that some media allow influence to take place across them better than others. The existence of a residual charge (Art. 61) can be explained either on the supposition that the dielectric is composed of heterogeneous particles which have unequal conducting powers, as Maxwell has suggested, or on the hypothesis that the molecules are actually subjected to a strain from which, especially if the stress be long continued, they do not recover all at once. Kohlrausch and others have pointed out the analogy between this phenomenon and that of the "elastic recovery" of solid bodies after being subjected to a bending or a twisting strain. A fibre of glass, for example, twisted by a certain force, flies back when released to almost its original position, a slight sub-permanent set remains, from which, however, it slowly recovers itself, the rate of its recovery depending upon the amount and duration of the original twisting strain. A quartz fibre never shows any sub-permanent set. Hopkinson has shown that it is possible to superpose several residual charges, even charges of opposite signs, which apparently "soak out" as the strained material gradually recovers itself. Perry and Ayrton have also investigated the question, and have shown that the polarization charges in voltameters exhibit a similar recovery. * Air condensers exhibit no residual charges. Nor do plates of quartz cut from homogeneous crystal.

When a condenser is discharged a sound is often heard. This was noticed by Lord Kelvin in the case of air condensers; Varley and Dolbear have constructed telephones in which the rapid charge and discharge of

* It would appear, therefore, probable that Maxwell's suggestion of heterogeneity of structure, as leading to residual electrification at the bounding surface of the particles whose electric conductivities differ, is the true explanation of the "residual" charge. The phenomenon of elastic recovery may itself be due to heterogeneity of structure. Glass itself is a mixture of different silicates. 
a condenser gave rise to musical tones and to articulate speech.

As to the precise nature of the molecular or mechanical operations in the dielectric when thus subjected to the stress of electrostatic induction, nothing is known. One pregnant experiment of Faraday is of great importance, by showing that induction is, as he expressed it, "an action of contiguous particles." In a glass trough (Fig. 157) is placed some oil of turpentine, in which are put some fibres of dry silk cut into small bits. Two wires pass into the liquid, one of which is joined to earth, the other

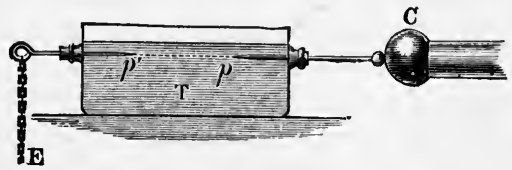

Fig. 157.

being put into connexion with the collector of an electrical machine. The bits of silk come from all parts of the liquid and form a quivering chain of particles from wire to wire, showing the electric lines of force. They at once disperse if the electric discharge is stopped. Faraday regarded this as typical of the internal actions in every case of influence across a dielectric, the particles of which he supposed to be "polarized," that is, to be turned into definite positions, each particle having a positive and a negative end. The student will perceive an obvious analogy, therefore, between the condition of the particles of a dielectric across which influence is taking place, and the molecules of a piece of iron or steel when subjected to magnetic induction. Instead of silk, crystals of sulphate of quinine may be used. Or finely-divided sulphide of antimony may be strewn on the bottom of a glass dish and covered with a layer of petroleum, to show the electric lines of force.

Siemens has shown that the glass of a Leyden jar is sensibly warmed after being several times rapidly charged and discharged. This obviously implies that 
molecular movement accompanies the changes of dielectric stress.

300. Electric Expansion.-Fontana noticed that the internal volume of a Leyden jar increased when it was charged. Priestley and Volta sought to explain this by suggesting that the attraction between the two charged surfaces compressed the glass and caused it to expand laterally. Duter showed that the amount of apparent expansion was inversely proportional to the thickness of the glass, and varied as the square of the potential difference. Quincke has recently shown that though glass and some other insulators exhibit electrical expansion, an apparent contraction is shown by resins and oily bodies under electrostatic stress. He connects with these properties the production of optical strain and of double refraction discovered by Kerr. (See Lesson on Electrooptics, Art. 525).

301. Submarine Cables as Condensers.-A submarine telegraph cable may act as a condenser, the ocean forming the outer coating, the internal wire the inner coating, while the insulating layers of guttapercha serve as dielectric. When one end of a submerged cable is connected to, say, the + pole of a powerful battery, electricity flows into it. Before any signal can be received at the other end, enough electricity must flow in to charge the cable to a considerable potential, an operation which may in the case of long cables require some seconds. Faraday predicted that this retardation would occur. It is, in actual fact, a "serions obstacle to rapid signalling through Atlantic and other cables. Professor Fleeming Jenkin has given the following experimental demonstration of the matter. Let a mile of insulated cable wire be coiled up in a tub of water (Fig. 158), one end $\mathrm{N}$ being insulated. The other end is joined up through a long-coil galvanometer $G$ to the + pole of a large battery, whose - pole is joined by a wire to the water in the tub. Directly this is done, the 
needle of the galvanometer will show a violent deflexion, electricity rushing through it into the interior of the cable, and a - charge being accumulated on the outside of it where the water touches the guttapercha. For perhaps an hour the flow will go on, though diminishing, until the cable is fully charged. Now remove the battery, and instead join up $a$ and $b$ by a wire; the charge in the cable will rush out through the galvanometer, which will

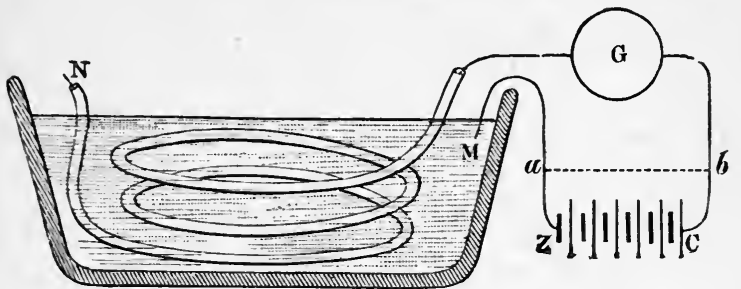

Fig. 158.

show an opposite deflexion, and the residual charge will continue "soaking out" for a long time.

Long land-lines carried overhead also possess a measurable capacity, and tend to retard the signals.

302. Use of Condensers.-To obviate this retardation and increase the speed of signalling in cables * several devices are adopted. Very delicate receiving instruments are used, requiring only a feeble current; for with the feebler batteries the actual charge given to the cable is less. In some cases a key is employed which, after every signal, immediately sends into the cable a charge of opposite sign, to sweep out, as it were, the charge left behind. Often a condenser of several microfarads' capacity is interposed in the circuit at each end of the cable to curb the signal, or make it shorter and sharper, and by its reaction assist the discharge. In duplex signalling (Art. 503) the resistance and electrostatic capa-

* The capacity of the "Direct" Atlantic cable from Ballinskelligs (Ireland) to Nova Scotia is 992 microfarads. 
city of the cable have to be met by balancing against them an "artificial cable" consisting of a wire of equal resistance, combined with a condenser of equal capacity. Messrs. Muirhead constructed for duplexing the Atlantic cable a condenser containing 100,000 square feet (over two acres of surface) of tinfoil. Condensers are also occasionally used on telegraph lines in single working to obviate disturbances from earth currents. They are constructed by placing sheets of tinfoil between sheets of mica or of paraffined paper, alternate sheets of foil being connected together. The paper is the finest bank-wove, carefully selected to be free from minute holes. Two thicknesses, drawn through a bath of the purest paraffin wax heated till it melts, are laid between each foil and the next ; care being taken to exclude air bubbles. When a sufficient number have been assembled hot they are put under pressure to cool, and afterwards adjusted. Smaii condensers of similar construction are used in connexion with induction coils (Fig. 135).

303. Practical Unit of Capacity.-Electricians adopt a unit of capacity, termed one farad, based on the system of electromagnetic units. A condenser of one

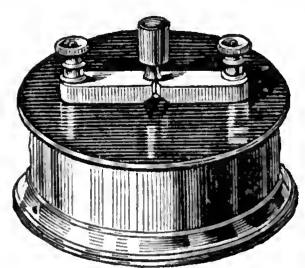

Fig. 159. farad capacity would be raised to a potential of one volt by a cliarge of one coulomb of electricity. ${ }^{*}$ In practice such a condenser would be too ellormous to be constructed; the earth itself, as an isolated sphere, has a capacity of only $\frac{7}{10000}$ of a farad. As a practical unit of capacity is therefore chosen the microfarad, or one millionth of a farad ; a capacity about equal to that of three miles of an Atlantic cable. Condensers of only $\frac{1}{3}$ microfarad capacity are about equal to one nantical mile of cable. They contain about 1200 square inches of foil. The

* See list of Practical Electromagnetic Units, Art. 354. 
dielectric in them is usually mica, in thin sheets. Their general form is shown in Fig. 159. The two brass pieces upon the ebonite top are connected respectively with the two series of alternate sheets of tinfoil. The plug between them serves to keep the condenser discharged when not in use.

Methods of measuring the capacity of a condenser are given in Art. 418.

304. Formulæ for Capacities of Conductors and Condensers. - The following formula give the capacity of condensers of all ordinary forms, in electrostatic units :-

Sphere: $($ radius $=r$. See Art. 271).

$$
\mathrm{K}=r \text {. }
$$

T'wo Concentric Spheres: (radii $r$ and $r$, dielectric capacity, $k$ ).

$$
\mathrm{K}=l \cdot \frac{r r^{\prime}}{r^{\prime}-r} .
$$

Cylinder : (length $=l$, radius $=r$ ).

$$
\mathrm{K}=\frac{l}{2 \log _{\epsilon} \frac{l}{r}} .
$$

Two Concentric Cylinders: (length $=l$, dielectric capacity $=k$, internal radius $=r$, external radius $=r^{\prime}$ ).

$$
\mathrm{K}=k \frac{l}{2 \log _{\epsilon} \frac{r^{\prime}}{r}} .
$$

Circular Disk: (radius $=r$, thickness negligible).

$$
\mathrm{K}=2 r / \pi \text {. }
$$

Two Circular Disks: (like air condenser, Art. 56, radii $=r$, surface $=\mathrm{S}$, thickness of dielectric $=b$, dielectric capacity $=k$ ).

or

$$
\begin{aligned}
& \mathrm{K}=k r^{2} / 4 b, \\
& \mathrm{~K}=k \mathrm{~S} / 4 \pi b .
\end{aligned}
$$


The latter formula applies to any two parallel disks of surface $\mathrm{S}$, whether circular or otherwise, provided they are large as compared with the distance $b$ between them. To calculate down to nicrofarads the numbers given by any of the above must be divided by 900,000 .

305. Energy of Discharge of Leyden Jar or Condenser.-It follows from the definition of potential, given in Art. 263, that in bringing up one + unit of electricity to the potential $\mathrm{V}$, the work done is $\mathrm{V}$ ergs. This assumes, however, that the total potential $\mathrm{V}$ is not thereby raised, and on this assumption the work ${ }^{*}$ done in bringing up $\mathrm{Q}$ units would be $\mathrm{QV}$ ergs. If, however, the potential is nothing to begin with, and is raised to $\mathrm{V}$ by the charge $Q$, the average potential during the operation is only $\frac{1}{2} \mathrm{~V}$; hence the total work done in bringing up the charge $\mathrm{Q}$ from zero potential to potential $\mathrm{V}$ is $\frac{1}{2} \mathrm{QV}$ ergs. Now, according to the principle of the conservation of energy, the work done in charging a jar or condenser with electricity is equal to the work which could be done by that quantity of electricity when the jar is discharged. Hence $\frac{1}{2} Q V$ represents also the energy of the discharge.

Since $Q=V K$, it follows that we may write $\frac{1}{2} Q V$ in the form $\frac{1}{2} \frac{Q^{2}}{K}$. That is to say, if a condenser of capacity $K$ is charged by having a charge $\mathrm{Q}$ imparted to it, the energy of the charge is proportional directly to the square of the quantity, and inversely to the capacity of the condenser.

306. Symbol for Condenser.-Electricians use as symbols for condensers in diagrams of electric circuits

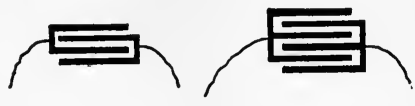

Fig. 160. those given in Fig. 160. The origin of these symbols is the alternate layers of tinfoils. The symbol on the right suggests six layers of foil, of which the first, third, and fifth

* If $\mathrm{Q}$ is given in coulombs and $\mathrm{V}$ in volts, the work will be expressed not in ergs but in joules (Art. 354). 
are joined together, and the second, fourth, and sixth are also joined together.

307. Capacities joined in Parallel. - To join two condensers together in parallel the positive foils of one are joined to the positive foils of the other, and their negative foils are also joined together. In Fig. 161 the two condensers $K_{1}$ and $K_{2}$ are joined in parallel. They will thus act simply like one large condenser of capacity $=\mathrm{K}_{1}+\mathrm{K}_{2}$. Any charge flowing in on the + side will divide between the two in porportion to their capacities.

If two equal Leyden jars are charged to the same potential, and then their inside and outside coatings are respectively joined, their united charge will be the same as that of a jar of equal thickness, but having twice the amount of surface.

If a charged Leyden jar is placed similarly in com-

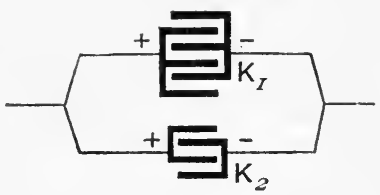

Fig. 161. munication with an uncharged jar of equal capacity, the charge will be shared equally between the two jars, and the passage of electricity from one to the other will be evidenced by the production of a spark when the respective coatings are put into communication. Here, however, half the energy of the charge is lost in the operation of sharing the charge, for each jar will have only $\frac{1}{2} \mathrm{Q}$ for its charge and $\frac{1}{2} \mathrm{~V}$ for its potential ; hence the energy of the charge of each, being half the product of charge and potential, will only be one quarter of the original energy. The spark which passes in the operation of dividing the charge is, indeed, evidence of the loss of energy ; it is about half as powcrful as the spark would have been if the first jar had been simply discharged, and it is just twice as powerful as the small sparks yielded finally by the discharge of each jar after the charge has becn shared between them.

The energy of a charge of the jar manifests itself, as 
stated above, by the production of a spark at discharge; the sound, light, and heat produced being the equivalent of the energy stored up. If discharge is effected slowly through a long thin wire of high resistance the air spark may be feeble, but the wire may be perceptibly heated. A wet string being a feeble conductor affords a slow and almost silent discharge; here probably the electrolytic conduction of the moisture is accompanied by an action resembling that of secondary batteries (Lesson 492) tending to prolong the duration of the discharge.

308. Capacities joined in Series.-If two condensers are joined in series they will act as a condenser having a lesser capacity than either of them separately. Their joint capacity in series will be the reciprocal of the sum of the reciprocals of their capacities separately.

Proof.-Let two condensers $K_{1}$ and $K_{2}$ be set in series (Fig. 162) between two points across which there is a difference of

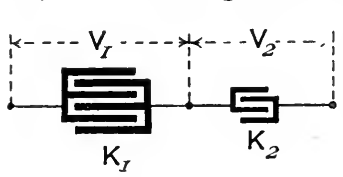

Fig. 162. potential V. This difference of potential will be divided between the two inversely in proportion to their capacities, seeing that the quantities of electricity that are displaced into and out of their respective coatings are necessarily equal. Or, if $Q$ be this quantity, and $\mathrm{K}_{3}$ the effective or joint capacity of the two together, to find the latter, we have :-

and

$$
\begin{aligned}
& \mathrm{Q}=\mathrm{V}_{1} \mathrm{~K}_{1}=\mathrm{V}_{2} \mathrm{~K}_{2}=\mathrm{VK}_{3} \quad \cdot \quad \cdot \quad . \\
& \mathrm{V}=\mathrm{V}_{1}+\mathrm{V}_{2} \cdot .
\end{aligned}
$$

From (1) we get

and

$$
\mathrm{V}_{1}=\mathrm{VK}_{3} / \mathrm{K}_{1} \text {, }
$$

$$
\mathrm{V}_{2}=\mathrm{VK}_{3} / \mathrm{K}_{2} \text {. }
$$

Inserting these in (2) we get

$$
\mathrm{V}=\mathrm{VK}_{3} / \mathrm{K}_{1}+\mathrm{VK}_{3} / \mathrm{K}_{2} ;
$$

whence, dividing down by $\mathrm{VK}_{3}$, we get

$$
\frac{1}{\mathrm{~K}_{3}}=\frac{1}{\mathrm{~K}_{1}}+\frac{1}{\mathrm{~K}_{2}} \text {. . . . Q.E.D. }
$$


Example.-If two condensers, respectively 3 and 2 microfarads, are joined in series, they will act as a single condenser of capacity $=1 /\left(\frac{1}{3}+\frac{1}{2}\right)=1 \frac{1}{6}$ microfarads.

309. Charge of Jars arranged in Cascade. Franklin suggested that a series of jars might be arranged, the outer coating of one being connected with the inner one of the next, the outer coating of the last being connected to earth. The object of this arrangement was that the second jar might be charged with the electricity repelled from the outer coating of the first, the third from that of the second, and so on. This "cascade" arrangement, however, is of no advantage, the sum of the charges accumulated in the series being only equal to that of one single jar if used alone. For if the inner coating of the first jar be raised to V, that of the outer coating of the last jar remaining at zero in contact with earth, the difference of potential between the outer and inner coating of any one jar will be only $\frac{1}{n} \mathrm{~V}$, where $n$ is number of jars. And as the charge in each jar is equal to its capacity $\mathrm{K}$, multiplied by its potential, the charge in each will only be $\frac{1}{n} \mathrm{KV}$, and in the whole $n$ jars the total charge will be $n \frac{1}{n} \mathrm{KV}$, or $\mathrm{KV}$, or equals the charge of one jar of capacity $\mathrm{K}$ raised to the same potential V.

\section{Lesson XXIV._Phenomena of Discharge}

310. Conductive Discharge.-An electrified conductor may be discharged in at least three different ways, depending on the medium through which the discharge is effected, and varying with the circumstances of the discharge. If the discharge takes place by the passage of a continuous current, as when electricity flows through a thin wire connecting the knobs of an influence machine, or joining the positive pole of a battery to the negative 
pole, the operation is termed a "conductive" discharge. Under some circumstances a conductive discharge takes the nature of an oscillation to and fro (Art. 515).

311. Disruptive Discharge.-It has been shown how influence across a non-conducting medium is always accompanied by a mechanical stress upon the medium; the tension along the electric lines of force increasing as the square of the intensity of the electric field. If this stress is very great the non-conducting medium will suddenly give way and a spark will burst across it. Such a discharge is called a "disruptive" discharge.

A very simple experiment will set the matter in a clear light. Suppose a metal ball charged with + electrification to be hung by a silk string above a metal plate lying on the ground. If we lower down the suspended ball a spark will pass between it and the plate when they come very near together, and the ball will then be found to have lost all its previous charge. It was charged with a certain quantity of electricity; and as it had, when suspended out of the range of other conductors, a certain capacity (numerically equal to its radius in centimetres), the electricity on it would be at a certain potential (namely $=\mathrm{Q} / \mathrm{K}$ ), and the charge would be distributed uniformly all over it. The plate lying on the earth would be all the while at zero potential. But when the suspended ball was lowered down towards the plate the previous state of things was altered. In the presence of the + charge of the ball the potential ${ }^{*}$ of the plate would rise, were it not that, by influence, just enough negative electrification appears on it to keep its potential still the same as that of the earth. The tension in the electric field will draw the + charge of the ball downwards, and alter the distribution of the charge, the surfacedensity becoming greater at the under surface of the ball

* The student must remember that, by the definition of potential in Art. 263, the potential at a point is the sum of all the separate quantities of electricity near it, divided each by its distance from the point. 
and less on the upper. The capacity of the ball will be increased, and therefore its potential will fall correspondingly. The layer of air between the ball and the plate is acting like the glass of a Leyden jar. The more the ball is lowered down the greater is the accumulation of the opposite kinds of charge on each side of the layer of air, and the tension across the layer becomes greater and greater, until the limit of the dielectric strength is reached; the air suddenly gives way and the spark tears a path across.

312. Convective Discharge.-A third kind of discharge, differing from either of those above mentioned, may take place, and occurs chiefly when electricity of a high potential discharges itself at a pointed conductor by accumulating there with so great a density as to electrify the neighbouring particles of air; these particles then flying off by repulsion, conveying away part of the charge with them. 'Such convective discharges may occur either in gases or in liquids, but are best manifested in air and other gases at a low pressure, in tubes exhausted by an air pump.

The discharge of a quantity of electricity in any of the above ways is always accompanied by a transformation of its energy into energy of some other kind,-sound, light, heat, chemical actions, and other phenomena being produced. These effects must be treated in detail.

313. Length of Spark.-Generally speaking, the length of spark between two conductors increases with the difference between their potentials. It is also found to increase when the pressure of the air is diminished. Riess found the distance to increase in a proportion a little exceeding that of the difference of potentials. Lord Kelvin confirmed this by measuring by means of an "absolute electrometer" (Art. 287) the difference of potential necessary to produce a spark discharge betweer two parallel plates at different distances. De la Rue and Muiller found with their great battery (Art. 186) 
that with a difference of potential of 1000 volts the striking distance of the spark was only 0127 centimetres (or about $\frac{1}{200}$ of an inch), and with a difference of 10,000 volts only $1 \cdot 369$. Their 11,000 silver cells gave a spark of 1.59 centim. (about $\frac{2}{3}$ of an inch) long. To produce a spark one mile long, through air at the ordinary pressure, would therefore require a difference of potential exceeding that furnished by $1,000,000,000$ Daniell's cells !

The length of the spark differs in different gases, being nearly twice as long in hydrogen as in air at the same density. Or to produce in hydrogen a spark as long as one in air requires less voltage. On the other hand, carbonic acid gas, whilst it is stronger than air for short sparks, is weaker for long ones.

The potential needful to produce a spark of given length in a given gas is independent of the kind of metal used as electrodes, but depends upon their shape. If points are used instead of balls it is found that, at equal voltage, points are best for long sparks, but are worst for short sparks.

According to Peace's observations a minimum potential of between 300 and 400 volts is necessary to start a spark, however short, in air. For sparks not under two millimetres in length the volts necessary to start a spark across a length of $l$ centimetres may be approximately expressed by the equation-

$$
\mathrm{V}=1500+30,000 l .
$$

The following table, calculated from the results of Heydweiller, gives the volts necessary to produce a spark in air at $15^{\circ} \mathrm{C}$. and 76 centimetres pressure between two spheres of various sizes. The figures must be increased 1 per cent for a fall of 3 degrees of temperature, or for a rise of 8 millimetres of pressure. 


\begin{tabular}{|c|c|c|c|c|}
\hline \multirow{2}{*}{ Radius of Balls. } & \multicolumn{3}{|c|}{ Distance between Balls (Centims.) } \\
\cline { 2 - 5 } & 0.1 & 0.5 & 1.0 & 1.5 \\
\hline $\begin{array}{c}\text { Centims. } \\
2.5\end{array}$ & $\begin{array}{c}\text { Volts. } \\
4500\end{array}$ & $\begin{array}{c}\text { Volts. } \\
18900\end{array}$ & $\begin{array}{c}\text { Volts. } \\
33840\end{array}$ & $\begin{array}{c}\text { Volts. } \\
47610\end{array}$ \\
\hline 1.0 & 4860 & 18030 & 32120 & 41160 \\
\hline 0.5 & 4950 & 17790 & 27810 & 32400 \\
\hline 0.25 & 4980 & 16200 & 20790 & 22980 \\
\hline
\end{tabular}

In rarefied air the spark is longer. Snow Harris stated that the length of spark was inversely proportional to the pressure, but this law is not quite correct, being approximately true only for pressures between that of 11 inches of mercury and that of 30 inches (one atmosphere). At lower pressures, as Gordon found, a greater difference of potential must be used to produce a spark than that which would accord with Harris's law. From this it would appear that thin layers of air oppose a proportionally greater resistance to the piercing power of the spark than thick layers, and possess greater dielectric strength.

Faraday, using two spheres of different sizes, found the spark-length greater when the smaller sphere was positive than when it was negative.

With rapidly-alternating differences of potential, smaller virtnal voltages suffice for the same spark-length, for the length depends on the maximum, not on the mean value. Using a ball of $1 \mathrm{~cm}$. diameter and a disk, Alexander Siemens found 3200 virtual volts to be needed at $0.1 \mathrm{~cm}$. distance, and 11,000 at $0.5 \mathrm{~cm}$. distance apart.

The dielectric strength of a gas appears to be weaker when field is varying than when it is steady. When the 
voltage is nearly high enough to produce a spark, reversing the poles will sometimes start a spark. Moreover, when once a spark has passed it is easier for a second one to follow on the same track. Probably the first spark procuces chemical dissociations in its path which do not instantly pass away.

Hertz made the singular observation that ultra-violet light (i.e. actinic waves) falling upon the kathode surface assist it to discharge (see Art. 531).

A perfect vacuum is a perfect insulator-no spark will cross it. It is possible to exhaust a tube so perfectly that none of our electric machines or appliances can send a spark through the vacuous space even over so short a distance as one centimetre.

On the other hand, a great increase of pressure also increases the dielectric strength of air, and causes it to resist the passage of a spark. Cailletet compressed dry air at 40 to 50 atmospheres' pressure, and found that even the spark of a powerful induction coil failed to cross a space of '05 centimetres' width.

314. Flames and Hot Air.-The arc produced by the passage of an electric current between two carbon poles is treated of in Art. 448. It is a species of flame which conducts the current from the tip of one carbon rod to the other, while volatilizing the carbon, and requires only some thirty to fifty volts for its maintenance. The alternate-current arc generated in air by high-frequency discharges at a potential of 10,000 to 50,000 volts is a different phenomenon, and is apparently an endothermic flame of nitrogen and oxygen burned together.

Sparks are longer and straighter through hot air than through cold. If air or other permanent gas is, however, heated in a closed vessel so that its density remains unaltered, the voltage needful to produce discharge remains the same; unless, indeed, the gas be heated to point of dissociation when discharge occurs at low voltage. 
Flames and currents of very hot air, such as those rising from a red-hot piece of iron, are extremely good conductors of electricity, and act even better than metallic points in discharging a charged conductor. Gilbert showed that an electrified body placed near a flame lost its charge; and the very readiest way to rid the surface of a charged body of low conducting power of a charge imparted to it by friction or otherwise, is to pass it through the flame of a spirit-lamp. Faraday found negative electrification to be thus more easily discharged than positive. Flames powerfully negatively electritied are repelled from conductors, though not so when positively electrified. Sir W. Grove showed that a current is set up in a platinum wire, one end of which touches the tip, and the other the base, of a flame.

Guthrie showed that a red-hot iron ball cannot be positively, but may be negatively charged. When whitehot it will retain neither kind of charge.

315. Mechanical Effects. - Chief amongst the mechanical effects of the disruptive spark discharge is the shattering and piercing of glass and other insulators. The dielectric strength of glass, though much greater than that of air, is not infinitely great. A slab of glass 3 inches thick has been pierced by the discharge of a powerful induction coil. The so-called "toughened" glass has a greater dielectric strength than ordinary glass, and is more difficult to pierce. A sheet of glass may be readily pierced by a spark from a large Leyden jar or battery of jars, by taking the following precautions :The glass to be pierced is laid upon a block of glass or resin, through which a wire is led by a suitable hole, one end of the wire being connected with the outer coating of the jar, the other being cut off flush with the surface. Upon the upper surface of the sheet of glass that is to be pierced another wire is fixed upright, its end being exactly opposite the lower wire, the other extremity of this wire being armed with a metal knob to receive the spark from 
the knob of the jar or discharger. To ensure good insulation a few drops of paraffin oil, or of olive oil, are placed upon the glass round the points where the wires touch it. A piece of dry wood similarly treated is split by a powerful spark. A layer of oil resists being pierced as much as a layer of air five or six times as thick would do.

If a spark is led through a tightly-corked glass tube containing water, the tube will be shattered into small pointed fragments by the sudden expansion of the liquid.

Lullin observed two curious effects when a piece of cardboard is perforated by a spark between two metal points. Firstly, there is a slight burr raised on each side, as if the hole had been pierced from the middle ontwards, as though the stress in the air had pulled at the card. Secondly, if the two points are not exactly opposite one another the hole is found to be nearer the negative point. But if the experiment is tried under the air pump in a vacuum, there is no such displacement of the hole; it is then midway exactly.

The mechanical action of the brush discharge at points is mentioned in Art. 47, and the mechanical effects of a current of electricity were described in Lesson XVI.

316. Chemical Effects.-The chemical actions produced by currents of electricity have been described in Lessons XIV. and XIX. Similar actions can be produced by the electric spark, and by the silent glow discharge (see Art. 319). Faraday showed, indeed, that electricity from all kinds of different sources produced the same kinds of chemical actions, and he relied upon this as one proof of the essential identity of the electricity produced in different ways. If sparks from an electric machine are received upon a piece of white blotting-paper moistened with a solution of iodide of potassium, brown patches are noticed where the spark has effected a chemical decomposition and liberated the iodine.

When a stream of sparks is passed through moist air 
in a vessel, the air is found to have acquired the property of changing to a red colour a piece of paper stained blue with litmus. This, Cavendish showed, was due to the presence of nitric acid, produced by the chemical union of the nitrogen and oxygen of the air. The effect is best shown with the stream of sparks yielded by a small induction coil (Fig. 135), in a vessel in which the air has been compressed beyond the usual atmospheric pressure.

Whenever an electric machine is giving out highvoltage discharges a peculiar orluur is perceived. This was formerly thought to be evidence of the existence of an electric "effluvium" or fluid ; it is now known to be due to the presence of ozone, a modified form of oxygen gas, which differs from oxygen in being denser, more active chemically, and in having a characteristic smell. The silent discharge of the influence machine and that of the induction coil are particularly favourable to the production of this substance.

The spark will decompose ammonia gas, and olefiant gas, and it will also cause chemical combination to take place with explosion, when passed through detonating mixtures of gases. Thus equal volumes of chlorine and hydrogen are exploded by the spark. So are oxygen and hydrogen gases, when mixed in the proportion of two volumes of the latter to one of the former. Even the explosive mixture of common coal gas mixed with from four to ten times its own volume of common air, can be thus detonated. A common experiment with the so-called electric pistol consists in filling a small brass vessel with detonating gases and then exploding them by a spark. The spark discharge is sometimes applied to the firing of blasts and mines in military operations.

317. Heating Effects. - The flow of electricity through a resisting medium is in every case accompanied by an evolution of heat. The laws of heating due to currents are given in Art. 427. The disruptive discharge is a transfer of electricity through a medium of great 
resistance and accompanied by an evolution of heat. A few drops of ether in a metallic spoon are easily kindled by an electric spark. The spark from an electric machine, or even from a rubbed glass rod, suffices to kindle an ordinary gas-jet. In certain districts of America, during the driest season of the year, the mere rubbing of a person's shoes against the carpet, as he shuffles across the floor, generates sufficient electrification to enable sparks to be drawn from his body, and he may light the gas by a single spark from his outstretched finger. Gunpowder can be fired by the discharge of a Leyden jar, but the spark should be retarded by being passed through a wet thread, otherwise the powder will simply be scattered by the spark.

The Electric Air-Thermometer, invented by Kinnersley, ${ }^{*}$ serves to investigate the heating powers of the discharge. It consists of a glass vessel enclosing air, and communicating with a tube partly filled with water or other liquid, in order to observe changes of volume or of pressure. Into this vessel are led two metal rods, between which is suspended a thin wire, or a filament of gilt paper; or a spark can be allowed simply to cross between them. When the discharge passes the enclosed air is heated, expands, and causes a movement of the indicating column of liquid. The results of observation with these instruments are as follows:-The heating effect produced by a given charge in a wire of given length is inversely proportional to the square of the area of the cross section of the wire. The total heat evolved is jointly proportional to the charge, and to the potential through which it falls. In fact, if the entire energy of the discharge is expended in producing heat, and in doing no other kind of work, then the heat developed will be the thermal

* This instrument differs in no essential respeet from that devised ninety years later by Riess, to whom the instrument is often aceredited. Riess, however, dedueed quantitative laws, while Kinnersley contented himself with qualitative observations. 
equivalent of $\frac{1}{2} \mathrm{QV}$ ergs, or $\frac{\mathrm{QV}}{2 \mathrm{~J}}$ calories; where $\mathrm{J}$ represents the mechanical equivalent of heat $(J=42$ million; since $42 \times 10^{6}$ ergs $=1$ calorie), and $\mathrm{Q}$ and $\mathrm{V}$ are expressed in C.G.S. units.

When a powerful discharge takes place through very thin wires, they may be heated to redness, and even fused by the heat evolved. Van Marum thus once heated 70 feet of wire by a powerful discharge. A narrow strip of tinfoil is readily fused by the charge of a large Leyden jar, or battery of jars. A piece of gold leaf is in like manner volatilized by a powerful discharge. Franklin utilized this property for a rude process of multiplying portraits or other patterns, which, being first cut out in card, were reproduced in a silhouette of metallic particles on a second card, by the device of laying above them a film of gold or silver leaf covered again with a piece of card or paper; a Leyden battery being then discharged through the leaf.

318. Luminous Effects.-The discharge exhibits many beautiful and varied luminous effects under different conditions. The spark of the disruptive discharge is usually a thin brilliant streak of light. When it takes place between two metallic balls, separated only by a short interval, it usually appears as a single thin and brilliant line. If, however, the distance be as much as a few centimetres, the spark takes an irregular zigzag form. In any case its path is along the line of least resistance, the presence of minute motes of dust floating in the air being quite sufficient to determine the zigzag character. Often the spark exhibits curious ramifications and forkings, of which an illustration is given in Fig. 163, which is drawn one-eighth of the actual size of the spark obtained from an electrical machine. Photograplss of lightning flashes almost always show similar branching. The branches always point toward the negative electrode. The discharge from a Leyden jar aftords a much brighter, 
shorter, noisier spark than the spark drawn direct from the collector of a machine. The length (see Art. 313) depends upon the potential, and upon the pressure and temperature of the air in which the discharge takes place. The brilliance depends chiefly upon the quantity of the discharge. The colour of the spark varies with the nature of the metal surfaces between which the discharge takes place; for the syark tears away in its passage small portions of the metal surfaces, and volatilizes them. Between copper or silver terminals the spark takes a green tint, while between iron knobs it is of a reddish hue.

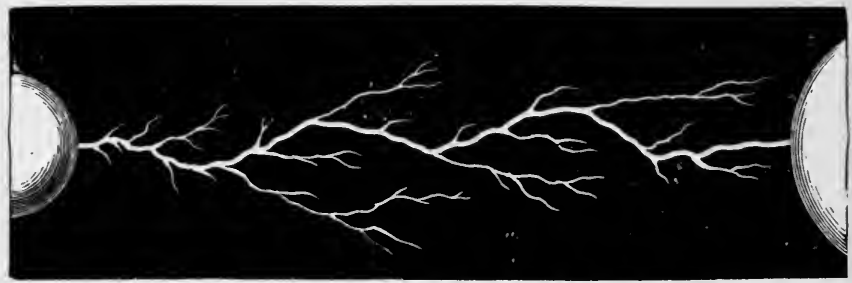

Fig. 163.

Examination with the spectroscope reveals the presence in the spark of the rays characteristic of the incandescent vapours of the several metals.

319. Brush Discharge: Glow Discharge.-If an electric machine is vigorously worked, but no sparks be drawn from its collector, a fine diverging brush of pale blue light can be seen (in a dark room) streaming from the brass ball at the end of it farthest from the collecting comb; a hissing or crackling sound always accompanies this kind of discharge. The brush discharge consists of innumerable fine twig-like ramifications, presenting a form of which Fig. 164 gives a fine example. The brightness and size of the brush is increased by holding a flat plate of metal a little way from it. With 
a smaller ball, or with a bluntly-pointed wire, the brush appears smaller, but is more distinct and continuous. When discharge is going on between two balls the brushes are never alike. At the positive ball or anode the brush discharge is larger and more ramified than at the negative ball. But the negative brush is more easily formed than the positive. Wheatstone found by using his rotating mirror that the brush discharge is really a series

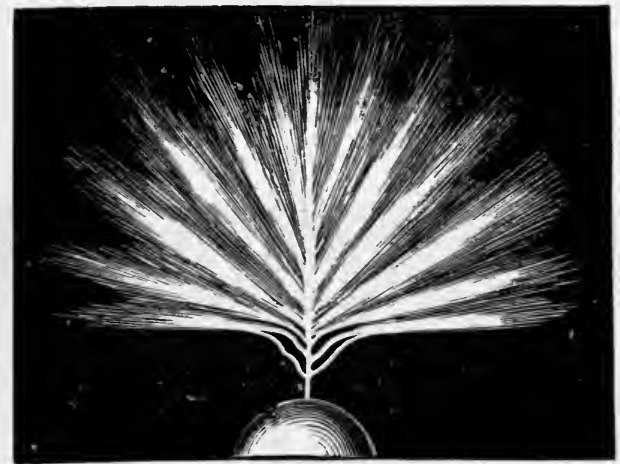

Fig. 164.

of successive partial sparks at rapid intervals. Metallic dust is in every case torn away from the electrode by the brush discharge.

If the blunt or rounded conductor be replaced by a pointed one, the brush disappears and gives place to a quiet and continnous glow where the electrified particles of air are streaming away at the point. If these convexion-streams are impeded the glow may once more give place to the brush. Where a negative charge is being discharged at a point, the glow often appears to be separated from the surface of the conductor by a dark space, where the air, without becoming luminous, still 
conveys the electricity. This phenomenon, to which Faraday gave the name of the "dark" discharge, is very well seen when electricity is discharged through rarefied air and other gases in vacuum tubes.

A spark discharge may degenerate into a brush if the surface of the electrode becomes pitted or roughened by frequent discharges. Hence in all spark experiments it is important to keep the discharging balls highly polished.

320. Discharges in Partial Vacua. - If the discharge takes place in glass tubes or vessels from which the air has been partially exhausted, many remarkable and beautiful luminous phenomena are produced. A common form of vessel is the "electric egg" (Fig. 137), a sort of oval bottle that can be screwed to an air pump, and

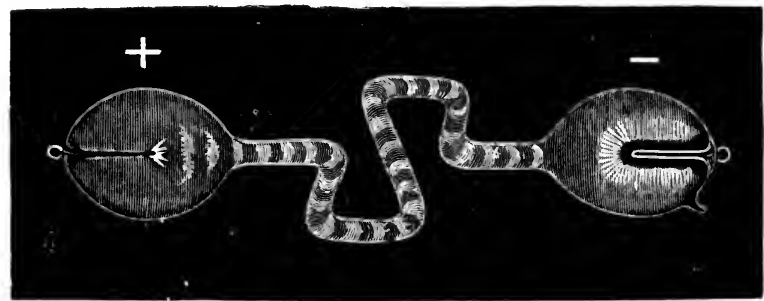

Fig. 165.

furnished with brass knobs to lead in the sparks. More often "vacuum tubes," such as those manufactured by the celebrated Geissler, are employed. These are merely tubes of thin glass blown into bulbous or spiral forms, provided with two electrodes of platinum wire fused into the glass, and sealed off after being partially exhausted of air by a mercurial air pump. Of these Geissler tubes the most useful consist of two bulbs joined by a narrow tube (Fig. 165), the luminous effects being usually more intense in the contracted portion. Such tubes are readily illuminated by discharges from an electrophorus or an 
influence machine ; but it is more common to work them with the spark of an induction coil (Fig. 135). A coil capable of throwing a $\frac{1}{2}$-inch spark in air will illuminate a vacuum tube 6 or 8 inches long. Where an alternatecurrent supply is available small transformers (Art. 228) wound to deliver $\frac{1}{50}$ ampere at 5000 volts serve admirably for lighting vacuum tubes.

Through such tubes, before exhaustion, the spark passes without any unusual phenomena being produced. As the air is exhausted the sparks become less sharply defined, and widen out to occupy the whole tube, becoming pale in tint and nebulous in form. The kathode exhibits a beautiful bluish or violet glow, separated from the conductor by a narrow dark space, while at the anode a single small bright star of light is all that remains. At a certain degree of exhaustion the light in the tube breaks up into a set of stria, or patches of light of a cup-like form, which vibrate to and fro between darker spaces. In nitrogen gas the violet aureole glowing around the kathode is very bright, the rest of the light being rosy in tint. In oxygen the difference is not so marked. In hydrogen gas the tint of the discharge is bluish, except where the tube is narrow, where a beautiful crimson may be seen. With carbonic acid gas the light is remarkably white. Particles of metal are torn off from the kathode, and projected from its surface. The kathode is also usually the hotter when made of similar dimensions to the anode. If the anode is heated and the kathode kept cool no discharge will pass. The luminosity disappears from the rarefied air in the neighbourhood of a red-hot platinum spiral inside the tube. If the kathode gets white-hot the glow disappears, and the gas conducts freely without shining. It is also observed that the light of these discharges in vacuo is rich in those rays which produce phosphorescence and fluorescence. Many beautiful effects are therefore produced by blowing tubes in uranium glass, which fluoresces with a fine green light, and by placing solutions of 
quinine or other fluorescent liquids in outer tubes of glass.

321. Phenomena in High Vacua.-Crookes has found that when exhaustion is carried to a very high degree the dark space separating the negative glow from the negative pole increases in width; and that across this space electrified molecules are projected in parallel paths normally from the surface of the kathode. If exhaustion be carried to such a high degree that the dark space fills the entire tube or bulb, the glass walls become beautifully phosphorescent. Diamonds, rubies, and even white powdered

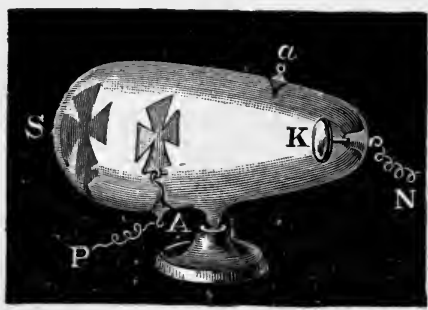

Fig. 166. alumina placed in the tubes become brilliantly phosphorescent if the kathode discharge is directed upon them. And if bodies (whether opaque or transparent) be interposed in front of the electrode, sharplydefined shadows of these bodies are projected upon the opposite wall of the vessel, as if they stopped the way fur some of the flying molecules, and prevented them from striking the opposite wall. In Fig. 166 the kathode $\mathrm{K}$ is a slightly convex disk of aluminium. In the path of the discharge is set a cross cut out of mica. Its shadow $\mathrm{S}$ appears on the end of the bulb, which phosphoresces all around the shadowed part. The anode may be either at $\mathrm{A}$ or $a$. Lightly-poised vanes are also driven round if placed in the path of the discharge. Crookes regarded this kathode discharge as exhibiting matter in an ultragaseous or radiant state. A disk placed in the line of the kathode discharge becomes thereby positively electrified. The kathode discharge is independent of the metal used as kathode, and is also independent of 
the position of the anode. Any restriction of space around the kathode tends to stop the discharge. Similar phenomena have been observed in vacuous tubes without any internal electrodes. Hertz discovered that these kathodic "rays" which will not pass through glass, mica, or any transparent substance, will pass through metal foil. Lenard, using a vacuum tube with a "window" of aluminium foil at one end, has succeeded in passing the kathodic rays out into the air (in which they cannot be produced at all), and finds them to retain their remarkable property of exciting phosphorescence.

In extremely high vacua there is an enormous resistance, apparently due to some difficulty in the electric discharge leaving the electrode. The molecular conductivity of the rarefied gas is itself very high. For an equal number of molecules it is higher than that of the metals.

Holtz has more recently produced "electric shadows," by means of discharges in air at ordinary pressure, between the poles of the influence machine (Fig. 41), the discharge taking place between a point and a disk covered with silk, on which the shadows are thrown.

322. Striæ.-The strice or stratifications have been examined very carefully by Gassiot, by Spottiswoode, and by De la Rue. The principal facts hitherto gleaned are as follow:-The striæ originate at the anode at a certain pressure, and become more numerous, as the exhaustion proceeds, up to a certain point, when they become thicker and diminish in number, until exhaustion is carried to such a point that no discharge will pass. J. J. Thomson found the column of striæ to exhibit a nearly constant electric resistance all along; though beyond it in the neighbourhood of the kathode the resistance was much greater. In a vacuum tube over 50 feet long the discharge was striated through whole length except near the kathode. If the kathode is moved forward the strix move with it. The striæ Hlicker even when the con- 
tinuous current from a battery of some thousands of cells (Art. 186) is used. There is a maximum of steadiness with a particular density of current. The striæ are hotter than the spaces between them. The number and position of the striæ vary, not only with the exhaustion, but with the difference of potentials of the electrodes. Each portion of the column of striæ acts as an independent discharge. When striæ are produced by the intermittent discharges of the induction coil, examination of them in a rotating mirror reveals that they move forward from the anode towards the kathode.

Schuster has shown that the discharge through gases is a process resembling that of electrolysis (Art. 237), being accompanied by breaking up of the gaseous molecules and incessant interchanges of atoms between them. The production of ozone (Art. 316) and the phenomena noticed at the kathode (Art. 321) give support to this view. Amongst other evidence is the striking discovery of Hittorf that quite a few cells can send a current through gas at ordinary pressures provided a spark-discharge is going on in the neighbourhood. J. J. Thomson finds that those gases which when heated are decomposed or molecularly dissociated, so that free atoms are present, are also good conductors. He regards chemical decomposition as an essential feature of gaseous discharge.

The discharges in vacuum tubes are affected by the magnet at all degrees of exhaustion, behaving like flexible conductors. Under certain conditions also, the discharge is sensitive to the presence of a conductor on the exterior of the tube, retreating from the side where it is touched. This sensitive state appears to be due to a periodic intermittence in the discharge; an intermittence or partial intermittence in the flow would also probably account for the production of striæ.

\section{Velocity of Propagation of Discharge.-} The earliest use of the rotating mirror to analyze phenomena of short duration was made by Wheatstone, who 
attempted by this means to measure "the velocity of electricity" in conducting wires. What he succeeded in measuring was not, however, the velocity of electricity, but the time taken by a certain quantity of electricity to flow through a conductor of considerable resistance and capacity. Viewed in a rotating mirror, a spark of definite duration would appear to be drawn out into an elongated streak. Such an elongation was found to be visible when a Leyden jar was discharged through a copper wire half a mile long; and when the circuit was interrupted at three points, one in the middle and one at each end of this wire, three sparks were obtained, which, viewed in the mirror, showed a lateral displacement, indicating (with the particular rate of rotation employed) that the middle spark took place $\frac{1}{1152000}$ of a second later than those at the ends. Wheatstone argued from this a velocity of 288,000 miles per second. But Faraday showed that the apparent rate of propagation of a quantity of electricity must be affected by the capacity of the conductor; and he even predicted that since a submerged insulated cable acts like a Leyden jar (see Art. 301), and has to be charged before the potential at the distant end can rise, it will retard the apparent flow of electricity through it. Professor Fleeming Jenkin says of one of the Atlantic cables that, after contact with the battery is made at one end, no effect can be detected at the other for two-tenths of a second, and that then the received current gradually increases, until about three seconds afterwards it reaches its maximum, and then dies away. This retardation is proportional to the square of the length of the cable, being proportional both to its capacity and to its resistance; hence it becomes very serious on long cables, reducing the speed of signalling. There is in fact no definite assignable "velocity of electricity." In the case of wires suspended in air the velocity of propagation of any rapid electrical vibration is equal to the velocity of light. But in the case of slow vibrations, like those of telephonic 
sounds being sent through land lines or cables, the velocity may be much less.

A very simple experiment will enable the student to realize the exceedingly short duration of the spark of a Leyden jar. Let a round disk of cardboard painted with black and white sectors be rotated very rapidly so as to look by ordinary light like a mere gray surface. When this is illuminated by the spark of a Leyden jar it appears to be standing absolutely still, however rapidly it may be turning. A flash of lightning is equally instantaneous; it is utterly impossible to determine at which end the flash begins.*

324. Electric Dust-Figures.-Electricity may creep slowly over the surface of bad conductors. Lichten-

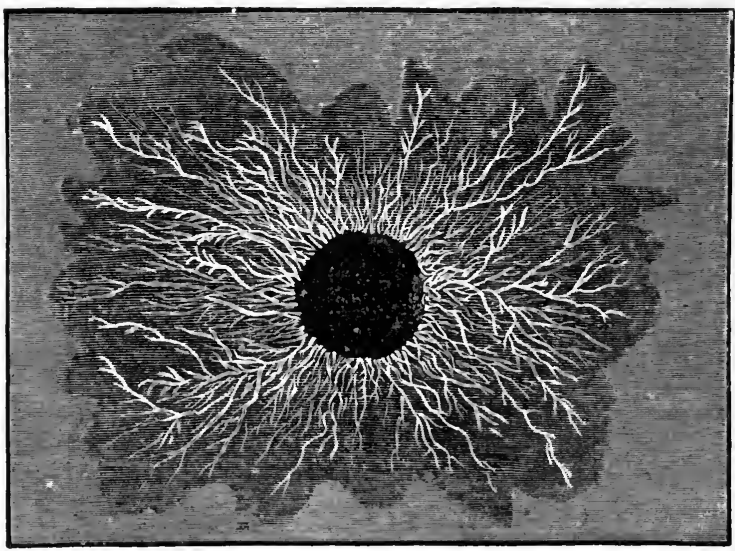

Fig. 167 .

berg devised an ingenious and easy way of investigating

* Sometimes the flash seems to strike downwards from the clonds, sometimes upwards from the earth. This is an optical illusion, resulting from the unequal sensitiveness to light of different portions of the retina of the eye. 
the distribution of electricity by means of certain electroscopic powders. Take a charged Leyden jar and write with the knob of it upon a cake of pitch or a dry sheet of glass. Then sift, through a bit of muslin, over the cake a mixture of powdered red lead and sulphur (vermilion and lycopodium powder answer equally well). The powders in this process rub against one another, the red lead becoming + , the sulphur -. Hence the sulphur will be attracted

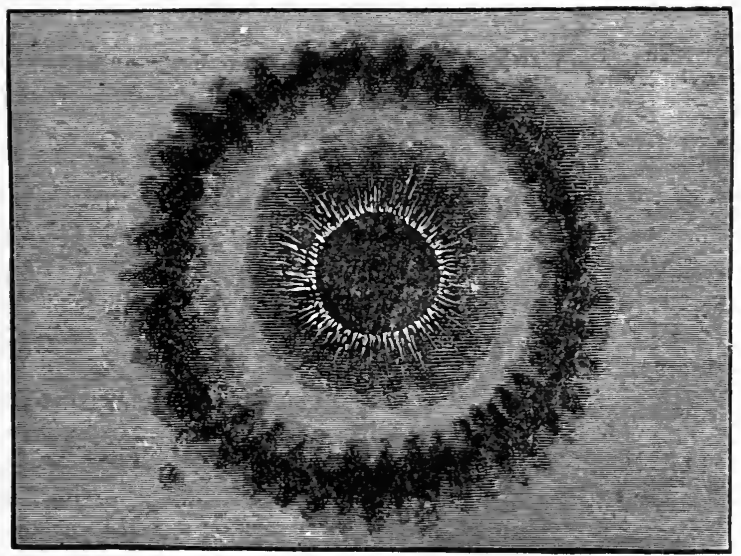

Fig. 168 .

to those parts where there is + electrification on the disk, and settles down in curious branching yellow streaks like those shown in Fig. 167. The red lead settles down in little red heaps and patches where the electrification is negative. These rounded red patches indicate that the - discharge has been of the nature of a wiml or silent discharge. The branching yellow streaks indicate that the positive discharge (as indeed may be heard) is of the nature of a brush. Fig. 168 shows the general appearance of the Lichtenberg's figure produced by holding the knob of 
the Leyden jar at the centre of a shellac plate that has previously been rubbed with flannel, the negative electrification being attracted upon all sides toward the central positive charge. These same powders may be used to investigate how surfaces have become electrified by rubbing, and how pyroelectric crystals (Art. 74) are electritied during cooling.

Powdered tourmaline, warmed and then sifted over a sheet of glass previously electrified irregularly, will show similar figures, though not so well defined.

Breath-figures can be made by electrifying a coin or other piece of metal laid upon a sheet of dry glass, and then breathing upon the glass where the coin lay, revealing a faint image of it on the surface of the glass.

F. Jervis-Smith finds that if a coin or engraving laid face-down upon a photographic dry-plate is sparked with an induction coil, the plate receives an invisible image which can be photographically developed.

325. Physiological Effects. - The physiological effects of the current have been described in Lesson XX. Those produced by the spark-discharge are more sudden in character, but of the same general nature. Death is seldom the direct result. The shock causes a sudden cessation of respiration, resulting in suffocation as from drowning. The bodies of persons struck by the lightning spark frequently exhibit markings of a reddish tint where the discharge in passing through the tissues has lacerated or destroyed them. Sometimes these markings present a singular ramified appearance, as though the discharge had spread in streams over the surface at its entry.

326. Dissipation of Charge.-However well insulated a charged conductor may be, and however dry the surrounding air, it nevertheless slowly loses its charge, and in a few days will be found to be completely discharged. The rate of loss of charge is, however, not uniform. It is approximately proportional to the difference of potential between the body and the earth. Hence the rate of loss 
is greater at first than afterwards, and is greater for highlycharged bodies than for those feebly charged. The law of dissipation of charge therefore resembles Newton's law of cooling, according to which the rate of cooling of a hot body is proportional to the difference of temperature between it and the surrounding objects. If the potential of the body be measured at equal intervals of time it will be found to have diminished in a decreasing geometric series; or the logarithms of the potentials at equal intervals of time will differ by equal amounts. The rate of loss is, however, greater at negatively-electrified surfaces than at positive.

This may be represented by the following equation :

$$
\mathrm{V}_{t}=\mathrm{V}_{o} \epsilon^{-p t} \text {, }
$$

where $\mathrm{V}_{o}$ represents the original potential and $\mathrm{V}_{t}$ the potential after an interval $t$. Here $\epsilon$ stands for the number $2 \cdot 71828$. . . (the base of the natural logarithms), and $p$ stands for the "coefficient of leakage," which depends upon the temperature, pressure, and humidity of the air. The same formula serves for the discharge of a condenser of capacity $K$ through a resistance $\mathrm{R}$; if $p$ is written for $1 / \mathrm{KR}$.

\section{Positive and Negative Electriflcation.-}

The student will not have failed to notice throughout this lesson frequent differences between the behaviour of positive and negative electrification. The striking dissimilarity in the Lichtenberg's figures, the displacement of the perforation-point in Lullin's experiment, the unequal tendency to dissipation at surfaces, the unequal action of heat on positive and negative charges, the remarkable differences in the various forms of brush and glow discharge, are all points that claim attention. Gassiot described the appearance in vacuum tubes as of a force emanating from the negative pole. Crookes's experiments in high vacua show molecules to be violently discharged from the negative electrode, the vanes of a little fly enclosed in such tubes being moved from the side struck by the negative discharge. Holtz found that 
when funnel-like partitions were fixed in a vacuun tube the resistance is much less when the open mouths of the funnels face the negative electrode. These matters are yet quite unaccounted for by any existing theory of electricity.

327a. Roentgen's Rays.-In 1895 Roentgen discovered that highly exhausted tubes, such as the Crookes tubes (Art. 321), when stimulated by electric discharges emit some invisible rays which have very remarkable properties. They excite brilliant Hlorescence on such substances as the platinocyanide of barium; they differ from ultra-violet light and other invisible kinds of radiation in being incapable of refraction, of polarisation, or of regular reflexion. They pass freely through aluminium, zinc, wood, paper, and flesh, but not through lead, platinum, glass, or bone. They also act on ordinary photographic plates. Hence it is possible by using a fluorescent screen to see, and by using sensitive plates to photograph, the shadows of such things as the bones in the living body, or the bullet in the barrel of a gun. It is found that these rays are given off, inside the Crookes tubes, from the solid surface - the glass or a metal target placed inside on purpose - against which the kathode rays are directed. Those substances which have highest atomic weights absorb the Roentgen rays best, or if used as targets emit them best. Hence the target should be of platinum, or uranium, or osmium.

\section{LESSON XXV.-Atmospheric Electricity}

328. The phenomena of atmospheric electricity are of two kinds. There are the well-known electrical phenomena of thunderstorms; and there are the phenomena of continual slight electrification in the air, best observed when the weather is fine. The phenomena of the Aurora constitute a third branch of the subject.

329. The Thunderstorm an Electrical Phenomenon.-The detonating sparks drawn from electrical machines and from Leyden jars did not fail to suggest to the early experimenters, Hauksbee, Newton, Wall, Nollet, and Gray, that the lightning flash and the thunderclap were due to electric discharges. In 1749, Benjamin Franklin, observing lightning to possess almost 
all the properties observable in electric sparks, ${ }^{*}$ suggested that the electric action of points (Art. 46), which was discovered by him, might be tried on thunderclouds, and so draw from them a charge of electricity. He proposed, therefore, to fix a pointed iron rod to a high tower. Before Franklin could carry his proposal into effect, Dalibard, at Marly-la-ville, near Paris, taking up the hint, erected an iron rod 40 feet high, by which, in 1752 , he drew sparks from a passing cloud. Franklin shortly after succeeded in another way. He sent up a kite during the passing of a storm, and found the wetted string to conduct electricity to the earth, and to yield abundance of sparks. These he drew from a key tied to the string, a silk ribbon being interposed between his hand and the key for safety. Leyden jars conld be charged, and all other electrical effects produced, by the sparks furnished from the clouds. The proof of the identity was complete. The kite experiment was repeated by Romas, who drew from a metallic string sparks 9 feet long, and by Cavallo, who made many important observations on atmospheric electricity. In 1753 Richmann, of St. Petersburg, who was experimenting with an apparatus resembling that of Dalibard, was struck by a sudden discharge and killed.

330. Theory of Thunderstorms. - Solids and liquids cannot be charged throughout their substance; if charged at all the electrification is upon their sirface (see Art. 31). But gases and vapuurs, being composed of

* Franklin enumerates specifically an agreement between electricity and lightning in the following respects :-Giving light; colour of the light; crooked direction; swift motion; being conducted by metals; noise in exploding; conductivity in water and ice ; rending imperfect conductors ; destroying animals; melting metals; firing inflammable substances; sulphureous smell (due to ozone, as we now know); and he had previously found that needles could be magnetized both by lightning and by the electric spark. He also drew attention to the similarity between the pale blue flame seen during thundery weather playing at the tips of the masts of ships (called by sailors St. Elmo's Fire), and the "glow" discharge at points. 
myriads of separate particles, can receive a bodily charge. The air in a room in which an electric machine is worked is found afterwards to be charged. The clouds are usually charged more or less with electricity, derived, probably, from evaporation going on at the earth's surface. The minute particles of water floating in the air become more highly charged. As they fall by gravitation and unite together, the strength of their charges increases. Suppose eight small drops to join into one. That one will have eight times the quantity of electricity distributed over the surface of a single sphere of twice the radius (and, therefore, of twice the capacity, by Art. 272) of the original drops; and its electrical potential will therefore be four times as great. Now a mass of cloud may consist of such charged spheroids, and its potential may gradually rise, therefore, by the coalescence of the drops, and the electrification at the lower surface of the cloud will become greater and greater, the surface of the earth beneath acting as a condensing plate and becoming charged, by influence, with the opposite kind of electrification. Presently the difference of potential becomes so great that the intervening strata of air give way under the strain, and a disruptive discharge takes place at the point where the air offers least resistance. This lightning spark, which may be more than a mile in length, discharges only the electricity that has been accumulating at the surface of the cloud, and the other parts of the clond will now react upon the discharged portion, producing internal attractions and internal discharges. The internal actions thus set up will account for the usual appearance of a thundercloud, that it is a well-defined flat-bottomed mass of cloud which appears at the top to be boiling or heaving up with continual movements.

331. Lightning and Thunder.-Three kinds of lightning have been distinguished by Arago: (i.) The Zigzag flash or "Forked lightning," of ordinary occurrence. The zigzag form is probably due either to the 
presence of solid particles in the air or to local electrification at certain points, making the crooked path the one of least resistance. (ii.) Sheet lightning, in which whole surfaces are lit up at once, is probably only the reflexion on the clouds of a flash taking place at some other part of the sky. It is often seen on the horizon at night, reflected from a storm too far away to produce audible thunder, and is then known as "summer lightning." (iii.) Globular lightning, in the form of balls of fire, which move slowly along and then burst with a sudden explosion. This form is very rare, but must be admitted as a real phenomenon, though some of the accounts of it are greatly exaggerated. Similar phenomena on a small scale have been produced (though usually accidentally) with electrical apparatus.

The sound of the thunder may vary with the conditions of the lightning spark. The spark heats the air in its path, causing sudden expansion and compression all round, followed by as sudden a rush of air into the partial vacuum thus produced. If the spark be straight and short, the observer will hear but one short sharp clap. If its path be a long one and not straight, he will hear the successive sounds one after the other, with a characteristic rattle, and the echoes from other clonds will come rolling in long afterwards. The lightning-flash itself never lasts more than $\frac{1}{100000}$ of a second, but sometimes is oscillatory in character (see Art. 515).

The damage done by a lightning-flash when it strikes an imperfect conductor appears sometimes as a disruptive mechanical disintegration, as when the masonry of a chimney-stack or church-spire is overthrown, and sometimes as an effect of heat, as when bell-wires and objects of metal in the path of the lightning-current are fused. The physiological effects of sudden discharges are discussed in Arts. 255 and 325.

The "return-stroke" experienced by persons in the neighbourhoor of a flash is explained in Art. 29. 


\section{Lightning Conductors.-The first sugges-} tion to protect property from destruction by lightning was made by Franklin in 1749, in the following words :-

"May not the knowledge of this power of points be of use to mankind, in preserving houses, churches, ships, etc., from the stroke of lightning, by directing us to fix on the highest parts of those edifices upright rods of iron made sharp as a needle, and gilt to prevent rusting, and from the foot of those rods a wire down the outside of the building into the ground, or round one of the shrouds of a ship, and down her side till it reaches the water? Would not these pointed rods probably draw the electrical fire silently out of a cloud before it came nigl enough to strike, and thereby secure us from that most sudden and terrible mischief?"

Maxwell proposed to corer houses with a network of conducting wires, without any main conductor, the idea being that then the interior of the building will, like Faraday's hollow cube (Art. 34), be completely protected from electric force. Much controversy has arisen of late respecting lightning-rods. Professor Oliver Lodge maintains the lightning flash to be of the nature of an electric oscillation (Art. 515) rather than a current. If so, the conductor of least resistance is not necessarily the best lightning - rod. Professor Lodge and the author independently, and for different reasons, recommend iron in preference to copper for lightning-rods.

The following points summarize the modern views on the subject :-

1. All parts of a lightning conductor should be of one and the same metal, avoiding joints as far as possible, and with as few sharp bends or corners as may be.

2. The use of copper for lightning-rods is a needless extravagance. Iron is far better. Ribbon is slightly better than round rorl ; but ordinary galvanized iron telegraph-wire is good enough.

3 . The conductor should terminate not merely at the highest point of a building, but be carried to all high points. It is unwise to erect very tall pointed rods projecting several feet above the roof.

4. A good deep wet "earth" should he provided, independent of gas o: water pipes, to which the conductor should be led down. 
5. If in any part the condnctor goes near a gas or water pipe it is better to connect them metallically than to leave them apart.

6. In ordinary buildings the conductor should be insulated away from the walls, so as to lessen liability of lateral discharge to metal stoves and things inside the house.

7. Connect all external metal-work, zinc spouts, iron crest ornaments, and the like, to each other, and to the earth, but not to the lightning conductor.

8. The cheapest way of protecting an ordinary house is to run common galvanized iron telegraph-wire up all the corners, along all the ridges and eaves, and over all the chimneys; taking them down to the earth in several places, to a moist stratum, and at each place burying a load of coke.

9. Over the tops of tall chimneys it is well to place a loop or arch of the lightning conductor, made of any stout and durable metal.

\section{Atmospheric Electricity. - In 1752 Le-} monnier observed that the atmosphere usually was in an electrical condition. Cavallo, Beccaria, Ceca, and others added to our knowledge of the subject, and more recently Quetelet and Lord Kelvin have generalized from more careful observations. The main result is that the air above the surface of the earth is usually, during fine weather, positively electrified, or at least that it is positive with respect to the earth's surface, the earth's surface being relatively negative. The so-called measurements of "atmospheric electricity" are really measurements of difference of potential between a point of the earth's surface, and a point somewhere in the air above it. In the upper regions of the atmosphere the air is highly rarefied, and conducts like the rarefied gases in Geissler's tubes (Art. 320). The lower air is, when dry, a nonconductor. The upper stratum is believed to be charged with + electricity, while the earth's surface is itself negatively charged; the stratum of denser air between acting like the glass of a Leyden jar in keeping the opposite charges separate. If we could measure the electric potential at different points within the thickness 
of the glass of a charged jar, we should find that the values of the potential changed in regular order from a + value at one side to a - value at the other, there being a point of zero potential about halfway between the two. Now, the air in fine weather always gives + indications, and the potential of it is higher the higher we go to measure it. Cavallo found higher electrification just outside the cupola of St. Paul's Cathedral than at a lower point of the building. Lord Kelvin found the potential in the island of Arran to increase from 23 to 46 volts for a rise of one foot in level; but the difference of potential was sometimes eight or ten times as much for the same difference of level, and changed rapidly, as the east wind blew masses of cloud charged with + or electricity across the sky. Joule and Kelvin, at Aberdeen, found the rise of potential to be equal to 40 volts per foot, or 1.3 volts per centimetre rise of level.

During fine weather a negative electrification of the air is extremely rare. Beccaria only observed it six times in fifteen years, and then with accompanying winds. But in broken weather and during rain it is more often - than + , and exhibits great fluctuations, changing from - to + , and back, several times in half an hour. A definite change in the electrical conditions usually accompanies a change of weather. "If, when the rain has ceased (said Ceca), a strong excessive $(t)$ electricity obtains, it is a sign that the weather will continue fair for several days."

334. Methods of Observation. - The older observers were content to affix to an electroscope (with gold leaves or pith-balls) an insulated pointed rod stretching out into the air above the ground, or to fly a kite, or (as Becquerel did) to shoot into the air an arrow communicating with an electroscope by a fine wire, which was removed before it fell. Gay-Lussac and Biot lowered a wire from a balloon, and found a difference of potential between the upper and lower strata of the air. None 
of these methods is quite satisfactory, for they do not indicate the potential at any one point. To bring the tip of a rod to the same potential as the surrounding air, it is necessary that material particles should be discharged from that point for a short time, each particle as it breaks away carrying with it a + or a - charge until the potentials are equalized between the rod and the air at that point. Volta did this by means of a small flame at the end of an exploring rod. Lord Kelvin has employed a "water-dropper," an insulated cistern provided with a nozzle protruding into the air, from which drops issue to equalize the potentials: in winter he uses a small roll of smouldering touch-paper. Dellmann adopted another method, exposing a sphere to influence by the air, and then insulating it, and bringing it within-doors to examine its charge. Peltier adopted the kindred expedient of placing, on or near the ground, a delicate repulsion-electrometer, which during exposure was connected to the ground, then insulated, then removed indoors for examination. This process really amounted to charging the electrometer by infuence with electrification of opposite sign to that of the air. The "quadrant" electrometer, described in Art. 288, and a "portable" electrometer on the attracted-disk principle, are now used for observations on atmospheric electricity. Using a water-dropping collector and a Kelvin electrometer, Everett made a series of observations in Nova Scotia, and found the highest + electrification in frosty weather, with a dry wind charged with particles of ice.

335. Diurnal Variations.-Quetelet found that at Brussels the laily indications (during fine weather) showed two maxima occurring in summer at 8 A.s. and 9 r.M., and in winter at 10 A.M. and 6 P.M. respectively, and two minima which in summer were at the hours of 3 P.M. and about midnight. $\mathrm{He}$ also found that in January the electricity was about thirteen times as strong as in June. At Kew there is a maximum at 8 A.x. in 
summer, and at 10 A.M. in winter; and a second minimum at 10 P.M. in summer and 7 P.M. in winter. The maxima correspond fairly with hours of changing temperature, the minima with those of constant temperature. In Paris, M. Mascart finds but one maximum, just before midnight: at sunrise the electricity diminishes until about 3 P.M., when it has reached a minimum, whence it rises till nightfall.

Our knowledge of this important subject is still very imperfect. We do not even know whether all the changes of the earth's electrification relatively to the air are due to causes operating above or below the earth's surface. Simultaneous observations at different places and at different levels are greatly wanted.

336. The Aurora.-In all the northern regions of the earth the Aurora borealis, or "Northern Lights," is an occasional phenomenon; and within and near the Arctic circle is of almost nightly occurrence. Similar lights are seen in the south polar regions of the earth, and are denominated Aurora australis. As seen in European latitudes, the usual form assumed by the aurora is that of a number of ill-defined streaks or streamers of a pale tint (sometimes tinged with red and other colours), either radiating in a fan-like form from the horizon in the direction of the (magnetic) north, or forming a sort of arch across that region of the sky, of the general form shown in Fig. 169. A certain flickering or streaming motion is often discernible in the streaks. Under very favourable circumstances the aurora extends over the entire sky. The appearance of an aurora is usually accompanied by a magnetic storm (Art. 159), affecting the compass-needles over whole regions of the globe. This fact, and the position of the auroral arches and streamers with respect to the magnetic meridian, directly suggest an electric origin for the light, - a conjecture which is confirmed by the many analogies found between auroral phenomena and 
those of discharge in rarefied air (Arts. 320 and 322). Yet the presence of an aurora does not, at least in our latitudes, affect the electrical conditions of the lower regions of the atmosphere. On September 1, 1859, a severe magnetic storm occurred, and auroræ were observed almost all over the globe; at the same time

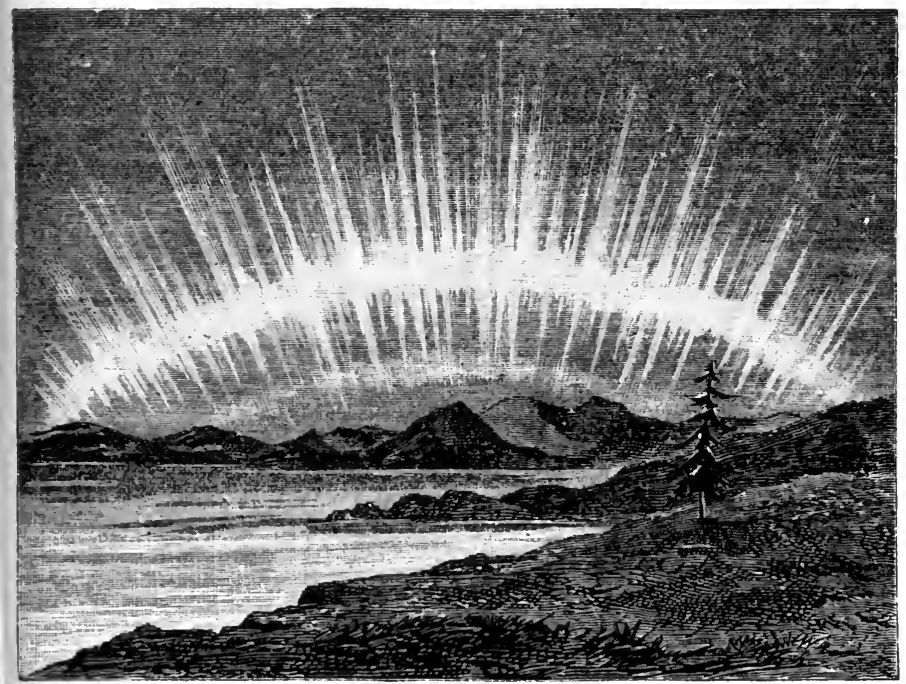

Fig. 169.

a remarkable outburst of energy took place in the photosphere of the sun; but no simultaneous development of atmospheric electricity was recorded. Aurora appear in greater frequency in periods of about $11 \frac{1}{2}$ years, which agrees pretty well with the cycles of maximum of magnetic storms (see Art. 159) and of sun-spots. 
The spectroscope shows the auroral light to be due to gaseous matter, its spectrum consisting of a few bright lines not referable with certainty to any known terrestrial substance, but having a general resemblance to those seen in the spectrum of the electric discharge through rarefied dry air.

The most probable theory of the aurora is that originally due to Franklin ; namely, that it is due to electric discharges in the upper air, in consequence of the differing electrical conditions between the cold air of the polar regions and the warmer streams of air and vapour raised from the level of the ocean in tropical regions by the heat of the sun.

According to Nordenskiold the terrestrial globe is perpetually surrounded at the poles with a ring or crown of light, single or double, to which he gives the name of the "aurora-glory." The outer edge of this ring he estimates to be at 120 miles above the earth's surface, and its diameter about 1250 miles. The centre of the auroraglory is not quite at the magnetic pole, being in lat. $81^{\circ}$ N., long. $80^{\circ} \mathrm{E}$. This aurora-glory usually appears as a pale arc of light across the sky, and is destitute of the radiating streaks shown in Fig. 169, except during magnetic and auroral storms.

An artificial aurora has been produced by Lemström, who erected on a mountain in Lapland a network of wires presenting many points to the sky. By insulating this apparatus and connecting it by a telegraph-wire with a galvanometer at the bottom of the mountain, he was able to observe actual currents of electricity when the auroral beam rose above the mountain. 


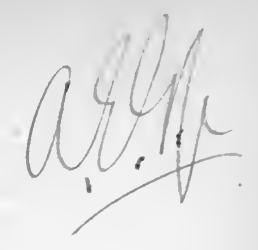

\section{CHAPTER V}

\section{ELECTROMAGNETICS}

\section{Lesson XXVI.-Magnetic Potential}

337. Electromagnetics. - That branch of the science of electricity which treats of the relation between electric currents and magnetism is termed Electromagnetics. In Arts. 128 to 140 the laws of magnetic forces were explained, and the definition of "unit pole" was given. It is, however, much more convenient, for the purpose of study, to express the interaction of magnetic and electromagnetic systems in terms not of "force" but of "potential"; i.e. in terms of their power to do work. In Art. 263 the student was shown how the electric potential due to a quantity of electricity may be evaluated in terms of the work done in bringing up as a test charge a unit of + electricity from an infinite distance. Magnetic potential can be measured similarly by the ideal process of bringing up a unit magnetic pole ( $\mathrm{N}$-seeking) from an infinite distance, and ascertaining the amount of work done in the operation. Hence a large number of the points proved in Lesson XXI. concerning electric potential will also hold true for magnetic potential. The student may compare the following propositions with the corresponding ones in Articles 263 to 268 :-

(a) The magnetic potential at any point is the work that must be spent upon a unit magnetic (N-seeking) 
pole in bringing it up to that point from an infinite distance.

(b) The magnetic potential at any point due to a system of magnetic poles is the sum of the separate magnetic potentials due to the separate poles.

The student must here remember that the potentials due to S-seeking poles will be of opposite sign to those due to $\mathrm{N}$-seeking poles, and must be reckoned as negative.

(c) The (magnetic) potential at any point due to a system of magnetic poles may be calculated (compare with Art. 263) by summing up the strengths of the separate poles divided each by its own distance from that point. . Thus, if poles of strengths $m^{\prime}, m^{\prime \prime}$, $m^{\prime \prime \prime}$, etc., be respectively at distances of $r^{\prime}, r^{\prime \prime}, r^{\prime \prime \prime}$, . ... from a point $P$, then the following equation gives the potential at $\mathrm{P}$ :-

$$
\begin{aligned}
\mathrm{V}_{\mathrm{P}} & =\frac{m^{\prime}}{r^{\prime}}+\frac{m^{\prime \prime}}{r^{\prime \prime}}+\frac{m^{\prime \prime \prime}}{r^{\prime \prime \prime}}+\ldots \ldots \ldots . . . . \\
\text { or } \mathrm{V}_{\mathrm{P}} & =\Sigma \frac{m}{r} .
\end{aligned}
$$

(d) The difference of (magnetic) potential between two points is the work to be done on or by a unit (N-seeking) pole in moving it from one point to the other. It follows that if $m$ units of magnetism are moved through a difference of potential $\mathrm{V}$, the work $\mathrm{W}$ done will be

$$
\mathrm{W}=m \mathrm{~V} .
$$

(e) Magnetic force on unit pole is the rate of change of (magnetic) potential per unit of length: it is numerically equal to the intensity of the field. Since by Art. 141,

$$
f=m \mathbf{H},
$$

and work is the product of a force into the length 
through which its point of application moves forward, it follows that

$$
\mathrm{W}=f l=m \mathrm{H} l .
$$

But

whence

$$
\mathrm{W}=m \mathrm{~V} ;
$$

and

$$
\mathrm{V}=\mathrm{H} l,
$$

$$
\mathrm{H}=\mathrm{V} / l \text {. }
$$

Example.-The difference of magnetic potential between two points 5 centims. apart along a magnetic field in which there are 6000 lines .per sq. cm., is 30,000 . Or, it would require 30,000 ergs of work to be expended to push a unit pole from one point to the other against the magnetic force.

(f) Equipotential surfaces are those (imaginary) surfaces surrounding a magnetic pole or system of poles, over which the (magnetic) potential has equal values. Thus, around a single isolated magnetic pole, the potential would be equal all round at equal distances; and the equipotential surfaces would be a system of concentric spheres at such distances apart that it would require the expenditure of one erg of work to move a unit pole up from a point on the surface of one sphere to any point on the next (see Fig. 146). Around any real magnet possessing two polar regions the equipotential surfaces would be much more complicated. Magnetic force, whether of attraction or repulsion, alvays acts across the equipotential surfaces in a direction normal to the surface; the magnetic lines of force are everywhere perpendicular to the equipotential surfaces.

Flux of Force. - From a single magnetic pole (supposed to be a point far removed from all other poles) the lines of force diverge radially in all directions. The space around may be conceived as thus divided up 
into a number of conical regions, each having its apex at that pole; and through each cone, as through a tube, a certain number of lines of force will pass. Such a conical space may be called a "tube" of force. The total number of magnetic lines within any tube of force is called the magnetic flux.* ${ }^{*}$ No matter where you cut across a tube of force, the cross-section will cut through the enclosed flux, though the lines diverge more widely as the tube widens. Hence,

(g) The magnetic flux across any section of a tube of force is constant wherever the section be taken.

In case the magnetism is not concentrated at one point, but distributed over a surface from which the tubes start, we shall have to speak of the "amount of magnetism " rather than of the "strength of pole," and in such a case the

(h) Magnetic density is the amount of magnetism per unit of surface. In the case of a simple magnetic shell over the face of which the magnetism is distributed with uniform density, the "strength" of the shell will be equal to the thickness of the shell multiplied by the surface-density.

338. Intensity of Field.-We have seen (Art. $115)$ that every magnet is surrounded by a certain "field," within which magnetic force is observable. We may completely specify the properties of the field at any point by measuring the strength and the direction of that force,- that is, by measuring the "intensity of the field" and the direction of the lines of force. The "intensity of the field" at any point is measured by the force with which it acts on a unit pole placed at that point. Hence, unit intensity of field is that intensity of field which acts on a unit pole with a force of one dyne. There is therefore a field of

* The magnetic flux is by some writers called the total induction; but the word induction ought to be kept for the operation of inducing. 
unit intensity at a point one centimetre distant from the pole of a magnet of unit strength. Suppose a magnet pole, whose strength is $m$, placed in a field at a point where the intensity is $\mathbf{H}$, then the force will be $m$ times as great as if the pole were of unit strength, and

$$
f=m \times \mathbf{H} \text {. }
$$

To aid the imagination by a graphic conception we adopt Faraday's notion of representing the properties of a magnetic field by supposing lines to be drawn so that they represent the direction and intensity of the field by the direction and density of the lines. This leads to the empirical rule to draw as many magnetic lines to the square centimetre (of cross section) as there would be dynes of force on unit pole. A field of $\mathrm{H}$ units means one where there would be $\mathbf{H}$ dynes on unit pole, or $\mathbf{H}$ lines per square centimetre. It follows that a unit magnetic pole will have $4 \pi$ lines of force proceeding from it: for there is unit field at unit distance away, or one magnetic line per square centimetre; and there are $4 \pi$ square centimetres of surface on a sphere of unit radius drawn round the pole. A magnet, whose pole-strength is $m$, has $4 \pi m$, or $12.57 \times m$, lines running through the steel, and diverging at its pole. The above-mentioned rule is the origin of the $4 \pi$ symbol which comes in so often into electromagnetic formula. Suppose a narrow crevasse between the faces of two opposing magnets, each having $\sigma$ units of magnetism per square centimetre of their pole surfaces. The field in the space between will have the value

$$
\mathrm{H}=4 \pi \sigma \text {. }
$$

339. Work done by Conductor carrying Current when it cuts across the Lines of a Magnetic Field.-By definition (Art. 263) it follows that the work W done in moving $Q$ units of electricity against an electromotive-force $\mathrm{V}$ is equal to $\mathrm{QV}$. Suppose 
that this electromotive-force is due to the conductor cutting $\mathrm{N}$ magnetic lines during time $t$. Then if the motion be uniform and the average current during the time is called $\mathrm{C}$, it follows that $\mathrm{Q}=\mathrm{C} t$. And the average electromotive-force is (see Art. 225) $=\mathbf{N} / t$. Inserting these values we get

or

$$
\begin{aligned}
& \mathrm{W}=\mathrm{C} t \times \mathbf{N} / t, \\
& \mathrm{~W}=\mathrm{CN} ;
\end{aligned}
$$

or, in words, the work done in moving a current across a magnetic flux is equal to the product of the current into the total number of magnetic lines cut. It will be noted that the work is the same whether the time is long or short. If $\mathrm{C}$ and $\mathbf{N}$ are in absolute (C.G.S) units, $\mathrm{W}$ will be in ergs.

340. Force exerted by Magnetic Field on Wire carrying Current.-If a wire is moved sideways across the lines of a magnetic field, through a distance $x$, it will sweep out an area equal to its own length $l$ multiplied by $x$. And if $\mathrm{H}$ is the number of magnetic lines per square centimetre the total number of lines cut will be $=\mathrm{H} l x$; and the work done if the wire carries current $\mathrm{C}$ will be $=\mathrm{CH} l x$. But if work $\mathrm{W}$ is done in moving the wire through distance $x$ the force $f$ exerted will be $W / x$. Hence the force on the wire will be

$$
f=\mathrm{CH} l ;
$$

or, in words, the force is proportional to the current, to the intensity of the field, and to the length of wire in the ficld. It is a force that tends to drag the wire laterally, acting at right angles to the wire and to the lines of the field.

This action is of course due to stresses going on in the medium, and is worthy of further thought. Consider the magnetic field in a gap between a large $\mathrm{N}$-pole and a similar S-pole. The lines will go nearly uniformly straight across. Let a current flow in a copper wire that lies across the field. In Fig. 170 the wire is seen end- 
ways, with the current flowing "up" or toward the observer. The result will be that the magnetic field of the current (Art. 202) will be superposed upon that of the magnets, and will perturb it: the form of the perturbed field being that shown. In such a field the stresses, which act as though the magnetic lines tended to shorten themselves, will have the eflect of urging the wire mechanically in the direction shown. This mechanical force acts on the matter of the wire, though due to the current.

In calculating by

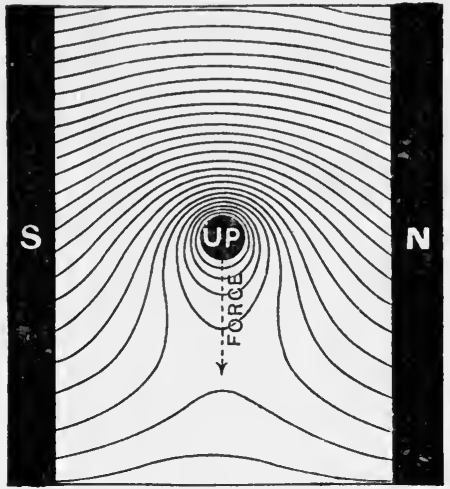

Fig. 170. the expression above, if $\mathrm{C}$ is given in amperes it must be divided by 10 .

341. Magnetomotive-force (or Total Magnetizing Force) of a Current circulating in a Spiral Conductor.-Let a conductor carrying a current of $\mathrm{C}$ amperes be coiled up in a spiral having $\mathrm{S}$ as the number of turns. It is known, and easily understood, that the total magnetizing force of such is proportional to the number of ampere-turns; for experiment shows that, for example, a current of 10 amperes circulating in a coil of 50 turns has precisely the same magnetic power as a current of 5 amperes in 100 turns, or as a current of 1 ampere in 500 turns. Each of these has 500 ampereturns.

To obtain the full expression let us find the work that would be done in the act of moving a unit magnet-pole around any closed path (Fig. 171) from any point $\mathrm{P}$ to 
the same point again, such path passing through all the turns of the magnetizing coil. The work done on a unitpole in moving it once around the closed path, against the magnetic forces of the system, is a measure of the power of that system to magnetize; or, in other words, is a measure of its magnetomotive-force. Such a closed path may lie, according to circumstances, either wholly in air, or

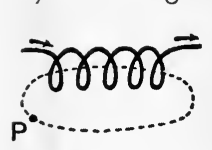

Fig. 171. partly in air partly in iron, or wholly in iron. The argument is entirely independent of any materials lying along the ideal path.

Now imagine this unit-pole, with its $4 \pi$ magnetic lines radiating out of it, to be passed along the closed path (Fig. 171) from P, through the spirals to $\mathrm{P}$ again. Each turn of the coil will cut each of the magnetic lines once, and therefore, by Arts. 338 and 339 , the total work done will be

$$
\mathrm{W}=4 \pi \mathrm{CS} / 10 \text {, }
$$

where we divide by 10 to bring amperes to C.G.S. units. Or, since $4 \pi=12.57$, we get the rule-the magnetomotiveforce ${ }^{*}$ of a coil is equal to 1.257 times the ampere-turns.

342. Intensity of Field in a long Tubular Coil, or Solenoid.-A spiral coil wound on a tube is called a solenoid. It has, when a current circulates in its coils, a magnetic field along the inside of it, and is, in fact, so long as the current circulates, a magnet without iron. This magnetic field, if the spiral is a very long one - say 20 times as long as the diameter of the spirals, is very uniform all along the interior, except just toward the ends, where it becomes weaker. To find the intensity of the field $\mathrm{H}$, we may remember that (Art. 337, e) the work done on a unit-pole in moving it through a length $l$ of field $\mathrm{H}$ is equal to $\mathrm{H} l$. But the work done in

* Since this magnetomotive-force is made up of a number of small elements distributed varionsly along the path it is sometimes called the line-integral of the magnetizing forces. 
moving it along the tubular coil of length $l$ is practically equal to that done around the closed path, since nearly all the forces are met along the part of the path inside. Hence we may equate $4 \pi \mathrm{CS} / 10$ to $\mathrm{H} l$; giving the result

$$
\mathrm{H}(\text { in gausses })=\frac{4 \pi}{10} \times \stackrel{\mathrm{CS}}{l} \text {, }
$$

or the intensity of the field in a long spiral is equal to $1 \cdot 257$ times the number of ampere-turns per centimetre of length.

At the mouth of a long spiral the intensity of the field is exactly half what it is midway between the ends.

343. Magnetic Field due to Indeflnitely Long Straight Current. Law of Inverse Simple Distance.-Consider a unit-pole at point $\mathrm{P}$ at a distance $r$ (Fig. 172) from an indefinitely long straight conductor carrying a current of $\mathrm{C}$ amperes. The force tending to make the pole circulate around the wire may be calculated very simply as follows. If the unit-pole were to be moved once around the wire on a circular path with radius $r$, each one of the $4 \pi$ magnetic lines that radiate from it would be cut once by the wire. Hence, by Art. 339, the work done in one such revolution would be equal

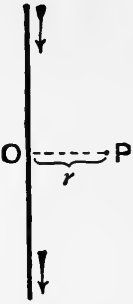

Fig. 172. to $4 \pi \mathrm{C} / 10$. But this work has been done by moving the unit, against the forces of the system, along a path the length of which is $2 \pi r$; wherefore

whence

$$
\mathrm{W}=f \times 2 \pi r=4 \pi \mathrm{C} / 10,
$$

$$
f=2 \mathrm{C} / 10 r .
$$

From this it appears that the force on unit-pole, and therefore the number of gausses or intensity of the field, is directly proportional to the current, and varies inversely as the simple distance from the wire.

Example.-The force exerted on a pole of 1200 units of 
magnetism at a distance of 4 centimetres from a long straight wire carrying current of 60 amperes will be 3600 dynes, or 3.52 grammes.

The fact that the force varies inversely as the simple distance, and not as the square, was experimentally discovered by Biot and Savart in 1820 .

Around such a straight conductor the magnetic field consists of a cylindrical whirl of circular lines (Art. 202), their density decreasing as their radius increases. Outside a straight wire carrying a 10 -ampere current the values of $\mathrm{H}$ are : 2 at $1 \mathrm{~cm} . ; 1$ at $2 \mathrm{~cm}$. ; 0.4 at $5 \mathrm{~cm}$., and so forth. The pole tends to move circularly around the wire.

344. Mutual Action of Magnet-pole and of Element of Current.-Consider an element of current, that is to say, an indefinitely

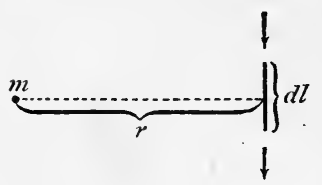

Fig. 173. short piece of a conductor traversed by a current. Calling the length ' $d l$, and the current $\mathrm{C}$, we have $\mathrm{C} d l$ as the magnetic value of the element with respect to all points in its equatorial plan. Suppose the element to be set (Fig. 173) at distance $r$ from a magnet-pole of $m$ units, and at right angles to the line joining them. Then, as the element is small compared with $r$, the law of inverse squares will hold good: the mutual force will be

$$
f=\frac{m \cdot \mathrm{C} d l}{10 \cdot r^{2}} .
$$

This will be neither an attraction nor a repulsion, but a force at right angles to the element and to the line joining it to $m$.

345. Magnetic Field due to Circular Current. - It is clesired to find the effect of a circular current (Fig. 174) at any point on the axis, at a distance $x$ from the centre. Suppose a unit-pole were placed at this point 
$\mathrm{P}$, only a fraction of the $4 \pi$ lines which radiate from it will pass through the circle; the number being proportional to the solid-angle (Art. 148) subtended at $\mathrm{P}$ by the circle, namely $2 \pi(1-\cos \theta)$, where $\theta$ is the angle between axis and slant distance $a$. Hence in bringing up the pole to this place, from an infinite distance, the work done by causing these lines to

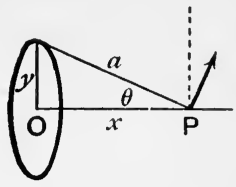

Fig. 174. cut across the wire carrying current $\mathrm{C}$ amperes will be (by Art. 339)

$$
\mathrm{W}=2 \pi \mathrm{C}(1-\cos \theta) / 10 .
$$

This represents the mutual energy of pole and current. To calculate the force at $\mathrm{P}$ we must differentiate this expression with respect to $x$, to ascertain the rate at which the mutual energy falls per unit length. For this purpose it will be convenient to substitute for $\cos \theta$ its value $x /\left(x^{2}+y^{2}\right)^{\frac{1}{2} .} \quad$ Substituting and differentiating we get

$$
f=d \mathrm{~W} / d x=\frac{2}{10} \pi \mathrm{C} y^{2} /\left(x^{2}+y^{2}\right)^{3} .
$$

Now $\left(x^{2}+y^{2}\right)^{3}$ is equal to $a^{3}$; whence the rule that the magnetic force at any point $\mathrm{P}$ on the axis varies directly as the current, and inversely as the cube of the slant distance. (Compare case of a bar-magnet, Art. 138.)

Another way of arriving at this result is as follows. Taking the expression found in Art. 344 for the action of an element of current, we may consider the effect of the topmost element of the ring (Fig. 174), situated at a slant distance $a=\sqrt{x^{2}+y^{2}}$. The elementary force $d f$ exerted on unit-pole at $\mathrm{P}$ by the element $\mathrm{C} d l$ will be at right angles to $a$ and to $d l$ (in direction of the arrow), and, by Art. 206, of the value

$$
d f=\mathrm{C} d l / 10 a^{2} .
$$

As the elements such as $d l$ are symmetrical around the axis we must resolve their oblique forces into two parts: 
part acting at right angles to the axis; which will disappear by mutually cancelling out in pairs, and part acting in the line of the axis, which will for each element be equal to the above expression multiplied by $\sin \theta$. So that the elementary axial force due to each element of length $d l$ will be

$$
d f=\mathrm{C} d l \cdot \sin \theta / 10 a^{2} ;
$$

or, since $\sin \theta=y / a$,

$$
d f=\mathrm{C} d l \cdot y / 10 a^{3} .
$$

But the total force $f$ due to all the elements will be the integral due to the sum of their lengths, and this integral length around the circle is $\int d l=2 \pi y$. Whence it at once follows that

$$
f=2 \pi \mathrm{C} y^{2} / 10 a^{3} .
$$

Note that if $P$ is pushed up to the centre of the circle $a=y$, and we get back to the rule for tangent galvanometer (Art. 212), $f=2 \pi \mathrm{C} / 10 r$.

Also note that for very great distances of $P$ from centre $a$ becomes sensibly equal to $x$, the force varying inversely as the cube of the axial distance.

This affords one way of varying the sensitiveness of tangent galvanometers, the needle with its scale being arranged to slide out along the axis of the coil. At a point P, such that $a=2 y$, the force of coil on needle is only $\frac{1}{8}$ of what it is at centre.

346. Moment of Circular Coil.-A circular coil carrying a current acts as a magnet whose axis is the axis of the coil. Its magnetic moment (Art. 135) will be the product of the current (in absolute units) into the area enclosed. Or, if $\mathrm{C}$ is in amperes, and $\mathrm{A}$ the total area of all the turns, its moment will be $\mathrm{AC} / 10$. If such a coil is placed in a field of intensity $\mathrm{H}$ it will tend to turn so as to place its axis along the direction of the field. If the angle between those directions is $\theta$ the torque (or turning-moment) will be $=\mathrm{ACH} \sin \theta / 10$. 
347. Potential due to a Solenoidal or Circuital Distribution of Magnetism.-A long thin uniformly magnetized magnet exhibits poles only at the two ends, and acts on external objects just as if there were two equal quantities of opposite kinds of magnetism collected at these two points. Such a distribution of magnetism is sometimes called solenoidal or circuital. The magnetic potential due to a solenoid, and all its magnetic effects, depend only on the position of its two poles, and on their strength, and not on the form of the bar between them, whether straight or curved. In Art. 337 (c) was given the rule for finding the potential due to a system of poles. Suppose the two poles of a solenoid have strengths $+m$ and $-m$ respectively, and that the distances of these poles from an external point $\mathrm{P}$ are $r_{1}$ and $r_{2}$, then the potential at $P$ will be

$$
\mathrm{V}_{\mathrm{P}}=m\left(\frac{1}{r_{1}}-\frac{1}{r_{2}}\right) \text {. }
$$

Suppose a magnet curled round until its $\mathrm{N}$ and $\mathrm{S}$ poles touch one another : it will not act as a magnet on an external object, and will have no "field"; for if the two poles are in contact, their distances $r_{1}$ and $r_{2}$ to an external point $\mathrm{P}$ will be equal, and

$$
\left(\frac{1}{r_{1}}-\frac{1}{r_{2}}\right) \text { will be }=0 .
$$

348. Potential due to a Magnetic Shell.-Gauss demonstrated that the potential due to a magnetic shell at a point near it is equal to the strength of the shell multiplied by the solid-angle subtended by the shell at that point; the "strength" of a magnetic shell being the product of its thickness into its surface-density of magnetization.

If $\omega$ represents the solid-angle subtended at the point $P$, and $i$ the strength of the shell, then

$$
\mathrm{V}_{\mathrm{P}}=\omega i \text {. }
$$

Proof. - To establish this proposition would require the integral calculus. But the fol-

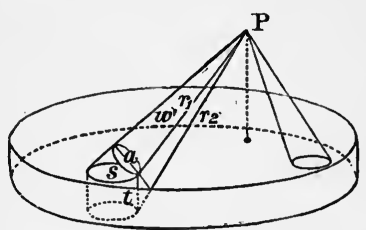

Fig. 175. lowing geometrical demonstration, though incomplete, must here suffice.

Let us consider the shell as composed, like that drawn, of a series of small elements of thickness $t$, and having each an area of surface $s$. The whole solid-angle subtended at $\mathrm{P}$ by the shell may 
likewise be conceived as made up of a number of elementary small cones, each of solid-angle $\omega$ : Let $r_{1}$ and $r_{2}$ be the distances from $P$ to the two faces of the element : Let a section be made across the small cone orthogonally, or at right angles to $r_{1}$, and call the area of this section $\alpha$ : Let the angle between the surfaces $s$ and $\alpha$ be called angle $\beta$ : then $s=a / \cos \beta$. Let $i$ he the "strength" of the shell (i.e. = its surface-density of magnetism $x$ its thickness); then $i / t=$ surface-density of magnetism, and $s i_{l}^{\prime} t=$ strength of either pole of the little magnet $=m$.

Now solid-angle $\dot{\omega}=\frac{\text { area of its orthogonal section }}{r^{2}}$

therefore

$$
=a / r^{2}
$$

and

Hence

$$
\begin{aligned}
& a=\omega r^{2}, \\
& s=\omega r^{2} / \cos \beta .
\end{aligned}
$$

But the potential at $\mathrm{P}$ of the magnet whose pole is $m$ will be

$$
\begin{aligned}
v & =m\left(\frac{1}{r_{1}}-\frac{1}{r_{2}}\right) \\
& =\omega i \frac{r^{2}}{t \cos \beta}\left(\frac{1}{r_{1}}-\frac{1}{r_{2}}\right)
\end{aligned}
$$

but $\frac{1}{r_{1}}-\frac{1}{r_{2}}=\frac{r_{2}-r_{1}}{r_{1} r_{2}}$, which we may write $\frac{r_{2}-r_{1}}{r^{2}}$

because $r_{1}$ and $r_{2}$ may be made as nearly equal as we please. And since $r_{2}-r_{1}=t \cos \beta$

$$
\begin{aligned}
& v=\omega i \frac{r^{2}}{t \cos \beta}\left(\frac{t \cos \beta}{r^{2}}\right) \\
& v=\omega i
\end{aligned}
$$

or the potential due to the element of the shell $=$ the strength of the shell $x$ the solid-augle subtended by the element of the shell. Hence, if $\mathrm{V}$ be.the sum of all the values of $v$ for all the different elements, and if $\omega$ be the whole solid-angle (the sum of all the small solid-angles such as $\dot{\omega}$ ),

$$
\mathrm{V}_{\mathrm{r}}=\omega i,
$$

or the potential due to a magnetic shell at a point is equal to the strength of the shell inultiplied by the solid-angle subtended by the whole of the shell at that point.

Hence $\omega i$ represents the work that would have to be done on or by a unit-pole, to bring it up from an infinite distance to the point $P$, where the shell subtends the solid-angle $\omega$. At a point $Q$ 
where the solid-angle subtended by the shell is different, the potential will be different, the difference of potential between $P$ and $Q$ being

$$
\mathrm{V}_{\mathrm{Q}}-\mathrm{V}_{\mathrm{P}}=i\left(\omega_{\mathrm{Q}}-\omega_{\mathrm{P}}\right) \text {. }
$$

If a magnet-pole whose strength is $m$ were brought up to $\mathrm{P}$, $m$ times the work would have to be done, or the mutual potential would be $=m \omega i$.

349. Potential of a Magnet-pole on a Shell.-It is evident that if the shell of strength $i$ is to be placed where it subtends a solid-angle $\omega$ at the pole $m$, it would require the expenditure of the same amount of work to bring up the shell from an infinite distance on the one hand, as to bring up the magnet-pole from an infinite distance on the other ; hence mwi represents both the potential of the pole on the shell and the potential of the shell on the pole. Now the lines of force from a pole may be regarded as proportional in number to the strength of the pole, and from a single pole they would radiate out in all directions equally. Therefore, if a magnet-pole was placed at $\mathrm{P}$, at the apex of the solid-angle of a cone, the number of lines of force which would pass through the solid-angle would be proportional to that solid-angle. It is therefore convenient to regard $m \omega$ as representing the number of lines of force of the pole which pass through the shell, and we may call the number so intercepted $\mathrm{N}$. Hence the potential of a magnet-pole on a magnetic shell is equal to the strength of the shell multiplied by the number of lines of force (due to the magnet-pole) which pass through the shell; or $\mathrm{V}=\mathrm{N} i$. If either the shell or the pole were moved to a point where a different number of lines of force were cut, then the difference of potential would be

$$
\mathrm{V}_{\mathrm{Q}}-\mathrm{V}_{\mathrm{p}}= \pm i\left(\mathrm{~N}_{\mathrm{Q}}-\mathrm{N}_{\mathrm{P}}\right) \text {. }
$$

To bring up a $\mathrm{N}$-seeking (or + ) pole against the repelling force of the $\mathrm{N}$-seeking face of a magnetic shell requires a positive amount of work to be done; and their mutual reaction would enable work to be done afterwards by virtue of their position : in this case then the potential is + . But in moving a N-seeking pole up to the S-seeking face of a shell work will be done by the pole, for it is attracted up ; and as work done by the .pole may be regarded as our doing negative work, the potential here will have a negative value.

Again, suppose we could bring up a unit $\mathrm{N}$-seeking pole against the repulsion of the $N$-seeking face of a shell of strength $i$, and should push it right up to the shell ; when it actually reached the plane of the shell the shell would occupy a whole horizon, or'half 
the whole space around the pole, the solid-angle* it subtended being therefore $2 \pi$, and the potential will be $+2 \pi i$. If we had begum at the S-seeking face the potential at that face would be $-2 \pi i$. It appears then that the potential alters its value by $4 \pi i$ on passing from one side of the shell to the other.

There is a reaction between pole and shell similar to that (Art. 121) between pole and pole.

If a $\mathrm{N}$-seeking pole be brought up to the $\mathrm{N}$-seeking face of a shell none of the lines of force of the magnet will cut the shell, but will be repelled out as in Fig. 72 ; whereas if a $\mathrm{N}$-seeking pole be brought up to the S-seeking face of a shell, large numbers of the lines will be run into one another; and the pole, as a matter of fact, will be attracted up to the shell, where as many lines of force as possible are cut by the shell. We may formulate this action by saying that a magnetic shell and a magnet-pole react on one another and urge one another in such a direction as to make the number of lines of force that are cut by the shell a maximum (Maxwell's Rule, Art. 204). Outside the attracting face of the shell the potential is - $-\omega i$, and the pole moves so as to make this negative quantity as great as possible, or to make the potential a minimum. Which is but another way of putting the matter as a particular case of the general proposition that bodies tend to move so that the energy they possess in virtue of their position tends to run down to a minimum.

350. Magnetic Potential due to Current.-The propositions concerning magnetic shells given in the preceding paragraphs derive their great importance because of the fact laid down in Art. 203 that circuits, traversed by currents of electricity, behave like magnetic shells. Adopting the electromagnetic unit of current (Art. 353), we may at once go back to Art. 347, and take the theorems about magnetic shells as being also true of closed voltaic circuits.

(a) Potential due to closed circuit (compare Art. 348).

The potential $\mathrm{V}$ due to a closed voltaic circuit (traversed by a current) at a point $\mathrm{P}$ near it, is equal to the strength of the current multiplied by the solid-angle $\omega$ subtended by the circuit at that point. If $\mathrm{C}$ be the strength of the current in electromagnetic units, then

$$
\mathrm{V}_{\mathrm{P}}=-\omega \mathrm{C} \text {. }
$$

(b) At a point $Q$, where the solid-angle subtended by the circuit is $\omega_{Q}$ instead of $\omega_{P}$, the potential will have a different value, the difference of potential being

$$
\mathrm{V}_{\mathrm{Q}}-\mathrm{V}_{\mathrm{P}}=-\mathrm{C}\left(\omega_{\mathrm{Q}}-\omega_{\mathrm{P}}\right) \text {. }
$$

* See note on Ways of Reckoning Angles, Art. 144 and Appendix A. 
(c) Mutual Potential of a Magnet-pole and a Circuit. - If a magnet-pole of strength $m$ were brought up to $\mathrm{P}, m$ times as much work will be done as if the magnet-pole had been of unit strength, and the work would be just as great whether the pole $m$ were brought up to the circuit, or the circuit up to the pole. Hence, the mutual potential will be

$$
-m \omega \mathrm{C} \text {. }
$$

But, as in Art. 349, we may regard $m \omega$ as representing the number of lines of force of the pole which are intercepted by and pass through the circuit, and we may write $\mathbf{N}$ for that number, and say

$$
\mathrm{V}=-\mathrm{CN} \text {, }
$$

or the mutual potential of a magnet-pole and a circuit is equal to the strength of the current multiplied by the number of the magnetpole's lines of force that are intercepted by the circuit, taken with reversed sign.

(d) As in the case of the magnetic shell, so with the circuit, the value of the potential changes by $4 \pi \mathrm{C}$ from a point on one side of the circuit to a point just on the other side ; that is to say, being $-2 \pi \mathrm{C}$ on one side and $+2 \pi \mathrm{C}$ on the other side, work equal to $4 \pi \mathrm{C}$ must be done in carrying a unit-pole from one side to the other round the outside of the circuit. The work done in thus threading the circuit along a path looped $\mathrm{S}$ times round it would be $4 \pi \mathrm{SC}$.

351. (e) Mutual Potential of two Circuits. - Two closed circuits will have a mutual potential, depending on the strengths of their respective currents, on their distance apart, and on their form and position. If their currents be respectively $\mathrm{C}$ and $\mathrm{C}^{\prime}$, and if the distance between two elements $d s$ and $d s^{\prime}$ of the circuits be called $r$, and $\epsilon$ the angle between the elements, it can be shown that their mutual potential is $=-\mathrm{CC}^{\prime} \iint \frac{\cos \epsilon}{r} d s a s^{\prime}$. This expression represents the work that would have to be done to bring up either of the circuits from an infinite distance to its present position near the other, and is a negative quantity if they attract one another. Now, suppose the strength of current in each circuit to be unity; their mutual potential will in that case be $\iint \frac{\cos \epsilon}{r} d s d s^{\prime}$, a quantity which depends purely upon the geometrical form and position of the circuits, and for which we may substitute the single symbol $M$, which we will call the "coefficient of mutual potential": we may now write the mutual potential of the two circuits when the currents are $\mathrm{C}$ and $\mathrm{C}^{\prime}$ as $=-\mathbf{C C}^{\prime} \mathrm{M}$. 
But we have seen in the case of a single circuit that we may represent the potential between a circuit and a unit-pole as the product of the strength of the current $-\mathrm{C}$ into the number $\mathrm{N}$ of the magnet-pole's lines of force intercepted by the circuit. Hence the symbol M must represent the number of each other's lines of force mutually intercepted by both circuits, if each carried unit current. If we call the two circuits $A$ and $B$, then, when each carries unit current, $\mathrm{A}$ intercepts $\mathrm{M}$ lines of force belonging to $\mathrm{B}$, and $B$ intercepts $M$ lines of force belonging to $A$.

Now suppose both currents to run in the same (clock-wise) direction; the front or S-seeking face of one circuit will be opposite to the back or $\mathrm{N}$-seeking face of the other circuit, and they will attract one another, and will actually $d o$ work as they approach one another, or (as the negative sign shows) negative work will be done in bringing up one to the other. When they have attracted one another up as much as possible the circuits will coincide in direction and position as nearly as can ever be. Their potential energy will have run down to its lowest minimum, their mutual potential being a negative maximum, and their coefficient of mutual potential $M$ having its greatest possible value. Two circuits, then, are urged so that their coefficient of mutual potential M shall have the greatest possible value. This justifies Maxwell's Rule (Art. 204), because M represents the number of lines of force mutually intercepted by both circuits. And since in this position each circuit induces as many lines of magnetic force as possible through the other, the coefficient of mutual potential $\mathrm{M}$ is also called the coefficient of mutual induction (Art. 454).

\section{Lesson XXVII.-The Electromagnetic System of Units}

352. Magnetic Units. -All magnetic quantities, strength of poles, intensity of magnetization, etc., are expressed in terms of special units derived from the fundamental units of length, mass, and time, explained in the Note on Fundamental and Derived Units (Art. 280). Most of the following units have been directly explained in the preceding Lesson, or in Lesson XI. ; the others follow from them.

Unit Magnet-Pole.-The unit magnetic pole is one of such a strength, that when placed at a distance of 1 centimetre (in air) from a similar pole of equal strength, repels it with a force of 1 dyne (Art. 141).

Magnetic Potential. -Magnetic potential being measured by work done in moving a unit magnetic pole against the 
magnetic forces, the unit of magnetic potential will be measured by the unit of work done on unit-pole.

Unit Difference of Magnetic Potential. - Unit difference of magnetic potential exists between two points when it requires the expenditure of one erg of work to bring a (N-seeking) unit magnetic pole from one point to the other against the magnetic forces. Magnetomotive-force, or magnetizing power, is measured in same units as difference of magnetic potential.

Intensity of Magnetic Field is measured by the force it exerts upon a unit magnetic pole : hence,

Unit Intensity of Field is that intensity of field which acts on a unit ( $\mathrm{N}$-seeking) pole with a force of 1 dyne. The name of gauss has been assignied to this unit. A field having an intensity of 6000 lines per square centimetre would be described as 6 kilogausses.

Magnetic Flux, or total induction of magnetic lines, is equal to intensity of field multiplied by area. Its unit will be one magnetic line, also called one maxwell.

Magnetic Reluctance (see Art. 376) is the ratio of magnetomotive-force to magnetic flux. Unit reluctance will be such that unit magnetomotive-force generates in it a flux of one line.

353. Electromagnetic Units. - The preceding magnetic units give rise to the following set of electrical units, in which the strength of currents, etc., are expressed in magnetic measure. They are sometimes called "absolute C.G.S." units. The relation of this "electromagnetic" set of units to the "electrostatic" set of units of Art. 283 is explained in Art. 359.

Unit Strength of Current. - A current has unit strength when one centimetre length of its circuit bent into an arc of one centimetre radius (so as to be always one centimetre away from the magnet-pole) exerts a force of one dyne on a unit magnet-pole placed at the centre (Art. 207).

Unit of Difference of Potential (or of Electromotive-force). Potential is work done on a unit of electricity; hence unit difference of potential exists between two points when it requires the expenditure of one erg of work to bring a unit of + electricity from one point to the other agaiust the electric force. Also, unit electromotiveforce is generated by cutting one magnetic line per second.

Unit of Resistance. - A conductor possesses unit resistance when unit difference of potential between its ends causes a current of unit strength to flow through it. 
Unit of Quantity of Electricity is that quantity which is conveyed by unit current in one second.

Unit of Capacity.-Unit capacity requires unit quantity to charge it to unit potential.

Unit of Induction.-Unit induction is such that unit electromotive-force is induced by the variation of the current at the rate of one unit of current per second.

354. Practical Units and Standards. ${ }^{*}$-Several of the above "absolute" units in the C.G.S. system would be inconveniently large and others inconveniently small for practical use. The following are therefore chosen as practical units :-

Resistance.-The Ohm, $=10^{9}$ absolute units of resistance (and theoretically the resistance represented by the velocity of one earth-quadrant per second, see Art. 357), but actually represented by the resistance of a uniform column of mercury 106.3 centimetres long and 14.4521 grammes in mass, at $0^{\circ} \mathrm{C}$. Such a column of mercury is represented by a "standard" ohm (see Appendix B).

Current. - The Ampere (formerly called the "weber"), $=10^{-1}$ absolute units; practically represented by the current which deposits silver at the rate of 0.001118 gramme per second (see Appendix B).

Electromotive-force. - The Volt, $=10^{8}$ absolute units, is that E.M.F. which applied to $1 \mathrm{ohm}$ will produce in it a current of 1 ampere; being $\frac{1000}{1434}$ of the E.M.F. of a Clark standard cell at $15^{\circ} \mathrm{C}$. (See Appendix C.)

Quantity. - The Coulomb, $=10^{-1}$ absolute units of quantity ; being the quantity of electricity conveyed by 1 ampere in one second.

Capacity. - The Farad, $=10^{-9}$ (or one one-thousandmillionth) of absolute unit of capacity; being the capacity of a condenser such as to be changed to a potential of 1 volt by 1 coulomb. The microfarad or millionth part of 1 farad $=10^{-15}$ absolute units.

Work. -The Joule, $=10^{7}$ absolute units of work (ergs), is represented by energy expended in one second by 1 ampere in $1 \mathrm{ohm}$.

Pover - The Watt, $=10^{7}$ absolute units of power (ergs per second), is power of a current of 1 ampere flowing

* The word " unit" expresses our conception in the abstract of a unit quantity, such as those defined in the preceding Articles. A "standard" is the concrete thing with which we compare quantities to be measured, such as a centimetre scale or a standard cell. 
ancler a pressure of 1 volt. It is equal to 1 joule per second, and is approximately $\frac{1}{46}$ of one horse-power.

Induction. - The Henry, $=10^{9}$ absolute units of induction, is the induction in a circuit when the electromotive-force induced in this circuit is 1 volt, while the inducing current varies at the rate of 1 ampere per second.

Seeing, however, that quantities a million times as great as some of these, and a million times as small as some, have to be measured by electricians, the prefixes mega- and micro- are sometimes used to signify respectively "one million" and "onemillionth part." Thus a megohm is a resistance of one million ohms, a microfarad a capacity of $\frac{1}{1000000}$ of a farad, etc. The prefix kilo- is used for "one thousand," and milli- for "onethousandth part"; thus a kilowatt is 1000 watts, and milli.ampere is the thousandth part of 1 ampere.

The "practical" system may be regarded as a system of units derived not from the fundamental units of centimetre, gramme; and second, but from a system in which, while the unit of time remains the second, the units of length and mass are respectively the earth-quadrant and $10^{-11}$ gramme.

355. Use of Index Notation.--Seeing that electricians have to deal with quantities requiring in some cases very large numbers, and in other cases very small numbers, to express them, a system of index notation is adopted, in order to obviate the use of long rows of ciphers. In this system the significant figures only of a quantity are put down, the ciphers at the end, or (in the case of a long decimal) at the beginning, being indicated by an index written above. Accordingly, we may write a thousand $(=10 \times 10 \times 10)$ as $10^{3}$, and the quantity 42,000 may be written $42 \times 10^{3}$. The British National Debt of $£ 770,000,000$ may be written $£ 77 \times 10^{7}$. Fractional quantities will have negative indices when written as exponents. Thus $\frac{1}{100}(=0.01)$ $=1 \div 10 \div 10=10^{-2}$. And so the decimal 0.00028 will be written $28 \times 10^{-5}$ (being $=28 \times \cdot 00001$ ). The convenience of this method will be seen by an example or two on electricity. The electrostatic capacity of the earth is $630,000,000$ times that of a sphere of one centimetre radius, $=63 \times 10^{7}$ (electrostatic) units. The resistance of selenium is about $40,000,000,000$, or $4 \times 10^{10}$ times as great as that of copper; that of air is about $10^{26}$, or

$$
100,000,000,000,000,000,000,000,000
$$

times as great. The velocity of light is about $30,000,000,000$ centimetres per second, or $3 \times 10^{10}$.

356. Dimensions of Magnetic and Electromagnetic Units.-The fundamental idea of "dimensions" is explained in 
Art. 284. A little consideration will enable the student to deduce for himself the following table:-

\begin{tabular}{|c|c|c|c|c|}
\hline & \multicolumn{3}{|c|}{ Units. } & Dimensions. \\
\hline & (Magnetic.) & & & \\
\hline$m$ & $\left\{\begin{array}{l}\text { Strength of pole } \\
\text { Quantity of magnetism }\end{array}\right\}$ & $=\sqrt{\text { force } \times(\text { distance })^{2}}$ & $=$ & $\mathrm{M}^{\frac{1}{2}} \mathrm{~L}^{\frac{3}{2}} \mathrm{~T}^{-1}$ \\
\hline $\mathrm{V}$ & Magnetic Potential & $=$ work $\div$ strength of pole & $=$ & $\mathrm{M}^{\frac{1}{2}} \mathrm{~L}^{\frac{1}{2}} \mathrm{~T}^{-1}$ \\
\hline $\mathbf{H}$ & Intensity of Field & $=$ force $\div$ strength of pole & $=$ & $\mathrm{M}^{\frac{1}{2}} \mathrm{~L}^{-\frac{1}{2}} \mathrm{~T}^{-1}$ \\
\hline $\mathrm{N}$ & Magnetic Flux & $=$ intensity $\times$ area & $=$ & $M^{\frac{1}{2}} \quad L^{\frac{3}{2}} \quad T^{-1}$ \\
\hline Z & $\begin{array}{l}\text { Reluctance } \\
\qquad \text { (Electromagnetic.) }\end{array}$ & $=$ flux $\div$ mag. potential & $=$ & $\mathrm{L}$ \\
\hline C & Current (strength) & $=$ intensity of field $\times$ length & $=$ & $M^{\frac{1}{2}} L^{\frac{1}{2}} T^{-1}$ \\
\hline $\mathbf{Q}$ & Quantity & $=$ current $\times$ time & $=$ & $M^{\frac{1}{2}} L^{\frac{1}{2}}$ \\
\hline $\begin{array}{l}\mathrm{V} \\
\mathrm{E}\end{array}$ & $\left.\begin{array}{l}\text { Potential } \\
\text { Electromotive-force }\end{array}\right\}$ & $=$ work $\div$ quantity & $=$ & $M^{\frac{1}{2}} L^{\frac{3}{2}} T^{-2}$ \\
\hline $\mathbf{R}$ & Resistance & $=$ E.M.F. $\div$ current & $=$ & $\mathrm{LT}^{-1}$ \\
\hline K & Capacity & $=$ quantity $\div$ potential & $=$ & $\mathrm{L}^{-1} \mathrm{~T}^{2}$ \\
\hline W & Power & $=$ current $\times$ potential & $=$ & $M L^{2} \mathrm{~T}^{-3}$ \\
\hline $\begin{array}{l}\mathrm{L} \\
\mathrm{M}\end{array}$ & $\left.\begin{array}{l}\text { Self-Induction } \\
\text { Mutual Induction }\end{array}\right\}$ & $=$ E.M.F. $\div$ current per sec. & $=$ & $\mathrm{L}$ \\
\hline
\end{tabular}

357. Resistance expressed as a Velocity.-It will be seen, on reference to the above table of "Dimensions" of electromagnetic units, that the dimensions of resistance are given as $\mathrm{LT}^{-1}$, which are the same dimensions (see Art. 284) as those of a velocity. Every resistance is capable of being expressed as a velocity. The following considerations may assist the student in forming a physical conception of this. Suppose we have a circuit composed of two horizontal rails (Fig. 176), CS and DT, 1 centim. apart, joined at $\mathrm{CD}$, and completed by means of a sliding piece $A B$. Let this variable circuit be placed in a uniform magnetic field of unit intensity, the lines of force being directed vertically downwards through the circuit. If, now, the slider be moved along towards ST with a velocity of $n$ centimetres per second, the number 
of additional lines of force embraced by the circuit will increase at the rate $n$ per second; or, in other words, there will be an-induced electromotive-force (Art. 225) impressed upon the circuit, which will cause a current to flow through the slider from A to B. Let the rails have no resistance, then the strength of the current will depend on the resistance of $A B$. Now let $A B$ move at such a

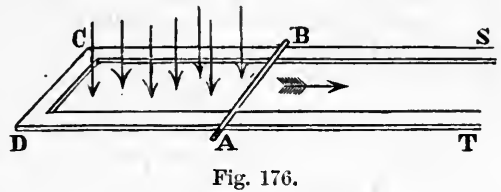

rate that the current shall be of unit strength. If its resistance be one "absolute" (electromagnetic) unit it need only move at the rate of 1 centim. per second. If its resistance be greater it must move with a proportionately greater velocity ; the velocity at which it must move to keep up a current of unit strength being numerically equal to its resistance. The resistance known as "one ohm" is intended to be $10^{9}$ absolute electromagnetic units, and therefore is represented by a velocity of $10^{9}$ centimetres, or ten million metres (one earth-quadrant) per second.

358. Evaluation of the Ohm. - The system of "practical" units was originally devised by a committee of the British Association, who also determined the value of the "ohm" by experiment in 1863, and constructed standard resistance coils of German-silver, called "B.A. Units" or "ohms."

There are several ways of measuring the absolute value of the resistance of a wire. One method (Joule's) is to measure the heat produced in it by a known current and calculate its resistance by Joule's law (Art. 427). Another method (Weber's) is to measure in absolute units the current that is sent through the wire by an electromotive-force which is also measured in some absolute way. The ratio of the latter to the former gives the value of the resistance. Weber's method involved spinning a coil in a magnetic field which would generate alternate currents. Kohlrausch used an induction coil to generate the E.M.F. Lorenz proposed a method in which a disk was spun. Foster a zero method in which the E.M.F. in the spinning coil was balanced. Lord Kelvin pro. posed to the British Association Committee a modification of Weber's method as follows. It being impracticable to give to a horizontal sliding-piece so high a velocity as was necessitated, the velocity which corresponded to the resistance of a wire was measured in the following way :-A ring. of wire (of many turns), 
pivoted about a vertical axis, as in Fig. 177 , was made to rotate very rapidly and uniformly. Such a ring in rotating cuts the lines of force of the earth's magnetism. The northern half of the ring, in moving from west toward east, will have (see Rule, Art. 225) an upward current induced in it, while the southern half, in crossing from east toward west, will have a downward current induced in it. Hence the rotating ring will, as it spins, act as its own galvanometer if a small magnet be hung at its middle ; the magnetic effect due to the rotating coil being proportional directly to the horizontal component of the earth's magnetism, to the velocity of rotation,

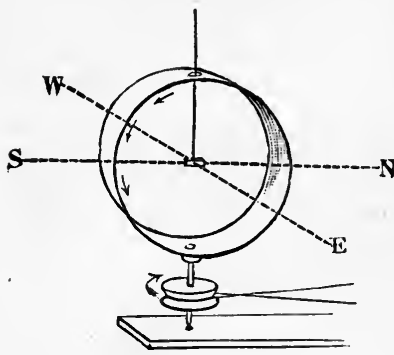

Fig. 177 . and to the number of turns of wire in the coil, and inversely proportional to the resistance of the wire of the coils. Hence, all the otherdata being known, the resistancecan be calculated and measured as a velocity. The earliest ohms or B.A. units were constructed by comparison with this rotating coil; but there being some doubt as to whether the B.A. unit really represented $10^{9}$ centims. per second, a redetermination of the ohm was suggested in 1880 by the British

Association Committee. At the first International Congress of Electricians in Paris 1881, the project for a redetermination of the ohm was endorsed, and it was also agreed that the practical standards should no longer be constructed in German-silver wire, but that they should be made upon the plan originally suggested by Siemens, by defining the practical ohm as the resistance of a column of pure mercury of a certain length, and of one millimetre of cross-section. The original "Siemens' unit" was a column of mercury one metre in length, and one square millimetre in section, and was rather less than an ohm $(0.9540$ B.A. unit). Acting on measurements made by leading physicists of Europe, the Paris Congress of 1884 decided that the mercury column representing the "legal" ohm should be 106 centimetres in length. This was, however, never legalized in this country or in America, as it was known to be incorrect. Lord Rayleigh's determination gave 106.21 centimetres of mercury, as representing the true theoretical ohm $\left(=10^{9}\right.$ absolute units $)$; and Rowland's determinations at Baltimore came slightly higher. The British Association Committee in 1892 agreed to lengthen it to $106 \cdot 3$ centims., and to define by mass instead of section. This was 
decided finally as the international ohm by the Congress of Chicago in 1893. These international units are now legalized in England and the United States. The order signed by Her Majesty in Council and issued through the Board of Trade is given in Appen$\operatorname{dix} B$.

The old B.A. unit is only 0.9863 of the true ohm ; the Siemens' unit is only 0.9408 .

359. Ratio of the Electrostatic to the Electromagnetic Units. - If the student will compare the Table of Dimensions of Electrostatic Units of Art. 283 with that of the Dimensions of Electromagnetic Units of Art. 356, he will observe that the dimensions assigned to similar units are different in the two systems. Thus, the dimensions of "Quantity" in electrostatic measure are $\quad \mathrm{M}^{\frac{1}{2}} \quad \mathrm{~L}^{\frac{3}{2}} \quad \mathrm{~T}^{-1}$, and in electromagnetic measure they are $\mathrm{M}^{\frac{1}{2}} \mathrm{~L}^{\frac{1}{2}}$. Dividing the former by the latter we get $\mathrm{LT}^{-1}$, a quantity which we at once see is of the nature of a velocity. This velocity occurs in every case in the ratio of the electrostatic to the electromagnetic measure of every unit. It is a definite concrete velocity, and represents that velocity at which two electrified particles must travel along side by side in order that their mutual electromagnetic attraction (considered as equivalent in so moving (Art. 397) to two parallel currents) shall just equal their mutual electrostatic repulsion (see Art. 260). This velocity, " $v$," which is of enormous importance in the electromagnetic theory of light (Art. 518), has been measured in several ways.

\begin{tabular}{|c|c|c|c|}
\hline UNIT. & Electrostatic. & Electromagnetic. & Ratio. \\
\hline Quantity & $M^{\frac{1}{2}} \quad \mathrm{I}^{\frac{3}{2}} \cdot \mathrm{T}^{-1}$ & $M^{\frac{1}{2}} L^{\frac{1}{2}}$ & $\mathrm{LT}^{-1}=v$ \\
\hline Potential & $\mathrm{M}^{\frac{1}{2}} \quad \mathrm{~L}^{\frac{1}{2}} \quad \mathrm{~T}^{-1}$ & $M^{\frac{1}{2}} \quad L^{\frac{3}{2}} \quad T^{-2}$ & $\mathrm{~L}^{-1} \mathrm{~T}=1 / v$ \\
\hline Capacity & L & $\mathrm{L}^{-1} \mathrm{~T}^{2}$ & $\mathrm{~L}^{2} \mathrm{~T}^{-2}=v^{2}$ \\
\hline Resistance . & $\mathrm{L}^{-1} \mathrm{~T}$ & $\mathrm{~L} \quad \mathrm{~T}^{-1}$ & $\mathrm{~L}^{-2} \mathrm{~T}^{2}=1 / v^{2}$ \\
\hline
\end{tabular}

(a) Weber and Kohlrausch measured the electrostatic unit of quantity and compared it with the electromagnetic unit of quantity, and found the ratio $v$ to be $=3 \cdot 1074 \times 10^{10}$ centims. per second.

(b) Lord Kelviu compared the two units of potential and found

$$
\begin{aligned}
v & =2.825 \times 10^{10}, \\
\text { and later, } & =2.93 \times 10^{10} .
\end{aligned}
$$


(c) Professor Clerk Maxwell balanced a force of electrostatic attraction against one of electromagnetic repulsion, and found

$$
v \quad=2.88 \times 10^{10} \text {. }
$$

(d) Professors Ayrton and Perry measured the capacity of a condenser electromagnetically by discharging it into a ballistic galvanometer, and electrostatically by calculations from its size, and found

$$
v=2.980 \times 10^{10} .
$$

The relocity of light according to latest values is-

$$
=2.9992 \times 10^{10} \text {; }
$$

so we take $v$ as $3 \times 10^{10}$, or thirty thousand million centimetres per second.

360. Rationalization of Dimensions of Units. - It seems absurd that there should be two different units of electricity ; still more absurd that one unit should be thirty thousand million centimetres per second greater than the other. It also seems absurd that the dimensions of a unit of electricity should have fractional powers, since such quantities as $\mathbf{M}^{\frac{1}{2}}$ and $\mathrm{L}^{\frac{8}{2}}$ are meaningless. These irrational things arise from the neglect to take account of the properties of the medium in applying the law of inverse squares to form definitions of the unit of electricity in the electrostatic system, and of the unit-pole in the magnetic system. If we were to insert the dielectric constant $k$ in the former, and the permeability $\mu$ in the latter, we might, if we knew the dimensions of these quantities, be able to rationalize the dimensional formula. But we do not know their dimensions. Rücker has, however, shown that they can be rationalized, and the two sets of units brought into agreement, ${ }^{*}$ by assuming that the product $k \mu$ has the dimensions of the reciprocal of the square of a velocity : or $v=1 / \sqrt{k \mu}$. If $k$ were the reciprocal of the rigidity of the ether, and $\mu$ its density, $v$ would represent the velocity of propagation of waves in it. Compare Art. 518 on electromagnetic theory of light.

361. Earth's Magnetic Force in Absolute Units.In making absolute determinations of current by the tangent galvanometer, or of electromotive-force by the spinning coil, it is needful to know the absolute value of the earth's magnetic field, or of its horizontal component. The intensity of the earth's magnetic force at any place is the force with which a magnet-pole of unit strength is attracted. As explained in Art. 153, it is usual to measure the horizontal component $\mathrm{H}$ of this force, and from this and the cosine of the angle of dip to calculate the total force, as the direct determination of the latter is surrounded with

* See Everett's Units and Physical Constants, 4th edition (1893), p. 208. 
difficulties. To determine $\mathrm{H}$ in absolute (or C.G.S.) units, it is necessary to make two observations with a magnet of magnetic moment M (Art. 135). In one of these observations the product $\mathrm{MH}$ is determined by a method of oscillations (Art. 133); in the second the quotient $\frac{\mathrm{M}}{\mathrm{H}}$ is determined by a particular method of deflexion (Art. 138). 'The square root of the quantity obtained by dividing the former by the latter will, of course, give $\mathrm{H}$.

(i.) Determination of $\mathrm{MH}$.- The time $\mathrm{T}$ of a complete oscillation to and fro of a magnetic bar is

$$
\mathrm{T}=2 \pi \wedge \sqrt{\frac{\mathrm{K}}{\mathrm{MH}}}
$$

where $\mathrm{K}$ is the "moment of inertia" of the magnet. This formula is, however, only true for very small arcs of vibration. By simple algebra it follows that

$$
\mathrm{MH}=\frac{4 \pi^{2} \mathrm{~K}}{\mathrm{~T}^{2}} .
$$

Of these quantities $\mathrm{T}$ is ascertained by a direct observation of the time of oscillation of the magnet hung by a torsionless fibre : and $\mathrm{K}$ can be either determined experimentally or by one of the following formule :-

For a round bar

$$
\mathrm{K}=w\left(\frac{l^{2}}{12}+\frac{a^{2}}{4}\right)
$$

$$
\text { For a rectangular bar } \quad \mathrm{K}=w \quad\left(\frac{l^{2}+l^{2}}{12}\right) \text {; }
$$

where $w$ is the mass of the bar in grammes, $l$ its length, $a$ its radius (if round), $b$ its breadth, measured horizontally (if rectangular).

(ii.) Determination of $\frac{M}{H^{\prime}}$ - The magnet is next caused to deflect a small magnetic needle in the following manner, " broadside on." The magnet is laid horizontally at right angles to the magnetic meridian, and so that its middle point is (magnetically) due south or due north of the small needle, and at a distance $r$ from its centre. Lying thus broadside to the small needle its $\mathrm{N}$-pole will repel, and its S-pole attract, the N-pole of the needle, and will exercise contrary actions on the S-pole of the needle. The total action of the magnet upon the needle will be to deflect the latter through an angle $\delta$, whose tangent is directly proportional to $\frac{M}{\bar{H}}$, and inversely proportional to the cube of the distance $r$; or

$$
\frac{\mathrm{M}}{\mathrm{H}}=r^{3} \tan \delta \text {. }
$$


Dividing the former equation by this, and taking the square root, we get

$$
\mathrm{H}=\frac{2 \pi}{\mathrm{T}} \sqrt{\frac{\mathrm{K}}{r^{3} \tan \delta^{\circ}}}
$$

\section{Lesson XXVIII._-Properties of Iron and Steel}

362. Magnetization of Iron.-When a piece of magnetizable metal is placed in a magnetic field, some of the lines of magnetic force run through it and magnetize it. The intensity of its magnetization will depend upon the intensity of the field into which it is put and upon the metal itself. There are two ways of looking at the matter, each of which has its advantages. We may think about the internal condition of the piece of metal, and of the number of magnetic lines that are running through it and emerging from it into the surrounding space. This is the modern way. Or we may think of the magnetism of the iron or other metal as something resident on the polar surfaces, and expressed therefore in units of magnetism. This is the old way. The fact that soft iron placed in the magnetic field becomes highly magnetic may then be expressed in the following two ways: (1) when iron is placed in the magnetic field, the magnetic lines run in greater quantities through the space now occupied by iron, for iron is very permeable to the lines of magnetic induction, being a good conductor of the magnetic lines; (2) iron when placed in the magnetic field develops strong poles on its end-surfaces, being highly susceptille to magnetization. Each of these ideas may be rendered exact by the introduction of appropriate coefficients.

363. Permeability. - The precise notion now attached to this word is that of a numerical coefficient. Suppose a magnetic force-due, let us say, to the circulation of an electric current in a surrounding coil-were to act on a space occupied by air, there would result a certain 
number of magnetic lines in that space. In fact, the intensity of the magnetic force, symbolized by the letter $\mathrm{H}$, is often expressed by saying that it would produce $\mathrm{H}$ magnetic lines per square centimetre in air. Now, owing to the superior magnetic power of iron, if the space subjected to this magnetic force were filled with iron instead of air, there would be produced a larger number of magnetic lines per square centimetre. This larger number expresses the degree of magnetization * or density of the magnetic flux in the iron; it is symbolized by the letter $\mathbf{B}$. The ratio of $\mathbf{B}$ to $\mathrm{H}$ expresses the permeability of the material. The usual symbol for the permeability is the Greek letter $\mu$. So we may say that the fluxdensity $\mathrm{B}$ is equal to $\mu$ times the magnetic force $\mathrm{H}$, or

$$
\mu=\mathrm{B} / \mathrm{H} \text {. }
$$

For example, a certain specimen of iron, when subjected to a magnetic force capable of creating, in air, 50 magnetic lines to the square centimetre, was found to be permeated by no fewer than 16,062 magnetic lines per square centimetre. Dividing the latter figure by the former gives as the value of the permeability at this stage of the magnetization 321 , or the permeability of the iron is 321 times that of air.

The permeability is always positive : for empty space it is 1 , for air it is practically 1 ; for magnetic materials it is greater than 1, for diamagnetic materials it is slightly less than 1 . In air, etc., $\mathrm{B}=\mathrm{H}$.

Where the magnetic lines emerge into the air at a polar surface they are of course continuous with the internal lines: the value of $\mathbf{B}$ just inside the polar surface is the same as that of $\mathbf{B}$ in the air just outside it.

The permeability of such non-magnetic materials as

* The actual number of magnetic lines that run through unit area of cross-section in the iron or other material-denoted by the symbol B-is called by several names - "the permeation," "the internal magnetization," or "the induction." The last name, unfortunately used by Maxwell and Hopkinson, is to be avoided. A better name is "flux-density." 
silk, cotton, and other insulators, also of brass, copper, and all the non-magnetic metals, is taken at 1 , being practically the same as that of the air.

This mode of expressing the facts is, however, complicated by the fact of the tendency in all kinds of iron to magnetic saturation. In all kinds of iron the magnetizability of the material becomes diminished as the actual magnetization is pushed further. In other words, when a piece of iron has been magnetized up to a certain degree, it becomes, from that degree on ward, less permeable to further magnetization, and thongh actual saturation is never reached, there is a practical limit beyond which it cannot well be pushed. Joule discovered this tendency to a limit. The practical linit of $\mathbf{B}$ in good wrought iron is about 20,000 lines per square centimetre, or in cast iron about 12,000. Using extraordinary magnetizing forces, Ewing has found it possible to increase B to 45,000 , and Du Bois has reached 60,000 lines per square centimetre. Manganese steel is curiously non-magnetic: Hopkinson found 310 as the maximum flux-density B.

364. Curves of Magnetization.-A convenient mode of studying the magnetic facts respecting any

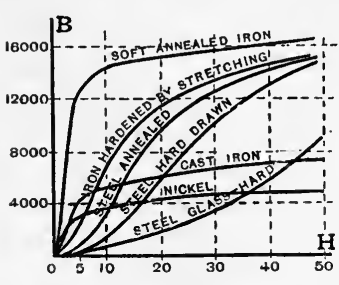

Fig. 17s. particular brand of iron is to plot on a diagram the curve of magnetization - i.e. the curve in which the values, plotted horizontally, represent the magnetic force $\bar{H}$, and the values plotted vertically those that correspond to the respective magnetization B. In Fig. 178 , which is modified from the researches of Ewing, are given five curves relating to soft iron, hardened iron, annealed steel, hard-drawn steel, and glass-hard steel. It will be noticed that all these curves have the same general form, and that there are three stages. (1) For small values of $H$ the values of 
B are small, and as $\mathrm{H}$ is increased $\mathrm{B}$ increases gradually. (2) The curve rises very suddenly, at least with all the softer sorts of iron. (3) The curve then bends over and becomes nearly horizontal, B increasing very slowly. When the magnetization is in the stage below the bend of the curve, the iron is said to be far from the state of saturation. But when the magnetization has been pushed beyond the bend of the curve into the third stage, the iron is said to be approaching saturation, because at this stage of magnetization it requires a large increase in the magnetizing force to produce even a very small increase in the magnetization. It will be noted that for soft wrought iron the stage of approaching saturation sets in when $B$ has attained the value of about 16,000 , or when $\mathrm{H}$ has been raised to about 50. The student is strongly advised to plot for himself similar curves from the subjoined table, which relates to the permeabilities of some samples of iron examined by Hopkinson.

\begin{tabular}{|c|c|c|c|c|c|}
\hline \multicolumn{3}{|c|}{ AnNealed Wrovght Iron. } & \multicolumn{3}{|c|}{ Grey Cast Iron. } \\
\hline B & $\mu$ & $\mathbf{H}$ & B & $\mu$ & $\mathbf{H}$ \\
\hline 5,000 & 3000 & $1 \cdot 66$ & 4,000 & 800 & 5 \\
\hline 9,000 & 2250 & 4 & 5,000 & 500 & 10 \\
\hline 10.000 & 2000 & 5 & 6,000 & 279 & $21 \cdot 5$ \\
\hline 11,000 & 1692 & $6 \cdot 5$ & 7,000 & 133 & 42 \\
\hline 12,000 & 1412 & $8 \cdot 5$ & 8,000 & 100 & 80 \\
\hline 13,000 & 1083 & 12 & 9,000 & 71 & 127 \\
\hline 14,000 & 823 & 17 & 10,000 & 53 & 188 \\
\hline 15,000 & 526 & $28 \cdot 5$ & 11,000 & 37 & 292 \\
\hline 16,000 & 320 & 50 & & & \\
\hline 17,000 & 161 & 105 & & & \\
\hline 18,000 & 90 & 200 & & & \\
\hline 19,000 & 54 & 350 & & & \\
\hline 20,000 & 30 & 666 & & & \\
\hline
\end{tabular}

It will be noted that at early stages of the magnetiza 
tion, in moderately weak fields where $\mathrm{H}$ is less than about 5 , the permeability has enormous values. But for values of $\mathrm{H}$ less than about 0.5 the permeability is quite small, usually about 300 .

The three stages observed in the magnetization are explained in Ewing's molecular theory (Art. 127).

If iron is compressed its permeability decreases; if subjected to tensile stress it is increased, provided the field is not too intense. Villari found that beyond a certain intensity tension diminishes the permeability.

365. Susceptibility.- Suppose a magnet to have $m$ units of magnetism on each pole; then if the length between its poles is $l$, the product $m l$ is called its magnetic moment, and the magnetic moment divided by its volume is called its intensity of magnetization; this term being intended, though based on surface-unit of pole strength, to convey an idea as to the internal magnetic state. Seeing that volume is the product of sectional area into length, it follows that if any piece of iron or steel of uniform section had its surface magnetism situated on its ends only, its intensity of magnetization would be equal to the strength of pole divided by the area of end surface. Writing I for the intensity of magnetization we should have

$$
\mathrm{I}=\frac{\text { mag. moment }}{\text { volume }}=\frac{m \times l}{s \times l}=\frac{m}{s} \text {. }
$$

Now, supposing this intensity of magnetization were due to the iron having been put into a magnetic field of intensity $\mathrm{H}$, the ratio between the resulting intensity of magnetization $I$ and the magnetizing force $H$ producing it is expressible by a numerical coefficient of magnetization, or susceptibility, $k$. We may write

$$
\text { or } \quad \begin{array}{ll}
\mathrm{I} & =k \mathrm{H}, \\
k & =\mathrm{I} / \mathrm{H} .
\end{array}
$$

This may be looked at as saying that for every 
magnetic line in the field there will be $k$ units of magnetism on the end surface. In magnetic substances such as iron, steel, nickel, etc., the susceptibility $k$ has positive values; but there are many substances such as bismuth, copper, mercury, etc., which possess feeble negative coefficients. These latter are termed "diamagnetic" bodies (Art. 369) and are apparently repelled by the poles of magnets. It was shown at end of Art. 338 that there are $4 \pi$ magnetic lines proceeding from each unit of pole magnetism. Hence if, as shown above, each line of force of the magnetising field produces $k$ units of magnetism there will be $4 \pi k$ lines added by the iron to each 1 line in the field, or the permeability of the iron $\mu$ is equal to $1+4 \pi k$. It follows that $\mathrm{B}=\mathrm{H}+4 \pi k \mathrm{H}$. This shows that $\mathrm{B}$ may go on increasing as long as $\mathrm{H}$ is increased, having no true limit. But since $k$ decreases as saturation sets in, the surface magnetization $\mathbf{I}$ (or $\mathbf{B}-\mathbf{H}$ to which it is proportional) may have a true limit. This maximum of $\mathbf{B}-\mathbf{H}$ appears to be about 21,360 in wrought iron, 15,580 in cast iron, and 5660 in nickel.

In the following table are given some figures from the researches of Bidwell on wrought iron.

\begin{tabular}{|c|c|c|c|c|}
\hline H & $k$ & I & $\mu$ & B \\
\hline $3 \cdot 9$ & $151^{\circ} 0$ & 587 & $1899 \cdot 1$ & 7390 \\
\hline $10 \cdot 3$ & $89 \cdot 1$ & 918 & $1121 \cdot 4$ & 11550 \\
\hline $40^{\circ}$ & $30 \cdot 7$ & 1226 & $386 \cdot 4$ & 15460 \\
\hline $115^{\circ}$ & $11 \cdot 9$ & 1370 & $150 \cdot 7$ & 17330 \\
\hline $208^{\circ}$ & $7 \cdot 0$ & 1452 & $88 \cdot 8$ & 18470 \\
\hline $427^{\circ}$ & $3 \cdot 5$ & 1504 & $45 \cdot 3$ & 19330 \\
\hline $585^{\circ}$ & $2 \cdot 6$ & 1530 & $33 \cdot 9$ & 19820 \\
\hline
\end{tabular}

Everett has calculated (from Gauss's observations) that the intensity of magnetization of the earth is only 0.0790 , or only $\frac{1}{1.800}$ of what it would be if the globe were 
wholly iron. In weak magnetic fields the susceptibility of nickel exceeds by about five times that of iron ; but in strong fields iron is more susceptible.

366. Measurement of Permeability.-There are several ways of measuring the permeability of iron: they all involve a measurement of $\mathrm{B}$.

(a) Magnetometer Methods. - The pole strength of long bars, when magnetized by a coil around them, can be measured by a magnetometer (Art. 138), and from this $\mathbf{N}$ is found by multiplying by $4 \pi$.

(b) Induction Methods.-Rings of iron which, having no poles, cannot be measured by the magnetometer are measured inductively. Upon the ring is wound a magnetizing coil, and also an exploring coil (Art. 232) which is connected to a ballistic galvanometer. On turning on or off the magnetizing current, or reversing it, induced currents are generated, giving a throw in the galvanometer proportional to the number of magnetic lines which have been made or destroyed. Iron rods can be examined by the same means.

(c) Traction Methods.-The pull needed to separate the two halves of a divided rod, or divided ring, is (Art. 384) proportional to the square of B. Bidwell and others have used this for measuring permeability.

(d) Optical Methods.-Du Bois has used a method based on Kerr's discovery (Art. 52 $)$ ) of magneto-optic rotation.

367. Residual Effects. - The retention of magnetism by steel, lodestone, hard iron, and even by soft iron if of elongated shape, has been already described (Art. 98). Some other residual effects must now be noted. It is found that if a new piece of iron or steel is subjected to an increasing magnetizing force, and then the magnetizing force is decreased to zero, some magnetism remains. If the results are plotted out in a curve it exhibits the following peculiarities. On first gradually increasing $\mathrm{H}$ from o, B rises as we have seen in Art. 364 . 
If when the curve has risen to $a(\mathrm{Fig} .179) \mathrm{H}$ is now decreased, the descending eurve does not follow the ascending curve, owing to the retention of the magnetism. When $\mathrm{H}$ has been reduced to zero the point $b$ is reached. This the residual value of $\mathbf{B}$ is called the remanence, and depends on the material, and on the degree to which $B$ was previously pushed. If now a reversed magnetizing force $-\mathrm{H}$ is applied it is found that it must be increased to a definite degree in order to demagnetize the iron and bring the curve down to $c$. The amount of reversed magnetic foree so needed is a measure of the retentivity of the material, and is known as the

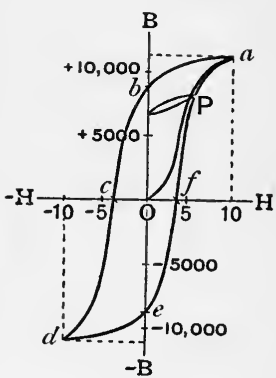

Fig. 179. coercive force. In hard steel it may amount to 100 ; in soft steel to 20 ; in soft iron to 2 or less. If the reversed magnetizing force is further increased, the curve descends from $c$ to $d$, the iron becoming magnetized with reversed polarity, and going toward saturation. On then diminishing the reversed force to zero, the curve turus to $e$, showing a negative remanence. On again increasing $\mathrm{H}$ as at first the curve ascends to $f$, and as the former value of $\mathrm{H}$ is reached comes up to $a$ again.

368. Cycles of Magnetization. Hysteresis.When $\mathrm{H}$ is thus carried through a cycle of increase and decrease, $\mathrm{B}$ also goes through a eycle; and as we have seen there is a lagging in the magnetization, evidenced in Fig. 179 by the formation of a closed loop in the curve. Warburg and Ewing, who have fully investigated the phenomenon, have remarked that the area enclosed indicates the waste of energy in the cycle of operations. In hard steel the areas of these loops are much wider thian in the case of soft iron. Ewing has given the name 
of Hysteresis to the subject of the lag of magnetic effects behind their causes. From his researches * also is taken the case of Fig. 180, a specimen of soft iron, the curve for which shows various loops. Ewing has devised a curve-tracer for recording the curves automatically. The

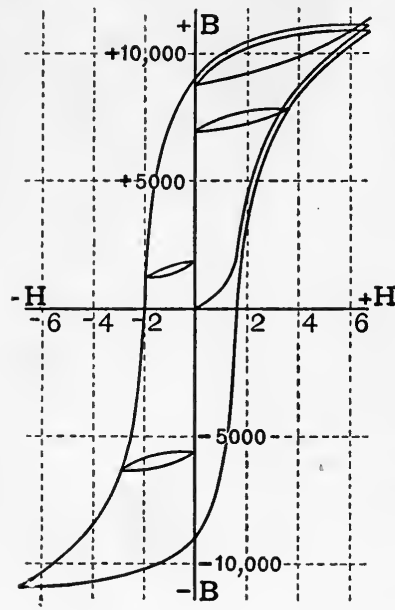

Fig. 180.

waste of energy per cubic centimetre in a cycle of strong magnetization may vary from 9000 ergs in annealed iron to 200,000 in glass-hard steel. If (as in the iron cores of alternate current transformers) the cycle is repeated 100 times a second the waste of power by hysteresis may heat the iron ; and it increases greatly with the frequency and with the degree to which the magnetization is pushed. If $\mathbf{B}$ does not exceed 5000, the power wasted at 100 cycles per second in every cubic foot of iron may be as low as 575 watts, but if $B$ is increased to 10,000 the waste becomes 1560 watts.

Since a smaller reversed force suffices to destroy magnetization than was required to produce it, all that is necessary in order to completely demagnetize iron is to subject it to a series of cycles of diminishing intensity.

Mechanical agitation tends to help the magnetizing forces to act, and lessens all residual and hysteresial effects.

Ewing has also shown that under constant magnetizing

* The student shoule not fail to consult Ewing's book, Magnetic Induction in Iron. 
force the magnetism will go on slowly and slightly increasing for a long time: this is called magnetic creeping, or viscous hysteresis.

\section{Lesson XXIX.—Diamagnetism}

369. Diamagnetic Experiments. - In 1778 Brugmans of Leyden observed that when a lump of bismuth was held near either pole of a magnet needle it repelled it. In 1827 Le Baillif and Becquerel observed that the metal antimony also could repel and be repelled by the pole of a magnet. In 1845 Faraday, using powerful electromagnets, examined the magnetic properties of a large number of substances, and found that whilst a great many are, like iron, attricted to a magnet, others are feebly repelled. To distinguish between these two classes of bodies, he termed those which are attracted paramagnetic, ${ }^{*}$ and those which are repelled diamagnetic. The property of being thus apparently repelled from a magnet he termed diamagnetism.

Faraday's method of experiment consisted in suspending a small bar of the substance in a powerful magnetic field between the two poles of an electromagnet, and observing whether the small bar was attracted into an axial position, as in Fig. 181, with its length along the line joining the two poles, or whether it was repelled into an equatorial position, at right angles to the line joining the poles, across the lines of force

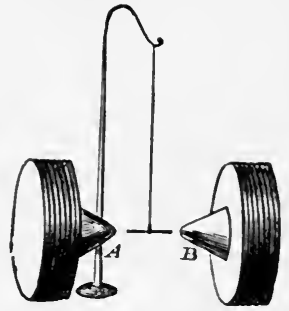

Fig. 181. of the field, as is shown by the position of the small bar in Fig. 182, suspended between the poles of an electromagnet constructed on Ruhmkorff's pattern.

* Or simply "magnetic." Some authorities use the term "ferro. magnetic." 


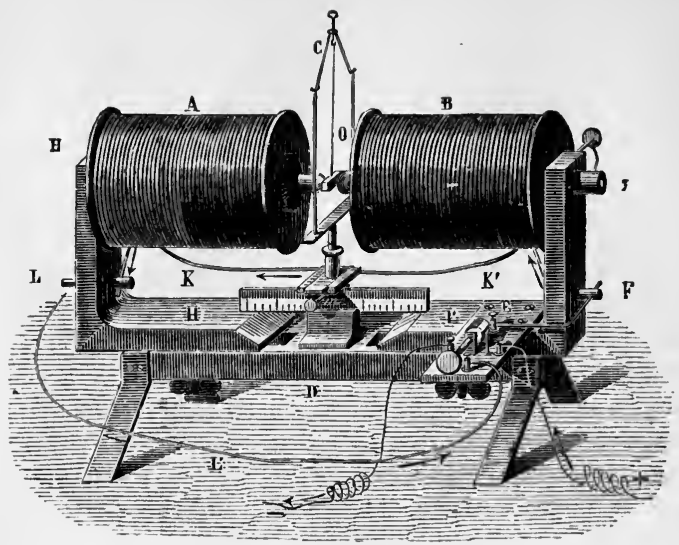

Fig. 1S2.

370. Results.-The following are the principal sub. stances examined by the method :-

\begin{tabular}{|l|l|}
\hline \multicolumn{1}{|c|}{ Paramagnetic. } & Diamagnetic. \\
\hline Iron & Bismuth \\
Nickel & Phosphorus \\
Cobalt & Antimony \\
Manganese & Thallium \\
Chromium & Zinc \\
Cerium & Mercury \\
Titanium & Lead \\
Platinum* & Silver \\
Many ores and salts & Copper \\
containing the & Gold \\
above metals & Water \\
Oxygen gas & Alcohol \\
Oxygen liquid & Tellurium \\
Ozone & Sulphur \\
\hline
\end{tabular}

* Chemically pure Platinum is diamagnetic, according to Wiedemann. 
Liquids were placed in glass vessels and suspended between the poles of the electromagnet. Almost all liquids are diamagnetic, except solutions of salts of the magnetic metals, some of which are feebly magnetic; but blood is diamagnetic though it contains iron. To examine gases bubbles are blown with them, and watched as to whether they were drawn into or pushed out of the field. Oxygen gas was found to be magnetic; ozone has been found to be still more strongly so. Dewar has found liquid oxygen sufficiently magnetic to rush in drops to the poles of a powerful magnet.

The diamagnetic properties of substances may be numerically expressed in terms of their permeability or their susceptibility (Arts. 363 and 365). For diamagnetic bodies the permeability is less than unity. For bismuth the value of $\mu$ is 0.999969 . The repulsion of bismuth is immensely feebler than the attraction of iron. Plïcker estimated the relative magnetic powers of equal weights of substances as follows :-

$\begin{array}{lcc}\text { Iron } & + & 1,000,000 \\ \text { Lodestone Ore } & + & 402,270 \\ \text { Ferric Sulphate } & + & 1,110 \\ \text { Ferrose Sulphate } & + & 780 \\ \text { Water } & - & 7 \cdot 8 \\ \text { Bismuth } & - & 23 \cdot 6\end{array}$

371. Apparent Diamagnetism due to surrounding Medium.-It is found that feebly magnetic bodies behave as if they were diamagnetic when suspended in a more highly magnetic fluid. A small glass tube filled with a weak solution of ferric chloride, when suspended in air between the poles of an electromagnet, points axially, or is paramagnetic ; but if it be surrounded by a stronger (and therefore more magnetic) solution of the same substance, it points equatorially, and is apparently repelled like diamagnetic bodies. All that the equatorial pointing of a body proves then is, that it is less magnetic than the medium that fills the surrounding space. 
A balloon, though it possesses mass and weight, rises through the air in obedience to the law of gravity, because the medium surrounding it is more attracted than it is. But it is found that diamagnetic repulsion takes place even in a vacuum: hence it would appear that the ether of space itself is more magnetic than the substances classed as diamagnetic.

372. Diamagnetic Polarity.-At one time Faraday thought that diamagnetic repulsion could be explained on the supposition that there existed a "diamagnetic polarity" the reverse of the ordinary magnetic polarity. According to this view, which, however, Faraday himself quite abandoned, a magnet, when its $\mathrm{N}$-pole is presented to the end of a bar of bismuth, induces in that end a $\mathrm{N}$-pole (the reverse of what it would induce in a bar of iron or other magnetic metal), and therefore repels it. Weber adopted this view, and Tyndall warmly advocated it, especially after discovering that the repelling diamagnetic force varies as the square of the magnetic power employed. It has even been suggested that when a diamagnetic bar lies equatorially across a field of force, its east and west poles possess different properties. The experiments named above suggest, however, an explanation less difficult to reconcile with the facts. It has been pointed out (Art. 363) that the degree to which magnetization goes on in a medium depends upon the magnetic permeability of that medium. Now, permeability expresses the number of magnetic lines induced in the medium for every line of magnetizing force applied. A certain magnetizing force applied to a space containing air or vacuum would induce a certain number of magnetic lines through it. If the space considered were occupied by a paramagnetic substance it would concentrate the magnetic lines into itself, as the sphere does in Fig. 183. But if the sphere were of a permeability less than 1, the magnetic lines would tend rather to pass through the air, as in Fig. 184. If the space considered were occupied by 
bismuth, the same magnetizing-force would induce in the bismuth fewer magnetic lines than in a vacuum. But those lines which were induced would still run in the same general direction as in the vacuum; not in the opposite direction, as Weber and Tyndall maintained. The result of there being a less induction through diamagnetic sub-

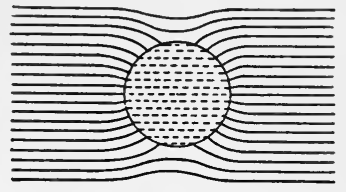

Fig. 183. stances can be shown to be that such substances will be urged from places where the magnetic force is strong to places where it is weaker.

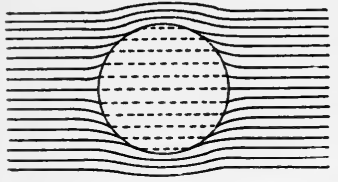

Fig. 184. This is why a ball of bismuth moves away from a magnet, and why a little bar of bismuth between the conical poles of the electromagnet (Fig. 182) turns equatorially so as to put its ends into the regions that are magnetically weaker. There is no reason to doubt that in a magnetic field of uniform strength a bar of bismuth would point along the lines of induction.

373. Magne-Crystallic Action.-In 1822 Poisson predicted that a body possessing crystalline structure would, if magnetic at all, have different magnetic powers in different directions. In 1847 Plücker discovered that a piece of tourmaline, which is itself feebly paramagnetic, behaved as a diamagnetic body when so hung that the axis of the crystal was horizontal. Faraday, repeating the experiment with a crystal of bismuth, found that it tended to point with its axis of crystallization along the lines of the field axially. The magnetic force acting thus upon crystals by virtue of their possessing a certain structure he named magne-crystallic force. Pliicker endeavoured to connect the magne-crystallic behaviour of crystals with their optical behaviour, giving the following 
law: there will be either repulsion or attraction of the optic axis (or, in the case of bi-axial crystals, of both optic axes) by the poles of a magnet; and if the crystal is a "negative" one (i.e. optically negative, having an extraordinary index of refraction less than its ordinary index) there will be repulsion, if a "positive" one there will be attraction. Tyndall has endeavoured to show that this law is insufficient in not taking into account the paramagnetic or diamagnetic powers of the substance as a whole. He finds that the magne-crystallic axis of bodies is in general an axis of greatest density, and that if the mass itself be paramagnetic this axis will point axially; if diamagnetic, equatorially. In bodies which, like slate and many crystals, possess cleavage, the planes of cleavage are usually at right angles to the magne-crystallic axis. Another way of stating the facts is to say that in non-isotropic bodies the inducerl magnetic lines do not necessarily rum in the same direction as the lines of the impressed magnetic field.

374. Diamagnetism of Flames.-In 1847 Bancalari discovered that flames are repelled from the axial line joining the poles of an electronagnet. Faraday showed that all kinds of flames, as well as ascending streams of hot air and of smoke, are acted on by the magnet, and tend to move from places where the magnetic forces are strong to those where they are weaker. Gases (except oxygen and ozone), and hot gases especially, are feebly diamagnetic. But the active repulsion and turning aside of flames may possibly be in part due to an electromagnetic action like that which the magnet exercises on the convexion-current of the voltaic arc (Art. 448) and on other convexion-currents. The electric properties of tlame are mentioned in Arts. 8 and 314. 


\section{Lesson XXX.-The Magnetic Circuit}

375. Magnetic Circuits. - It is now generally recognized that there is a magnetic circuit law similar to the law of $\mathrm{Ohm}$ for electric circnits. Ritchie, Sturgeon, Joule, and Faraday dimly recognized it. But the law was first put into shape in 1873 by Rowland, who calculated the flow of magnetic lines through a bar by dividing the "magnetizing force of the helix" ly the "resistance to lines of force" of the iron. In 1882 Bosanquet introduced the term magnetomotive-force, and showed how to calculate the reluctances of the separate parts of the magnetic circuit, and, by adding them, to obtain the total reluctance. *

The law of the magnetic circuit may be stated as follows :-

$$
\begin{aligned}
\text { Nagnetic Flux } & =\frac{\text { magnetomotive-force }}{\text { reluctance }}, \\
\text { or } \mathbf{N} & =\frac{\mathrm{M}}{\mathrm{Z}} .
\end{aligned}
$$

376. Reluctance.-As the electric resistance of a prismatic conductor can be calculated from its length, cross-section, and conductivity, so the magnetic reluctance of a har of iron can be calcnlated from its length, crosssection, and permeability. The principal difference between the two cases lies in the circumstance that whilst in the electric case the conductivity is the same for small and large currents, in the magnetic case the permeability is not constant, but is less for large magnetic fluxes than for small ones.

Let the length of the bar be $l$ centims., its section $\mathbf{A}$ sq. cms., and its permeability $\mu$. Then its reluctance

* This useful tern, far preferable to "magnetic resistance," was intrnduced by Oliver Heaviside. The term reluctivity is sometimes used for the specific reluctance; it is the reciprocal of permeability. 
will be proportional directly to $l$, and inversely to $A$ and $\mu$. Calling the reluctance $Z$ we have

$$
\mathrm{Z}=l / \mathrm{A} \mu \text {. }
$$

Example.-An iron bar $100 \mathrm{~cm}$. long and 4 sq. cms. in cross-section is magnetized to such a degree that $\mu=320$ : then $\mathrm{Z}$ will be 0.078 .

The reluctance of a magnetic circuit is generally made up of a number of reluctances in series. We will first take the case of a closed magnetic circuit (Fig. 185) made up of a curved iron core of length $l_{1}$, section $A_{1}$, and

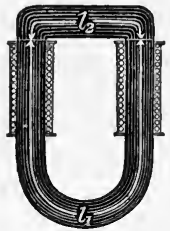

Fig. 185 . permeability $\mu_{I}$; and an armature of length $l_{2}$, section $A_{2}$, and permeability $\mu_{2}$, in contact with the ends of the former. In this case the reluctance is

$$
Z=\frac{l_{1}}{A_{1} \mu_{1}}+\frac{l_{2}}{A_{2} \mu_{2}} \text {. }
$$

377. Calculation of Exciting Power.-Passing on to the more difficult case of a circuit made up partly of iron and partly of air, we will suppose the armature to be moved to a distance, so that there are two air-gaps in the circuit, each gap of length $l_{3}$ (from iron to iron), and section $\mathrm{A}_{3}$ (equal to area of pole face). This will introduce an additional reluctance $2 l_{3} / \mathrm{A}_{3}$, the permeability for air being $=1$. It will also have the effect of making part of the magnetic flux leak out of the circuit.

By Art. 341, if the exciting power consists of $\mathrm{C}$ amperes circulating in $\mathrm{S}$ spirals around the core, the magnetomotive-force will be $4 \pi \mathrm{CS} / 10$. Applying

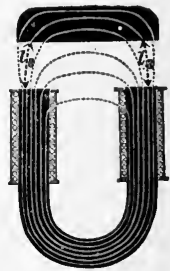

Fig. 186. this to the preceding example, dividing the magnetomotive-force by the reluctance, we get for the magnetic flux- 


$$
\mathbf{N}=\frac{4 \pi \mathrm{CS}}{10\left\{\frac{l_{1}}{\mathrm{~A}_{1} \mu_{1}}+\frac{l_{2}}{\mathrm{~A}_{2} \mu_{2}}+\frac{2 l_{3}}{\mathrm{~A}_{3}}\right\}}
$$

But more often the calculation is wanted the other way round, to find how many ampere-turns of excitation will be needed to produce a given flux through a magnetic circuit of given size. Two difficulties arise here. The permeability will depend on the degree of saturation. Also the leakage introduces an error. To meet the first difficulty approximate values of $\mu$ must be found. Suppose, for example, it was intended to produce a flux of 1,000,000 lines through an iron bar having a section of 80 sq. centims., then $B$ will be 12,500 , and reference to the table in Art. 364 shows that if the bar is of wrought iron $\mu$ will be about 1247. To meet the second difficulty we must estimate (from experience) an allowance for leakage. Suppose we find that of all the lines created in the $U$-shaped part only the fraction $1 / v$ gets through the armature, then to force $\mathbf{N}$ lines through the armature we must generate $\nu \mathrm{N}$ lines in the U-shaped piece, where $v$ is the coefficient of allowance for leakage, an improper fraction increasing with the width of the gaps.

We then proceed to calculate in parts as follows:-

$\left.\begin{array}{c}\text { Ampere-turns needed to drive } \mathrm{N} \text { lines } \\ \text { through iron of armature }\end{array}\right\}=\mathbf{N} \times \frac{l_{1}}{\mathrm{~A}_{1} \mu_{1}} \div 1.257$.

$\left.\begin{array}{c}\text { Ampere-turns needed to drive } \mathrm{N} \text { lines } \\ \text { through two gaps }\end{array}\right\}=\mathrm{N} \times \frac{2 l_{3}}{\mathrm{~A}_{3}} \div 1.257$.

$\left.\begin{array}{c}\text { Ampere-turns needed to drive } \nu \mathrm{N} \text { lines } \\ \text { through iron of magnet core }\end{array}\right\}=\nu \mathrm{N} \times \frac{l_{2}}{\mathrm{~A}_{2} \mu_{2}} \div 1 \cdot 25 \%$.

Then adding up, we get :-

Total ampere-turns needed $=\mathrm{N}\left\{\frac{l_{1}}{\mathrm{~A}_{1} \mu_{1}}+\frac{\nu l_{2}}{\mathrm{~A}_{2} \mu_{2}}+\frac{2 l_{3}}{\mathrm{~A}_{3}}\right\} \div 1 \cdot 257$.

Formulæ similar to this have been used by Hopkinson and by Kapp in designing electromagnets for dynamos.

378. Effect of Air-Gap in Circuit.-Air having 
no remanence the presence of a gap in the iron circuit tends to make residual magnetism unstable, as though the polar magnetism on the end-faces had a self-demagnetizing effect. In fact it is very difficult to give a permanent magnetism to short pieces of metal. Further, the low permeability of air necessitates enormous magnetomotive-forces, compared with those required for iron, to produce a given flux. The effect is to shear over to the right the curves of magnetization, seeing that a greater $\mathrm{H}$ is needed to attain an equal value of $\mathbf{B}$. Joints in the magnetic circuit have the same kind of effect.

The reason why the pull exerted by an electromagnet on its armature falls off so very greatly when the armature is moved away to a short distance is the diminution of the magnetic flux caused by the great reluctance of the air-gap thus introduced into the circuit.

379. General Law of Electromagnetic Systems.-Consider an electromagnetic system consisting of any number of parts-iron masses, coils carrying currents, air, masses of other materials, whether magnetic or diamagnetic - in any given configuration. Any change in the configuration of the parts will in general produce either an increase or a decrease in the magnetic flux. For example, if the armature of an electromagnet is allowed to move up toward the poles, or the needle of a galvanometer is allowed to turn, there will be a betterment of the magnetic circuit, and the magnetic flux through the coils will be increased. Magnetic circuits always tend to close up and become as compact as possible. On the contrary, if we pull away the armature from an electromagnet the magnetic reluctance is increased, and the flux diminished; and this action is resisted by the reaction of the system. All these things may be summed up in the following general law :-

Every electromagnetic system tends so to change the configuration of its parts as to make the maynetic flux a maximum. 
Suppose (the external magnetizing forces remaining the same) a motion of any part through a distance $d x$ results in a decrease of flux $d \mathbf{N}$, then the force resisting such motion will be proportional to $d \mathbf{N} / d x$.

380. Law of the Electromagnet.-Before the law of the magnetic circuit was understood many attempts were made to find algebraic fornulæ to express the relation between the strength of current and the amount of magnetism produced. Lenz and Jacobi suggested that the magnetism of an electromagnet was proportional to the current and to the number of turns of wire in the coil-in other words, is proportional to the ampere-turns. Or in symbols

$$
m=a \mathrm{CS},
$$

where $a$ is a constant depending on the quantity, quality, and form of iron. This rule is, however, only trine when the iron core is still far from being "saturated." If the iron is already strongly magnetized a current twice as strong will not donble the magnetization in the iron, as Joule showed in 1847.

Miiller gave the following approximate rule:-The strength of an electromagnet is proportional to the angle whose tangent is the strength of the magnetizing current; or

$$
m=\mathrm{A} \tan ^{-1} \mathrm{C} \text {, }
$$

where $\mathrm{C}$ is the magnetizing current, and $\mathrm{A}$ a constant depending on the construction of the particular magnet. If the student will look at Fig. 121 and imagine the divisions of the horizontal tangent line OT to represent strengths of current, and the number of degrees of arc intercepted by the oblique lines to represent strengths of magnetism, he will see that even if OT be made infinitely long, the intercepted angle can never exceed $90^{\circ}$.

Another formula, known as Frölich's, is-

$$
m=a \frac{\mathrm{C}}{1+b \mathrm{C}},
$$

where $a$ and $b$ are constants depending on the form, quality, and quantity of the iron, and on the winding of the coil. The constant $b$ is the reciprocal of that number of amperes which would make $m$ equal to half possible maximum of magnetism.

The author's variety of this formula expresses the number of magnetic lines $\mathrm{N}$ proceeding from the pole of the electromagnet-

$$
\mathrm{N}=\mathrm{Y} \frac{\mathrm{C}}{\mathrm{C}+\mathrm{C}^{\prime}},
$$


where $\mathrm{Y}$ represents the maximum number of magnetic lines that there would be if the magnetizing current were indefinitely increased and the iron core saturated, and $\mathrm{C}^{\prime}$ stands for that number of amperes which would bring the magnetism up to half-saturation.

None of these empirical formulæ are as useful as the rational formula at the end of Art. 377.

\section{LESsON XXXI._Electromagnets}

381. Electromagnets.-In 1820, almost immediately after Oersted's discovery of the action of the electric current on a magnet needle, Arago and Davy independently discovered how to magnetize iron and steel by inserting needles or strips into spiral coils of copper wire around

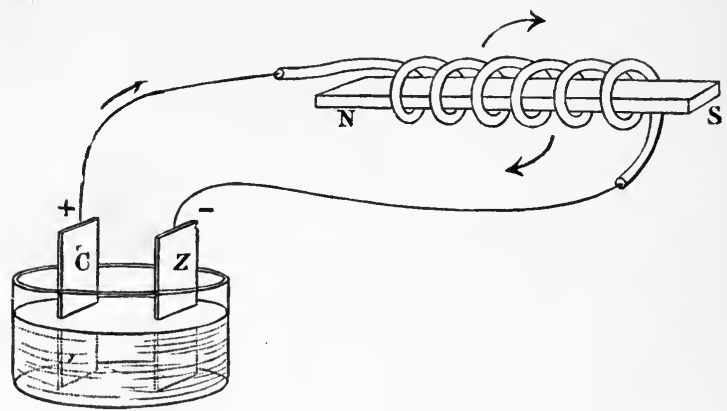

Fig. 187 .

which a current was circulating. The method is shown in the simple diagram of Fig. 187, where a current from a single cell is passed through a spiral coil of insulated copper wire, in the hollow of which is placed a strip of iron or steel, which is thereby magnetized. The separate turns of the coil must not touch one another or the central bar, otherwise the current will take the shortest road open to it and will not traverse the whole of the coils. To prevent such short-circuiting by contact the 
wire of the coil should be overspun with silk or cotton (in the latter case insulation is improved by varnishing it or by steeping the cotton covering in melted paraffin wax) or covered with a layer of guttapercha. If the bar be of iron it will be a magnet only so long as the current flows; and an iron bar thus surrounded with a coil of wire for the purpose of magnetizing it by an electric current is called an Electromagnet. Sturgeon, who gave this name, applied the discoveries of Davy and Arago to the construction of electromagnets far more powerful than any magnets previously made. His first electromagnet was a horse-shoe (Fig. 188) made of a rod of iron about 1 foot long and $\frac{1}{2}$ inch in diameter coiled with a single stout copper wire of only 18 turns. With the current from a single cell it lifted $9 \mathrm{lbs}$. ; but with a more powerful battery it lifted $50 \mathrm{lbs}$.

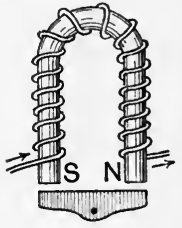

Fig. 188. It was first shown by Henry that when electromagnets are required to work at the distant end of a long line they must be wound with many turns of fine wire. The great usefulness of the electromagnet in its application to electric bells and telegraphic instruments lies in the fact that its magnetism is under the control of the current; when circuit is "made" it becomes a magnet, when circuit is "broken" it ceases to act as a magnet. Moreover, it is capable of being controlled from a distance, the current being "made" or "broken" at a distant point of the circuit by a suitable key or "switch."

382. Polarity and Circulation of Current.By applying Ampère's Rule (Art. 197) we can find which end of an electromagnet will be the $\mathrm{N}$-seeking pole ; for, imagining ourselves to be swimming in the current (Fig. 187), and to face towards the centre where the iron bar is, the $\mathrm{N}$-seeking pole will be on the left. It is convenient to remember this relation by the following rules: -Looking at the S-seeking pole of an electromagnet, the 
magnetizing currents are circulatiny round it in the same. cyclic direction as the hands of a clock move; and, looking at the $N$-seeking pole of an electromagnet, the maynetizing currents are circulating round it in the opposite cyclic
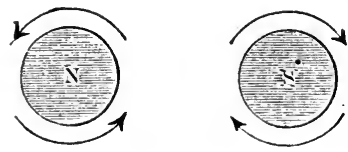

Fig. 189. direction to that of the hands of a clock. Fig. 189 shows this graphically. These rules are true, no matter whether the beginning of the coils is at the end near the observer, cr at the farther end from him, i.e. whether the spiral be a right-handed screw, or (as in Fig. 187) a left-handed screw. It will be just the same thing, so far as the magnetizing power is concerned, if the coils begin at one end and run to the other and back to where they began; or they may begin half-way along the bar and run to one end and then back to the other: the one important thing to know is which way the current flows round the bar when you look at it end-on. The corkscrew rule (Art. 198) leads to the same result.

Suppose an iron core to be wound with a right-handed coil, and that it exrent is introduced at some point, and to How both ways, it will produce

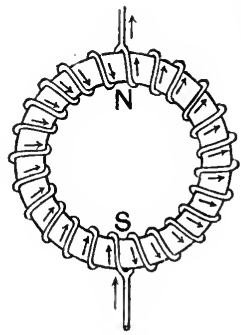

Fig. 190. oppositely-directed magnetizing actions in the two points, and there will be consequent poles (Art. 120) at the point of entrance. In Fig. 190 an iron ring with a righthandedly-wound closed coil is shown. There will be a double s-pole at the point where the current enters, and a clouble N-pole where it leaves the windings.

383. Construction of Electromagnets. The most useful form of electromannet is that in which the inon core is bent into the form of a horse-shoe, so that both poles nay be applied to one iron armature. In this 
case it is usual to divide the coils into two parts wound on bobbins, as in Figs. 64 and 191. The electromagnet depicted in Fig. 192 is of a form adapted for laboratory experiments, and has movable coils which are slipped on over the iron cores. The cores are united at the bottom by a stout iron yoke. Sometimes only one coil is wound on the yoke part. A special form of electromagnet devised by Ruhmkorff for experiments on diamagnetism is shown in Fig. 182.

Many special forms * of electromagnet have been devised for special purposes. To give a very powerful attraction at very short distances, a short cylindrical electromagnet surrounded by an outer iron tube, united at

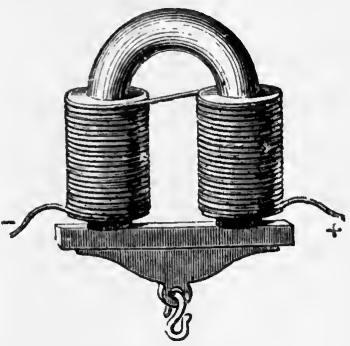

Fig. 191. the bottom by iron to the iron core, is found best; the iron jacket constituting a return path for the magnetic lines. This form is known as an iron-clad magnet. To attract iron across a wide gap which offers much reluctance, a horse-shoe shape with long cores should be chosen ; for it needs long cores to wind on enough wire to provide sufficient exciting power to drive the flux across the gap. To give a gentle pull over a long range a solenoid (Art. 385), or long tubular coil, having a long movalle iron core is used. For giving a very quick-acting magnet the coils should not be wound all along the iron, but only round the poles. As a rule the iron parts, including the-yoke and armature, should form as nearly as possible a closed magnetic circuit. The cross-sections of yokes should be thicker than those of the cores.

* For descriptions of these, as well as for cliscussion of all other matters relating to the subject, see the author's treatise on The Electromagnet and Electromagnetic Mechanism. 
384. Lifting-power of Electromagnets.-The tractive force of an electromagnet depends not only on its magnetic strength, but also upon its form, and on the

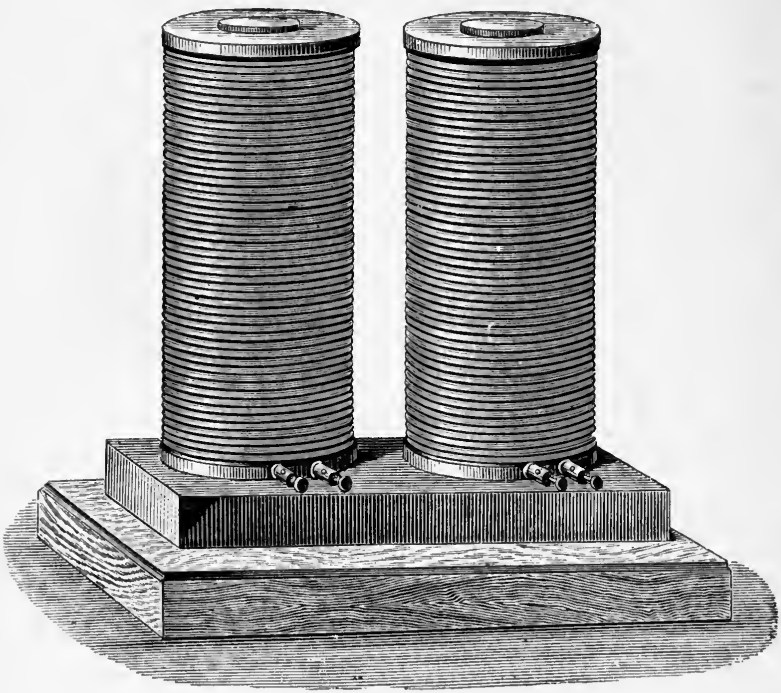

Fig. 192.

shape of its poles, and on the form of the soft iron armature which it attracts. It should be so arranged that as many lines of force as possible should run through the armature, and the armature itself should contain a sufficient mass of iron. Joule designed a powerful electromagnet, capable of supporting over a ton. The maximum attraction he could produce between an electromagnet and its armature was 200 lbs. per square inch, or about 13,800,000 dynes per square centimetre. Bidwell has found the attraction to go up to $226.3 \mathrm{lbs}$. per square 
inch when the wrought-iron core was saturated up to 19,820 magnetic lines to the square centimetre. The law of traction is that the pull per square centimetre is proportional to the square of the number of lines per square centimetre : or in symbols

$$
\mathrm{P}=\frac{\mathrm{B}^{2} \mathrm{~A}}{8 \pi},
$$

where $\mathrm{P}$ is the pull in dynes, and $\mathrm{A}$ the area in square centims. In the following table are given the values of the tractive force for different stages of magnetization.

\begin{tabular}{|r|r|c|c|}
\hline $\begin{array}{c}\text { B } \\
\text { lines per } \\
\text { sq. cm. }\end{array}$ & $\begin{array}{c}\text { Dynes } \\
\text { per } \\
\text { sq. centim. }\end{array}$ & $\begin{array}{c}\text { Grammes } \\
\text { per sq. } \\
\text { centim. }\end{array}$ & $\begin{array}{c}\text { Pounds } \\
\text { per } \\
\text { sq. inch. }\end{array}$ \\
\cline { 2 - 3 } 1,000 & 39,790 & $40 \cdot 56$ & $\cdot 577$ \\
2,000 & 159,200 & $162 \cdot 3$ & $2 \cdot 308$ \\
3,000 & 358,100 & $365 \cdot 1$ & $5 \cdot 190$ \\
4,000 & 636,600 & $648 \cdot 9$ & $9 \cdot 228$ \\
5,000 & 994,700 & 1,014 & $14 \cdot 39$ \\
6,000 & $1,432,000$ & 1,460 & $20 \cdot 75$ \\
7,000 & $1,950,000$ & 1,987 & $28 \cdot 26$ \\
8,000 & $2,547,000$ & 2,596 & $36 \cdot 95$ \\
9,000 & $3,223,000$ & 3,286 & $46 \cdot 72$ \\
10,000 & $3,979,000$ & 4,056 & $57 \cdot 68$ \\
12,000 & $5,730,000$ & 5,841 & $83 \cdot 07$ \\
14,000 & $7,800,000$ & 7,950 & $113 \cdot 1$ \\
16,000 & $10,170,000$ & 10,390 & $147 \cdot 7$ \\
18,000 & $12,890,000$ & 13,140 & $186 \cdot 8$ \\
20,000 & $15,920,000$ & 16,230 & $230 \cdot 3$ \\
\hline
\end{tabular}

It will be noted that doubling B makes the pull four times as great. One curious consequence of this law is that to enlarge its poles weakens the pull of an electromagnet or magnet. In some cases - bar magnets for example-their tractive power is increased by filing down or rounding the poles so as to concentrate $\mathbf{B}$. 
385. Solenoid.-Without any central core of iron or steel a spiral coil of wire traversed by a current acts as an electromagnet (though not so powerfully as when an iron core is placed in it). Such a coil is sometimes termed a solenoid. A solenoid has two poles and a neutral equatorial region. Ampère found that it will attract magnets and be attracted by magnets. It will attract another solenoid; it has a magnetic field resembling

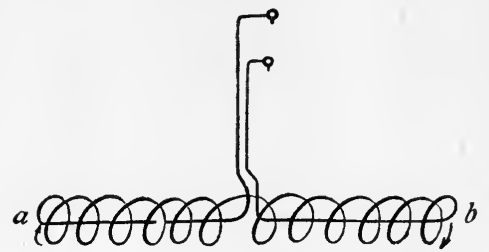

Fig. 193. generally that of a bar magnet. If so arranged that it can turn round a vertical axis, it will set itself in a North and South direction along the magnetic meridian. Fig. 193 shows a solenoid arranged with pivots, by which it can be suspended to a "table," like that shown in Fig. 198.

With an iron core the solenoid becomes far more powerful. The effect of the iron core is by its greater permeability to multiply the number of magnetic lines as well as to concentrate them at definite poles. The student has been told (Art. 202) that the lines of force due to a current flowing in a wire are closed curves, approximately circles (Figs. 115 and 195), round the wire. If there were no iron core many of these little circular lines of force would simply remain as small closed curves around their own wire ; but, since iron has a permeability hundreds of times greater than air, wherever the wire passes near an iron core the magnetic lines alter their shape, and instead of being little circles around the separate wires, run through the iron core from end to end, and round outside from one end of the coil back to the other. A few of the magnetic lines do this when there is no iron ; almost all of them do this when there is iron, and when 
there is iron there are more lines to flow back. ${ }^{*}$ Hence the electromagnet with its iron core has enormously stronger poles than the spiral coils of the circuit would have alone.

In Art. 342 it was shown that the intensity of the magnetic field down the middle of a solenoid of length.l, having $\mathrm{S}$ spirals, carrying $\mathrm{C}$ amperes, is-

$$
\mathrm{H}=\frac{4 \pi}{10} \times \frac{\mathrm{CS}}{l} .
$$

Since the area enclosed is $\pi r^{2}$, the flux down the solenoid (without iron) will be

$$
\mathbf{N}=\frac{4 \pi^{2} r^{2}}{10 l} \times \text { CS. }
$$

And, since $4 \pi$ magnetic lines go to one unit of magnetism, the solenoid (without iron) will act as though it had as the magnetism at its pole-

$$
m=\frac{\pi r^{2}}{10 l} \mathrm{CS} .
$$

It will be noticed that for any solenoid of given length and radius the three magnetic quantities $\mathbf{H}$ (internal field), $\mathbf{N}$ (magnetic flux), and $m$ (strength of poles) are proportional to the amperes of current and to the number of turns in the coil. The product which thus comes into all electromagnet formulæ is called the number of ampereturns.

A solenoirl with a movable iron plunger is sometimes called a sucking-maynet. The iron core tends to move into the position in which it best completes (Art. 379) the magnetic circuit. If the core is much longer than the coil, the pull increases as the end of the core penetrates

* But, in the case of a permanent steel horseshoe magnet, bringing up the iron keeper, though it concentrates the lines through the poles, does not increase the total number of lines through the bend of the $\mathrm{U}$. 
down the coil, diminishing quickly as the core emerges. Short iron cores are only pulled while at the mouth of the coil ; the maximum pull being when about half their length has entered.

386. The Winding of Electromagnets.-The exact laws governing the winding of electromagnets are somewhat complicated; but it is easy to give certain rules which are approximately true. Evepy electromagnet shows the same general set of facts that with small exciting power there is little magnetism produced, with larger exciting power there is more magnetism, and that with very great exciting power the iron becomes practically saturated and will take up very little additional magnetism. It follows at once that if the electromagnet is destined to be used at the end of a long line through which only a small current (perhaps only $\frac{1}{100}$ ampere) will flow, the requisite number of ampere-turns to excite the magnetism will not be attained unless many turns of wire are used and as the current is small a fine wire may be used

It may be noted that when electromagnets are wound with many turns of fine wire, these coils will add to the electric resistance of the circuit, and will tend to diminish the current. Herein lies a difference in construction of telegraphic and other instruments; for while electromagnets with "long coils," consisting of many turns of fine wire, must be used on long circuits where there is great line resistance, such an instrument would be of no service in a laboratory circuit of very small resistance, for the resistance of a long thin coil would be disproportionately great: here a short coil of few turns of stout wire would be appropriate (see Art. 192).

It is the nature of the line, according to whether it is of high resistance or low, which governs the questions how the coil shall be wound and how the battery shall be grouped.

Similar electromagnets of different sizes must have 
ampere-turns proportional to their linear dimensions if they are to be raised to equal degree of saturation.

As the magnetism of the magnet depends on the number of ampere-turns, it should make no matter whether the coils are bigger than the core or whether they enwrap it quite closely. If there were no magnetic leakage this would be true in one sense; but for an equal number of turns large coils cost more and offer higher resistance. Hence the coils are wound as closely to the iron core as is consistent with good insulation. Also the iron is chosen as thick as possible, as permeable as possible, and forming as compact a magnetic circuit as possible, so that the magnetic resistance may be reduced to its utmost, giving the greatest amount of magnetism for the number of ampere-turns of excitation. This is why horse-shoeshaped electromagnets are more powerful than straight electromagnets of equal weight ; and why also a horseshoe electromagnet will only lift about a quarter as much load if one pole only is used instead of both.

As the coils of electromagnets grow hot with the current, sufficient cooling surface must be allowed, or they may char their insulation. Each square centimetre of surface warmed $1^{\circ} \mathrm{C}$. above the surrounding air can get rid of about 0.0029 watt. If $50^{\circ}$ above the surrounding air be taken as the safe limit of rise of temperature, and the electromagnet has resistance $r$ and surface $s$ sq. cms., the highest permissible current will be $0.38 \sqrt{s / r}$ amperes.

387. Polarized Mechanism.-An electromagnet moves its armature one way, no matter which way the current flows. Reversing the current makes no difference. There are, however, two ways of making a mechanism that will cause an armature to move in either sense at will. (a) The armature's movement is controlled by an adjusted spring so as to be in an intermediate position when a weak current is flowing. Then sending a stronger current will move the armature one way, and weakening 
or stopping the current will make it move the other way. (b) A polarized armature or tongue (i.e. one that is independently magnetized) is placed between the poles of the electromagnet instead of opposite them. The direction in which it tends to move will be reversed by reversing the current in the circuit of the electromagnet.

388. Growth of Magnetism.-It requires time to magnetize an iron core. This is mainly due to the fact that a current, when first switched on, does not instantly attain its full strength, being retarded by the self-induced counter-electromotive-foree (Art. 458); it is partly due to the presence of transient reverse eddy-currents (Art. 457) induced in the iron itself. Faraday's large electromagnet at the Royal Institution takes about two seconds to attain its maximum strength. The electromagnets of large dynamo machines often take ten minutes or more to rise to their working stage of magnetization.

When electromagnets are used with rapidly-alternating currents (Art. 470) there are various different phenomena, for which the student is referred to Art. 47\%.

\section{ILESSON XXXII.-Electrodynamics}

389. Electrodynamics.-In 1821 , almost immediately after Oersted's discovery of the action of a current on a magnet, Ampère discovered that a current acts upon another current, apparently attracting it * or repelling it according to certain definite laws. These actions he inrestigated by experiment, and from the experiments he built up a theory of the force exerted by one current on another. That part of the science which is concerned with the force which one current exerts upon another he termed Electrodynamics. It is now known that these

\footnotetext{
* It would be more correct to speak of the force as acting on conductors carrying currents, than as acting on the currents themselves.
} 
actions are purely magnetic, and are due to stresses in the intervening medium. The magnetic field around a single conductor consists of a magnetic whirl (Art. 202), and any other conductor carrying a current when brought into the field of the first is acted upon by it. Fig. 194 shows the field due to two parallel straight current con-

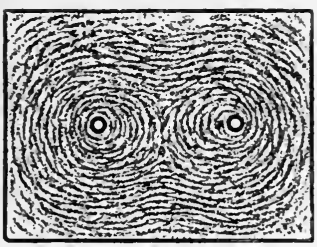

Fig. 194.

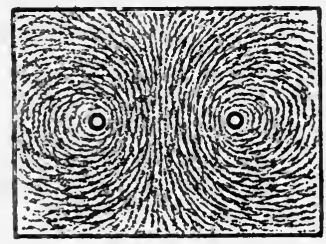

Fig. 195.

ductors, which were passed through holes in a sheet of glass on which iron filings were sprinkled. In Fig. 194 the currents flow in the same direction; in Fig. 195 in opposite directions. In the first case the stresses in the field (Art. 119) tend to pull them together, in the second to push them apart.*

390. Laws of Parallel and Oblique Circuits.The following are the laws discovered by Ampère:-

(i.) Two parallel portions of a circuit attract one another if the currents in them are flowing in the same direction, and repel one another if the currents flow in opposite directions.

This law is true whether the parallel wires be parts of two different circuits or parts of the same circuit. The separate turns of a spiral coil, like Fig. 193, when traversed by a current attract one another; such a coil, therefore, shortens when a current is sent through it. But this is equally well explained

* See article by the author in the Philosophical Magazine, November 1878, p. 348. 
by the general law of electromagnetic systems (Art. 379), because shortening will reduce the reluctance of the magnetic circuit and increase the flux.

(ii.) Two portions of circuits crossing one another obliquely attract one another if both the currents run either towards or from the point of crossing, and repel one another if one runs to and the other from that point.

Fig. 196 gives three cases of attraction and two of repulsion that occur in these laws.

(iii.) When an element of a circuit exerts a force on another element of a circuit, that force always tends to urge
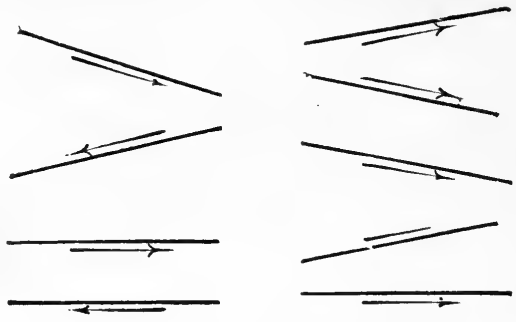

Fig. 196.

the latter in a direction at right angles to its own direction. Thus, in the case of two parallel circuits, the force of attraction or repulsion acts at right angles to the currents themselves.

An example of laws ii. and iii. is afforded by the case shown in Fig. 197. Here two currents $a b$ and $c d$ are movable round $\mathrm{O}$ as a centre. There will be an apparent repulsion between $a$ and $d$ and between $c$ and $b$, while in the other quadrants there will be an apparent attraction, $a$ attracting $c$, and $b$ attracting $d$. 
The foregoing laws may be summed up in one, by saying that two portions of circuits, however situated, set up stresses in the surrounding medium tending to set them so that their currents flow as nearly in the same path as possible.

(iv.) The force exerted between

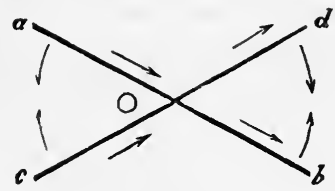

Fig. 197 . two parallel portions of circuits is proportional to the product of the strengths of the two currents, to the length of the portions, and inversely proportional to the simple distance between them.

391. Ampère's Table.-In order to observe these attractions and repulsions, Ampère devised the piece of

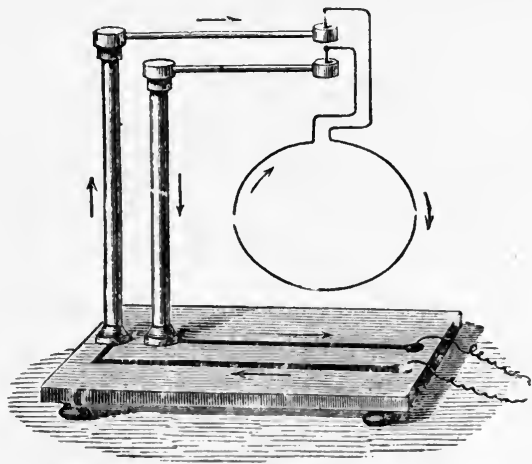

Fig. 198.

apparatus known as Ampère's Table, shown in Fig. 198, consisting of a double supporting stand, upon which wires, shaped in different ways, can be so hung as to be capable of rotation. The ends of the suspended wires 
dip into two mercury cups, so as to ensure good contact, while allowing freedom to move.

By the aid of this piece of apparatus Ampère further demonstrated the following points :-

(a) A circuit doubled back upon itself, so that the current flows back along a path close to itself, exerts no force upon external points.

(b) A circuit bent into zigzags or sinuosities produces the same magnetic effects on a neighbouring piece of circuit as if it were straight.

(c) There is in no case any force tending to move a conductor in the direction of its own length.

(d) The force between two conductors of any form is the same, whatever the linear size of the system, provided the distances be increased in the same proportion, and that the currents remain the same in strength.

The particular case, given in Fig. 199, will show the value of these experiments. Let $\mathrm{AB}$ and $\mathrm{CD}$ represent two wires carrying currents, lying neither parallel nor in the same plane. It follows from $(b)$ that if we replace the portion $\mathrm{PQ}$ by the crooked wire

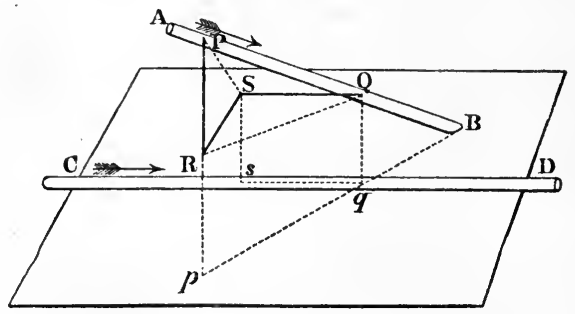

Fig. 199.

PRSQ, the force will remain the same. The portion PR is drawn vertically downwards, and as it can, by $(c)$, experience no force in the direction of its length, this portion will neither be attracted nor repelled by $C D$. In the portion RS the current runs at right angles to CD, and this portion is neither attracted nor repelled by $\mathrm{CD}$. In the portion $\mathrm{SQ}$ the current runs parallel to $\mathrm{CD}$, and in the same direction, and will therefore be attracted downwards. On the whole, therefore, $\mathrm{PQ}$ will be urged towards $\mathrm{CD}$. The 
portions PR ard RS will experience forces of rotation, however, $\mathrm{P}$ being urged round $R$ as a centre towards $C$, and $R$ being urged horizontally round $\mathrm{S}$ towards $\mathrm{C}$. These actions would tend to make $A B$ parallel with $C D$.

392. Ampère's Theory.-From the four preceding experimental data, Ampère built up an elaborate mathematical theory, assuming that, in the case of these forces acting apparently at a distance across empty space, the action took place in straight lines between two points, the total attraction being calculated as the sum of the separate attractions on all the different parts.

The briefest summary must suffice. If we deal first with two parallel elements of length $d l_{1}$ and $d l_{2}$ carrying currents $\mathrm{C}_{1} \mathrm{C}_{2}$, and set at right angles to the distance $r$ joining them, their mutual force will be

$$
d f=-\mathrm{C}_{1} \mathrm{C}_{2} d l_{1} d l_{2} / 100 r^{2} .
$$

If, however, they are not parallel or in one plane, let $\phi$ be the angle they make with one another, while $\theta_{1}$ and $\theta_{2}$ are the angles they make with $r$; when

$$
d f=-\mathrm{C}_{1} \mathrm{C}_{2} d l_{1} d l_{2}\left(\cos \phi-\frac{3}{2} \cos \theta_{1} \cdot \cos \theta_{2}\right) / 100 r^{2} .
$$

By integrating this expression one obtains the forces for circuits of any given dimensions. For example, for two parallel straight conductors of lengths $l_{1} l_{2}$, if these lengths are great compared with the distance $r$ between them, we have

$$
f=-2 \mathrm{C}_{1} \mathrm{C}_{2} l_{1} l_{2} / 100 r .
$$

The researches of Faraday have, however, led to other views; the mutual attractions and repulsions being regarded as due to actions taking place in the medium which fills the space around and between the conductors. All these so-called electrodynamic actions are merely magnetic actions.

An interesting experiment, showing an apparent mutual self-repulsion between contiguous portions of the circuit, was devised by Ampere. A trough divided by a partition into two parts, and made of non-conducting materials, is filled with mercury. Upon it floats a 
metallic bridge formed of a bent wire, of the form shown in Fig. 200, or consisting of a glass tube filled siphonwise with mercury. When a current is sent through the

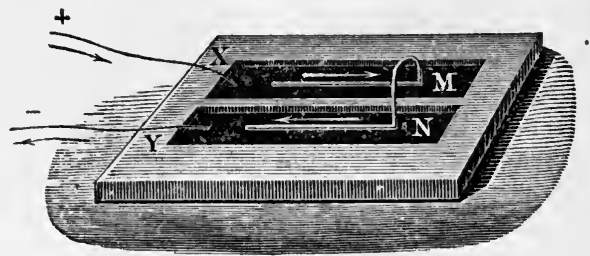

Fig. 200.

floating conductor from $\mathrm{X}$ over $\mathrm{MN}$, and out at $\mathrm{Y}$, the floating bridge is observed to move so as to increase the area enclosed by the circuit. But the force would be

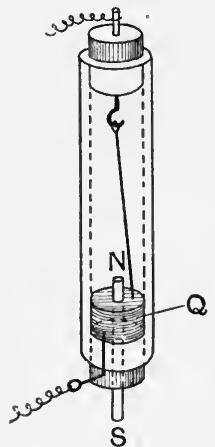

Fig. 201. diminished indefinitely if the two parallel parts could be made to lie quite close to one another.

393. Electromagnetic Rotations.-Continuous rotation can be produced between a magnet and a circuit, or letween two parts of one circuit, provided that one part of the circuit can move while another part remains fixed, or that the current in one part can be reversed. The latter device is adopted in the construction of electric motors (Art. 443). The former alternative is applied in some historic apparatus for showing rotations, a sliding-contact being made between one part of the circuit and another. Several different forms of rotation-apparatus were devised by Faraday and by Ampère. One of Faraday's is shown in Fig. 201, in which a wire carrying a current is jointerl at the top and dips into a cup of 
mercury surrounding the pole of a magnet. On switching on the current the wire at once begins to walk round the pole with a motion that continues until the current is switched off.

A pole of a magnet can also be made to rotate round a current; and if a vertical magnet be pivoted so as to turn around its own axis it will rotate when a current is led into its middle region and out at either end. If the current is led in at one end and out at the other there will be no rotation, since the two poles would thus be urged to rotate in opposite ways. Liquid conductors too can exhibit electromagnetic rotations. Let a cylindrical metallic vessel connected to one pole of a battery be filled with mercury or dilute acid, and let a wire from the other pole dip into its middle, so that a current may flow radially from the centre to the circumference, or vice versa; then, if this be placed upon the pole of a powerful magnet, or if a magnet be held vertically over it, the liquid may be seen to rotate.

394. Electrodynamometer. - Weber devised an instrument known as an electrodynamometer for measuring the strength of currents by means of the electrodynamic action of one part of the circuit upon another part. It is a sort of galvanometer, in which, instead of a needle, there is a small coil suspended. One form of this instrument, in which both the large outer and small inner coils consist of two parallel coils of many turns, is shown in Fig. 202. The inner coil CD is suspended with its axis at right angles to that of the outer coils $\mathrm{AA}, \mathrm{BB}$, and is supported bifilarly (see Art. 130) by two fine metal wires. If one current flows round both coils in either direction the inner bobbin tends to turn and set its coils parallel to the outer coils; the sine of the angle through which the suspending wires are twisted being proportional to the square of the strength of the current.

If $\mathrm{G}$ be the "principal constant" (Art. 213) of the large coils, and $g$ the "moment" of the small coils (Art. 346) when carrying 
unit current, and $\mathrm{C}_{2} \mathrm{C}_{2}$ the currents in them, the torque (or turning moment) will be

$$
=\mathrm{GgC}_{2} \mathrm{C}_{2} / 100 \text {. }
$$

The chief advantage of this instrument over a galvanometer is, that it may be used for alternating currents; a

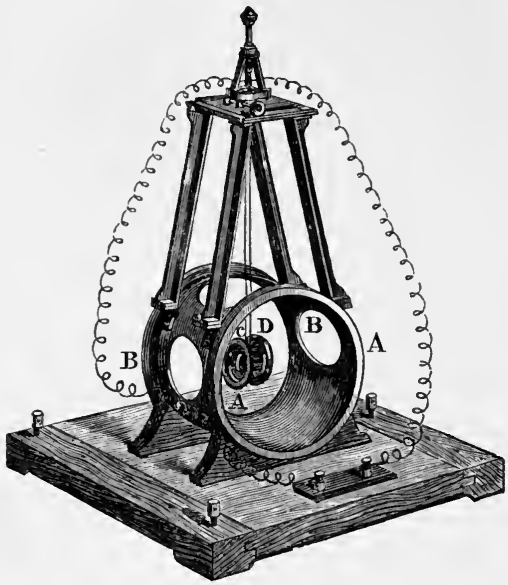

Fig. 202.

current in one direction being followed by a reverse current, perhaps thousands of times in a minute. Such currents hardly affect a galvanometer needle at all; the needle simply quivers in its place without turning.

\section{Siemens's Electrodynamometer.}

- In Siemens's dynamometer (Fig. 203), much used for measurement of strong currents, whether of the continuous or the alternating kind, one coil is fixed permanently, whilst the otlier coil, of one or two turns, dipping with its ends in mercury cups, is hung at right angles, and controlled by a spiral spring below a torsionhead. When current passes the movable coil tends to 
turn parallel to the fixed coils, but is prevented; the torsion index being turned until the twist on the spring balances the torque. The angle through which the index has had to be turned is proportional to the product of $\mathrm{C}_{1} \mathrm{C}_{2}$, the currents in the fixed and movable coils.

For use of dynamometer as wattmeter, see Art. 438.

396. Kelvin's Current Balances. - Joule, Mascart, Lord Rayleigh, and others have measured currents by balances in which gravity was opposed to the attraction or repulsion of two coils. Of such balances the most perfect are those of Lord Kelvin,

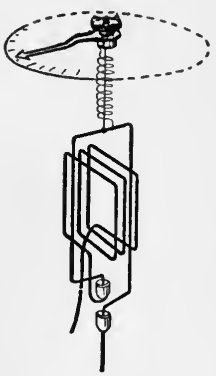

Fig. 203. the principle of which is outlined in Fig. 204. There are four fixed coils, ABCD, between which is suspended, by a flexible metal ligament of fine wires, at the ends of a

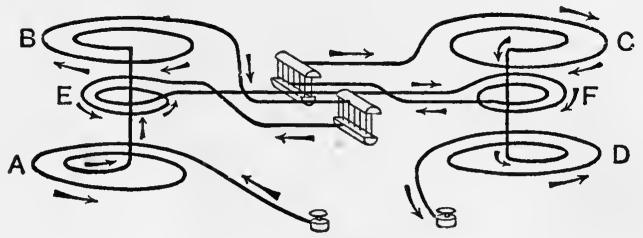

Fig. 204.

light beam, a pair of movable coils, $\mathrm{E}$ and $\mathrm{F}$. The current flows in such directions through the whole six that the beam tends to rise at $\mathrm{F}$ and sink at $\mathrm{E}$. The beam carries a small pan at the $\mathrm{F}$ end, and a light arm, not shown in Fig. 204, but shown in Fig. 205, along which, as on a steel-yard, a sliding weight can be moved to balance the torque due to the current. The current is proportional to the square-root of this torque, since the 


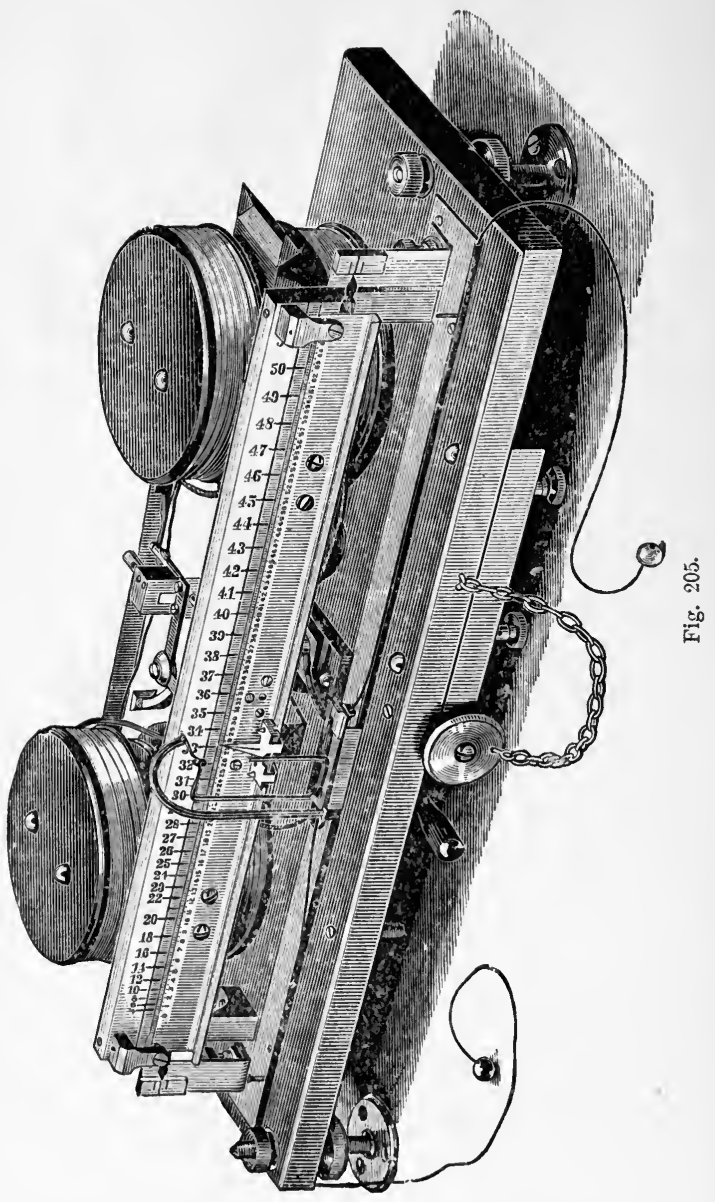


force is proportional to the product of the current in the fixed and movable coils as in all electrodynamometers.

Lord Kelvin has designed a whole range ${ }^{*}$ of these instruments : -a centi-ampere balance reading from 0.01 to 1 ampere; a deciampere balance reading from 0.1 to 10 ; a deka-ampere balance reading from 1 to 100 ; a hekto-ampere balance reading from 6 to 600 ; and a kilo-ampere balance reading up to 2500 amperes. The centi-ampere balance is shown in Fig. 205, in which the sliding weight is carried on the base of the pointer (shown white), and when at the zero of the scale just balances the weight in the V. shaped pan. Any current passing through the coils causes the beam to tilt and the pointer is moved (by means of a.self-releasing slider attached to cords) until it is again horizontal (as shown by the black pointer at either end). With a certain pair of weights the fixed scale gives the current in decimal parts of an ampere ; but by the use of other weights a wider range is obtained.

The "ampere-standard" instrument, and the "voltstandard" instruments of the Board of Trade, kept at Whitehall as legal standards for Great Britain (see Appendix B), are current balances of special construction, designed by Major Cardew.

397. Electromagnetic Actions of Convexion Currents.-According to Faraday a stream of particles charged with electricity acts magnetically like a true conduction current. ' This was first proved in 1876 by Rowland, who found a charged disk rotated rapidly to act upon a magnet as a feeble circular current would do. Convexion currents, consisting of streams of electrified particles, are also acted upon by magnets. The convective discharges in vacuum-tubes (Art. 320) can be drawn aside by a magnet, or caused to rotate around a magnet-pole. The brush discharge (Art. 319) when taking place in a strong magnetic field is twisted. The electric arc (Art. 448) also behaves like a flexible conductor, and can be attracted or repelled laterally by a nuagnet. Two stationary positively-electrified particles repel one another, but two

* For a fuller account of these Current Balances, and of the Wattmeters on the same principle, see Gray's Absolute Measurements in Electricity and Magnetism, from which Fig. 205 is taken. 
parallel currents attract one another (Art. 390), and if electrified particles flowing along act like currents, there should be an (electromagnetic) attraction between two electrified particles moving along side by side through space. According to Maxwell's theory (Art. 518) the electrostatic repulsion will be just equal to the electromagnetic attraction when the particles move with a velocity equal to the velocity of light.

Hall discovered in 1879 that when a powerful magnet is made to act upon a current flowing along in a strip of very thin metal, the equipotential lines are no longer at right angles to the lines of flow of the current in the strip. This action appears to be connected with the magnetic rotation of polarized light (Art. 526), the coefficient of this transverse thrust of the magnetic field on the current being feebly + in gold, strongly + in bismuth, and - in iron, and immensely strong negatively in tellurium. It was shown by the author, and about the same time by Righi, that those metals which manifest the Hall effect undergo a change in their electric resistance when placed in the magnetic field. The resistance of bismuth increases so greatly that it affords a way of measuring the strength of magnetic fields.

398. Ampère's Theory of Magnetism.-Ampère, finding that solenoids (such as Fig. 193) act precisely as magnets, conceived that all magnets are simply collections of currents, or that around every individual molecule of a magnet an electric current is ceaselessly circulating. We know that such currents could not flow perpetually if there were any resistance to them, and we know that there is resistance when electricity flows from one molecule to another. As we know nothing about the interior of molecules themselves, we cannot assert that Ampère's supposition is impossible. Since a whirlpool of electricity acts like a magnet, there seems indeed reason to think that magnets may be merely made up of rotating portions of electrified matter. 


\section{CHAPTER VI}

MEASUREMENT OF CURRENTS, ETC.

Lesson XXXIII._Ohm's Law and its Consequences

399. Law of Dr. Ohm.-In Art. 191 the law discovered by Dr. G. S. Ohm was stated in the following terms :-The strength of the current varies directly as the electromotive-force, and inversely as the resistance of the circuit.

Using the units adopted by practical electricians, and explained in Art. 354, we may now restate Ohm's law in the following definite manner:-The number of amperes of current flowing through a circuit is equal to the number of volts of electromotive-force divided by the number of ohms of resistance. Or,

$$
\begin{aligned}
\text { amperes } & =\text { volts } \div \text { ohms, } \\
\mathrm{C} & =\mathrm{E} / \mathrm{R} .
\end{aligned}
$$

The above is the simplest way of stating the law, but in its application it is not quite so simple. If we apply it to a whole circuit we must consider both the total $\mathbb{E}$ and the total R. For if a number of cells are used and the circuit be inade up of a number of different parts through all of which the current must flow, we have to take into account not only the electromotive-forces of the cells, but their resistances, as well as the resistances of other parts of the 
circuit. For example, the current may flow from the zinc plate of the first cell through the liquid to the carbon plate, then through a connecting wire or screw to the next cell, through its liquid, through the connecting screws and liquids of the rest of the cells, then through a wire to a galvanometer, then through the coils of the galvanometer, then perhaps through an electrolytic cell, and finally through a return wire to the zinc pole of the battery. In this case there are a number of separate electromotive-forces all tending to produce a flow, and a number of different resistances, each obstructing the flow and adding to the total resistance. If in such a case we knew the separate values of all the different electromotiveforces and all the different resistances that are in series we could ealculate what the current would be, for it would have the value-

$$
\begin{aligned}
& \mathrm{C}=\frac{e^{\prime}+e^{\prime \prime}+e^{\prime \prime \prime}+e^{\mathrm{iv}}+\ldots \ldots}{r^{\prime}+r^{\prime \prime}+r^{\prime \prime \prime}+r^{\mathrm{iv}}+\ldots \ldots} \\
& \mathrm{C}=\frac{\text { Total electromotive-force }}{\text { Total resistance }}
\end{aligned}
$$

Example.-Let there be 5 cells in series each having $e=1 \cdot 4$ voits, and each an internal $r=0.4 \mathrm{ohm}$; and let the external part of circuit have resistance 3 ohms. Total $\mathrm{E}=7$ volts ; total $\mathrm{R}=5$ ohms. Current $\mathrm{C}$ will be $1 \frac{2}{\mathrm{~s}}$ aimperes.

If any one of the cells were set wrong way round its electromotive-force would oppose that of the other cells; an opposing electromotive-force must therefore be subtracted, or reckoned as negative in the algebraic sum. The "polarization" (Arts. 175 and 487) which occurs in battery cells and in electrolytic cells after working for some time is an opposing electromotive-force, and diminishes the total of the electromotive-forces in the circuit. So, also, the induced back E.M.F. which is set up when a current from a battery drives an electric notor (Art. 444) reduces the strength of the working 
current; in such case, if $\mathrm{E}$ is the electromotive-force of the battery, $e$ the opposing electromotive-force, and $R$ the total resistance, we shall have

$$
\mathrm{C}=\frac{\mathrm{E}-e}{\mathrm{R}} .
$$

Example.-Suppose the battery to generate current at 25 volts, and the motor to generate a back electromotive-force of 20 volts, and the total resistance to be $2 \frac{1}{2} \mathrm{ohms}$, there will be a current of 2 amperes.

But we may apply Ohm's law to a part of a circuit. If $e$ represents the difference of potential between two ends of a conductor of resistance $r$, the current $\mathrm{C}$ in it must be $=e / r$. Or, to put it the other way round, the electromotive-force needed to drive $\mathrm{C}$ amperes through a resistance of $r$ ohms will be $e=r \mathrm{C}$ volts.

Consider the case of a circuit of which the resistance is made up of two parts, an external resistance $R$ consisting of wires, lamps, etc., and of a smaller resistance $r$ internal to the battery or dynamo (viz. the resistance of the liquids in the cells, or of the wire of the armature). Then if $\mathrm{E}$ is the whole electromotive-force we shall have as current

$$
\mathrm{C}=\frac{\mathrm{E}}{\mathrm{R}+r},
$$

or

$$
\mathrm{C}(\mathrm{R}+r)=\mathrm{E} \text {; }
$$

or again

$$
\mathrm{CR}+\mathrm{Cr}=\mathrm{E} \text {. }
$$

This means in words that the total volts may be considered as being employed partly in driving the current through the external resistance $R$, partly in driving the current through the internal resistance $r$. This latter part of the electromotive-force is called the lost volts; the remainder being the useful or externally available volts, that would be measurable by a voltmeter (Art. 220) set across the terminals. If we call the available volts $\mathrm{V}$ we mav write $\mathrm{V}=\mathrm{CR}$, whence 


$$
\mathrm{V}=\mathrm{E}-\mathrm{C} r
$$

or in words : the volts as measured at the terminals of a cell or dynamo are less than the whole E.M.F. generated therein; being equal to the whole E.M.F. less the lost volts. The lost volts being proportional to internal resistance it is obviously best to keep all internal resistances as low as possible. Only when the cell is giving no current are the external volts $\mathrm{V}$ equal to the whole E.M.F. ; for when $\mathrm{C}=0, \mathrm{Cr}$ is also $=0$.

Example.-A dynamo is designed to generate its currents with an electromotive-force of 105 volts. The internal resistance of its armature is $\frac{1}{30} \mathrm{ohm}$. When it is giving out current of 120 amperes, the lost volts will be $120 \times \frac{1}{30}$ $=4$ volts. Consequently the volts available in the external circuit will be only 101 .

Since $\mathrm{C}=\frac{\mathrm{E}}{\mathrm{R}+r}=\frac{\mathrm{V}}{\mathrm{R}}$ it follows also that $\mathrm{V}=\mathrm{E} \frac{\mathrm{R}}{\mathrm{R}+r}$.

400. Resistance.-Resistance is the name given to that property of materials by virtue of which they obstruct the steady flow of electricity through them, and fritter down into heat the energy of the current. It is found that the resistance of a metal wire, if kept at an unvarying temperature, is the same whether a large current or a small current be flowing through it. For example, if a wire has a resistance such that when a difference of potential of 10 volts is applied to its ends a current of 2 amperes flows through it (its resistance being $5 \mathrm{ohms}$ ), it will be found that if 1 volt is applied the current will be 0.2 amperes, the ratio between volts and amperes being 5 as before.

The unit of resistance, or ohm, is a standard chosen in order that the resistances of other conductors may be expressed in definite numbers. The definition of it is given in Art. 354. It is convenient to remember that 100 yards of ordinary iron telegraph-wire has roughly a resistance about $1 \mathrm{ohm}$. 
Resistances in a circuit may be of two kinds-first, the resistances of the conductors (metals, alloys, liquids) themselves; second, the resistances due to imperfect contact at points. The latter kind of resistance is affected by pressure, for when the surfaces of two conductors are brought into more intimate contact with one another, the current passes more freely from one conductor to the other. The contact-resistance of two copper conductors may vary from infinity down to a small fraction of an ohm, according to the pressure. The variation of resistance at a point of imperfect contact is utilized in telephone transmitters (Art. 512). The conduction of powdered metals is remarkable. A loose heap of filings scarcely conducts at all, owing to the want of cohesion, or to the existence of films of air or dust. But it becomes instantly a good conductor if an electric spark is allowed to occur anywhere within a few yards of it (see Art. 521). The resisting films of air are broken down by minute internal discharges in the mass. A very slight agitation by tapping at once makes the powder non-conductive.

For the purpose of regulating the flow of currents, and for electrical measurements (Art. 411), variable resistances are employed. Resistance coils (Art. 414) are sets of coils made each of a definite value in ohms, of which one or more can be inserted in the circuit at will. Rheostats consist of easily-adjustable resistances, the length of wire in circuit

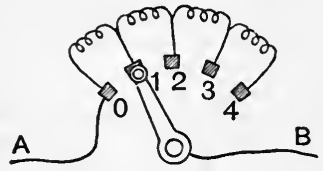

Fig. 206. being varied by turning a handle. In some cases the rheostat wire is wound off and on to a roller. In others a handle (Fig. 206) moving over a number of metal studs varies the amount of resistance-wire through which the current must flow. Carbon rheostats consist of a number of little plates of hard carbon, about 3 inches 
square, arranged in a pile, with a screw to reduce their resistance by squeezing them together into better contact.

401. Laws of Resistance.-The following are the laws of the resistance of conductors :-

(i.) The resistance of a conducting wire is proportional to its length. If the resistance of a mile of iron telegraph-wire be $17 \mathrm{ohms}$, that of 50 miles will be $50 \times 17=850$ ohms.

(ii.) The resistance of a conducting wire is inversely proportional to the area of its cross-section, and therefore in the usual round wires is inversely proportional to the square of its diameter. Ordinary telegraphwire is about $\frac{1}{6}$ of an inch thick; a wire twice as thick would conduct four times as well, liaving four times the area of cross-section; lience an equal length of it would have only $\frac{1}{4}$ the resistance.

(iii.) The resistance of a conducting wire of given length and thickness depends upon the material of which it is made-that is to say, upon the specific resistance of the material.

If the length of a wire be $l$ centimetres, and its area of section A square centimetres, and the specific resistance of the material be $\rho$, then its resistance $\mathrm{R}$ will be

$$
\mathrm{R}=l \rho / \mathrm{A} .
$$

Example.-Find the resistance of a platinoid wire of section 0.004 sq. cm., and $200 \mathrm{~cm}$. long; $\rho=32.5 \times 10^{-6}$. $\mathrm{R}=1 \cdot 625$ ohms.

402. Conductance and Resistance.-The term conductance is used as the inverse of resistance; a conductor whose resistance is $r$ ohms is said to have a conductance of $1 / r$ " mhos." When a number of conductors are in parallel with one another their united conductance is the sum of their separate conductances.

The conductance of a prism of which the length is 
$1 \mathrm{~cm}$. and its area of section is 1 sq. $\mathrm{cm}$., is called its conductivity or specific conductance.

The resistance of a prism of length $1 \mathrm{~cm}$. and section $1 \mathrm{sq} . \mathrm{cm}$. is sometimes called its resistivity or specific resistance.

403. Speciflc Resistance.-The specific resistance of a substance is most conveniently stated as the resistance (in millionths of an ohm) of a centimetre cube of the substance. The Table on p. 404 also gives the relative conductance when that of copper is taken as 100 .

Aluminium is a better conductor than silver, weight for weight.

It is found that those substances that possess a high conducting power for heat are also the best conductors of electricity, but the ratio of these conductivities is not constant ; it varies as the absolute temperature.

Liquids fall under three heads : (1) molten metals and alloys, which conduct simply as metals; (2) fused salts and solutions of salts and acids, which conduct only by electrolysis (Art. 487) ; (3) insulators, such as the oils, turpentine, etc., and bromine. Liquid electrolytes are worse conductors than metals ; gases, including steam, are perfect non-conductors, except when so rarefied as to admit of discharge by convexion through them (Art. 320).

404. Effects of Heat on Resistance.-Changes of temperature affect temporarily the conducting power of metals. Nearly all the pure metals increase their resistance about 0.4 per cent for a rise of $1^{\circ} \mathrm{C}$. in temperature, or about 40 per cent when warmed $100^{\circ}$. When cooled in liquid oxygen the resistance was found by Wroblewski to fall greatly. A copper wire which at $0^{\circ}$ had a resistance of $17.5 \mathrm{ohms}$ fell to $1.65 \mathrm{ohms}$ at $-201^{\circ} \mathrm{C}$. Dewar and Fleming find all pure metals to lower their resistance as though at $-274^{\circ} \mathrm{C}$. (absolute zero of temperature) they would become perfect conductors. The resistance of carbon, on the other hand, diminishes on heating. The filament of a glow-lamp, 


\section{TABLE OF SPECIFIC RESISTANCE}

\begin{tabular}{|c|c|c|c|}
\hline Substance. & $\begin{array}{l}\text { Specific Resistance } \\
\text { (microhms of } \\
1 \mathrm{~cm} \text {. cube). }\end{array}$ & $\begin{array}{c}\text { Resistance } \\
\text { (ohms) of metre } \\
\text { length l sq. } \\
\text { mm. Section. }\end{array}$ & $\begin{array}{c}\text { Relative } \\
\text { Conductance. }\end{array}$ \\
\hline 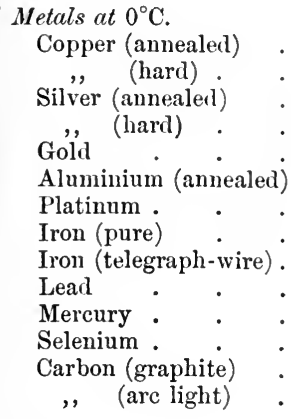 & $\begin{array}{c}1 \cdot 570 \\
1 \cdot 603 \\
1 \cdot 492 \\
1 \cdot 620 \\
2 \cdot 077 \\
2 \cdot 889 \\
8 \cdot 982 \\
9 \cdot 638 \\
15 \\
19 \cdot 63 \\
94 \cdot 34 \\
6 \times 10^{10} \\
2400 \text { to } 42000 \\
\text { abont } 4000\end{array}$ & $\begin{array}{l}\cdot 0157 \\
\cdot 0160 \\
\cdot 0149 \\
\cdot 0162 \\
\cdot 0208 \\
\cdot 0289 \\
\cdot 0898 \\
\cdot 0964 \\
\cdot 15 \\
\cdot 1963 \\
\cdot 9434\end{array}$ & 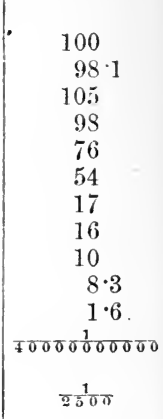 \\
\hline $\begin{array}{l}\text { Alloys. } \\
\text { German-silver } \\
\text { (Cu 60, Zn 26, Ni 14) } \\
\text { Platinum-silver. } \\
\text { (Pt } 67, \mathrm{Ag} 33) \\
\text { Platinoid. } \\
\text { (Cu 59, Zn } 25 \cdot 5 \text {, } \\
\quad \text { Ni 14, W } 55) \\
\text { Manganin. } \\
\text { (Cu 84, Ni 12, Mn } 3 \cdot 5)\end{array}$ & $\begin{array}{r}20 \cdot 76 \\
2 \cdot 4 \\
32 \cdot 5\end{array}$ & $\begin{array}{l}\cdot 2076 \\
\cdot 024 \\
\cdot 325 \\
\cdot 475\end{array}$ & $\begin{array}{l}7 \cdot 6 \\
6 \cdot 5 \\
4 \cdot 8\end{array}$ \\
\hline $\begin{array}{l}\text { Liquids at } 18^{\circ} \mathrm{C} . \\
\text { Pure Water } \\
\text { Dilute } \mathrm{H}_{2} \mathrm{SO}_{4}, 5 \% \\
\text {,, } \mathrm{H}_{2} \mathrm{SO}_{4}, 30 \% \\
,, \quad \mathrm{H}_{2} \mathrm{SO}_{4}, 80 \% \\
,, \quad \mathrm{ZnSO}_{4}, 24 \% \\
,, \quad \mathrm{HNO}_{3}, 30 \%\end{array}$ & $\begin{array}{c}26.5 \times 10^{8} \\
486 \times 10^{4} \\
137 \times 10^{4} \\
918 \times 10^{4} \\
214 \times 10^{5} \\
129 \times 10^{4}\end{array}$ & & $\begin{array}{l}\text { less than one } \\
\text { millionth part }\end{array}$ \\
\hline $\begin{array}{l}\text { Insulators. } \\
\text { Glass at } 20^{\circ} \mathrm{C} . \\
\text { Glass at } 200^{\circ} \mathrm{C} \text {. } \\
\text { Guttaperel } 24^{\circ} \mathrm{C} \text {. }\end{array}$ & $\begin{array}{r}91 \times 10^{18} \\
22 \cdot 7 \times 10^{12} \\
4.5 \times 10^{20}\end{array}$ & & $\begin{array}{l}\text { less than one } \\
\text { billionth }\end{array}$ \\
\hline
\end{tabular}


which when cold was 230 ohms, was only 150 when white hot. German-silver and other alloys do not show so much change, hence they are used in making standard resistance coils. The temperature-coefficient of German-silver is only 0.00044 for $1^{\circ}$ C., or $\frac{1}{10}$ that of the pure metals. Platinoid and platinum-silver have about 0.00011 for their coefficient. Weston has found alloys of manganese, copper, and nickel, which have a small negative coefficient. Those liquids which only conduct by being electrolyzed (Art. 234) conduct better as the temperature rises. The effect of light in varying the resistance of selenium is stated in Art. 529. The property of changing resistance with temperature is now used for measuring furnace temperatures in Callendar's platinum pyrometer. The bolometer used by Langley in researches on radiant heat depends on the same property.

405. Insulators.-The name insulators is given to materials which have such high resistances that they can be used as non-conductors. They differ much in their mechanical qualities as well as in their insulation-resistance. They may be classed under several heads: (1) Vitreous, including glass of all kinds and slags ; (2) Stony, including slate, marble, stoneware, steatite, porcelain, mica, asbestos ; (3) Resinous, including shellac, resin, beeswax, pitch, various gums, bitumen, ozokerit ; (4) Elastic, including india-rubber, guttapercha, ebonite; (5) Oily, including various oils and fats of animal and vegetable origin, as well as solid paraffin and petroleum oil ; (6) Cellulose, including dry wood and paper, and preparations of paper, such as "fibre" and celluloid. All these materials decrease their resistance enormously as the temperature rises, and in general become fairly good conductors as soon as any chemical change begins; some of them (as glass) conduct as electrolytes so soon as they soften.

The name insulators is also used for the insulating 
supports of stoneware, porcelain, or glass on which telegraph wires are carried (Art. 497).

406. Typical Circuit.-Let us consider the typical case of the circuit shown in Figr. 207, in which a battery, $\mathrm{ZC}$, is joined up in circuit with a galvanometer by means of wires whose resistance is $R$. The total electromotiveforce of the battery we will call $\mathrm{E}$, and the total internal resistance of the liquids in the cells $r$. The resistance of the galvanometer coils may be called G. Then, by Ohm's law :-

$$
\mathrm{C}=\frac{\mathrm{E}}{\mathrm{R}+r+\mathrm{G}} .
$$

The internal resistance $r$ of the liquids of the battery bears an important relation to the external resistance of

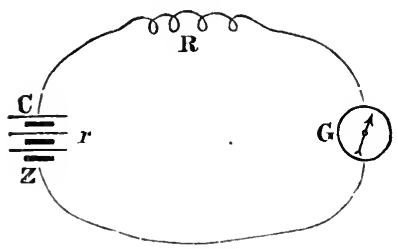

Fig. 207. the circuit (including $\mathrm{R}$ and $\mathrm{G}$ ), for on this relation depends the best way of arranging the battery cells. Suppose, for example, that we have a battery of 50 small Daniell's cells at our disposal, of which we may reckon the electromotiveforce as one volt (or, more accurately, 1.07 volt) each, and each having an internal resistance of two ohms. If we have to use these cells on a circuit where there is already of necessity a high resistance, we should couple them up "in series" rather than in parallel. For, supposing we have to send our current through a line of telegraph 100 miles long, the external resistance $R$ will be (reckoning $13 \mathrm{ohms}$ to the mile of wire) at least 1300 ohms. Through this resistance a single such cell would give a current of less than one milliampere, for here $\mathrm{E}=1, \mathrm{R}=1300, r=2$, and therefore $\mathrm{C}=\frac{\mathrm{E}}{\mathrm{R}+r}=\frac{1}{1300+2}=\frac{1}{1302}$ of an ampere, a current far too weak to work a telegrapl instrument. 
With fifty such cells in series we should have $\mathrm{E}=\mathbf{5 0}$, $r=100$, and then

$\mathrm{C}=\frac{50}{1300+100}=\frac{50}{1400}=\frac{1}{28}$ of an ampere, or over 35 milliamperes. In telegraph work, where the instruments require a current of 5 to 10 milli-amperes to work them, it is usual to reckon an additional Daniell's cell for every 5 miles of line, each instrument in the circuit being counted as having as great a resistance as 10 miles of wire.

If, however, the resistance of the external circuit be small, such arrangements must be made as will keep the total internal resistance of the battery small. Suppose, for example, we wish merely to heat a small piece of platinum wire to redness, and use stout copper wires to connect it with the battery. Here the external resistance may possibly not be as much as $1 \mathrm{ohm}$. In that case a single cell would give a current of $\frac{1}{3}$ of an ampere (or 333 milli-amperes) through the wire, for here $\mathrm{E}=1$, $\mathrm{R}=1$, and $r=2$. But 10 cells would only give half as much again, or 476 milli-amperes, and fifty cells only 495 milli-amperes, and with an infinite number of such cells in series the current could not possibly be more than 500 milli-amperes, because every cell, though it adds 1 to $\mathrm{E}$, adds 2 to $\mathrm{R}$. It is clear then that though linking many cells in series is of advantage where there is the resistance of a long line of wire to be overcome, yet where the external resistance is small the practical advantage of adding cells in series soon reaches a limit.

But suppose in this second case, where the external resistance of the circuit is small, we reduce also the internal resistance of our battery by linking cells together in parallel, joining several zincs of several cells together, and joining also their copper poles together (as suggested in Art. 192), a different and better result is attained. Suppose we thus join up four cells. Their electromotive-force will be no more, it is true, than that 
of one cell, but their resistance will be but $\frac{1}{4}$ of one such cell, or $\frac{1}{2}$ an ohm. These four cells would give a current of 666 milli-amperes through an external resistance of $1 \mathrm{ohm}$, for if $\mathrm{E}=1, \mathrm{R}=1$, and the internal resistance be $\frac{1}{4}$ of $r$, or $=\frac{1}{2}$, then

$\mathrm{C}=\frac{\mathrm{E}}{\mathrm{R}+r}=\frac{2}{3}$ of an ampere, or 666 milli-amperes.

If we arrange the cells of a battery in $n$ files of $m$ cells in series in each file (there being $m \times n$ similar cells altogether), the electromotive-force of each file will be $m$ times the electromotive-force $\mathrm{E}$ of each cell, or $m \mathrm{E}$; and the resistance of each file will be $m$ times the resistance $r$ of each cell, or $m r$. But there being $n$ files in parallel the whole internal resistance will be only $\frac{1}{n}$ of the resistance of any one file, or will be $\frac{m}{n} r$, hence, by Ohm's law, such a battery would give as its current

$$
\mathrm{C}=\frac{m \mathrm{E}}{\frac{\mathrm{m}}{\mathrm{n}} r+\mathrm{R}} .
$$

407. Best Groupings of Cells.-If the question arises as to the best way of grouping a given number of cells, it must be replied that there are sereral best ways.

(1) Grouping for best Economy.-So group the cells that their united internal resistance shall be very small compared with the external resistance. In this case the materials of the battery will be consumed slowly, and the current will not be drawn off at its greatest possible strength; but there will be a minimum waste of energy (Art. 435).

(2) Grouping for greatest Current.-It can be shown mathematically that, for a given battery of cells, the way of grouping them that will give the largest steady current when they are required to work through a given external resistance $\mathrm{R}$, is so to choose $m$ and $n$, that the internal resistance $\left(\frac{m}{n} r\right)$ shall equal the external resistance. The 
student should verify this rule by taking examples and working them out for different groupings of the cells. Although this arrangement gives the strongest current it is not the most economical ; for if the internal and external resistances be equal to one another, the useful work in the outer circuit and the useless work done in heating the cells will be equal also, half the energy being wasted.

(3) Grouping for quickest Action.-If there are electromagnets, or other objects possessing self-induction (Art. 458 ) in the circuit, which would tend to prevent the current rising quickly to its proper value, the best grouping to cause the current to rise as quickly as possible is one that will make the internal resistance higher than the external, namely, put all the cells in series (see Art. 460).

408. Long and Short Coil Instruments.-The student will also now have no difficulty in perceiving why a "long-coil" galvanometer, or a "long-coil" electromagnet, or instrument of any kind in which the conductor is a long thin wire of high resistance, must not be employed on circuits where both $\mathrm{R}$ and $r$ are already small. He will also understand why, on circuits of great length, or where there is of necessity a high resistance and a battery of great electromotive force is employed, "shortcoil" instruments are of little service, for though they add little to the resistances, their few turns of wire are not enough to produce the required action with the small currents that circulate in high-resistance circuits. He will understand, too, why "long-coil" instruments are here appropriate as multiplying the effects of the currents by their many turns, their resistance, though perhaps large, not being a serious addition to the existing resistances of the circuit. The main point to grasp is that it is the nature of the line, whether of high resistance or low, which determines not only the grouping of the battery, but also what kind of winding is appropriate in the instruments.

409. Divided Circuits. - If a circuit divides, as in Fig. 208, into two branches at A, uniting together again 
at $\mathrm{B}$, the current will also be divided, part flowing through one branch, part through the other. Any branch which serves as a by-pass to another branch is termed a shunt. The relative strengths of current it the two branches

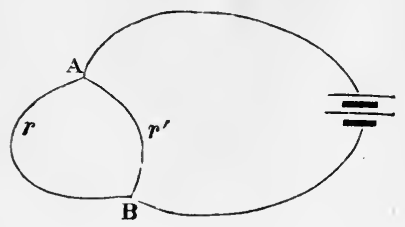

Fig. 20s.

will be proportional to their conductances, i.e. inversely proportional to their resistances.* Thus, if $r$ be a wire of 2 ohms resistance and $r^{\prime} 3 \mathrm{ohms}$, then current in $r$ : current in $r^{\prime}=r^{\prime}: r=3: 2$, .

or, $\frac{3}{5}$ of the whole current will flow through $r$, and $\frac{2}{5}$ of the whole current through $r^{\prime}$.

The joint resistance of the divided circuit between $\mathrm{A}$ and $B$ will be less than the resistance of either branch singly, because the current has now two paths. In fact, the joint conductance will be the sum of the two separate conductances. And if we call the joint resistance $R$, it follows that

$$
\frac{1}{\mathrm{R}}=\frac{1}{r}+\frac{1}{r^{\prime}}=\frac{r^{\prime}+r}{r r^{\prime}},
$$

whence $\mathrm{R}=\frac{r r^{\prime}}{r^{\prime}+r}$, or, in words, the joint $r e$ sistance of a divided conductor is equal to the product of the two separate resistances divided by their sum. This is sometimes called the law of shunts, because each of the branches may be regarded as a shunt to the other. A simple construction for finding the value graphically is given in Fig. 209. Let lines representing the two resistances $r$ and $r^{\prime}$ be erected at the ends of any base line, and the diagonals

* There is a popular fallacy that an electric current "always takes the line of least resistance." It never does, though part of the current may flow that way. It divides between the various paths in proportion to their easiness. It is only spark discharges which pierce a non-conductor that can be said to take the line of least resistance. 
drawn as shown. The perpendicular at the point of their intersection will be the joint resistance $R$.

In case there are three or more branches all in parallel, as in Fig. 210, the rule may be generalized as follows :-

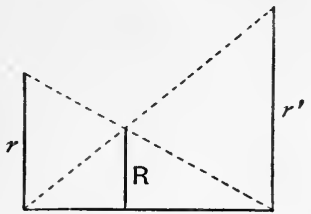

Fig. 209.

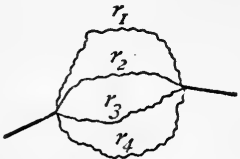

Fig. 210.

The joint resistance of any number of conductors in parallel is the reciprocal of the sum of the reciprocals of the separate resistances.

Kirchhoff has given the following important laws, both of them deducible from Ohm's law :-

(i.) In any branching network of wires the algebraic sum of the currents in all the wires that meet in any point is zero.

(ii.) When there are several electromotive-forces acting at different points of a circuit, the total electromotiveforce round the circuit is equal to the sum of the resistances of its separate parts multiplied each into the strength of the current that flows through it.

410. Current Sheets.-When a current enters a solid conductor it no longer flows in one line but spreads out and flows through the mass of the conductor. When a current is led into a thin plate of conducting matter it spreads out into a current sheet and flows through the plate by stream-lines in directions that depend upon the form of the plate and the position of the pole by which it returns to the battery. Thus, if wires from the two poles of a battery are brought into contact with two neighbouring 
points $A$ and $B$ in the middle of a very large flat sheet of tinfoil, the current flows through the foil not in one straight line from $\mathrm{A}$ to $\mathrm{B}$, but in stream-lines, which start out in all directions from $A$, and curl round to meet in $B$, in curves very like those of the "lines of force" that run from the N-pole to the S-pole of a magnet (Fig. 67). When the earth is used as a return wire to conduct the telegraph currents (Fig. 274), a similar spreading of the currents into current sheets occurs.

\section{Lesson XXXIV.-Electrical Measurements}

411. Measurement of Resistance.-The practical electrician has to measure electrical resistances, electromotive-forces, and the capacities of condensers. Each of these several quantities is measured by comparison with ascertained standards, the particular methods of comparison varying, however, to meet the circumstances of the case. Only a few simple cases can be here explained.

Ohm's law shows us that the strength of a current due to an electromotive-force falls off in proportion as the resistance in the circuit increases.

(a) Method of Substitution.-It is therefore possible to compare two resistances with one another by finding out in what proportion each of them will cause the current of a constant battery to fall off. Thus, suppose in Fig. 207 we have a standard battery of a few Daniell's cells, joined up in circuit with a wire of an unknown resistance $\mathrm{R}$, and with a galvanometer, we shall obtain a current of a certain strength, as indicated by the galvanometer needle experiencing a certain deflexion. If we remove the wire $\mathrm{R}$, and substitute in its place in the circuit wires whose resistances we lnow, we may, by trying, find one which, when interposed in the path of the current, gives the same deflexion on the galvanometer. This wire and the one we called $R$ offer equal resistance to the current. This 
method of substitution of equivalent resistances was further dereloped by Wheatstone, Jacobi, and others, when they proposed to employ as a standard resistance a long thin wire coiled upon a wooden cylinder, so that any desired length of the standard wire might be thrown into the circuit by unwinding the proper number of turns of wire off the cylinder, or by making contact at some point at any desired distance from the end of the wire. This form of rheostat was found, however, to be less accurate than the resistance coils described below.

(b) Method of Proportional Deflexion. - The method explained above can be used with any galvanometer of sufficient sensitiveness, but if a tangent galvanometer is available the process may be shortened by calculation. Suppose the galvanometer and an unknown resistance $\mathrm{R}$ to be included in the circuit, as in Fig. 207, and that the current is strong enough to produce a deflexion $\delta$ : Now substitute for $R$ any known resistance $R^{\prime}$, which will alter the deflexion to $\delta^{\prime}$; then (provided the other resistances of the circuit be negligibly small) it is clear that since the strengths of the currents are proportional to $\tan \delta$ and $\tan \delta^{\prime}$ respectively, the resistance $\mathrm{R}$ can be calculated by the inverse proportion.

$$
\tan \delta: \tan \delta^{\prime}=\mathrm{R}^{\prime}: \mathrm{R} .
$$

(c) Method of Differential Galvanometer.-With a differential galvanometer (Art. 217), and a set of standard resistance coils, it is easy to measure the resistance of a conductor. Let the circuit divide into two branches, as in Fig. 211, so that part of the current flows through the unknown resistance and round one set of coils of the galvanometer, the other part of the current being made to flow through the known resistances and then round the other set of coils in the opposing direction. When we have succeeded in matching the unknown resistance by one equal to it from amongst the known resistances, the currents in the two branches will be equal, and the needle 
of the differential galvanometer will show no deflexion. This null method is very reliable.

(d) Bridge Method. - The best of all the ways of measuring resistances is, however, with the important instrument known as Wheatstone's Bridge, described below in Art. 413.

(e) Condenser Methods.-To measure very high resistances the plan may be adopted of charging a condenser

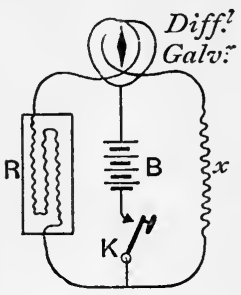

Fig. 211. from a standard battery for a definite time through the resistance, and then ascertaining the accumulated charge by discharging it through a ballistic galvanometer (Art. 218). Or in another method the condenser is allowed to discharge itself slowly through the high resistance, and the time taken by the potential to fall through any given fraction of its original value is observed. This time is proportional to the resistance, to the capacity, and to the logarithm of the given fraction.

412. Fall of Potential along a Wire. - To understand the principle of Wheatstone's Bridge we must explain a preliminary point. If the electric potential of different points of a circuit be examined by means of an electrometer, as explained in Art. 289, it is found to decrease all the way round the circuit from the + pole of the battery, where it is highest, down to the - pole, where it is lowest. If the circuit consist of one wire of uniform thickness, which offers, consequently, a unifcrm resistance to the current, it is found that the potential falls uniformly ; if, however, part of the circuit resists more than another, it is found that the potential falls mcst rapidly along the conductor of greatest resistance. If with a suitable voltmeter we explore the fall of potential between two points $a$ and $b$ of a circuit (Fig. 212), we shall find in every case the fall of potential proportional to the resistance 
between those two points. For $\mathrm{V}=\mathrm{CR}$, and therefore, for the same $\mathrm{C}$, the $\mathrm{V}$ across any part is proportional to the $\mathrm{R}$ of that part. We know, for example, that when we have gone round the circuit to a point where the potential has fallen through half its value, the current has at that point gone through half the resistances. The best way to measure a very large current is to measure (with sensitive voltmeter arrangement of galvanometer) the drop of potential it produces when sent through a known very low

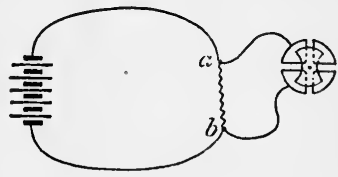

Fig. 212. resistance such as a strip of platinoid having exactly $\frac{1}{1000}$ ohm resistance between two measured points. To measure a very small resistance, it should be put in series with another known very small resistance, and the drops of potential when the same current flows through both are compared : the resistance of each being as the drop in potential between its ends.

413. Wheatstone's Bridge.-This instrument, invented by Christie, and applied by Wheatstone to measure resistances, consists of a system of conductors shown in diagram in Fig. 213. This circuit of a battery is made to branch at $\mathrm{P}$ into two parts, which reunite at $\mathrm{Q}$, so that part of the current flows through the point $M$, the other part through the point $\mathrm{N}$. The four conductors D, C, B, A, are spoken of as the "arms " of the "balance" or "bridge" ; it is by the proportion subsisting between their resistances that the resistance of one of them can be calculated when the resistances of the other three are known. When the current which starts from $\mathrm{C}$ at the battery arrives at $\mathrm{P}$, the potential will have fallen to a certain value. The potential of the current in the upper branch again falls to $M$, and continues to fall to $Q$. The potential of the lower branch falls to $\mathrm{N}$, and again falls till it reaches the value at $Q$. Now if $N$ be the same 
proportionate distance along the resistances between $\mathrm{P}$ and $\mathrm{Q}$, as $\mathrm{M}$ is along the resistances of the upper line between $\mathrm{P}$ and $\mathrm{Q}$, the potential will have fallen at $\mathrm{N}$ to the same value as it has fallen to at $\mathrm{M}$; or, in other words, if the ratio of the resistance $\mathrm{C}$ to the resistance $\mathrm{D}$

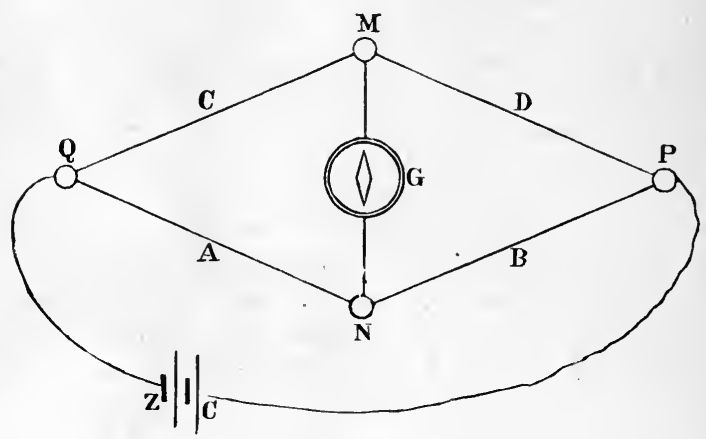

Fig. 213.

be equal to the ratio between the resistance $\mathrm{A}$ and the resistance $\mathrm{B}$, then $\mathrm{M}$ and $\mathrm{N}$ will be at equal potentials. To find out whether they are at equal potentials a sensitive galvanometer is placed in a branch wire between M and $\mathrm{N}$; it will show no deflexion when $\mathrm{M}$ and $\mathrm{N}$ are at equal potentials ; or when the four resistances of the arms "balance" one another by being in proportion, thus :-

$$
\mathrm{A}: \mathrm{C}:: \mathrm{B}: \mathrm{D} \text {. }
$$

If, then, we know what $\mathrm{A}, \mathrm{B}$, and $\mathrm{C}$ are, we can calculate $\mathrm{D}$, which will be

$$
\mathrm{D}=\frac{\mathrm{B} \times \mathrm{C}}{\mathrm{A}} .
$$

Example.-Thus if A and $\mathrm{C}$ are (as in Fig. 216) 10 ohms and 100 ohms respectively, and $B$ be 15 ohms, D will be $15 \times 100 \div 10=150$ ohms. 
414. Resistance Coils. - Wires of standard resistance are now sold by instrument-makers under the name of Resistance Coils. They consist of coils of some alloy, German-silver, platinum-silver, or platinoid (see Art. 404), wound with great care, and adjusted to such a length as to have resistances of a definite number of ohms. In order to avoid selfinduction, and the consequent sparks (see Art. 458 ) at the opening or closing of the circuit,

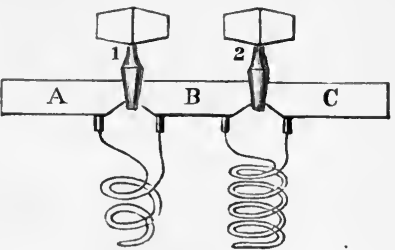

Fig. 214. they are wound in the peculiar non-inductive manner indicated in Fig. 214, each wire (covered with silk or paraffined-cotton) being doubled on itself before being coiled up. Each end of a coil is soldered to a solid brass

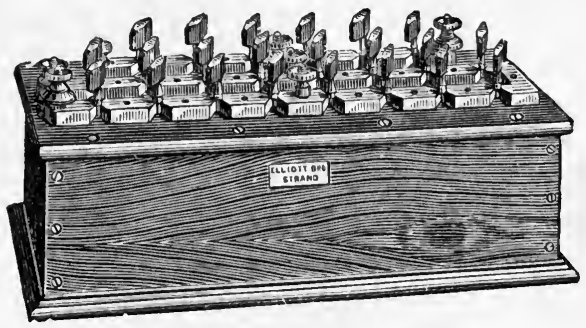

Fig. 215.

piece, as coil 1 to $\mathrm{A}$ and $\mathrm{B}$, coil 2 to $\mathrm{B}$ and $\mathrm{C}$; the brass pieces being themselves fixed to a block of ebonite (forming the top of the "resistance box"), with sufficient room between them to armit of the insertion of stont wellfitting plugs of brass. Fig. 215 shows a complete resistance box, as fitted up for electrical testing, with the plugs in their places. So long as the plugs remain in, the 
current flows through the solid brass pieces and plugs without encountering any serious resistance; but when any plug is removed, the current can only pass from the one brass piece to the other by traversing the coil thus thrown into circuit. The series of coils chosen is usually of the following numbers of ohms' resistance-1, 2, 2,$5 ; 10,20,20,50 ; 100,200,200,500 ; \ldots$ up to 10,000 ohms. By pulling out one plug any one of these can be thrown into the circuit, and any desired

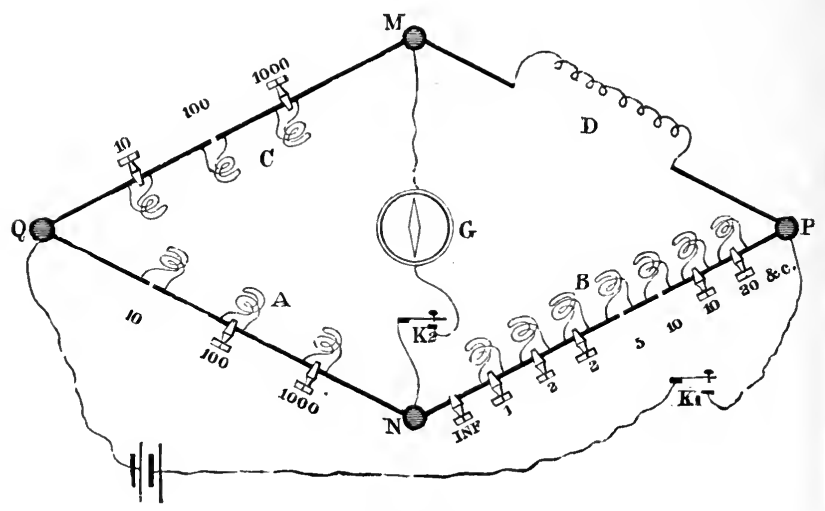

Fig. 216.

whole number, up to 20,000 , can be made up by pulling out more plugs; thus a resistance of 263 ohms will be made up as $200+50+10+2+1$ by unplugging those five coils.

It is usual to construct Wheatstone's bridges with some balancing resistance coils in the arms $\mathrm{A}$ and $\mathrm{C}$, as well as with a complete set in the arm $B$. The advantage of this arrangement is that by adjusting $\mathrm{A}$ and $\mathrm{C}$ we determine the proportionality between $\mathrm{B}$ and $\mathrm{D}$, and can, in certain cases, measure to fractions of an ohm. Fig. 216 shows a 
more complete scheme, in which resistances of 10,100 , and $1000 \mathrm{ohms}$ are included in the arms $\mathrm{A}$ and $\mathrm{C}$.

Example.-Suppose we had a wire whose resistance we knew to be between 46 and 47 ohms, and wished to measure the fraction of an ohm, we should insert it at $\mathrm{D}$, and make A 100 ohms and C $10 \mathrm{ohms}$; in that case D would be balanced by a resistance in B 10 times as great as the wire D. If, on trial, this be found to be $464 \mathrm{ohms}$, we know that $\mathrm{D}=464 \times 10 \div 100=46.4 \mathrm{ohms}$.

415. Other Patterns of Bridge.-In practice the bridge is seldom or never made in the lozenge-shape of the diagrams.

Post-Office Bridge.-The resistance-box of Fig. 215 is, in itself, a complete "bridge" of the Post-Office pattern, the appropriate connexions being made by screws at various points. In using the bridge the battery circuit should always be completed by depressing the key $\mathrm{K}_{1}$ before the key $\mathrm{K}_{2}$ of the galvanometer circuit is depressed, in order to avoid the sudden violent "throw" of the galvanometer needle, which occurs on closing circuit in consequence of self-induction (Art. 458).

Dial Bridge. - To avoid errors arising from the different numbers of plugs in use, the coils of a bridge are sometimes arranged in dials - the units in one, the tens of ohms in another, and so forth-each dial having but one plug, or a movable arm like Fig. 206.

Metre Bridge.-This is a simple form very useful for measuring resistances not exceeding a few hundred ohms. Upon a long board is stretched over a scale one metre long a uniform thin wire of German-silver or other alloy, its ends being joined to stout pieces of copper. A, B, C, and $\mathrm{D}$ are four resistances joined as shown by stout strips of copper. When the wire from the galvanometer is slid along the wire to such a point that there is no current, it follows that

$$
\mathrm{A}+a: \mathrm{B}+b=\mathrm{C}: \mathrm{D} \text {. }
$$


Foster's method of measuring small differences of resistance is to get balance at a certain point along the wire, then interchange $A$ and $B$, and again get balance at another point. The resistance of the piece of wire be-

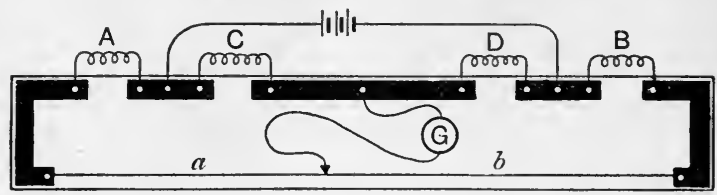

Fig. 217.

tween the two points will then be equal to the difference of the resistances $\mathrm{A}$ and $\mathrm{B}$.

In a simpler way of using the bridge, $\mathrm{A}$ and $\mathrm{B}$ are replaced by strips of no appreciable resistance, so that

$$
a: b:: \mathrm{C}: \mathrm{D} \text {. }
$$

If $\mathrm{D}$ is the unknown resistance and $\mathrm{C}$ a known resistance, the ratio of the lengths $a$ and $b$ at once enables the unknown resistance to be calculated.

For further details of bridge methods consult Gray's Absolute Measurements in Electricity and Magnetism, Kempe's Electrical Measurement, or Ayrton's Practical Electricity.

416. Measurement of Electromotive-F'orce. -There being' no easy absolute method of measuring electromotive-forces, they are usually measured relatively, by comparison with the electromotive-force of a standard cell, such as Clark's (Art. 188). The methods of comparison are various; only five are here mentioned.

(a) Reduced Deflexion Method.-Call E the electromotiveforce of the battery to be measured, and $E^{\prime}$ that of a standard battery. Join $\mathrm{E}$ with a galvanometer, and let. it produce a deflexion of $\delta_{1}$ degrees through the resist- 
ances of the circuit; then add enough resistance $r$ to bring down the deflexion to $\delta_{z}$ degrees-say 10 degrees less than before. Now substitute the standard battery in the circuit and adjust the resistances till the deflexion is $\delta_{1}$ as before, and then add enough resistance $r^{\prime}$ to bring down the deflexion to $\delta_{2}$. Then

$$
r^{\prime}: r=\mathrm{E}^{\prime}: \mathrm{E}
$$

since the resistances that will reduce the strength of the current equally will be proportional to the electromotiveforces. (Not recommended.)

(b) Potentiometer Method.-If the poles of a standard battery are joined by a long thin wire, the potential will fall uniformly from the + to the - pole. Hence, by

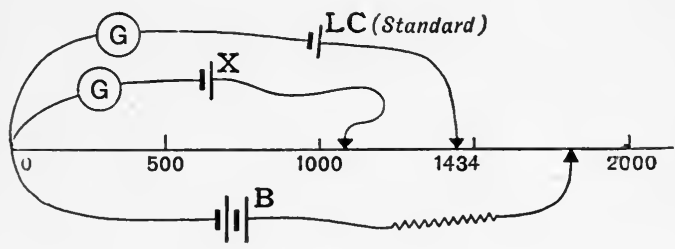

Fig. 218.

making contacts at one pole and at a point any desired distance along the wire, any desired proportional part of the whole electromotive-force can be taken. This proportional part may be balanced against the electromotiveforce of any other battery as follows:-Let a uniform thin wire of platinoid or German-silver be stretched over a scale divided into say 2000 parts. Connect a Clark standard cell IC through a sensitive galvanometer, as shown in Fig. 218, to make contact at the 1434 division of the scale. Then connect a single accumulator cell B, or two Daniells, or a Grove cell with a sliding contact, and move it up and down until a point is found 
such that the galvanometer shows that the Clark cell is balancel. Then connect the cell $\mathrm{X}$ whose E.M.F. is to be measured, and slide its contact along the wire until it also is balanced. Suppose it balances at 1024 of the scale, its E.M.F. will be 1.024. A single galvanometer will suffice if the wire to $\mathrm{X}$ is joined in between $\mathrm{G}$ and the Clark cell.

(c) Voltmeter Method.-If a galvanometer be constructed so that the resistance of its coils is several thousand ohms (in comparison with which the internal resistance of a battery or dynamo machine is insignificant), it will serve to measure electromotive-forces ; for the strength of current through it will depend only on the electromotive-force between the ends of the coil. (See Art. 220 on Voltmeters.)

(d) Condenser Method.-A condenser of known capacity is charged from a standard cell, and then discharged through a ballistic galvanometer (Art. 218). The cell to be compared is then substituted for the standard cell. The E.M.F. is proportional to the throw of the galvanometer.

(e) Electrometer Method.-The electromotive-force of a battery may be measured directly as a difference of potentials by a quadrant electrometer. In this case the circuit is never closed, and no current flows.

417. Measurement of Internal Resistance of Cells.-This may be done in several ways.

(a) Coudenser Method.-As in (d) of preceding Article, observe throw of galvanometer from condenser charged by the cell. Then shunt the cell with a suitably high resistance $R$ and take another charge and discharge. If the two throws are called $d_{1}$ and $d_{2}$, the internal resistance will be $=\mathrm{R}\left(d_{1}-d_{2}\right) / d_{2}$.

(b) Half-deflexion Method.-Place the cell in series with a galvanometer the resistance of which is $\mathrm{G}$, and a resistance-box in which there is unplugged a resistance $R$ such that the deflexion is conveniently large. Now in- 
crease the resistance in the box until it is seen by the deflexion that the current has been reduced to half what it was. If this added resistance is called $a$, then by Ohm's law it follows that the internal resistance is $=a-(\mathrm{R}+\mathrm{G})$. This method is suitable for very high internal resistances.

(c) Method of Opposition.-Take two similar cells and join them in opposition to one another, so that they send no current of their own. Then measure their united resistance just as the resistance of a wire is measured. The resistance of one cell will be half that of the two.

(d) Mance's Method.-Place the cell itself in one arm of the Wheatstone's bridge, and put a key where the battery usually is, adjust the resistances till the permanent galvanometer deflexion is the same whether the key be depressed or not. When this condition of things is attained the battery resistance is balanced by those of the other three arms. (Not a reliable method.)

(e) Alternate Current Method.-If greater accuracy is required in the opposition method, the cells in opposition may be placed in one of the arms of a Wheatstone's bridge in which instead of the usual battery is inserted the secondary coil of a small induction coil (without condenser), and with which a telephone receiver is used instead of a galvanometer. The ceasing of the buzzing in the telephone corresponds to nul deflexion. By this means we avoid the disturbance of the balance of the opposing cells which occurs if continuous currents are used. This method is also excellent for measuring resistances of liquids.

418. Measurement of Capacity.-The capacity of a condenser may be measured by comparing it with the capacity of a standard condenser-such as the $\frac{1}{3}$ microfarad condenser (Fig. 159)-in one of the following ways :- 
(a) Electrometer Method.-Charge the condenser of unknown capacity to a certain potential ; then make it share its charge with the condenser of known capacity, and measure the potential to which the charge sinks; then calculate the original capacity, which will bear the same ratio to the joint capacity of the two as the final potential bears to the original potential.

(b) Ballistic Galvanometer Method.-Charge eaclı condenser to equal differences of potential from the same cell or battery, and then discharge each suecessively through a ballistic galvanometer (Art. 188). The throw of the needle will be proportional in each case to the charge, and therefore to the capacity.

The law of the ballistic galvanometer is :-

$$
\mathrm{KV}=\mathrm{Q}=\frac{\mathrm{H}}{\mathrm{G}} \times \frac{\mathrm{T}}{\pi} \sin \frac{1}{2} a,
$$

where $Q$ is the quantity of electricity (in C.G.S. units), $\mathrm{H}$ the magnetic field, by the constant of the galvanometer, $\mathrm{T}$ the period of one complete swing of the needle, and $a$ the angle of first swing. The factor $\mathrm{H} / \mathrm{G}$ may be eliminated by passing a steady current $\mathrm{C}$ to produce a steady deflexion $\beta$; when

$$
\mathrm{C}=\frac{\mathrm{H}}{\mathrm{G}} \tan \beta \text {. }
$$

Combining this with the preceding, we have

$$
\mathrm{Q}=\frac{\mathrm{CT} \sin \frac{1}{2} a}{\pi \tan \beta} .
$$

If $\alpha$ and $\beta$ are both small this becomes

$$
\mathrm{Q}=\mathrm{CT} a / 2 \pi \beta \text {. }
$$

If $\mathrm{C}$ is in amperes, $\mathrm{Q}$ will be in coulombs.

(c) Bridge Method.-Connect the two condensers $\mathrm{K}_{1}$ and $\mathrm{K}_{2}$ in two arms of a Wheatstone's bridge and adjust the 
resistances so that there is no deflexion on charge or discharge (Fig. 219). Then $K_{1}: K_{2}:: r_{2}: r_{1}$, the laryer capacity acting as a smuller resistance.

(d) Potential-divider nul Method.-Two resistances $r_{1}$ and $r_{2}$ are joined in series to the + and - poles of a battery. The middle point between $r_{1}$ and $r_{2}$ is connected to one of the terminals of $K_{1}$ and also of $K_{2}$. The free terminals

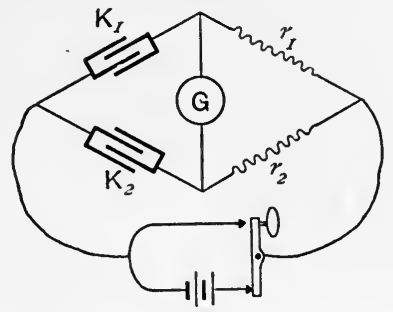

Fig. 219.

of $K_{1}$ and $K_{2}$ are momentarily joined to the + and poles of the battery respectively and receive charges of opposite sign. They are then connected; and if of equal amount the charges will neutralize each other. The resistances $r_{1}$ and $r_{2}$ are adjusted until this condition is satisfied, as shown by nul deflexion when the key of a galvanometer circuit across their terminals is depressed. Then $\mathrm{K}_{1}: \mathrm{K}_{2}:: r_{2}: r_{1}$.

(e) Tuning-fork Method.-A tuning-fork acting as a vibrating two-way switch charges and discharges the condenser $n$ times per second, allowing to pass VK $n$ coulombs per second or VK $n$ amperes. The apparent resistance $r$ of this combination is $1 / \mathrm{K} n$, and can be measured by a Wheatstone bridge, whence $\mathrm{K}=1 / n r$.

(f) Loss of Charge Method.-This is the same as the last method in Art. 411 e, a known high resistance being used. 


\title{
CHAPTER VII
}

\author{
THERMO-ELECTRICITY
}

\section{Lesson XXXV.-Thermo-Electric Currents}

419. Seebeck Effect.-In 1822 Seebeck discovered that a current may be produced in a closed circuit by heating a point of contact of two dissimilar metals. If a piece of bismuth and a piece of antimony be soldered together, and their free ends connected with a short-coil galvanometer, it is found that if the junction be warmed to a temperature higher than that of the rest of the circuit, a current flows in the direction from bismuth to antimony across the heated point ; the current being proportional to the excess of temperature. If the junction is cooled below the temperature of the rest of the circuit a current in the opposite direction is observed. The electromotive-force thus set up will maintain the current so long as the excess of temperature of the heated point is kept up ; heat being all the while absorbed in order to maintain the energy of the current. Such currents are called Thermo-electric currents, and the electromotive-force producing them is known as Thermo-electromotive-force.

420. Peltier Effect.-In 1834 Peltier discovered a phenomenon which is the converse of that discovered by Seebeck. He found that if a current of electricity from a battery be passed through a junction of dissimilar 
metals the junction is either heated or cooled, according to the direction of the current. Thus a current which passes through a bismuth-antimony pair in the direction from bismuth to antimony absorbs heat in passing the junction of these metals, and cools it; whereas, if the current flow from antimony to bismuth across the junction it evolves heat, and the junction rises in temperature. It is clear that if bismuth is positive with respect to antimony, any current that may be caused to flow from bismuth to antimony is aided by the electromotive force at that junction; whilst any current flowing from antimony to bismuth will meet with an opposing electromotive-force. In the latter case the current will do

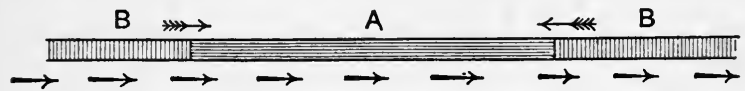

Fig. 220.

work and heat the junction; in the former the current will receive energy at the expense of the junction, which will give up heat. In Fig. 220, the feathered arrows, at the junctions represent the Peltier electromotive-forces, and the plain arrows the direction of the current.

This phenomenon of heating (or cooling) by a current, where it crosses the junction of two dissimilar metals (known as the "Peltier effect," to distinguish it from the ordinary heating of a circuit where it offers a resistance to the current, which is sometimes called the "Joule effect "), is utterly different from the evolution of heat in a conductor of high resistance, for $(a)$ the Peltier effect is reversible ; the current heating or cooling the junction according to its direction, whereas a current meeting with resistance in a thin wire heats it in whichever direction it flows; and $(b)$ the amount of heat evolved or absorbed in the Peltier effect is proportional simply to the current, not to the square of the current as the heat of resistance is. 
The complete law of the heat developed in a circuit will therefore require to take into account any Peltier effects which may exist at metal junctions in the circuit. If the letter $\mathrm{P}$ stand for the difference of potential due to the heating of the junction, expressed as a fraction of a volt, then the complete law of heat is

$$
\mathrm{U}=0 \cdot 24 \times\left(\mathrm{C}^{2} \mathrm{R} t \pm \mathrm{PC} t\right)
$$

which the student should compare with Joule's law in Art. 427. The quantity called $\mathrm{P}$ is also known as the coefficient of the Peltier effect; it has different values for different pairs of metals, and is numerically equal to the number of ergs of work which are evolved as heat at a junction of the particular metals by the passage of one absolute unit (10 coulombs) of electricity through the junction.

421. Thermo-electric Laws.-The thermo-electric properties of a circuit are best studied by reference to

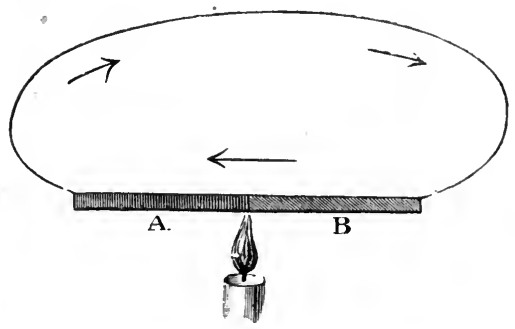

Fig. 221. the simple circuit of Fig. 221, which represents a bismuth - antimony pair united by a copper wire. If all parts of the circuit are at one temperature, even though there may lie at the junctions electromotive-forces as suggested above, there will be no current, since the electromotive-forces are in equilibrium. But when a junction is heated this equilibrium no longer exists, and there will be a resultant electromotive-force. It is found to obey the following laws:- 
(i.) The thermo-electromotive-force is, for the same pair of metals, proportional (through limited ranges of temperature) to the excess of temperature of the junction over the rest of the circuit.

(ii.) The total thermo-electromotive-force in a circuit is the algebraic sum of all the separate thermo-electromotiveforces in the various parts.

It follows from this law that the various metals can be arranged, as Seebeck found, in a series, according to their thermo-electric power, each one in the series being thermoelectrically positive (as bismuth is to antimony) toward one lower down.

422. Thermo-electric Power.-In the following table is shown the thermo-electric series of metals, together with the thermo-electric power of each when cold. The term thermo-electric power of a metal means the electromotive-force per degree (centig.) for a pair made of that metal with the standard metal (lead). In the table the numbers are microvolts per degree.

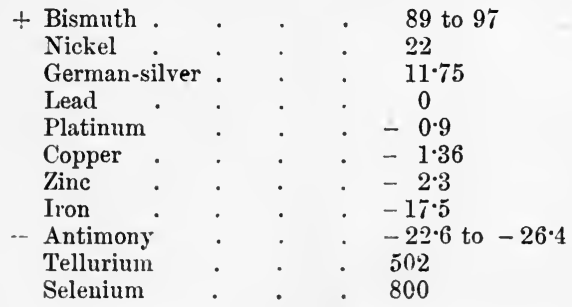

A very sinall amount of impurity may make a great difference in the thermo-electric power of a metal, and some alloys, and some of the metallic sulphides, as galena, exhibit extreme thermo-electric power.

The electromotive-forces due to heating single pairs of metals are very small indeed. If the junction of a copper-iron pair be raised $1^{\circ} \mathrm{C}$. above the rest of the circuit its electromotive-force is only $16 \cdot 14$ microvolts. 
That of the more powerful bismuth-antimony pair is for $1^{\circ}$ C., about 117 microvolts. Thermo-electric power varies, however, with temperature : for example, that of iron is really $-17.5+0.049 t$ (where $t$ is the mean temperature of the two junctions), iron becoming less negative when hot. Copper is $-1.36-0.01 t$, becoming more negative. There will be obviously one particular temperature or neutral point, at which their powers will be equal.

423. Thermo-electric Inversion.-Cumming discovered that in the case of iron and other metals an inversion of their thermo-electric properties may take place at a high temperature. In the case of the copperiron pair the temperature of $275^{\circ}$ is a neutral point; below that temperature the current flows through the hotter junction from the copper to the iron; but when the circuit is above that temperature iron is thermoelectrically positive to copper. The neutral point for a zinc-iron pair is about $200^{\circ}$. The inversion is easily shown by heating the junction of two long strips of these

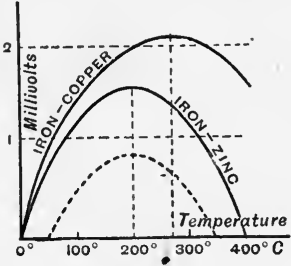

Fig. 222. metals, riveted together in a $\mathrm{V}$-form, and watching the effect on a galvanometer connected to their other ends. There will at first be a deflexion which will go on increasing until the temperature of $200^{\circ}$ is attained, but on further heating the junction the deflexion diminishes and at about $400^{\circ}$ reverses, the current flowing the other way. Fig. 222 shows graphically the curves obtained with iron-zinc and iron-copper pairs when one junction is kept at $0^{\circ}$ while the other is heated. The dotted line is for the iron-zinc pair when one junction is kept at $50^{\circ}$ and the other heated.

424. Thermo-electric Diagram. - The facts of thermo-electricity are best studied by means of the dia- 
grams suggested by Lord Kelvin and constructed by Professor Tait. In that given in Fig. 223 the horizontal divisions represent the temperatures; the vertical distances indicating the thermo-electric power, in microvolts per degree. These powers are measured with respect to the metal lead, which is taken as the standard of zero at all temperatures, because, while with other metals there

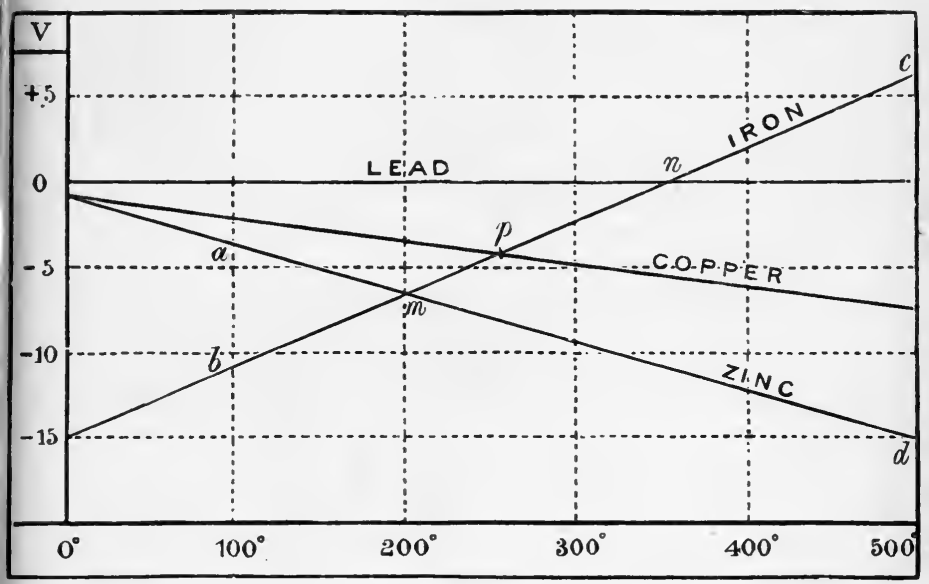

Fig. 223.

appears to be a difference of potentials between the metal hot and the same metal cold, hot lead brought into contact with cold lead shows no perceptible thermoelectric difference.

An example will illustrate the usefulness of the diagram. Let a circuit be made by uniting at both ends a piece of iron and a piece of zinc; and let the two junctions be kept at $0^{\circ}$ and $100^{\circ}$ respectively by melting ice and boiling water. Then the total electromotive-force round the circuit is represented by the area $a, 0,-15, b$. 
The slope of the lines for the various metals represents the property referred to above, of an electromotive-force between differently-heated portions of the same metal accompanied by an absorption or evolution of heat when the current flows from a hotter to a colder portion of the same metal. This effect, known as the Thomson effect from its discoverer Sir W. Thomson (Lord Kelvin), is opposite in iron to what it is in copper or zinc. Copper when hot is negative compared with copper that is cold. Hence if a current is sent from a hot to a cold part of a piece of copper it encounters an opposing electromotiveforce. Hence when a current of electricity flows from a hot to a cold point in copper it evolves heat; and it absorbs heat when it flows from a cold point to a hot point in the copper. In iron a current flowing from a hot point to a cold point absorbs heat.

The thermo-electromotive-force of a pair, of which the junctions are at temperatures $\mathrm{T}$ and $t$ respectively, and of which $n$ is the temperature of the neutral point, may be conveniently expressed by the following formula:-

$$
\mathrm{E}=p(\mathrm{~T}-t)\left\{1-\frac{\frac{1}{2}(\mathrm{~T}+t)}{n}\right\} ;
$$

where $p$ is the volts per degree $\left(\right.$ at $0^{\circ}$ ) as given in the table (Art. 422).

425. Thermo-electric Piles.-The electromotiveforce of a bismuth-antimony pair, when the junctions are kept at $0^{\circ}$ and $100^{\circ}$, is only 0.0115 volt. In order to increase the electromotive-force of thermo-electric pairs it is usual to join a number of pairs of metals (preferably bismuth and antimony) in series, but so bent that the alternate junctions can be heated as shown in Fig. 224 at $\mathrm{BBB}$, whilst the other set AAA are kept cool. The various electromotive-forces then all act in the same direction, and the current is increased in proportion to the number of pairs of junctions. Powerful thermoelectric batteries have been made by Clamond-an iron- 
galena battery of 120 pairs affording a strong current; but it is extremely difficult to maintain them in effective action for long, as they fail after continued use, probably owing to a permanent molecular change at the junctions. In the hands of Melloni the thermo-electric pile or thermopile, constructed of many small pairs of antimony and bismuth united in a compact form, proved an excellent electrical thermometer when used in conjunction with a sensitive short-coil astatic galvanometer. For the

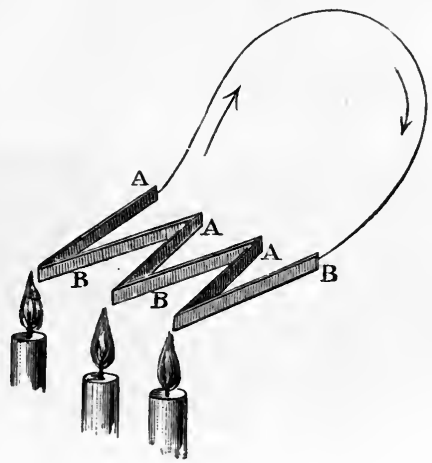

Fig. 22 !.

detection of excessively small differences of temperature the thermopile is an invaluable instrument, the currents being proportional to the difference of temperature between the hotter set of junctions on one face of the thermopile and the cooler set on the other face. The arrangement of a thermopile with the old astatic galvanometer is shown in Fig. 225.

A still more sensitive arrangement for detecting minute heating due to radiation consists in suspending between the poles of a powerful magnet a closed circuit having a bismuth-antimony junction in it. Sturgeon 
proposed a thermo-galvanometer on this plan in 1835.

In the radio-micrometer of Vernon Boys (1889) a loop of wire, suspended by a delicate quartz fibre between the

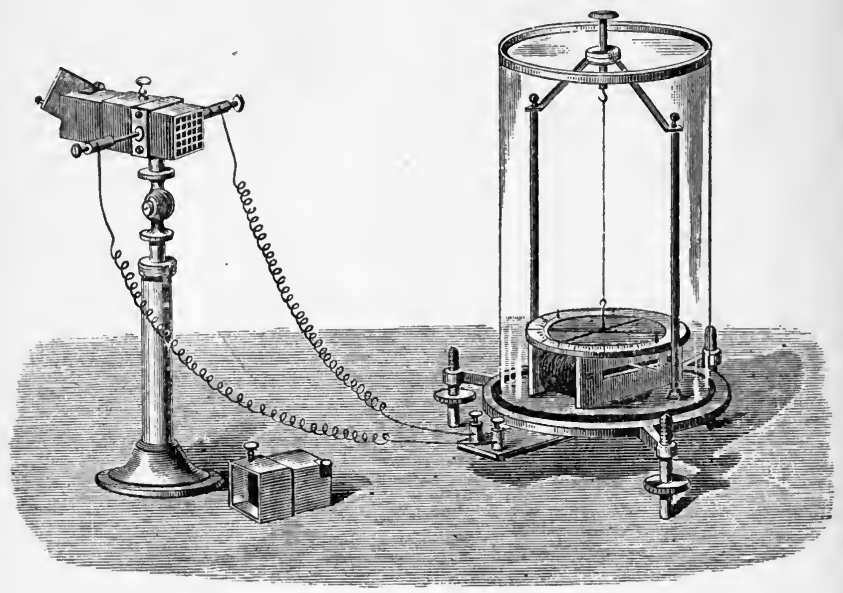

Fig. 225.

poles of a magnet (like the coil in Fig. 126) has its circuit closed at its lower end by a piece of antimony and a piece of bismuth (or alloys of these metals) soldered to a minute disk of copper foil. A rise of temperature of the copper foil even so small as one millionth of a degree will generate a current in the loop and give a deflexion over one division of the scale. With an instrument of this kind the radiant heat of a candle can be detected at a distance of two miles. 


\section{CHAPTER VIII}

HEAT, POWER, AND LIGHT, FROM ELECTRIC CURRENTS

\section{Lesson XXXVI.-Heating Effects. of Currents}

426. Heat and Resistance.-A current may do work of various kinds, chemical, magnetic, mechanical, and thermal. In every case where a current does work that work is done by the expenditure of part of the energy of the current. We have seen that, by the law of $\mathrm{Ohm}$, the current produced by a given battery is diminished in strength by anything that increases the external resistance. But the current may be diminished, in certain cases, by another cause, namely, the setting up of an opposing electromotive-force at some point of the circuit. Thus, in passing a current through an electrolytic cell (Art. 237) there is a diminution due to the opposing electromotive-force ("polarization") which is generated while the chemical work is being done. So, again, when a current is used to drive an electric motor (Art. 443), the rotation of the motor will itself generate a back E.M.F., which will diminish the current. Whatever current is, however, not expended in this way in external work is frittered down into heat, either in the battery or in some part of the circuit, or in both. Suppose a quantity of electricity to be set flowing round a closed circuit. If there were no resistance to stop it it would circulate for ever; just as a waggon set rolling along a 
circular railway should go round for ever if it were not stopped by friction. When matter in motion is stopped by friction the energy of its motion is frittered down by the friction into heat. When electricity in motion is stopped by resistance the energy of its flow is frittered down by the resistance into heat. Heat, in fact, appears wherever the circuit offers a resistance to the current. If the terminals of a battery be joined by a short thick wire of small resistance, most of the heat will be developed in the battery and so wasted; whereas, if a thin wire of relatively considerable resistance be interposed in the outer circuit, it will grow hot, while the battery itself will remain comparatively cool.

427. Laws of Development of Heat: Joule's Law.-To investigate the development of heat by a

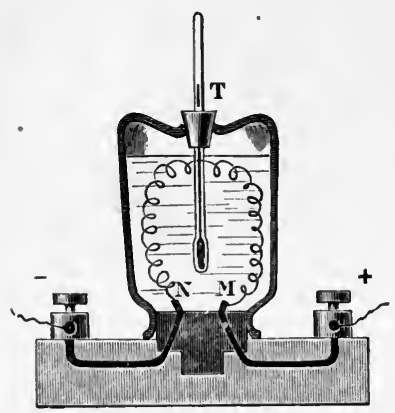

Fig. 226. current, Joule and Lenz used instruments on the principle shown in Fig. 226. A thin wire joined to two stout conductors is enclosed within a glass vessel containing alcohol, into which also a thermometer dips. The resistance of the wire being known, its relation to the other resistances can be calculated. Joule found that the number of units

of heat developed in a conductor is proportional-

(i.) to its resistance ;

(ii.) to the square of the strength of the current; and

(iii.) to the time that the current lasts.

The equation expressing these relations is known as Joule's Law, and is -

$$
\mathrm{U}=\mathrm{C}^{2} \mathrm{R} t \times 0 \cdot 24,
$$


where $\mathrm{C}$ is the current in amperes, $\mathrm{R}$ the resistance in ohms, $t$ the time in seconds, and $U$ the heat in calories; one calorie being the amount of heat that will raise 1 gramme of water through $1^{\circ}$ C. of temperature (Art. 281).

This equation is equivalent to the statement that $a$ current of one ampere flowing through a resistance of one ohm develops therein 0.24 calories per second. The proof of this rule is given in Art. 439. The heat produced thus by the degradation of energy in a resistance is sometimes called the "ohmic" heat to distinguish it from the reversible Peltier effect (Art. 420).

The electric unit of heat, the joule, is only 0.24 of an ordinary heat-unit or calorie, and 1 calorie will be equal to $4 \cdot 2$ joules.

The second of the above laws, that the heat is, cateris paribus, proportional to the square of the strength of the current, often puzzles young students, who expect the heat to be proportional to the current simply. Such may remember that the consumption of zinc is, cceteris paribus, also proportional to the square of the current; for, suppose that in working through a high resistance (so as to get all the heat developed outside the battery) we double the current by doubling the number of battery cells, there will be twice as much zinc consumed as before in each cell, and as there are twice as many cells as at first the consumption of zinc is four times as great as before.

428. Favre's Experiments. - Favre made a series of most important experiments on the relation of the energy of a current to the heat it develops. He ascertained that the number of calories evolved when 33 grammes (1 equivalent) of zinc are dissolved in dilute sulphuric acid (from which it canses hydrogen to be given off) is 18,682 . This figure was arrived at by conducting the operation in a vessel placed in a eavity of his calorimeter, an instrument resembling a gigantic thermometer filled with mercury, the expransion of which was proportional to the beat imparterl to it. When a Snee's cell was introduced into 
the same instrument, the solution of the same amount of zinc was observed to be accompanied by the evolution of 18,674 calories (i.e. an amount almost identical with that observed before), and this amount was the same whether the evolution took place in the battery-cell when the circuit was closed with a short thick wire, or whether it took place in a long thin wire placed in the external circuit. He then arranged 5 Smee's cells in series, in cavities of the calorimeter, and sent their current round a small electric motor. The amount of heat evolved during the solution of 33 grammes of zinc was then observed in three cases: (i.) when the motor was at rest; (ii.) when the motor was running round and doing no work beyond overcoming the friction of its pivots ; (iii.) when the motor was employed in doing 13,124,000 grammecentimetres $\left(=12,874 \times 10^{6} \mathrm{ergs}\right)$ of work, by raising a weight by a cord running over a pulley. The amounts of heat evolved in the circuit in the three cases were respectively, 18,667, 18,657, and 18,374 calories. In the last case the work done accounts for the diminution in the heat wasted in the circuit. If we add the heat-equivalent of the work done to the heat evolved in the latter case, we ought to get the same value as before. Dividing the $12,874 \times 10^{6}$ ergs of work by Joule's equivalent $\left(42 \times 10^{6}\right)$, we get as the heat-equivalent of the work done 306 calories. Now $18,374+306=18,680$, a quantity which is almost identical with that of the first observation, and quite within the limits of unavoidable experimental error.

429. Rise of Temperature. - The elevation of temperature in a resisting wire depends on the nature of the resistance. A very short length of a very thin wire may resist just as much as a long length of stout wire. Each will cause the same number of units of heat to be evolved, but in the former case, as the heat is spent in warming a short thin wire of small mass, it will get very hot, whereas in the latter case it will perluaps only warm to an imperceptible degree the mass of the long thick wire, which, moreover, has a larger surface to get rid of its heat. If the wire weigh $w$ grammes, and have a specific capacity for heat $s$, then $\mathrm{U}=s w \theta$, where $\theta$ is the rise of temperature in degrees (Centigrade). Hence if none of the heat were radiated a way

$$
\theta=0.24 \times \frac{\mathrm{C}^{2} \mathrm{R} t}{s w} .
$$


Since the resistance of metals increases as they rise in temperature, a thin wire heated by the current will resist more, and grow hotter and hotter until its rate of loss of heat by conduction and radiation into the surrounding air equals the rate at which heat is supplied by the current.

The following pretty experiment illustrates the laws of heating. The current from a few cells is sent through a chain made of alternate links of silver and platinum wires. The platinum links glow red-hot while the silver links remain comparatively cool. The explanation is that the specific resistance of platinum is about six times that of silver, and its eapacity for heat about half as great; hence the rise of temperature in wires of equal thickness traversed by the same current is roughly twelve times as great for platinum as for silver.

Thin wires heat much more rapidly than thick, the rise of temperature in different parts of the same wire (carrying the same current) would be, for different thicknesses, inversely proportional to the fourth power of the diameters if they had equal surfaces for radiation.

Thus, suppose a wire at any point to become reduced to half its diameter, the cross-section will have an area $\frac{1}{4}$ as great as in the thicker part. The resistance here will be 4 times as great, and the number of heat units developed will be 4 times as great as in an equal length of the thicker wire. But 4 times the amount of heat spent on $\frac{1}{4}$ the amount of metal would warm it to a degree 16 times as great: and the thin wire has only half as much surface for getting rid of heat. But the hotter a body becomes the more freely does it radiate heat to things around it. For wires of given material, the current needed to raise them to an equal temperature varies as the square root of the cube of the diameter. This law applies to the sizes of wires used as safety-fuses in electric lighting. These are pieces of tin wire interposed in the circuit to melt if by any chance the current becomes abnormally strong. 
430. Cardew's Voltmeter.-The current flowing through a long thin wire of platinum when it is made to

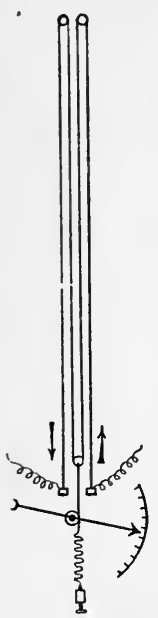

Fig. 22\%. connect two points on a circuit will measure the potential difference between these two points. Owing to its becoming warmed it will expand, and its expansion may be made to move a hand over a dial graduated to read volts (Fig. 227).

431. Electric Cautery.-For surgical purposes a thin platinum wire, heated redhot by a current, is sometimes used instead of a knife, as, for example, in the operation of amputating the tongue for cancer. Platinum is chosen on account of its infusibility, but even platinum wires are fused by the current if too strong. Carbon alone, of conductors, resists fusion.

432. Blasting by Electricity.-In consequence of these heating effects, electricity can be applied in blasting and mining to ignite the charges. Stout conducting wires are carried from an appropriate battery at a distance to a special fuze, in which a very thin platinum wire is joined in the circuit. This wire gets hot when the current flows, and being laid amidst an easily combustible substance to serve as a priming, ignites this and sets fire to the charge of gunpowder. Torpedoes can thus be exploded beneath the water, and at any desired distance from the battery.

433. Electric Welding.-If two wires or rods of metal are held together with sufficient force while a very large current is passed through them, much heat is developed at the junction, so that they soften and hecome welded together. The processes of electric welding have been perfected by Elihu Thomson, who has utilized for this purpose alternate-current transformere (Art. 480) to 
produce currents of many hundred amperes at a pressure of a few volts.

A singular effect is noticed when two iron rods connected to the poles of a powerful source at 50 or more volts are dipped into water. The rod which serves as kathode is observed to be corered with a luminous layer, and it presently becomes red-hot. Guthrie, who first investigated this phenomenon in 1876, ascribed the heating to the resistance of a film of hydrogen. Recently it has been made the basis of a welding method.

434. Electric Cooking.--Since public supplies of electricity became common, electric stoves, ovens, and heaters for cooking, stewing, etc., have become articles of commerce. The heating is effected by passing currents through resistance wires embedded in cement or other suitable insulating material.

\section{Lesson XXXVII.-Electric Energy: its Supply and Measurement}

435. Electric Energy.-An electric current conveys energy from a battery or dynamo to some other part of the circuit, where it is transformed back into work,-mechanical, chemical, or thermal work. We must inquire into this electrical energy, and into the rate at which it is generated or transformed.

Power is the rate at which energy is being received or spent. It may be expressed in foot-pounds per second or in ergs per second. James Watt considered a horse capable on the average of working at the rate of 550 footpounds per second (against gravity). As 1 foot $=30.48$ centimetres, and the force of $1 \mathrm{lb} .(=453.6$ grammes $\times 981)$ $=445,000$ dynes nearly, it follows that 1 horse-power is worth $7,460,000,000$ (or $746 \times 10^{7}$ ) ergs per second.

If a quantity of electricity $Q$ is moved through a difference of potential $\mathrm{V}$, it follows from the definition 
(Art. 263) that the work done is equal to QV. If this is occurring in a battery or dynamo, $\mathrm{QV}$ represents electrically the work (chemical or mechanical) done on the system, or the energy received (electrically) by the system. Now, suppose this operation to have occupied time $t$, the rate at which the energy is being imparted to the circuit will be $\mathrm{QV} / t$. But (Art. 162) $\mathrm{Q} / t=\mathrm{C}$. Hence the power given to the circuit is equal to $\mathrm{CV}$.

This justifies the statement that the power of an electric current to perform useful work, whether in lighting, heating, or producing mechanical actions, is proportional both to the strength of the current, and to the electromotiveforce which drives it. In other words, power is proportional to both amperes and volts jointly. Similarly the power of a steam engine is proportional not only to the quantity of steam it uses, but also to the pressure at which the steam is supplied. The electric unit of power will then be the power of a current of 1 ampere driven by an electric pressure of 1 volt. This unit is known as 1 volt-ampere, or 1 watt.

Since 1 volt $=10^{8}$ absolute units of E.M.F. (Art. 354) and 1 ampere $=10^{-1}$ absolute units of current (Art. 354), it follows that 1 watt $=10^{7}$ absolute units of power (i.e. $10^{7}$ ergs per second). But 1 horse-power $=746 \times 10^{7}$ ergs per second (see above). Hence 1 H.-P. $=746$ watts.

One thousand watts is called 1 kilowatt. The kilowatt is therefore approximately $1 \frac{1}{3}$ H.P.

To find the number of watts of power supplied by any dynamo or battery, multiply the number of amperes of current by the number of volts at which the current is driven. The same rule serves to calculate the power electrically delivered to any motor, lamp, accumulator, or other means of spending electric energy.

Horse-power $=\mathrm{C} \times \mathrm{V} \div 746$.

Example.-If a current of 20 amperes is supplied to a big arc-lamp at a pressure of 56 volts, find the amount of power absorbed therein. Ans. 1120 watts or $1 \frac{1}{2}$ H.P. 
436. Intake and Output of Power.-At any generator battery, dynamo, or thermopile, power is taken in to the electric circuit. At any motor or lamp, or at any part in the circuit where chemical work (electroplating, decomposing gases, or charging accumulators) is being done or at any place where heat is being evolved, power is being given out by the electric circuit. At every place where energy is coming in to the circuit there will be an electromotive-force in the same direction as the current, and helping to drive it. At every part where energy is being given out by the circuit there will be an electromotive-force in a direction opposed to the current.* The word output, $\uparrow$ as applied to dynamos, etc., means the number of watts or kilowatts which the machine supplies or can supply. For example, a dynamo capable of supplying 300 amperes "at" 100 volts (meaning with an available E.M.F. of 100 volts) is said to have an output of 30 kilowatts.

437. Power - Measurement.-To measure the power given electrically to any part $a b$ of a circuit by an unvarying current,. it suffices to measure the current with an ampere-meter

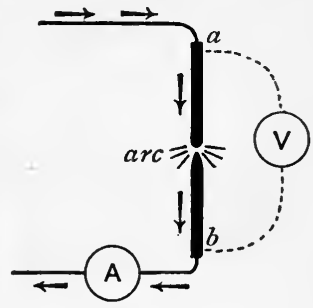

Fig. 22S. (Art. 221), and the potentials across the part with a voltmeter (Arts. 220, 290), the latter being of course con-

* Consider the mechanical analogue of transmission of power from one pulley to another pulley by a belt. The effort in the driving pulley is in the same direction as the motion of the belt. The effort in the driven pulley is opposed in direction to the motion. (See also Art. 24S.)

This fundamental principle accounts for the back-electroinotive-forces observed in motors, and in accumulators while being charged. Because of it we know (Art. 166) that the seat of the main electromotive-force in a voltaic cell is at the surface of the zinc, and that (Art. 422) bismuth is thermo-electrically positive to antimony.

† The word output, as applied to central station work, is sometimes used in sense of total outflow of amperes irrespective of voltage. 
nected as a shunt as in Fig. 228. The product of volts and amperes gives the watts. Or a wattmeter may be used as below.

438. Wattmeters.-The product of amperes and volts may be measured directly by means of a wattmeter. This name is given to a variety of electrodynamometer (Art. 394) in which the fixed and movable coils constitute two separate circuits, one being a thick wire of low resist-

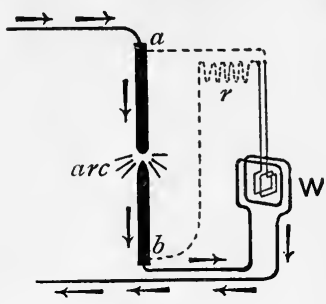

Fig. 229. ance to carry the amperes, the other being, or including, a thin wire of high resistance (as in voltmeters) to receive a current proportional to the volts. The latter circuit is to be connected as a shunt to the part $a b$ of the circuit in which the supplied power is to be measured. In Fig. 229, as in Fig. 228, the part $a b$ is an arclamp. The auxiliary resistance $r$ is introduced into the thin-wire circuit of the instrument, the whole current flowing through the thick-wire circuit.

Wattmeters are made both on the pattern of Siemens's dynamometer (Art. 395) and on that of Kelvin's balances (Art. 396).

When power-measurements have to be made on alternate-current circuits, separate instruments must not be used, as in Art. 437, to measure volts and amperes. For, owing to the differences of phase (Art. 472) between voltage and current, the apparent walts, got by multiplying the separate readings, will be in excess of the true watts as measured by a wattmeter.

439. Power wasted in Heating.-If a current $\mathrm{C}$ is driven through a resistance $R$, the volts neeled will (by Ohm's law) he

$$
\mathrm{V}=\mathrm{CR} \text {. }
$$

The power $\mathrm{CV}$ so expended will merely heat the resistance. 
Substitute for $\mathrm{V}$ its value as above, and we have

$$
\text { Watts wasted }=\mathrm{CV}=\mathrm{C}^{2} \mathrm{R}=\mathrm{V}^{2} / \mathrm{R} \text {. }
$$

Or, if the expenditure goes on for $t$ seconds, the amount of energy turned into heat (joules) will be

$$
\text { Energy }=\mathrm{QV}=\mathrm{C} t \mathrm{~V}=\mathrm{C}^{2} \mathrm{R} t \text {. }
$$

The nett power of a dynamo or battery is always less than its gross power, because of internal resistance. If $r$ be the internal resistance, and $\mathrm{E}$ the whole electromotiveforce, the nett or available volts $\mathrm{V}=\mathrm{E}-\mathrm{Cr}$. The gross power will be EC watts. But the nett power will be $\mathrm{VC}=\mathrm{EC}-\mathrm{C}^{2} r$. Or, the available watts equal the total watts generated, less the watts wasted in internal heating.

To prove Joule's law of heating as given in Art. 427, it may be remembered that the mechanical equivalent of heat is 42 million ergs to 1 calorie (Joule's equivalent), or $\mathrm{W}=\mathrm{JU}$, where $\mathrm{W}$ is the work in ergs, $U$ the heat in calories, and $J=4 \cdot 2 \times 10^{7}$. Hence $\mathrm{U}=\mathrm{C}^{2} \mathrm{R} t / \mathrm{J}$. But to reduce the work to ergs we must multiply $\mathrm{C}^{2} \mathrm{R} t$ by $10^{7}$; whence $\mathrm{U}=\mathrm{C}^{2} \mathrm{R} t \times 0.24$.

440. Distribution of Electric Energy.-Electric energy is now distributed on a large scale for lighting, motive power, and heating. Large Central Stations or Power-houses are erected at convenient spots, with steamengines or turbines (if water-power is available) to drive generating machinery (dynamos and alternators). From the power-house distributing mains of copper go out, consisting of feeders leading into the network of conductors that runs from house to house.

Supply systems may be classified according to whether they operate at a low voltage (or low pressure), i.e. from 100 volts (or under) to 300 volts; high voltage, i.e. from 300 to 3000 volts; or extra high voltage, over 3000 volts. The low-voltage systems generally use continuous currents, the high - voltage systems generally (but not necessarily) use alternate currents, and transformers (Art. 
480) to transform to low pressure at the consumers' houses.

Example. -The City of London Electric Lighting Company generates alternate currents at a little over 2000 volts at its power-house on the south side of the Thames, and sends these currents through the feeders to sub-stations in the city, where they are transformed down to currents twenty times as large at a pressure of 100 volts ; at which low pressure they supply the network of mains and house branches, which are laid in conduits under the streets.

Since the power of a current depends on the voltage at which it is supplied, the unit of supply recognized in law is based on the unit of power, the watt (Art. 435), and is defined as 1000 watts supplied for one hour (i.e. 1 kilowatt-hour) or its equivalent. The maximum price which the English Board of Trade permits the supply company to charge the consumer for 1 unit is eightpence.

441. Conditions of Electric Supply.-Electric energy is almost always supplied under one of two standard conditions, either-

(a) at Constant Voltage, or

(b) with Constant Current.

In the former case the circuit is branched, and the current is supplied (usually at 100 volts) to all the lamps or motors in parallel (Art. 409), each lamp, etc., being independent of all others ; and the current varying precisely in proportion to the demand.

In the latter case, seldom used except for strings of arc-lamps, the circuit is undivided, and the current (usually 10 amperes) flows through all the lamps in series (Art. 168). If lamps are turned out (by short-circuiting them) the voltage must be reduced to keep the current constant.

442. Supply Meters.-Meters for measuring the supply to the houses of consumers are of several kinds.

(a) Chemical Meters.-The current or a known fraction 
of it is passed through an electrolytic cell, there to deposit copper (Edison's method) or dissolve zinc (Jehl's improved Edison). The amount of chemical action is proportional to the ampere-hours.

(b) Integrating Meters. - A uniformly-going clock drives a counting apparatus through an intermediate gear operated by the current (or by the watts), this intermediate gear being such that when current is small counting is small, when current large counting is large. An integrating disk-and-roller, or an integrating cam, is a usual mechanism, its operation being controlled by the motion of an ampere-meter or wattmeter.

(c) Motor Meters.-If the current passes through the armature of a small motor (Art. 443) having a constant field, and having its speed controlled purely by fluid friction (by a fan) or by eddy-current friction (in a copper conductor revolving between magnet poles, Art. 457), its speed will at every instant be proportional to the current. Hence such a motor attached to a suitable counting-train of wheels will serve as a meter, the total number of revolutions being proportional to the amperehours. In Perry's meter (1893) the revolving part is a copper bell immersed in mercury, revolving around a central magnet pole (as the wire does in Fig. 201), and surrounded by an external S-pole with ribbed projections to promote eddy-currents. In Shallenberger's meter for alternate currents the motor drives a fan. In Elihu Thomson's meter, which records the watt-hours, the revolving armature is of fine wire and high resistance, connected as shunt, while the fixed coils that serve as ficld-magnet take the whole current supplied. So the torque is proportional to the watts; while a copper disk revolving between magnet poles, by its drag keeps the speed proportional to the torque. 
(d) Retarded Clocks.-Current may be made to act upon the rate of a clock, by flowing in a coil under the pendulum bob if the latter is a magnet. Any force added thins to gravity or subtracted from it will cause the clock to gain or lose. Ayrton and Perry proposed to measure the supply by the total time gained or lost by a clock. In Aron's meter, of which this is the principle, there is a double clock with two pendulums, only one of which is acted on by the current. A train of counting wheels is geared to record the difference between the two.

\section{Lesson XXXVIII.-Electric Motors (Electromagnetic Engines)}

443. Flectric Motors-Electromagnetic engines, or motors, are machines in which the motive power is derived from electric currents by means of their electromagnetic action. In 1821 Faraday showed a simple case (Art. 393) of rotation produced between a magnet and a current of electricity. Barlow produced rotation in a star-wheel, and Sturgeon in a copper disk, when traversed radially by a current while placed between the poles of a horse-shoe magnet. In 1831 Henry, and in 1833 Ritchie, constructed small engines producing rotation by electromagnetic means. Fig. 230 shows a modification of Ritchie's motor. An electromagnet DC is poised upon a vertical axis between the poles of a fixed magnet (or electromagnet) SN. A current, generated by a suitable battery, is carried by wires which terminate in two mercury-cups, A, B, into which dip the ends of the coil of the movable electromagnet CD. When a current traverses the coil of CD it turns so as to set itself in the line between the poles NS, but as it swings round, the wires that dip into the mercury-cups pass from one cup 
to the opposite, so that, at the moment when $\mathrm{C}$ approaches $\mathrm{S}$, the current in CD is reversed, and $\mathrm{C}$ is repelled from $\mathrm{S}$ and attracted round to $\mathrm{N}$, the current through $\mathrm{CD}$ being thus reversed every half turn. In larger motors the mercury-cup arrangement is replaced by a commutator (devised by Sturgeon), consisting of a copper tube, slit into two or more parts, and touched at opposite points by a pair of metallic springs or "brushes."

In another early form of motor, devised by Froment, bars of iron fixed upon the circumference of a rotating cylinder are attracted up towards an electromagnet, in which the current is automatically broken at the instant when each bar has come close up to its poles. In a third kind, an electromagnet is made to attract a piece of soft iron alternately up and down, with a motion like the piston of a steam-engine, which is con-

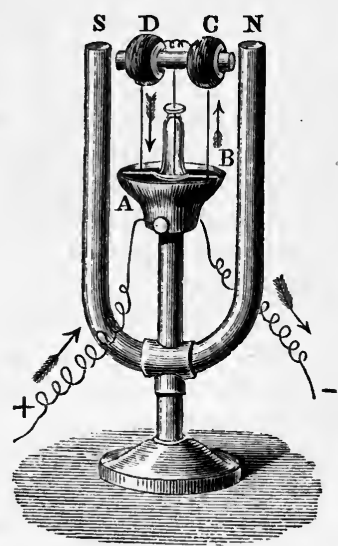

Fig. 230. verted by a crank into a rotatory motion. In these cases the difficulty occurs that, as the attraction of an electromagnet falls off rapidly at a distance from its poles, the attracting force can only produce effective motion through very small range. Page from 1838 to 1850 designed various motors, in some of which iron plungers were sucked into hollow tubular coils of wire in which currents were caused to circulate at recurring intervals.

In 1839 Jacobi propelled a boat along the river Neva at the rate of $2 \frac{1}{4}$ miles per hour with an electromagnetic engine of about one horse-power, worked by a battery of 64 large Grove's cells. 
Jacobi appears to have been the first to recognize, about 1850, that the action of the electric motor is the simple converse of that of the dynamo or generator. Every magneto-electric generator or dynamo, such as is used in electric lighting, can also work as a motor, giving out mechanical power when supplied with electric currents from some other source. Indeed the dynamos designed as generators make far more efficient motors than any of the older sorts of electromagnetic engines, which were little more than toys.

In 1882 an iron screw-boat capable of carrying 12 persons, and driven by two such motors, with a power of about 3 horse-power, the current being furnished by 45 accumulators, was worked upon the Thames at a speed of 8 miles per hour. There is now a whole flotilla of electric launches on the Thames.

444. Modern Electric Motors.-These are of two kinds: (1) those for use with continuous currents; (2) those for use with alternate currents. The former are constructed precisely on the plan of continuous current dynamos (Art. 462) having fixed field magnets and rotating armature. The armature is dragged round by the mutual action of the currents flowing in the copper conductors and the magnetic field in which the conductors lie. As explained in Art. 340, the force acting laterally on the conductors is proportional to the product of current and field. Hence if very powerful field-magnets are employed, a great torque (or turning moment) can be produced without requiring too great a current to be sent into the armature. The two factors of mechanical rotatory power are torque (= angular force) and angular speed. If the field of the motor is maintained constant the torque is proportional to the current and the speed is proportional to the volts. If $\mathrm{E}$ is the electromotive-force generated (in direction opposing the current, see Art. 436) in the revolving armature, and $\mathrm{C}$ the current supplied to it, the electrical and mechanical expressions for the power 
(watts) imparted to the armature are

$$
\mathrm{CE}=a n \mathrm{~T},
$$

where $n$ is revolutions per second, $\mathrm{T}$ the torque, and $a$ a coefficient depending on the units chosen.

If the armature current is supplied from mains at constant voltage, strengthening the magnetic field has the effect of slowing speed, for equal power ; and weakening the field quickens the speed. Alternate-current motors are described in Arts. 484 to 486 .

445. Effliency of Motors.-If an ampere-meter be included in.the circuit with a battery and a motor, it is found that the current is weaker when the motor is working than when the motor is standing still, and that the faster the motor runs the weaker does the current become. This is due to the E.M.F. generated in the revolving armature of the motor, which necessarily (Art. 436) opposes the current. If the motor only exerts a small back electromotive-force it cannot utilize much of the power of the current. If $\mathrm{V}$ be the volts at which the current is supplied, and $\mathrm{E}$ the counter-electromotive-force generated by the motor, and $\mathrm{C}$ the current, then $\mathrm{VC}=$ gross power supplied, EC = nett power utilized; and dividing the latter by former we get, as the electrical efficiency of the motor, the ratio $\frac{\mathrm{E}}{\mathrm{V}}$.

Example.-Suppose $\mathrm{V}=100$ volts and $\mathrm{E}=90$ volts, the efficiency will be 90 per cent.

To make the efficiency as high as possible the motor should be so arranged (either by strengthening its magnetic field, or by letting it run faster) that $\mathrm{E}$ is very nearly equal to $\mathrm{V}$. In that case the motor will utilize nearly all the energy that flows to it. But since, by Ohm's law, the current is $=(\mathrm{V}-\mathrm{E}) / r$, where $r$ is the internal resistance of the motor, it follows that when $\mathrm{E}$ becomes nearly equal to $\mathrm{V}$ the current will be reduced to 
a small fraction of what it would be if the motor were at rest. The diagram (Fig. 231) makes the matter plainer. Let the line OV represent by its length the volts of supply $\mathrm{V}$, and let $\mathrm{OE}$ represent the volts generated in the armature, proportional to speed and to field. On OV describe the square OVWX, and

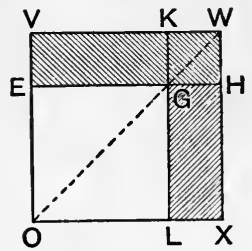

Fig. 231. draw the diagonal and the lines $\mathrm{EH}$, KL. Then the area EVWH is proportional to the gross power, being $\mathrm{V}(\mathrm{V}-\mathrm{E})$, and area GLXH is proportional to the nett power, being $\mathrm{E}(\mathrm{V}-\mathrm{E})$. These two areas become more nearly equal, though both become small, when $\mathrm{E}$ is increased to be nearly equal to $\mathrm{V}$. The area GLXH, the nett output of the motor, is a maximum when $\mathrm{E}=\frac{1}{2} \mathrm{~V}$; but then the efficiency would be only 50 per cent.

The fact that when $\mathrm{E}$ is small the current is enormous is of great advantage in the starting of motors; for at starting the great rush of current (which would destroy them if it lasted) produces a great torque, and the motor soon gets up speed and so cuts down the current to the working amount.

446. Electric Locomotion.-Motors placed on cars or on separate locomotives can propel them singly or in trains provided the requisite power is supplied. This may be done in several ways:-

(a) A battery of charged accumulators is carried on the car.

(b) Current is furnished from a power-house, to a third rail insulated from earth. From this the current is picked up by the car, the ordinary rails being used as return conductor.

(c) Current is furnished from a power-house to an overhead line, with which the car makes contact as it runs by means of a trolley-wheel fixed on a long rod above the car.

(d) Current is picked up by the car from conductors laid in a 
slot-conduit in the road between the rails, by means of a contact-piece let down into the slot.

(e) Current is picked up by the car from studs slightly projecting from the surface of the road between the rails, by means of a long skate fastened under the car, each stud being automatically connected to the underground mains as the car comes up and disconnected as it passes on.

Plan (a) is uneconomical, owing to expense of accumulators. Plan $(b)$ is used in several heavy electric railways in England, using locomotives of 200 to 400 horse-power. Plan $(c)$ is used for tramways of which there are now thousands of miles. Plan $(d)$ is used in New York, Berlin, and other cities where overhead wires are not allowed. Plan (e) is being introduced in Paris and other cities and is cheaper than the slot-conduit.

447. Electric Transmission of Power.-Power may be transmitted to great distances electrically from a generator at one end of the circuit to a motor at the other. A mountain stream may be made to turn a turbine which drives a dynamo or alternator, the currents from which are conveyed to some centre of population by insulated wires to the motor which reconverts the electrical power into mechanical power. Scores of such examples are now at work. In the striking demonstration at Frankfort, in 1891,140 horse-power was conveyed from the Falls of the Neckar at Lauffen, 117 miles away, through three wires only 4 millimetres in diameter, with a nett efficiency of 74 per cent, including all losses.

Fig. 232 illustrates the case of a simple transmission between two machines. In one the electromotive-force drives the current, in the other the electromotive-force opposes the current. The first acts as generator (by the principle of Art. 436), the second as motor. If their respective electromotive-forces are $\mathrm{E}_{1}$ and $\mathrm{E}_{2}$ the electrical efficiency of the transmission is the ratio $\mathrm{E}_{2} / \mathrm{E}_{1}$.

The power lost in the line by reason of its resistance is the chief difficulty to face in such transmissions, owing 
to the prohibitive price of copper for carrying large currents without overheating. The watts wasted in a line of resistance $R$ are (Art. 439) $=C^{2} R$. The gross watts utilized are (Art. 435) $=\mathrm{CV}_{\mathrm{M}}$, where $\mathrm{V}_{\mathrm{M}}$ is the volts at the motor end. Hence the power that must be poured in to the sending end of the line is $\mathrm{C}^{2} \mathrm{R}+\mathrm{CV}_{\mathrm{M}}$ watts. Now it will be obvious that one may keep the $\mathrm{C}^{2} \mathrm{R}$ loss constant

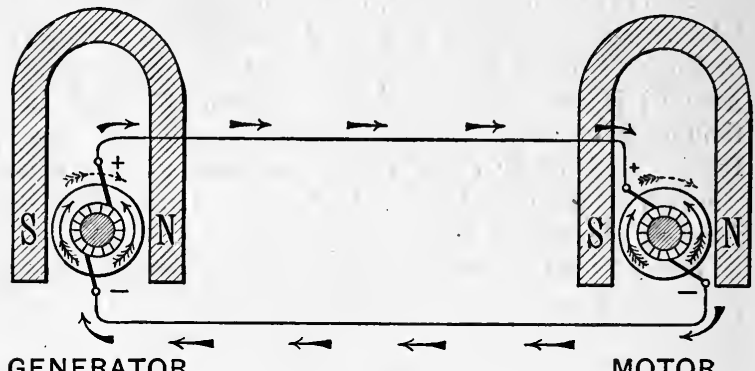

Fig. 232.

and yet increase the power that is transmitted by increasing $V_{M}$ the voltage at the motor-using in fact a highvoltage motor, and of course a high-voltage generator to correspond. To put the matter in another way. Let $\mathrm{V}_{\mathrm{G}}$ be the volts at the generator end of the line, $\left(\mathrm{V}_{\mathrm{a}}-\mathrm{V}_{\mathrm{M}}\right) \mathrm{R}$ will be $=\mathrm{C}$. Now we may keep $\mathrm{C}$ constant (and therefore the $\mathrm{C}^{2} \mathrm{R}$ loss constant) and yet increase the voltages, provided $V_{G}-V_{M}$ remains as before.

Example.-Suppose a line of copper-wire 20 miles long has resistance of $100 \mathrm{ohms}$. A current of 6 amperes in it will waste 3600 watts or nearly 5 horse-power. To send 6 amperes through 100 ohms requires a difference of potentials of 600 volts. Suppose $V_{G}=1000$ and $\mathrm{V}_{\mathbf{M}}=400, \mathrm{~V}_{\mathbf{a}}-\mathrm{V}_{\mathbf{M}}=600$. The watts sent in are $\mathrm{CV}_{\mathrm{G}}=6000$, and the watts delivered are $\mathrm{CV}_{\mathrm{M}}=2400$. Of 8 horse-power put in only about $3 \frac{1}{4}$ are delivered, the 
efficiency being $\mathrm{V}_{\mathbf{M}} / \mathrm{V}_{\mathrm{O}}=40$ per cent. Now suppose $V_{G}$ increased to 2000 volts, and $V_{M}$ to 1400 . $\quad V_{G}-V_{M}=$ 600 , as before. $\mathrm{C}=6$ amperes, as before. $C^{2} R$ loss is 3600 watts, as before. But watts sent in are now 12,000 (over 16 H.P.), and the watts delivered are 8400 (111 H.P.). Whilst the efficiency is now 70 per cent.

It is therefore clear that high voltage is the secret of success in the electrical transmission of energy, whether for lighting or power, to long distances. In the transmission of energy from the Falls of Tivoli to light the city of Rome sixteen miles away, a pressure of 5000 volts is successfully used. At the great power station of Niagara the currents are distributed in the district at 2250 volts, but for transmission to Buffalo, 16 miles distant, the voltage is raised to 11,000 .

\section{Lesson XXXIX.-Electric Light}

448. The Electric Arc.-If two pointed pieces of carbon are joined by wires to the terminals of a powerful voltaic battery or other generator of electric currents, and are brought into contact for a moment and then drawn apart to a short distance, a kind of electric flame called the arc or "voltaic" arc is produced between the points of carbon, and a brilliant light is emitted by the whitehot points of the carbon electrodes. This phenomenon was first noticed by Humphry Davy in 1800, and its explanation appears to be the following :-Before contact the difference of potential between the points is insufficient to permit a spark to leap across even $\frac{1}{10000}$ of an inch of air-space, but when the carbons are made to touch, a current is established. On separating the carbons the spark at parting volatilizes a small quantity of carbon between the points. Carbon vapour being a partial conductor allows the current to continue to flow across the gap, provided it be not too wide; but as the carbon vapour has a very high resistance it becomes intensely heated by the passage of the current, and the carbon points 
also grow hot. Since, however, solid matter is a better radiator than gaseous matter, the carbon points emit far more light than the arc itself, though they are not so hot. The temperature of the are is simply determined by the temperature at which carbon volatilizes; about $3500^{\circ} \mathrm{C}$. according to Violle. In the arc the most infusible substances, such as flint and diamond, melt ; and metals such as gold and platinum are even vaporized readily in its

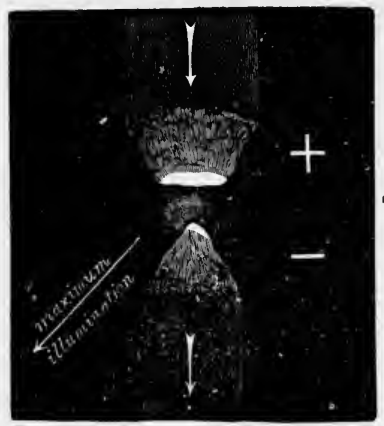

Fig. 233. intense heat. When the are is produced in the air the carbons slowly burn away by oxidization. It is observed, also, that particles of carbon are volatilized off ' and torn away from the + electrode, which becomes hollowed out to a cup-shape, or crater, and if the gap between the carbons is small some of these particles are deposited on the - electrode, which assumes a pointed form, as shown in Fig. 233.

The resistance of the arc may vary, according to circumstances, from $0.2 \mathrm{ohm}$ upwards, according to the length and section of the flame. The are also exerts an opposing electromotive-force of its own, amounting to about 35 volts when the are is silent. If air gets to the white hot crater the arc becomes unstable and hisses, and the back electromotive-force is much lower. The seat of this back electromotive-force is at the surface of the crater where the work of volatilizing the carbon is being done.

To produce an electric light satisfactorily a minimum electromotive-force of 40 to 50 volts is necessary if continuous currents are used. With alternate currents 30 to 35 volts suffice. The usual eurrent for arc lamps of 1000 to 2000 candle-power is from 5 to 10 amperes. With 
weaker currents or smaller electromotive-forces it is impracticable to maintain a steady arc. For search-lights on board ship and for lighthouses, arc lights of greater power are produced by using thicker carbons and supplying them with currents of 20 to 100 or more amperes. The common size of carbon rod in use is 10 or 11 millimetres in diameter: the consumption is roughly 1 inch per hour, the + carbon consuming much faster than the - carbon. Enclosed arcs from which free access of air is excluded, consume their carbons about twenty times slower. The internal resistance of ordinary Daniell's or Leclanché's cells is too great to render them serviceable for producing arc lights. A battery of 40 to 60 Grove's cells (Art. 182) will not last more than 2 or 3 hours. A dynamo-electric machine (Arts. 461 to 469 ) is the generator of currents in practical electric lighting. The quantity of light emitted by an arc lamp differs in different directions, the greatest amount being emitted (when the + carbon is at the top) at an angle of about $45^{\circ}$ downwards. Most of it comes from the white-hot crater, very little from the negative point. In the alternate-current arc the carbon points are alike and emit equal light. The current must not alternate more slowly than 40 periods per second. The total quantity of light emitted, when the current is supplied at a fixed voltage, is not quite proportional to the current, but increases in a somewhat higher ratio. Doubling the current makes rather more than twice as much light.

449. Arc Lamps.-Davy employed wood charcoal for electrodes to obtain the arc light. Pencils of hard gas-carbon were later introduced by Foucault. In all the more recent arc lamps, pencils of a more dense and homogeneous artificial coke-carbon are used. These consume away more regularly, and less rapidly, but still some automatic contrivance is necessary to push the points of the carbons forward as fast as neeled. The mechanism of the arc lamp should "strike" the arc by causing the 
pencils to touch, and then separate them to the requisite distance, about 5 millimetres; the mechanism should also "feed" the carbons into the arc as fast as they are consumed, and it should also cause

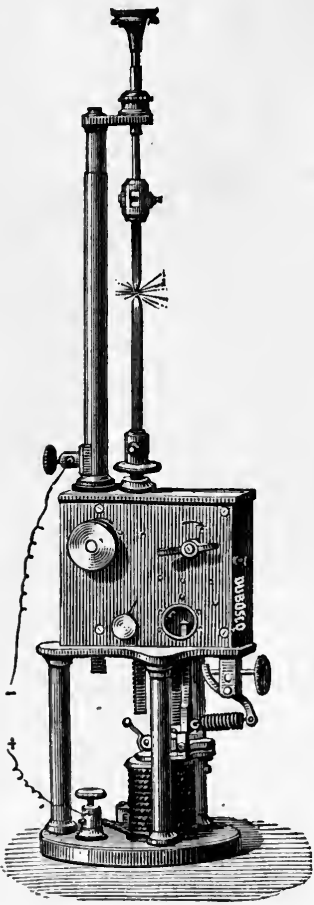

Fig. 234. the points to approach or recede automatically in case the are becomes too long or too short; it should further bring the carbons together for an instant to strike the arc again if by any chance the flame goes out. Arc Lamps or "regulators," fulfilling these conditions, have been invented by a number of persons. The earliest was invented in 1847 by W.E. Staite. Arc lamps may be classified as follows :-

(a) Clockwork Lamps.-Fig. 234 shows the regulator of Foucanlt as constructed by Duboseq; in this lamp the carbon-holders are propelled by a train of clockwork wheels actuated by a spring. An electromagnet at the base, through which the current runs, attracts an armature and governs the clockwork. If the current is too strong the armature is drawn down, and the clockwork draws the carbons farther apart. If the current is weakened by the increase of the resistance of the arc as the carbons burn away, the armature is drawn upwards by a spring, and a second train of wheels comes into play and moves the carbons nearer together. Clockwork arc 
lamps have also been devised in which the weight of the carbon-holders drives the clockwork mechanism. Of this class was Serrin's lamp, which from 1855 to the present time has been largely used for lighthouses, and for the optical lantern.

(b) Brake-wheel Lamps.-Another mechanism for regulating the rate of feeding the carbon into the arc consists in the addition of a brake-wheel; the brake which stops the wheel being actuated by an electromagnet which allows the wheel to run forward a little when the resistance of the arc increases beyond its normal amount. In Fig. 235 $\mathrm{B}$ is the brake-wheel, $\mathrm{L}$ the lever which governs it, $\mathrm{C}$ an iron core of the coil $\mathrm{S}$ inserted in the circuit. When current is switched on, the core is drawn up, causing $\mathrm{L}$ to grip $\mathrm{B}$ and turn it a little, so parting the carbons and striking the arc.

(c) Solenoid Lamps.-In this class of arc lamp one of the carbons is attached to an iron plunger capable of sliding vertically up or down inside a hollow coil or solenoid, which, being traversed by the current, regulates

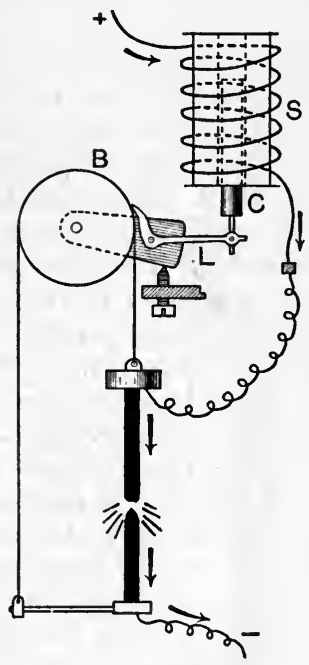

Fig. 235 . the position of the earbons and the length of the arc. Siemens employed two solenoids acting against one another differentially, one being a main-circuit coil, the other being a fine-wire coil connected as a shunt to the arc. The shunt coil acts as a voltmeter to watch the arc and feed the carbons forward when the volts rise above the normal, it being set to control the feeding mechanism. (d) Clutch Lamps.-A somewhat simpler device is that 
of employing a clutch to pick up the upper carbonholder, the lower carbon remaining fixed. In this kind of lamp the clutch is worked by an electromagnet, through which the main current passes. If the lamp goes out the magnet releases the clutch, and the upper carbon falls by its own weight and touches the lower carbon. Instantly the current starts round the electromagnet, which causes the clutch to grip the carbon-holder, and raise it to the requisite distance. Should the arc grow too long, the lessening attraction on the clutch automatically permits the carbon-holder to advance a little.

(e) Motor Lamps.-Sometimes little electric motors are used to operate the carbons automatically.

450. Grouping of Arc Lamps.-If the condition of supply is constant voltage the arc lamps must be set in parallel; if the arc lamps are to be run in series, the same current flowing in succession through each of the lamps, then the supply must be of a current of unvarying strength. In this case a shunt circuit is necessary in each lamp to work the regulating gear.

451. Electric Candles. - To obviate the expense and complication of such regulators, electric candles have been suggested. Fig. 236 depicts Jablochkoff's candle, consisting of two parallel pencils of hard carbon separated by a thin layer of plaster of Paris and supported in an upright holder. The arc plays across the summit between the two carbon wicks. In order that both carbons may consume at equal rates, alternating currents must be employed.

452. Incandescent Lamps or GlowLamps.-Arc lamps of an illuminating power of less than 100 candles are very unsteady and Fig. 236 . uneconomical. For small lights it is both simpler and cheaper to employ a thin continuous wire or filament of some infusible conductor, heated to whiteness by passing a current through it. Thin wires of platinum 
or iridium have repeatedly been suggested for this purpose, but they cannot be kept from risk of fusing. Edison in 1878 devised a lamp consisting of a platinum spiral combined with a short-circuiting switch to divert the current from the lamp in case it became overheated. Swan in February 1879 publicly showed a carbon wire lamp in a vacuous bulb. Edison in October 1879 devised a vacuum lamp with a coiled filament made of lamp black and tar carbonized. Swan in January 1880 prepared filaments from cotton thread parchmentized in sulphuric acid, and afterwards carbonized. Edison in 1880 substituted a flat strip of carbonized bamboo for a filament.

Modern glow-lamps mostly have thin carbon wires prepared from parchmentized cellulose, which is then carbonized in a closed vessel. Sometimes the filaments are "flashed" over with surface carbon by being momentarily heated electrically in a carbonaceous atmosphere. They are mounted upon platinum supports in a.glass bulb through which the platinum wires pass out, and into which they are sealed, the bulbs being afterwards exhausted of air and other gases, the vacuum being made very perfect by the employment of special mercurial air-pumps. The bulbs should be heated during exhaustion to drive out residual gases. Carbon is the only suitable material for the conductor because of its superior infusibility and higher resistance. It also has the remarkable property, the reverse of that observed in metals, of offering a lower resistance when hot than when cold. Two common forms of glow-lamp are shown in Fig. 237: the typical form used by Swan in England, and the typical form perfected by Edison in America. The resistance of such lamps varies according to size and length of the filament. A modern 16 candle-power lamp for use on a 100 -volt circuit will take about 0.6 ampere. That is to say, its resistance when hot will be about 166 olmms (or over 200 ohms when cold), and it will absorb about 60 watts. This is at the 
rate of less than 4 watts per' candle. Used so, it will

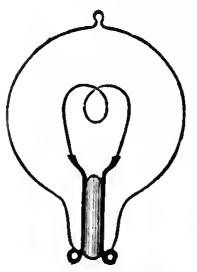

Fig. 237. last on the average over 1000 hours of burning. Lamps are made to give equal light and use less current, by using a thinner and rather shorter filament; but then they do not last so long. The surface disintegrates in time if forced to emit too much light. The power required to operate 12 such 60 watt lamps will be 720 watts, or nearly 1 horse-power.

The following table gives some data about a 10-candle 50 -volt lamp if used at different voltages :

\begin{tabular}{|c|c|c|c|c|c|}
\hline Volts. & Ainperes. & Watts. & $\begin{array}{c}\text { Candle } \\
\text { power. }\end{array}$ & $\begin{array}{c}\text { Watts per } \\
\text { candle. }\end{array}$ & $\begin{array}{c}\text { Probable } \\
\text { life(hours). }\end{array}$ \\
\cline { 1 - 4 } & & & & & \\
\hline 58 & $0 \cdot 77$ & 37 & 8 & $4 \cdot 3$ & 3200 \\
50 & $0 \cdot 81$ & $40 \cdot 5$ & 10 & $4 \cdot 05$ & 1500 \\
53 & $0 \cdot 87$ & $46 \cdot 4$ & $14 \cdot 5$ & $3 \cdot 2$ & 800 \\
55 & $0 \cdot 92$ & $50 \cdot 6$ & $18 \cdot 5$ & $2 \cdot 7$ & 480 \\
58 & $0 \cdot 99$ & $57 \cdot 5$ & $25 \cdot 5$ & $2 \cdot 2$ & 250 \\
61 & $1 \cdot 06$ & $64 \cdot 7$ & $35 \cdot 5$ & $1 \cdot 8$ & 150 \\
\hline
\end{tabular}

The light increases as about the sixth power of the volts; the energy consumed is only as the second power. But raising the volts a little shortens the life enormously.

For special lamps of larger candle-power, up to 800 or 1000, thin filaments cannot be used. In these flat strips or thick wires of carbon are used; they give out, for equal expenditure of power, much less light than an arc lamp.

453. Grouping of Glow-Lamps.-Glow-lamps are usually grouped in parallel (Fig. 238) between mains kept at constant voltage. A common value for the 
difference of potential between the + and - mains is 100 volts. The current in the mains subdivides and flows through each lamp independently. When any lamp is switched on it does not diminish the current in the others, but by opening an additional path simply causes proportionately more current to flow from the source of supply. The method of

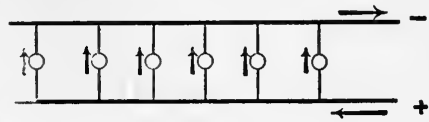

Fig. 238. grouping in series (Art. 168) is seldom used for glowlamps; each lamp then requires an automatic cut-out to prevent the rest of the row from being extinguished in case one lamp goes out.

Three-wire systems, in which a third or neutral wire is introduced between the + and the - main, have been devised to enable

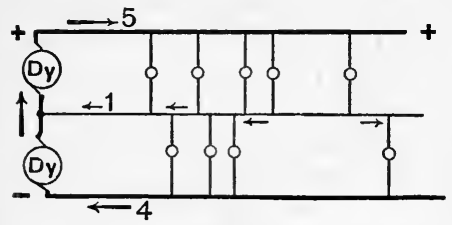

Fig. 239. higher voltages to be used, and thereby enable twice as many lamps to be lit with little additional expenditure in copper. To render the lamps on one side of the circuit (Fig. 239) independent of those on the other, in case an equal number do not happen to be switched on at the same time, the middle wire (which only need be thick enough to carry a current equal to the difference between the currents in the two outer wires) is carried back to the station and kept at mean potential between the two outer wires by the use of two dynamos instead of one.

453a. Nernst Lamps.-Prof. Nernst has devised lamps in which a thin thread of magnesia or other earthy oxide is rendered incandescent. Such threads do not conduct until first raised to a dull red heat: but they consume less than 1 watt per candle. 


\title{
CHAPTER IX
}

\author{
INDUCTANCE
}

\section{Lesson XL.-Mutual Induction}

454. Mutual Induction.-Mutual induction between two circuits, a primary and a secondary, was briefly considered in Art. 224. Let us now consider the electromotive-forces so induced. Suppose the primary coil to have $S_{1}$ spirals, and the secondary coil $S_{2}$ spirals. At first let them be arranged (by use of an iron core or by geometric juxtaposition) so that all the magnetic lines evoked by the primary coil pass through all the spirals of the secondary coil ; both coils being placed close together upon a suitable core of laminated iron.

By Art. 377 the magnetic flux due to current $\mathrm{C}$ in the primary coil will be

$$
\mathrm{N}=4 \pi \mathrm{CS}_{1} / 10 \mathrm{Z},
$$

where $\mathrm{Z}$ is the reluctance (Art. 376) of the magnetic circuit. The total amount of cutting magnetic lines by the $\mathrm{S}_{2}$ spirals of the secondary, when current $\mathrm{C}$ is turned off or on, will be

$$
\mathrm{S}_{2} \mathrm{~N}=4 \pi \mathrm{CS}_{1} \mathrm{~S}_{2} / 10 \mathrm{Z} \text {. }
$$

Hence it follows that the amount of cutting of magnetic lines (i.e. the induction in the secondary circuit) due to turning on or off 10 amperes (=1 C.G.S. unit of current) in the primary, will be $4 \pi \mathrm{S}_{1} \mathrm{~S}_{2} / \mathrm{Z}$. This quantity 
is denoted for brevity by the symbol M. If the primary and secondary coils are not so arranged that all the magnetic lines due to the one pass through the spirals of the other, then $\mathrm{M}$ will have a less value than $4 \pi \mathrm{S}_{1} \mathrm{~S}_{2} / \mathrm{Z}$.

The practical unit for coefficients of mutual induction is the same as for those of self-induction, namely the henry (Art. 354), and is $10^{9}$ C.G.S. units. Hence to bring $M$ to henries we must divide the above value by $10^{9}$.

If the current in the primary is varying at the rate $d \mathrm{C} / d t$, the electromotive-force $\mathrm{E}_{2}$ thereby induced in the secondary circuit will be

$$
\mathrm{E}=-\mathrm{M} \cdot d \mathrm{C} / d t,
$$

where $\mathrm{E}$ will be in volts if $\mathrm{M}$ is expressed in henries, $\mathrm{C}$ in amperes, and $t$ in seconds.

The value of $\mathbf{M}$ for the small induction coils used in telephone work is usually about 0.01 henry; for a Ruhmkorff eoil capable of giving a spark 10 centimetres long it may be as much as 5 henries.

Example.-Suppose in a spark-coil the value of $\mathrm{M}$ is 8 henries, and the primary current changes by an amount of 1 ampere in one ten-thousandth of a second (owing to the quick-acting break), the electromotive-force induced in the secondary during that ten-thousandth of a second will be 80,000 volts.

To measure a coefficient of mutual induction, there are several methods, some of which depend on the use of Wheatstone's bridge ; but the best method is one due to Carey Foster. In this the quantity of electricity discharged from a condenser of known capacity $\mathrm{K}$ shunted by a resistance $p$ in the primary circuit is balanced against the quantity discharged in the secondary circuit by regulating a resistance $q$ in the latter. Then $\mathrm{M}=\mathrm{K} p q$.

455. Induced Currents of Higher Orders.Joseph Henry, an independent discoverer of magnetoelectric induction, discovered that the variations in the 
strength of the secondary current could induce tertiary currents in a third closed circuit, and that variations in the tertiary currents might induce currents of a fourth order, and so on. A single sudden primary current produces two secondary currents (one inverse and one direct), each of these produces two tertiary currents, or four tertiary currents in all. But with alternating or periodic there are the same number of secondary and tertiary fluctuations as of primary; but the currents of the second, fourth, etc. orders will be inverse in the direction of their flow to those of the first, third, fifth, etc.

456. Lenz's Law.-In Art. 223 it was explained how an increase in the number of magnetic lines through a circuit (as by pushing in a magnet) tended to set up an inverse current, or one flowing in such a direction as is opposed to the magnetism. Similarly a decrease in the magnetic lines (as by withdrawing the magnet) tends to set up currents that will pull the magnet back. Again, in Art. 379, it was laid down that a circuit traversed by a current experiences a force tending to move it so as to include the greatest possible number of magnetic lines-offorce in the embrace of the circuit. But if the number of lines be increased, during the increase there will be an opposing (or negative) electromotive-force set up, which will tend to stop the original current, and therefore tend to stop the motion. If there be no current to begin with, the motion will generate one, which being in a negative direction, will tend to diminish the number of lines passing through the circuit, and so stop the motion. Lenz, in 1834, summed up the matter by saying that in all cases of electromagnetic induction the induced currents have such a direction that their reaction tends to stop the motion which produces them. This is known as Lenz's law : it is a particular case of the more general law applicable to all electromagnetic systems, namely, that every action on such a system, which, in producing a change in its configuration or state, involves a transforma- 
tion of energy, sets up reactions tending to preserve unchanged the configuration or state of that system. (Compare Arts. 204 and 379.)

457. Eddy-Currents Induced in Masses of Metal.-In 1824 Gambey found that a compass-needle oscillating in its box came to rest sooner if the bottom of the box were made of metal than if of wood. Arago investigated the matter, and found a copper plate under the needle most effective in damping its motions. He then rotated a copper disk in its own plane underneath a compass-needle, and found that the needle was dragged round as by some invisible friction. A copper disk suspended over a rotating magnet was found to be dragged by it. Attempts were made to account for these phenomena-known as Arago's rotations-by supposing there to be a sort of magnetism of rotation, until Faraday proved them to be due to induction. A magnet moved near a solid mass or plate of metal induces in it currents, which, in flowing through it from one point to another, have their energy eventually frittered down into heat, and which, while they last, produce (in accordance with Lenz's law) electromagnetic forces tending to stop the motion. These currents, circulating wholly within the nietal, are called eddy-currents. If a cube or ball of good conducting metal be set spinning between the poles of such an electromagnet as Fig. 182 , and the current be suddenly turned on, the spinning metal stops suddenly. In a copper disk revolving between the poles of a magnet (Fig. 240) there are a pair of eddies in the

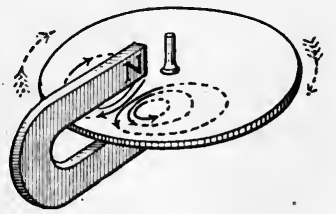

Fig. 240. part passing between the poles, and these currents tend to pull the disk back. In fact, any conductor moved forcibly across the lines of a magnetic field experiences a mechanical resistance due to the induced currents which oppose its 
motion. Foucault showed ${ }^{*}$ that if, by sheer force, a disk be kept spinning between the poles of a powerful electromagnet it will become hot in consequence of the eddy currents induced in it.

The eddy-current drag on a moving conductor (sometimes called the magnetic friction) is a force proportional to the speed and proportional to the square of the magnetic field ; for the force (Art. 340) is proportional to the product of field and current, and the current (circulating round a given path) is proportional both to field and to speed. Hence eddy-current drag is employed in some forms of electric supply meter (Art. 442) to control the speed of the moving part.

Alternating electric currents also set up eddy-currents in masses of metal near them ; for this reason the iron cores of transformers (Art. 480) and of dynamo armatures (Art. 463) must be carefully laminated, otherwise there will be heating and waste of energy.

Further, eddy-currents in any mass of metal between a primary and a secondary circuit will tend to set up in the secondary tertiary electromotive forces opposing those set up by the primary. Hence interposed sheets of metal act as induction-screens.

\section{Lesson XLI.-Self-Induction}

458. Self-Induction.-It has been pointed out in Art. 224 how when a current in a circuit is increasing or diminishing, it exercises an inductive effect upon any neighbouring circuit; this inductive effect being due to the change in the magnetic field surrounding the varying current. But since the magnetic lines surrounding a current may, as they move inwards or outwards from the wire, cut across other parts of the same circuit, it is evident

* Hence some writers call the eddy-currents "Foucault's currents," though they were known years before Foucault's experiments were made. 
that a current may act inductively on itself. The selfinductive action is great if the circuit consists of a coil of many turns, and is still greater if the coil possesses an iron core. Suppose a coil of wire to possess S spirals, and that it generates a magnetic flux through these spirals of $\mathrm{N}$ lines when current $\mathrm{C}$ is turned on. Then it is clear that turning on the current will have the same effect as if a magnet of $\mathbf{N}$ lines were suddenly plunged into the coil ; and turning off the current will have the same effect as if the magnet were suddenly withdrawn. Now (Art. 225) the current induced by plunging a magnet into a coil is an inverse current tending to push it out, while that induced by withdrawing the magnet is a direct current, tending to attract it back. It follows that the selfinduced electromotive-force on turning the current on will tend to oppose the current, and prevent it growing as quickly as it otherwise would do, while that induced on stopping the current will tend to help the current to continue flowing. In both cases the effects of selfinduction is to oppose change: it acts as an electromagnetic inertia.

In the case supposed above, where the coil has $\mathrm{S}$ turns, the total cutting of magnetic lines in the operation will $=\mathrm{S} \times \mathrm{N}$, provided all the lines thread through all the spirals. Let the symbol $\mathrm{L}$ be used to represent the total amount of cutting of lines by the circuit when a current of 1 ampere is suddenly turned on or off in it. Clearly $\mathrm{L} \times \mathrm{C}=\mathrm{S} \times \mathrm{N}$. This quantity $\mathrm{L}$ is called " the inductance" of the circuit. It was formerly called "the coefficient of self-induction" of the circuit. The unit of induction is called the henry, and corresponds to a cutting of $10^{9}$ magnetic lines when 1 ampere is turned on or off.

Since (in circuits without iron cores) $\mathbf{N}$ is proportional to $\mathrm{S}$, it follows that $\mathrm{L}$ is proportional to $\mathrm{S}^{2}$. Or since (see Art. 377) $\mathbf{N}=4 \pi \mathrm{CS} / 10 \mathrm{Z}$, and the total cutting of lines by the $\mathrm{S}$ spirals (if all the lines pass through all the 
spirals) is $\mathrm{S} \times \mathrm{N}$, hence the induction, when 10 amperes are turned on or off will be

$$
\mathrm{L}=4 \pi \mathrm{S}^{2} / \mathrm{Z},
$$

which may be expressed in henries by dividing by $10^{9}$. If all the lines do not pass through all the spirals the value of $l_{1}$ will be less than this.

The self-induced electromotive-force will depend upon the rate at which the current is changing; for if the total cutting SN take place in time $t$, it follows (Art. 225) that

$$
\mathrm{E}=-\mathrm{SN} / t=-\mathrm{LC} / . t \text {. }
$$

But since the rate at which the current changes is not uniform, $\mathrm{E}$ is also not uniform. If in an element of time $d t$ the current charges by an amount $d \mathrm{C}$, the rate of charge of the current is $d \mathrm{C} / d t$, and the self-induced electromotiveforce is $=-\mathrm{L} \cdot d \mathrm{C} / d t$.

The formal definition of the henry (Art. 354) is based on the above expression in order that it may apply to circuits with iron cores as well as to circuits without them.

The energy of the magnetic field surrounding the current is equal to $\frac{1}{2} \mathrm{LC}^{2}$, since while the field is growing up to have LC lines in total, the average value of the current is $\frac{1}{2} \mathrm{C}$.

To measure a coefficient of self-induction there are several methods :-

(a) Alternate-current method.-The volts $\mathrm{V}$ required to send current $\mathrm{C}$ at frequency $n$ through coil having resistance $R$ and coefficient of self-induction $L$ are $\mathrm{V}=\mathrm{C} \sqrt{\mathrm{R}^{2}+4 \pi^{2} n^{2} \mathrm{~L}^{2}}$; or, if the resistance is negligible, $\mathrm{V}=2 \pi n \mathrm{CL}$, whence $\mathrm{L}=\mathrm{V} / 2 \pi n \mathrm{C}$ (see Art. 472).

(b) Bridge methods.-Of several bridge methods the best is Maxwell's. Let balance be obtained in usual way; key in battery circuit being put down before key in galvanometer circuit (Art. 415). Then press the keys in 
reverse order, when the presence of self-induction in one of the four arms will upset balance, the needle giving a kick $a$ proportional to the self-induction. Now introduce in the same arm an additional small resistance $r$, such that when keys are again operated in the usual order there is a small permanent deflexion $\delta$. If the periodic time of swing of the needle be $\mathrm{T}$ the following formula then holds :- $\mathrm{L}=\mathrm{T} r \alpha / 2 \pi \delta$.

(c) Secohmmeter method.-Ayrton and Perry invented an instrument which alternately makes and breaks the battery circuit of the bridge and only allows the galvanometer to be in operation during a short interval of time $\mathrm{T}$ immediately after each making of the battery circuit (the galvanometer at other times being short-circuited). As the current is increasing during this interval, the self-induction $L$ of a coil placed in one of the arms of the bridge acts as though there were an additional resistance $r$ in that arm. The formula is then, $\mathrm{L}=\mathrm{T} r$. As $\mathrm{L}$ is then the product of seconds and ohms, Ayrton and Perry proposed for the unit (now called the henry) the name of secohm.

459. Effects of Inductance.-The presence of inductance in a circuit affects the currents in several ways. The special choking-effect on alternate currents is dealt with in Art. 474. The effects on battery currents are also important. So long as the current is not changing in strength inductance has no effect whatever; but while the current is starting or while it is dying away the presence of inductance greatly affects it. In all cases inductance tends to oppose any change in the strength of the current; as may be foreseen from Lenz's law (Art. 456). When a current is increasing in strength inductance causes it to increase more slowly. When a current is dying away inductance tends to prolong it.

The existence of inductance in a circuit is attested by the so-called extra-current, which makes its appearance as a bright spark at the moment of breaking circuit. If 
the circuit be a simple one, and consist of a straight wire and a parallel return wire, there will be little or no inductance ; but if the circuit be coiled up, especially if it be coiled round an iron core, as in an electromagnet, then on breaking circuit there will be a brilliant spark, and a person holding the two ends of the wires between which the circuit is broken may receive a shock, owing to the high electromotive-force of this self-induced extra current. This spark represents the energy of the magnetic field surrounding the wire suddenly returning back into the circuit. The extra-current on " making" circuit is an inverse current, and gives no spark, but it prevents the battery current from rising at once to its full value. The extra-current on breaking circuit is a direct current, and therefore keeps up the strength of the current just at the moment when it is about to cease. To avoid the perturbing effects of inductance, resistance-coils are always coiled back upon themselves (Art. 414).

Even when a circuit consists of two parallel straight wires there is a magnetic field set up between them, giving inductive reactions. The coefficient of self-induction for two wires of length $l$ and radius $a$ at an axial distance $b$ apart in air is

$$
\mathrm{L}=l\left(\frac{\mu}{2}+4 \log _{\epsilon} \frac{b}{a}\right) 10^{-9} ;
$$

where $\mathrm{L}$ is in henries; $a, b$ and $l$ in centimetres; and $\mu$ the permeability of the wire.

460. Helmholtz's Equation. Time-constant.From thai which precedes it is clear that whenever a current is turned on there is a variable period while the current is growing up to the value which it will reach when steady, namely the value as determined by Ohm's law. But during the variable period Ohm's law is no longer applicable.

Von Helmholtz, who investigated mathematically the effect of self-induction upon the strength of a current, 
deduced the following important equations to express the relation between the inductance of a circuit and the time required to establish the current at full strength :-

Let $d t$ represent a very short interval of time, and let the current increase during that short interval from $\mathrm{C}$ to $\mathrm{C}+d \mathrm{C}$. The actual increase during the interval is $d \mathrm{C}$, and the rate of increase in strength is $d \mathrm{C} / d t$. Hence, if the inductance be $\mathrm{L}$, the electromotive-force of self-induction will be $-\mathrm{L} d \mathrm{C} / d t$, and, if the whole resistance of the circuit be $\mathrm{R}$, the strength of the opposing extra-current will be $-\frac{\mathrm{I}_{4}}{\mathrm{R}} \cdot \frac{d \mathrm{C}}{d t}$ during the short interval $d t$; and hence the actual strength of current flowing in the circuit during that short interval instead or being (as by Ohm's law it would be if the current were steady) $\mathrm{C}=\mathrm{E} / \mathrm{R}$, will be

$$
\mathrm{C}=\frac{\mathrm{E}}{\mathrm{R}}-\frac{\mathrm{L}}{\mathrm{R}} \cdot \frac{d \mathrm{C}}{d t} .
$$

To find out the value to which the current will have grown after a time $t$ made up of a number of such small intervals added together, requires an application of the integral calculus, which at once gives the following result :-

$$
\mathrm{C}=\frac{\mathrm{E}}{\mathrm{R}}\left(1-\epsilon^{-\mathrm{R} t / \mathrm{L}}\right),
$$

(where $\epsilon$ is the base of the natural logarithms).

Put into words, this expression amounts to saying that after a lapse of $t$ seconds the self-induction in a circuit on making contact has the effect of diminishing the strength of the current by a quantity, the logarithm of uhose reciprocal is inversely proportional to the inductance, and directly proportional to the resistance of the circuit and to the time that has elapsed since making circuit.

The quantity $L / R$, the reciprocal of which appears in the exponential expression, is known as "the time-constant" or "persistence" of the circuit. It is the time required by 
the current to rise to a certain fraction, namely $(\epsilon-1) / \epsilon$, -or 0.634 - of its final value.

A very brief consideration will. show that in those cases where the circuit is so arranged that the inductance $L$ is small as compared with the resistance $R$, so that the time-constant is small, the term $\left(\epsilon^{-\mathrm{R} t / \mathrm{L}}\right)$ will vanish from the equation for all appreciable values of $t$.

On the other hand if $\mathrm{L}$ is great compared with $\mathrm{R}$, the current during its growth will be governed almost entirely by the inductance, and not by the resistance of the circuit, which will art as though its resistance were $=\mathrm{L} / \mathrm{t}$.

These matters are graphically depicted in Fig. 241, in which there are two curves of rise of current. Consider a circuit having $\mathrm{E}=10$ volts, $\mathrm{R}=1 \mathrm{ohm}, \mathrm{L}=10$ henries. The steady current will be 10 amperes; but at the end

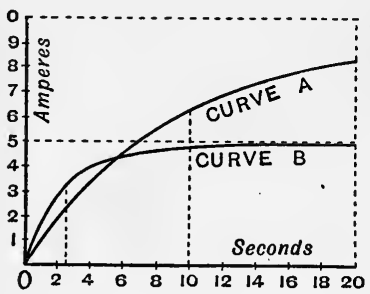

Fig. 241. of 1 second, as may be calculated by Helmholtz's equation, the current is only 0.95 of an ampere! In 2 seconds it is $1 \cdot 81$, in 5 seconds $3 \cdot 95$, in 10 seconds 6.34 amperes (see curve $A$ ). At the end of a whole minute it is only $9 \cdot 975$ amperes. Suppose now we increase the resistance to $2 \mathrm{ohms}$, and reduce the inductance to 5 henries. The final value of the current will be only 5 amperes instead of 10 ; but it will rise more quickly than before (see curve B). At the end of 1 second it will be $1 \cdot 647$ ampere, in 2 seconds $2 \cdot 755$, in 10 seconds 4.91 amperes. We conclude that for all apparatus that is required to be rapid-acting (relays, telephones, chronographs, etc.) it is much more important to keep down the inductance than the resistance of the circuit. We also see that the rule (Art. 407) so often given, about making the resistance of a battery equal to that of the rest of the circuit, is quite wrong for cases of rapid action. If the circuit has self-induction as well as resistance then it is better to group the cells of the battery so as to have higher resistance, namely put them all in series.

In fact everything goes on as though at time $t$ after 
"make" there were two currents flowing in opposite directions at once; one the ordinary current flowing from the first at full strength, the other the extra-current having the value $-\frac{\mathrm{E}}{\mathrm{R}} \epsilon^{-\mathrm{R} t / \mathrm{L}}$; the actual current being the difference between the two.

At "break" of circuit everything goes on as if, the ordinary current having dropped suddenly to zero, there was superposed an extra-current having the value $+\frac{\mathrm{E}}{\mathrm{R}} \epsilon^{-\mathrm{R} t / \mathrm{L}} ;$ but here, since there is introduced into the circuit a resistance of unknown amount (the resistance along a spark being indefinite) the calculation becomes impracticable. We know that $R$ is very great; hence we know that the variation will be more sudden, and that the self-induced E.M.F. at "break" is much greater than that at "make." The self-induced E.M.F. would be represented by the expression $\mathrm{E}_{t}=\mathrm{E} \epsilon^{-\mathrm{r} t / \mathrm{L}}$. This expression should be compared with that for the E.M.F. of discharge of a condenser of capacity $K$ through a resistance $R$ (see also Art. 326), which is $\mathrm{V}_{t}=\mathrm{V}_{0} \epsilon^{-t / \mathrm{kr}}$. From this it appears that in the case of a condenser discharge $K R$ acts as the time-constant $L / R$ does in the case of self-induction.

The actual quantity of electricity conveyed by the "extra-current" is equal to that which would be conveyed by current of strength $E / R$ of lasting for time $L / R$; or $=\mathrm{EL} / \mathrm{R}^{2}$. At the "make" of the circuit the retardation causes the flow of electricity to be lessened by the amount $q=\mathrm{EL} / \mathrm{R}^{2}$. The energy which is stored up outside the wire while the current grows up from 0 to its final value $\mathrm{C}$ is equal to $\frac{1}{2} q \mathrm{E}=\frac{1}{2} \mathrm{LC}^{2}$. 


\section{CHAPTER X}

\section{DYNAMOS AND TRANSFORMERS}

Lesson XLII.-Magneto-electric and Dynamo-electric Generators

461. Simple Magneto-electric Machines. Faraday's discovery of the induction of currents in wires by moving them across a

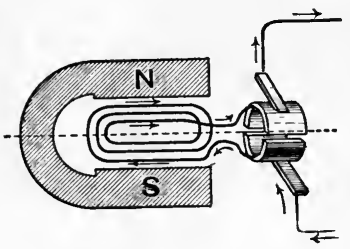

Fig. 242. magnetic field suggested the construction of magnetoelectric machines to generate currents in place of voltaic batteries, and Faraday himself constructed the first of such machines (Fig. 132) in 1831. In the early attempts of Pixii (1833), Saxton, and Clarke, bobbins of insulated wire were fixed to an axis and spun rapidly in front of the poles of strong steel magnets. But, since the currents thus generated were alternately inverse and direct currents, a commutator (which rotated with the coils) was fixed to the axis to turn the successive currents all into the same direction. Fig. 242 illustrates the plan adopted by Sturgeon in 1836, using a split tube of copper to commute the connection to the outer circuit at each half-turn. In the figure the wire coil is supposed to be spun around a longi- 
tudinal axis; the upper portion coming towards the observer. The arrows show the direction of the induced currents delivered by the commutator to the contactsprings or brushes. The little magneto-electric machines, still sold by opticians, are on this principle. Holmes and Van Malderen constructed more powerful machines, the latter combin-

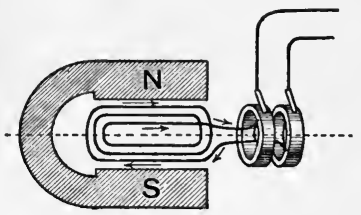

Fig. 243. ing around one axis sixty-four separate coils rotating between the poles of forty powerful magnets.

In 1856 Werner Siemens devised an improved armature, in which the coils of wire were wound shuttle-wise upon a grooved iron core, which concentrated the magnetic lines in a powerful field between the poles of a series

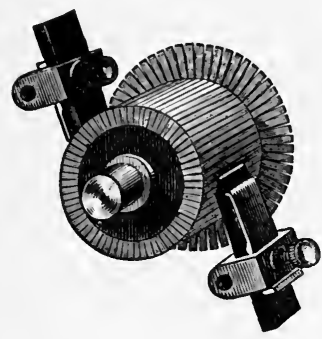

Fig. 244. of adjacent steel magnets. The next improvement, due to Wilde, was the employment of electro-magnets instead of steel magnets for producing the field in which the armature revolved; these electro-magnets being excited by currents furnished by a small auxiliary magnetoelectric machine, also kept in rotation. If instead of commuting the currents the ends of the revolving coil are connected to a pair of contact rings, on each of which presses a brush, the machine will deliver alternate currents. Fig. 243 illustrates a primitive form of alternator. It will be seen that if the induced E.M.F. in the wires as they move past the $\mathrm{N}$-pole towards the observer is from left to right, the two contact rings will alternately become + and - at each half-turn.

462. Dynamo-electric Machines.-The name 
dynamo-electric machine, or, briefly, dynamo, is given to any machine for converting mechanical power into electrical power by the operation of producing relative motion between magnets and conductors. The part which acts as magnet is termed the field-magnet. In continuous-current generators it usually stands still ; in some alternators it is inade to revolve. Its function is to provide a large number of magnetic lines. The part which acts as the active conductor, cutting the magnetic lines and having electromotive force induced in it, is termed the armature. In continuous current generators, the armature revolves between the poles of the field-magnet. In some alternators it is stationary. In the early machines the magnetism of the field magnets was independently excited. Various suggestions were made by Hjorth, Murray, S. A. Varley, and others to use the currents generated in the armature to excite the field-magnets. This was done in 1867 by Varley, Werner Siemens, and Wheatstone; the small current induced by the feeble residual magnetism being sent around the electromagnet to exalt its magnetism, and prepare it to induce still stronger currents. The name of dynamo-electric machines is given (it was first applied in 1867 to Wilde's machine) to such generators to distinguish them from those in which permanent steel magnets are employed. In either case the current is due to magneto-electric induction ; and in either case also the energy of the currents so induced is derived from the dynamical power of the steam-engine or other motor which performs the work of moving the rotating coils of wire in the magnetic field. But the name has been extended to all generators, whether selfexciting or not. In all of them the electromotive-force generated is proportional to the number of turns of wire in the rotating armature, and to the speed of revolution. When currents of small electromotive-force but of con. siderable strength are required, as for electroplating, the rotating armatures of a generator must be made with 
small internal resistance, and therefore of a few turns of stout wire or ribbon of sheet copper. For producing currents at a high electromotive-force the armature must consist of many turns of wire or of rods of copper suitably connected, and it must revolve in a very powerful magnetic field.

463. Continuous-current Dynamos. - The dynamos of different makers differ in the design of their field-magnets and in the means adopted for securing continuity in the induced currents. Most continuous-current dynamos have a simple field magnet with two poles: but many large machines are made with four, six, or eight poles. But the modern armature is complex. A simple coil, such as Fig. 242, with its 2-part commutator will not yield a steady current; for twice in each revolution the E.M.F. dies away to zero. The coils must be grouped so that some of them are always active. In most dynamos the armature winding is constructed as a closed coil, the wire being wound on a ring core of iron (Pacinotti's core with teeth, Gramme's core without teeth), or as a drum over a cylindrical core (Siemens's or Von Hefner's plan), or having the coils arranged flat as a disk (Desrozier's plan). In all these cases the convolutions are joined up so that (like the ring winding in Fig. 190) the coil is endless. If the current is brought in at one side of such a coil and taken out at the other side there will be two paths through the coil. As the coil spins between the poles of the magnet the electromotive-forces induced in the ascending and descending parts will tend to send the currents in parallel through these parts; and consequently contact-brushes must be set to take off the currents from the revolving coils at the proper places. The brushes are, however, set in contact not with the coils themselves but with a commutator, Fig. 244, consisting of a number of copper bars, insulated from one another, and joined on to the armature coil at regular intervals. Consider, for example, a Gramme ring made 
as it were of a number of bobbins wound upon a ring core of iron wire. Each bobbin constitutes one section of the winding, and they are all joined together, the end of one section to the beginning of the next, and each such junction is joined down to a bar of the commutator. The current cannot pass from one bar of the commutator to the next without traversing the intervening section of

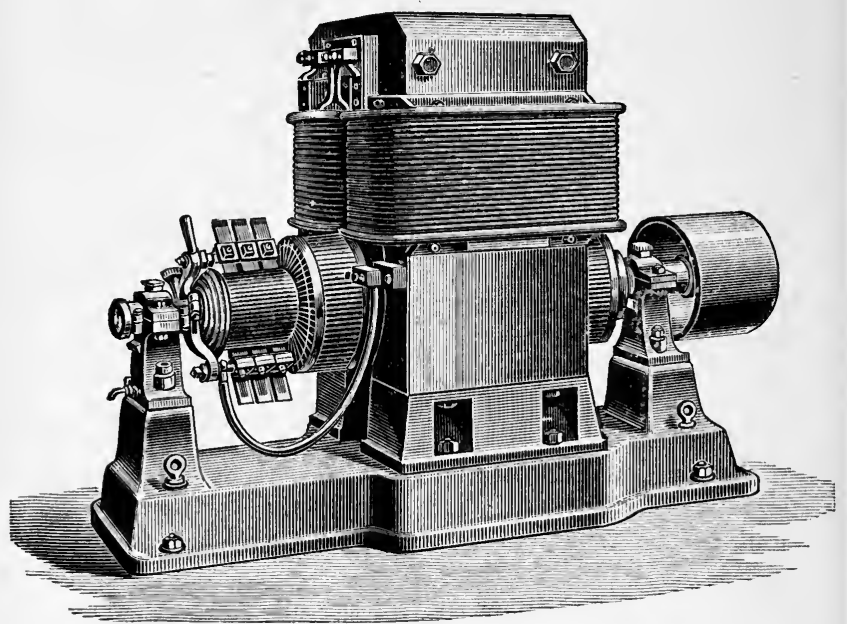

Fig. 245.

the windings. The commutator revolves with the armature; while the brushes, which are clamped in suitable holders, press against its surface, and are set in such a position that the current passes into them with as little sparking as possible. It is found that to prevent sparking the brushes must be set a little in advance of the diameter that is symmetrical between the poles: for the current in each section of the winding is reversed as it passes under the brush, and for sparkless reversal needs to 
be moving at that instant in a magnetic field of sufficient strength. The current in the armature exercises a magnetizing action, and tends to distort the magnetic field in the direction of the rotation. To prevent serious distortion and sparking, the field-magnet is made very powerful and massive. The "brushes" that receive the current were originally bunches of springy wires: in modern machines they are built up of copper strip or copper gauze, or consist of small blocks of carbon. Fig. 245 depicts a bipolar type of dynamo, having a vertical magnet of massive wrought iron magnetized by currents flowing in coils wound upon the two limbs. Below, between the polar surfaces which are bored out to receive it, is the revolving armature (in this case a drum-armature) with the commutator and brushes. The core of the armature is built up of thin iron disks lightly insulated from one another, to prevent eddy-currents. All large modern dynamos are now made multipolar, with 4, 6, 8, or more poles ; bipolars being only used in small sizes. All continuous-current dynamos will run as motors (Art. 443), if supplied with current at the proper voltage.

For fuller descriptions of dynamos, and technical details of construction, the reader is referred to the author's treatise on Dynamo-electric Machinery.

464. Dynamo Calculations.-In a 2-pole dynamo if $\mathbf{N}$ be the total number of magnetic lines sent by the field-magnet through the armature, $S$ the number of wires or conductors in series on the armature, counted all round, and $n$ the number of revolutions per second, the electromotive-force generated by the spinning armature will be

$$
\mathrm{E}=n \mathrm{SN} / 10^{8},
$$

for the cutting per second of magnetic lines is proportional to each of these three quantities, and we divide by $10^{\mathrm{s}}$ to bring to volts. As with batteries (Art. 171), so with dynamos, if there is an internal resistance $r$, the available 
volts at the terminals $\mathrm{V}$ will be less than the whole volts generated by an amount equal to $r \mathrm{C}$, the lost volts.

$$
\mathrm{V}=\mathrm{E}-r \mathrm{C} \text {. }
$$

As the electrical efficiency of the machine is the ratio $\mathrm{V} / \mathrm{E}$, it is evident that $r$ should be as low as possible.

Example.-A dynamo having $\mathrm{N}=7,170,000, \mathrm{~S}=120$, running at 780 revs. per min. (=13 revs. per sec.) will generate an electromotive-force of 111 volts. If $r=0.033 \mathrm{ohm}$, then when $\mathrm{C}=210$ amperes, $r \mathrm{C}=7$ volts. Hence $\mathrm{V}=104$ volts.

The current $\mathrm{C}$ which a dynamo yields depends on the resistance, etc., of the circuit it supplies. The maximum current it can supply is limited by several considerations, such as the heating of its parts, the sparking at the brushes, which becomes serious if too much current is drawn from the machine, the mechanical strength of its parts, and also the power of the driving-engine.

The gross output of a dynamo is the number of amperes multiplied by the total electromotive-force generated, or CE. The nett output is the number of amperes multiplied by the volts at terminals, or CV. These numbers are turned to horse-power by dividing by 746 .

The commercial efficiency of a dynamo is the ratio between the nett output and the mechanical power applied to drive the machine.

All the armature conductors of a dynamo are subject, when the machine is running, to a mechanical drag opposing the rotation. This is due to the action between the magnetic field and the current (Art. 340).

A little power is wasted by eddy-currents (Art. 457), and by hysteresis (Art. 368) in the armature core, and also a little by eddy-currents (Art. 463) in the moving masses of metal, so diminishing the efficiency: but in well-constructed machines such losses are slight.

To calculate the field-magnet windings the formulæ of 
Arts. 377 and 399 must be applied (see exercise 21 on Chap. V.).

465. Excitation of Field-Magnets. - There are several modes of exciting the magnetism of the fieldmagnets, giving rise to the following classification :-

1. Magneto Machine, with permanent steel magnets.

2. Separately-excited Dynamo; one in which the currents used to excite the field-magnets are furnished by a separate machine called an "exciter."

3. Separate-coil Dynamo, with a separate coil wound on the armature to generate the exciting current.

4. Series-Dynamo, wherein the coils of the field-magnet are in series with those of the armature and the external

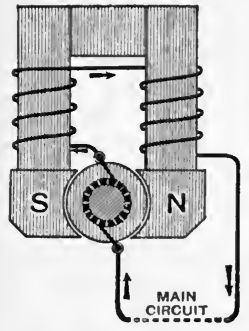

Fig. 246.

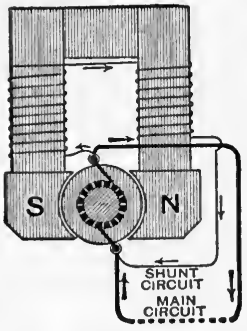

Fig. 247.

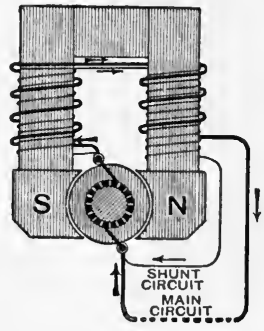

Fig. 248.

circuit (Fig. 246), and consist of a few turns of thick wire.

5. Shunt-Dynamo, in which the coils of the fieldmagnet form a shunt to the main circuit; and, being made of many turns of thin wire, draw off only a small fraction of the whole current (Fig. 247).

6. Compound-Dynamo, partly excited by shunt coils, partly by series coils (Fig. 248).

The last three modes are illustrated in the accompanying diagrams. Each variety of winding has certain advantages depending on conditions of use. 
466. Characteristic Curves.-To study the behaviour of various types of dynamo, Hopkinson devised the method of characteristic curves, wherein the two elements of output - the volts and the amperes-are plotted out. If a series-dynamo is examined with amperemeter and voltmeter, while run at constant speed on various loads, its performance will be found to give a curve like $\mathrm{OQV}$ in Fig. 249, where the external volts are plotted

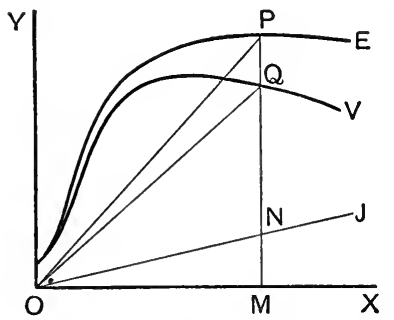

Fig. 249. vertically, the amperes horizontally. This curve is the external characteristic. The volts rise as the current is increased, because of the increase of magnetization, but when this is near saturation they fall again because of internal resistance and sundry reactions. At any point such as $Q$ the resistance of the external circuit is represented by the slope of the line QO (i.e. by the trigonometrical tangent of the angle QOX), since tan QOX is equal to QM/OMI (= the volts divided by the amperes). If line OJ be drawn so that $\tan J O X$ is equal to the internal resistance, then $\mathrm{MN}$ will represent the lost volts when the current=OMI. Adding to $\mathrm{QM}$ a piece $\mathrm{PQ}=$ MN, we obtain PMI as the corresponding value of the total electromotive-force. In this way, from the curve $\mathrm{OV}$ we can construct the total characteristic OE. It will be evident that if the total resistance (i.e. the slope of the line $\mathrm{OP}$ ) be increased $\mathrm{P}$ will come down the curve toward $O$, and there will be a certain point at which any further increase in the slope will produce a sudden drop of volts and amperes to almost zero. This is a peculiarity of series machines; when running at a given speed they cease to yield any current if the resistance exceeds a certain critical value, depending in each machine on its construction. 
For a shunt-dynamo the characteristic has a different form. When the machine is on open circuit, giving no current externally, the shunt circuit is fully at work exciting the magnet. The curve $\mathrm{YV}$ of volts at terminals begins at a high value, and as the current is increased by diminishing the resistance, the voltage gently falls. Part of this drop is due to internal resistance; part is due to armature reactions and magnetic distortion; and part to the reduction of the shunt current. If, as before, we draw OJ to represent by its

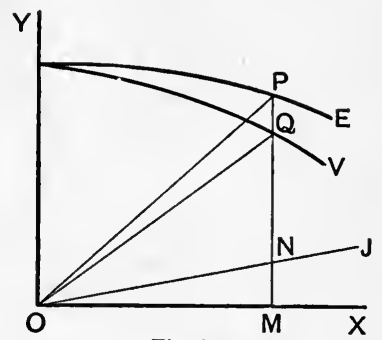

Fig. 250. slope the internal resistance, we can find the lost volts MN and add these on above $Q$, so obtaining $P$, a point on the total electromotive-force curve. This also drops slightly. If a shunt-dynamo be short-circuited, its magnetisn is at once reduced to almost zero. To regulate the voltage of a shunt-dynamo a suitable rheostat (Fig. 206) may be introduced into its shunt circuit, to vary the exciting current.

467. Constant Voltage Machines.-For glowlamp lighting, machines are needed that will maintain the voltage constant, whether the current going to the mains be small or large. The current that flows out of the machine will regulate itself exactly in proportion to the demand; more flowing when more lamps are turned on, provided the potential difference between the mains is kept constant. For this purpose neither a series-dynamo nor a shunt-dynamo (driven at a constant speed) will suffice; thongh by hand-regulation, as above, a shunt-dynamo may be used. It will be noted that, while in shunt-machines the characteristic drops as the current is increased, in series-machines the curve rises. 
Consequently, by using a compound-winding, consisting of a shunt-winding (to give the proper voltage an open circuit) and a few coils of thick wire, in series with the main circuit (to raise the excitation in proportion to the output), the voltage may be kept remarkably constant. By over-compounding, with more series windings the dynamo may be made to maintain a constant voltage at some distant point in the circuit.

468. Constant Current Machines - Series Lighting.-To maintain an unvarying current in a series of lamps, as is frequently wanted for lighting with arc lamps (Art. 448), special dynamos are used known as arc-lighting machines. The best known of these are the Brush and the Thomson-Houston dynamos. Both have open-coil armatures (in which the coils are not grouped in a closed circuit), with special commutators, and automatic devices to regulate the output, the one by shunting the exciting current, the other by shifting the brushes. The current may thus be kept at 10 amperes, while the volts change (according to the number of lamps in circuit) from 50 to 2000 or more.

469. Unipolar Machines.-There is another class of dynamo-electric machines, differing entirely from any of the preceding, in which a coil or other movable conductor slides round one pole of a magnet and cuts the magnetic lines in a continuous manner without any reversals in the direction of the induced currents. Such machines, sometimes called "uni-polar" machines, have, however, very low electromotive-force, and are not practical. Faraday'is disk-machine (Fig. 132) belonged to this class.

\section{Lesson XLIII.-Alternate Currents}

470. Periodic Currents.-We have seen that the revolving of a simple coil in a magnetic field sets up 
electromotive-forces, which change in direction at every half-turn, giving rise to alternate currents. In each whole revolution there will be an electromotive-force which rises to a maximum and then dies away, followed immediately by a reversed electromotive-force, which also grows to a maximum and then dies away. Each such complete set of operations is called a period, and the number of periods accomplished in a second is called the frequency or periodicity of the alternations, and is symbolized by the letter $n$. In 2-pole machines $n$ is the same as the number of revolutions per second ; but in multipolar machines $n$ is greater, in proportion to the number of pairs of poles. By revolving in a uniform field the electromotive-forces set up are proportional to the sine of the angle through which the coil has turned from the position in which it lay across the field. If in this position the flux of magnetic lines through it were $\mathbf{N}$, and the number of spirals in the coil that enclose the $\mathbf{N}$ lines be called $\mathbf{S}$, then the value of the induced electromotiveforce at any time $t$ when the coil has turned through angle $\theta(=2 \pi n t)$ will be

$$
\mathrm{E}_{\theta}=2 \pi n \mathrm{SN} \sin \theta \div 10^{s} \text {, }
$$

or, writing $\mathrm{D}$ for $2 \pi n \mathrm{SN} / 10^{8}$, we have

$$
\mathrm{E}_{\theta}=\mathrm{D} \sin \theta .
$$

In actual machines the magnetic fields are not uniform, nor the coils simple loops, so the periodic rise and fall of the electromotive-forces will not necessarily follow a simple sine law. The form of the impressed waves will depend on the shape of the polar faces, and on the form and breadth of the coils. But in most cases we are sufficiently justified in assuming that the impressed electromotive-force follows a sine law, so that the value at any instant may be expressed in the above form, where $\mathrm{D}$ is the maximum value or amplitude attained by $\mathrm{E}$, and $\theta$ an angle of phase upon an imaginary circle of 
reference. Consider a point $\mathrm{P}$ revolving clock-wise round a circle. If the radius of this circle be taken as unity, PM will be the sine of the angle $\theta$, as measured from $0^{\circ}$. Let the circle be divided into any number of equal angles, and let the sines be drawn similarly for each. Then let these sines be plotted out at equal distances apart along the horizontal line, as in Fig. 251, giving us the sine curve.

In Fig. 251 one revolution of $\mathrm{P}$ around the circle of reference corresponds to one complete alternation or cycle

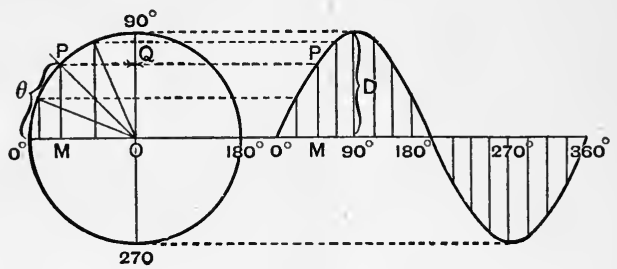

Fig. 251.

of changes. The value of the electromotive-force (which varies between $+\mathrm{D}$ and $-\mathrm{D}$ as its maximum values) may be represented at any moment either by the sine PM or by projecting $\mathrm{P}$ on to the vertical diameter, giving $\mathrm{OQ}$. As $\mathrm{P}$ revolves, the point $\mathrm{Q}$ will oscillate along the diameter.

The currents which result from these periodic or alternating electromotive-forces are also periodic and alternating; they increase to a maximum, then die away and reverse in direction, increase, die away, and then reverse back again. If the electromotive-force completes 100 such cycles or reversals in a second, so also will the current.

471. Virtual Volts and Virtual Amperes.Measuring instruments for alternate currents, such as electrodynamometers (Art. 395), Cardew voltmeters (Art. 
430), and electrostatic voltmeters (Art. 290), do not measure the arithmetical average values of the amperes or volts. The readings of these instruments, if first calibrated by the use of continuous currents, are the square roots of the means of the squares of the values. They measure what are called virtual amperes or virtual volts. The mean which they read (if we assume the currents and voltages to follow the sine law of variation) is equal to 0.707 of the maximum values, for the average of the squares of the sine (taken over either 1 quadrant or a whole circle) is $\frac{1}{2}$; hence the square-rootof-mean-square value is equal to $1 \div \sqrt{2}$ times their maximum value. If a voltmeter is placed on an alternating circuit in which the volts are oscillating between maxima of +100 and -100 volts, it will read $70 \cdot 7$ volts ; and 70.7 volts continuously applied would be required to produce an equal reading. If an alternate current amperemeter reads 100 amperes, that means that the current really rises to +141.4 amperes and then reverses to -141.4 amperes; but the effect is equal to that of 100 continuous amperes, and therefore such a current would be described as 100 virtual amperes.

472. Lag and Lead.-Alternating currents do not always keep step with the alternating volts impressed

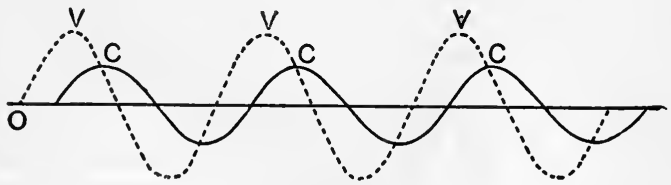

Fig. 252.

upon the circuit. If there is inductance in the circuit the currents will lag: if there is capacity in the circuit they will lead in phase. Fig. 252 illustrates the lag produced by inductance. The impulses of current, represented by 
the blacker line, occur a little later than those of the volts. But inductance has another effect of more importance than any retardation of phase; it produces reactions on the electromotive-force, choking the current down. While the current is increasing in strength the reactive effect of inductance tends to prevent it rising. To produce a current of 40 amperes in a resistance of $1 \frac{1}{2}$ ohms would require-for continuous currents-an E.M.F. of 60 volts. But an alternating voltage of 60 volts will not be enough if there is inductance in the circuit reacting against the voltage. The matter is complicated by the circumstance that the reactive impulses of electromotive-force are also out of step: they are in fact exactly a quarter period behind the current. If an alternate current of $\mathrm{C}$ (virtual) amperes is flowing with a frequency of $n$ cycles per second through a circuit of inductance $\mathrm{L}$, the reactive electromotive-force * will be $2 \pi n \mathrm{LC}$ (virtual) volts. If, for example, $\mathrm{L}=0.002$ henry, $n=50$ periods per second, and $\mathrm{C}=40$ amperes, the reactive electromotive-force will be $25 \cdot 1$ volts. Now if we wish to drive the 40 (virtual) amperes not only through the resistance of $1 \frac{1}{2}$ ohms but against this reaction, we shall require more than 60 volts. But we shall not require $60+25 \cdot 1$ volts, since the reaction is out of step with the current. Ohm's law is no longer adequate. To find out what volts will be needed we have recourse to geometry.

Plot out (Fig. 253) the wave-form OAbd, to correspond to the volts necessary to drive the current through the resistance, if there were no inductance. The ordinate $a \mathrm{~A}$ may be taken to scale as 60 . This we may call the current curve. Then plot out the curve marked $-p \mathrm{LC}$ to represent the volts needed to balance the reaction of

* This is calculated as follows (from Art. 45s), $\mathrm{E}=\mathrm{L} d \mathrm{C} / d t$. Now $\mathrm{C}$ is assumed to be a sine function of the time having instantaneous value $\mathrm{C}_{0} \sin 2 \pi n t$; where $\mathrm{C}_{0}$ is the maximum value of $\mathrm{C}$. Differentiating this with respect to time we get $d \mathrm{C} / d t=2 \pi n \mathrm{C}_{0} \cos 2 \pi n t$. The "virtual" values of cosine and sine being equal, we have for $\mathrm{E}$ the value $2 \pi n \mathrm{LC}$, but differing by $\frac{1}{4}$ period from the current in phase. 
the inductance. Here $p$ is written for $2 \pi n$. The ordinate at $\mathrm{O}$ is 25.1 ; and the curve is shifted back one quarter of the period : for when the current is increasing at its greatest rate, as at $O$, the self-inductive action is greatest. Then compound these two curves by adding

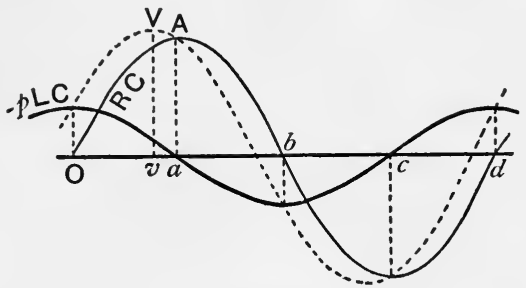

Fig. 253.

their ordinates, and we get the dotted curve, with its maximum at V. This is the curve of the volts that must be impressed on the circuit in order to produce the current. It will be seen that the current curve attains its maximum a little after the voltage curve. The current lags in phase behind the volts. If $\mathrm{O} d$ is the time of one complete period, the length $v a$ will represent the time that elapses between the maxima of volts and amperes. In Fig. 254 the same facts are represented in a revolving diagram of the same sort as Fig. 251. The line $\mathrm{OA}$ represents the working

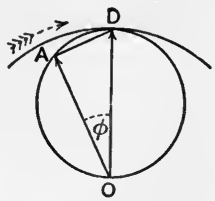

Fig. 254. volts $R \times C$, whilst the line $A D$ at right angles to $O A$ represents the self-induced volts $p \mathrm{LC}$. Compounding these as by the triangle of forces we have as the impressed volts the line OD. The projections of these three lines on a vertical line while the diagram revolves around the centre $\mathrm{O}$ give the instantaneous values of the three quantities. The angle $\mathrm{AOD}$, or $\phi$, by which the current lags behind the impressed volts is termed the 
angle of lag. However great the inductance or the frequency, angle $\phi$ can never be greater than $90^{\circ}$. If $\mathrm{OA}$ is 60 and $\mathrm{AD}$ is $25 \cdot 1$, OD will be 65 volts. In symbols, the impressed volts will have to be such that $\mathrm{E}^{2}=(\mathrm{RC})^{2}+(p \mathrm{LC})^{2}$. This gives us the equation

$$
\mathrm{C}=\frac{\mathrm{E}}{\sqrt{\mathrm{R}^{2}+p^{2} \mathrm{~L}^{2}}} \text {. }
$$

The denominator which comes in here is commonly called the impedance.

473. Maxwell's Law.-In Figs. 255 and 256 the angle of lag is seen to be such that $\tan \phi=p \mathrm{LC} / \mathrm{RC}$ or

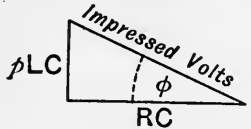

Fig. 255.

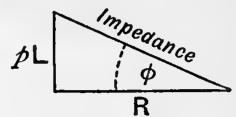

Fig. 256 .

$=p \mathrm{~L} / \mathrm{R} . \quad$ And it is evident that the effect of the inductance is to make the circuit act as if its resistance instead of being $\mathrm{R}$ was increased to $\sqrt{\mathrm{R}^{2}+p^{2} \mathrm{~L}^{2}}$. In fact the alternate current is governed not by the resistance of the circuit but by its impedance. At the same time the current is lagging as if the angle of reference were not $\theta$ but $\theta-\phi$, so that the equation for the instantaneous values of $\mathrm{C}$, when $\mathrm{E}=\mathrm{D} \sin \theta$, is

$$
\mathrm{C}=\frac{\mathrm{D} \sin (\theta-\phi)}{\sqrt{\mathrm{R}^{2}+p^{2} \mathrm{~L}^{2}}} .
$$

This is Maxwell's law for periodic currents as retarded by inductance. As instruments take no account of phase but give virtual values the simpler form preceding is usually sufficient.

The effect of capacity introduced into an alternate current circuit is to produce a lead in phase, since the reaction of a condenser instead of tending to prolong the 
current tends to drive it back. The reactance is therefore written as $-1 / p \mathrm{~K}$, and the angle $\phi$ will be such that $\tan$ $\phi=-1 / p \mathrm{KR}$. The impedance will be $\sqrt{\mathrm{R}^{2}+1 / p^{2} \mathrm{~K}^{2}}$.

If both inductance and capacity are present, tan

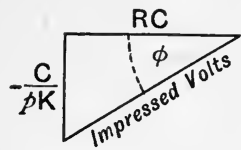

Fig. 257.

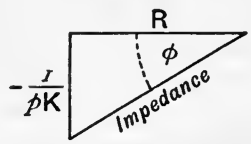

Fig. 258.

$\phi=(p \mathrm{~L}-1 / p \mathrm{~K}) / \mathrm{R}$; the reactance will be $p \mathrm{~L}-1 / p \mathrm{~K}$; and the impedance $\sqrt{\mathrm{R}^{2}+(p \mathrm{~L}-1 / p \mathrm{~K})^{2}}$.

Since capacity and inductance produce opposite effects they can be used to neutralize one another. They exactly balance if $\mathrm{L}=1 / p^{2} \mathrm{~K}$. In that case the circuit is noninductive and the currents simply obey Ohm's law.

474. Choking Coils.-It will be seen that if in a circuit there is little resistance, and much reactance, the current will depend on the reactance. For example if $p(=2 \pi n)$ were, say, 1000 and $\mathrm{L}=10$ henries while $\mathrm{R}$ was only $1 \mathrm{ohm}$, the resistance part of the impedance would be negligible and the law would become

$$
\mathrm{C}=\frac{\mathrm{E}}{p \mathrm{~L}} .
$$

Self-induction coils with large inductance and small resistance are sometimes used to impede alternate currents, and are called choking coils, or impedance coils.

If the current were led into a condenser of small capacity (say $\mathrm{K}=\frac{1}{10}$ microfarad, then $1 / p \mathrm{~K}=10,000$ ), the current running in and out of the condenser would be governed only by the capacity and frequency, and not by the resistance, and would have the value-

$$
\mathrm{C}=\mathrm{E} p \mathrm{~K} \text {. }
$$

475. Alternate-current Power.-If to measure 
the power supplied to a motor, or other part of an alternate current circuit, we measure separately with amperemeter and voltmeter the amperes and volts, and then multiply together the readings, we obtain as the apparent watts a value often greatly in excess of the true watts, owing to the difference in phase, of which the instruments take no account. The true power (watts) is in reality $\mathrm{W}=\mathrm{CV} \cos \phi$, where $\mathrm{C}$ and $\mathrm{V}$ are the virtual values, and $\phi$ the angle of lag. But the latter is usually an unknown quantity. Hence recourse must be had to a suitable watt-meter ; the usual form being an electrodynamometer (Art. 438) specially constructed so that the high-resistance circuit in it shall be non-inductive.

Whenever the phase-difference (whether lag or lead) is very large the current, being out of step with the volts, is almost wattless. This is the case with currents flowing through a choking-coil or into a condenser, if the resistances are small.

476. High Frequency Currents.-The reactive effects of inductance and capacity increase if the frequency is increased. The frequency used in electric lighting is from 50 to 120 cycles per second. If high frequencies of 1000 or more cycles per second are used the reactions are excessive. In such cases the currents do not flow equally through the cross-section of the conducting wire, but are confined mainly to its outer surface, even thick rods of copper offering great impedance. Even at a frequency of 100 the current at a depth of 12 millimetres from the surface is (in copper) only about $\frac{1}{7}$ of its value in the surface layers. In iron wires the depth of the skin for $\frac{1}{7}$ value is about 1 millimetre. For such rapid oscillations as the discharge of a Leyden jar, where the frequency is several millions, the conducting skin is probably less than $\frac{1}{100}$ of a millimetre thick. Hollow tubes in such cases conduct just as well as solid rods of same outer diameter. The conductance is proportional not to section but to perimeter. 
Whenever a current is not distributed equally in the cross-section of any conductor there is a real increase in the resistance it offers; the heating effect being a minimum when equally distributed. The fact that the oscillatory currents are greatest at the skin gives the strongest support to the modern view that the energy in an electric circuit is transmitted by the surrounding medium and not through the wire (see Art. 519 on energy-paths).

477. Alternate - current Electromagnets.When an alternate current is sent through a coil it produces an alternating magnetic field. An iron core placed in the alternating field will be subjected to a periodic alternating magnetization. Electromagnets for alternate currents must have their iron cores laminated to avoid eddy-currents; and owing to their choking action are made with fewer turns of wire than if designed for continuous currents of equal voltage. They repel sheets of copper owing to the eddy-currents which they set up in them; the phase of these eddy-currents being retarded by their self-induction. Elihu Thomson, who studied these repulsions, constructed some motors based on this principle. A solenoid, with a laminated iron plunger, if supplied with alternate currents at constant voltage, has the remarkable property of attracting the core with much greater force when the core is protruding out than when it is in the tube. This also is owing to the choking action.

\section{ILesson XLIV.-Alternate-current Generators}

478. Alternators.-The simple alternator (Fig. 243), with its two slip-rings for taking off the current, is merely typical. In practice machines are wanted which will deliver their currents at pressures of from 1000 to 5000 volts, with frequencies of from 50 to 120 cycles per second. 
Slower frequencies are unsuitable for lighting, though applicable for power transmission. High voltages are common with alternate currents because (when using transformers) of the economy (Art. 447) thereby effected in the copper mains. Under these conditions almost all alternators are designed as multipolar machines; and as the perfect insulation required in the armatures is more readily attained if these parts are stationary it is common to fix them, and instead to rotate the field-magnet. The latter is separately excited with a small continuous current led in through slip-rings. One advantage of alternate-current machines over continuous - current dynamos is that there is no commutator.

Amongst the various types of alternators may be mentioned the following:-(1) Magnet rotating internally and consisting of a number of poles, alternately $\mathrm{N}$ and $\mathrm{S}$, pointing radially outwards; armature external, fixed, and consisting of a number of coils wound either upon an iron ring or upon inwardly-projecting iron poles, or set against the inner face of an iron core, or embedded in holes just within the face of an iron core. In all cases where iron cores are used in armatures it is carefully laminated. (2) Magnet fixed externally and consisting of a number of alternate poles pointing radially inwards; armature internal, revolving, consisting of a number of coils wound either upon the surface of a cylindrical iron core, or fixed upon radially projecting poles. (3) Magnet fixed externally and consisting of two crowns of alternate poles, alternately $\mathrm{N}$ and $\mathrm{S}$, projecting toward one another and nearly meeting, so making a number of magnetic fields between them; armature revolving, and without iron, consisting of a number of flat coils mounted together as a sort of star disk, revolve in the narrow gaps between the poles. (4) "Inductor" machines having all magnetizing and inductive coils fixed, the only revolving part being a wheel to which masses of iron are affixed. (5) Another form due to Mordey, 
used in England, is depicted in Fig. 259. The thin armature coils are fixed, in an external stationary ring, between two crowns of poles revolving on each side of them. These poles are, however, all N-poles on one side, and all S-poles on the other, being projections of two massive iron pole-pieces fixed on the shaft against a huge

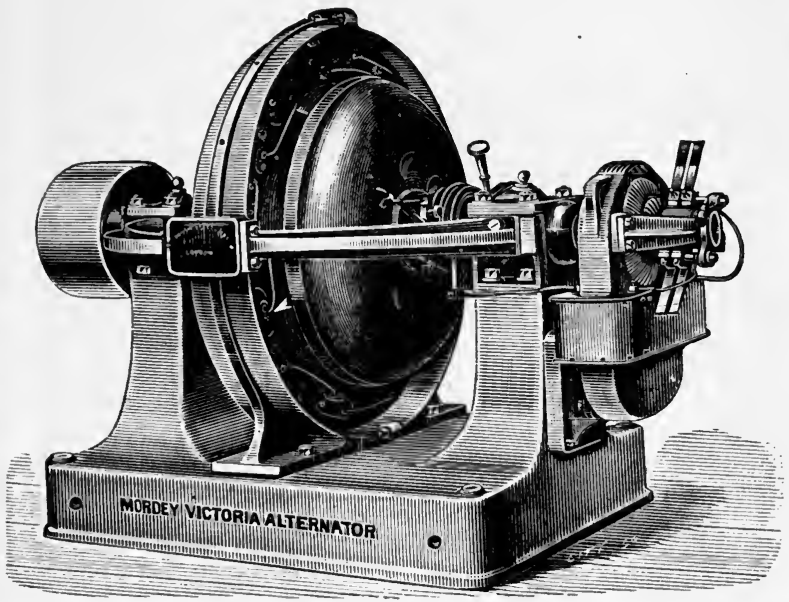

- Fig. 259.

internal bobbin, thus constituting a solid simple form of field-magnet. On the end of the shaft is a small continuous-current dynamo as exciter.

In Fig. 260 is given a view of the central generating station for the electric lighting of the city of London. Two kinds of alternators (Thomson-Houston and Mordey). are used. The cut shows one of the latter driven by an 800 horse-power steam-engine. Each of these machines has 40 poles in each crown, and can deliver 250 amperes at 2200 volts. 


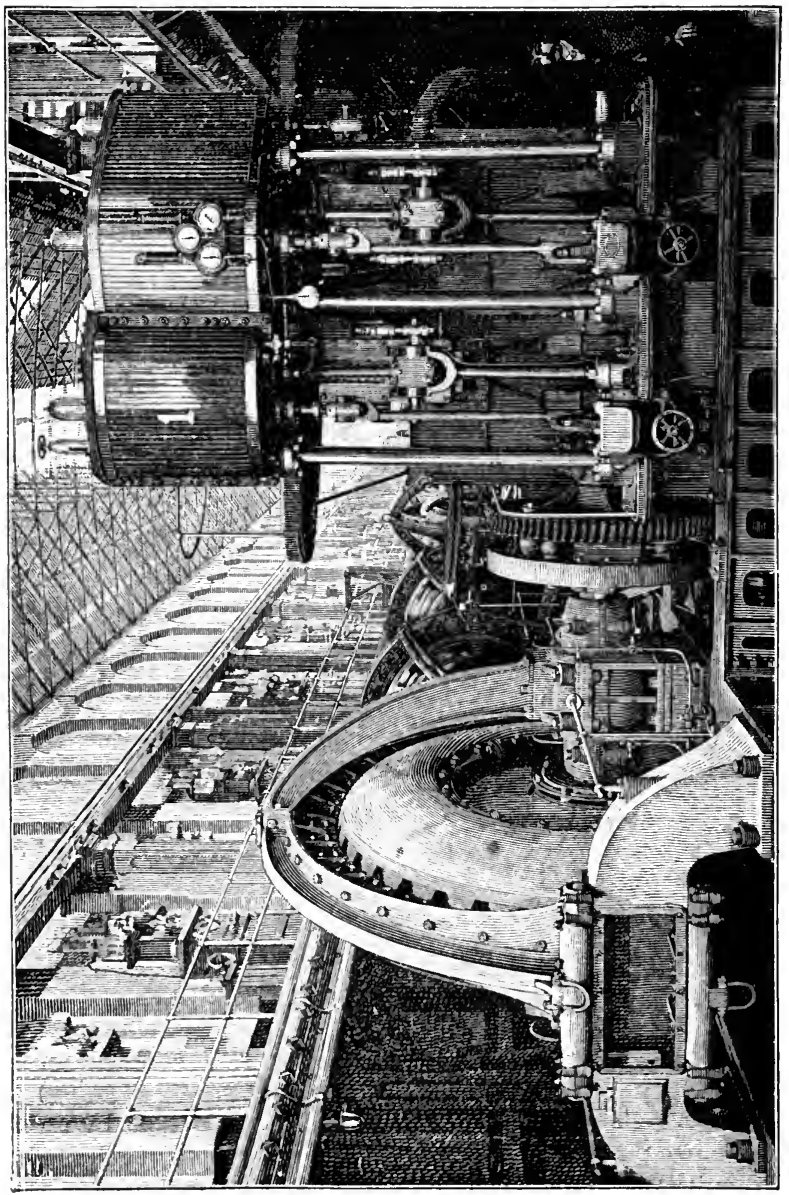

焉 
479. Coupling of Alternators.-In the use of two or more alternators on one circuit a peculiarity arises that does not exist with continuous-current dynamos, owing to differences of phase in the currents. If two alternators driven by separate engines are running at the same speed and at equal voltage, it will not do to join their circuits by merely switching them to the mains if they are not also in phase with one another; or serious trouble may occur. In central station work it is usual to run several machines all in parallel. Now if two machines are feeding into the same mains each is tending to send current back to the other; and if their electromotive forces are at any instant unequal, that with the greater will tend to send its current the opposite way through the other. To explain what occurs consider Fig. 261, which is a revolving diagram of the same kind as Figs. 251 and 254. If the two alternators are

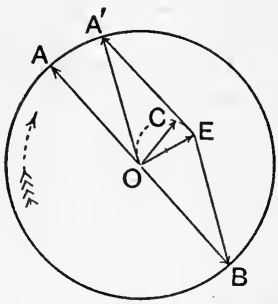

Fig. 261.

exactly in step they will both be sending a pulse of current toward the mains at the same moment, but, so far as the circuit connecting them is concerned, these impulses will be exactly opposed. Let $\mathrm{OA}$ and $\mathrm{OB}$ represent these two exactly-opposed impulses. Now suppose one of the two machines to gain a little on the other, $\mathrm{OA}$ shifting forward to $\mathrm{OA}^{\prime}$. The two electromotive-forces no longer balance, but will have a resultant $\mathrm{OE}$ tending to make a current oscillate through the two machines, this current being out of phase both with the leading machine $\mathrm{A}$ and with the lagging machine $B$. But this local current will itself lag a little in phase behind $\mathrm{OE}$ because of the inductance in its path. Let the phase of the current then be indicated by OC, which is set back a little. There is now a current surging to and fro between the two machines, and it is obviously more nearly in phase with 
$\mathrm{OA}$ than with $\mathrm{OB}$. This means that in the leading machine $A$ the volts and amperes are more nearly in phase with one another than in the lagging machine $B$. Reference to Arts. 436 and 445 will at once show that the current is helping to drive $B$ as a motor, and that a greater mechanical effort will be thrown on A, which is acting more as a generator. Hence this interchange of current tends automatically to bring up the lagging machine and to load the leading machine. They will come back into phase. All alternators of good construction suitably driven will run together in parallel, even though their electromotive-forces are unequal. On the other hand, if two alternators are joined in series, the resulting current, when they are ever so little out of phase, tends to load the lagging machine and hasten the leading one till they get into complete opposition of phase, one running entirely as generator, the other entirely as motor. This is excellent for transmission of power from an alternator at one end of a line to a synchronous alternator at the other: the two machines keep step at all loads. But they will not run together in series if both are to act as generators, unless rigidly coupled together on the same shaft.

To prevent accidents arising from too sudden a transfer of current between two machines it is usual in lighting stations to employ a synchronizer, a device to indicate the phases of the alternations. When an alternator is to be switched into circuit (in parallel with one or more others) the operator does not turn the switch until (speed and volts being both right) the electromotive-force of the machine has come exactly into identical phase with that of the circuit into which it is to be introduced.

\section{Lesson XLV.-Transformers}

480. Alternate-Current Transformers.-Transformers are needed in the distribution of currents to a distance, because glow-lamps in the houses need low 
pressures of 50 to 100 volts, whilst for economy of copper in the mains it is necessary that the generators should work at high pressures of 1000 to 5000 or more volts. The principle of transformation was briefly touched in Art. 228. Alternate-current transformers are simply induction-coils having well-laminated iron cores, usually of thin, soft sheet-iron strips piled together, and shaped so as to constitute a closed magnetic circuit. Upon the cores are wound the primary coil to receive the alternating current, and a secondary coil to give out other alternating currents. Usually the primary consists of many turns of fine copper wire, very well insulated, to receive a small current at high pressure ; and the secondary of a few turns of thick copper wire or ribbon, to give out a much larger current at low pressure.

To transform down from about 2000 volts to 100 volts, the ratio of the windings will be $20: 1$. Whatever the ratio of the voltages, the currents will be about in the inverse ratio, since, apart from the inevitable small losses in transformation, the power put in and taken out will be equal. Taking the above case of a transformer having $20: 1$ as the ratio of its windings, if we desire to take out of the secondary 100 amperes at 50 volts, 'we must put into the primary at least 5 amperes at 1000 volts.

In scattered districts a small transformer is provided for each house, the lamps being in the low pressure cir-

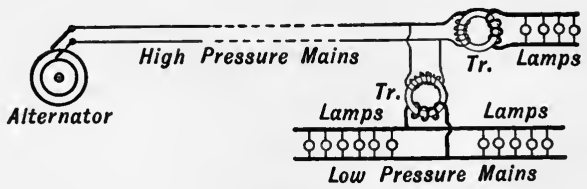

Fig. 262.

cuit. In cities large transformers are placed in substations, from which issue the low-pressure mains distributing the current to the houses. Fig. 262 shows 
in diagram the use of transformers on a distributing system.

481. Elementary Theory of Transformers.If the primary volts are maintained constant, the secondary volts will be nearly constant also, and the apparatus becomes beautifully self-regulating, more current flowing into the primary of itself when more lamps are turned on in the secondary circuit. This arises from the choking effect of self-induction in the primary. If no lamps are on the secondary circuit the primary coil simply acts as a choking-coil. When all the lamps are on, the primary acts as a working-coil to induce currents in the secondary. When only half the lamps are on, the primary acts partly as a choking-coil and partly as a working-coil.

Let $V_{1}$ be the volts at the primary terminals, $V_{2}$ those at the secondary terminals; $\mathrm{S}_{1}$ the number of turns in the primary coil, $\mathrm{S}_{2}$ the number in the secondary ; $r_{1}$ the internal resistance in the primary, $r_{2}$ that of the secondary. Call the ratio of transformation $k=\mathrm{S}_{1} / \mathrm{S}_{2}$. The alternations of magnetism in the core will set up electromotive-forces $\mathrm{E}_{1}$ and $\mathrm{E}_{2}$ in the two coils strictly proportional to their respective numbers of turns (if there is no magnetic leakage); so $\mathrm{E}_{2}=\mathrm{E}_{1} / k$; and since (apart from small hysteresis losses) $\mathrm{E}_{1} \mathrm{C}_{1}=\mathrm{E}_{2} \mathrm{C}_{2}$, it follows that $\mathrm{C}_{1}=\mathrm{C}_{2} / k$. The volts lost in primary are $r_{1} \mathrm{C}_{1}$, those in secondary $r_{2} \mathrm{C}_{2}$. Hence we may write

$$
\begin{aligned}
& \mathrm{V}_{1}=\mathrm{E}_{1}+r_{1} \mathrm{C}_{1}, \\
& \mathrm{~V}_{2}=\mathrm{E}_{2}-r_{2} \mathrm{C}_{2} .
\end{aligned}
$$

Writing the first as $\mathrm{E}_{1}=\mathrm{V}_{1}-r_{1} \mathrm{C}_{1}=\mathrm{V}_{1}-r_{1} \mathrm{C}_{2} / k$, and inserting $\mathrm{E}_{1} / k$ for $\mathrm{E}_{2}$ in the second equation, we get

$$
\mathrm{V}_{2}=\frac{\mathrm{V}_{1}}{k}-\left(\frac{r_{1}}{k^{2}}+r_{2}\right) \mathrm{C}_{2} ;
$$

which shows that everything goes on in the secondary as 
though the primary had been removed, and we had substituted for $V_{1}$ a fraction of it in proportion to the windings, and at the same time had added to the internal resistance an amount equal to the internal resistance of the primary, reduced in proportion to the square of the ratio of the windings. We also see that to keep the secondary volts constant the primary generator must be so regulated as to cause the primary volts to rise slightly when much current is being used. The currents in the two coils are in almost exact opposition of phase; they reach their maxima at the same instant, flowing in opposite senses round the core. The efficiency of well-constructed transformers is very high, the internal losses being a very small percentage of the working load.

482. Continuous-current Transformers (Motor-dynamos).-To transform continuous currents from one voltage to another it is necessary to employ a rotating apparatus, which is virtually a combination of a motor and a generator. For example, a motor receiving a current of 10 amperes at 1000 volts may be made to drive a dynamo giving out nearly 200 amperes at 50 volts. Instead of using two separate machines, one single armature may be wound with two windings and furnished with two commutators; the number of turns in the windings being proportioned to the voltages, and their sectional areas to the amperes. Such motor-dynamos are in use. The elementary theory of these is the same as that in Art. 481, $\mathrm{E}_{1}$ and $\mathrm{E}_{2}$ now standing for the electromotive-forces respectively induced in the two windings on the revolving armature.

483. Continuous-alternate Transformers.Revolving machinery equivalent to a combination of a continuous-current dynamo and an alternator may be used to transform continuous currents into alternating, or vice versa, one part acting as motor to drive, the other as generator. In this case also two separate machines need not always be used. Fig. 263 represents in diagram 
a simple rotatory converter having both a split-tube commutator to collect contin-

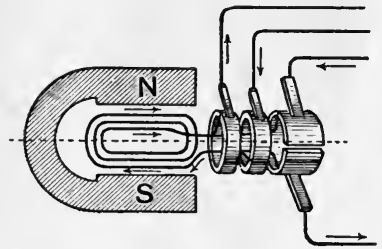

Fig. 263. uous currents, and a pair of slip-rings or alternating currents. Such a machine may convert continuous currents into alternating, or alternating into continuous. Or it may act as a motor if supplied with either kind of current; or

may, if driven mechanically, generate both kinds of current at the same time.

\section{Lesson XLVI.-Alternate-current Motors}

484. Alternate-current Motors.-We have seen (Art. 479) that one alternator can drive another as a motor, the two machines in series working in synchronism. There are two disadvantages in such motors-(i) that they are not self-starting, but must be brought up to speed before the current is applied; (ii) that their field-magnets must be separately excited. Other forms of motor have consequently been sought. Ordinary continuous-current motors, if made with laminated iron magnets, will work, though not well, with alternating currents.

The modern alternate-current motor has developed from the proposals of Borel (1887), Ferraris (1888), and Tesla (1888) to employ two or more alternating currents in different phases.

485. Polyphase Currents. - It is obviously possible, by placing on the armature of an alternator two separate sets of coils, one a little ahead of the other, to obtain two alternate currents of equal frequency and strength, but differing in phase by any desired degree. Gramme, indeed, constructed alternators with two and 
with three separate circuits in 1878 . If two equal alternate currents, differing in phase by one-quarter of a period, are properly combined, they can be made to produce a rotatory magnetic field. And in such a rotatory field conductors can be set rotating, as was first suggested by Baily in 1879 . Consider an ordinary Gramme ring (Fig. 264) wound with a continuous winding. If a single alternating current were introduced at the points $\mathrm{AA}^{\prime}$ it would set up an oscillatory magnetic field, a N-pole growing at $\mathrm{A}$, and a $\mathrm{S}$-pole at $\mathrm{A}^{\prime}$, then dying away and reversing in direction. Similarly, if another

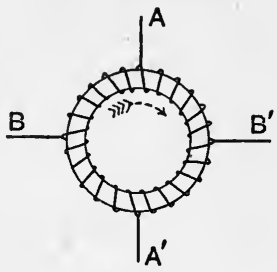

Fig. 264. alternate current were introduced at $\mathrm{BB}^{\prime}$, it would produce another oscillatory magnetic field in the $\mathrm{BB}^{\prime}$ diameter. If both these currents are set to work but timed so that the $\mathrm{BB}^{\prime}$ current is $\frac{1}{4}$ period behind the

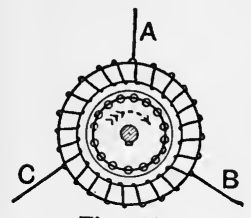

Fig. 265. $\mathrm{AA}^{\prime}$ current, then they will combine to produce a rotatory magnetic field, though the coil itself stands still. This is quite analogous to the wellknown way in which a rotatory motion, without any dead points, can be produced from two oscillatory motions by using two cranks at right angles to one another, the impulses being given $\frac{1}{4}$ period one after the other. The above combination is called a di-phase system of currents. If the $\mathrm{BB}^{\prime}$ current is $\frac{1}{4}$ period later than the $\mathrm{AA}^{\prime}$ current, the rotation in Fig. 265 will be right-handed. Another way of generating a rotatory field is by a tri-phase system * (or so-called "dreh-strom") of currents. Let $\mathbf{3}$ alternate currents, differing from one another by $\frac{1}{3}$ period (or $120^{\circ}$ ), be led

* Tri-phase currents were used in the famous Frankfort transmission of power in 1891. See Art. 447. 
into the ring at the points $\mathrm{A} \mathrm{B} \mathrm{C.} \mathrm{The} \mathrm{current} \mathrm{flows} \mathrm{in}$ first at $\mathrm{A}$ (and out by $\mathrm{B}$ and $\mathrm{C}$ ), then at $\mathrm{B}$ (flowing out by $C$ and $A$ ), then at $C$ (out by $A$ and $B$ ), again producing a revolving magnetic field. This is analogous to a 3 -crank engine, with the cranks set at $120^{\circ}$ apart.

There are several ways of combining the circuits that receive the currents of the various phases. For example, the windings of Fig. 264 might be divided into four separate coils, each having one end joined to a common junction, and the four outer ends joined respectively to the four line wires. Or the windings of Fig. 265 might be arranged as three separate coils, each having one end joined to a common junction, and with the three outer ends joined respectively to the three line wires. Such arrangements would be called star groupings, as distinguished from the mesh groupings of the cuts. Also the coils, in whichever way grouped, need not be wound upon a ring. The two-phase coils of Fig. 264 might be wound upon four inwardly-projecting pole-pieces; and the three-phase coils of Fig. 265 might be wound upon three inwardly-projecting pole-pieces. Or in larger multipolar machines a three-phase set of coils might be arranged upon a set of six, nine, twelve, or more projections, in regular succession.

For generating two-phase (or three-phase) currents the alternators must be designed with two (or with three) separate sets of windings in the armature; these separate sets of windings being so spaced out as to come into inductive operation in regular succession. There will thus be two (or three) independent circuits of equal voltage which may be then connected up in either a stargrouping or a mesh-grouping as described above. To transmit the two-phase currents four line-wires are usually employed. For transmitting three-phase currents three wires suffice.

486. Properties of the Rotatory Field Asynchronous Motors.-In such rotating inagnetic 
fields masses of metal at once begin to rotate. A magnet or mass of iron, pivoted centrally, can take up a synchronous motion, but may require to be helped to start. Any pivoted mass of good conducting metal, such as copper, will also be set in motion, and will be self-starting, but will not be synchronous. In such a centred mass, or rotor, eddy-currents are set up (just as in Arago's rotations, Art. 457), which drag the metal mass and tend to turn it. The strength of these currents in the rotating part depends on the relative speed of the field and the rotor. If the rotor were to revolve with speed equal to the revolving field, the eddy-currents would die away, and there would be no driving force. The rotor actually used in such motors consists of a cylindrical core built up of thin iron disks, over which is built up a sort of squirrel cage of copper rods joined together at their ends into a closed circuit. In some forms (designed by Brown) the rods are inserted in holes just below the surface of the core. The rotor need not have any commutator or sliprings, and is entirely disconnected from any other circuit. It receives its currents wholly by induction. Such asynchronous motors start with considerable torque (or turning moment) and have a high efficiency in full work. Similar motors for use with ordinary or single-phase alternate currents are now in use. To start them it is necessary to split the alternate current into two currents differing in phase. This is done by the use of a divided circuit, in the two branches of which different reactances are introduced. If in one branch there is a chokingcoil to offer inductance, the current in that branch will be retarded; if in the other there is a condenser, the current in this branch will be accelerated in phase. Combining these two currents a rotatory field is produced for starting the movement. When once the motor has started a further turn of the switch simply puts on the alternate current, as at $\mathrm{AA}^{\prime}$ in Fig. 264, and it continues to be driven, though the impulse is now only oscillatory. 


\section{CHAPTER XI}

\section{ELECTRO-CHEMISTRY}

\section{LESSON XLVII._Electrolysis}

487. Electromotive-force of Polarization.The simple laws of definite chemical action due to the current having been laid down in Lesson XIX., it remains to consider the relations between the chemical energy and its electrical equivalent. Whenever an electrolyte is decomposed by a current, the resolved ions have a tendency to reunite, that tendency being commonly termed "chemical affinity." Thus when zinc sulphate $\left(\mathrm{ZnSO}_{4}\right)$ is split up into $\mathrm{Zn}$ and $\mathrm{SO}_{4}$ the zinc tends to dissolve again into the solution, and so spread the potential energy of the system. But zinc dissolving into sulphuric acid sets up an electromotive-force of definite amount; and to tear the zinc away from the sulphuric acid requires an electromotive-force at least as great as this, and in an opposite direction to it. So, again, when acidulated water is decomposed in a voltameter, the separated hydrogen and oxygen tend to reunite and set up an opposing electromotive-force of no less than 1.47 volts. This opposing electromotive-force, which is in fact the measure of their "chemical affinity," is termed the electromotive-force of polarization. It can be observed in any water voltameter (Art. 243) by simply disconnecting the wires from the battery and joining them to a galvanometer, when a 
current will be observed flowing back through the voltameter from the hydrogen electrode toward the oxygen electrode. The polarization in a voltaic cell (Art. 175) produces an opposing electromotive-force in a perfectly similar way.

Now, since the affinity of hydrogen for oxygen is represented by an electromotive-force of 1.47 volts, it is clear that no cell or battery can decompose water at ordinary temperatures unless it has an electromotive-force of at least 1.47 volts. With every electrolyte there is a similar minimum electromotive-force necessary to produce complete continuous decomposition.

488. Theory of Electrolysis.-Suppose a current to convey a quantity of electricity $Q$ through a circuit in which there is an opposing electromotive-force $\mathrm{E}$ : the work done in moving $Q$ units of electricity against this electromotive-force will be equal to $\mathrm{E} \times \mathrm{Q}$. (If $\mathrm{E}$ and $Q$ are expressed in "absolute " C.G.S. units, $E \times Q$ will be in ergs.) The total energy of the current, as available for producing heat or mechanical motion, will be diminished by this quantity, which represents the work done against the electromotive-force in question.

But we can arrive in another way at an expression ior this same quantity of work. The quantity of electricity in passing through the cell will deposit a certain amount of metal: this amount of metal could be burned, or dissolved again in acid, giving up its potential energy as heat, and, the mechanical equivalent of heat being known, the equivalent quantity of work can be calculated. $\mathbf{Q}$ units of electricity will cause the deposition of $\mathrm{Q} z$ grammes of an ion whose absolute electro-chemical equivalent is z. [For example, $z$ for hydrogen is 0001038 gramme, being ten times the amount (see table in Art. 240) deposited by one coulomb, for the coulomb is $\frac{1}{10}$ of the absolute C.G.S. unit of quantity.] If $\mathrm{H}$ represents the number of heat units evolved by one gramme of the substance, when it enters into the combination in ques- 
tion, then $\mathrm{Q} z \mathrm{H}$ represents the value (in heat units) of the chemical work done by the flow of the $\mathrm{Q}$ units ; and this value can immediately be translated into ergs of work by multiplying by Joule's equivalent J $\left(=42 \times 10^{6}\right)$. [See Table on page 512.]

We have therefore the following equality :-

$$
\begin{aligned}
\mathrm{EQ} & =\mathrm{Q} z \mathrm{HJ} ; \text { whence it follows that } \\
\mathrm{E} & =2 \mathrm{HJ} ; \text { or, in words, the electromotive-force }
\end{aligned}
$$
of any chemical reaction is equal to the product of the electrochemical equivalent of the separated ion into its heat of combination, expressed in dynamical units.

Examples. * - (1) Electromotive-force of Hydrogen tending to unite with Oxgyen. For Hydrogen $z=\cdot 0001038 ; \mathrm{H}$ (heat of combination of one gramme) $=34000$ grammedegree-units ; $J=42 \times 10^{6}$.

$.0001038 \times 34000 \times 42 \times 10^{6}=1.48 \times 10^{8}$ "absolute" units of electromotive-force, or $=1.48$ volts.

(2) Electromotive-force of Zinc dissolving into Sulphuric Acid. $z=\cdot 00337 ; \mathrm{H}=1670$ (according to Julius Thomsen); $J=42 \times 10^{6}$.

$$
\begin{aligned}
\cdot 00337 \times 1670 \times 42 \times 10^{6} & =2 \cdot 364 \times 10^{8} . \\
\text { or } & =2 \cdot 364 \text { volts. }
\end{aligned}
$$

(3) Electromotive-force of Copper dissolving into Sulphuric Acid. $z=00327 ; \mathrm{H}=909.5 ; \mathrm{J}=42 \times 10^{6}$.

$$
\begin{aligned}
\cdot 00327 \times 909 \cdot 5 \times 42 \times 10^{6} & =1 \cdot 249 \times 10^{8} . \\
\text { or } & =1.249 \text { volts. }
\end{aligned}
$$

(4) Electromotive-force of a Daniell's Cell. Here zinc is dissolved at one pole to form zinc sulphate, the chemical action setting up a + electromotive-force, while at the other pole copper is deposited by the current out of a

* The figures given in these examples as well as those on p. 512 for the heat of combination must be taken as only approximate. The heat of combination is different at different temperatures, and the heat evolved by the salt dissolving in water must also be taken into account. Exact figures have not yet been ascertained. In fact von Helmholtz showed that the expression $z \mathrm{HJ}$ is incomplete, and that to it should be added a term $\theta \cdot d \mathrm{E} / d \theta$, wherein $\theta$ is the absolute temperature of the cell. 
solution of copper sulphate, thereby setting up an opposing (or -) electromotive-force. That due to zinc is shown above to be +2.364 volts, that to deposited copper to be $-1 \cdot 249$. Hence the net electromotiveforce of the cell is (neglecting the slight electromotiveforce where the two solutions touch) $2 \cdot 364-1 \cdot 249=$ $1 \cdot 115$ volts. This is nearly what is found (Art. 181) in practice to be the case. It is less than will suffice to electrolyze water, though two Daniell's cells in series electrolyze water easily.

Since 1 horse-power-hour $=746$ watt - hours $=746$ ampere-hours at 1 volt, it follows that at $\mathrm{V}$ volts the number of ampere-hours will $=746 \div \mathrm{V}$. Now as the weight of zinc consumed in a cell is 1.213 grammes per ampere-hour (when there is no waste), the consumption will be as follows :-

$\left.\begin{array}{l}\text { Weight of zinc used } \\ \text { per horse-power-hour }\end{array}\right\}=\frac{746}{\mathrm{~V}} \times 1 \cdot 213 \mathrm{grm} .=\frac{2}{\mathrm{~V}} \mathrm{lbs}$.

Hence the quantity of zinc that must be consumed to generate 1 horse-power-hour in any battery of cells cannot be less than $2 \mathrm{lbs} . \div$ the available volts of a single cell of the battery.

Example.-If a new cell can be invented to give 2 volts at its terminals when in full work, a battery of such cells, however arranged, will consume $1 \mathrm{lb}$. of zinc per hour per horse-power, or 1.34 lbs. per "unit" of supply (or kilowatt-hour).

An equivalent quantity of exciting and depolarizing chemicals will also be used, and these will increase the total cost per unit. It is clear that as a source of public supply primary batteries consuming zinc can never compete in price with dynamos driven by steam. The actual cost of coal to central stations in London is from 1 to $1 \frac{1}{2}$ pence per " unit"; and the maximum legal price that a supply company may charge in Great Britain for electric energy is eightpence per "unit." See Art. 440.

489. Electro-Chemical Power of Metals.-The accompanying Table gives the electromotive-force of the 
different metals as calculated (Art. 488) from the heat evolved by the combination with oxygen of a portion of the metal equivalent electro-chemically in amount to one gramme of hydrogen. The figures in the second column are in calories. The figures in the third column are calculated from those in the second by multiplying by the electro-chemical equivalent of hydrogen, and by Joule's equivalent $\left(42 \times 10^{6}\right)$ and dividing by $10^{8}$, to reduce to volts. The electromotive-forces as observed (in dilute sulphuric acid) are added for comparison.

\begin{tabular}{|c|c|c|c|c|}
\hline \multirow{2}{*}{ Substance. } & \multirow{2}{*}{$\begin{array}{c}\text { Heat of Oxi- } \\
\text { dation of } \\
\text { Equivalent. }\end{array}$} & \multicolumn{2}{|c|}{ I. M.F. calculated. } & \multirow{2}{*}{$\begin{array}{l}\text { E.M.F. } \\
\text { observed. }\end{array}$} \\
\hline & & $\begin{array}{l}\text { Relatively } \\
\text { to Oxygen. }\end{array}$ & $\begin{array}{l}\text { Relatively } \\
\text { to Zinc. }\end{array}$ & \\
\hline Potassium & 69,800 & $3 \cdot 01$ & $+1 \cdot 18$ & $+1 \cdot 13$ \\
\hline Sodium . . . & 67,800 & $2 \cdot 91$ & $+1 \cdot 09$ & \\
\hline Zinc. . . . . & 42,700 & $1 \cdot 83$ & 0 & 0. \\
\hline Iron . . . . & 34,120 & $1 \cdot 55$ & -0.28 & \\
\hline Hydrogen . . . & 34,000 & $1 \cdot 47$ & -0.36 & \\
\hline Lead. . . & 25,100 & $1 \cdot 12$ & -0.71 & -0.54 \\
\hline Copper . & 18,760 & 80 & -1.08 & $-1 \cdot 047$ \\
\hline Silver . & 9,000 & $\cdot 39$ & $-1 \cdot 44$ & \\
\hline Platinum & 7,500 & $\cdot 33$ & $-1 \cdot 50$ & $-1 \cdot 53$ \\
\hline Carbon . . & 2,000 & .09 & $-1 \cdot 74$ & \\
\hline Oxygen. . & & 0 & $-1 \cdot 83$ & $-1 \cdot 85$ \\
\hline $\begin{array}{l}\text { (Nitric Acid) } \\
\text { (Black Oxide of }\end{array}$ & $-6,000$ & -0.26 & $-2 \cdot 09$ & $-1 \cdot 94$ \\
\hline Manganese) & $-6,500$ & -0.29 & $-2 \cdot 12$ & $-2 \cdot 23$ \\
\hline (Peroxide of Lead) & $-12,150$ & -0.52 & $-2 \cdot 35$ & $-2 \cdot 52$ \\
\hline (Ozone) & $-14,800$ & -0.63 & $-2 \cdot 46$ & $-2 \cdot 64$ \\
\hline Acid). . . & $-25,070$ & $-1 \cdot 09$ & $-2 \cdot 92$ & -3.03 \\
\hline
\end{tabular}

The order in which these metals are arranged is in fact nothing else than the order of oxidizability of the metals (in the presence of dilute sulphuric acid); for that metal tends most to oxidize which can, by oxidizing, give out the most energy. It also shows the order in which 
the metals stand in their power to replace one another (in a solution containing sulphuric acid). In this order, too, the lowest on the list are the metals deposited first by an electric current from solutions containing two or more of them: for that metal comes down first which requires the least expenditure of energy to separate it from the elements with which it was combined.

490. General Laws of Electrolytic Action.In addition to Faraday's quantitative laws given in Art. 240, the following are important :-

(a) Every electrolyte is decomposed into two portions, an anion and a kation, which may be themselves either simple or compound. In the case of simple binary compounds, such as fused salt $(\mathrm{NaCl})$, the ions are simple elements. In other cases the products are often complicated by secondary actions. It is even possible to deposit an alloy of two metals-brass, for example-from a mixture of the cyanides of zinc and of copper.

(b) In binary compounds and most metallic solutions, the metal is deposited by the current where it leaves the cell, at the kathode.

(c) Aqueous solutions of salts of the metals of the alkalies and alkaline earths deposit no metal, but evolve hydrogen owing to secondary action of the metal upon the water. From strong solutions of caustic potash and soda Davy succeeded in obtaining metallic sodium and potassium, which were before unknown. If electrodes of mercury are employed, an amalgam of either of these metals is readily obtained at the kathode. The so-called ammonium-amalgam is obtained by electrolyzing a warm, strong solution of salammoniac between mercury electrodes.

(d) Metals can be arranged in a definite series according to their electrolytic behaviour; each metal on the list behaving as a kation (or being "electropositive") when electrolyzed from its compound in preference to one lower down on the list. In such a series the oxidiz- 
able metals, potassium, sodium, zinc, etc., come last; the less oxidizable or "electronegative" metals preceding them. The order varies with the nature, strength, and temperature of the solution used.

(e) From a solution of mixed metallic salts the least electropositive metal is not deposited first, if the current is so strong relatively to the size of the kathode as to impoverish the solution in its "neighbourhood. To deposit alloys a solution must be found in which both metals tend to dissolve with equal electromotive-forces.

$(f)$ The liberated ions appear only at the electrodes.

$(g)$ For each electrolyte a minimum electromotive-force is requisite, without which complete electrolysis cannot be effected. (See Art. 491.)

(h) If the current be of less electromotive-force than the requisite minimum, electrolysis may begin, and a feeble current flow at first, but no ions will be liberated, the current being completely stopped as soon as the opposing electromotive-force of polarization has risen to equality with that of the electrolyzing current.

(i) There is no opposing electromotive-force of polarization when electrolysis is effected from a dissolving anode of the same metal that is being deposited at the kathode. The feeblest cell will suffice to deposit copper from sulphate of copper if the anode be a copper plate.

$(j)$ Where the ions are gases, pressure affects the conditions but slightly. Under 300 atmospheres acidulated water is still electrolyzed; but in certain cases a layer of acid so dense as not to conduct collects at the anode and stops the current.

(k) The chemical work done by a current in an electrolytic cell is proportional to the minimum electromotive-force of polarization.

(l) Although the electromotive-force of polarization may exceed this minimum, the work done by the current in overcoming this surplus electromotive-force will not appear as chemical work, for no more of the ion will be 
liberated ; but it will appear as an additional quantity of heat (or "local heat") developed in the electrolytic cell.

(m) Ohm's law holds good for electrolytic conduction.

(n) Amongst the secondary actions which may occur the following are the chief :

(1) The ions may themselves. decompose; as $\mathrm{SO}_{4}$ into $\mathrm{SO}_{3}+\mathrm{O}$. (2) The ions may react on the electrodes; as when acidulated water is electrolyzed between zinc electrodes, no oxygen being liberated, owing to the affinity of zinc for oxygen. (3) The ions may be liberated in an abnormal state. Thus oxygen is frequently liberated in its allotropic condition as ozone, particularly when permanganates are electrolyzed. The "nascent" hydrogen liberatecl by the electrolysis of dilute acid has peculiarly active chemical properties. So also the metals are sometimes deposited abnormally: copper in a black pulverulent film; antimony in roundish gray masses (from the terchloride solution) which possess a curious explosive property. When a solution of lead is electrolyzed a film of peroxide of lead forms upon the anode. If this be a plate of polished metal placed horizontally in the liquid beneath a platinum wire as a kathode, the deposit takes place in symmetrical rings of varying thickness, the thickest deposit being at the centre. These rings, known as Nobili's rings, exhibit all the tints of the rainbow, owing to interference of the waves of light occurring in the film. The colours form, in fact, in reversed order, the "colours of thin plates" of Newton's rings.

\section{Hypotheses of Grotthuss and of Clau-} sius.-A complete theory of electrolysis must explainfirstly, the transfer of electricity, and secondly, the transfer of matter, through the liquid of the cell. The latter point is the one to which most attention has been given, since the "migration of the ions" (i.e. their transfer through the liquid) in two opposite directions, and their appearance at the electrodes only, are salient facts.

The hypothesis put forward in 1805 by Grotthuss serves fairly, when stated in accordance with modern terms, to explain these facts. Grotthuss supposes that, when two metal plates at different potentials are placed 
in a cell, the first effect produced in the liquid is that the molecules of the liquird arrange themselves in innumerable chains, in which every molecule has its constituent atoms pointing in a certain direction; the atom of electropositive substance being attracted toward the kathode, and the fellow atom of electronegative substance being attracted toward the anode. (This assumes that the constituent atoms grouped in the molecule retain their individual electric properties.) The diagram of Fig. 266 shows, in the case of hydrochloric

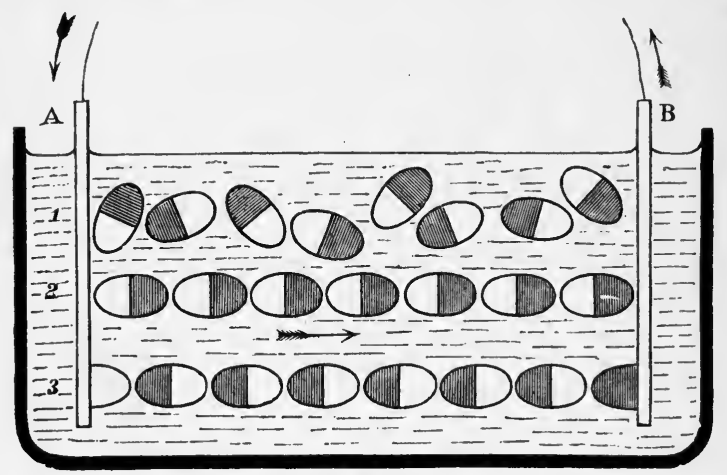

Fig. 266.

acid, a first row of molecules distributed at random, and secondly grouped in a chain as described. The action which Grotthuss then supposes to take place is that an interchange of partners goes on between the separate atoms all along the line, each $\mathrm{H}$ atom uniting with the $\mathrm{Cl}$ atom belonging to the neighbouring molecule, $a+$ half molecule of hydrogen being liberated at the kathode, and a - half molecule of chlorine at the anode. This action would leave the molecules as in the third row, and would, when repeated, result in a double migration of hydrogen atoms in one direction and of chlorine atoms 
in the other; the free atoms appearing only at the electrodes, and every atom so liberated discharging a certain definite minute charge of electricity upon the electrode where it was liberated.*

Clausius sought to bring the ideas of Grotthuss into conformity with the modern kinetic hypothesis of the constitution of liquids. He supposes that in the nsual state of a liquid the molecules are always gliding about amongst one another, and their constituent atoms are also in novement, continually separating and recombining into similar groups, their movements taking place in all possible directions throughout the liquid. But under the influence of an electromotive-force these actions are controlled in direction, so that when, in the course of the usual movements, an atom separates from a group it tends to move either towarl the anode or kathode; and if the electromotive-force in question be powerful enough to prevent recombination, these atoms will be permanently separated, and will accumulate around the electrorles. This theory has the advantage of accounting for a fact easily observed, that an electromotive-force less than the minimun which is neederl to effect complete electrolysis may send a feeble current through an electrolyte for a limited time, until the opposing electromotive-force has reached an equal value. Von Helinholtz, who gave the name of electrolytic cunvexion to this phenomenon of partial electrolysis, assumed that it takes place by the agency of uncombined atoms previously existing in the liquid.

491a. Migration of the Ions.--So far as explained it might be supposed that the migrations of the constituents along the molecular chains during electrolysis was merely a continually repeated exchange of partners between the two sets of ions, the anions and kations travelling thus at equal rates, in opposite directions, toward the anode and kathode respectively. There are, however, some

* Mr. G. J. Stoney has reckoned, from considerations founded on the size of atoms (as calculated by Ioschmidt and Iord Kelvin), that for every chemical bond ruptured, a charge of 10-20 of a coulomb is transferred. [E. Budde says $17 \times 10-20$ conlomb.] This quantity would appear therefore to be the natural atomic charge or unit. To tear one atom of hydrogen from a hydrogen compound this amount of electricity must be sent through it. To liberate an atom of zinc, or any nther divalent metal from its compound, implies the transfer of twice this amount of electricity. 
additional facts to be observed by experiment which indicate that the anions and kations travel at different rates, and that each ion has, under given circumstances, its own specific rate of migrating. Hittorf, who first drew attention to these facts, tabulated the observed ionic velocities. Since then Kohlrausch, Arrhenius, Ostwald and others have shown that this property is intimately connected with the conductivity of the electrolyte, and with the phenomena of solubility, of osmotic pressure, and of vapour pressure. In fact, a whole new chapter of electro-chemistry has thus been opened out.

The fundamental experiment upon which is based the

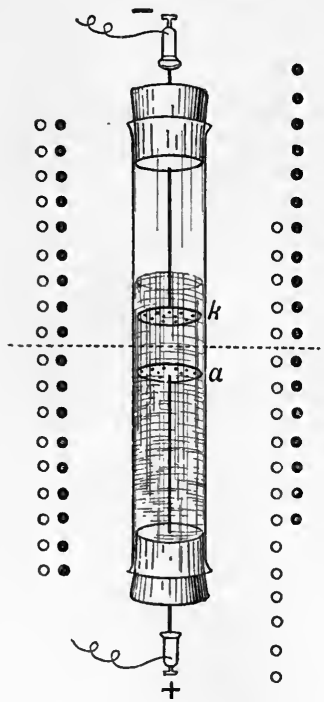

Fig. 266a. modern conception of the velocity of migration of the ions is an - exceedingly simple one. Let a - simple glass tube about a foot - long and an inch in internal diameter be provided with wellfitting corks at its two ends, as in Fig. 266a. In this is placed a nearly concentrated and slightly acidified solution of copper sulphate to be electrolysed. Through the corks pass two stout copper wires each furnished at the end with a round disk of sheet-copper, perforated with holes to permit of circulation of liquid. The upper one $k$, which serves as kathode, is just immersed below the surface of the liquid; the other $a$, which is the anode, is placed two or three inches lower down in the liquid. The current from a few cells of battery is then sent upwards through the electrolyte, the current being so regulated that it is not too strong, otherwise bubbles of gas will be given off and dis- 
turb the experiment. Copper will, of course, be plated upon the upper or kathode plate, an equal amount of copper being dissolved off the lower or anode plate. After half an hour or so it will be seen that, immediately under the kathode, the blue liquid has become quite colourless, and if the experiment is continued, the surface of separation between the colourless liquid at the top and the blue liquid below it will be found to have moved steadily downward. the current is sent downwards no such phenomenon can be seen, owing to the descent by gravity of the heavier blue liquid). The colourless liquid is simply water slightly acidulated. There are two ways of explaining that which has occurred. One is that, in some way, in addition to the ordinary electrolysis in which the ions $\mathrm{Cu}$ and $\mathrm{SO}_{4}$ have been transferred in opposite directions, there has been a bodily transfer toward the anode of the $\mathrm{CuSO}_{4}$ which was in solution. The other mode of explanation is that the ions $\mathrm{Cu}$ and $\mathrm{SO}_{4}$ have travelled with different velocities; the $\mathrm{Cu}$ travelling upward more slowly than the $\mathrm{SO}_{4}$ downward. In the diagrams to the left and right of the apparatus in Fig. $266 a$ are shown some rows of dots for the purpose of illustrating the relative numbers of the ions in the upper and lower parts of the liquid. The black dots show the kations $(\mathrm{Cu})$, and the white ones the anions $\left(\mathrm{SO}_{4}\right)$. Before electrolysis begins the solution is alike, as shown on the left; there being 9 anions and 9 kations (that is 9 of $\mathrm{CuSO}_{4}$ ) in each part, upper and lower. Suppose that electrolysis has gone on for so long a time that 6 of the kations have been dissociated and plated on the upper disk, and that 6 of the anions have been likewise liberated and carried down to the anode, there to combine with fresh copper. Now, if the observed state of things is represented by the diagram on the right, it will be seen that while in the upper layer there are $5 \mathrm{CuSO}_{4}$ molecules, in the lower there are $7 \mathrm{CuSO}_{4}$ molecules. together with the $6 \mathrm{SO}_{4}$ which have gone to dissolve fress wpper. If the migrations of anions 
and kations had been equal there would have been 6 $\mathrm{CuSO}_{4}$ in each layer. If the anions alone had migrated, downwards, there would have been $15 \mathrm{CuSO}_{4}$ below and $3 \mathrm{CuSO}_{4}$ above. If the kations alone had moved, upwards, there would have been $9 \mathrm{CuSO}_{4}$ in the upper layer, leaving 3 of the original $\mathrm{CuSO}_{4}$ in the lower, together with the newly-formed $6 \mathrm{CuSO}_{4}$. If, however, the diagram on the right represents the facts, either $2 \mathrm{CuSO}_{4}$ must have been bodily transferred into the lower layer from the upper, or else the transfer of ions must have been unequal, 4 anions going downwards into the lower layer, while 2 kations have gone upward into the upper layer. In other words, $\frac{2}{6}$ or 0.33 of the total displacement has been that of the copper ions, while $\frac{4}{6}$ or 0.66 has been that of the $\mathrm{SO}_{4}$ ions. These numbers Hittorf called the migration constants: they state the relative velocities with which the ions migrate. The numbers vary with the concentration of the solution. Thus, in the case of copper sulphate, if the solution contains 2 gramme-equivalents per litre the migration constant for the anion is about $0 \cdot 725$, while if it contain only $\frac{1}{25}$ as much per litre the constant falls to 0.638 . If this number for the anion be called $n$, then that of the kation will obviously be $1-n$. If we denote by $u$ and $v$ the actual velocities with which the kations and anions respectively travel under a potential gradient of 1 volt per centimetre of length of the electrolyte, we clearly may write the equation

$$
\frac{u}{v}=\frac{1-n}{n} \text {. }
$$

Further the relative velocity of the ions past one another will be $u+v$. If by using a stronger battery we cause a greater fall of potential per centimetre than 1 volt, the actual velocities will be proportionally greater, but the ratio of $u$ to $v$ will remain as before. The actual velocities $u$ and $v$ Kohlrausch derluced from the specific conductivities of the liquids. For if 0.0001038 grammes 
be the electro-chemical equivalent of hydrogen, and if there be $\mathrm{N}$ gramme-equivalents of the dissolved electrolyte in 1 cubic centimetre of the solution, then $\mathrm{N} \div 0.0001038$ will be the number of coulombs of electricity concerned in electrolysing this amount of the solution; and if the ions are dragged past one another with a speed of $u+v$ (centimetres per second) the How of electricity in one second across unit area will be $(u+v) \mathrm{N} \div 0.0001038$. Now if the fall of potential across a length of $x$ centimetres be called $\mathrm{V}$, the potential gradient being therefore $\mathrm{V} \div x$, the current will be equal to this multiplied by the specific conductivity $k$; and equating these we have-

$$
u+v=0.0001038 \frac{k}{\mathrm{~N}} \cdot \frac{\mathrm{V}}{x} ;
$$

or for a potential gradient of 1 volt $\left(=10^{8}\right.$ C.G.S.) per centimetre-

$$
u+v=10380 \frac{k}{\mathrm{~N}} ;
$$

or finally-

$$
u+v=10380000 \frac{k}{u},
$$

where $u$ is the number of gramme-equivalents per litre. Kohlrausch determined the values of the molecular conductivity $k \div n$ for many solntions. He found it to increase with dilution; becoming constant for each salt at very extreme dilutions. He also found that the values of this velocity came out the same for the same ion when used in different chemical combinations. Thus for hydrogen at $18^{\circ} \mathrm{C}$, and under a gradient of 1 volt per centimetre, the ionic velocity is 0.00320 centimetres per second ; that of sodium 0.00045 ; that of silver 0.00057 .

According to Arrhenius, the electrolysing current does not require to split the molecules; he regards the act of solution as ionising the dissolved salt producing free ions, each having its associated + or - charge. 


\section{LESSON XLVIII.-Accumulators}

492. Accumulators or Secondary Batteries. -A voltameter, or series of voltameters, whose electrodes are thus charged respectively with hydrogen and oxygen,

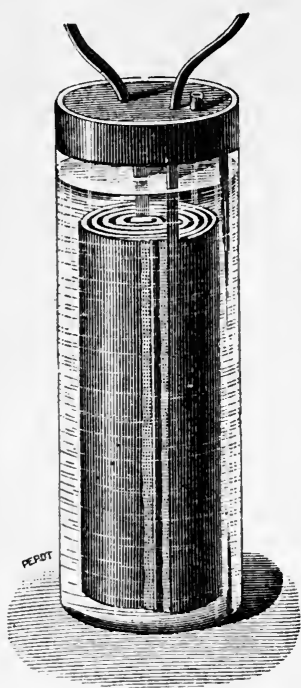

Fig. 26\%. will serve as secondary batteries, in which the energy of a current may be stored up and again given out. Ritter, who in 1803 constructed a secondary pile, used electrodes of platinum. It will be seen that such cells do not accumulate or store electricity; what they accumulate is energy, which they store in the form of chemical work. A secondary cell resembles a Leyden jar in that it can be charged and then discharged. The residual charges of Leyden jars, though small in quantity and transient in their discharge, yet exactly resemble the polarization-charges of voltameters. Varley found 1 sq. centim. of platinum foil in dilute acid to act as a condenser of about 63 microfarads' capacity, when polarized to a potentialdifference of 1 volt. Gaston Planté, in 1860, devised a secondary cell consisting of two pieces of sheet lead rolled up (without actual contact) as electrodes, dipping into dilute sulphuric acid, as in Fig. 267. To "form" or prepare the learl it was charged with currents which after a time were reversed in direction, and after a further time again reversed, until, after several reversals, it became coated with a semi-porous film of brown dioxide of lead 
on the anode plate; the kathode plate assuming a spongy metallic state presenting a large amount of surface of high chemical activity. When such a secondary battery, or accumulator, is charged by comnecting it with a dynamo (shunt-wound), or other powerful generator of currents, the anode plate becomes peroxidized, while the kathode plate is deoxidized by the hydrogen that is liberated. The plates may remain for many days in this condition, and will furnish a current until the two lead surfaces are reduced to a chemically inactive state. The electromotive-force of such cells is from $2 \cdot 0$ to 1.85 volts during discharge. Planté ingeniously arranged batteries of such cells so that they can be charged in parallel, and discharged in series, giving (for a short time) strong currents at extremely high voltages. Faure, in 1881, modified - the Planté accumulator by giving the two lead plates a preliminary coating of red-leal (or minium). When a eurrent is passed through the cell to charge it, the redlead is peroxidized at the anode, and reduced-first to a condition of lower oxide, then to the spongy metallic state-at the kathode, and thus a greater thickness of the working substance is provided, and takes far less time to "form" than is the case in Planté's cells. In modern accumulators the red-lead (or litharge), freshly mixed with dilute sulphuric acid to the form of a paste, is pressed into the holes of a leaden grid, shaped so as to

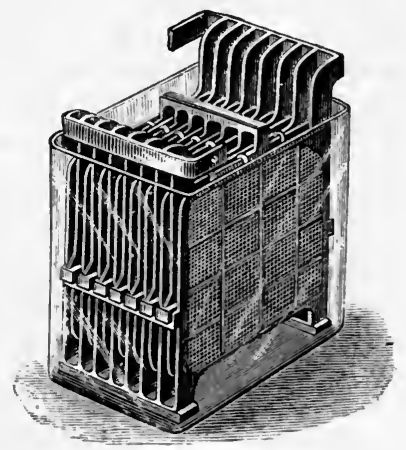

Fig. 268. give it a good mechanical attachment. During the subsequent process of "formation" the hardened paste is reduced on one plate and peroxidized on the other. A 
cell of the kind known as the E.P.S. cell is shown in Fig. 268. Accumulators are still made on the Planté method from metallic lead, which is first finely divided on its surface by some mechanical or chemical means, and then "formed" by prolonged charging. Cells of this type are not so subject to disintegration as paste cells, and may be discharged at a greater rate. To keep accumulators in good condition they should be charged up every day till full (known by bubbles rising) and not be discharged too quickly. The density of acid should never be allowed to exceed $1 \cdot 21$ nor fall below $1 \cdot 15$.

493. Grove's Gas Battery.-Sir W. Grove devised a cell in which platinum electrodes, in contact respectively with hydrogen and oxygen gas, replaced the usual zinc and copper plates. Each of these gases is partially occluded by the metal platinum, which, when so treated, behaves like a different metal.

Attempts have been made to generate electricity on a larger scale by means of gas batteries. Mond and Langer found that the greatest E.M.F. to be obtained from a cell of hydrogen and oxygen, with finely-divided platinum as collectors, was 0.97 , the difference between this and the theoretical, $1 \cdot 47$, being lost in heat generated by the condensation of the gases by the platinum.

\section{Llesson XLIX.—Electrodeposition}

494. Electrometallu rgy.-The applications of electro-chemistry to the industries are threefold. Firstly, to the reduction of metals from solutions of their ores, the process is useful in the accurate assay of certain ores, as, for example, of copper ; secondly, to the copying of types, plaster casts, and metal-work by kathode deposits of metal ; thirdly, to the covering of objects made of baser metal with a thin film of another metal, such as gold, silver, or nickel. All these operations are included under the general term of electrometallurgy. 
Pure aluminium is now produced in large quantities by the electrolysis of fused cryolite, which is a double fluoride of aluminium and sodium, pure alumina being added from time to time.

Copper of a high degree 'of purity is produced on a large scale by suspending anodes of impure copper in a solution of copper sulphate and electrolytically depositing pure copper on the kathodes. The impurities such as arsenic being more electronegative than copper are left in the bath.

495. Flectrotyping.-In 1836 De la Rue observed that in a Daniell's cell the copper deposited out of the solution upon the copper plate which served as a kathode took the exact impress of the plate, even to the seratches upon it. In 1839 Jacobi in St. Petersburg, Spencer in Liverpool, and Jordan in London, independently developed ont of this fact a method of obtaining, by the electrolysis of copper, impressions (in reversed relief) of coins, stereotype plates, and ornaments. A further improvement, due to Murray, was the employment of moulds of plaster or wax, coated with a film of plumbago in order to provide a conducting surface upon which the deposit could be made. Bronze in the form of a fine powder is much used instead of plumbago, being a better conductor. Jacobi gave to the process the name of galvano-plastic, a term generally abandoned in favour of the term electrotyping or electrotype process.

Electrotypes of copper are easily made by hanging a suitable mould in a cell containing a nearly saturated and slightly acidulated solution of sulphate of copper, and passing a current of a battery through the cell, the mould metallized on its surface being the kathode, a plate of copper being employed as an anode, dissolving gradually into the liquid at a rate exactly equal to the rate of deposition at the kathode. This use of a separate cell or "bath" is more convenient than producing the electrotypes in the actual cell of a Daniell's battery. The 
process is largely employed at the present day to reproduce repoussé and chased ornament and other works of art in facsimile, and to multiply copies of wood blocks for printing. Almost all the illustrations in this book, for example, are printed from electrotype copies, and not from the original wood blocks, which would not wear so well. In all deposition processes success largely depends on having the proper current-density. To deposit metals that are more positive than hydrogen, such as zinc or chromium, it is advisable to use concentrated solutions and high current-densities. For metals that are less positive, such as copper and silver, the current-density may be less. To procure a good tough deposit of copper the current should not exceed 15 amperes per square foot of kathode surface. If a more rapid deposit is required, a solution of nitrate of copper should be used and kept in rapid agitation.

To deposit iron (by the process known as acierage, or steel-facing) a very large sheet of iron is used as anode, and the liquid used is simply a solution of salammoniac in water. This solution is "charged" with iron by passing the current for a little time through the bath prior to inserting the object to be steel-faced.

496. Electroplating.-In 1801 Wollaston observed that a piece of silver, connected ${ }^{\prime}$ with a more positive metal, became coated with copper when put into a solution of copper. In 1805 Brugnatelli gilded two silver medals by making them the kathodes of a cell containing a solution of gold. Messrs. Elkington, about the year 1840 , introduced the commercial processes of electroplating. In these processes a baser metal, such as German silver (an alloy of zinc, copper, and nickel), is covered with a thin film of silver or gold, the solutions employed being, for electro-gilding, the double cyanide of gold and potassium, and for electro-silvering the double cyanide of silver and potassium.

Fig. 269 shows a battery and a plating-vat containing 
the silver solution. As anode is hung a plate of metallic silver which dissolves into the liquid. To the kathode

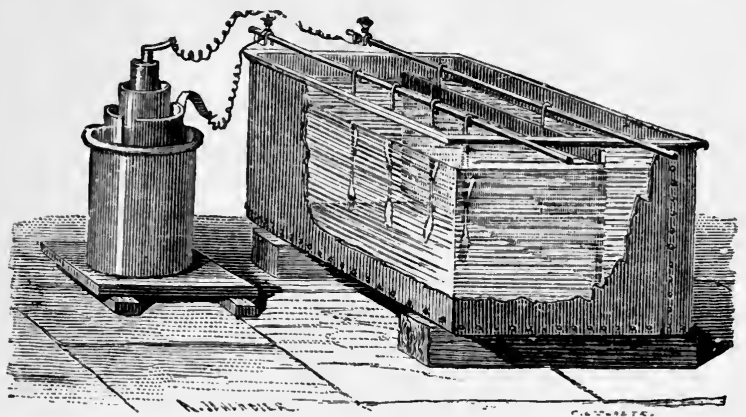

Fig. 269.

are suspended the spoons, forks, or other articles which are to receive a coating of silver. The addition of a minute trace of bisulphide of carbon to the solution causes the deposited metal to have a bright surface. If the current is too strong, and the deposition too rapid, the deposited metal is grayish and crystalline.

In gilding base metals, such as pewter, they are usually first copper-coated. The gilding of the insides of jugs and cups is effected by filling the jug or cup with the gilding solution, and suspending in it an anode of gold, the vessel itself being connected to the - pole of the battery.

In silvering or gilding objects of iron it is usual first to plate them with a thin coating of copper deposited from an "alkaline" copper bath containing an ammoniacal solution of cyanide of copper. Brass is deposited also from an ammoniacal solution of the mixed cyanides of copper and rinc. In the deposition of nickel a solution of the double sulphate of nickel and ammonium is used: the anode being a sheet of rolled (or cast) nickel.

Except on the very small scale batteries are now 
seldom used for electrotyping and plating. A shuntwound dynamo designed to give a large output of current at 5 to 10 volts pressure is generally preferred.

496a. Other Electrolytic Processes. - The electrolytic action of the current is now commercially employed for other purposes than the deposition of metals. By the electrolysis of chloride of potassium under suitable conditions chlorate of potash is now manufactured in large quantities. Bleaching liquors containing hypochlorites can also be produced from chlorides. Caustic soda is prepared by electrolysis of common salt; and several electrolytic methods of disinfecting sewage have been proposed.

It has also been shown that the slow processes of tanning can be accelerated by the aid of electric currents, the action being probably osmotic rather than electrolytic.

It seems probable that in the future the use of electric currents will enter largely into the chemical manufactures.

496b. The Electric Furnace.-If two stout rods of carbon are introduced into a crucible lined with magnesia or other refractory substance, and an arc (Art. 448) is formed between them, the internal temperature exceeds that of any other artificial source, enabling many chemical actions to be produced that are otherwise unattainable. Thus if lime mixed with coke is heated in the electric furnace there is produced calcium carbide $\mathrm{CaC}_{2}$, which when mixed with water yields acetylene gas. Many most refractory compounds, such as the oxides of titanium and chromium, can thus be reduced. It is not established whether the reduction of aluminium in the electric furnace is partly electrolytic or whether it is purely chemical. Aluminium oxide is mixed with charcoal and placed between the ends of two thick carbon rods in a closed firebrick furnace lined with charcoal. A current of several thousand amperes is passed between the carbon rods, and the aluminium ore is melted and parts with its oxygen to carbon. The liberated aluminium is commonly allowed to alloy with some other metal, such as copper. 


\section{CHAPTER XII}

TELEGRAPHY

\section{Lessox L.-Electric Telegraphs}

497. The Electric Telegraph.-It is difficult to assign the invention of the telegraph to any particular inventor. Lesage (Geneva, 1774), Lomond (Paris, 1787), and Sir F. Ronalds (London, 1816) invented systems for transmitting signals through wires by observing at one end the divergence of a pair of pith-balls when a charge of electricity was sent into the other end. Cavallo (London, 1795) transmitted sparks from Leyden jars through wires "according to a settled plan." Soemmering (Munich, 1808) established a telegraph in which the signals were made by the decomposition of water in voltameters; and the transmission of signals by the chemical decomposition of substances was attempted by Coxe, $R$. Smith, Bain, and others. Ampère (Paris, 1821) suggested that a galvanometer placed at a distant point of a circuit might serve for the transmission of signals. Schilling and Weber (Göttingen, 1833) employed the deflexions of a galvanometer needle moving to right or left to signal an alphabetic code of letters upon a single circuit. Cooke and Wheatstone (London, 1837) brought into practical application the first form of their needle telegraph. Henry (New York, 1831) utilised the attraction of an electromagnet to transmit signals, the movement of the armature 
producing audible sounds according to a certain code. Morse (New York, 1837) devised a telegraph in which the attraction of an armature by an electromagnet was made to mark a dot or a dash upon a moving strip of paper. Steinheil (Munich, 1837) discovered that instead of a return-wire the earth might be used, contact being made to earth at the two ends by means of earth-plates (see Fig. 274) sunk in the ground. Gintl (1853) and Stearns (New York, 1870) devised methods of duplex signalling. Stark (Vienna) and Bosseha (Leyden, 1855) invented diplex signalling, and Heaviside (London, 1873) and Edison (Newark, N.J., 1874) invented quadruplex telegraphy. Varley (London, 1870) and Elisha Gray (Chicago, 1874) devised harmonic telegraphs. For fastspeed work Wheatstone devised his automatic transmitter, in which the signs which represent the letters are first punched by machinery on strips of paper ; these are then run at a great speed through the transmitting instrument, which telegraphs them off at a much greater rate than if the separate signals were telegraphed by hand. Hughes devised a type-printing telegraph. Wheatstone invented an $\mathrm{ABC}$ telegraph in which signals are spelled by a hand which moves over a dial. Cowper (1876) and Elisha Gray (1893) invented autographic writing telegraphs. For cable-working Lord Kelvin invented his mirror galvanometer and his delicate siphon-recorder. It is impossible in these Lessons to describe more than one or two of the simple ordinary forms of telegraph instrument now in use in Great Britain. Students desiring further information should consult the excellent manuals on Telegraphy by Messrs. Preece and Sivewright, and by Mr. Culley.

498. Single-Needle Instrument.-The singleneedle instrument (Fig. 270) consists essentially of a vertical galvanometer, in which a lightly-hung magnetic needle is deflected to right or left when a current is sent, in one direction or the other, around a coil surrounding 
the needle; the needle visible in front of the dial is but an index, the real magnetic needle being behind. A code of movements agreed upon comprises the whole alphabet in combinations of motions to right or left. In order to send currents in either direction through the circuit, a "signalling-key" or "tapper" is usually employed. The tapper at one end of the line works the instrument at the other; but for the sake of convenience it is fixed to the receiving instrument. In Fig. 270 the two protruding levers at the base form the tapper, and

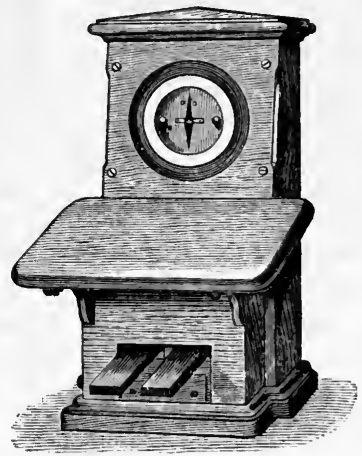

Fig. $2 \% 0$. by depressing the right-hand one or the left-hand one, currents are sent in either direction at will.

The principle of action will be made more clear by

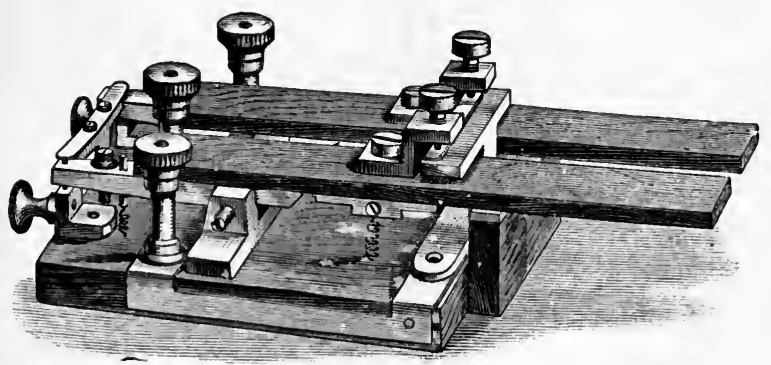

Fig. 271.

reference to Fig. 2il, which shows a separate signalling key. The two horizontal levers are respectively in communication with the "line," and with the return-line 
through "earth." When not in use both levers spring up against a cross strip of metal joined to the zinc pole of the battery. At their farther end is another cross strip, which communicates with the copper $($ or + ) pole of the battery. On depressing the "line" key the current runs through the line and back by earth, or in the positive direction. On depressing the "earth" key (the line-key remaining in contact with the zinc-connected strip), the current runs through the earth and back by the line, or in the negative direction. Telegraphists ordinarily speak of these as positive and negative currents respectively.

499. The Morse Instrument.-The most widely used instrument at the present day is the Morse. It con-

\section{LOCAL BATTERY}

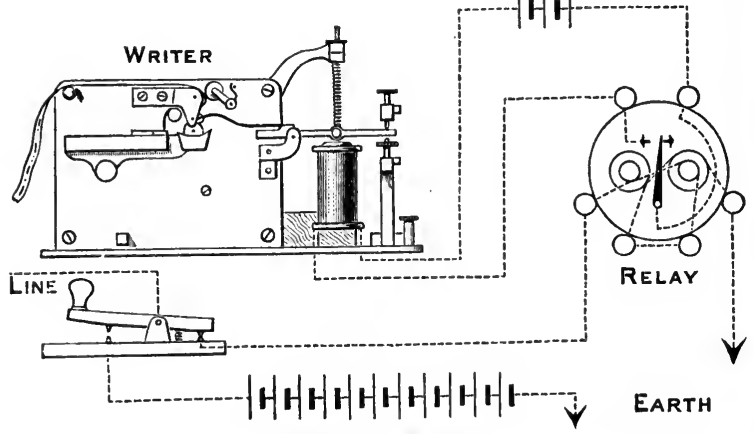

SENDING BATTERY

Fig. 272.

sists essentially of an electromagnet, which, when a current passes through its coils, draws down an armature for a short or a long time. It may either be arranged as a "sounder," in which case the operator who is receiving the message listens to the clicks, and notices whether the 
intervals between them are long or short; or it may be arranged as an "embosser," to print dots and dashes upon a strip of paper drawn by clockwork through the instrument. In the most modern form, however, the Morse instrument is arranged as an "ink-writer" (Fig. 272), in which the attraction of the armature downwards lifts a little inky wheel and pushes it against a ribbon of paper. If the current is momentary it prints a mere dot. If the current continues to flow for a longer time while the ribbon of paper moves on the ink-wheel marks a dash. The International Morse code, or alphabet of dots and dashes, is as follows:-
A. -
B $-\ldots$
$\mathrm{K}-\cdot-$
$\mathrm{U} \ldots-$
$\mathrm{C}-.$.
$\mathrm{L} . \cdots$
$\mathrm{V} \ldots-$
$\mathrm{D}-$. .
M - -
W. - -
E.
F... -
$\mathrm{N}-$.
$\mathrm{X}-\ldots-$
$\mathrm{G}-$ -
$\mathrm{O}-\cdots$
$\mathrm{H} \ldots$...
P. - - .
$\mathrm{Y}-\cdot--$
I . .
$\mathrm{Q}$
Full stop . . . .
J
R.- .
S ...
Repetition .. - . .
J
Hyphen - .... -
Apostrophe

The American Morse code differs in many respects from the International code, the signals for some of the letters depending

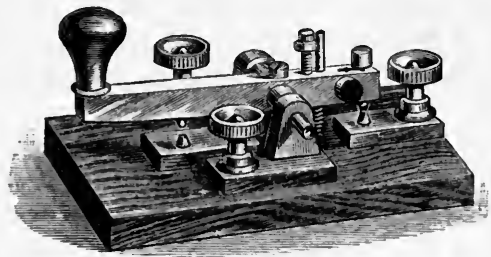

Fig. 273 .

on the length of the spacings between the dots and dashes; and more than four marks are used to form some of the letters.

The Morse key is shown in Fig. 273. The line wire 
is connected with the central pivot A. A spring keeps the front end of the key elevated when not in use, so that the line wire is in communication through the rear end of the key with the receiving instrument or relay. Depressing the key breaks this communication, and by putting the line wire in communication with the sending battery transmits a current through the line.

500. Open and Closed Circuit Working.European telegraphs work oll the open-eircuit plan,

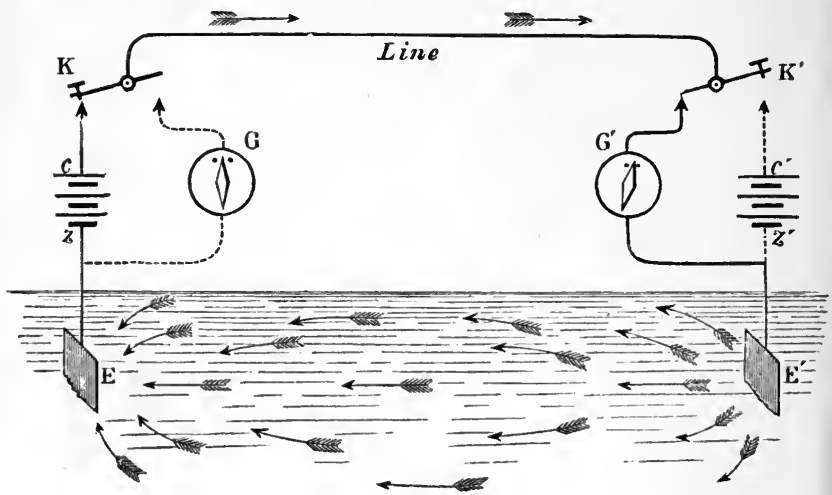

Fig. 274 .

the battery being out of circuit when no message is being sent. American telegraphs are usually on the closedcircuit plan, the current being always on until interrupted to send signals. In the open-circuit method, as it is necessary that a line should be capable of being worked from either end, a battery is used at each, and the wires so connected that when at either end a message is being received, the battery circuit at that end shall be open. Fig. 274 shows the simplest possible case of such an arrangement. At each end is a battery $\approx c$, one pole of 
which is put to earth, and the other communicates with a Morse key K. This key is arranged (like that in Fig. 273) so that when it is depressed to send a signal through the line it quits contact with the receiving instrument at its own end. The current flowing through the line passes through $\mathrm{K}^{\prime}$ and enters a receiving instrument $\mathrm{G}^{\prime}$ at the distant end, where it produces a signal, and returns by the earth to the battery whence it started. A similar battery and key at the distant end suffice to transmit signals in the opposite direction to $\mathrm{G}$ when $\mathrm{K}$ is not depressed. The diagram is drawn as if $\mathrm{G}$ were a simple galvanometer; but the arrangement would perfectly suit the Morse instrument, in which it is only required at either end to send long and short currents without reversing the direction, as with the needle instruments.

501. Relays.-In working over long lines, or where there are a number of instruments on one circuit, the currents are often not strong enough to work the recording instrument directly. In such a case there is interposed a relay or repeater. This instrument consists of an electromagnet round which the line current flows, and whose delicately poised armature, when attracted, makes contact for a local circuit in which a local battery and the receiving Morse instrument are included. The principle of the relay is, then, that a current too weak to do the work itself may set a strong local current to do its work for it.

In Fig. 272 the Morse

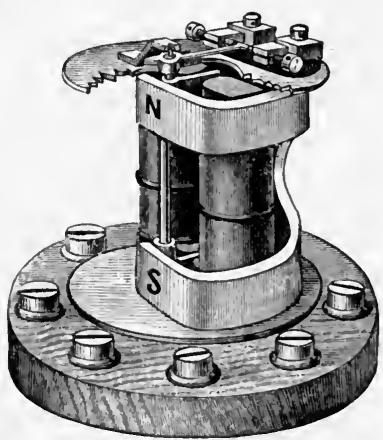

Fig. 275 . receiver (an "ink-writer") $M$ is placed in a local circuit with a local battery LB and a relay of the British Post- 
Office pattern. Whenever a current in the line circuit moves the tongue of the relay it closes the local circuit, and causes the Morse to record either a dot or a dash upon the strip of paper. A view of the P.O. standard relay itself is given in Fig. 275 . It is of the "polarized" kind (Art. 387); a permanent steel magnet of compact shape being used to magnetize tongues that are pivoted between the poles of the electromagnet.

502. Faults in Telegraph Lines.-Faults may occur in telegraph lines from several causes; either from the breakage of the wires or conductors, or from the breakage of the insulators, thereby short-circuiting the current through the earth before it reaches the distant station, or, as in overhead wires, by two conducting wires touching one another. Various modes for testing the existence and position of faults are known to telegraph engineers; they depend upon accurate measurements of resistance or of capacity. Thus, if a telegraph cable part in mid-ocean it is possible to calculate the distance from the shore end to the broken end by comparing the resistance that the cable is known to offer per mile with the resistance offered by the length up to the fault, and dividing the latter by the former.

503. Duplex and Quadruplex Telegraphy.To send two messages through one wire, one from each end, at the same time, is known as duplex working. There are two distinct methods of arranging apparatus for duplex working. The first of these, known as the difierential method, involves the use of instruments wound with differential coils, and is applicable to special cases. The second method of duplex working, known as the bridge method, is capable of much more general application. The diagram of Fig. 276 will explain the general principle. The first requirement in duplex working is that the instrument at each end shall only move in response to signals from the other end, so that an operator at $\mathrm{R}$ may be able to signal to the distant instrument $\mathrm{MI}^{\prime}$ without his 
own instrument $\mathbf{M}$ being affected, $\mathbf{M}$ being all the while in circuit and able to receive signals from the distant operator at $R^{\prime}$. To accomplish this the circuit is divided at $R$ into two branches, which go, by $A$ and $B$ respectively, the one to the line, the other through a certain resistance $P$ to the earth. If the ratio between the resistances in the arms $\mathrm{RA}$ and $\mathrm{RB}$ is equal to the ratio of the resistances of the line and of $\mathrm{P}$, then, by the principle of Wheatstone's Bridge, no current will pass through M. So M does not show any currents sent from $R$; but $M^{\prime}$ will show them, for the current on arriving at $\mathrm{C}$ will divide into two parts, part flowing round to the earth by $R^{\prime}$, the other

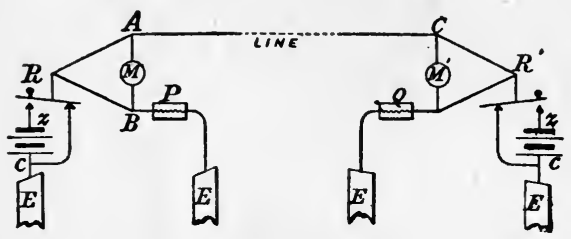

Fig. 276.

part flowing through $\mathbf{M}^{\prime}$ and producing a signal. If, while this is going on, the operator at the distant $R^{\prime}$ depresses his key and sends an equal current in the opposite direction, the flow through the line will cease ; but M will now show a signal, because, although no current flows through the line, the current in the branch RA will now flow down through $\mathrm{M}$, as if it had come from the distant $R^{\prime}$, so, whether the operator at $R$ be signalling or not, $M$ will respond to signals sent from $R^{\prime}$. In duplexing long lines and cables condensers are employed in the arms RA and RB of the bridge; and instead of a mere balancing resistance at $\mathrm{P}$ and $\mathrm{Q}$ there is used an "artificial cable," a combination of condensers and resistances to imitate the electrical properties of the actual line or cable between the stations, 
The Diplex method of working consists in sending two messages at once through a wire in the same direction. To do this it is needful to employ instruments which work only with currents in one given direction. The method involves the use of polarized relays, which, being themselves permanently magnetized, respond therefore only to currents in one direction.

The Quadruplex method of working combines the duplex and the diplex methods. On one and the same line are used two sets of instruments, one of which (worked by a polarized relay) works only when the direction of the current is changed, the other of which (worked by a non-polarized relay adjusted with springs to move only with a certain minimum force) works only when the strength of the current is changed and is independent of their direction.

\section{Lesson LI.-Cable Telegraphy}

504. Submarine Cables.-Telegraphic communieation between two countries separated by a strait or

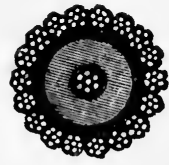

Fig. 277.

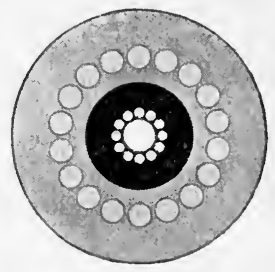

Fig. 278.

ocean is carried on through cables, sunk to the bottom of the sea, which carry conducting wires carefully protected hy an outer sheath of insulating and protecting materials. The conductor is usually of purest copper wire, weighing from 70 to 400 lbs. per nautical mile, made in a seven- 
fold strand to lessen risk of breaking. Figs. 277 and 278 show respectively, in their natural size, sections of the first Atlantic cable laid in 1857 and of one of the most recent. In the latter, which is of the usual type of cable for long lines, the core is protected first by a stout layer of guttapercha, to secure perfect insulation, and then by an outer sheathing, in which are embedded a number of strong steel wires. The shore ends are even more strongly protected by external wires.

505. Speed of Signalling through Cables.Signals transmitted through long cables are retarded, the retardation being due to two causes.

Firstly, The self-induction of the circuit prevents the current from rising at once to its height, the retardation being expressed by von Helmholtz's equation (Art. 460).

Secondly, The cable in its insulating sheath, when immersed in water, acts laterally like a Leyden jar of enormous capacity (as explained in Art. 301), and the first portions of the current, instead of flowing through, remain in the cable as an electrostatic charge on the surface of the guttapercha. For every separate signal the cable must be at least partially charged and then discharged. Culley states that when a current is sent through an Atlantic cable from Ireland to Newfoundland no effect is producel on the most delicate instrument at the receiving end for two-tenths of a second, and that it requires three seconds for the current to gain its full strength, rising in an electric wave which travels forward through the cable. The strength of the current falls gradually also when the circuit is broken. The greater part of this retardation is due to electrostatic charge, not to electromagnetic self-induction. The number of signals that can be sent through a cable in a given time is less as the capacity and resistance are greater. The time required to trausmit a given number of signals through a cable of capacity $\mathrm{K}$ and resistance $\mathrm{R}$ is proportional to $\mathrm{KR}$ : so the retardation is proportional to the square of 
the length of the cable. The various means adopted to get rid of this retardation are explained in Art. 302. It is usual to insert in the circuit at each end of the cable a condenser of several microfarads, through which the signals pass. The tendency of the condenser to discharge helps to curb the signals and make each shorter and sharper. It is theoretically possible (compare Art. $473)$ to compensate capacity by self-induction; but as the eapacity of a cable is lateral, not longitudinal, and distributed all along it, the self-induction coils to compensate the retardation wonld have to be applied as shunts at intervals. A cable with a self-inductive shunt or leak at a point near its middle transmits signals nore rapidly than one not so compensated.

506. Receiving Instruments for Cables.-The mirror-galvanometer of Lord Kelvin (Art. 215) was devised for cable signalling, the movements of the spot of light sweeping over the scale to a short or a long distance sufficing to signal the dots and dashes of the Morse code. Lord Kelvin's Siphon Recorder is an instrument which writes the signals upon a strip of paper by the following ingenious means:-The cable communicates with a delicately-suspended coil of wire that hangs between the poles of a powerful magnet. To the suspended coil is attached a fine siphon of glass suspended by a silk fibre, one end of which dips into an ink vessel. The ink makes marks upon a strip of paper (moved by clockwork vertically past the siphon), friction being obviated by giving the siphon a continual minute vibration. The siphon record is a wavy line having little bends above or below the central line of the strip for dots or dashes.

\section{Lesson LII.-Miscellaneous Telegraphs}

507. Multiplex Telegraphs.--Varley proposed to send messages by transmitting electrically musical tones, 
interrupted to sound as dots and dashes. This necessitated the transmission of currents either rapidly alternating or rapidly intermittent. Gray, who constructed harmonic telegraphs on this plan, found it possible to transmit five or six messages simultaneously in one line.

By using at each end of a line two synchronously revolving distributing switches, it is possible to send several messages at once throngh a line ; the distributors (invented by Delany) causing each transmitting instrument to be in circuit with its corresponding receiving instrument for a small fraction of a second at regular short intervals.

508. Electric Bells. - The common form of Electric Trembling Bell (invented 1850 by John Mirand) consists of an electromagnet, which moves a hammer backward and forward by alternately attracting and releasing it, so that it beats against a bell. The arrangements of the instrument are shown in Fig. 279, in which $\mathrm{E}$ is the electromagnet and $\mathrm{H}$ the hammer. A battery, consisting of one or two Leclanché cells placed at some convenient point of the circuit, provides a current when required. By touching the "push" $P$, the circuit is completed, and a current flows along the line and round the coils of the electromagnet, which forthwith attracts a small piece of soft iron attached to the lever, which terninates in the hammer H. The lever is itself included in the circuit, the current entering it above and quitting it at $\mathrm{C}$ by a contact-breaker, consisting of a spring tipped with platinum resting against the platinum tip of a screw, from which a return wire passes back to the zine pole of the battery. As soon as the lever is attracted forward the circuit is broken at $\mathrm{C}$ by the spring moving away from contact with the screw ; hence the current stops, and the electromagnet ceases to attract the armature, but the momentum of the hammer carries it forward. Immediately afterwards, however, the hammer falls back, again establishing contact at $\mathrm{C}$, whereupon the armature is once 
more attracted forward, and so on. The push $\mathrm{P}$ is shown in section in Fig. 280. It usually consists of a cylindrical knob of ivory or porcelain capable of moving loosely through a hole in a circular support of porcelain or wood,

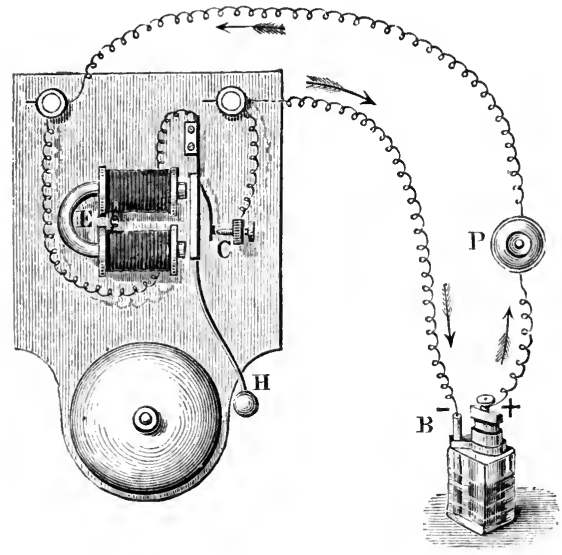

Fig. 27 .

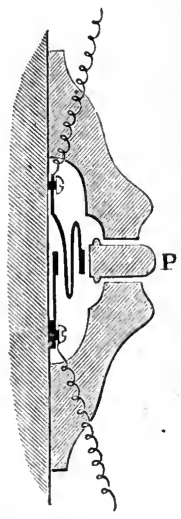

Fig. 280.

and which, when pressed, forces a platinum-tipped spring against a metal pin, and so makes electrical contact between the two parts of the interrupted circuit. Bells having a polarized armature, and without any break, are used as call-bells for telephones; the generator being a small magneto alternator like Fig. 243, driven by a handle.

509. Electric Clocks and Chronographs.Clocks may be either driven or controlled by electric currents. Bain, Hipp, and others have devised electric clocks of the first kind, in which the ordinary motivepower of a weight or spring is abandoned, the clock being driven by its pendulum, the "bot)" of which is an electromagnet alternately attracted from side to side. The diff- 
culty of maintaining a perfectly constant battery current has prevented such clocks from coming into use.

Electrically controlled clocks, governed by a standard central clock, have proved a more fruitful invention. In these the standard timekeeper is constructed so as to complete a circuit periodically, once every minute or half minute. The transmitted currents set in movement the hands of a system of dials placed at distant points, by causing an electromagnet placed behind each dial to attract an armature, which, acting upon a ratchet wheel by a pawl, causes it to move forward through one tooth at each specified interval, and so carries the hands round at the same rate as those of the standard clock.

Electric chronographs are used for measuring very small intervals of time. A stylus fixed to the armature of an electromagnet traces a line upon a piece of paper fixed to a cylinder revolving by clockwork. A current sent through the coils of the electromagnet moves the armature and causes a lateral notch in the line so traced. Two currents are marked by two notches; and from the interval of space between the two notches the interval of time which elapsed between the two currents may be calculated to the ten-thousandth part of a second if the speed of rotation is accurately known. The velocity with which a cannon ball moves along the bore of the cannon can be measured thus.

509a. Telegraphy across Space.-It is possible to telegraph to considerable distances without using connecting wires. There are three methods: (1) by conduction through the earth or sea, using at each station a base-line of telegraph wire earthed at both ends ; (2) by electromagnetic induction between coil and coil (see Art. 224) at a distance, using alternating currents ; (3) by employing Hertz waves (Arts. 520-21), detecting them at the distant station on the plan invented by Oliver Lodge of relaying the signals by a coherer to a telegraphic receiver or sounder. This is the plan used by Marconi and by Slaby. 


\title{
CHAPTER XIII
}

\author{
TELEPHONY
}

\section{Lesson LIII.-Electric Telephones}

510. Early Telephones. - The first successfu] attempt to transmit sounds electrically was made in 1861 by Reis, who succeeded in conveying musical and other tones by an imperfect telephone. In this instrument the voice was caused to act upon a point of loose contact in an electric circuit, and by bringing those parts into greater or less intimacy of contact (Art. 400), thereby varied the resistance offered to the circuit. The transmitting part of Reis's telephone consisted of a battery and a contact-breaker, the latter being formed of a tympanum or diaphragm of stretched membrane, capable of taking up sonorous vibrations, and having attached to it a thin elastic strip of platinum, which, as it vibrated, beat to and fro against the tip of a platinum wire, so making and breaking contact wholly or partially at each vibration in exactly the same manner as is done with the carbon contacts in the modern transmitters of Blake, Berliner, etc. The receiving part of the instrument consisted of an iron wire fixed upon a sounding-board and surrounded by a coil of insulated wire forming part of the circuit. The rapid magnetization and demagnetization of such an iron core will produce audible sounds (Art. 124). If the current vary, the iron wire 
is partially magnetized or demagnetized, giving rise to corresponding vibrations of varying amplitudes and forms; hence such a wire will serve perfectly as a receiver to reproduce speech if a good transmitter is used. Reis himself transmitted speech with his instrument, but only imperfectly, for all tones of speech cannot be transmitted by abrupt interruptions of the current, to which Reis's transmitter is prone when spoken into, owing to the extreme lightness of the contact : they require gentle undulations, sometimes simple, sometimes complex, according to the nature of the sound. The vowel sounds are produced by periodic and complex movements in the air ; the consonants being for the most part non-periodic. Reis also devised a second receiver, in which an electromagnet attracted an elastically-supported armature of iron, which vibrated under the attraction of the more or less interrupted current.

In 1876 Elisha Gray devised a transmitter in which a variable water-resistance (made by a platinum wire dipping into water) was acted upon by the voice. $\mathrm{He}$ designed an electro-magnetic receiver.

Telephone receivers were invented by Varley and Dolbear, in which the attraction between the oppositelyelectrified armatures of a condenser is utilised in the production of sounds. Dolbear's receiver consists merely of two thin metal disks, separated by a very thin air-space. As the varying currents flow into and out of this condenser the two disks attract one another more or less strongly, and thereby vibrations are set up which correspond to the vibrations of the original sound.

In 1876 Graham Bell invented the magneto-telephone. In this instrument the speaker talks to an elastic plate of thin sheet iron, which vibrates and transmits its every movement electrically to a similar plate in a similar telephone at a distant station, causing it to vibrate in an identical manner, and thereby to emit identical sounds. The transmission of the vibra- 
tions depends upon the principles of magneto-electric induction explained in Lesson XVIII. Fig. 281 shows Bell's Telephone in section. The disk $\mathrm{D}$ is placed behind a conical mouthpiece, to which the speaker places his mouth or the hearer his ear. Behind the disk is a magnet AA running the length of the instrument; and upon its front pole, which nearly touches the disk, is fixed a small bobbin, on which is wound a coil $\mathrm{C}$ of fine insulated wire, the ends of the coil being connected with the terminal screws FF. One such instrument is used to

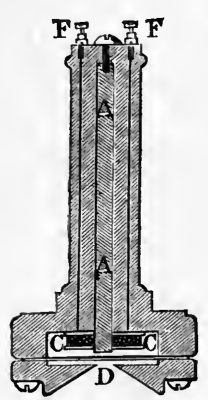

Fig. 281. transmit, and one to receive the sounds, the two being connected in simple circuit. No battery is needed, for the transmitting instrument itself generates the induced currents as follows: The magnet AA induces a certain number of magnetic lines through the coil C. Many of these pass into the iron disk. When the iron disk in vibrating moves toward the magnet-pole, more magnetic lines meet it ; when it recedes, fewer lines meet it. Its motion to and fro will therefore alter the number of lines which pass through the hollow of the coil $\mathrm{C}$, and will therefore (Art. 225) generate in the wire of the coils currents whose strength is proportional to the rate of change in the number of the lines. Bell's instrument, when used as a transmitter, may therefore be regarded as a sort of vibrating dynamo, which pumps currents in alternate directions into the wire. At the distant end the currents as they arrive flow round the coils either in one direction or the other, and therefore either add momentarily to or take from the strength of the magnet. When the current in the coils is in such a direction as to reinforce the magnet, the magnet attracts the iron disk in front of it more strongly than before. If the current is in the opposite direction the disk is less attracted and flies back. 
Hence, whatever movement is imparted to the disk of the transmitting telephone, the disk of the distant receiving telephone is forced to repeat, and it therefore throws the air into similar vibrations, and so reproduces the sound. Bell's method of transmitting was soon abandoned (except for very short lines). In modern telephonic work Reis's plan of using a separate transmitter with a battery is universal, the Bell instrument being used as a receiver only and not as a transmitter.

511. Edison's Transmitter.-Edison constructed a transmitting instrument, in which the vibrations of the voice, actuating a diaphragm of mica, made it exert more or less compression on a button of prepared lamp-black placed in the circuit. The resistance of this is affected by pressure of contacts; hence the varying pressures due to the vibrations cause the button to offer a varying resistance to any current flowing (from a battery) in the circuit, and vary its strength accordingly. This varying current may be received as before in an electromagnetic receiver of the type described above, and there set up corresponding vibrations. This instrument also has been abandoned in favour of transmitters of the microphone type. Edison also invented a receiver of singular power, which depends upon a curious fact discovered by himself, namely, that if a platinum point presses against a rotating cylinder of moist chalk, the friction is reduced when a current passes between the two. And if the point be attached to an elastic disk, the latter is thrown into vibrations corresponding to the fluctuating currents coming from the speaker's transmitting instrument.

512. Microphones.-Hughes, in 1878, discovered that a loose contact between two conductors, forming part of a circuit in which a small battery and a receiving telephone are included, may serve to transmit sounds without the intervention of any specific tympanum or diaphragm like those of Reis and Edison, because the smallest vibrations will affect the resistance (Art. 400) at 
the point of loose-contact. The Microphone (Fig. 282) embodies this principle. In the form shown in the figure, a small thin pencil of carbon is supported loosely between two little blocks of the same substance fixed to a sounding-board of thin pine-wood, the blocks being connected with one or two small cells and a Bell receiver. The amplitude of the vibrations emitted by the receiver may be much greater than those of the original sounds, and therefore the microphone may serve, as its name

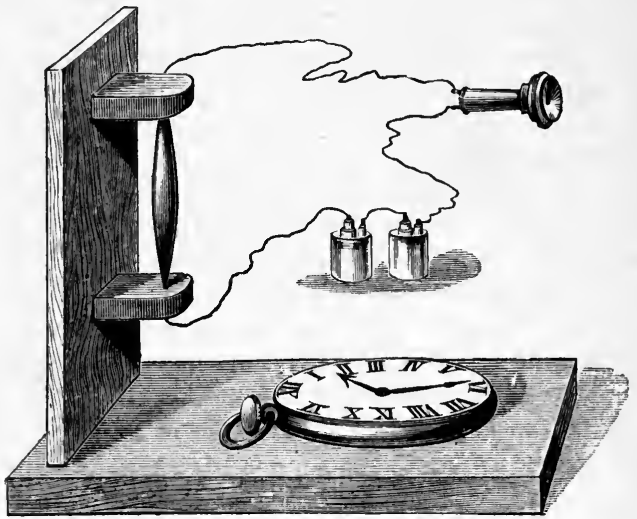

Fig. 282.

indicates, to magnify minute sounds, such as the ticking of a watch or the footfalls of an insect, and render them audible. In modern telephony microphones under the name of carbon transmitters are in general use. In the Blake transmitter a pin of platinum is pressed by a light spring against a polished plug of hard carbon, forming a delicate contact through which the current flows. This electrical mechanism is mounted behind a metal disk to take up the vibrations of the speaker's voice. In the Hunnings loud-speaking transinitter granulated coke car- 
bon is placed loosely between two metal surfaces, so that the current flows through the loose particles. The voice acts on all the loose contacts at once.

For all long line work the microphone transmitter is included with a battery of one or two cells in a small local circuit of low resistance, in which is inserted the primary wire of a small transformer or induction coil. The secondary wire of this transformer is a coil of fine wire of many turns, which transmits through the line and return circuits much smaller currents at a higher voltage.

513. Telephone Exchanges.--For enabling a large number of subscribers to communicate by telephone with one another, the lines from each subscriber's instrument are brought to a central office known as a telephone exchange. Here each line terminates on a switch-board which is so arranged that the operator can in an instant make a connexion from the line of any one subscriber to that of any other, so that these two can talk together.

514. Hughes' Induction Balance.-The extreme sensitiveness of Bell's receiver (Art. 510) to the feeblest currents has suggested its employment to detect currents too weak to affect the most delicate galvanometer. The

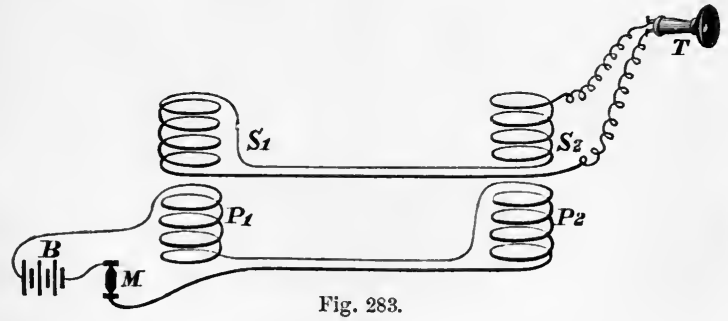

currents must be intermittent, or alternating, or they will not keep the disk of the telephone in vibration. Hughes applied this property of the telephone to an instrument named the Induction Balance (Fig. 283). A small 
battery $\mathrm{B}$, connected with a microphone $\mathrm{M}$, passes through two coils of wire $\mathrm{P}_{1}, \mathrm{P}_{2}$, wound on bobbins fixed on a suitable stand. Above each of these primary coils are placed two secondary coils, $S_{1}, S_{2}$, of wire, of the same size, and of exactly equal numbers of turns of wire. The secondary coils are joined to a receiver $\mathrm{T}$, and are wound in opposite directions. The result of this arrangement is that whenever a current either begins or stops flowing in the primary coils, $\mathrm{P}_{1}$ induces a current in $\mathrm{S}_{1}$, and $\mathrm{P}_{2}$ in $\mathrm{S}_{2}$. As $\mathrm{S}_{1}$ and $\mathrm{S}_{2}$ are wound in opposite ways, the two currents thus induced in the secondary wire neutralize one another, and, if they are of equal strength, balance one another so exactly that no sound is heard in the telephone. But a perfect balance cannot be obtained unless the resistances and the coefficients of mutual induction and of self-induction are alike. If a flat piece of silver or copper (such as a coin) be introduced between $S_{1}$ and $P_{1}$, there will be less induction in $S_{1}$ than in $S_{2}$, for part of the inductive action in $P_{1}$ is now spent on setting up currents in the mass of the metal (Art. 457), and a sound will again be heard in the telephone. But balance can be restored by moving $\mathrm{S}_{2}$ farther away from $\mathrm{P}_{2}$, until the induction in $S_{2}$ is reduced to equality with $S_{1}$, when the sounds in the telephone again cease. It is possible by this means to test the relative conductivity of different metals which are introduced into the coils. It is even possible to detect a counterfeit coin by the indication thus afforded of its conductivity. The induction balance has also been applied in surgery by Graham Bell to detect the presence of a bullet in a wound, for a lump of metal may disturb the induction when some inches distant from the coils. 


\title{
CHAPTER XIV
}

\author{
ELECTRIC WAVES
}

\section{Lesson LIV.-Oscillations and Waves}

515. Electric Oscillations.-If a charged condenser or Leyden jar is discharged slowly through a conductor of high resistance, such as a nearly dry linen thread, the charge simply dies away by a discharge which increases in strength at first and then gradually dies away. If, however, the condenser is discharged through a coil of wire of one or more turns (the spark being taken between polished knobs to prevent premature partial discharges by winds or brushes), the effect is wholly different, for then the discharge consists of a number of excessively rapid oscillations or surgings. This is in consequence of the self-induction of the circuit, by reason of which (Art. 458) the current once set up tends to go on. The first rush more than empties the condenser, and charges it the opposite way ; then follows a reverse discharge, which also overdoes the discharge, and charges the condenser the same way as at first, and so forth. Each successive oscil-

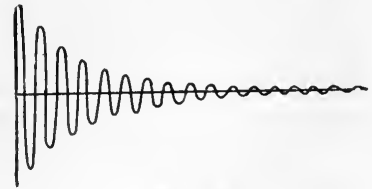

Fig. 2 S.

lation is feebler than the preceding, so that after a number of oscillations the discharge dies away as in Fig. 284. The 
spark of a jar so discharged really consists of a number of successive sparks in reverse directions. One proof of this, as pointed out by Henry in 1842 from the experiments of Savery, is that if jar discharges through a coil are used to magnetize steel needles, the direction of the magnetization is anomalous, being sometimes one way, sometimes the other.

That a discharge ought under certain conditions to become oscillatory was noted by von Helmholtz. Lord Kelvin in 1855 predicted these conditions. If the capacity of the condenser is $\mathrm{K}$ (farads), the resistance of the circuit $R$ (ohms), and its inductance $L$ (henries), there will be oscillations if

$$
\mathrm{R}<\sqrt{4 \mathrm{~L} / \mathrm{K}} ;
$$

and there will be no oscillations if

$$
\mathrm{R}>\sqrt{4 \mathrm{~L} / \mathrm{K}} .
$$

In the former case the frequency $n$ of the oscillations will be such that

$$
2 \pi n=\sqrt{\frac{1}{\mathrm{KL}}-\frac{\mathrm{R}^{2}}{4 \mathrm{~L}^{2}}}
$$

Example. - If $\mathrm{K}=0.01$ microfarad, $\mathrm{L}=0.00001$ henry, and $\mathrm{R}=0, n=503,000$.

If $\mathrm{R}$ is small $n$ is nearly equal to $1 \div 2 \pi \sqrt{\mathrm{KL}}$.

The oscillations can be made slower by increasing either $\mathrm{K}$ or L. The oscillations of an ordinary Leyden jar discharge may last only from a ten-thousandth to a tenmillionth of a second. By using coils of well-insulated wire and large condensers, Lodge has succeeded in slowing down the oscillations to 400 a second; the spark then emitting a musical note. Iron is found to retain its magnetic properties even for oscillations of the frequency of one million per second.

Feddersen subsequently examined the spark of a Leyden jar by means of a rotating mirror, and found that 
instead of being a single instantaneous discharge, it exhibited definite fluctuations. ${ }^{*}$ With very small resistances in the circuit, there was a true oscillation of the electricity backward and forward for a brief time. The period of the oscillations was found to be proportional to the square root of the capacity of the condenser. With a certain higher resistance the discharge became continuous but not instantaneous. With a still higher resistance the discharge consisted of a series of partial intermittent discharges, following one another in the same direction. Such sparks when viewed in the rotating mirror showed a series of separate images at nearly equal distances apart.

516. Electric Waves.-Though the increasing and dying away of currents, for example in cables, is sometimes loosely described as of "waves" of current, these phenomena are very different from those of true electric or electromagnetic waves propagated across space. In the case of true electric waves, portions of the energy of the current or discharge are thrown off from the conductor and do not return back to it, but go travelling on in space. If a current increases in strength the magnetic field around it also increases, the magnetic lines enlarging from the conductor outward, like the ripples on a pond. But as the current is decreased the magnetic lines all return back and close up upon the conductor; the energy of the magnetic field returns back into the system. But if for currents slowly waxing and waning we substitute electric oscillations of excessive rapidity, part of their energy radiates off into the surrounding medium as electromagnetic wares, and only part returns back. As will be presently set forth, these waves possess all the optical properties of light-waves, and can be reflected, refracted, polarized, etc.

It is a fundamental part of the modern views of electric action that while an electric displacement (Art. 57) is

* 'These electric oscillations were examined also by Schiller, Orerbeck, Blaserna, and others, notably by Hertz; see Art. 520 below. 
being produced in a dielectric, the effect in surrounding space is the same as if there had been a conductive instead of an inductive transfer of electricity. Maxwell gave the name of displacement-current to the rate of change of the displacement. Experiment proves that displacement-currents, while they last, set up magnetic fields around them ; just as convexion-currents (Art. 397) and conduction-currents do.

517. Resonance. - The circumstance that when certain definite relations exist between the capacity and inductance of a circuit and the frequency of the periodic currents, the choking reactions of these properties neutralize one another, has been already alluded to in Art. 473. And we have seen (Art. 515) that a circuit with a certain self-induction, capacity, and resistance tends to oscillate electrically at a certain frequency. If it be placed in a medium through which electric waves of that frequency are passing in such a position that the electric and electromagnetic fields of the successive waves can induce currents in it, each wave will give a slight impulse to the readily-excited oscillations, which will grow in intensity, just as small impulses given to a pendulum at the right times will make it swing violently.

The following experiment of Oliver Lodge beautifully illustrates this phenomenon of resonance, and at the same

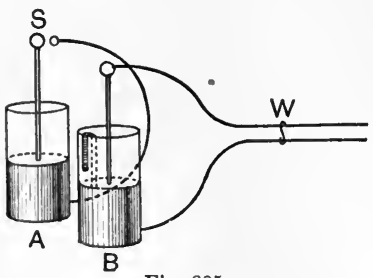

Fig. 285 . time the production of waves by an oscillatory discharge. Two Leyden jars, Fig. 285, are placed a little way apart from one another. One of them, charged from an influence machine not shown, is provided with a bent wire to serve as a discharging circuit, with a spark-gap $\mathrm{S}$ between the polished knobs at the top. The second jar is provided with a circuit of wire, the inductance of which 
can be adjusted by sliding in or out a cross-piece IV hooked upon the other portions. A strip of tinfoil is brought up from the inner coating over the lip of this jar, but does not quite touch the outer coating. If the two circuits are properly tuned together, whenever a spark passes in the gap at the top of $A$, surgings will be set up in the circuit of $B$ which will cause the jar to overflow, producing a spark at the end of the strip.

\section{Lesson LV.-The Electromagnetic Theory of Light}

518. Maxwell's Theory.-In 1867 Clerk Maxwell put forward the theory that the waves of light are not mere mechanical motions of the ether, but that they are electrical undulations. These undulations are partly electrical and partly magnetic, oscillating electrical displacements being accompanied by oscillating magnetic fields at right angles to them, whilst the direction of propagation of the wave is at right angles to both. According to this theory the phenomena of electromagnetism and the phenomena of light are all due to certain modes of motion in the ether, electric currents and magnets being due to streams and whirls or other bodily movements in the substance of the ether, while light is due to vibrations to and fro in it.

An electric displacement in its growth or decay produces a magnetic force at right angles to itself; it also produces (by the peculiar action known as induction) an electric force which is propagated at right angles both to the electric displacement and to the magnetic force. Now it is known that in the propagation of light the actual displacements or vibrations which constitute the so-called ray of light are executed in directions at right angles to the direction of propagation. This analogy is an important point in the theory, and immediately suggests the question whether the respective rates 
of propagation are the same. Now the velocity of propagation of electromagnetic induction is that velocity " $v$ " which was shown (Art. 359) to represent the ratio between the electrostatic and the electromagnetic units, and which (in air) has been found to be

$2.9857 \times 10^{10}$ centimetres per second.

And the velocity of light (in air) has been repeatedly measured (by Fizeau, Cornu, Michelson, and others), giving as the approximate value

\section{$2.9992 \times 10^{10}$ centimetres per second.}

From the equations for the propagation of a disturbance in an electromagnetic medium, having dielectric coefficient $k$ (Art. 295) and permeability $\mu$ (Art. 363), it was calculated by Maxwell that the velocity ought to be numerically $=1 / \sqrt{k \mu}$. And, as we have seen, this quantity enters into the ratio of the units (Art. 360), and can be calculated from them. It follows that if there are two transparent media of equal permeability but different dielectric capacities, the velocities in them ought to vary relatively inversely as $\sqrt{k}$. But the ratio of the velocities of light in them is called their refractive index. Hence if Maxwell's theory is true, the dielectric capacity of ordinary transparent media ought to be equal to the square of the refractive index. Experiments by Gordon, Boltzmann, and others, show this to be approximately true for waves of very great wave-length. The values are shown below. For gases the agreement is even closer.

\begin{tabular}{|c|c|c|}
\hline - & $k$. & (Index) ${ }^{2}$ \\
\hline Flint Glass & $3 \cdot 162$ & $2 \cdot 796$ \\
\hline Bisulphide of Carbon & $1 \cdot 812$ & $2 \cdot 606$ \\
\hline Sulphur (mean) & $4 \cdot 151$ & $4 \cdot 024$ \\
\hline Paraffin . & $2 \cdot 32$ & $2 \cdot 33$ \\
\hline
\end{tabular}


Another consequence of the theory is that all conductors, since they dissipate the energy of the currents set up in them, ought to be opaque to light. Metallic conductors are, except when in very thin films. But electrolytic liquids are not opaque, the mechanism of their conduction being different (Art. 490). In some crystalline bodies which conduct electricity better in one direction than in another, the opacity to light differs correspondingly. Coloured crystals of tourmaline conduct electricity better across the long axis of the crystal than along that axis. Such crystals are much more opaque to light passing along the axis than to light passing across it. And, in the case of rays traversing the crystal across the axis, the vibrations across the axis are more completely absorbed than those parallel to the axis: whence it follows that the transmitted light will be polarized.

519. Energy Paths.-From Maxwell's equations Poynting in 1883 drew the conclusion that in all cases where energy is transferred in an electric system it flows parallel to the surfaces of both electric and magnetic equipotentials. What we call an electric current along a wire is rather a transfer of energy by an invisible mechanism in the medium outside the wire. Wherever in the wire there is resistance, wasting energy by degrading it into heat, at that point energy flows in laterally from the medium. According to this view, the service of the wire is merely to guide the energy flow going on outside it.* We know that when a current is started much energy is spent in building up around the conductor a magnetic field, the amount spent being $\frac{1}{2} \mathrm{LC}^{2}$ (Art. 458). When the circuit is "broken" this energy flows on laterally into the wire, giving rise to the so-called extra-current sparks. According to Poynting's view, which has been independently elaborated by Heaviside, all the energy flows in similarly. In the case of the transfer of energy in an alternate-current transformer from the coils of the primary

* See particularly Oliver Lodge's Modern Views of Electricity. 
circuit to those of the secondary, it is pretty obvious that the flow of energy must take place laterally to the copper wires ; and it also takes place laterally to the iron wires of the core, though this is not so obvious.

520. Researches of Hertz.-In $1888 \mathrm{Hertz}$ found the most convincing experimental proofs of Maxwell's theory, and succeeded in producing electromagnetic waves in a way which permitted him to examine their propagation through space, and to show that, while they were much larger than ordinary waves of light, they possessed the same properties, travelled at the same speed, and were capable of being reflected, refracted, polarized, etc.

Of the power of oscillatory discharges to propagate disturbances in the surrounding space something was

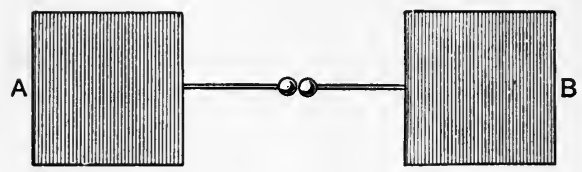

OSCILLATOR.

Fig. 286.

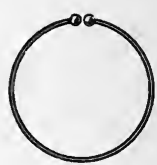

RESONATOR.

Fig. 287.

already known. Henry had shown that they set up other sparks in distant conducting circuits. It had been discovered ${ }^{*}$ that a spark-gap in the exciting circuit was necessary. Fitzgerald had definitely proposed to start waves by the oscillatory discharges of small condensers. But no one had systematically followed out the phenomena of propagation of the waves.

Hertz employed to start the waves an apparatus called an oscillator (Fig. 286), consisting of two metallic conductors (balls or plates) united by a metal rod, at the middle of which was interposed a spark-gap between two well-polisherl knobs. And to detect the waves at a

* See paper by the author in the Philosophical Magazine (September 1876). 
distance he employed a resonator, simply a circle or square of wire, having in it a spark-gap capable of minute adjustment. In one experiment the oscillator consisted of two rinc plates $A$ and $B$ (Fig. 286) with sides $40 \mathrm{~cm}$. long mounted $60 \mathrm{~cm}$. apart, and having stout copper wires leading to a spark-gap between very brightly polished brass balls. A dry wood stand was a sufficient insulator. The resonator to match was a circle $35 \mathrm{~cm}$. in radius. To experiment with this apparatus the oscillator is joined to a small induction coil. When

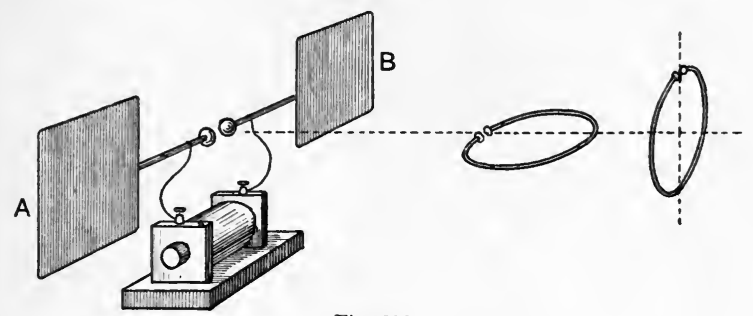

Fig. 2e8.

a spark snaps across the gap it sets up a temporary conducting path for the surgings that follow. For a rush of current from left to right overcharges the right-hand plate, and so there follows a rush back from right to left, and so on. Each spark sent by the coil across the gap consists of a dozen or so oscillations, each lasting about 1/100,000,000 of a second, the period being determined (Art. 515) by the capacity and inductance of the apparatus ; the discharges surging backward and forward from $\mathrm{A}$ to $\mathrm{B}$ until they die out (Fig. 284). Let the line drawn horizontally in Fig. 288 be termed the base line, and let the line $\mathrm{AB}$ be termed the line of oscillation. Then if the resonator is placed with its centre on the base line at a few feet away from the oscillator and is turned into various positions, various effects are observed. If the resonator is set 
edge-on vertically, no sparks are observed in it whatever the situation of the gap in the circle. If it is laid edgeon horizontally sparks pass between the balls of the resonator. These are brightest when the gap-space is nearest toward the oscillator, so that the induced spark is parallel to the primary spark. If the resonator be now turned broadside on to the oscillator it will be found that there are sparks when the gap is at the top or bottom of the circle-so that the sparks are parallel to the primary spark; but there are none if the gap is at the side. The primary spark does not here induce sparks at right angles to itself.

The reflexion of electric waves was observed in various ways. If right opposite the oscillator, Fig. 286, is set a large metal sheet as a reflector, to send back the waves that pass along the base line, stationary nodes will be produced at regular intervals. If the resonator is put broadside-on, with its gap at the highest point, and moved along the base line till it lies flat against the reflector, there will in this position be no sparks ; but if it is slowly moved back from the sheet sparks will show, will come to a maximum, then die out as the first node is reached at about $180 \mathrm{~cm}$. from the reflector. Passing this node the sparks will begin again, nodes occurring at equal intervals apart along the base line. By using large parabolic mirrors Hertz showed that these electric waves can be reflected and brought to a focus exactly as light-waves can be. Hertz also showed refraction with a prism of pitch ; and polarization by means of gratings of parallel wires.

Later Tesla showed that the Hertzian effects could be much augmented by increasing the suddenness of the spark by using a magnetic field to blow it out. Elihu Thomson uses an air-blast across the spark-gap for the same purpose.

521. Detectors of Electric Waves.-The Hertz spark-gap resonator is only one means of detecting electric 
waves. A prepared frog's leg (Art. 255) may be used instead of a spark-gap. A sensitive vacuum-tube, especially if primed by application with a battery of some hundreds of small cells not quite able of themselves to start a spark, forms a good explorer. Electrometers; thin wires capable of expanding when heated by the induced currents; and galvanometers in circuit with the gap, are amongst the possible means. Best of all is Lodge's device of a tube partly filled with metallic filings, inserted in circuit with a galvanometer and a single cell. The resistance of the filings is very great, and little current flows, until an electric wave impinges upon the tube, when at once the filings conduct (compare Art. 400 on conductance of powers). On lightly tapping the tube the filings fall back into their former state. Using such a detector, called a coherer, and an oscillator consisting of a highly polished brass ball between two smaller balls, Lodge has shown how these electric waves can pass hundreds of feet through walls and floors of houses. This invention is the basis of so-called wireless telegraphy; the coherer current being used in turn to operate a telegraphic receiver.

522. Properties of Electric Waves.-The universal equation connecting frequency $n$, wave-length $\lambda$, and velocity of propagation $v$ is : $v=n \lambda$. Taking $v$ (in air) as $3 \times 10^{10}$ (cms. per sec.) as the velocity of light, and the measured length of the red waves (the longest visible) as 0.000076 , it follows that the frequency of oscillation of these must be no' less than $395 \times 10^{12}$. The waves artificially produced by electric oscillations are of much lower frequency than these, and their wave length proportionally longer. Their wave-length depends on the size of the apparatus used as oscillator, just as the note emitted by an iron cylinder when struck on its end depends on the length of the cylinder. The wave-length of waves emitted from an oscillator consisting of a wire with a small capacity at each end is twice the length of the wire. That of waves emitted from 
a sphere (Fig. 289) of diameter $d$ is $2 \pi d / \sqrt{3}$ or $3.6 d$; but they die out after about 1 vibration. If a spark-gap is made between two knobs across the diameter of a

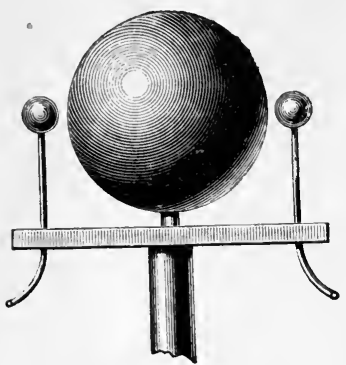

Fig. 289. hollow cylinder, the wavelength of the waves emitted from the end of the cylinder is about equal to its diameter, and the vibrations are numerous before all the energy has been radiated away. Using symmetrical pairs of condensers carefully adjusted, Ebert has obtained oscillations that do not die out till after 20,000 periods.

The currents produced in wires by oscillations of such enormous frequency are only skin-currents (Art. 476), the inner part of the wire being idle. Hence for such currents the impeding resistance of a stout copper wire may be millions of ohms. One evidence of this is afforded by the tendency to lateral discharge. This is readily shown by connecting between the Leyden jars of an influence machine a loop of stout copper wire bent as in Fig. 290. When a discharge takes place between the knobs, there will be an oscillatory current set up

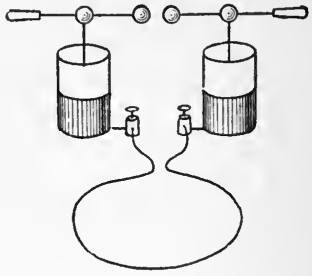

Fig. 290. between the outer coatings also; and this oscillatory current rather than flow along the metal loop will jump as a spark across the parts that lie nearest together. The tendency of lightning to produce lateral discharges is relied upon by Oliver Lodge in his contention as to the oscillatory character of the flash.

523. Travelling of Waves along Wires.-If an 
oscillatory spark is sent into one end of a long wire, by the time that the second pulsation reaches its maximum the first will have travelled a certain distance which may be called the wave-length of the disturbance. According to Maxwell's theory the velocity of propagation will be equal to that of light, the energy really travelling through the air, and settling down laterally into the wire. It appears from experiment that the velocity of a wave guided by a wire is the same as that of a wave travelling, in free air. That the speed of travelling is independent of the thickness or materials of the wire was proved in 1870 by Von Bezold using the device of Fig. 291. Let an oscillatory discharge be sent by a wire at $\mathrm{G}$ into a rectangular circuit $A B C D$, having a spark-gap $\mathrm{PQ}$ midway between $\mathrm{B}$ and $\mathrm{D}$. It is evident that if $G$ is midway between

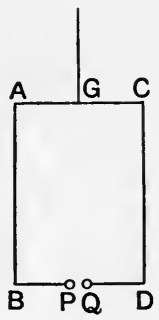

Fig. 291.

$\mathrm{A}$ and $\mathrm{C}$ the impulses will arrive simultaneously at $\mathrm{P}$ and $Q$ if both sides of the system are alike; and there will be no spark. If now one side, say $\mathrm{CD}$, be made of iron and the other, $\mathrm{AB}$, of copper, it will be found that still the discharge must be led in at $G$, exactly midway if there is to be no spark.

Lesson LVI._Other Relations between Light and Electricity

524. Electro - optical Phenomena. - Of late years several important relations have been observed between electricity and light. These observations may be classified under the following heads :-

(i.) Production of double refraction by dielectric stress.

(ii.) Rotation of plane of polarization of a wave of light 
on traversing a transparent medium placed in a magnetic field, or by reflexion at the surface of a maynet.

(iii.) Change of electric resistance, exhibited by selenium and other bodies during exposure to light.

(iv.) Photo-chemical excitation of electromotive forces.

(v.) Relation between refractive index and dielectric capacity of transparent bodies.

(vi.) Electric effect of ultra-violet light.

It was announced by Mrs. Somerville; by Zantedeschi, and others, that steel needles could be magnetized by exposing portions of them to the action of violet and ultra-violet rays of light ; the observations were, however, erroneous.

Bidwell has found that light falling upon a recently demagnetized piece of iron produces an instantaneous revival of magnetism.

525. Electrostatic Optical Stress.-In 1875 Dr. Kerr of Glasgow discovered that glass when subjected to a severe electrostatic stress undergoes an actual strain, which can be observed by the aid of a beam of polarized light. In the original experiment two wires were fixed into holes drilled in a slab of glass, but not quite meeting, so that when these were placed in connexion with the terminals of an induction coil or of an influence machine the accumulating charges on the wires subjected the intervening dielectric to an electrostatic tension along the electric lines of force. The slab when placed between two Nicol prisms as polarizer and analyzer * exhibited double refraction, as if it had been subjected to a pull and had expanded along the direction of the electric force. Bisulphide of carbon and other insulating liquids exhibit similar phenomena, but fatty oils of animal and

* A ray of light is said to be polarized if the vibrations take place in one plane. Ordinary light can be reduced to this coudition by passing it throngh a suitable polarizing apparatus (such as a Nicol prism, a thin slice of tourmaline crystal, etc.). 
vegetable origin exhibit an action in the negative direction, as if they had contracted along the electric lines. It is found that the difference of retardation between the ordinary and extraordinary waves per unit thickness of the dielectric is proportional to the square of the resultant electric force. The axis of double refraction is along the line of the electric force. Quincke has pointed out that these phenomena can be explained by the existence of electrostatic expansions and contractions, stated in Art. 300.

526. Magneto-optic Rotation of the Plane of Polarization of Light.-In 1845 Faraday discovered that a ware of light polarized in a certain plane can be twisted round by the action of a magnet, so that the vibrations are executed in a different plane. The plane in which a beam is polarized can be detected by observing it through a second Nicol prism (or tourmaline), for each such polarizer is opaque to waves polarized in a plane at right angles to that plane in which it would itself polarize light. Faraday caused a polarized beam to pass throngh a piece of a certain "heavy glass" (consisting chiefly of borate of lead), lying in a powerful magnetic field, between the poles of a large electromagnet, through the coils of which a current could be sent. In the path of the emerging beam was placed as analyzer a second Nicol prism which had been turned round until all the light was extinguished. In this position its own plane of symmetry was at right angles to the plane of polarization of the beam. On completing the circuit, light was at once seen through the analyzing Nicol prism, proving that the waves had been twisted round into a new position, in which the plane of polarization was no longer at right angles to the plane of symmetry of the analyzer. But if the analyzing Nicol prism was itself turned round, a new position could be found (at right angles to the plane of polarization of the waves) at which the light was once more extinguished. The direction of the magneto-optic rotation 
of the plane of polarization is the same (for diamagnetic media) as that in which the current flows which produces the magnetism. Verdet discovered the important law that, with a given material, the amount of rotation is proportional to the strength of the magnetic force $\mathrm{H}$. In case the waves do not pass straight along the direction of the field, the amount of rotation is proportional to the cosine of the angle $\beta$ between the direction of the beam and the lines of force. It is also proportional to the length $l$ of the material through which the waves pass. These laws are combined in the equation for the rotation $\theta$ :

$$
\theta=w \cdot \mathrm{H} \cdot \cos \beta \cdot l,
$$

where $w$ is a coefficient which represents the specific magnetic rotatory power of the given substance, and is known as Verdet's constant. Now, $\mathrm{H} \cdot \cos \beta \cdot l$ is the difference of magnetic potential between the point $A$ where the wave enters and $B$ where it leaves the medium. Hence

$$
w=\frac{\theta}{V_{B}-V_{\mathrm{A}}} .
$$

The value of Verdet's constant for yellow sodium light, at $18^{\circ} \mathrm{C}$., has been carefully determined. Its value (in radians per unit fall of magnetic potential) is, in bisulphide of carbon $1.222 \times 10^{-5}$; in water $0.375 \times 10^{-5}$; in heavy glass $2 \cdot 132 \times 10^{-5}$. For diamagnetic substances the coefficient is usually positive; but in the case of many magnetic substances, such as solutions of ferric chloride, has a negative value (i.e. in these substances the rotation is in the opposite direction to that in which the magnetizing current flows). The phenomenon discovered by Hall (Art. 397) appears to be intimately related to the phenomenon of magneto-optic rotation. For light of different colours the rotation is not equal, but varies very nearly inversely as the square of the wave-length.

Gases also rotate the plane of polarization of light in 
a magnetic field with varying amounts; coal-gas and carbonic acid being more powerful than air or hydrogen ; oxygen and ozone being negative. The rotation is in all cases very slight, and varies for any gas in proportion to the quantity of gas traversed. H. Becquerel has shown that the plane of the natural polarization of the sky does not coincide with the plane of the sun, but is rotated by the influence of the earth's magnetism through an angle which, however, only reached $59^{\prime}$ of arc at a maximum on the magnetic meridian.

We have seen (Arts. 126, 397, and 398) what evidence there is for thinking that magnetism is a phenomenon of rotation, there being a rotation of something around an axis lying in the direction of the magnetization. Such a theory would explain the rotation of the plane of polarization of a ray passing through a magnetic field. For a ray of plane-polarized light may be conceived of as consisting of a pair of (oppositely) circularly-polarized waves, in which the right-handed rotation in one ray is periodically counteracted by an equal left-handed rotation in the other ray; and if such a motion were imparted to a medium in which there were superposed a rotation (such as we conceive to take place in every magnetic field) about the same direction, one of these circularlypolarized rays would be accelerated and the other retarded, so that, when they were again compounded into a single planepolarized ray, this plane would not coincide with the original plane of polarization, but would be apparently turned round through an angle proportional to the superposed rotation.

527. Kerr's Effect.-Dr. Kerr showed in 1877 that a ray of polarized light is also rotated when reflected at the surface of a magnet or electromagnet. When the light is reflected at a pole the plane of polarization is turned in a direction contrary to that in which the magnetizing current flows. If the light is reflected at a point on the side of the magnet it is found that when the plane of polarization is parallel to the plane of incidence the rotation is in the same direction as that of the magnetizing current; but that, when the plane of polarization is perpendicular to the plane of incidence, the rotation is in the same direction as that of the 
magnetizing current only when the incidence exceeds $75^{\circ}$, being in the opposite direction at lesser angles of incidence.

528. Kundt's Effect.-Kundt found that the plane of polarization of light-waves is also rotatel if the light is passed through a film of iron so thin as to be transparent, if placed transversely in a magnetic field.

529. Photo-electric Properties of Selenium. -In 1873 Willoughby Smith announced the discovery (by J. E. Mayhew) that the element selenium possesses the abnormal property of changing its electric resistance under the influence of light. Ordinary fused or vitreous selenium is a very bad conductor; its resistance being nearly forty-thousand-million $\left(3.8 \times 10^{10}\right)$ times as great as that of copper. When carefully annealed (by keeping for some hours at a temperature of about $220^{\circ} \mathrm{C}$, just below its fusing point, and subsequent slow cooling) it assumes a crystalline condition, in which its electric resistance is considerably reduced. In the latter condition, especially, it is sensitive to light. Adams found that greenish-yellow rays were the most effective. He also showed that the change of electric resistance varies directly as the square root of the illumination, and that the resistance is less with a high electromotive-force than a low one. In 1879, Graham Bell and Sumner Tainter devised "selenium cells," in which annealed selenium is formed into narrow strips between the edges of broad conducting plates of brass; thus securing both a reduction of the transverse resistance and a large amount of surfaceexposure to light. Thus a cell, whose resistance in the dark was 300 ohms, when exposed to sunlight had a resistance of but $150 \mathrm{ohms}$. This property of selenium these investigators applied in the construction of the Photophone, an instrument which transmits sounds to a distance by means of a bean of light reflected to a distant spot from a thin mirror thrown into vibrations by the voice; the beam falling, consequently, with 
varying intensity upon a receiver of selenium connected in circuit with a small battery and a Bell telephone receiver (Art. 510) in which the sounds are reproduced by the variations of the current.

Similar properties are possessed, to a smaller degree; by tellurium. Carbon is also sensitive to light.

530. Photo-chemical Cells.-About the middle of the present century Becquerel showed that when two plates of silver, coated with freshly-deposited chloride of silver, are placed in a cell with water and connected with a galvanometer, a current is observed to pass when light falls upon one of the two plates, the exposed plate acting as an anode; and Minchin has more recently shown the efficiency of other photo-chemical combinations. Some of these are very sensitive to electric waves of greater wavelength.

531. Photo-electric Loss of Charge.-In 1887 Hertz made the discovery that a spark starts more readily between the balls of a discharger when illuminated by light that is rich in violet and ultra-violet rays (magnesium light, arc light, or spark of induction coil) than when not so illuminated. The effect varies with different metals, with their cleanuess, the nature of the surrounding gas, with the kind of charge, and with the polarization of the light. In ultra-violet light freshly polished zinc in air rapidly discharges a negative charge, but not a positive one. On the other hand the peroxides, in an atmosphere of hydrogen, when so illuminated readily discharge positive charges. The effect is stronger when the plane of the vibration of the incident waves is at right angles to the surface than when the polarization is in a parallel plane. The phenomenon appears to be due to the small light-waves stimulating chemical reactions which do not occur except (Art. 322) by a species of electric exchange. In a strong magnetic field no such discharges occur. Hallwachs charged clean zinc plates positively by exposure to ultra-violet light. 


\begin{tabular}{|c|c|c|c|c|c|}
\hline $\begin{array}{c}\theta \text { in } \\
\text { Degrees. }\end{array}$ & $\begin{array}{c}\theta \text { in } \\
\text { Radians. }\end{array}$ & Sine $\theta$. & Tangent $\theta$. & $\begin{array}{l}\text { Solid Angle } \\
2 \pi(1-\cos \theta) .\end{array}$ & $\begin{array}{c}\text { Complement } \\
\text { of } \theta=\phi .\end{array}$ \\
\hline $0^{\circ}$ & 0 & 0 & 0 & 0 & $90^{\circ}$ \\
\hline 1 & .0175 & $\cdot 0175$ & $\cdot 0175$ & .000957 & 89 \\
\hline 2 & $\cdot 0349$ & $\cdot 0349$ & $\cdot 0349$ & .003837 & 88 \\
\hline 3 & 0524 & $\cdot 0523$ & $\cdot 0524$ & $\cdot 00861$ & 87 \\
\hline 4 & $\cdot 0698$ & 0698 & •0699 & .01532 & 86 \\
\hline 5 & $\cdot 0873$ & $\cdot 0872$ & $\cdot 0875$ & $\cdot 02391$ & 85 \\
\hline 6 & $\cdot 1047$ & $\cdot 1045$ & $\cdot 1051$ & $\cdot 03441$ & 84 \\
\hline$i$ & $\cdot 1222$ & $\cdot 1219$ & $\cdot 1228$ & $\cdot 04683$ & 83 \\
\hline 8 & $\cdot 1396$ & $\cdot 1392$ & $\cdot 1405$ & $\cdot 06115$ & 82 \\
\hline 9 & $\cdot 1571$ & - 1564 & $\cdot 1584$ & .07735 & 81 \\
\hline 10 & $\cdot 1745$ & $\cdot 1737$ & $\cdot 1763$ & $\cdot 09545$ & 80 \\
\hline 11 & $\cdot 1920$ & $\cdot 1908$ & $\cdot 1944$ & $\cdot 1154$ & 79 \\
\hline 12 & •2094 & 2079 & $\cdot 2126$ & $\cdot 1373$ & 78 \\
\hline 13 & $\cdot 2269$ & .2250 & $\cdot 2309$ & $\cdot 1610$ & $\pi 7$ \\
\hline 14 & $\cdot 2444$ & $\cdot 2419$ & $\cdot 2493$ & $\cdot 1866$ & 76 \\
\hline 15 & 2618 & $\cdot 2588$ & $\cdot 2680$ & $\cdot 2140$ & 75 \\
\hline 16 & $\cdot 2793$ & $\cdot 2756$ & 2868 & $\cdot 2434$ & 74 \\
\hline 17 & $\cdot 2967$ & $\cdot 2924$ & $\cdot 3057$ & 2745 & 73 \\
\hline 18 & $\cdot 3142$ & $\cdot 3090$ & $\cdot 3249$ & $\cdot 3075$ & $\pi 2$ \\
\hline 19 & $\cdot 3316$ & $\cdot 3256$ & $\cdot 3443$ & $3+23$ & $i 1$ \\
\hline 20 & $\cdot 3491$ & $\cdot 3420$ & 3640 & 3789 & ro \\
\hline 21 & $\cdot 3665$ & $\cdot 3584$ & •3839 & 4173 & 69 \\
\hline 22 & $\cdot 3840$ & 3746 & $\cdot 4040$ & $\cdot 4575$ & 68 \\
\hline 23 & $\cdot 4014$ & '3907 & $\cdot 4245$ & $\cdot 4994$ & 67 \\
\hline 24 & $\cdot 4189$ & $\cdot 4067$ & $\cdot 4452$ & $\cdot 5431$ & 66 \\
\hline 25 & $\cdot 4363$ & $\cdot 4226$ & $\cdot 4663$ & ${ }^{\circ} 5886$ & 65 \\
\hline 26 & $\cdot 4538$ & $\cdot 4384$ & $\cdot 4877$ & $\cdot 6358$ & 64 \\
\hline 27 & $\cdot 4712$ & $\cdot 4540$ & $\cdot 5095$ & 6848 & 63 \\
\hline 28 & $\cdot 4887$ & $\cdot 4695$ & $\cdot 5317$ & •7354 & 62 \\
\hline 29 & $\cdot 5062$ & $\cdot 4845$ & $\cdot 5543$ & $\cdot \pi s t \tau$ & 61 \\
\hline 30 & $\cdot 5236$ & $\cdot 5000$ & .5774 & 8417 & 60 \\
\hline 31 & $\cdot 5411$ & $\cdot 5150$ & 6309 & $\cdot 8974$ & 59 \\
\hline 32 & $\cdot 5585$ & $\cdot 5299$ & $\cdot 6249$ & $\cdot 954 \bar{\imath}$ & 58 \\
\hline 33 & $\cdot 5760$ & $\cdot 5446$ & $\cdot 6494$ & $1 \cdot 0136$ & $5 \%$ \\
\hline 34 & $\cdot 5934$ & $\cdot 5592$ & $\cdot 6745$ & $1 \cdot 0741$ & 56 \\
\hline 35 & $\cdot 6109$ & $\cdot 5736$ & $\cdot 7002$ & $1 \cdot 1362$ & 55 \\
\hline 36 & $\cdot 6283$ & $\cdot 5878$ & $\cdot 7265$ & $1 \cdot 1999$ & 54 \\
\hline 37 & $\cdot 6458$ & $\cdot 6018$ & $\cdot 7536$ & $1 \cdot 2652$ & 53 \\
\hline 38 & $\cdot 6632$ & $\cdot 6157$ & 7813 & $1 \cdot 3319$ & 52 \\
\hline 39 & $\cdot 6807$ & $\cdot 6293$ & 's098 & $1 \cdot 4002$ & 51 \\
\hline 40 & $\cdot 6981$ & $\cdot 6428$ & ·8391 & $1 \cdot 4700$ & 50 \\
\hline 41 & $\cdot 7156$ & $\bullet 6561$ & •S693 & $1 \cdot 5412$ & 49 \\
\hline 42 & $\cdot 7330$ & $\cdot 6691$ & •9004 & $1 \cdot 6138$ & 48 \\
\hline 43 & $* 505$ & $\cdot 6820$ & $\cdot 9325$ & 1.6879 & 47 \\
\hline 44 & 7679 & 6947 & 9657 & $1 \cdot 6634$ & 46 \\
\hline \multirow[t]{2}{*}{45} & $\cdot 7854$ & 7071 & $1 \cdot 0000$ & $1 \cdot 8402$ & 45 \\
\hline & & Cosine $\phi$ & Cotangent $\phi$ & $2 \pi(1-\sin \phi)$ & $\phi$ in Degrees \\
\hline
\end{tabular}




\section{ANGLES AND SOLID ANGLES}

\begin{tabular}{|c|c|c|c|c|c|}
\hline $\begin{array}{c}\theta \text { in } \\
\text { Degrees. }\end{array}$ & $\begin{array}{c}\theta \text { in } \\
\text { Radians. }\end{array}$ & Sine $\theta$ & Tangent $\theta$. & $\begin{array}{l}\text { Solid Angle } \\
2 \pi(1-\cos \theta) .\end{array}$ & $\begin{array}{c}\text { Complement } \\
\text { of } \theta=\phi .\end{array}$ \\
\hline $45^{\circ}$ & 7854 & $\ulcorner 071$ & $1 \cdot 0000$ & $1 \cdot 8402$ & $45^{\circ}$ \\
\hline 46 & $\cdot 8029$ & $\cdot 7193$ & 1.0355 & $1 \cdot 9185$ & 44 \\
\hline 47 & 8203 & $\cdot 7314$ & 1.0724 & $1 \cdot 9980$ & 43 \\
\hline 48 & $\cdot 8378$ & $\cdot 7431$ & $1 \cdot 1106$ & $2 \cdot 0789$ & 42 \\
\hline 49 & $\cdot 8552$ & 7547 & $1 \cdot 1504$ & $2 \cdot 1610$ & 41 \\
\hline 50 & 8727 & $\cdot 7660$ & $1 \cdot 1918$ & $2 \cdot 2444$ & 40 \\
\hline 51 & .8901 & $\cdot 7772$ & $1 \cdot 2349$ & $2 \cdot 3290$ & 39 \\
\hline 52 & 9076 & $\cdot 7880$ & $1 \cdot 2799$ & $2 \cdot 4149$ & 38 \\
\hline 53 & $\cdot 9250$ & $\cdot 7986$ & $1 \cdot 32 \pi 0$ & $2 \cdot 5019$ & 37 \\
\hline 54 & $\cdot 9425$ & $\cdot 8090$ & $1 \cdot 3764$ & $2 \cdot 5900$ & 36 \\
\hline 55 & .9599 & $\cdot 8192$ & $1 \cdot 4282$ & $2 \cdot 6793$ & 35 \\
\hline 56 & $\cdot 9774$ & -8290 & $1 \cdot 4826$ & $2 \cdot 7696$ & 34 \\
\hline 57 & .9948 & $\cdot 8387$ & $1 \cdot 5399$ & $2 \cdot 8611$ & 33 \\
\hline 58 & 1.0123 & $\cdot 8481$ & $1 \cdot 6003$ & $2 \cdot 9536$ & 32 \\
\hline 59 & 1.0298 & 8572 & $1 \cdot 6643$ & $3 \cdot 0472$ & 31 \\
\hline 60 & $1 \cdot 0472$ & $\cdot 8660$ & $1 \cdot 7321$ & $3 \cdot 1416$ & 30 \\
\hline 61 & 1.0647 & $\cdot 8746$ & $1 \cdot 8041$ & $3 \cdot 23 ; 0$ & 29 \\
\hline 62 & $1 \cdot 0821$ & $\cdot 8830$ & $1 \cdot 8807$ & $3 \cdot 3334$ & 28 \\
\hline 63 & $1 \cdot 0996$ & .8910 & $1 \cdot 9626$ & $3 \cdot 4307$ & 27 \\
\hline 64 & $1 \cdot 1170$ & -8988 & $2 \cdot 0503$ & $3 \cdot 5288$ & 26 \\
\hline 65 & $1 \cdot 1345$ & $\cdot 9063$ & $2 \cdot 1445$ & $3 \cdot 62 \div 8$ & 25 \\
\hline 66 & $1 \cdot 1519$ & $\cdot 9136$ & $2 \cdot 2460$ & $3 \cdot 7276$ & 24 \\
\hline 67 & $1 \cdot 1694$ & $\cdot 9205$ & $2 \cdot 3559$ & $3 \cdot 8281$ & 23 \\
\hline 68 & $1 \cdot 1868$ & $\cdot 9272$ & $2 \cdot 4751$ & 3.9295 & 22 \\
\hline 69 & $1 \cdot 2043$ & 9336 & $2 \cdot 6051$ & $4 \cdot 0315$ & 21 \\
\hline 70 & $1 \cdot 2217$ & $\cdot 9397$ & $2 \cdot 7475$ & $4 \cdot 1342$ & 20 \\
\hline 71 & $1: 2392$ & 9455 & $2 \cdot 9042$ & $4 \cdot 2376$ & 19 \\
\hline 72 & $1 \cdot 25 t 6$ & $\cdot 9511$ & $3.0 \pi \pi$ & $4 \cdot 3416$ & 18 \\
\hline 73 & $1 \cdot 2741$ & $\cdot 9563$ & $3 \cdot 2709$ & $4 \cdot 4462$ & 17 \\
\hline 74 & $1 \cdot 2916$ & $\cdot 9613$ & $3 \cdot 4874$ & $4 \cdot 5513$ & 16 \\
\hline 75 & $1 \cdot 3090$ & $\cdot 9659$ & $3 \cdot 7321$ & $4 \cdot 6570$ & 15 \\
\hline 76 & $1 \cdot 3265$ & 9703 & 4.0108 & $4 \cdot 7632$ & 14 \\
\hline 77 & $1 \cdot 3439$ & $\cdot 9744$ & $4 \cdot 3315$ & $4 \cdot 8698$ & 13 \\
\hline 78 & $1 \cdot 3614$ & $\cdot 9782$ & $4 \cdot 7046$ & 4.9768 & 12 \\
\hline 79 & 1.3788 & $\cdot 9816$ & $5 \cdot 1446^{\circ}$ & $5 \cdot 0843$ & 11 \\
\hline 80 & $1 \cdot 3963$ & $\cdot 9848$ & $5 \cdot 6713$ & $5 \cdot 1921$ & 10 \\
\hline 81 & $1 \cdot 4137$ & $\cdot 9877$ & $6 \cdot 3138$ & $5 \cdot 3003$ & 9 \\
\hline 82 & $1 \cdot 4312$ & $\cdot 9903$ & $7 \cdot 1154$ & $5 \cdot 4087$ & 8 \\
\hline 83 & 1.4486 & $\cdot 9926$ & $8 \cdot 1444$ & $5 \cdot 5174$ & 7 \\
\hline 84 & $1 \cdot 4061$ & $\cdot 9945$ & $9 \cdot 5144$ & $5 \cdot 6264$ & 6 \\
\hline 85 & $1 \cdot 4835$ & •9962 & $11 \cdot 4301$ & $5 \cdot 7356$ & 5 \\
\hline 86 & $1 \cdot 5010$ & $\cdot 9976$ & $14 \cdot 3007$ & $5 \cdot 8449$ & 4 \\
\hline 87 & 1.5184 & $\cdot 9986$ & $19 \cdot 0811$ & $5 \cdot 9543$ & 3 \\
\hline 88 & $1 \cdot 5359$ & •9994 & $28 \cdot 6363$ & 6.0639 & 2 \\
\hline 89 & $1 \cdot 5534$ & $\cdot 9999$ & $57 \cdot 2900$ & $6 \cdot 1735$ & 1 \\
\hline 90 & $1 \cdot 5708$ & $1 \cdot 0000$ & $\infty$ & $6 \cdot 2832$ & 0 \\
\hline & & Cosine $\phi$ & Cotangent & $2 \pi(1-\sin$ & $\phi$ in Deg \\
\hline
\end{tabular}




\title{
APPENDIX B
}

\author{
Order in Council on Electrical Units and \\ Standards (Abstract)
}

Dated the 23rd day of August 1894

Whereas by "The Weights and Measures Act, 1889," it is among other things enacted that the Board of Trade shall from time to time cause such new denominations of standards for the measurement of electricity as appear to them to be required for use in trade to be made and duly verified.

And whereas it has been made to appear to the Board of Trade that new denominations of standards are required for use in trade based upon the following units of electrical measurement, viz. :-

1. The $o h m$, which has the value $10^{9}$ in terms of the centimetre and the second of time, and is represented by the resistance offered to an unvarying electric current by a column of mercury at the temperature of melting ice, 14.4521 grammes in mass of a constant cross sectional area and of a length of $106 \cdot 3$ centimetres.

2 . The ampere, which has the value $\frac{1}{10}$ in terms of the centimetre, the gramme, and the second of time, and which is represented by the unvarying electric current which, when passed through a solution of nitrate of silver in water, in accordance with the specification appended hereto, deposits silver at the rate of 0.001118 of a gramme per second.

3 . The volt, which has the value $10^{8}$, in terms of the centimetre, the gramme, and the second of time, being the electrical pressure that, if steadily applied to a conductor whose resistance is one ohm, will produce a current of one ampere, and which is represented by $0.6974\left(\frac{1}{10 \frac{0}{4}} \frac{0}{4}\right)$ of the 
electrical pressure at a temperature of $15^{\circ} \mathrm{C}$. between the poles of the voltaic cell known as Clark's cell, set up in accordance with the specification appended hereto.*

And whereas they have caused the said new denominations of standards to be made and duly verified,

Now THEREFore, Her Majesty, by virtue of the power $\mathbf{v} \epsilon$ sted in Her by the said Act, by and with the advice of Her Privy Council, is pleased to approve the several denominations of standards set forth in the schedule hereto as new denominations of standards for electrical measurement.

\section{SCHEDULE}

\section{I.-STandard of Electrical Resistaxce}

A standard of electrical resistance denominated one ohm being the resistance between the copper terminals of the instrument marked "Board of Trade Ohm Standard Verified 1894 " to the passage of an unvarying electrical current when the coil of insulated wire forming part of the aforesaid instrument and connected to the aforesaid terminals is in all parts at a temperature of $15 \cdot 4^{\circ} \mathrm{C}$.

\section{II.-Standard of Electrical CurRent}

A standard of electrical current denominated one ampere being the current which is passing in and through the coils of wire forming part of the instrument marked "Board of Trade Ampere Standard Verified 1894" when on reversing the current in the fixed coils the change in the forces acting upon the suspended coil in its sighted position is exactly balanced by the force exerted by gravity in Westminster upon the iridio-platinum weight marked $A$, and forming part of the said instrument.

\section{III.-Standard of Electrical Presscre}

A standard of electrical pressure denominated one volt heing one hundredth part of the pressure which when applied between the terninals forming part of the instrument marked "Board of Trade Volt Standard Verified 1894" causes that

* See Appendix C. 
rotation of the suspended portion of the instrument which is exactly measured by the coincidence of the sighting wire with the image of the fiducial mark $A$ before and after application of the pressure and with that of the fiducial mark $B$ during the application of the pressure, these images being produced by the suspended mirror and observed by means of the eyepiece.

The coils and instruments are deposited at the Board of Trade Standardizing Laboratory, Whitehall, London. 


\section{APPENDIX C}

Official Specification for the Preparation of the

Clark Cell

\section{Definition of the Cell}

The cell consists of zinc or an analgam of zine with mereury and of mercury in a neutral saturated solution of zine sulphate and mercurous sulphate in water, prepared with mercurous sulphate in excess.

\section{Preparation of the Materials}

1. The Mercury. - To secure purity it should be first treated with acid in the usual manner, and subsequently distilled in vacuo.

2. The Zinc.-Take a portion of a rod of pure redistilled zinc, solder to one end a piece of copper wire, clean the whole with glass paper or a steel burnisher, carefully removing any loose pieces of the zinc. Just before making up the cell dip the zine into dilute sulphuric acid, wash with distilled water, and dry with a clean cloth or filter paper.

3. The Mercurous Sulphate.-Take mercurous sulphate, purchased as pure, mix with it a small quantity of pure mercury, and wash the whole thoroughly with cold distilled water by agitation in a bottle ; drain off the water, and repeat the process at least twice. After the last washing, drain off as much of the water as possible.

4. The Zinc Sulphate Solution.-Prepare a neutral saturated solution of pure ("pure recrystallized") zinc sulphate by 
mixing in a flask distilled water with nearly twice its weight of crystals of pure zinc sulphate, and adding zinc oxide in the proportion of about 2 per cent by weight of the zinc sulphate crystals to neutralise any free acid. The crystals should be dissolved with the aid of gentle heat, but the temperature to which the solution is raised should not exceed $30^{\circ} \mathrm{C}$. Mercurous sulphate treated as described in (3) should be added in the proportion of about 12 per cent by weight of the zinc sulphate crystals to neutralise any free zinc oxide remaining, and the solution filtered, while still warm, into a stock bottle. Crystals should form as it cools.

5. The Mercurous Sulphate and Zine Sulphate Paste.-Mix the washed mercurous sulphate with the zinc sulphate solution, adding sufficient crystals of zinc sulphate from the stock bottle to ensure saturation, and a small quantity of pure mercury. Shake these up well together to form a paste of the consistence of cream. Heat the paste, but not above a temperature of $30^{\circ} \mathrm{C}$. Keep the paste for an hour at this temperature, agitating it from time to time, then allow it to cool; continue to shake it occasionally while it is cooling. Crystals of zinc sulphate should then be distinctly visible, and should be distributed throughout the mass; if this is not the case add more crystals from the stock bottle, and repeat the whole process.

This method ensures the formation of a saturated solution of zinc and mercurous sulphates in water.

\section{To set up the Cell}

The cell may conveniently be set up in a small test tube of about 2 centimetres diameter, and 4 or 5 centimetres deep. Place the mercury in the bottom of this tube, filling it to a depth of say 0.5 centimetre. Cut a cork about 0.5 centimetre thick to fit the tube; at one side of the cork bore a hole through which the zinc rod can pass tightly; at the other side bore another hole for the glass tube which covers the platinum wire; at the edge of the cork cut a nick through which the air can pass when the cork is pushed into the tube. Wash the cork thoroughly with warm water, and leave it to soak in water for some hours before use. Pass the zinc rod about 1 centimetre through the cork.

Contact is made with the mercury by means of a platinum wire about No. 22 gange. This is protected from contact with the other materials of the cell by being sealed into a glass tube. The ends of the wire project from the ends of the 
tube ; one end forms the terminal, the other end and a portion of the glass tube dip into the mercury.

Clean the glass tube and platinum wire carefully, then heat the exposed end of the platinum red hot, and insert it in the mercury in the test tube, taking care that the whole of the exposed platinum is covered.

Shake up the paste and introduce it without contact with the upper part of the walls of the test tube, filling the tube above the mercury to a depth of rather more than 1 centimetre.

Then insert the cork and zinc rod, passing the glass tube through the hole prepared for it. Push the cork gently down until its lower surface is nearly in contact with the liquid. The air will thus be nearly all expelled, and the cell should be left in this condition for at least 24 hours before sealing, which should be done as follows :

Melt some marine glue until it is fluid enough to pour by its own weight, and pour it into the test-tube above the cork, using sufficient to cover completely the zinc and soldering. The glass tube containing the platinum wire should project some way above the top of the marine glne.

The cell may be sealed in a more permanent manner by coating the marine glue, when it is set, with a solution of sodium silicate, and leaving it to harden.

The cell thus set up may be mounted in any desirable manner. It is convenient to arrange the mounting so that the cell may be immersed in a water bath up to the level of, say, the upper surface of the cork. Its temperature can then be determined more accurately than is possible when the cell is in air.

In using the cell sudden variations of temperature should as far as possible be avoided.

The form of the vessel containing the cell may be varied. In the $\mathrm{H}$-form, the zinc is replaced by an amalgam of 10 parts by weight of zine to 90 of mercury. The other materials should be prepared as already described. Contact is made with the amalgam in one leg of the cell, and with the mercury in the other, by means of platinum wires sealed through the glass. 


\section{PROBLEMS AND EXERCISES}

\section{QUESTIONS ON CHAPTER I}

1. In what respects does an electrified body differ from a non-electrified body?

2. Name some of the different methods of producing electrification.

3. $\Lambda$ body is charged so feebly that its electrification will not perceptibly move the leaves of a gold-leaf electroscope. Can you suggest any means of ascertaining whether the charge of the body is positive or negative?

4. How would you prove that the production of a positive charge is accompanied by the production of an equal negative charge.

5. Describe an experiment to prove that moistened thread conducts electricity better than dry thread.

6. Why do we regard the two electric charges produced simultaneously by rubbing two bodies together as being of opposite kinds?

7. Explain the action of the electrophorus. Can you suggest any means for accomplishing by a rotatory motion the operations of lifting up and down the cover of the instrument so as to obtain a continuous supply instead of an intermittent one?

8. Deseribe the state of the medium between two oppositely charged bodies, and state how you would determine the direction of the lines of force at any point.

9. Explain the Torsion Balance, and how it can be used to investigate the laws of the distribution of electricity. 
10. Describe what takes place as an electrified conducting ball is made to approach a large condueting surface. Show by diagram the direction and relative number of the lines of foree.

11. Two small balls are charged respectively with +24 and -8 units of electricity. With what force will they attract one another when placed at a distance of 4 centinietres from one another? Ans. 12 dynes.

12. If these two balls are then made to touch for an instant and then put back in their former positions, with what force will they act on each other?

Ans. They will repel one another with a force of 4 dynes.

13. Enumerate the essential parts of an influence machine ; and explain how they operate to produce electrification.

14. Take the diagrammatic representation of the Wimshurst machine (Fig. 40) and fill in the lines of electric foree, showing their direction and relative number.

15. Explain the action of the Leyden jar by the consideration of electric displacement.

16. Deseribe four different ways of electrifying a tourmaline crystal.

17. Zinc filings are sifted through a sieve made of copper wire upon an insulated zinc plate joined by a wire to an electroscope. What will be observed?

18. Explain the prineiple of an air-condenser; and state why it is that the two oppositely charged plates show less signs of electrification when placed near together than when drawn apart from one another.

19. There are four Leyden jars A, B, C, and D, of which A, $B$, and $D$ are of glass, $C$ of guttapercha. $A, B$, and $C$ are of the same size, $D$ being just twice as tall and twice as wide as the others. A, C, and D are of the same thickness of material, but B is made of glass only half as thiek as A or D. Compare their eapacities.

Ans. Take capacity of $\mathrm{A}$ as 1 ; that of $\mathrm{B}$ will be 2 ; that of $\mathrm{C}$ will be $\frac{3}{4}$; and that of $\mathrm{D}$ will be 4 .

20. How would you show that a bar made half of zine and half of copper is capable of producing electrification?

21. How would you prove that there is no electrification within a closed conductor? 
22. What prevents the charge of a body from escaping away at its surface?

23. Explain the action of Hamilton's mill.

24. Two brass balls mounted on glass stems are placed half an inch apart. One of them is gradually charged by a machine until a spark passes between the two balls. State exactly what happened in the other brass ball and in the intervening air up to the moment of the appearance of the spark.

25. Define electric density. A charge of 248 units of electricity was imparted to a sphere of 4 centimetres radius. What is the density of the charge? Ans. 1.23 nearly.

\section{QUESTIONS ON CHAPTER II}

1. A dozen steel sewing-needles are hung in a bunch by threads through their eyes. How will they behave when hung over the pole of a strong magnet?

2. Explain the operation of an iron screen in protecting a galvanometer needle from magnets in its vicinity, and state why it is not perfectly effectual.

3. Of what material, and of what shape, would you make a magnet which is required to preserve its magnetism unaltered for a very long time? Describe the process of tempering.

4. What is meant by the resultant magnetic force at a point?

5. Six magnetized sewing-needles are thrust vertically through six little floats of cork, and are placed in a basin of water with their N-pointing poles upwards. How will they affect one another, and what will be the effect of holding over them the S-pointing pole of a magnet?

6. What distinction do you draw between magnets and magnetic matter?

7. On board an iron ship which is laying a submarine telegraph cable there is a galvanometer used for testing the continuity of the cable. It is necessary to screen the magnetized needle of the galvanometer from being affected by the magnetism of the ship. How can this be done?

8. How would you prove two magnets to be of equal strength? 
9. The force which a magnet-pole exerts upon another magnet-pole decreases as you increase the distance between them. What is the exact law of the magnetic force, and how is it proved experimentally?

10. Describe the behaviour of Ewing's model of molecular magnetism in a magnetic field, and show how it corresponds with the behaviour of iron when magnetized. Divide the process of magnetizing into three successive stages.

11. What force does a magnet-pole, the strength of which is 9 units, exert upon a pole whose strength is 16 units placed 6 centimetres away?

Ans. 4 dynes.

12. How would you place a long magnet so that one of its poles deflects a compass while the other does not affect it?

13. Distinguish between the "strength" of a magnet and its "magnetic moment."

14. Describe an instrument for comparing the relative values of magnetic forces. How would you use it to compare the magnetic moments of two magnets? If their distances from the magnetometer are respectively 20 centimetres and 30 centimetres, what is the ratio of their magnetic moments?

Ans. $8: 27$.

15. Two magnets have the same pole strength, but one is twice as long as the other. The shorter is placed 20 centimetres from a magnetometer (using the end-on method); state at what distance the other must be placed in order that there may be no deflexion.

Ans. $25 \cdot 198$ centimetres.

16. A pole of strength 40 units acts with a force of 32 dynes upon another pole 5 centimetres away. What is the strength of that pole?

Ans. 20 units.

17. It is desired to compare the magnetic force at a point 10 centimetres from the pole of a magnet with the magnetic force at 5 centimetres' distance. Describe four ways of doing this.

18. Explain the phenomenon of Consequent Poles.

19. In what direction do the lines of magnetic induction (or "lines of force") run in a plane in which there is a single magnetic pole? How would you arrange an experiment by which to test your answer?

20. What is a Magnetic Shell? What is the law of the potential due to a magnetic shell? 
21. A steel bar-magnet suspended horizontally, and set to oscillate at Bristol, made 110 complete oscillations in five minutes ; the same needle when set oscillating horizontally at St. Helena executed 112 complete oscillations in four minutes. Compare the horizontal component of the force of the earth's magnetism at Bristol with that at St. Helena.

Ans. $\mathrm{H}$ at Bristol : $\mathrm{H}$ at St. Helena : : 484 : 784.

22. Supposing the dip at Bristol to be $70^{\circ}$ and that at St. Helena to be $30^{\circ}$, calculate from the data of the preceding question the total force of the earth's magnetism at St. Helena, that at Bristol being taken as 0.48 unit. Ans. 0.307.

23. A small magnetic needle was placed magnetically north of the middle point of a strong bar-magnet which lay (magnetically) east and west. When the magnet was 3 feet away from the needle the deflexion of the latter was $2^{\circ}$ : when moved up to a distance of 2 feet the deflexion was $6^{\circ} 30^{\prime}$; and when only 1 foot apart the deflexion was $43^{\circ}$. Deduce the law of the total action of one magnet on another.

24. Describe how the daily irregularities of the earth's magnetism are registered at different stations for comparison.

\section{QUESTIONS ON CHAPTER III}

1. Show that the total of the differences of potential by contact in three simple voltaic cells joined in series is three times as great as the difference of potential in one cell, the materials being the same in each.

2. Classify the different methods of preventing polarization in voltaic cells, and state the advantages and disadvantages of using a strong depolarizer, such as chromic acid.

3. On what does the internal resistance of a battery depend? Is there any way of diminishing it?

4. A current of 10 amperes flows for half an hour, find the total quantity of electricity that passes. Also define the unit by which the quantity is measured. Ans. 18,000 coulombs.

5. State from what source the energy yielded by a voltaic cell is derived.

6. How is local action in a voltaic cell minimised ?

7. Twenty-four similar cells are grouped together in four 
rows of six cells each; compare the electromotive-force and the resistance of the battery thus grouped, with the electromotive-force and the resistance of a single cell.

Ans. The E.M.F. of the battery is six times that of one cell. The total internal resistance is one and a half times that of one cell.

8. Describe a form of cell that could be used as a standard of E.M.F. State the essential qualities of such a cell.

9. A piece of silk-covered copper wire is coiled round the equator of a model terrestrial globe. Apply Ampère's rule to determine in which direction a current must be sent through the coil in order that the model globe may represent the condition of the earth magnetically.

Ans. The current must flow across the Atlantic from Africa to America, and across the Pacific from America toward India; or, in other words, must flow always from east toward west.

10. A current of $\cdot 24$ amperes flows through a circular coil of seventy-two turns, the (average) diameter of the coils being 20 centinietres. What is the strength of the magnetic field which the current produces at the centre of the coil?

Ans. 1·08.

11. Show the direction of the lines of force about a conductor carrying a current (1) when the conductor is straight; (2) when it is bent into the form of a ring ; (3) when it is wound on a cylinder many times round. What do you mean by the direction of the lines of force?

12. Suppose a current passing through the above coil produced a deflexion of $35^{\circ}$ upon a small magnetic needle placed at its centre (the plane of the coils being in the magnetic meridian), at a place where the horizontal component of the earth's magnetic force is ' 23 units. Calculate the strength of the current in amperes. (Art. 213).

Ans. 0.035.

13. The current generated by a dynamo-electric machine was passed throngh a large ring of stout copper wire, at the centre of which hung a small magnetic needle to serve as a tangent galvanometer. When the stean engine drove the armature of the generator at 450 revolutions per minute the deflexion of the needle was $60^{\circ}$. When the speed of the engine was increased so as to produce 900 revolutions per minute the deflexion was $74^{\circ}$. Compare the strength of the currents in the two cases. Ans. The current was twice as great as before, for tan $74^{\circ}$ is almost exactly double of $\tan 60^{\circ}$. 
14. State a general law which will enable you to find the way in which the different parts of a magnetic system tend to move.

15. Deduce the law of the force on a magnetic pole due to a current flowing along a long straight conductor.

16. Describe four ways of controlling the needle of a galvanometer.

17. What is meant by a "null method" of observation?

18. Why is the needle of a tangent galvanometer made very short?

19. You are supplied with an ammeter and a voltmeter for the purpose of ascertaining the current supplied to an electrolytic bath, and the voltage at which it is supplied. Show how you would join them up.

20. The current from two Grove's cells was passed through a sine-galvanometer to measure its strength. When the conducting wires were of stout copper wire the coils had to be turned through $70^{\circ}$ before they stood parallel to the needle. But when long thin wires were used as conductors the coils only required to be turned through $9^{\circ}$. Compare the strength of the current in the first case with that in the second case when flowing through the thin wires which offered considerable resistance. Ans. Currents are as 1 to $\frac{1}{6}$, or as 6 to 1 .

21. A plate of zinc and a plate of copper are respectively united by copper wires to the two screws of a galvanometer. They were then dipped side by side into a glass containing dilute sulphuric acid. The galvanometer needle at first showed a deflexion of $28^{\circ}$, but five minutes later the deflexion had fallen to $11^{\circ}$. How do you account for this falling off ?

22. Classify liquids according to their power of conducting electricity. In which class would melted pewter come?

23. Name the substances produced at the anode and kathode respectively during the electrolysis of the following substances : - Water, dilute sulphuric acid, sulphate of copper (dissolved in water), hydrochloric acid (strong), iodide of potassium (dissolved in water), chloride of tin (fused).

24. A current is sent through three electrolytic cells, the first containing acidulated water, the second sulphate of copper, the third contains a solution of silver in cyanide of potassiun. How much copper will have been deposited in the second cell 
while $2 \cdot 268$ grammes of silver have been deposited in the third cell? And what volume of mixed gases will have been given off at the same time in the first cell?

Ans. 6656 grammes of copper and $351^{\circ} 4$ cubic centimetres of mixed gases.

25. A current passes by platinum electrodes through three cells, the first containing a solution of blue vitriol (cupric sulphate), the second containing a solution of green vitriol (ferrous sulphate), the third containing a solution of ferric chloride. State the amounts of the different substances evolved at each electrode by the passage of 1000 coulombs of electricity.

Ans. First Cell, $\left\{\begin{array}{l}\text { Anode } 0829 \text { gramme of oxygen gas. } \\ \text { Kathode } 3281 \text { gramme of copper. }\end{array}\right.$

Second Cell, $\{$ Anode $\cdot 0829$ gramme of oxygen.

Third Cell, $\left\{\begin{array}{l}\text { Anode } \cdot 3673 \text { gramme of chlorine. } \\ \text { Kathode } \cdot 1935 \text { gramme of iron. }\end{array}\right.$

26. The ends of a coil of fine insulated wire are connected with the terminals of a galvanometer. A steel bar-magnet is pushed slowly into the hollow of the coil and then withdrawn suddenly. What actions will be observed on the needle of the galvanometer?

27. Round the outside of a deep cylindrical jar are coiled two separate pieces of fine silk-covered wire, each consisting of many turns. The ends of one coil are fastened to a battery, those of the other to a sensitive galvanometer. When an iron bar is poked into the jar a momentary current is observed in the galvauometer coils, and when it is drawn out another momentary current, but in an opposite direction, is observed. Explain these observations.

28. A casement window has an iron frame. The aspect is north, the hinges being on the east side. What happens in the frame when the window is opened?

29. Explain the construction of the induction coil. What are the particular uses of the condenser, the automatic break, and the iron wire core?

30. It is desired to measure the strength of the field between the poles of an electromagnet which is excited by a current from a constant source. How could you apply Faraday's discovery of induetion-currents to this purpose?

31. A small battery was joined in circuit with a coil of fine wire and a galvanometer, in which the current was found to 
produce a steady but small deflexion. An unmagnetized iron bar was now plunged into the hollow of the coil and then withdrawn. The galvanometer needle was observed to recede momentarily from its first position, then to return and to swing beyond it with a wider are than before, and finally to settle down to its original deflexion. Explain these actions, and state what was the source of the energy that moved the needle.

32. A tangent galvanometer, whose "constant" in absolute units was 0.08 , was joined in circuit with a battery and an electrolytic cell containing a solution of silver. The current was kept on for one hour; the deflexion observed at the beginuing was $36^{\circ}$, but it fell steadily during the hour to $34^{\circ}$. Supposing the horizontal component of the earth's magnetic force to be $\cdot 23$, calculate the amount of silver deposited in the cell during the hour, the absolute electro-chemical equivalent of silver being 0.01134 .

Ans. 0.526 gramme.

33. A piece of zinc, at the lower end of which a piece of copper wire is fixed, is suspended in a glass jar containing a solution of acetate of lead. After a few hours a deposit of lead in a curious tree-like form ("Arbor Saturni") grows downwards from the eopper wire. Explain this.

34. Explain the conditions under which electricity excites muscular contraction. How can the converse phenomenon of currents of electricity produced by muscular contraction be shown?

35. A certain piece of apparatus has two terminals on each side. To these a pair of wires, $A$ and $B$, are attached at one side, and another pair at $\mathrm{C}$ and $\mathrm{D}$. Examination with a voltmeter shows that the potential of $A$ is higher than that of $B$, and that of $\mathrm{C}$ higher than that of $\mathrm{D}$. Yet examination with an ampere-meter shows that a current is flowing from $\mathrm{B}$ to $\mathrm{A}$ through the apparatus, and another current from $\mathrm{C}$ to D through the other part of the apparatus. By which circuit is the energy coming in, and by which is it going ont?

36. Show that if $\mathrm{N}$ magnetic lines are withdrawn from a circuit of resistance $R$, the quantity of electricity thereby transferred around the circuit (i.e. the time integral of the induced current) will be $Q=N / R$. (See Art. 225.)

37. The strength of the field between the poles of a large electromagnet was deternined by the following means:-A small circular coil, consisting of 40 turns of fine insulated wire, mounted on a handle, was comnected to the terminals of a long-coil galvanometer having a heavy needle. On inverting 
this coil suddenly, at a place where the total intensity of the earth's magnetic force was 48 unit, a deflexion of $6^{\circ}$ was shown as the first swing of the galvanometer needle. The sensitiveness of the galvanometer was then reduced to $\frac{1}{100}$ by means of a shunt. The little coil was introduced between the poles of the electromagnet and suddenly inverted, when the first swing of the galvanometer needle reached $40^{\circ}$. What was the strength of the field between the poles?

Ans. $315 \cdot 7$ units.

\section{QUESTIONS ON CHAPTER IV}

1. Define the unit of electricity as derived in absolute terms from the fundamental units of length, mass, and time.

2. At what distance must a small sphere charged with 28 units of electricity be placed from a second sphere charged with 56 units in order to repel the latter with a force of 32 dynes?

Ans. 7 centimetres.

3. Suppose the distance from the earth to the moon to be (in round numbers) $383 \times 10^{8}$ centimetres; and that the radius of the earth is $63 \times 10^{7}$ centimetres, and that of the moon $15 \times 10^{7}$ centimetres; and that both moon and earth are charged until the surface density on each of them is of the average value of 10 units per square centimetre. Calculate the electrostatic repulsion between the moon and the earth.

4. A small sphere is electrified with 24 units of + electricity. Calculate the force with which it repels a unit of + electricity at distances of $1,2,3,4,5,6,8$, and 10 centimetres respectively. Then plot out the "curve of force" to seale; measuring the respective distances along a line from left to right as so many centimetres from a fixed point as origin; then setting out as vertical ordinates the amounts you have calculated for the corresponding forces; lastly, connecting by a curved line the system of points thus found.

5. Define electrostatic (or electric) "potential" ; and calculate (by the rule given in italics in Art. 263) the potential at a point $\mathrm{A}$, which is at one corner of a square of 8 centimetres' side, when at the other three corners $\mathrm{B}, \mathrm{C}, \mathrm{D}$, taken in order, charges of $+i 6,+34$, and +24 units are respectively placed. Ans. 8 (very nearly).

6. A small sphere is electrified with 24 units of + electricity. Calculate the potential due to this charge at points $1,2,3,4,5,6,8$, and 10 centinetres' distance respectively. 
Then plot out the "curve of potential" to scale, as described in Question 4.

7. A small sphere charged with 100 units of electricity is dipped into a bath of oil having a dielectric capacity 2, find the force it would exert on a unit charge 5 centimetres away.

Ans. 2 dynes.

8. Distinguish between the surface density at a point and the potential at that point due to neighbouring charges.

9. What are equipotential surfaces? Why is the surface of an insulated conductor an equipotential surface? Is it always so?

10. Show that the capacity of an isolated sphere in air of radius $\nu$ has a capacity equal to $\nu$ units. What is the electrostatic unit of capacity?

11. Why is the potential of the earth due to charges that we produce practically equal to zero?

12. A sphere whose radius is 14 centimetres is charged until the surface density has a value of 10 . What quantity of electricity is required for this ? Ans. 24,640 units (nearly).

13. In the above question what will be the potential at the surface of the sphere? (See Art. 269.) Ans. 1760 (very nearly).

14. In the case of question 12 , what will be the electric force at a point outside the sphere and indefinitely near to its surface? (Art. 276.) Ans. $125 \cdot 7$ (very nearly).

15. Suppose a sphere whose radius is 10 centimetres to be charged with 6284 units of electricity, and that it is then caused to share its charge with a non-electrified sphere whose radius is 15 centimetres, what will the respective charges and surface-densities on the two spheres be when separated?

Ans. Small sphere, $q=2513 \cdot 6, \rho=2$ : Large sphere, $q=3770 \cdot 4, g=1.33$.

16. A charge of +8 units is collected at a point 20 centimetres distant from the centre of a metallic sphere whose radius is 10 centimetres. It induces a negative electrification at the nearest side of the sphere. Find a point inside the sphere such that if 4 negative units were placed there they would exercise a potential on all external points exactly equal to that of the actual negative electrification. (See Art. 275.)

Ans. The point must be on the line between the outside positive charge and the centre of the sphere and at 5 centims. from the surface. 
17. Two large parallel metal plates are charged both positively but unequally, the density at the surface of $A$ being +6 , that at the surface of $B$ being +3 . They are placed 2 centimetres apart. Find the foree with which a +unit of electricity is urged from A towards B. Find also the work done by a + unit of electricity in passing from $\mathrm{A}$ to $\mathrm{B}$.

Ans. Electric force from A towards $\mathrm{B}=18.85$ dynes; work done by unit in passing from $\mathrm{A}$ to $\mathrm{B}=37 \cdot 5$ ergs.

18. What is meant by the dimensions of a physical quantity? Deduce from the Law of Inverse Squares the dimensions of electricity; and show by this means that electricity is not a quantity of the same physical dimensions as either matter, energy, or force.

19. Explain the construction and principles ef action of the quadrant electrometer. How could this instrument be made self-recording?

20. Describe the construction of an electrostatic voltmeter, and state some of the advantages that this instrument possesses.

21. One of the two coatings of a condenser is put to earth, to the other coating a charge of 5400 units is imparted. It is found that the difference of potential thereby produced between the eoatings is 15 (eleetrostatic) units. What was the capacity of the condenser?

Ans. 360 .

22. What is the meaning of specific inductive capacity? Why does hot glass appear to have a higher specific inductive capacity than cold glass?

23. Describe a method of mapping out the lines of force in an electrostatic field.

24. Two condensers of eapacity 4 and 6 respectively are placed in parallel; and in series with them is placed another condenser having a capacity of 5 mierofarads. Find the capacity of the whole combination. Ans. $3 \cdot \dot{3}$.

25. Compare the phenomenon of the residual eharge in a Leyden jar with the phenomenon of polarisation in an electrolytic cell.

26. A condenser was made of two flat square metal plates, the side of each of them being 35 centimetres. A sheet of indiarubber 4 centim. thiok was placed between them as a 
dielectric. The specific inductive capacity of indiarubber being taken as $2 \cdot 25$, calculate the capacity of the condenser. Ans. 548.8 electrostatic units.

27. Calculate (in electrostatic units) the capacity of a mile of telegraph cable, the core being a copper wire of 18 centim. diameter, surrounded by a sheathing of guttapercha $\cdot 91$ centim. thick. $[k$ for guttapercha $=2 \cdot 46 ;$ one mile $=160,933$ centims.]

Ans. 82,164 units.

28. A Leyden jar is made to share its charge with two other jars, each of which is equal to it in capacity. Compare the energy of the charge in one jar with the energy of the original charge. Ans. One-ninth as great.

29. A series of Leyden jars of equal capacity are charged " in cascade." Compare the total energy of the charge of the individual jars thus charged, with that of a single jar charged from the same source.

30. Classify the various modes of discharge, and state the conditions under which they occur.

31. Suppose a condenser, whose capacity is 10,000 charged to potential 14, to be partially discharged so that the potential fell to 5. Calculate the amount of heat produced by the discharge, on the supposition that all the energy of the spark is converted into heat. Ans. ${ }^{\circ} 020357$ of a unit of heat.

32. How do changes of pressure affect the passage of electric sparks through air?

33. Describe some of the properties of matter in its ultragaseous or radiant state.

34. Why are telegraphic signals through a submerged cable retarded in transmission, and how can this retardation be obviated?

35. How is the difference of potential betwcen the earth and the air above it measured? and what light do such measurements throw on the periodic variations in the electrical state of the atmosphere?

36. What explanation can be given of the phenomenon of a thunderstorm?

37. What are the essential features which a lightning-conductor must possess before it can be pronouneed satisfactory? And what are the reasons for insisting on these points?

38. How can the duration of an electric spark be 'measured? 


\section{QUESTIONS ON CHAPTER V}

1. Define magnetic potential, and find the (magnetic) potential due to a bar-magnet 10 centimetres long, and of strength 80 , at a point lying in a line with the magnet poles and 6 centimetres distant from its $\mathrm{N}$-seeking end. Ans. $8 \cdot \dot{3}$.

2. A N-seeking pole and a S-seeking pole, whose strengths are respectively +120 and -60 , are in a plane at a distance of 6 centinietres apart. Find the point between them where the potential is $=0$; and through this point draw the curve of zero potential in the plane.

3. Define "intensity of the magnetic field." A magnet whose strength is 270 is placed in a uniform magnetic tield whose intensity is $\mathbf{1 6 6}$. What are the forees which act upon its poles? Ans. +45 dynes and -45 dynes.

4. Define "intensity of magnetization." A rectangular bar-magnet, whose length was 9 centimetres, was magnetized until the strength of its poles was 164 . It was 2 centimetres broad and 5 centimetre thick. Supposing it to be uniformly magnetized throughout its length, what is the intensity of the magnetization?

Ans. 164.

5. A certain electric motor has 100 conductors on its armature, each earrying 10 amperes. The number of lines of foree passing through the armature is 500,000. Find the work (in ergs) done in one revolution of the armature.

As eaeh conductor cuts the lines twice in one revolution the answer will be 100,000,000 ergs.

6. Find the torque (see Art. 136) on the armature described in the last question. Note that with the above data the torque is independent of the radius of the armature, for the force on each conductor is proportional to the strength of the field, and this is inversely proportional to the radius if $\mathbf{N}$ remains the same. Ans. $\frac{100,000,000}{2 \pi}$ dyne-centimetres.

7. A current whose strength in "absolute" electromagnetic units was equal to 0.05 traversed a wire ring of 2 centimetres' radius. What was the strength of field at the centre of the ring? What was the potential at a point $\mathrm{P}$ opposite the middle of the ring and 4 centimetres distant from the circum. ference of the ring. Ans. $f=\cdot 1571 ; \mathrm{V}= \pm 0.0421$. 
8. (a) A spiral of wire of 1000 turns carries a current of 1 ampere. Find the total magnetomotive force which it exerts. Ans. 1257.

(b) If the spiral were 1 metre in length and 1 centimetre in diameter, find the force on a unit pole placed (1) in its centre ; (2) at its end. Ans. 12.57 dynes and 6.28 dynes.

9. What limits are there to the power of an electromagnet?

10. What is the advantage in using an iron core in an electro-magnet?

11. A rod of soft iron, $0.32 \mathrm{~cm}$. in diameter and 1 metre long, is uniformly overwound from end to end with an insulated copper wire making 637 turns in one layer. Find (using Bidwell's data in Art. 365) what strength of poles this rod will acquire when a current of 5 amperes is sent through the coil. Ans. $98 \cdot 9$ units.

12. Enunciate Maxwell's rule concerning magnetic shells, and from it deduce the laws of parallel and oblique currents discovered by Ampère.

13. A circular copper dish is joined to the zinc pole of a small battery. Acidulated water is then poured into the dish, and a wire from the carbon pole of the battery dips into the liquid at the middle. A few scraps of cork are thrown in to render any movement of the liquid visible. What will occur when the $\mathrm{N}$-seeking pole of a strong bar-magnet is held above the dish?

14. Roget hung up a spiral of copper wire so that the lower end just dipped into a cup of mercury. When a strong current was sent through the spiral it started a continuous dance, the lower end producing bright sparks as it dipped in and out of the mercury. Explain this experiment.

15. It is believed, though it has not yet been proved, that ozone is more strongly magnetic than oxygen. How could this be put to proof?

16. What is meant by the permeability of a substance? State some substances in which it is constant, and some in which it varies. iron.

17. Describe a method of measuring the permeability of

18. A ring of iron is wound with two coils. One coil is connected to a ballistic galvanometer, and on connecting the 
other to a battery a throw of the needle of 160 scale divisions is observed. The current is then broken and there is a throw of 40 divisions in the opposite direction. Why are the two throws not equal? What change has taken place in the iron? How would you bring it back to its original condition?

19. Sketch a closed hysteresis curve for hard steel, for which, when $\mathrm{H}$ is raised to $100, \mathrm{~B}=12,800$, and for which the remanence is 9500 and the coercive force 40.

20. An iron bar 30 centimetres long and 10 square centimetres in sectional area is bent into the shape of a horse-shoe for the purpose of making an electromagnet which shall have a pull of 66 kilograms upon its armature (a bar 12 centimetres long and 10 square centimetres in section) when it is $\frac{1}{2}$ inch away from its poles. Find the number of ampere turns required, assuming a leakage of one-third of the lines of force.

Taking the formula:-

$$
\frac{\mathrm{B}^{2}}{8 \pi} \times 20 \text { sq. cms. of pole face }=66,000 \times 981 \text { dynes, }
$$

we get $B=9000$. From the table, Art $364, \mu_{1}$ for the armature $=2250, B$ for the horse - shoe $=1.5 \times 9000=13,500$, so that $\mu_{2}=900$, then ampere-turns $=$

$90,000\left\{\frac{12}{10 \times 2250}+\frac{1.5 \times 30}{10 \times 900}+\frac{2 \times 0 \cdot 5 \times 2 \cdot 54}{10}\right\} \div 1 \cdot 257=18,930$.

21. What thickness of copper wire must be used to wind the above magnet in order to obtain 18,930 ampere-turns, the winding on each cylindrical bobbin having a mean diameter of 7 centimetres, if the pressure at the terminals of the magnet is intended to be 100 volts.

If $r$ is the resistance of one turn, and $s$ the number of turns, $r=\frac{\mathrm{E}}{c s}=\frac{100}{18,930}$; but we know that $r=\frac{7 \times \pi}{d^{2} \times \frac{1}{4} \pi} \times 1 \cdot 6 \times 10^{-6}$.

Hence diameter of wire, $d=\sqrt{\frac{18,930 \times 7 \times 4 \times 1.6}{10^{6} \times 100}}=0.092$ ems.

N.B.-The thickness of wire is independent of the number of turns (except in so far as this affects the mean diameter of the bobbin), but the greater the number of turns the less will be the number of watts expended.

22. What is the object of "polarizing" the armature of a magnet in a piece of mechanism, such as a relay?

23. Describe the construction of a current-balance, and the mode of using it. 


\section{QUESTIONS ON CHAPTER VI}

1. The resistance of telegraph wire being taken as 13 ohms per mile, and the E.M.F. of a Leclanché cell as 1.4 volt, calculate how many cells are needed to send a current of 12 milli-amperes through a line 120 miles long; assuming that the instruments in circuit offer as much resistance as 20 miles of wire would do, and that the return current through earth meets with no appreciable resistance. Ans. 16 cells.

2. 50 Grove's cells (E.M.F. of a Grove $=1.8$ volt) are united in series, and the circuit is completed by a wire whose resistance is $15 \mathrm{ohms}$. Supposing the internal resistance of each cell to be $0.3 \mathrm{ohm}$, calculate the strength of the current.

Ans. 3 amperes.

3. The current running through an ineandescent filament of earbon in a lamp was found to be exactly 1 ampere. The difference of potential between the two terminals of the lamp while the current was flowing was found to be 30 volts. What was the resistance of the filament?

4. Define specific resistance. Taking a specific resistance of copper as 1642, calculate the resistance of a kilometre of copper wire whose diameter is 1 millimetre. Ans. $20^{\circ} 9 \mathrm{ohms}$.

5. On measuring the resistance of a piece of No. 30 B.W.G. (eovered) copper wire, 18.12 yards long, I found it to have a resistance of 3.02 ohms. Another coil of the same wire had a resistance of $22.65 \mathrm{ohms}$; what length of wire was there in the coil ? Ans. $135 \cdot 9$ yards.

6. Calculate the resistance of a copper conductor one square eentimetre in area of cross-section, and long enough to reach from Niagara to New York, reckoning this distance as 480 kilometres.

Ans. $78 \cdot 8 \mathrm{ohms}$.

7. Find the drop in volts if 400 amperes is passed through this conductor. What would be the waste of power (in watts)? Ans. 31,520 volts, 12,608,000 watts.

8. The resistance from plate to plate in a certain electrolytic bath is 0.9 of an olm. You wish to pass through it the strongest current you can get from 20 Daniell's cells, each with a resistance of one ohm. How would you group the cells? Ans. 4 in series, 5 rows in parallel. 
9. The specific resistance of guttapercha being $3.5 \times 10^{23}$, calculate the number of coulombs of electricity that would leak in one century through a sheet of guttapercha one centimetre thick and one metre square, whose faces were covered with tinfoil and joined respectively to the poles of a battery of 100 Daniell's cells.

Ans. $9 \cdot 7$ coulomb.

10. Six Daniell's cells, for each of which $\mathrm{E}=1 \cdot 05$ volts, $r=$ $0.5 \mathrm{ohm}$, are joined in series. Three wires, $\mathrm{X}, \mathrm{Y}$, and $\mathrm{Z}$, whose resistances are severally 3,30 , and $300 \mathrm{ohms}$, can be inserted between the poles of the battery. Determine the current which flows when each wire is inserted separately; also determine that which flows when they are all inserted at once in parallel. Ans. Through X 1.05 amperes. $\begin{array}{lll}\text { Through Y } & 0 \cdot 1909 & \text {, } \\ \text { Through Y } & 0 \cdot 0207 & , \\ \text { Through all three } 1 \cdot 105 & ,\end{array}$

11. Calculate the number of cells required to produce a current of 50 milli-amperes, through a line 114 miles long, whose resistance is $12 \frac{1}{2} \mathrm{ohms}$ per mile, the available cells of the battery having each an internal resistance of $1.5 \mathrm{ohm}$, and an E.M.F. of 1.5 volt. Ans. 50 cells.

12. You have 20 large Leclanché cells (E.M.F. $=1.5$ volt, $r=0.5 \mathrm{ohm}$ each) in a circuit in which the external resistance is 10 ohms. Find the strength of current which flows $(a)$ when the cells are joined in simple series; $(b)$ all the zincs are united, and all the carbons united, in parallel arc ; $(c)$ when the cells are arranged two abreast (i.e. in two files of ten cells each); $(d)$ when the cells are arranged four abreast. Ans. (a) $1.5 ;(b) 0.1496 ;(c) 1 \cdot 2 ;(d) 0.702$ ampere.

13. With the same battery how wonld you arrange the cells in order to telegraph through a line 100 miles long, reckoning the line resistance as $12 \frac{1}{2} \mathrm{ohms}$ per mile ?

14. Show that, if we have a battery of $n$ given cells each of resistance $r$ in a circuit where the external resistance is $R$, the strength of the current will be a maximum when the cells are coupled up in a certain number of rows equal numerically to $\sqrt{n r \div \mathrm{R}}$.

15. Two wires, whose separate resistances are 28 and 24 , are placed in parallel in a circuit so that the current divides, part pessing through one, part through the other. What resistance do they offer thus to the current?

Ans. 12.92 ohms. 
16. Using a large bichromate cell of practically no internal resistance, a deflexion of $9^{\circ}$ was obtained upon a tangent galvanometer (also of small resistance) through a wire whose resistance was known to be 435 ohms. The same cell gave a deflexion of $5^{\circ}$ upon the same galvanometer when a wire of unknown resistance was substituted in the circuit. What was the unknown resistance? Ans. 790 ohms.

17. In a Wheatstone's bridge in which resistances of 10 and 100 ohms respectively were used as the fixed resistances, a wire whose resistance was to be determined was placed : its resistance was balanced when the adjustable coils were arranged to throw $281 \mathrm{ohms}$ into circuit. What was its resistance?

Ans. $28 \cdot 1$ ohms.

18. Describe the method of using a metre bridge to measure resistances.

19. Give the proof of Foster's method of measuring small differences of resistance from the consideration of Ohm's law.

20. To find the voltage of a dynamo you connect to its brushes the ends of a German-silver wire 120 feet long, wound on an insulating cylinder, and find that when one terminal of a Daniell's cell (1.05 volt) is joined to a point on the wire, and the other terminal in series with a galvanometer is connected to another point $1 \mathrm{ft}$. from the first, no deflexion is observed. What is the voltage of the dynamo?

Ans. 126 volts.

21. A battery of 5 Leclanché cells was connected in simple circuit with a galvanometer and a box of resistance coils. A deflexion of $39^{\circ}$ having been obtained by adjustment of the resistances, it was found that the introduction of 150 additional ohms of resistance bronght down the deflexion to $22^{\circ}$. Assuming the galvanometer to have $140 \mathrm{ohms}$ ' resistance, find the internal resistance of the battery.

Ans. 10 ohms.

22. How are standard resistance coils wound, and why? What materials are they made of, and why?

23. Three very small Daniell's cells gave, with a sine galvanometer (itself of no appreciable resistance), a reading of $57^{\circ}$. On throwing $20 \mathrm{ohms}$ into the circuit the galvanometer reading fell to $25^{\circ}$. Calculate the internal resistance of the cells. Ans. $6 \cdot 6 \mathrm{ohms}$ each.

24. A length of telegraph cable was plunged in a tub of water and then charged for a minute from a battery of 120 
Daniell's cells. The cable was then discharged through a long-coil galvanometer "with a needle of slow swing. The first swing was $40^{\circ}$. A condenser whose capacity was $\frac{1}{3}$ microfarad was then similarly charged and discharged; but this time the first swing of the needle was only $14^{\circ}$. What was the capacity of the piece of cable? Ans. 0.934 microfarad.

25. Using an absolute electrometer, Lord Kelvin found the difference of potential between the poles of a Daniell's cell to be 0.00374 electrostatic units (C.G.S. system). The ratio of the electrostatic to the electromagnetic unit of potential is given in Art. 359, being $=1 / v$. The volt is defined as $10^{8}$ electromagnetic units. From these data calculate the E.M.F. of a Daniell's cell in volts. Ans. 1.115 volt.

26. The radius of the earth is approximately $63 \times 10^{7}$ centimetres. The ratio of the electrostatic to the electromagnetic unit of capacity is given in Art. 359. The definition of the farad is given in Art. 354. Calculate the capacity of the earth (regarded as a sphere) in microfarads.

Ans. 700 microfarads (nearly).

27. The electromotive-force of a Daniell's cell was determined by the following process :-Five newly-prepared cells were set up in series with a tangent galvanometer, whose constants were found by measurement. The resistances of the circuit were also measured, and found to be in total $16.9 \mathrm{ohms}$. Knowing the resistance and the absolute strength of current, the E.M.F. could be calculated. The deflexion obtained was $45^{\circ}$, the number of turns of wire in the coil 10, the average radius of the coils 11 centimetres, and the value of the horizontal component of the earth's magnetism at the place was $0 \cdot 18$ G.C.S. units. Deduce the E.M.F. of a Daniell's cell. Ans. $1.0647 \times 10^{8}$ G.C.S. units, or 1.0647 volt.

28. Apply the formula of the ballistic galvanometer (Art. $418, b)$ to determine the number of magnetic lines cut by an exploring coil (Art. 366, b) when the magnetism in the core on which it is wound is suddenly reversed. If $R$ is the resistance of the circuit, $Q=2 \mathrm{~N} / \mathrm{R}$. Hence the answer is $\mathbf{N}=$ RT $\sin \frac{1}{2} \alpha / 2 \pi \mathrm{S}$, where $\mathrm{S}$ is the number of turns in the exploring coil.

29. Suppose a copper disk to revolve in a field produced by a fixed coil closely surrounding its circumference. In circuit with the coil is a small battery and a resistance wire. In the wire are found two points such that the fall of potential between them is equal to the volts generated between the 
centre and circumference of the revolving disk. By balancing these with a galvanometer Lorenz was able to calculate in absolute measure the resistance of the wire. If $M$ be the coefficient of mutual induction between the circumference of the disk and the surrounding coil, and $\mathrm{T}$ the period of revolution of the disk, show that $\mathrm{R}$ the resistance between the points $=\mathrm{M} \div \mathrm{T}$.

Ans. Since $\mathrm{N}$ the magnetic flux through the disk $=\mathrm{MC}$, and $\mathrm{E}=\mathrm{N} / \mathrm{T}$, and $\mathrm{C}=\mathrm{E} / \mathrm{R}$, it follows that $\mathrm{CR}=$ $\mathrm{MC} / \mathrm{T}$, whence $\mathrm{R}=\mathrm{M} / \mathrm{T}$.

Q.E.D.

\section{QUESTIONS ON CHAPTER VII}

1. A strong battery-current is sent, for a few moments, through a bar made of a piece of antimony soldered to a piece of bismuth. The battery is then disconnected from the wires and they are joined to a galvanometer which shows a deflexion. Explain this phenomenon.

2. A long strip of zinc is connected to a galvanometer by iron wires. One junction is kept in ice, the other is plunged into water of a temperature of $50^{\circ} \mathrm{C}$. ' Calculate, from the table given in Art. 422, the electromotive-force which is producing the current. Ans. 690 microvolts.

3. When heat is evolved at a junction of two metals by the passage of a current, how would you distinguish between the heat due to resistance and the heat due to the Peltier effect.

4. Lord Kelvin discovered that when a current flows through iron it absorbs heat when it flows from a hot point to a cold point; but that when a current is flowing through copper it absorbs heat when it flows from a cold point to a hot point. From these two facts, and from the general law that energy tends to run down to a minimim, deduce which way a current will flow round a circuit made of two half-rings of iron and copper, one junction of which is heated in hot water and the other cooled in ice.

5. Give a curve showing the increase and decrease of the thermo-electromative-force as a junction of iron and copper is raised from $0^{\circ} \mathrm{C}$. to $400^{\circ} \mathrm{C}$., and explain it by means of the thermoelectric diagram of Professor Tait. 


\section{QUESTIONS ON CHAPTER VIII}

1. Calculate by Joule's law the number of calories developed in a wire whose resistance is $4 \mathrm{ohms}$ when a steady current of 0.14 ampere is passed through it for ten minutes.

Ans. 11.2 calories.

2. Why does the platinum wire in a Cardew voltmeter, when a steady voltage is applied to it, rise to a certain temperature and then remain at that temperature without alteration?

3. Show from the definitions of the horse-power and of the watt, and from the relations between the pound and the gramme, the foot and the centimetre, that there are 746 watts in one horse-power.

4. Explain why you would expect the heat produced in a conductor to be proportional to the square of the eurrent.

5. Describe the construction of a watt meter and explain how you would connect it up to measure the power supplied to an electric motor.

6. Explain why it is advantageous to distribute electric energy at a high voltage. There is already laid a copper main having a resistance of 0.5 of an ohm along which it is desired to transmit 4 kilowatts, and to deliver it at the far end at a pressure of 100 volts. Which would be the more efficient method of the two following, to send $40 \mathrm{amps}$. at an initial pressure of 120 volts, or to send a current at a pressure of 2400 volts, using a transformer with an efficiency of 85 per cent ?

Ans. The latter method would have an efficiency of $84 \cdot 9$ per cent, the former of $83 \cdot \dot{3}$ per cent.

7. Mention some of the principles upon which supply meters have been designed.

8. An electric motor is supplied at a pressure of 100 rolts : the armature resistance is $0.01 \mathrm{ohm}$. When it is supplying 20 horse-power, what is its electrical efficiency?

$$
\text { Ans. } 98.5 \text { per cent. }
$$

9. Show under what cireumstances an electric motor is most efficient.

10. Enumerate the principal parts of an are lamp. 
11. Why in a continuous-current arc lamp is the current usually sent downwards rather than upwards?

12. Why does the filament of an incandescent lamp get hotter than the platinum leading-in wires?

13. Explain by a diagram the system of three-wire distribution; and point out its advantage over a two-wire distribution.

14. A current of 9 amperes worked an electric arc light, and on measuring the difference of potential between the two carbons by an electrometer it was found to be 50 volts. What was the amount of horse-power absorbed in this lamp?

Ans. 0.603 H.P.

\section{QUESTIONS ON CHAPTER IX}

1. The reluctance of the core of a certain transformer is 0.002 . Find the coefficient of mutual induction between the primary and secondary coils which have 1000 and 50 turns respectively, assuming no magnetic leakage.

Ans. 0.314 henry.

2. A battery current is sent through the primary of this transformer. State from Lenz's law the direction (relatively to this current) of the E.MI.F.'s induced in both the primary and secondary, $(a)$ when the current is starting, $(b)$ when it is ceasing.

3. Foucault set the heavy bronze wheel of his gyroscope spinning between the poles of a powerful electromagnet, and found that the wheel grew hot. What was the cause of this? Where did the heat come from?

4. You try to turn a copper disk between the poles of a magnet. If you move it slowly it goes quite easily, if you try to move it quickly it resists. Why is this? What is the force required to turn it proportional to ?

5 . The shunt coil of a certain dynamo has a resistance of $40 \mathrm{ohms}$. It is switched on to a battery of accumulators yielding 100 volts, and one second afterwards the current has risen to 0.9825 of an ampere. Find the coefficient of self-induction of the shunt coil. Assume $\log 0 \cdot 607=\overline{1} \cdot 783$ and $\log \epsilon=0 \cdot 434$. Ans. 80 henries.

6. If a battery of 10 cells each of 1.4 volt and 2 ohms' 
resistance be applied to a circuit which has a resistance of 5 ohms and inductance 0.1 henry, find what modes of grouping the cells are best, $(a)$ to give the largest steady current, $(b)$ to give the largest current at the end of $\frac{1}{1000}$ second, $(c)$ to give the largest amount of external work relatively to the weight of zinc consumed.

Ans. (a) 5 in series, 2 rows in parallel. (b) All in series. (c) All in parallel.

\section{QUESTIONS ON CHAPTER X}

1. What devices are employed in continuous current dy. namos to obtain $(a)$ a current continuously in one direction, (b) a current of uniform strength ?

2. Apply Fleming's Rule (Art. 226) to determine which way the electromotive-forces will operate in a ring armature (gramme) wound right-handedly over the core revolving righthandedly in a horizontal magnetic field having the $\mathrm{N}$-pole on the right hand.

Ans. The induced E.M.F.'s tend to make the currents climb, in both the ascending and descending halves, toward the highest point of the ring.

3. A dynamo's field magnet gives a flux of 9,000,000 lines. How many conductors must there be on the armature in order that the dynamo may generate 108 volts when driven at a speed of 600 revolutions per minute?

Ans. 120.

4. You have an engine which will drive a dynamo at a fairly constant speed at all loads. How would you excite the dynamo if it were intended for lighting by incandescent lamps ? Nake a diagrammatic sketch of all necessary connections, including the lamp circuit.

5. Take the equation $\mathrm{E}=a \sin (2 \pi n t)$. Let $a=140$ and $n=100$. Now take different values for $t$, beginning $t=0005$ of a second, then $t=001$, taking 20 different values until $t=\cdot 01$. Fill in the values in the above equation and find the corresponding 20 values of $\mathrm{E}$. Then plot on squared paper taking ${ }^{\circ} \mathrm{E}$ as ordinate and $t$ as abscisse. The result will be a curve like that shown in Fig. 251.

6. Repeat the process of the last question, taking the equation $\mathrm{C}=b \sin (2 \pi n t-\phi)$, where $b=20, n=100$, and $\phi=0.5$ 
radian. Plot the results upon the same paper as the curve in the last equation was plotted. One curve represents the E.M.F. at each instant, the other the lagging current.

7. An alternating pressure of 100 (virtual) volts following a sine law with a frequency of 100 per second is applied to the ends of a coil having a resistance of $8 \mathrm{ohms}$ and a coefficient of self-induction of 0.005 henry, find the current that will flow and the angle of lag.

Ans. Current $=11 \cdot 6$ amperes; lag $=22$ degrees.

8. An alternate-current magnet with properly-laminated core has a coil of 160 turns, and a coefficient of self-induction of 0.005 of a henry. What alternating voltage of frequency 100 per second must be applied to it in order to obtain 4800 ampere-turns, assuming the resistance to be negligible?

Ans. $47 \cdot 1$.

9. How much resistance must be put in eircuit with the coils of this magnet in order that the angle of lag may be $45^{\circ}$ ? Ans. 3·14.

10. An alternate-current transformer is designed to give out 40 amperes at a pressure of 50 volts at its secondary terminals. No. of windings 300 primary ; 12 secondary. Resistances 12 ohms, primary; 0.014 ohm, secondary. Find the coefficient of transformation, and the volts that must be applied at the prinary terminals.

Ans. Coefficient of transformation is 25 ; volts at primary terminals 1283 .

11. State the principles upon which continuons-current transformers are made. Why is it necessary to have a moving part in continuous-current transformers and not in alternatecurrent transformers?

12. Enumerate three distinct kinds of alternate-current motors, and state which kind is synchronous and which not.

13. An alternate-current synchronous motor is supplied from the street mains. It is found that when fully loaded it takes more current than when lightly loaded, though it always goes at the same speed and the volts remain constant. Explain how this comes about.

14. How can you produce a rotatory magnetic field? Describe some of its properties. 


\section{QUESTIONS ON CHAPTER XI}

1. It is found that a single Daniell's cell will not electrolyze acidulated water, however big it may be made. It is found, on the other hand, that two Daniell's cells, however small, will suffice to produce continuous electrolysis of acidulated water. How do you account for this?

2. From the table of electro-chemical equivalents (Art. 240) calculate how many coulombs it will take to deposit one grain of the following metals:-Copper (from sulphate), silver, nickel, gold. Ans. Cu 3058, Ag 891, Ni 3286, Au 1473.

3. A battery of 2 Grove's cells in series yields a current of 5 amperes for 2 hours, how much zine will be consumed, assuming no waste?

Ans. $24 \cdot 26$.

4. Calculate the E.M.F. of a Daniell's cell from considerations of the heat value of the combinations which take place and the quantity of the elements consumed, taking the heat value for zinc in sulphuric acid as 1670 and that for copper as $909 \cdot 5$. Ans. $1 \cdot 11$ volts.

5. Describe the construction and working of a modern secondary battery.

6. Most liquids which conduct electricity are decomposed (except the melted metals) in the act of conducting. How do you account for the fact observed by Faraday that the amount of matter transferred through the liquid and deposited on the electrodes is proportional to the amount of electricity transferred through the liquid?

7. Describe the process for multiplying by electricity copies of engravings on wood-blocks.

8. How would you make arrangements for silvering spoons of nickel-bronze by electro-deposition?

\section{QUESTIONS ON CHAPTER XII}

1. Sketch an arrangement by which a single line of wire can be used by an operator at either end to signal to the other; the condition of working being that whenever you are 
not sending a message yourself your instrument shall be in circuit with the line wire, and out of circuit with the battery at your own end.

2. What advantages has the Morse instrument over the needle instruments introduced into telegraphy by Cooke and Wheatstone?

3. Explain the use and construction of a relay.

4. Show, from the law of traction (Art. 384), that the change of attracting force resulting from a change in the number of magnetic lines that enter an armature will be greater if the system is polarized (i.e. magnetized to begin with) than if it is non-polarized.

Ans. Since $f \propto \mathrm{N}^{2}$, it follows that $f+d f$ will be proportional to $(\mathrm{N}+d \mathrm{~N})^{2}$. Expanding, and subtracting the former, and neglecting the small term $(d N)^{2}$, we find $d f \propto 2 \mathrm{~N} . d \mathrm{~N}$; which shows that, for a given $d \mathrm{~N}$, $d f \propto \dot{N}$.

5. It is desirable in certain cases (duplex and quadruplex signalling) to arrange telegraphic instruments so that they will respond only to currents which come in one direction through the line. How can this be done?

6. It is wished to make a sort of duplex telegraph by using one set of instruments that work with continuous currents, the other set with rapidly-alternating currents, at the same time on the same line. To carry out this idea there must be found $(a)$ an apparatus which will let continuous currents flow through it, but will choke off alternate currents; $(b)$ an apparatus which will transmit alternate currents, but cut off continuous currents. What apparatus will do these things ?

7. A battery is set up at one station. A galvanometer needle at a station eighty miles away is deflected through a certain number of degrees when the wire of its coil makes twelve turns round the needle ; wire of the same quality being used for both line and galvanometer. At 200 miles the same deflexion is obtained when twenty-four turns are used in the galvanometer-coil. Show by calculation $(a)$ that the internal resistance of the battery is equal to that of 40 miles of the line-wire; $(b)$ that to produce an equal deflexion at a station 360 miles distant the number of turns of wire in the galvanometer-coil must be 40 .

8. Suppose an Atlantic cable to snap off short during the process of laying. How can the distance of the broken end from the shore end be ascertained? 
9. Suppose the copper core of a submarine cable to part at some point in the middle without any damage being done to the outer sheath of guttapercha. How could the position of the fault be ascertained by tests made at the shore end ?

10. Explain the construction and action of an electric bell.

11. Describe and explain how electric currents are applied in the instruments by which very short intervals of time are measured.

\section{QUESTIONS ON CHAPTER XIII}

1. Explain the use of Graham Bell's telephone (1) to transmit vibrations ; (2) to reproduce vibrations.

2. Describe a form of telephone in which the vibrations of sound are transmitted by means of the changes they produce in the resistance of a circuit in which there is a constant electromotive-force.

3. Two coils, A and B, of fine insulated wire, made exactly alike, and of the same number of windings in each, are placed upon a common axis, but at a distance of 10 inches apart. They are placed in circuit with one another and with the secondary wire of a small induction-coil of Ruhmkorff's pattern, the connections being so arranged that the currents run round the two coils in opposite directions. A third coil of fine wire, C, has its two ends connected with a Bell's telephone, to which the experimenter listens while he places this third coil between the other two. He finds that when $\mathrm{C}$ is exactly midway between $A$ and $B$ no sound is audible in the telephone, though sounds are heard if $\mathrm{C}$ is nearer to either $\mathrm{A}$ or B. Explain the cause of this. He also finds tilat if a bit of iron wire is placed in A silence is not obtained in the telephone until $C$ is moved to a position nearer to $B$ than the middle. Why is this ? Lastly, he finds that if a disk of brass, copper, or lead is interposed between $A$ and $C$, the position of silence for $\mathrm{C}$ is now nearer to $\mathrm{A}$ than the middle. How is this explained?

\section{QUESTIONS ON CHAPTER XIV}

1. What apparatus would you use to produce electric oscillations? Show how you would operate it, and explain why the oscillations take place. 
2. Explain how electric oscillations in a condenser circuit produce electric waves in the surrounding medium.

3. The capacity of an air-condenser is 0.001 of a microfarad. It is charged and then discharged through a circuit having a self-induction of 0.004 of a henry and a resistance of $4 \mathrm{ohms}$. Find the frequency of the vibration.

Ans. $n=159100$.

4. Under what circumstances do oscillations not take place when a condenser is discharged?

5. If the frequency of oscillation of a Hertz oscillator is $3,000,000$ per second, find the length of the waves it will produce.

Ans. 10,000 centimetres.

6. Explain the action of a resonator.

7. Give the reasons which exist for thinking that light is an electromagnetic phenomènon.

8. How is the action of magnetic forces upon the direction of the vibrations of light shown? and what is the difference between magnetic and diamagnetic media in respect of their magneto-optic properties?

9. It was announced by Willoughby Smith that the resistance of selenium is less when exposed to light than in the dark. Describe the apparatus you would employ to investigate this phenomenon. How would you proceed to experiment if you wished to ascertain whether the amount of electric effect was proportional to the amount of illumination? 


\section{INDEX}

\section{N.B.-The Numbers refer to the Numbered Paragraphs.}

ABsolute Electrometer, 287

Galvanometer, 213

units, 353

Accumulators, 492 (see also Condenser)

used in locomotion, 446

Action at a distance, 25, 64, 299

in medium, 5, 13, 64, 279, 299

Aether (see Ether)

Air condeuser, 56, 294, 359

Air-gap, 378

Air, resistance of, 313,326

Alini, Giovanni, experiments on Animals, 255

Alternate currents, 162, 461, 470

Alternate current magnet, 388,477 method of measuring resistance, 417

motors, 484

power, 475

Alternators, 478

Aluminium, reduction of, 494

Amalgam, electric, 44 ammonium-, sodium-, etc., 490

Amalgamating zinc plates, 174

Amber, 2

Amœba, the sensitiveness of, 256

Ammeter, 221

Ampère, André Marie, Theory of Electro-dynamics, 392

"Ampère's Rule," 197, 382

Laws of currents, 390,391 suggest a Telegraph, 497

Table for Experiments, 391

Theory of Magnetisın, 398

Ampere, the, 162, 207, 354

meter, 221

-turns, 341, 377 (and p. 589)
Amplitude of E.M.F., 470

Angle of lag, 472,473

Angles, Ways of Reckoning, 144, Appendix A

Solid, 148, Appendix A

Animal Electricity, 76, 257

Anion, 239, 491

Annual variations of magnet, 157

Anode, 170, 236

Anoinalous inagnetization, 373

Aperiodic galvanometer, 219

Apparent watts, 438, 472, 475

resistance, $417 e, 458 c, 472$

Appropriating brush, 50

Arago, Francois Jean

classitication of lightning, 331

on magnetic action of a voltaic current, 202, 381

on magnetic rotations, 457

Arc, the electric, theory of, 448

Arc lamps, 449

light, 448

Arc-lighting machines, 468

Armature of magnet, 103

of dynamo-electric machine, 462

Armstrong, Sir Wm., his Hydroelectric Machine, $\mathbf{4 8}$

Astatic magnetic needles, 201

Galvanometer, 201, 211, 215

Asynchronons motors, 486

Atmospheric Electricity, 72, 328

Atoms, charge of, 491 (footnote)

Attracted-disk Electrometers, 28t

Attraction and repulsion of electrified bodies, $2,4,22,24$, 74,262 


\section{The Numbers refer to the Numbered Paragraphs.}

Attraction and repulsion of currents, 389

and repulsion of magnets, 84 , 88,337

due to influence, 24

Aurora, the, 158, 159, 161, 336

Ayrton (W. E.) and Perry (John)

ammeter, 221

on contact electricity, 80

on dielectric capacity, 298

secohmmeter, $458(c)$

value of " $v$, " 359

voltmeter, 221

Ayrton and Mather galvanometer, 216

Azimuth Compass, 149, 151

B. A. Unit, 358

Back E.M.F., 445

Back Stroke, 29

Bain, Alex., his Chemical Writing

Telegraph, 246

Balance methods, 139, 411, 413 et seq. Wheatstone's, 413

Ballistic Galvanometer, 218, 418

Bancalari on flames, 374

Barrett, William F., on magnetic contraction, 124

Batteries, voltaic, 168, 179, 193

secondary, 492 list of, 189

Battery of Leyden jars, 62

Beccaria, Father G., on electric distillation, 251

on atmospheric electricity, 333

Becquerel, Antoine Cesar, on atmospheric electricity, 334

on diamagnetism, 369

Becquerel, Edmond, on photo-voltaic currents, 530

Becquerel, Henri, on magneto-optic rotation, 526

Bell, Alexander Graham, his Telephone, 510

Uses induction balance to detect bullet, 514

The Photophone, 529

Bells, electric, 508

Bennet, Abraham, his doubler, 49

Electroscope, 16, 28

Best grouping of cells, 192, 407

Bichromate Battery, 180, 189

Bidwell, Shelford, on magnetic contraction, 124

on susceptibility, 365

on lifting power, 384

Effect of light on magnets, 524
Bifilar Súspension, 130, 209, 288

Biot, Jean Baptiste, experiment with hemispheres, 33

Law of inagnetic distribution, 153

on atmospheric electricity, $\mathbf{3 3 4}$

Bismuth, diamagnetic properties of, 94,370

change of resistance in mag. netic field, 397

Blasting by electricity, 316, 432

Blood, conducting power of, 256

Board of Trade Standards, Appen$\operatorname{dix} B$

Board of Trade Unit, 440

Bolometer, 404

Boltzmann, Ludwig, on Dielectric capacity, 297, 298, 518

Boracite, 74

Bosanquet, R. H. M., magnetic cir. cuit, 375

"Bound" electricity, 27, 79

Boyle, Hon. Robert, 2 (footnote)

Boys, Charles Vernon, radio-micrometer, 425

Brake-wheel are lamps, 449

Branched circuit, 409

Brass, deposition of, 490

Breaking a magnet, 116

Breath-figures, 324

Bridge, Wheatstone's, 413

British Association Unit, 358

Broadside-on method, 138

Brown, C. E. L., on motor, 486

Brugmans discovers magnetic repul. sion of bismuth, 369

Brush, Charles $F$., his dynamo, 468

Brush discharge, 319, 324

Brushes, 463

Bunsen's Battery, 183, 189

CABLE, Atlantic, 301 (footnote), 302, 323,504

submarine, 504

" as condenser, 301, 323

Cabot, Sebastian, on magnetic declination, 151

Cadmium in standard cell, 188

Cailletet on resistance of air, 313

Calc-spar, 75

Calibration of Galvanometer, 211

Callan, induction coil, 229

Battery, 183 (footnote)

Callaud's Battery, 187

Callender's pyrometer, 404

Calomel cell, 188 


\section{The Numbers refer to the Numbered Paragraphs.}

Calories and joules, 427, 439

Candles, electric, 451

Canton, John, discovers electrostatic induction, 22

on electric amalgam, 44

Capacity, definition of, 271

in alternate circuit, 473

measurement of, 418

of cable, 301 et seq.

of condenser, 58, 294, 304, 473

of conductor, $40,55,272,304$

of Leyden jar, 58,294

of liquid condenser, 492

specific inductive, $25,56,295$, 304

unit of (electrostatic), 272

unit of (practical), 303

Capillary Electrometer, 253, 292

Carbon plates and rods, 183 (footnote) filaments, 452

Carbons for arc lamps, 449

Cardew, Philip, his voltmeter, 430, 471

Carhart, Henry S., on standard cells, 188

Carnivorous Plants, sensitive to electricity, 256

Carré, $F$., Dielectric machine, 45

Carriers, 49

on magnets of cast metal, 106

Cars, electric, 446

Cascade arrangement of jars, 309

Cautery by electricity, 431

Cavallo Tiberius, his attempt to telegraph, 497

his pith-ball electroscope, 4

on atmospheric electricity, 333

Cavendish, Hon. H., on Specific Inductive capacity, 295, 296

on nitric acid produced by sparks, 316

Ceca, Father, on atmospheric electricity, 333

Cell, voltaic, 166

Cells, classification of, 180

grouping of, 192, 407

list of, 189

Centi-ampere balance, 396

Central stations, 440,478

Circuit, 166, 406

Magnetic, 375

points of, where energy gained

and lost, 248, 436

Circuits, branched, 243, 409

Circuital magnetism, 118, 347

Circular current, $\mathbf{3 4 5}$

City of London central station, 478
Clange of configuration, law of, 204, 379

Characteristic curves, 466

Charge, electric, 8 resides on surface, 32 residual of Leyden jar, 61, 299 of accumulator, 492

Chart, magnetic, 154 (frontispiece)

Chemical action, E.M.F. of, 488

Cheluical actions in the battery, 172 laws of, $178,240,488$

of spark discharge, 316 outside the battery, 234,487

Chemical test for weak currents, 246 , 316

depolarization, 180

Chimes, electric, 46

Choking-coils, 474

Choking-effect, $459,473,474$

Chromic solution, 183

Chronograph, electric, 509

Clamond's thermopiles, 425

Clark, Latimer, his standard cell, 188, and Appendix C

Classification of cells, 180

Clausius, $R$., theory of Electrolysis, 491

Cleavage, electrification by, 68

Clock diagram, 470,472

Clocks, electric, 509

Closed circuit, cell for, 176,181

Closed-circuitmethod of Telegraphy, 500

Closed-coil armature, 463

Cobalt, magnetism of, 93

Coefficient of Magnetic induction (see Permeability)

of Magnetization (see Susceptibility)

of mutual-induction (or potential), 351, 454

of self-induction, 458

Coercive force, 96,367

Colour of spark, 318

Columbus, Cristofero, on magnetic variation, 151

Combs on influence machine, 42, 50

Combustion a source of electrification, 70

heat of, 488

Commercial efficiency of dynamo, 464

Commutator, 443, 461, 463

Compass (magnetic), Mariner's, 87 , 149

error due to iron ship, 149

Compound circuit, 192, 243, 409 


\section{The Numbers refer to the Numbered Paragraphs.}

Compound dynamo, 465, 467 magnets, 104

Condensation, 56

Condenser, 56, 294, 303 capacity of, how measured, 418

discharge of, 326,515

in alternate circuit, 473,474

liquid, 492

method of measuring a resist. ance, 411,417

standard, 303

use of, 229,302

Condensing electroscope, 79

Conductance, 402, 404

Conduction, 7, 30, 171, 402, 404, 476 by liquids, 234,404

of gases, 171, 322

Conductivity, $171,322,346,348,402$, 404

Conductor cutting lines, 225, 339, 353,355

Conductors and Non-conductors, 8, $2 \tau, 30,402$ et seq.

Conductors electrified by rubbing, 13 opaque, 518

Consequent Poles, 117, 120, 382

Constant-current dynamos, 468 voltage dynamos, 467

Contact Electricity, 79, 163

Series of metals, 80

rings, 461

of surfaces, 12

Continuous-alternate transformers, 483

currents, 162

current dynamos, 463

current transforiners, 482

electrophorus, 26,49

Contraction due to magnetism, 124

Control of galvanometer, 209

Convective discharge, 312

Convexion of electricity, 49, 312, 397 eurrents, 397

induction machines (see Influence machines)

streams at points, 38, 47, 274, 329

Cooking by electricity, 434

Cooling and heating of junction by current, 419

"Corkscrew Rule," 198

Cost of power derived from electricity, 440

Coulomb, Torsion Balance, 18, 132

Law of Inverse Squares, 19, $129,132,261,270$
Coulom $\dot{b}$ on distribution of charge, 38,273

Coulomb, the, 162, 354

how many electrostatic units, 262 (footnote)

Couple, magnetic, 136

Coupling of alternators, 479

Creeping, stopped by paraffin, 183 magnetic, 368

Crookes, William, on shadows in electric discharge, 321

on repulsion from negative electrode, 327

Crown of cups, 165

Cruickshank's 'Trough Battery, 180

Crystallization, 69

Crystals, electricity of, 74,75

dielectric properties of, 297 magnetism of, 373

Cumming, James, invents galvanoneter, 200

thermo-electric inversion, 423

Cuneus' discovery of Leyden jar, 60

Curbing telegraphic signals, 302

Current, effects due to, 167

Electricity, 162

strength of, 171, 190

", unit of, 162, 207

Current, is the magnetic whirl, 202 balance, 396, and Appendix B slieets, 410

Currents, very large, measurement of, 412

Curvature affects surface-density, 38,274

Curve-tracer, 368

- Curves, magnetic (see Magnetic Figures)

Curves of magnetization, 364 characteristic of dynamos, 466

Cuthbertson, John, his electric machine, 41

Cycles of magnetization, 368 of alternate currents, 470

Cylinder Electrical machine, 42

DAILY variations of compass, 156

Dalibard's lightning-rod, 329

Damping galvanometers, 219

Daniell, John F., his cell, 181, 181

D'A rsonval, galvanometers, 216

Davy's (Marié) Battery, 193

Davy, Sir Humphry, magnetization

by current, 381

discovers electric light, $44 \mathrm{~S}$

electrolyzes caustic alkalies, 490 (c) 


\section{The Numbers refer to the Numbered Paragraphs.}

De Haldat, magnetic writing, 122

De la Rive's Floating Battery, 205

De la Rue, Chloride of Silver Battery, 186,313

on electrotyping, 495

on length of spark, 313

Dead-beat galvanometers, 219

Declination, Magnetic, 151 variations of, 151, 155

Decomposition of water, 235 of alkalies, $490(\mathrm{c})$

De-electrification by flame, 314

Deflexions, unethod of, 131, 136

Deflexion of galvanometer, 210

Dellmann's electrometer, 286

Demagnetize, how to, 368

Density (surface) of charge, 38, 273 magnetic, 134, 337

Depolarization, mechanical, 180 chernical, $180,182,183$ electro-chemical, 180, 181

Deposition of uetals, 494

Deviation of compass, 149

Devar, James, on currents generated by light in the eye, 257

his capillary electrometer, 253 magnetic properties of iron at $200^{\circ}, 111$

oxygen magnetic, 370

Dewar and Fleming, resistance at low temperature, 404

Diagram, thermo-electric, 424

Dial bridge, 415

Diamagnetic polarity, 369

Diamagnetism, 94, 369 of flames, 374

of gases, 370,374

Diaphragm currents, 254

Dielectric capacity, 295 to 299 capacity, effect on intensity of field, 262,298

coefficient, 283, 517 strength, 315

Dielectrics, 10, 25, 57, 295

Difference of potential, 265 magnetic potential, 337

Differential galvanometer, 217, 411

Dimensions of units, 356

1)i-phase currents, 485

Dip, or Inclination, 152 variation of, 155

Diplex signalling, 503

Dipping Needle, 152

"Direct" and "inverse" current, 223

Direction of induced E.M.F., 226, 456
Discharge affected by magnet, 322

brush, 319,324

by evaporation, 251

by flame, 8,314

by points, $47,319,329$

by water dropping, 334

conductive, 310

convective, 47,312

disruptive, 311

effects of, $47,315,316,317$

glow, 319, 329 (footnote)

limit of, 273

oscillatory, 515

sensitive state of, 322

striated, 320

through gas at low voltage, 322

velocity of, 323

Discharger, Discharging-tongs, 59 Universal, 62

Disk armature, 463

Displacement, electric, 57 currents, 516

Disruption, electrification by, 68

Dissectable Leyden jar, 63

Dissipation of Charge, 326

Dissociated gases condrct, 322

Distillation, electric, 251

Distribution of Electricity, 31 to 38 , 273,274

of Magnetism, 117, 134

Distribution by transformers, 480

Distribution of energy, 440

Distortion of dymamo-field, 463

Divided circuits, 409

Tonch, 101

Dolbear, A. E., his telephone, 299, 510

Donbler, the, 26,49

Donble refraction by electric stress, 524,525

Double Touch, 102

Dreh-stron, 485

Drop of voltage in mains, 412, 447

Dry cells, 184, 189, 193

Dry-Pile, 193, 291

Du Bois, limit of magnetization, 363 measurement of permeability, 366

Duboscq, Jules, his lamp, 449

Du Fay's experiments, '5, 30

Duplex Telegraphy, 302, 503

Duration of Spark, 323

Dust, allaying, 54

Duter on Electric Expansion, 300

Dynamic Electricity (see Current Electricity) 
The Numbers refer to the Numbered Paragraphs.

Dynamos, 461

as motors, 443,463

Dynamometer, 394

Dyue, the (unit of force), 281

Earth, the, a magnet, 95

currents, 302

electrostatic capacity of, 303

intensity of magnetization, 365

magnetic force in absolute units, 361

used as return wire, 497

Earth's magnetism (see Terrestrial Magnetism)

Earth, potential, 269

Ebert, $H$., on oscillations, 522

Eddy-currents, $457,477,486$

Edison, Thomas Alva, electric lamp, 452

carbon telephone, 511

meter for currents, 244, 442

quadruplex telegraphy, 503

Edlund on galvanic expansion, 249

Eel, electric (Gymnotus), 76

Efficiency of transmission, 447

of dynamos, 464

of motors, 445

of transformers, 481

Electric Air-Thermometer, 317

Cage, 37

Candle, 451

Clocks, 509

Displacement, 57

Distillation, 251

Egg, the, 232, 320

Expansion, 300

Field, 13, 16, 20, 22, 24, 262, $279,299,524,525$

Force, 169 (footnote), 266

(Frictional) machines, 42

Fuze, 316, 429, 432

Images, 275

Kite, 329

Light, 448

Lines of Force, 13, 16, 20, 22, 24, 299

Mill or Fly, 47

Oscillations, 515

Osmose, 250

Pistol, 316

Shadows, 321

Shock, 254

Stress, 13, 16, 20, 22, 24, 63, 279

Waves, 515

Wind, 4i, 324

Electrics, 2
Electricity, theories of, 7, 327 word first used, 2 (footnote)

Electro-capillary phenomena, 253

Electro-chemical Depolarization, 180

equivalents, 240,489

power of metals, 489

Electro-chemistry, $4 \mathrm{S7}$ deposition, 494

Electrodes, 236 unpclarizable, 257

Electrodynamics, 389

Electrodynamometer, 394

Electrolysis, 237, 487

in discharge, 322

laws of, 240,490

of copper sulphate, 238

of water, 236,487

theory of, 491

Electrolytes, 236, 487

Electrolytic condenser, 492 convexion, 491

Electromagnet, alternate current: 477

Electroinagnets, 107, 381

laws of, 380

calculations for, 375,376 (and see p. 589)

Electromagnetic engines (see Motors!

Electromagnetic systems, law of, 204,379

system of units, 352

theory of Light, 517

waves, 515

Electromagnetics, 337

Electromagnetism, 337

Electrometallurgy, 494

Electrometer, absolute, 287

attracted-disk, 287

capillary, 253, 292

Dellmann's, 286

Peltier's, 286, 334

portable, 287

quadrant (Lord Kelvin's), 288

repulsion, 286

torsion, 18

trap-door, 287

Electromotive-force, 169, 487

induced, 222

measurement of, 416

unit of, 354

Electromotive intensity, 266, 283

Electromotors, 443, 484

Electro-Optics, 524

Electrophorus, 26

continuous, 26,49

Electroplating, 496 


\section{The Numbers refer to the Numbered Paragraphs.}

Electroplating, dynamos for, 462

Electroscopes, 14

Bennet's gold-leaf, 16, 28

Bohnenberger's, 16, 291

Fechner's, 291

Gilbert's straw-needle, 15

Hankel's, 291

Henley's quadrant, 17

Pith-ball, 3, 4

Volta's condensing, 79

Electroscopic powders, 31, 47, 299, 324

Electrostatic Optical Stress, 525 voltmeter, 290

Electrostatics, 8, 259

Electrotyping, 495

Element of Current, 344

Elwell-Parker alternator, 478

End-on method, 138

Energy, 1, 64

of magnetic field, 202

of charge of Leyden jar, 305

of electric current, 435

paths, 518

points in circuit where it is lost or gained, 248,436

supply and measurement of, 435

Equator, Magnetic, 86

Equipotential surfaces, 267

magnetic, 337 (f)

Equivalents, electro-chemical, 240

Erg, the (unit of work): 281

Ether, 1, 7, 64, 517

Evaporation produces electrification, 71,330

discharge by, 251

Everett, James D., on atmospheric electricity, 334

on exact reading of galvanometer, 214 (footnote)

on intensity of magnetization of earth, 365

Ewing, James A., on limit of magnetization, 363

curves of magnetization, 364

theory of magnetism, 127

Exchanges, telephone, 513

Excitation of Field-magnets, 465

Exciting power, 377

Expansion, electric, 300,525

Extra-current, 459

Failure and exhaustion of batteries, 172

Fall of potential along a wire, 289, 412
Farad, the (unit of capacity), 303, 354

Faraday, Michael, molecular theory of electricity, 7

chemical theory of cell, 178

dark discharge, 319

diamagnetism, 369, 373, 374

discovered inductive capacity, 25, 296, 298

discovery of magneto-induction, 222

Disk machine, 227

electro-magnetic rotation, 393

experiment on dielectric polarization, 299

gauze-bag experiment, 34

hollow-cube experiment, 34

ice-pail experiment, 37

laws of electrolysis, 240, 242

length of spark, 313

Magnetic lines-of-force, 119

magnetism in crystals, $\mathbf{3 7 3}$

on Arago's rotations, 457

on dissipation of charge, 314

on electrodynamics, 392

on identity of different kinds

of electricity, $245,246,316$

predicted retardation in cables, 301

Ring, 228

rotation of plane of polarized light, 526

voltameter, 242

Faure, Camille, his Secondary Battery, 492

Favre's experiments on heat of currents, 428

Fechner's electroscope, 291

Feddersen, $W_{\text {., }}$ on electric oscillations, 514

Feeders, 440

Ferromagnetic substances, 369

Field, electric, 13, 16, 20, 22, 24, 262, $279,299,525$

magnetic, $115,202,337,462,526$

Field-1nagnet, 462

Field-magnets, excitation of, 465

Field-plate, 50

Figures, magnetic (see Magnetic figures)

electric, 31, 299, 324

Filament of incandescent lamps, 452

Filings for mapping fields, 121

Fire of St. Elino, 329 (footnote)

Flame, currents of, 314

diamagnetism of, 374

discharge by, 8,314

produces electrification, 70 


\section{The Numbers refer to the Numbered Paragraphs.}

"Flashing" filaments, 452

Fleming and Dewar, resistance at low temperature, 404

Fleming, John Ambrose, his Battery, 193

rule as to direction of E.M.F., 226

Flux, magnetic, 142, 337, 363, 377

density, 363 (footnote)

Fontana on electric expansion, 300

Force, electric, 169 (footnote), 266, 276,277

electromotive (see Electromotive-force)

magnetic, 91, 169 (footnote), 337

near a straight conductor, 207 , 343

on conductor in field, 340, 341

Form, effect of, on retentivity, 98

on lifting power, 114

"Forming" accumulator plates, 492

Foster, George Carey, his evaluation of ohm, 358

method of testing, 415

Foucault, Léon, his Regulator Lamp, 449

Interrupter, 229

Foucault - currents (see Eddy-currents)

Franklin, Benjamin, discovered action of points, mentioned in, $38(c), 47,329$

cascade arrangement of Ley den jars, 309

Electric chimes, 47

Electric kite, 329

Electric portraits, $\mathbf{3 1 7}$

his charged pane of glass, 55

invents lightuing conductors, 329,332

kills turkey by electric shock, 254

One-fluid theory of electricity, 7

on seat of charge, 63

theory of the aurora, 336

Frankfort, transmission of power to, 447, 485 (footnote)

"Free" electricity, 27, 79 (footnotu)

Frequency, 470, 476

of oscillations, 515,520

Friction produces electrification, 2,12

Frog's legs, contractions of, 163, 255

Frölich, Otto, on electromagnet, 380

Froment's motor, 443

Fuel, zinc as, 166

Fuses, 316, 429, 432
Fusing of wires, 429

" G" of galvanometer, 213

Galvani, Aloysius, observed movements of frog's leg, 163

on preparation of frog's limbs, 255

on Animal Electricity, 257

Galvanic Batteries (see Voltaic Batteries)

Electricity (see Current Electricity)

Taste, 254

Galvanism (see Current Electricity)

Galvanometer, 208

absolute, 213

astatic, 211,215

ballistic, 218,418

constant of, 213

damping of, 219

D'Arsonval's, 216

dead beat, 219

differential, 217, 411

Du Bois Reymond's, 257

reflecting (Lord Kelvin's), or mirror, 215

sine, 214

tangent, 212

Von Helmholtz's, 212 (footnote)

Galvanoplastic (see Electrotyping)

Galvanoscope, 199

Gas Battery, 493

Gases, dissociated, conduct, 322

resistance of, $171,314,322$

Gassiot, J. P., on striæ, 322,327

Gaugain, 'Tsan Mothée

on Pyroelectricity, 74

Tangent Galvanometer, 212 (footnote)

Gauss, $C . F$., invented absolute measurement, 352

magnetic force of the earth, 361

magnetic observations, 365

on magnetic shell, 348

Gay-Lussac, on atmospheric electricity, 334

Geissler's tubes, 320

Generators of alternate currents, 473 continuous currents, 463

Gernez on electric distillation, 251

Gibson and Barcley on dielectric capacity of paraffin, 297

Gilbert, Dr. William, discovers electrics, 2

discovered magnetic reaction, 91 


\section{The Numbers refer to the Numbered Paragraphs.}

Gilbert, Dr. William, discovers that the earth is a magnet, 95 , 150

heat destroys magnetism, 109

his balanced-needle electroscope, 15

his terrella, 95

observation of moisture, 10

observations on magnets, 86

on de-electrifying power of flaine, 314

on magnetic figures, 119

on magnetic substances, 92

on magnetic permeability, 97

on methods of magnetization, 105,106

Glass, a conuluctor when hot, 31

Globular lightning, 331

Glow Discharge, 319, 329 (footnote)

lainps, 452

Guld-leaf Electroscope (see Electroscope)

Gordon, J. E. H., on magneto-optic rotatory power, 526

on dielectric capacity, 297, 298

on length of spark, 313

Gramme, Zenobe Théophile, his ring. armature, 463

Gravity Battery, 187

Gray, Andrew, Absolnte Measurements in E. and M., 136 (footnote), 287 (footnote), 396 (footnote)

Gray, Stephen, discovers conduction, 30

on lightning, 329

Grid of accumulator, 492

Grotthuss' theory, 172, 491

Grouping of are lamps, 450

cells, 192, 407

glow-lamps, 453

Grove, Sir William R., his Gas Battery, 493

Crove's Battery, 182

magnetic experiment, 124

on electric property of Hame, 314

Guard-ring, Gnard-plate, 273, 287

Guericke, Otto von, discovered electric repulsion, 4

invents electric machine, 41

observes electric sparks, 11

Gunpowiler fired by electricity, 316, 317,432

Guthrie, Frederick, effect of heat on discharge, 314

heating of kathode in water, 433
Gymnotus (electric eel), 76, 246

HaLF deflexion method, 417

Hall, Edward $H$., his effect, 397

Hankel, Wilhelm G., his electroscope, 291

Hardening of steel, 108

Harris, Sir $W$. Snow, his unit Leyden jar, 285

attracted-disc electrometer 287

on length of spark, 313

Hauksbee, Francis, on thunderstorms, 329

Hä̈y, The Abbé, his astatic method, 201

Heat and resistance, 426, 439

of combination, 488

effect of, on magnets, 109, 111

$\begin{array}{ll}, & \text { batteries, } 194 \\ , & \text { Geissler tube, } 320 \\ , & \text { resistance, } 404\end{array}$

emission, 386, 429

Heat, unequal action of, on + and charges, 314,327

Heating of coils, 386,429

Heating effects of currents, 182, 426, 439

due to magnetization, 124,368

effect of sparks, 317

local, at electrodes, 491

Heaviside, Oliver, reluctance, $3 \mathbf{7 5}$ (footnote)

- on energy paths, 518

- on quadruplex telegraphy, 497

Helmholtz, Hermann L. F. von, on effect of current on sight, 254

Electrolytic convexion, 491

Equations of self-induction, 460

Galvanometer, 212 (footnote)

Hemihedry in crystals, 75

Henry, Joseph, invented the "sounder," 497

on induced currents of higher orders, 455

IIenry, the, 354, 454, 458

Hertz, Heinrich, on effect of ultraviolet waves, 313,531

kathode rays, 321

researches on electrie waves, 520

Heydweiller, on length of spark, 313

High frequency, $476,515,520$

Hittorf, on discharge, 322 


\section{The Numbers refer to the Numbered Paragraphs.}

Holtz, W., his electric machine, 53

on electric shadows, 321

on tubes having unilateral resistance, 327

Hopkinson, John, on dielectric capacity of glass, 297

on residual charge and its return, 299

on magnetization, 364

his characteristic curves, 466

Horizontal coinponent of magnetism, $136,153,361$

Horse power and watts, 435

Hot glass, a conductor, 31

Hughes, David Edward, the Printing Telegraph, 497

the Microphone, 512

magnetic balance, 140

induction balance, 514

Humboldt, Alexander von, on electric eels, 76

discovers galvanic sinell, 254

produced electric contractions

in fishes, 255

Hunter, Dr. John, on effect of current on sight, 254

Hydroelectric machine, 48

Hysteresis, 367, 368

IDIostatic method of using voltmeter, 290

Images, electric, 275

Impedance, $4 \pi 2$

(Impedance) coils, 474

Incandescent lamps, 452

Inclination (or Dip), 152

variation of, 155

Index Notation, 355

Inductance, 458

Induced charges of electricity, 22 currents, 222

Induction (electrostatic) of charges, (see Influence)

(magnetic) lines of, 96

(magnetic) of magnetism, 96

(magneto-electric) of currents, 222

(volta-electric) of currents by currents (see Self induction, Mutual induction)

the, meaning the internal magnetization, 363 (footnote)

Induction-coil or Inductorium, 229

Induction-con vexion machines, 49

Inductive-capacity, specific, 25,56 , 295, 299

Inertia, electromagnetic, 458
Influence, 22

Influence-machine, 49-54

Insulators, 10, 30, 405

Intensity of current, 190 (footnote) of earth's magnetic force, 153, 358,361

of magnetic field, 338

of magnetization, 365

Internal resistance, $171,406,417$ of armatures, 462

International ohm, 358

"Inverse" and "direct" currents, 223

Inverse Squares, Law of, 19, 129, $148,261,270$

Inversion, Thermo-electric, 423

Ions, 239

Ironclad magnet, 883

Iron, properties of, 362

Iron rods red-hot in water, 433

Isoclinic lines, 154

Isogonic lines, 154

Isolated, 271

JA BLOCHKOFF, PAUL, his battery, 193 electric candle, 451

Jacobi, Moritz Hermann, on local action, 174

discovers galvanoplastic process, 495

his boat propelled by electricity, 443

on electromagnet, 380

theory of electromotors, 443

Jar, Leyden, 59

capacity of, 58,294,
304
cascade arrangement
of, 309
discharge of, 59,310,
515
discovery of, 60
energy of charge of,
305 seat of charge of, 63
,$\quad$ spark of, 318,323
theory of, 294
U',
Unit, 285

Jenkin, Fleeming, on cable as condenser, 301

on retardation in cables, 323

Joints in magnetic circuit, 378

Joule, James Prescott, on effects of magnetization, 124

evaluation of ohm, 358

Law of heat of current, $42 \%$, 439 
The Numbers refer to the Numbered Paragraphs.

Joule, James Prescott, limit of magnetization, 363

magnetic circuit, 375

Mechanical equivalent of heat, 439,488

on atmospheric electricity, 333

on lifting-power of electromagnet, 384

Joule effect, 420

Joule, the, 354, 439

KAPP, GISBERT, on magnetic circuit, 377

Kathode, 170, 236

Kathodic " rays," 321

Kation, 239, 491

Keeper, 103

Kelvin, Lord (Sir William Thomson)

Attracted-disk Electrometer, 79,287

Compass, 149

Current Balances, 396

Divided-ring Electrometer, 79

Electric convexion of heat (Thomson effect), 424

Evaluation of ohm, 358

" $v$," 359

Modified Daniell's cell, 187

on atmospheric electricity, 333

on electric images, 275

on electrostatics, 287 (footnote)

on length of spark, 313

on nomenclature of magnetic poles, 89 (footnote)

on sounds in condensers, 299

predicts electric oscillations, 515

proof of contact electricity, 79

Quadrant Electrometer, 2s8

Replenisher (or Mouse Mill), $49,287,288$

Thermo-electric diagram, 424

Water-dropping Collector, $\mathbf{3 3 4}$

Kerr, Dr. John, Electro-optic discoveries, 300,525

Magneto-optic discoveries, $125,366,527$

Kerr's effect, 527

Kinnersley, Elijah, Electric Thermometer, 317

Kirchhoff, Gustav, Laws of Branched

Circnits, 409

Kite, the electric, 329
Kohlrausch, Friedrich, on residual charge, 299

on electro-chemical equivalent, 240

on evaluation of $\mathrm{ohm}, 358$

Kundt, August, his effect, 528

LAG and lead, 472

Lagging of nagnetization, 368

Lamellar magnetization, 118

Laminated magnets, 104

Lamination of cores, $457,463,477,480$

Lamps, arc, 449

Lamps, incandescent, 452

Langley's, S. P., his bolometer, 404

Law, cell, 180

Laws of electrolysis, 490

of inverse sqnares, 19, 129, $14 \mathrm{~S}, 261,270$

of electro-magnetic system, 204, 379

Lead, used in accumulators, 492

no Thomson-effect in, 424

Lead and lag, in phase, 472

Lead of brushes, 463

Leakage, magnetic, 377

photoelectric, 531

rate of electric, 326

Le Bailliff, diamagnetisn, 369

Leclanché, Georges, his celr, 184

Lemonnier discovers atmospheric electricity, 333

Lenard, Philipp, aluminium "window," 321

Length of spark, 313

Lenz's Law, 456

Lenz on electromagnet, 380

Leyden jar, 55

prevention of piercing spark, 62

oscillatory discharge of, 515

resonance between two, 517 seat of charge in, 63

Leydens (see Condensers)

Lichtenberg's figures, 324

Life of Lamps, 452

Lifting-power of magnets, 113, 114 of electromagnets, $\mathrm{s} 4$

Light affects resistance, 529

affects a magnet, 524

Electric, 488

Electromagnetic theory of, 1, 518

polarised, rotated by magnet, $125,526,527,528$

velocity of, 359,518

Lightning, 11, 329, 331 . 


\section{The Numbers refer to the Numbered Paragraphs.}

Lightning conductors, 35, 332

duration of, 323,331

best methods of protection from, 332

Limit of heating of electromagnet, 386

magnetization, 363

Lines-of-force, electric, 13, 16, 20, $22,24,268$

magnetic, 96, 119, 337

Line-integral, 341 (footnote)

Lippmann, G., Capillary Electrometer, 253,292

Liquids as conductors, 234, 490, 518 resistance of, 403,404

Liquid condensers, 492

"Local Action" in batteries, 1\%8

Locomotion, electric, 446

Lorlestone, 84

Lodge, Oliver, on resonance, 517

his oscillator, 521

his detector or coherer, 521

London, city of, Central Station, 478

"Long" and "short" coils for mag. nets, 386

Long and short coil instruments, 403

Lorenz, L., on evaluation of ohm, 358

Loss of charge, 326, 531

Louis $X V$. electrifies 700 monks, 254

Lullin's experiment, 315

Luminous effects of spark, 318

Machine, Electric, 42

alternate-current, 478

cylinder, 42

dynamo-electric, 461

Holtz's, 53

hydro-electrical, 48

influence, 49

magneto-electric, 461

plate, 43

'Toepler's or Voss, 51

Wimshurst, 52

Winter's, 43

Magne-crystallic action, 373

Magnet, breaking a, 116

Magnets, natural and artificial, 84, 85

Magnetic actions of current, 195, 338,389

attraction and repulsion, s8, 121,389

cage, 97

creeping, 368
Magnetic circuit, 375

field, 115, 202, 389

" rotatory, 485,486

figures, 119, 120, 121, 202, 389 theory of, 142

flux, 337, 377

flux density, 363 (footnote)

force, $91,337(a)$

" measurement of, 130

hysteresis, $367,368,464$

induction, 96, 363 (footnote)

iron-ore, 84

lag, alleged, 368

lines-of-force, $96,119,120,121$, $349,362,373,377,389,464$

lines-of-force of current, 202, 389

maps, 154

meridian, 151

metals, 93, 362, 369

model (Ewing's), 127

moment, 135, 346, 361

needle, 87,149

oxide of iron, 84,183 (footnote)

paradox, a, 143

permeability, $96,363,366$, 518

pole, unit, 141, 352

potential, $337,347,348$

proof-plane, 232

saturation, 112, 363

scree'n, 97

Beetz, on, 126

shell, 11s, 203, $337(h), 348$

" force due to, 345

", potential due to, 348

storms, 158, 336

substances, $92,362,369$

susceptibility, 365

units, 352

writing, 122

Magnetism, $8 \dot{4}$

action of, on light, 125, 126

destruction of, 109

distribution of, 117

lamellar, 118

laws of, $89,128,337$

of gases, 370,374

permanent, 98

phenourenon of rotation, 526

residual, 112, 364

solenoidal, 118,347

temporary, 98,112

terrestrial, 95, 150

theories of, $99,126,526$

unit of, 141,352

Magnetite, 84 


\section{The Numbers refer to the Numbered Paragraphs.}

Magnetization, anomalous, 373 coefticient of (see Suscepti bility)

cycles of, $36 \%, 368$

intensity of, 365

lamellar, 118

inechanical effects of, 124

methods of, 100-107

solenoidal, 118, 347

sound of, 124,510

time needed for, 388

Magneto-electricity, S2, 222, 461

Magneto-electric machines, 461

Magnetographs, 160

Magnetometer, 137

self-registering, 160

Magnetomotive-force, 341,375

Magneto-optic Rotations, 524

Magnets, sce also electromagnet

action of light on, 524

artificial, 85

compound, 104

forms of, 103

lamellar, 118

laminated, 104, 477

methods of making, 100-107

natural, 84, 103

power of, 114

unvarying, 110

Mance, Sir Henry, his method, 417

Manganese steel, 363

Manganin, 404

Maps, magnetic, 154

Mariner's Compass, 149

Marked pole, 88

Murum heating by discharge, 31 i

Mascurt, E., on atmospheric electricity, 335

Matteucci, Carlo, on pliysiological effects, 76,256

on electromotive - force in muscle, 257

Maynooth Battery (see Callan's Battery)

Maxwell, James Clerk, Electro-magnetic theory of light, 397 , 518

Law of alternate currents, 473

Law of electromagnetic systein, 204, 349, 379

measurement of " $\mathrm{v}$, , 359

on Electric Images, 275

on protection from lightning, 35,332

on residual charge of jar, 299

rule for action of current on magnet, 204, 349
Maxwell, James Clerk, Theorem of equivalent Magnetic shell, 203,351

Theory of Magnetism, 126

Measurement of capacity, 418

of currents, 221, 395, 412

of E.M.F., 416

of internal resistance, 417

of magnetic forces, 130

of inutual induction, 454

of permeability, 366

of power, 437

of resistance, 411,412

of self-induction, 458

Mechanical depolarization, 180

effects of discharge, 47,315

" of magnetization, 124

", in dielectric, 299, 525

Medical Äplications of Electricity, 258

Medium, action in, $5,13,279$

elasticity and density of, 360

energy paths in, 519

velocity of waves in, 359,518

Mega-, 354

Megohm, 354

Meidinger's Battery, 1S7

Melloni, Macedonio, his thermopile, 425

Mendenhall, T. C., U.S. Geodetic

Survey, 155

Meridian, Magnetic, 151

Metallo-chromy, 490

Metals, electro-chemical power of, 489

electro-deposition of, 494

refining by electricity, 494 specific resistance of, 403

Meter Bridge, 415

Meters, 442

Metric system, the, 280

Mho, the, 402

Mica, dielectric capacity of, 296

Micro-, 354

Microfarad, the, 283, 354

condenser, 303

Microphone, the, 512

Milli-, 354

Milli-ainpere, 354

Mimosa, the electric behaviour of, 256

Minotto's cell, 187

Mirror Galvanometer, 215

Molecular action of magnetism, 126

actions of current, 249

theory of Electric action, 7

Ioment of Couple, 136 
The Numbers refer to the Numbered Paragraphs.

Moment of circular coil, 346 of inertia, 361

magnetic, 135, 361

Morse, Samuel F. B., his Telegraph instrument, 499

Morse Alphabet, the, 499

Mordey's alternator, 478

Motion, law of, in magnetic field, 204,379

Motor-dynamos, 482

Motors, 443

alternate-current, 484

Moulton, John Fletcher, on sensitive state, 322

Mouse-inill (see Replenisher)

Müller, Johannes, on strength of electromagnets, 380

Multicellular voltmeter, 290

Multiplier, Schweigger's, 200

Muscular contractions, 255, 257

Musschenbroek, Peter van, discovery of Leyden jar, 60

on Magnetic Figures, 121

Mutual induction, 454

potential, 351

NAPOLEON IIT'S cell, 193

Navigation, electric, 443

Needle, magnetic, 87

telegraph, 498

Negative electrification, 5,327

Network mains, 440

Neutralizing brush, 50

Newton, Sir Isaac, observations on action and reaction, 91

his lodestone, 114

suggests elertric origin of lightning, 11, 329

suggests glass for electric machines, 41

Niagara Falls, transmission of power from, 447

Niaudet, Alfred, his cell, 184

Nickel, 93, 364

Nobili, Leopoldo, on muscular con. tractions, 76

on currents of animal electricity, 257

discovers Nobili's rings, 490

Non-conductors, 10,405

Non-electrics, 3

North and south, 89, 150

magnetic pole, the, 89,150

Null methods, 210, 289, $411(c), 413$, $416(b), 417(e), 418(d)$

Obuique currents, laws of, 390
Oersted, Hans Christian, discovers magnetic action of current, 195, 196,202

Ohm, Dr. Georg Simon, 190

"Ohm's Iaw," 191, 399

Ohm, the, 354, and Appendix B evaluation of, 358

Oil, dielectric strength of, 315

One-fluid theory of electricity, 7

Opposition method, 417

Optical strain, electrostatic, 525 rotation, electromagnetic, 526 , 527,528

Oscillations, electric, 332, 515

method of, 210

method of (for electrostatics), 133 (footnote)

method of (for magnetic measurement), 133, 134, 361

Oscillator, 520,522

Oscillograph, $216 a$

Osmose, electric, 250

Other sources of electrification than friction, 12,65

Output of dynamo, 464

Over-compounding, 467

Overhead line for tramcars, 446

Oxygen magnetic, 370

Ozone, 237, 316, 329 (footnote)

Pacinotiti's armature, 463

Page, Charles $G$., discovers magnetic sounds, 124

Parallel, capacities in, 307 cells in, 168, 406

circuits, laws of, 390

lamps in, 453

resistances in, 409

running of alternators, 479

Paramagnetic bodies, 369

"Passive" state of iron, 183

Pathological dose of current, 258

Peace, on length of spark, 313

Péclet, electrification by rubbing, 73

Peltier, Athanase, his electrometer, 286,334

heating effect at junctions, 420

theory of thunderstorms, 330

Peltier effect, 420

Penetrative power of discharge, 315

Periodic current, 470

Periodicity (see Frequency)

of aurora and magnetic storms.

$158, \mathrm{~J} 59,336$

Permeability, 96, 363, 518

measurement of, 366 
The Numbers refer to the Numbered Paragraphs.

Perry, John, his meter, 442

Persistence (see Time-constant)

Phase, 470, 472

Phosphorescence caused by discharge, 320, 321

Photo-chemical excitation, 530

Photographic plate affected by discharge, 324

Photophone, 529

Photo-voltaic property of selenium, 529

Physiological actions, 254, 325

Piercing glass, prevention of, 62

Piezo-electricity, 75

Plane, the proof-, 32

", for magnetism, 232

Planté, G̈aston, secondary cells, 492 globular lightning, 331

Plants, electricity of, 77,256

Plate condenser, 56, 295, 304 electrical machine, 43

Platinoid, 404

Plücker, Julius, on diamagnetism, etc., 370,373

Poggendorff, J. C., his cell, 180 method of measuring E.M.F., 416

Points, density of charge on, 38,274 discharge at, $42,45,46,47,274$, 329

Poisson, magne-crystallic action, 373

Polarity, diamagnetic, 369 magnetic, $90,116,126$

Polarization (electrolytic) in battery cells, 175,487

of Voltameter, 487, 492

remedies for, 180

rotation of plane of, $526 \mathrm{et} \mathrm{seq}$.

Polarized mechanism, 387 relay, 501

Poles of magnets, 86, 134 of pyroelectric crystals, 74 of voltaic battery, 168

Polyphase currents, 485

Porous cell, ISO

Porret's phenomenon, 250

Portable electrometer, 287

Portative force, 114

Post-Office Bridge, 415 relay, 501

Positive and negative electrification, 5,327

Potential, electric, 40, 263

," zero, 4n, 264

of conducting sphere, 269

galvanometers, 220

magnetic, $337,347,348$
Potential, magnetic, due to current, 351

- mutual, of two circuits, 352, 357

Potential-divider nul method, 418

Potentiometer, 416

Pouillet, Claude S. M., sine galvanometer, 214

tangent galvanometer, 212

Powdered metals, conduction of, 400

sensitiveness to sparks, 521

Powders, electroscopic, 31, 47, 299, 324

Power, 435

transmission of, 447

Power-houses, 440

Poynting, John Henry, on energypaths, 519

Practical units, 354

Preece, William Henry, telegraphy, 497

Pressure produces electrification, 75 effect on electrolysis, 490

(voltage), 169

Priestley, Joseph, on electric expansion, 300

on influence, 26 (footnote)

Prime conductor, 42

Printing telegraphs, 497

Proof-plane, 32

magnetic, 232

Protoplasm, electric property of, 256

Pyroelectricity, 74

Pyrometer, 404

QuADRANT electrometer (Lord $\mathrm{Kel}$ vin's), 288

electroscope (Henley's), 17

Quadruplex telegraphy, 503

"Quantity" arrangement of cells, etc., 192,407

of electricity, unit of, 21, 262, 354

Quartz fibre, 299

Quartz, no residual charge from, 299

as insulator, 30, 299

Quetelet, E., on atmospheric electricity, 333, 335

Quincke, Georg, on diaphragm currents, 252

on electric expansion, 300

on electro-optic phenomena 525

Quinine, use of, for mapping fields, 299 


\section{The Numbers refer to the Numbered Paragraphs.}

RADIANT state of matter, 321

Radio-micrometer, 425

Rate of change of current, 454, 472 (footnote)

Ratio of electrostatic to electromagnet units, 283, 359

Ray, electric (torpedo), 76

Rays, kathodic, 321 current balance, 396

Rayleigh, Lord, determination of ohin, 358

Reactance, 473

Reciprocal accumulation, 49

Recording instruments, 160, 334

Redistribution of charge, 39

Reduction of metals, 494

Reflecting galvanometer, 215

Reflexion of electric waves, 520

Refractive index, 518

Registering magnetographs and electrometers, 160,334

Reis, Philipp, invention of teleplione, 510

Relays, 501

Reluctance, 375

Reluetivity, 375 (footnote)

Remanence, 367

Replenisher, 49, 287, 288

Repulsion and attraction of electrified bodies, $2,4,22,24,74$, 262

and attraction, experiments on, 47

and attraction of currents, $338,389,394$

and attraction of magnets, 84 , 88

Repulsion electrometers, 286

Residual charge of Leyden jar, 61, 299

$", \quad$ of cable, 301
of Voltaineter, 492

Resinous eleetricity, 5

Resistance and heat, 426

Resistance, 30, 171, 400, 426 affected by temperature, 404

$$
\begin{array}{ll}
,, & \text { light, 529 } \\
, & \text { magnetism, } 397
\end{array}
$$

as a velocity, 357

bridge or balance, 413

coils, 414

internal, of cell, 192, 407, 417

$$
,, \quad, \quad \text { measurement }
$$

laws of, 400
Resistance, magnetic, 375

measurement of, 411 et seq.

of gases, 171, 322

of glow lamps, 452

of liuman body, 255

of liquids, 171, 403

of vacuum, 321

specific, 403

to alternate currents, 476

units of, 352 et seq.

Resistivity, 402

Resonance, 517 .

Resonator, 520

Resultant magnetic force, 115

Retardation of currents throngh cables, 301, 323, 505

Retentivity (magnetic), 98, 367

Return shock or stroke, 29, 331

Reversal of influence machines, 53

Reversibility of processes in circuit, 248,436

Reversing-switch, 230, 498

Reymond, Du Bois, his galvanometer, 257 .

on animal electricity, 257

unpolarizable electrodes, 257

Rheostats, 400

Rheometer, )

Rheoscope, see footnote to $20 \mathrm{~s}$

Rheotrope,

Riess, Peter, on electric distribution, 38

on length of spark, 313

electric thermometer, 317

Ritchie, magnetic circuit, 375

his motor, 443

Ritter, Johann Wilhelm, on action of current on sight, 254

his secondary pile, 492

on subjective galvanic sounds, 254

on the sensitive plant, 256

Roentgen, Wilhelm Conrad, his rays, $327 a$

Rolling friction, 12, 73

Romagnosi, Dr., discovers magnetic action of current, 195

Romas, De, his electric kite, 329

Ronalds, Sir Francis, invented a telegraph, 497

Rotation of plane of polarization, 526

Rotations, electromagnetic, 393

Arago's, 457

Rotatory maynetic field, 485

Roughness of surface as depolarizer, 180

Rouland, Henry A., on electric convexion, 397 


\section{The Numbers refer to the Numbered Paragraphs.}

Rowland, Henry A., on magnetic circuit, 375

Rücker, Arthur William, on rationalization of dimensions, 360

Rücker and Thorpe, magnetic survey, 154

Ruhmkorffs electromagnet, 369 induction coil, 229 coil, mutual induction of, 454

St. Elmo's Fire, 329 (footnote)

Safety-fuses, 429

Salts, electrolysis of, 238,490

Sanderson, J. Burdon, on electric sensitiveness of carnivorous plants, 256

Saturation, magnetic, 112, 363 et seq.

Satery, 85

Sawdust battery, 187

Schallenberger's ineter, 442

Sehuckert, ammeter, 221

Schuster, Arthur, on electrolysis of gases, 322

Schveigger's multiplier, 200

Screening, magnetic, 96 inductive, 514 of eddy-currents, 457,514

Secohm, 458

secondary actions in electrolysis, 490

Secondary batteries, 492

Secular variations of magnetic elements, 155

Seebeck, Thomes Johann, effect, 419

Selenium, photo-electric properties of, 529

resistance of, 403 (table), 529

Self-exciting influence machine, 50 dynamo, 462

Self-induction, 455,472 in electric discharge, 515

Self-recording instruments, 160,334

Semaphore, Henley's, 17

Sensitive plant, behaviour of, 256

Series, arc lamps in, 450 capacities in, 308

cells in, 168,406

dynamos, 465

resistances in, 406

Serrin, Victor, his lamp, 440

Sharlows, electric, 47 in partial vacuum, 321

Sheet conductor, flow of electricity in, 410

Shell, magnetic, 118, 203, 350 potential due to, 348

Shielding, magnetic, 97
Shock, electric, 254, 325

Shunt, 215, 409 coil in are lamps, 449 dynamo, 465

Shuttle armature, 461

Siemens, Alexander, on length of spark, 313

Siemens, Werner, on dynamos, 461 mercury unit, $358^{\circ}$ electrod y namometer, 395 shuttle-wound armature, 461 heating in Leyden jar, 299

Sight affected by current, 254

Silurus, the, 76

Sine gal vanometer, 214

Sine law, 476

Single-fluid cells, 180

Single-needle instrument, 498

Single touch, 100

Siphon recorder, 506

Skew-symmetry of crystals, 75

Skin effect, 476

Skin, E.M.F. in the, 257

Smee, Alfred, his Battery, 180

Smith, Frederick John, effect on photographic plate, 324

Smith, Willoughby, on selenium, 529

Soap-bubble, electrified, 4

Sodium hy electrolysis, 490

Solenoid are lamps, 449

Solenoid, 385 magnetizing force of, 341

Solid angles, 148 (Appendix A)

Solidification, 69

Sound of magnetization, 124, 510

Sounder, the, 497

Sources of electricity, 12, 65

Spark, 11, 46, 47, 310 duration of, 323 lengtl of, 48, 313, 329

Sparking at commutator, 463

Specific resistance, 403 inductive capacity, 25,56 , 295,299

Speed of motor, 444 of signalling, 301, 302, 323

Sphere, distribution of charge over, 38,273 et seq. potential of, $269,2 \pi 1$ capacity of, 271

Spiral shortens itself, 390

Spottiswoode, William, on strix, 322

Square root of mean square, 471

Standard cells, 188 effect of temperature on, 194

Standards, 354

Steel hardening, 108 


\section{The Numbers refer to the Numbered Paragraphs.}

Steel, properties of, 362 facing, 495

Stewart, Balfour, on atmospheric electricity, 335

on magnetic storms, 158

Storms, magnetic, 159

Straight conductor force near, 207, 343

Strain, dielectric, 64, 299, 525

Strength of current, 171, 190, 354 of current in magnetic measure, 206, 207, 353 et seq.

of dielectric, 299,311

of magnet pole, 112, 352

of magnetic shell, 348

Stress, electric, 13, 16, 20, 22, 24, 63, $279,299,311,525$

electric, optical effect of, 525 magnetic, 119, 340, 389

Striæ in vacuum tubes, 320, 322

Sturgeon, William, his commutator, 461

electro-magnets, 381

on magnetic circuit, 375

induction coil, 229

Subinarine telegraphs, 504

Sucking-magnet, 385

Sulphur as depolarizer, 185

Sulphuretted hydrogen, iron negative to copper in, 80

Sulzer's experiment, 254

Supply meters, 442

Surface contact, 12

density of charge, 38,273

limit of, 273

of magnetism, 134, 337

Surgical applications, 258

Susceptibility, 365

Suspended-coil galvanometers, 216

Swammerdam's frog experiment, 255

Swan's incandescent lamp, 452

Symmer, on two kinds of electrification, 5

Synchronizing, 479

TAIT, PETER GUTHRIE, electrification by evaporation of sul. phate of copper solution, 71 heating of iron electrode, 433 thermo-electric diagram, 424

Tangent galvanometer, 212

Tapper, 498

of angle of lag, 473

Taste affected by current, 254

Telegraph, electric, 497

Bain's chemical, 246

Morse's instrument, 499
Telegraph, needle instrument, 498

Telegraphy, diplex, 503

duplex, 503

quadruplex, 503

submarine, 504

Telephone, Philipp Reis's, 510

currents of, 255

Dolbear's, 299, 511

Edison's (carbon), 511

Graham Bell's (articulating), 510

Varley's (condenser), 299, 511

Exchanges, 513

Temperature affects resistance, 194, 404

affected by resistance, 426

effect on length of spark, 313 , 314

of the arc, 448

Tempering of steel, 108

'Tension, electric, 13, 16, 20, 22, 24, 63,273 (footnote), 279, 299, 311, 525

Terquem, A., parrot-cage experiment, 34

Terrestrial Magnetism, 95, 150, 361, 365

Test for weak currents (chemical), 246,316

for weak currents (physiological), 255

Testing for faults, 502

Tetanization produced by interrupted currents, 256

Theories of Electricity, 7, 327, and preface, ix

Theories of Magnetism, 99, 126

$\begin{array}{ll}\text {," Ampère's, } 398 \\ " & \text { Ewing's, } 127 \\ \text { ", } & \text { Waxwell's, } 126 \\ \text { Weber's, 126, } 127\end{array}$

'Theory of Electrolysis, Grotthuss's and Clausius's, 491

Theory of Earth's magnetism, 161 of Light, 518

$\left.\begin{array}{l}\text { Thermo-electric currents, } \\ \text { Thermo-electricity, }\end{array}\right\}$

Thermo-electric Diagram, 424

Thermo-electromotive Series, 424

Thermopile, 425

Thompson, Silvanus Phillips, on magnetic figures due to currents, 202,389

on positive and negative states, 327

on opacity of tourmaline, 518

Thomson, Joseph J., on Contact Electricity, 81 
The Numbers refer to the Numbered Paragraphs.

Thomson, Joseph J., on conductivity of gases, 322

Thomson, Sir William (see Kelvin, Lord) effect, 424

Thomson, Elihu, his meter, 442 on alternate-current magnets, 477

on welding, 433

Thonsson-Houston dynamos, 468, 478

Thorpe and Rücker, magnetic survey, 154

Three-wire system, 453

Thunder, 11, 331

Thunderstorms, 329

Theory of, 330

Time-constant, 460

Tinfoil Condensers, 55, 302

Tivoli, transmission of power from, 447

Toepler, A., his Influence Machine, 51

Tongs, Discharging, 59

Torpedo (electric fish), 76,246

Torpedoes, fuzes for firing, 316, 432

Torque, 136

Torque of motor, 444

Torsion affected by magnetization, 124

Torsion Balance, or ? Coulomb's,

Torsion Electrometer, $\} \quad 18,132$

Torsion method, 209, 210

Tourmaline, 74, 324, 518

Transformers, 228, 480 for vacuum tubes, 320

Transmission of power, 447, 479

Tri-phase, 485

Trolley wheel for tramcars, 446

Trowbridge, on magnetization at $-100^{\circ}$

C., 111

Tube of force, $337(\mathrm{~g})$

Tuning-fork method, 418

Two-fluid cells, 181 theory, 7

Two kinds of Electrification, 5, 6

Magnetic poles, 89

Tyndall, John, diamagnetic polarity, 372 niagne-crystallic action, 373

ULTRA-GaseOUS MATTER, 321

Ultra-violet waves, 313

discharge by, 531

effect on metal, 531

Unit, Board of Trade, 440, 488

Unit jar, 285
Units and standards, Board of Trade (see Appendix B)

electromagnetic, 352 et seq.

electrostatic, 283 et seq.

fundamental and derived, 281, 282

ratio of electrostatic to electromagnetic, 262 (footnote), 283,359

Unipolar Machines, 469

Universal Discharger, 62

Unvarying magnets, 110

Upward, his cell, 193

Ure, $D r$., on animal electricity, 255

"v," 359, 518

Vacuum, induction takes place through, 64, 96, 97

partial, spark in, 11, 320

spark will not pass through, 313,321

tubes, 320,321

"Variation," the (see Declination)

Variation of Declination and Dip,

annual, 157

diurnal, 156

geographical, 151, 154

secular, 155

of electritication of the atmo. sphere, 335

Varley, Cromwell Fleetwood, his galvanometer, 316

on capacity of polarization, 492

telegraph, 497

Varley, Samuel Alfred, his telephone, 299,510

early dynamo, 462

Vegetables, Electricity of, it

carnivorous, sensitiveness of, 256

Velocity of discharge, 323

of light, 359,518

of electric waves, 518

of rubbing, electrification depends on, 73

resistance as a, 357

Verdet's constant, 526

Vibration produces Electrification, $6 \bar{\imath}$

Vibrator for measuring capacity, 418

Villari, Emilio, effect of tension, 364

Violet waves (see Cltra-violet)

Virtual volts and amperes, 471

Vitreous electricity, 5

Volt, 169, 354 


\section{The Numbers refer to the Numbered Paragraphs.}

Volta, Alessandro, his Electrophorus, 26

Condensing Electroscope, 79

Contact Series, 80

Crown of Cups, 165

on Atmospheric Electricity, 334

on Contact Electricity, 79, 163

on Electric Expansion, 300

on Electrification due to combustion, 70

Subjective Sounds due to Current, 254

Volta's Law, 80, 163, 170

Voltaic Pile, 164

Voltaic Electricity (see Current Electricity)

are, 448

battery, 168,178 ; pile, 164

cell, simple, 166

Voltameter, 242, 243, 244, 4S7

Voltmeter, 220

Carde'w's, 430

electrostatic, 290

Voss machine, 51

WALKER, CHARLES V., used sulphur in cell, 185

Warburg, E., on hysteresis, 368

Water, Electrolysis of, 235,487

Water-dropping, discharge by, 334

Watt, the, 354, 435

Wattmeter, 438

Watts, true and apparent, 475

Waves, electric, 515

Weber, the, 354

Weber, Wilhelm, the Electro-dynamorneter, 394

on diamagnetic polarity, 372

evaluation of ohm, 358 of " $v$, , 359

theory of magnetisin, 126, 127
Welding, 433

Weston, Edward, voltmeter, 220 standard cell, 188

temperature coefficient of alloys, 404

Wheatstone, Sir Charles, on the brush discharge, 319

Automatic Telegraph, 497

Dynamo-electric Machines, 462

on supposed velocity of electricity, 323

Wheatstone's Bridge or Balance, 413

Whirls, magnetic, 202, 389

Wiedemann, Gustav, on effect of magnetism on torsion, 124

dianlagnetism. 370 (footnote)

Wilde, Henry, Dynamo-electric

Machine, 462

Wilcke, A., electrophorus, 26 (footnote)

Wimshurst, James, Influence machine, 52

Wind, electric, 47, 324

Winding of electromagnets, 375 et seq., 385,386 (and see page 5S9)

Window, aluminium, 321

Wöler's cell, 193

Wollaston's Battery, 180

Work by conductor cutting lines, 339

Wroblewski, resistance of, at low temperatures, 404

ZA MBovi's Dry Pile, 16, 193, 291

Zanotti, experiment on grasshopper, 255

Zero Potential, 40, 264

Zero of temperature, resistance near, 404

Zinc as fuel, 166

\section{THE END}

Printed by R. \& R. CLARK, LIMITED, Edinburgh. 


\section{MACMILLAN \& CO.'S SCIENCE CLASS BOOKS.}

\section{Adapted to the South Kensington Syllabuses.}

Practical Plane and Solid Geometry for Elementary Students. By J. HarRison, M.I.M.E., etc. New Edition, 1903, revised. 2s. 6d.

Practical Plane and Solid Geometry By J. Harrison, M.Inst.M.E., etc., and G. A. Baxandall. Part 1. Elementary, 25. 6d. Part II. Advanced, 4s. 6d.

Geometrical Drawing and Design. By J. Humphrey Spanton. 2s. 6d.

Practical Exercises in Geometry. By W. D. Eggar, M.A. 2s. 6d.

Test Papers in Practical Plane and Solid Geometry. Elementary Stage. By George Grace, B.Sc. (Lond.). 24 Tests printed on Cartridge Paper. 6d.

Machine Construction for Beginners. By F. CAstLe, M.I.M.E. [In preparation.

Building Construction for Beginners. By J. W. RiLey. 2s. 6d.

Bullding Construction for Advanced Students. By J. W. RILEY. [In preparation.

An Elementary Course of Mathematics. Comprising Arithmetic, Algebra, and Euclid. By H. S. Hall, M.d., and F. H. Stevens, M.A. 2s. 6d.

Graduated Test Papers in Elementary Mathematics.. By the Rev. WALTER J. Wood, B.A. is.

Elementary Practical Mathematics. By F. Castle, M.I.M.E. 3s, 6d.

Practical Mathematics for Beginners. Adapted to the new Elementary South Kensington Syllabus. By F. CASTLE, M.I.M.E. 2S. 6 d.

Manual of Practical Mathematics. By F. CAstle. 6s.

Mathematical Tables. By F. CAstle. Sewed. 2 d.

Elementary Mechanics of Solids. By W. T. A. EmTAGE, M.A., Director of Public Instruction in Mauritius. 2s. $6 \mathrm{~d}$.

Meohanics for Beginners. By W. Gallatly, B.A. 2s. 6 d.

Mechanics for Beginners. By Rev. J. B. Lock, M.A. Part I. Mechanics of Solids. 2s. $6 \mathrm{~d}$.

Hydrostatics for Beginners. By F. W. Sanderson, M.A. 2s. 6d.

Lessons in Applied Mechanics. By Prof. J. H. Cotterill, F.R.S., and J. H.

Slade. 5s. 6d.

Applied Mechanics for Beginners. By J. Duncan, Wh. Ex., A.M.I.M.E. 2s. 6d. Elementary Lessons in Heat, Light, and Sound. By D. E. Jones, B.Sc. 2s. 6d.

Lessons in Heat and Light. By D. E. Jones, B.Sc. 3s. 6d.

Practical Exercises in Heat. A Laboratory Course for Schools of Science and

Colleges. By E. S. A. Robson, M.Sc. (Vict.). 2s, 6d.

Practical Exercises in Light. By R. S. CLAY, D.Sc. 2s. 6d.

Heat for Advanced Students. By E. Edser, A.R.C.Sc. 4s. 6d.

Light for Advanced Students. By E. Edser, A.R.C.Sc. 6s.

Elementary Physics. By Balfour Stewart, F.R.S. New Edition, thoroughly revised. 4s. 6ul. Questions, 2S.

Electricity and Magnetism for Beginners. By F. W. SANDERSon, M.A. 2s. 6d. Magnetism and Electricity for Beginners. By H. E. HAdLEy, B.Sc. (Lond.). 2s. $6 \mathrm{~d}$.

Practical Exercises in Magnetism and Electricity. Being a Laboratory Course for Schools of Science. By H. E. HADLEY, B.Sc. 2s. $6 \mathrm{~d}$.

Elementary Lessons in Electricity and Magnetism. By Prof. Silvanus P. ThомpSon, F.R.S. New Edition. 4s. 6d.

Introductory Chemistry for Intermediate Schools. By Lionel M. Jones, B.Sc. $2 s$.

Chemistry for Beginners. By Sir Henkv Roscoe, F.R.S., assisted by J. LunT,

B.Sc. New Edition, Revised. 2s. 6d.

MACMILLAN AND CO., LTD., LONDON. 


\section{MACMILLAN \& CO.'S SCIENCE CLASS BOOKS.}

\section{Adapted to the South Kensington Syllabuses.}

The Elements of Chemistry. By Prof. Ira Remsen. 2s. 6d.

Inorganic Chemistry for Advanced Students. By Sir H. E. Roscoe, F.R.S., and Dr. A. HARDEN. 4s. 6d.

Chemical Problems. By Prof. T. E. Thorpe, F.R.S. With Key. $2 s$.

Chemical Arithmetic. By S. Lupton, M.A. With 1200 Problems. 4s. 6 d.

Inorganic Chemistry. By Prof. IRA Remsen. 6s. 6d.

Chemistry for Organised Schools of Science. By S. Parrish, B.Sc., A.R.C.S

(Lond.), with Introduction by Dr. ForsyTH. 2s. 6d.

Practical Inorganic Chemistry. By G. S. Turpin, M.A., D.Sc. 2s. 6d.

Practical Inorganic Chemistry for Advanced Students. By Chapman Jonzs. F.I.C., F.C.S. 2s. 6 d.

The Junior Course of Practical Chemistry. By F. Jones, F.C.S. 2s. 6d.

Organic Chemistry for Beginners. By G. S. Turpin, M.A., D.Sc. 2s. 6d.

Organic Chemistry. By Prof. Ira Remsen. 6s. 6d.

Practical Organic Chemistry for Advanced Students. By J. B. Coнen, Ph.D. 3s. $6 \mathrm{~d}$.

Theoretical Organic Chemistry. By J. B. Cонеn, Ph. D. 6s.

Geology for Beginners. By W. W. Watrs, M.A., F.G.S. 2s. 6d.

Physiology for Beginners. By Sir Michael Foster, K.C.B., and Dr. L. E. Shore. 25. $6 \mathrm{~d}$.

Lessons in Elementary Physiology. By the Right Hon. T. H. HuxLey, F.R.S. 45. 6d. Questions, 1s. 6d.

An Introduction to Nature-Study. By ERnest Stenhouse, B.Sc. 3s. 6d.

A Manual of Zoology. By the late T. Jefrery PARker, D.Sc., F.R.S., and

W. A. Haswell, M.A., D.Sc., F.R.S. Illustrated. ros. 6 d.

Botany for Beginners. By Eknest Evans, Burnley Technical School. 2s. 6d.

Coal Mining for Beginners. By Morgan W. Davies, M.I.M.E. [In preparation.

A Text-Book of Elementary Metallurgy. By A. H. Hionns. 3s. Questions, rs. Steam, Gas, and Oll Engines. By JoHn Perry, F.R.S. 7s. 6d. net.

Experimental Science : Physiography (Section I.). By Prof. R. A. Gregory and

A. T. Simmons, B.Sc. 2s. 6 d.

Physiography for Beginners. By A. T. Simmons, B.Sc. 2s. 6d.

Physiography for Advanced Students. By A. T. Simmons, B.Sc. 4S. 6d.

Elementary Lessons in Astronomy. By Sir Norman Lockyer, K.C.B., F.R.S.

New Edition. 5s. 6d.

Elementary Lessons in the Science of Agricultural Practice. By H. TANner,

F.C.S. 3s. 6d.

Hygiene for Beginners. By E. S. Reynolds, M.D. 2s. 6d.

Science of Common Life (Experimental Hygiene). By A. T. Simmons, B.Sc., and

E. Stenhouse, B.Sc. 2s. 6d.

Hyglene for Students. By E. F. Willoughby, M.B. Adapted to the Advanced

Stage of the Scuth Kensington Syllabus. New and Revised Edition. 4S. 6d.

$$
\text { MACMILLAN AND CO., LTD., LONDON. }
$$






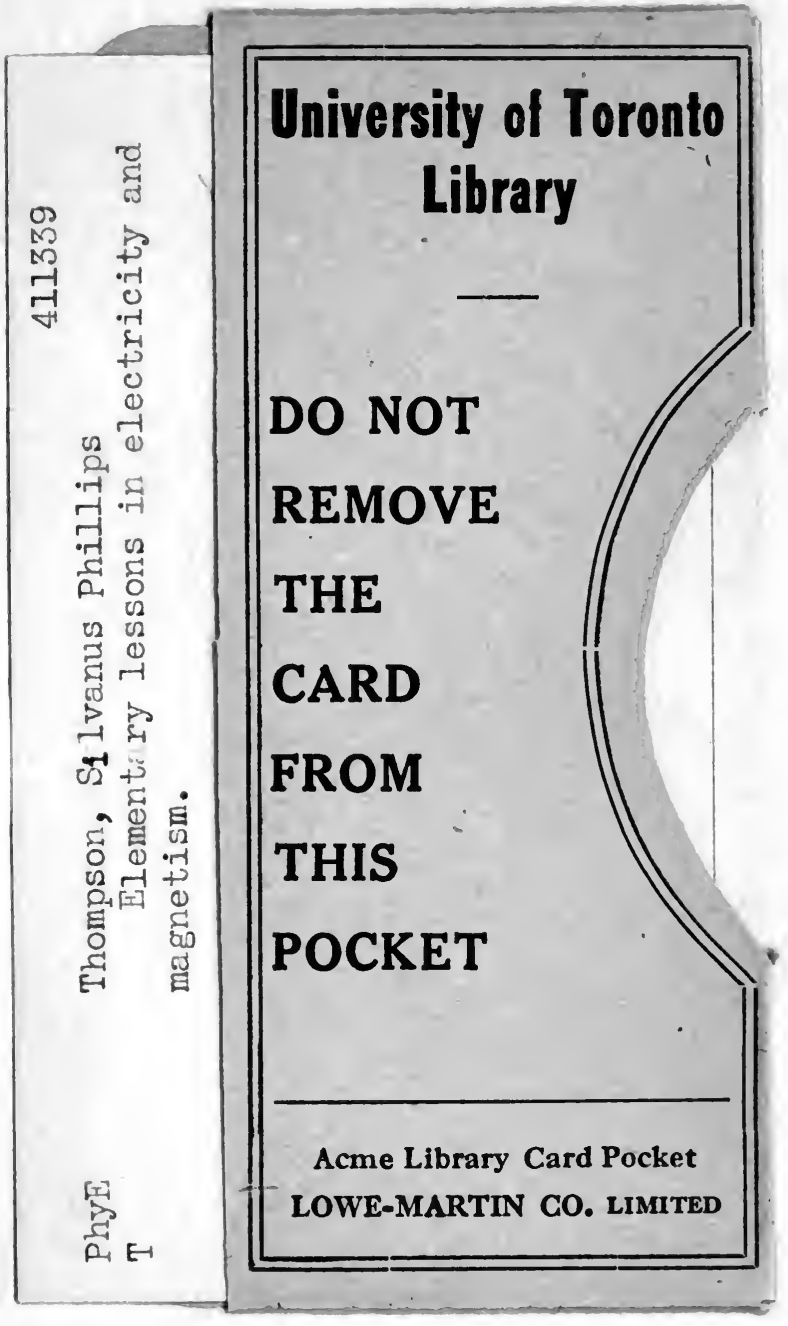


